
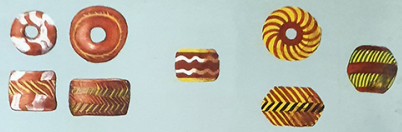

\title{
THE GLASS BEADS OF ANGLO-SAXON ENGLAND c. $\mathrm{AD} 400-700$
}

(9) (2)

MARGARET GUIDO

Edited by Martin Welch

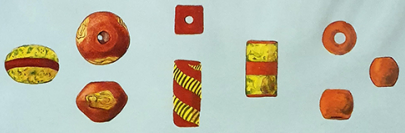


Reports of the Research Committee of the Society of Antiquaries of London, No. 58

$$
\begin{gathered}
\text { The Glass Beads of } \\
\text { Anglo-Saxon England } \\
\text { c.AD } 400-700
\end{gathered}
$$




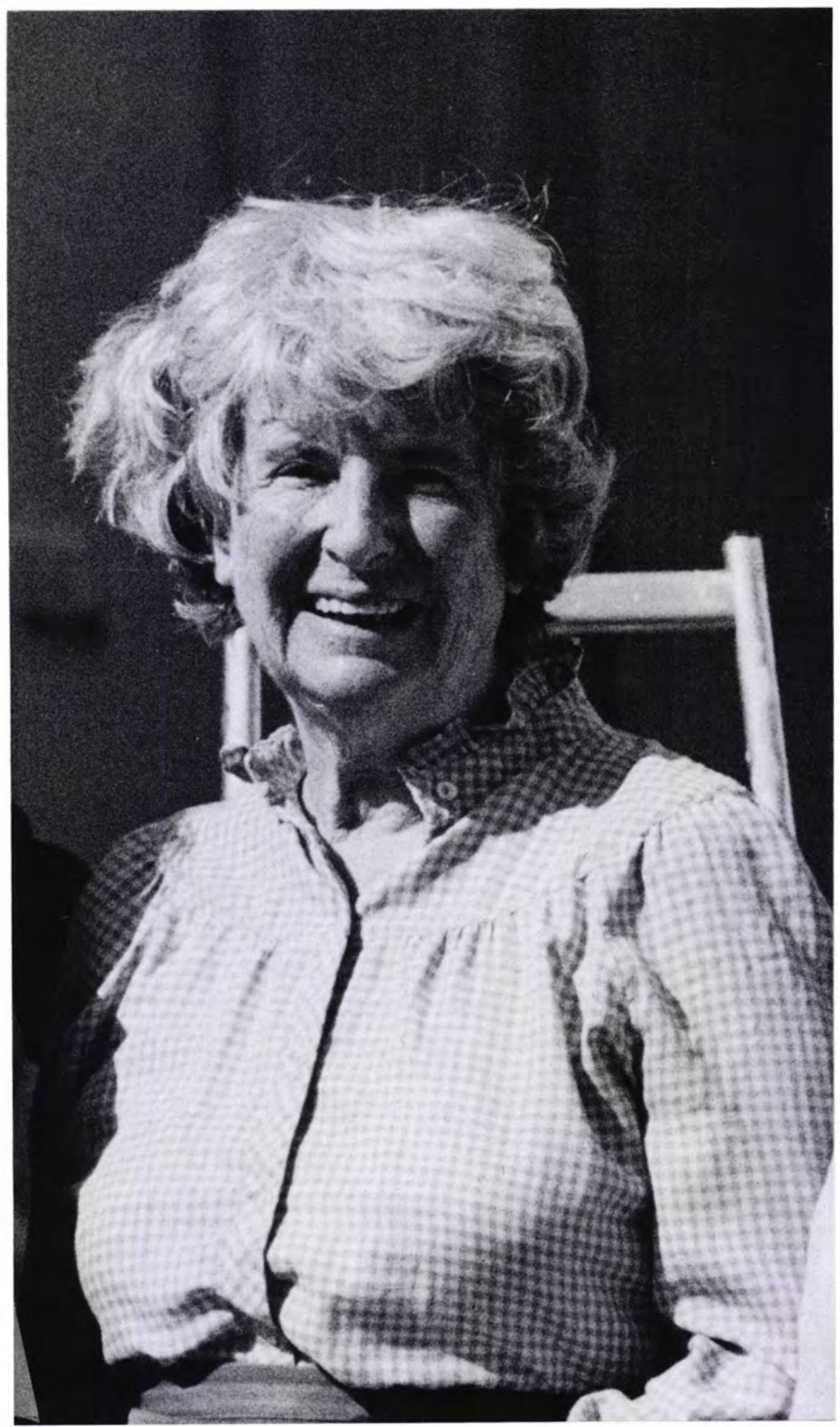

Cecily Margaret Guido, 1912-1994 (photograph by John Smith) 


\title{
The Glass Beads of ANGLO-SAXON ENGLAND c.AD $400-700$
}

\author{
A PRELIMINARY VISUAL CLASSIFICATION OF \\ THE MORE DEFINITIVE AND DIAGNOSTIC TYPES
}

\section{Margaret Guido}

Edited by Martin Welch

With contributions by

Justine Bayley, Julian Henderson and Martin Welch

The Boydell Press

for

The Society of Antiquaries of London 


\title{
This book is published with the generous assistance of the Trustees of Cecily Margaret Guido's Estate
}

\author{
First published 1999 \\ by \\ The Boydell Press \\ an imprint of Boydell \& Brewer Ltd \\ PO Box 9, Woodbridge, Suffolk IPI 2 3DF, UK \\ and at PO Box 41026, Rochester NY 14604-4126, USA \\ website: http://www.boydell.co.uk \\ for \\ The Society of Antiquaries of London \\ (3) The Society of Antiquaries of London 1999 \\ All Rights Reserved. Except as permitted under current \\ legislation, no part of this work may be photocopied, \\ stored in a retrieval system, published, performed in \\ public, adapted, broadcast, transmitted, recorded or \\ reproduced in any form or by any means, without the \\ prior permission of the copyright owner.
}

ISBN 0851157181

ISSN 0953-7163

A catalogue record for this book is available from the British Library

Library of Congress Cataloging-in-Publication Data

Guido, Margaret.

The glass beads of Anglo-Saxon England c. AD 400-700 : a preliminary visual classification of the more definitive and diagnostic types / Margaret Guido ; edited by Martin Welch ; with contributions by Justine Bayley, Julian Henderson, and Martin Welch.

p. cm. - (Reports of the Research Committee of the Society

of Antiquaries, ISSN $0953-7163 ; 56$ )

Includes bibliographical references.

ISBN 0-85115-718-1 (hardback : acid-free paper)

1. Glass beads, Anglo-Saxon - Expertising. I. Welch, Martin G.

II. Title. III. Series: Reports of the Research Committee of the

Society of Antiquaries of London; no. 56.

NK5440.B34G85 1999

$748.8^{\prime} 5^{\prime} 094209015-\mathrm{dc} 21$

$98-56389$

This publication is printed on acid-free paper

Printed in Great Britain by

St Edmundsbury Press Ltd, Bury St Edmunds, Suffolk 


\section{Contents}

List of Figures vii

List of Plates vii

List of Maps vii

Preface ix

Acknowledgements $\quad$ xi

PART I Descriptive ANALYsis

Introduction: Glass beads in early Anglo-Saxon contexts $\quad 1$

by Martin Welch

Chapter 1 Colourless and pale translucent glass beads $\quad 11$

$\begin{array}{lll}\text { Chapter } 2 & \text { 'Black' glass beads } & 17\end{array}$

$\begin{array}{lll}\text { Chapter } 3 \text { White (or blue-white) glass beads } & 30\end{array}$

Chapter 4 Opaque yellow glass beads 36

Chapter 5 Green and turquoise glass beads $\quad 41$

$\begin{array}{lll}\text { Chapter } 6 & \text { Blue glass beads } & 47\end{array}$

Chapter 7 Crimson or pink glass beads $\quad 55$

$\begin{array}{llll}\text { Chapter } & 8 & \text { Terracotta or brick-red glass beads } & 58\end{array}$

$\begin{array}{lll}\text { Chapter } 9 & \text { Opaque orange glass beads } & 68\end{array}$

$\begin{array}{lll}\text { Chapter } 10 & \text { Millefiori and mosaic glass beads } & 70\end{array}$

Chapter 11 Annular glass beads with coloured surface spirals $\quad 74$

$\begin{array}{lll}\text { Chapter } 12 & \text { Annular glass beads with fine twist decoration } & 76\end{array}$

$\begin{array}{lll}\text { Chapter } 13 & \text { Gold and silver-in-glass beads } & 78\end{array}$

Chapter 14 Technological aspects of Anglo-Saxon glass beads $\quad 81$

by Julian Henderson, with an Appendix by Justine Bayley

Conclusion: A future for Anglo-Saxon glass bead studies? 94

by Martin Welch

Part II The schedules

Introduction $\quad 131$

Topographical index $\quad 136$

$\begin{array}{lll}\text { Schedule } 1 \text { Colourless and pale translucent glass beads } & 153\end{array}$

$\begin{array}{lll}\text { Schedule } 2 \text { 'Black' glass beads } & 170\end{array}$

$\begin{array}{lll}\text { Schedule } 3 & \text { White (or blue-white) glass beads } & 191\end{array}$

$\begin{array}{lll}\text { Schedule } 4 \text { Opaque yellow glass beads } & 211\end{array}$

$\begin{array}{lll}\text { Schedule } 5 & \text { Green and turquoise glass beads } & 230\end{array}$

$\begin{array}{lll}\text { Schedule } 6 & \text { Blue glass beads } & 255\end{array}$ 
Schedule 7 Crimson or pink glass beads

$\begin{array}{llll}\text { Schedule } & 8 & \text { Terracotta or brick-red glass beads } & 283\end{array}$

Schedule 9 Opaque orange glass beads 328

Schedule 10 Millefiori and mosaic glass beads $\quad 330$

Schedule 11 Annular glass beads with coloured surface spirals 332

Schedule 12 Annular glass beads with fine twist decoration 338

$\begin{array}{ll}\text { Schedule } 13 \text { Gold and silver-in-glass beads } & 340\end{array}$

$\begin{array}{ll}\text { Bibliography } & 345\end{array}$ 


\section{Illustrations}

\section{FrontisPiece}

Cecily Margaret Guido

\section{Figures}

1 Anglo-Saxon glass beads: main diagnostic shapes 13

2 Anglo-Saxon glass beads: main diagnostic decorative motifs 15

Plates

between pages 241 and 242

1 Colourless and pale translucent glass beads (Schedule 1) and 'black' glass beads (Schedule 2)

2 'Black' glass beads (Schedule 2)

3 'Black' glass beads (Schedule 2) and white (or blue-white) glass beads (Schedule 3)

4 Opaque yellow glass beads (Schedule 4) and green and turquoise glass beads (Schedule 5)

5 Blue glass beads (Schedule 6)

6 Crimson or pink glass beads (Schedule 7) and terracotta or brick-red glass beads (Schedule 8)

7 Terracotta or brick-red glass beads (Schedule 8) and opaque orange glass beads (Schedule 9)

8 Millefiori and mosaic glass beads (Schedule 10), annular glass beads with coloured surface spirals (Schedule 11), annular glass beads with fine twist decoration (Schedule 12) and gold and silver-in-glass beads (Schedule 13)

\section{MAPS}

1 Colourless translucent glass beads (Schedule 1) 97

2 'Black' melon, sub-melon or lobed beads (Schedule 2) 98

3 'Black' annular beads with broken white girth band (Schedule 2) 99

4 'Black' annular beads: (a) with white or yellow wave; (b) with blue wave 100 (Schedule 2)

5 'Black' annular beads: (a) with white zigzags; (b) with yellow or green 101 zigzags; (c) with red zigzags (Schedule 2)

6 Large 'black' decorated beads: (a) plano-convex (Schedule 2) 102

7 Large 'black' decorated beads: (b) biconvex (Schedule 2) 103 
8 'Black' globular beads with complex crossed waves and eyes or spots (Schedule 2)

9 'Black' globular beads with coloured specks ('crumb' beads) (Schedule 2) 105

10 Opaque white short cylinder beads, single or double (Schedule 3) 106

11 Opaque white beads with light blue or turquoise crossed waves, without 107 dots, single or double (Schedule 3)

12 Opaque white beads with light blue crossed waves and blue dots (Schedule 3)

13 Opaque white beads with light blue crossed waves and red dots (Schedule 3)

14 Opaque white beads with green or terracotta waves, with or without dots (Schedule 3)

15 Opaque yellow annular, globular and barrel-shaped beads (Schedule 4)

16 Opaque yellow beads with coloured crossed waves, with or without dots, single or double (Schedule 4)

17 Green segmented or wound beads (Schedule 5)

18 Blue melon, sub-melon and lobed beads (Schedule 6) 114

19 Blue beads with white and coloured specks (Schedule 6) 115

20 Blue beads with red spots and with white or yellow spots (Schedule 6) 116

21 Pink, crimson or purple beads (Schedule 7) 117

22 Terracotta melon and sub-melon beads (Schedule 8) 118

23 Terracotta cylinder beads with coloured combing, including green and $\quad 119$ yellow (Schedule 8)

24 Terracotta cylinder beads with coloured combing, including green and $\quad 120$ yellow (Schedule 8)

25 Terracotta, mainly bun-shaped, beads with twisted (reticella) and related decoration, excluding green and yellow (Schedule 8)

26 Terracotta, mainly bun-shaped, beads with green and yellow twisted bands (Schedule 8)

27 Terracotta, mainly bun-shaped, beads with green and yellow marbling (Schedule 8)

28 Terracotta cylinder beads, round, square or polygonal, with green and yellow bands and green and yellow marbling (Schedule 8)

29 Opaque orange beads (Schedule 9)

30 Millefioribeads (Schedule 10) 126

31 Beads with coloured surface spirals: (a) white annular beads with light blue 127 surface spirals and (b) with other colour surface spirals (Schedule 11)

32 Annular beads with fine twist decoration (Schedule 12) 


\section{Preface}

Cecily Margaret (Peggy) Guido (1912-1994) developed a childhood interest in Roman coins, which led to an active involvement in archaeology in the 1930s. She participated in excavations directed by Mortimer and Tessa Wheeler and obtained a Diploma in Prehistory at University College London. During her marriage to Stuart Piggott, she developed further her skills as a field archaeologist, proving to be a resourceful and dedicated director of rescue excavations in the difficult conditions of wartime and immediately post-war Britain. Her husband's appointment to Edinburgh University led her to excavate in Scotland, adding to her earlier experience in southern England. A subsequent marriage to a Sicilian academic, Luigi Guido, saw her interests move to the Mediterranean region and her publication of a number of valuable guidebooks to southern Italy and Sicily, as well as a volume on Sardinia, which was commissioned for the celebrated Ancient Peoples and Places series.

Her return to live permanently in southern England saw a new phase in her archaeological career, stemming from her realization that glass jewellery, and especially beads, provided an unjustly neglected field of study. Her pioneering study of British and Irish glass beads in the prehistoric and Roman periods (Guido 1978) acknowledged the encouragement she had received from Donald Harden and George Boon among others, and demonstrated an awareness that glass beads had the potential to reveal much about those who wore them as display items, while the technology of their manufacture and the identification of evidence for their production were equally important aspects, which could be linked to issues of their trade and exchange. Nor did she neglect to keep abreast with the latest developments in scientific analysis of ancient glass and glassmaking. She set about the task of systematically collecting data on British and Irish glass beads on to a card index from publications, museum collections and information from field archaeologists. At the same time, she played an active role in the establishment of the Bead Study Trust, which included assisting in the cataloguing and reordering of the Beck collection of beads at the Cambridge University Museum.

Initially, her collection of records on the glass beads of the Anglo-Saxon period had the modest aim of assisting her in differentiating these from beads of the preceding periods. She hoped to be able to pick out prehistoric and Roman beads when they occurred as unstratified or redeposited stray finds on a multi-period site. Over time, however, she had gathered so much information that it seemed wasteful not to go further and produce the present and sadly posthumous volume. She felt a need to familiarize herself with the bead types used in the late Roman and early medieval periods across northern Europe and undertook museum study tours for that purpose. Despite the fact that she was undertaking pioneering work here, characteristically she always felt diffident in the presence of specialists on Anglo-Saxon 
cemeteries. This might seem strange when one considers her contribution as an excavator of such sites, for example, her role in the élite team which defined, recorded and lifted the Sutton Hoo ship burial grave deposit in 1939 and in another such team which rescued the first thirty-one graves from the well-known cemetery at Petersfinger near Salisbury in 1948 (Leeds and Shortt 1953, preface). Nevertheless, growing recognition of her expertise led many field archaeologists to make their recently excavated glass beads available to her and over the years she contributed quite a number of specialist reports on Anglo-Saxon beads. While it is sad that she did not live to see the present study in print, she had at least read and approved the typescript of her main text (Chapters 1 to 13) and the Introduction before her final illness. We can only hope that she would have been pleased with the final result.

Martin Welch 


\section{Acknowledgements}

This book has been long in gestation and preparation and many individuals have contributed to its completion and publication. Cecily Margaret Guido would have offered her gratitude to the British Academy for financial support, to the many museum curators and excavators in this country and across Europe who assisted her collection of data, to other scholars who shared their specialist knowledge with her and to her many friends in Devizes, whose effective support was so important to her. Mrs E L Machin in particular deserves personal mention as friend, confidante and German language translator.

Turning Margaret Guido's manuscript into a book was largely the task of Martin Welch, as academic editor, and he would like firstly to acknowledge the important scientific contribution of Julian Henderson and Justine Bayley, whose work appears in Chapter 14. There is also the assistance of Dido Clark, who helped check many references for schedule entries and augmented the author's entries in the schedules against the records for the then unpublished sites of Lechlade (Gloucestershire) and Mucking (Essex). Kikar Singh and Nicholas Griffiths were responsible for most of the line and colour drawings, while Kikar Singh prepared the final artwork for publication. Martin Welch would particularly like to thank the Trustees of Cecily Margaret Guido's Estate for their generosity in funding the cost of preparing the distribution maps, produced in the Drawing Office of the Department of Geography, University College London, and for covering the cost of providing additional line and colour drawings for the production of the colour plates. Finally, Sue Hirst generously acted as an academic referee and provided additional assistance in advising the academic editor as well as organizing and supervising additional illustrations and final paste-up, while the late Janet Clayton and subsequently Kate Owen as Managing Editors for the Society of Antiquaries of London must be thanked for their patient support and professional judgement in seeing this book through the press. Any remaining errors and infelicities are the sole responsibility of Martin Welch.

It is surely appropriate that this book be dedicated to the memory of its author, Cecily Margaret Guido, a kind and generous scholar, whose contribution to British and European archaeology, and, in particular, to the study of glass beads, has yet to receive its full due. 



\section{PART I \\ Descriptive ANalysis}





\title{
INTRODUCTION
}

\section{GLASS BEADS IN EARLY ANGLO-SAXON CONTEXTS}

\author{
Martin Welch
}

The migration of Angles, Saxons, Jutes and other Germanic peoples from the northern Netherlands, north-west Germany and southern Scandinavia to settle in eastern and southern Britain began the process of creating England from postRoman Britain in the fifth and sixth centuries. This involved a major change in the material culture of lowland Britain as well as alterations to its political, linguistic and social structure. This process is evidenced by much more than just simple linkages between artefacts excavated in England and those found in the continental AngloSaxon homelands. It is also marked by the arrival of new burial practices associated with those artefacts and the first appearance of a distinctive continental building type, the Grubenhaus. Abandoned and depopulated regions within the continental homelands, as revealed by fieldwork in Angeln near Schleswig (Müller-Wille et al 1988), match the foundation of new cemeteries and settlements in lowland Britain (Welch 1992). Study of the furnished inhumations and pottery cremation urns of Anglo-Saxon burial grounds has developed over the last two centuries since the pioneering excavations of Bryan Faussett and James Douglas in the second half of the eighteenth century (Hawkes 1990a).

The past three decades of the present century have seen a fuller appreciation of the benefits of giving equal value to all the components of Anglo-Saxon burial assemblages when studying them. Similar attention is also now being paid to the range of artefacts recoverable from settlement contexts. Beads commonly occur in relatively large numbers in cemeteries and great care is now routinely accorded to recording their relative positions within a grave in three dimensions. The aim is to reconstitute as precisely as possible the sequence in which the beads were strung. Inhumation burials of fully dressed individuals have revealed a variety of positions in which strings of beads were worn and preserved and mineralized textile impressions on brooches and other metal items can provide evidence for the attachment of strings to dress fittings. Festoons of beads strung between pairs of brooches that 
fastened a peplos costume (or tubular dress) at the shoulders over the collar bones are particularly common in the better-equipped costumes of the fifth and sixth centuries (Owen-Crocker 1986), but they might also have been worn as true necklaces placed over the head and around the neck. Strings of beads have also been recorded around the lower arms, functioning as bracelets, or near the pelvis and beside or between the upper legs as if suspended from a waist belt, to mention some of the most common alternatives.

The majority of Anglo-Saxon female graves contain beads, both those which include a brooch or brooches and those which lack such metal dress fittings. Yet, although a typical feature of female costume, finds of one or two beads can occur in male assemblages. Larger than average beads in adult male graves are often associated with that most prestigious of weapons, the two-edged iron sword or spatha: they are, therefore, known as sword-beads (Evison 1967 and 1975; Menghin 1983). Beads can also be recovered from cremation urns, but are usually badly distorted by the heat of the pyre and sometimes fused to the bone (Richards 1987, 81, 199-200; McKinley 1994), as the corpse seems to have been laid out fully dressed.

Anglo-Saxon beads were not necessarily made of glass, for a variety of other materials were available, above all amber, which was particularly popular in the sixth century. Indeed, amber beads occur in such quantities that it seems probable that the bulk of this amber was imported from the Baltic region (Huggett 1988, 64-6, fig 1). Rock crystal, probably imported from the Rhineland, occurs in burials of the fifth and sixth centuries (Huggett 1988, 70, fig 4), while amethyst, quite possibly traded from India via the Mediterranean (Meaney 1981, 76), has come to be regarded as a type-fossil indicating seventh-century burial (Huggett 1988, 66-8, fig 2). Beads of jet or related materials are relatively rare and seem likely in most cases to represent reused Roman beads, while other natural materials such as bone, shell and fossils could be turned into beads. Metal fittings also occur on relatively early bead strings as beads, wire rings, pendants or end-stops, but metal beads are a more typical feature of seventh-century bead strings (Hyslop 1963, 191, 198-200). Still, even those sixth-century bead strings dominated by amber beads usually contain at least a few glass beads, and the study of glass-bead shapes, together with the colour range present on both translucent and opaque monochrome glass beads, and the decoration on polychrome opaque glass beads, provides us with material that can be ordered and analysed.

To date, the study of Anglo-Saxon glass beads from contexts between the fifth and early eighth centuries has appeared principally in the form of discussion of the beads from individual cemeteries within published cemetery reports. Alison Cook's report on the beads from the Portway, Andover (Hampshire), site (Cook and Dacre 1985), Sue Hirst's on the Sewerby (Humberside/Yorkshire) beads, with contributions by Leo Biek and other scientists at the Ancient Monument Laboratories on the results of chemical analyses (Hirst 1985), and Vera Evison on the beads from the Buckland, Dover (Kent), cemetery (Evison 1987) are three excellent recent 
examples. Such publications have made an important contribution over the last decade or so, but, inevitably, these reports have concentrated on the particular range of bead types recovered by excavation at their individual sites, rather than seeking to establish full regional, national or international patterns. In many cases such reports will turn out to have provided a firm basis for local and regional patterns of beadwearing in burial contexts. On the other hand, while we have so few well-excavated and adequately published cemeteries there is a real danger of relying too heavily on their bead corpus. Rather, we should seek to place their material within the larger context of the finds from this period, drawing on earlier and usually less wellpublished excavations preserved in museum collections across the country.

The present study represents the first extensive description and discussion of early Anglo-Saxon glass beads. It is based on Margaret Guido's first-hand examination of glass beads over several decades in museums throughout Britain as well as in Europe and Scandinavia. She recorded her observations and sketches of each bead on a card. Wherever possible, the beads were handled and studied under good light conditions, but, inevitably, there were occasions when beads could not be taken off public display. In such cases, a combination of poor display lighting and the impossibility of handling the beads hindered exact description. It also made it difficult for her to comment on the exact amount of translucency of these beads. Invitations by excavators to examine and provide specialist reports on beads from often stillunpublished sites provided another valuable batch of data to add to the museum material and the growing corpus of published reports on beads now in the public domain. She attempted to provide a visual classification of glass beads recovered from Anglo-Saxon contexts datable between the fifth and early eighth centuries, which follows on naturally from her earlier study of prehistoric and Roman glassbead types (Guido 1978). There is the obvious desirability of being able to differentiate earlier prehistoric and Roman glass beads from similar ones manufactured and buried in the post-Roman period, either when they were recovered as unstratified finds, or where the museum records provide inadequate information about their archaeological contexts.

It should be noted, though, that the emphasis of students of the archaeology of the Anglo-Saxon migrations has turned away from the recent tendency to stress continuity and overlap of settlement in late Roman Britain. There is increasing acceptance that there must have been a significant time lapse separating the disappearance of most aspects of Roman material culture from the first phase of AngloSaxon cemetery and settlement foundation (Welch 1992). Roman coinage ceased to be imported in any significant quantity after c.AD 402, and it is clear that wheelthrown pottery production and marketing had ceased in Roman Britain before the Anglo-Saxons arrived. If it had not, we can be sure that the Anglo-Saxons would have been as eager to have acquired it and been buried with it, as were their Frankish counterparts at the end of the fourth and throughout the fifth century in north-east Gaul. We lose most of the dating indicators on which Roman archaeologists have 
relied and are forced to turn to an alternative chronology based on metalwork dress fittings introduced from the continent by Anglo-Saxon settlers. These include fifthcentury belt sets associated with late Roman imperial service and especially the army, as well as north German brooch forms whose ornamentation was heavily influenced by such Roman metalwork. Coin-dated grave contexts from cemeteries in north-east Gaul provide the framework for this chronology, which recently has been shifted somewhat later than hitherto within the fifth century (compare Böhme 1974 and 1986 with Böhme 1987).

This is not to deny the presence of Germanic peoples and other barbarians in late Roman Britain, as also earlier in the Roman period, mostly within the context of Roman army units (Böhme 1986). Burials of 'foreigners' often stand out from the general uniformity of late Roman cemeteries. This is certainly the case at the Lankhills School site, which was one of the extra-mural cemeteries of Roman Winchester (Guido 1978, 38-9, 101-2; Clarke 1979). One such group at Lankhills seems likely to represent Sarmatians recruited in the central Danube region, who brought their families with them. Similarly, an 'officer' of east Germanic, possibly Gothic, origin has been identified from an isolated grave at Kingsholm, Gloucester (Böhme 1986, 503, Abb. 25; Hills and Hurst 1989). It does seem probable, though, that most, if not all, these troops had crossed the Channel to Gaul within the first two decades of the fifth century, if not still earlier.

The De Excidio attributed to Gildas, and other later written sources such as the Historia Brittonum, seem to imply that Saxon mercenaries were hired by a British ruler or tyrant, who felt a need to recruit military expertise from across the North Sea in the absence of effective Roman army units. The extreme rarity and normal absence of complete or near-complete late Romano-British wheel-thrown pottery from early Anglo-Saxon cemeteries and settlements suggests that, by then, lowland Britain no longer possessed pottery industries and many other aspects of Roman material culture had also ceased to exist. The archaeological case for a continuity of significant Germanic occupation in eastern Britain from the third and fourth centuries into the fifth century, as proposed by the late J N L Myres (1977 and 1986) and others since the 1940s, has proved less than convincing. Attempts to date the pottery urns at the Anglo-Saxon cemeteries of Caistor-by-Norwich (Norfolk) and Sancton (Humberside/Yorkshire) and elsewhere earlier than the fifth century founder from the lack of any support from associated datable metalwork in the form of fourthcentury brooches or other dress fittings. Similarly, the so-called 'Romano-Saxon' pottery can now be seen to be a Romano-British ware with no demonstrable connection with the Saxons either in Britain or in North Germany (Gillam 1979; Roberts 1982; Welch 1992).

The schedules of glass beads that accompany this study provide a preliminary database from which the distribution and date range of glass beads of any given shape, colour or decoration can be established. It is hoped that this will prove a useful tool for future researchers preparing reports on glass beads from early 
Anglo-Saxon sites. The date range of deposition of particular bead types can be suggested from other associated finds, such as brooches and belt sets, in closed-find assemblages, usually graves. We can also use well-recorded graves to establish the extent to which particular beads were either kept as heirlooms or recycled after scavenging finds from old settlements or burials. This enables us to begin to comment meaningfully on whether particular glass beads were imported across the North Sea and the Channel or else might have been made within this country. If manufacture within Britain is suspected we need to establish from the distribution of finished products the region(s) in which we might hope to recover the workshop evidence we still lack. The means by which glass beads were acquired for members of the small rural farming communities represented by the vast majority of excavated early Anglo-Saxon cemeteries and settlements also needs consideration. We can compare the distribution patterns of bead types with those for other glass artefacts, notably glass beakers, bowls and other vessels (for example, Harden 1956 and 1971; Evison 1972, 1981 and 1982a; Huggett 1988, 72-4, fig 7), as well as consider the distribution patterns of related materials used in bead manufacture such as amber, rock crystal, amethyst and other semi-precious gemstones (for example, Meaney 1981; Huggett 1988; Welch 1991).

Written sources refer to Roman-period glass production in the eastern Mediterranean basin, including Alexandria in Egypt, Syria, Palestine and Phoenicia. Craftsmen of Jewish and Syrian origins seem to have migrated throughout the Roman empire, setting up glass workshops in Italy, Spain and Gaul (in present-day France, Belgium and the German Rhineland). For example, there is evidence for their presence in the Rhineland at Cologne and Andernach, on the Mosel at Trier and on the Rhône at Lyon. As there seems to have been no break in the production of glass vessels in the Rhineland and the Meuse valley within Gaul between the fourth and the seventh centuries, there is no reason why the production of glass beads should not have continued both there and in Italy and Spain throughout the same period. Where primary glass production from raw materials had broken down, glass scrap recovered from deserted Roman settlements or imported cullet might continue to provide sufficient glass to continue bead production. Thus, the small amounts of antimony recovered in a few of the glass beads from the Alton (Hampshire) cemetery could be regarded as possibly due to reused Roman glass in the cullet for their manufacture (Evison 1988a, 58).

There is also excavated evidence for glass-bead workshops on the continent in both the late Roman and Merovingian periods. At Trier two successive production areas were discovered. These appear to have been destroyed in $c .260$ and $c .375$ respectively, and were then sealed under rubble, while the latest glass beads from the workshop area were attributed to the first part of the fifth century (Loeschcke 1925). Its publisher drew attention to the contrast between the beads from these Trier workshops and the typical beads of the Roman empire. Both in terms of their preference for dark colours and the patterns used to decorate them, these Trier beads mark 
a complete break with previous Roman forms. He explained them in terms of a Roman craftsman's response to Germanic and barbarian taste. The most popular colours for the applied decoration on the dark background were yellow and red, as also occurred among beads from the late Roman fort at Nijmegen (Netherlands) on the Rhine frontier. Sky blue was another colour used in this way. The influerce of Germanic barbarian taste on glass-bead production in the Rhineland frontier zone as early as the mid-third century should not cause too much surprise and the Trier bead production must be considered in any evaluation of developments in the centuries immediately preceding the Anglo-Saxon settlement of post-Roman Britain.

The glass and amber beads that accompany the adult female in a still not fully published late Roman multiple burial pit within the Roman town of Canterbury (Cakebread Robey V) include a significant number of garishly decorated 'black' glass beads as well as plainer 'Roman' glass beads (Bennett 1980, 407, figs 3 and 4). It is difficult to offer a precise date for this deposit, which marks the breakdown of the normal Roman prohibition on burial within a settlement. The very end of the fourth century is the earliest probable date, but, in view of the metalwork parallels for the pins and other dress fittings, it is unlikely that this family group was buried more than a decade or so into the fifth century. It seems probable that this group substantially antedates the mid-fifth-century date currently favoured for the earliest phase of Anglo-Saxon settlement represented by Grubenhäuser in Canterbury.

Another glass-bead factory broadly contemporary with the Trier workshop has been located just outside the Roman municipium of Tibiscum (Theiss) near the late Roman Danubian frontier in present-day Romania (Benea 1983 and 1997). Glass waste, rejects and beads were recovered in quantity in a market area immediately outside the Roman town. Presumably these workshops produced beads which matched the tastes of both Roman provincials and barbarians. There are a number of beads in the Roman tradition here, but alongside these, and often on a 'black' ground, there were beads with crossed-wave designs or with specks or mosaics as well. Many of these could have been produced in the fourth century after the Romans had moved the frontier south of the Danube, leaving the native craftsmen to expand the repertoire of their workshops. The material from this site has yet to be published fully.

Our present understanding of the bead types being worn and buried beyond the frontiers of the Roman empire owes a great deal to Magdalena Mączyńska, whose study of beads in the central European Barbaricum during the Roman Iron Age and the earliest phase of the Migration period provide the foundations for all future research (Tempelmann-Mączyńska 1985). For the Merovingian period in western Europe, we owe a similar debt to the researches of Ursula Koch, and in particular to her report on the beads from the rich cemetery at Schretzheim in south-west Germany (Koch 1977). The colour plates from volume one, combined with the close dating achieved for the phasing of the cemetery, provide us with a basis for 
discussion of the glass-bead types of the sixth and seventh centuries, which she has since developed further (for example, Koch 1997).

Returning to the production evidence, there are two sites rather closer to England than either Trier or Tibiscum. These are the excavated bead workshops at Jodenstraat 26 in Maastricht (Netherlands) and at Rothulfuashem near Leiden (Netherlands), both of which contained a quantity of waste glass, beads, glass rods and various signs of work in connection with them (Panhuysen et al 1990, 217-18, Afb. 21; Sablerolles et al 1997; Bloemers et al 1981, 132). Production dates from the sixth century and the sixth to seventh centuries respectively have been proposed for these sites. Certainly the repertoire at Rothulfuashem c.AD 600 (van Klaveren 1988 and 1993) includes bead types that are well represented in early Anglo-Saxon sites. Dark blue annular beads, white annulars, triple-segmented opaque yellow beads, double and triple terracotta-coloured beads with white crossed waves, and white beads with blue crossed waves, were recovered there. The Rothulfuashem parallels will be discussed in greater detail under the relevant chapter headings below. It is unfortunate that only a very summary account has so far appeared in print of this important site, and its full publication is awaited with interest.

There is extensive excavated evidence for bead-making workshops in Scandinavia dating to the eighth century or later, for example, at Eketorp on Öland (Sweden) and Ribe in Jutland (Denmark), to name just two of the best-known sites. These have been discussed by Bencard with others (1978), by Bencard and Jørgensen (1990) and by Agneta Lundström (1976 and 1981), while Ulf Näsman (1984) has published a detailed study of the northern trade and market in millefiori and so-called 'reticella' glass vessels (for comment on reticella, see pages $64-6$ below). He argues that these were made in both Ireland and Belgium, though production was not exclusive to either of these regions, and that they were traded via Frisia to Scandinavia. Relatively few of these were made in, or exported to, England, though they are being recovered increasingly on Middle Saxon settlement sites (Evison 1982b, 1983 and 1988b; Webster and Backhouse 1991, 88, no. 66x), and there may have been rather more contact with Ireland than used to be thought.

Excavated glass kilns are extremely rare and the only ones known in Europe for this period appear to be those dated to the late sixth and seventh centuries at Torcello in Italy (Leciejewicz et al 1977). The vessel glass forms consisted principally of bottles and stemmed beakers, but coloured mosaic tesserae were also recovered among the finds (Evison 1982b, 8).

The conversion of the Anglo-Saxons and the establishment of monasteries in England coincides with the first archaeological evidence for glass manufacture here, which may have included bead production. Prior to the second half of the seventh century the nearest thing we have to evidence for local production is the occasional presence in graves of beads damaged in manufacture, which we would normally expect to have been rejected and dumped as wasters. Of course, the distribution pattern of certain beads as finished products certainly suggests manufacture within 
England, while there are Anglo-Saxon bead types that are extremely rare on the continent but particularly common in East Anglia (for example, flat annular beads, often white, with a coloured surface spiral on each face). Then there are terracotta-red beads of various shapes, but always decorated with green and yellow inlay, often with so-called 'reticella' ornament (here called herringbone or twist). Similar cases have been made, on distributional grounds, for glass-vessel manufacture in Kent during the sixth and seventh centuries (for example, Evison 1982a; Bruce-Mitford 1974, 346-8). The use of glass enamelling on Anglo-Saxon metalwork in eastern England during the sixth century (Brown 1981; Scull 1985) has been documented, and there is a strong probability that the hanging-bowls found in Anglo-Saxon burials between the mid-sixth and the mid-seventh century were produced in British workshops within the Anglo-Saxon provinces (Brenan 1991).

Written references to glass-workers being brought over from the continent to produce window glass and vessel glass for a pair of Anglo-Saxon monasteries in north-east England in the seventh and eighth centuries can now be supported by a growing corpus of excavated evidence for glass workshops in documented monasteries and other 'high-status' sites that we suspect are monastic communities. In the Historia Abbatum (cap. 5), Bede describes how in c.AD 676 Benedict Biscop sent 'representatives to Gaul to bring back glass-makers, craftsmen as yet unknown in Britain, to glaze the windows of the church, its side chapels and upper storey' at his newly founded monastery at Wearmouth, 'and not only did they complete the work that was asked of them, but they also taught and instructed the English race in their skill'. Coloured window glass has been excavated at Benedict Biscop's monasteries of Jarrow and Monkwearmouth in Tyne and Wear (Cramp 1970a, 1970b, 1975; Webster and Backhouse 1991, 138-9, no. 105a). While no glass furnace was found at either site, there was evidence for the manufacture of window glass at Jarrow, and fragments of vessel glass, millefiori rods, beads and inlay from the debris of the monastic guesthouse at Jarrow indicate that other types of glass was being worked on site. Yet, in a letter to Bishop Lul at Mainz dated to 764, Abbot Cuthbert of Wearmouth and Jarrow could request that Lul send him 'any man in your diocese who can make vessels of glass well . . . because we are ignorant and destitute of that art' (Tangl 1955, 250-2, no. 116; Whitelock 1979, 831-2, no. 185), which suggests that the Northumbrian Angles were still dependent on short visits by foreign glassmasters a century later.

Similar potential evidence for glass manufacture is now accumulating from a number of other Middle Saxon period monasteries and 'high-status' settlements. The colours of the Jarrow window glass are paralleled at Brandon (Suffolk) and the double monastery at Whitby (Yorkshire) in both window and vessel glass (Webster and Backhouse 1991, 87-8, no. 66v-y, and 143-4, no. 107f-j). Millefiori rods and glass tessera fragments of the type recovered at Jarrow, Monkwearmouth and Whitby (Cramp 1970a, fig la-f; Webster and Backhouse 1991, 145, no. 107o-p) certainly suggest that the glass materials that could be used to make beads were 
readily available. There are also the rich glass finds recovered from York Minster, Flixborough (Lincolnshire) and Barking Abbey (Essex) (Webster and Backhouse 1991, 90-2, no. 67m-t, 94-5, no. 69, 146-7, no. 108).

The very active production of glass beads in early Christian Ireland has been recognized for at least half a century. As yet though, there has been no overall synthesis of the rich excavated workshop evidence there. This would be of particular value to scholars studying the archaeology of the west and north-west regions of Britain in the Anglo-Saxon period, where 'foreign' beads might belong to recognizable Irish types, or else be confused with Anglo-Saxon beads of a very similar type. Hencken $(1950,132-45)$ was among the first scholars to consider Irish beads of the early Christian period. Since his discussion of the bead workshop evidence from Lagore (Co. Meath), other production centres have been recognized in Ireland.

From the Iron Age onwards, Irish beads show advanced and original technique and design. They underwent a remarkable renaissance with the presumed arrival of foreign craftsmen at the time of the early ecclesiastical foundations there. Imported skills were quickly learnt by native glassworkers, who were inspired by them to brilliant inventions and elaborations. They produced a variety of glass inlays for brooches, shrines, patens and chalices, as well as for disc mounts on hanging-bowls, and so on (Youngs 1989, 173-4, 201-8). It was at this period that millefiori glass, previously used in Ireland within the Roman period, came to prominence once more. Irish craftsmen also came to mainland Britain and their work is recognizable on both ecclesiastical and secular sites within the kingdom of Dalriada founded in the seventh century by Irish Scotti in what is now western Scotland. At excavated fortified centres such as Dunadd (Argyllshire), Anglo-Saxon metalwork has been found alongside Irish glasswork (Campbell and Lane forthcoming; for the metalwork, see Campbell and Lane 1993).

More sites in Ireland have produced evidence for bead-making in this period than in the whole of Britain. Glass waste, rods and reject beads survive to be excavated together with crucibles and metal implements employed in their manufacture. Relevant workshop sites are at Garranes (Co. Cork), attributed to the sixth to seventh centuries (O'Riordain 1941-2, 116-21, figs 14 and 15; Youngs 1989, 201-2); Scotch Street, Armagh (Co. Armagh), of the sixth to ninth centuries (Hamlin and Lynn 1988, 57-61; Lynn 1988; Youngs 1989, 203-6); Ballinderry Crannog 2 (Co. Offaly) of the eighth to ninth centuries (Lawlor 1925); Moynaugh Loch (Co. Meath) of the seventh to eighth centuries (Youngs 1989, 201 and 205); and, in Dalriada, at Dunadd (Argyllshire), of the seventh century and later (Campbell and Lane forthcoming). A recently reported early Christian period site at Dunmisk (Co. Tyrone) is important for providing evidence for glass-making from primary raw materials in place of the more customary remelted glass (J Henderson, pers comm). The presence of glass rods in numerous other Irish sites suggest the possibility of local bead manufacture at these settlements.

There is no obvious reason why the technology for bead manufacture from 
coloured glass tesserae or millefiori glass rods imported for the purpose should have been beyond the skills of Anglo-Saxon craftsmen. Imported and old Roman vessel glass, as well as glass tesserae scavenged from mosaics, would provide alternative sources. We may yet locate the workshops that demonstrate the ability to work glass. A case based on indirect evidence can be made for at least three groups of glass beads as local products (see Chapters 7, 8 and 12 below, and Guido and Welch forthcoming).

Nevertheless, it seems probable that a significant proportion of the glass beads recovered from early Anglo-Saxon contexts were imported as trade goods via the Frankish continent. The rulers of Kent and a Jutish province on the Isle of Wight and southern Hampshire may have been the 'middlemen' in a monopolistic trade between the Franks and other Anglo-Saxon kingdoms in the sixth and early seventh centuries. It was during the seventh century that this monopoly was replaced by a series of direct arrangements between individual Anglo-Saxon and Frankish kings. These are represented archaeologically by major trading settlements at Southampton, London, Ipswich, York and elsewhere outside Kent (Huggett 1988; Welch 1991). The study of early Anglo-Saxon glass beads is still in its infancy, but it is hoped that this preliminary study, in combination with scientific analysis, will provide the foundations for further research. 


\title{
CHAPTER 1
}

\section{Colourless AND PALE TRANSLUCENT GLASS BEADS}

\author{
MONOCHROME \\ i Colourless translucent beads in various forms \\ ii Light-coloured annular, globular, biconical and double beads (excluding \\ smokey yellow forms) \\ iii Light blue-green translucent melon and sub-melon beads \\ iv Smokey yellow beads (excluding melon and sub-melon forms) \\ $\mathrm{v}$ Smokey yellow melon and sub-melon beads \\ Polychrome \\ vi Decorated translucent beads: (a) on a light blue-green ground \\ (b) on a smokey yellow ground \\ vii Light-coloured translucent annular or globular beads with coloured parallel \\ or spiral bands (excluding lobed forms) \\ viii Light yellowish-green beads with decoration (mostly square-sectioned form) \\ ix Light-coloured decorated lobed or horned beads
}

The information that we can glean from pale translucent glass beads is much less than is the case for the opaque versions. There are two obvious reasons. Firstly, the translucent beads present us only with forms, most of which continue a repertoire already current in the Roman period. On the whole, very little visual distinction can be observed between translucent beads of Roman and Anglo-Saxon date, and as yet there is no clear analytical difference detectable between light colours: tones of green, yellow, blue-green, and so on. Thus, we have to look for forms that differ from the Roman ones and which would appear, therefore, to have been introduced into lowland Britain at the time of the Anglo-Saxon settlement. It is only types iii and $\mathrm{v}$ listed above (ie, the small melon and sub-melon beads) that have no clear Roman period ancestry. (See Figure 1 for main diagnostic shapes in Anglo-Saxon beads.)

Secondly, in contrast to the opaque beads, decoration is very rare on pale translucent beads. Consequently, the other main criteria for classification are absent (see Figure 2 for main diagnostic decorative motifs). In certain decorated classes, for 
example, those with surface spirals or a fine twist (see Chapters 11 and 12 respectively), pale coloured translucent glass is occasionally used as the basis. On the other hand, for beads enclosing a metal foil (Chapter 13: gold or silver-in-glass), colourless translucent glass was normally employed, but, in all probability, none of these was ever made in lowland Britain. The most interesting small category are the decorated square-section beads of type viii (not illustrated). They are mostly fragmentary and we must await the discovery of further complete examples to elucidate their cultural background.

In subsequent chapters, the darker tones of translucent glass are classified with their individual colours together with their opaque equivalents.

\section{MONOCHROME}

i Colourless translucent beads (Plate 1, Schedule 1, pp 153-5) The earliest glass of this type apparently came from Egypt (Daily Telegraph, 26 September 1989), at the temple of Deir al-Bahri near Luxor, and dates to c.1497-1488 BC. Such glass had previously been misidentified as rock crystal, but is in fact a soda-lime-silica glass used as a substitute for quartz. This was evidently adopted in both Greece and Etruria from the fifth century BC (Harden 1968b and $1969,60-2)$. It was little used for beads in Roman times, but had a limited renaissance during the fifth to sixth centuries AD.

Very little, however, can be gleaned from the schedule. The beads reveal not a type so much as a variety of forms. A number of beads have been omitted from the schedule, since many, though described in reports as small globular beads, are in fact much-damaged remains of metal-foil enclosed beads (see Chapter 13), of which only the inner part now exists.

\section{ii Light-coloured anNular, globular, Biconical and DOUble BEADS (EXCLUDING SMOKEY YELLOW FORMS) \\ (Plate 1, Schedule 1, pp 155-9)}

Glass of this very pale translucent variety could have been made from recycled bottle glass of Roman date. Not much can be shown from a perusal of the schedule here. They were not uncommon in East Anglia during the late fifth to early sixth centuries, by which time they were also to be found in the Upper Thames region at Abingdon (Berkshire) and other sites. One from Alfriston (East Sussex) may be among the earliest recorded. Some greenish-blue or light yellow roughly squaresectioned beads come from Lechlade (Gloucestershire), some of which belong within the sixth century, but others occur in firmly dated seventh-century contexts at both Lechlade and Buckland, Dover. 


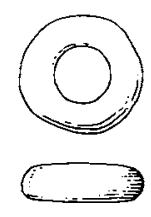

thin annular
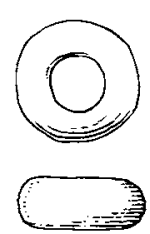

thick annular
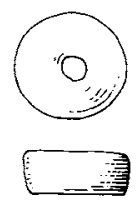

disc

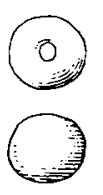

globular
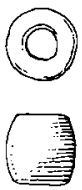

barrel
(2)

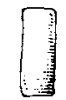

cut cylinder
0

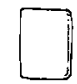

squaresection cylinder (o)<smiles>c1ccccc1</smiles>

polygonal cylinder
(2)

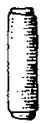

(0)

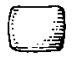

short cylinder
(0)<smiles>C1=C[C@H]2CC[C]1C2</smiles>

short double cylinder

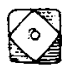

筫

polyhedral
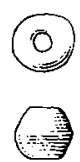

small biconical
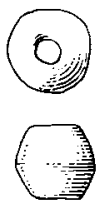

large biconical

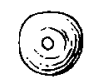

$\mathrm{a}$
7

long biconical
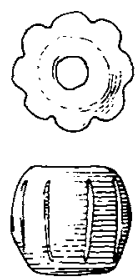

melon or gadrooned
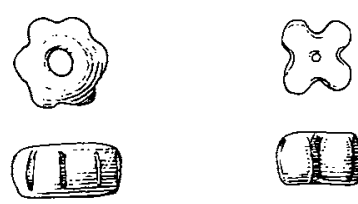

sub-melon

('nicked')
(क)

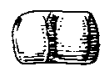

lobed melon
(6)

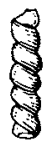

segmented (wound)
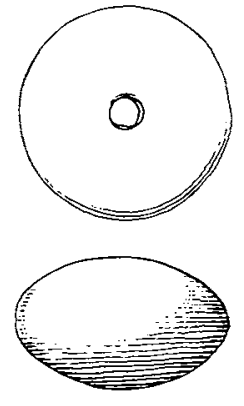

biconvex
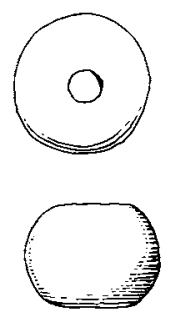

bunshaped (o)<smiles>c1ccc2ccccc2c1</smiles>

segmented (globular)

Figure 1 Anglo-Saxon beads: main diagnostic shapes 


\section{iii LIGHT BLUE-GREEN TRANSLUCENT MELON AND SUB-MELON BEADS}

(Plate 1, Schedule 1, pp 159-61)

Glass of this colour is so common, and its production covered so long a span of time, that it is necessary to study the forms rather than the glass itself. It was mostly used for melon and sub-melon forms, whose distribution is biased towards the southeast, east and north-east coastal regions. Among the earliest finds was a bead from the late Roman multiple burial at Canterbury (a context of c.AD 400) referred to in the Introduction ( $p$ 6). A later fifth-century string comes from grave 15 at Wallingford (Berkshire) in the Upper Thames valley dated by the associated applied saucer brooches and a fifth-century cremation context occurs at Spong Hill (Norfolk). There are plenty of sixth-century examples, and seventh-century ones from Chamberlain's Barn II (Bedfordshire) and Charlton (Wiltshire). A continental origin might be sought in the Rhineland, as they occur at Krefeld-Gellep in a cemetery horizon (Stufe II) conventionally dated c.AD 450-525 (for example, grave 404 (Pirling 1966, 156, Taf. 33.12)). (See also type v below.)

\section{iv SMOKEY YELLOW BEADS (EXCLUDING MELON AND SUB-MELON FORMS) (Plate 1, Schedule 1, pp 161-3)}

The same date (ie, the late fifth to sixth century and a seventh-century context at Buckland, Dover) applies to the popularity of these glass beads as to those in type iii above. There are several examples in Kent and Suffolk as well as a small cluster around Salisbury in south Wiltshire.

\section{$\checkmark$ SMOKEY YELLOW MELON AND SUB-MELON BEADS}

(Plate 1, Schedule 1, pp 163-4)

It is not possible to differentiate analytically between these beads and those in type iii above, and they should be studied together.

\section{POLYCHROME}

\section{vi Decorated translucent beads: (a) ON A light Blue-green} GROUND (Plate 1, Schedule 1, pp 164-6)

As the decoration on these beads varies so much, they cannot be regarded as a true class. Decoration on translucent light-coloured glass appears on beads in England by the late fifth century, but it was never much favoured, though it continued into the sixth and even the seventh century.

\section{vi Decorated translucent BeAds: (b) ON A SMOKEy Yellow} GROUND (Plate 1, Schedule 1, p 166)

Never very popular, at present these beads cannot usefully be separated from those of via, discussed briefly above, though one example does come from a context dated to the first half of the fifth century at Colchester (Essex). 

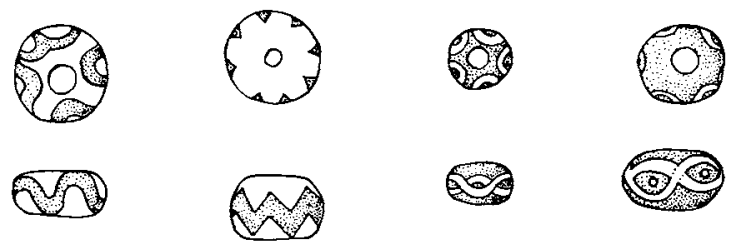

1

2

3

4
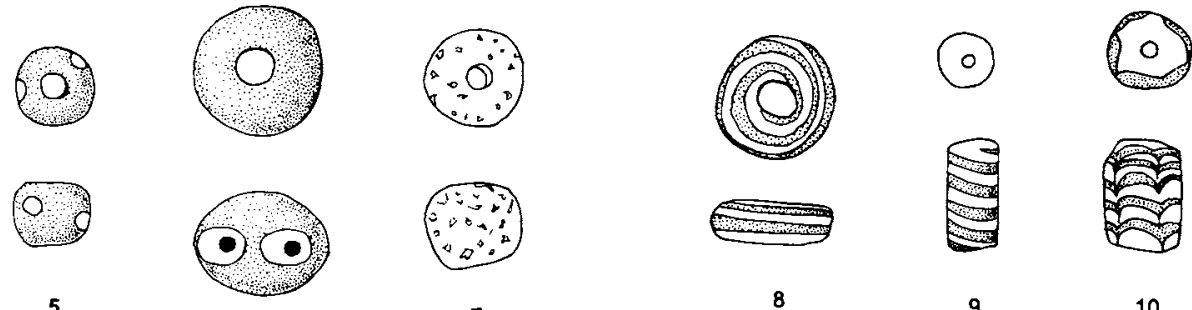

7

8
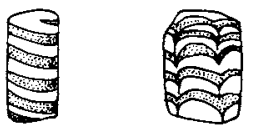

9

10

6
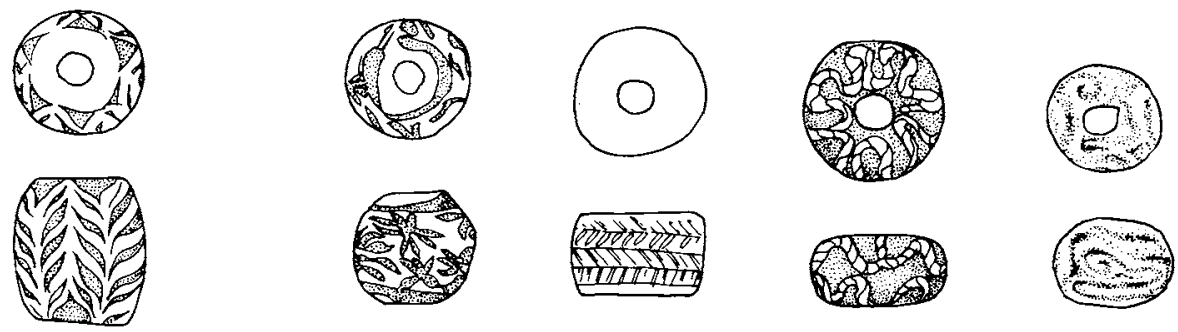

12

13

14

15

Key:

1 wave; 2 zigzag; 3 crossed waves; 4 crossed waves and spots; 5 large spots; 6 eyes; 7 speckled (often many colours and touching specks); 8 surface spiral; 9 spirally wound bands; 10 combed bands (one direction); 11 combed bands (alternate directions); 12 millefiori; 13 reticella herringbone or chevron; 14 twisted cable decoration; 15 marbling in two colours

Figure 2 Anglo-Saxon beads: main diagnostic decorative motifs 
vii Light-COLOURED TRANSLUCENT ANNULAR OR GLOBULAR BEADS WITH COLOURED PARALLEL OR SPIRAL BANDS (EXCLUDING LOBED Forms) (Plate 1, Schedule 1, pp 167-8)

These appear to be a recognizable type and to have been current probably by the fifth century at Milton Keynes. So far, it has not been possible to identify an origin for them on the continent, though they appear not to be unusual and to occur in fifth-century graves (Birte Brugmann, pers comm). It should be noted that one atypical example on an opaque ground comes from Lyminge in south-east Kent.

\section{viii Light yellowish-GREEN BEADS With DECORATION (MOSTLY SQUARE-SECTIONED FORM) (Plate 1, Map 1, Schedule 1, p 168)} These rare beads are often decorated with random lines of red and yellow and quite a few of them are square-sectioned (none illustrated). The latter are noticeably earlier and their manufacture may belong within the late Roman period, possibly continuing into the early fifth century. Their distribution is relatively circumscribed in north Buckinghamshire, Cambridgeshire and Essex (see Map 1). Related beads, though not square-sectioned, were excavated at the ring-fort of Eketorp II (fifth to seventh centuries) on Olland (Sweden) in the Baltic (information from UNäsman).

\section{ix LIGHT-COLOURED DECORATED LOBED OR HORNED BEADS}

(Plate 1, Schedule 1, pp 168-9)

The main characteristic of these beads are lobes decorated with either wound lines or red tips. The earliest example comes from an early fifth-century grave from Mucking (Essex). Clearly, these are related to the type vii beads above, and also occur in fifth-century graves on the continent (Birte Brugmann, pers comm). In England, it is noticeable that they are confined to the south and the Thames valley. 


\section{CHAPTER 2}

\section{'BLACK' GLASS BEADS}

Monochrome
i $\quad$ Small 'black' annular and globular beads
ii
iii

Polychrome

iv 'Black' annular beads with broken white girth band

v 'Black' annular beads: (a) with white or yellow wave (some with girth band)

(b) with blue wave

vi 'Black' annular beads: (a) with white zigzags

(b) with yellow or green zigzags

(c) with red zigzags

vii Large 'black' decorated beads: (a) plano-convex

(b) biconvex

viii 'Black' (or dark) globular beads with complex crossed waves and eyes or spots (and related beads)

ix 'Black' globular beads with white or coloured crossed waves, with or without eyes or spots

$\mathrm{x} \quad$ 'Black' beads with a few spots (mostly annular beads)

xi 'Black' globular beads with coloured specks ('crumb' beads)

xii 'Black' decorated long cylinder beads

xiii Long fluted beads banded in 'black' and white (not scheduled)

xiv Small cylindrical dark beads with unmarvered yellow ends and central band (not scheduled)

xv Large dark globular beads with several lines of coloured zigzags

xvi Tall drum-shaped dark beads with several lines of coloured zigzags

The beads here described as 'black' may actually be of varied dark colours: blue, dark green, brown and purple (see Chapters 5, 6 and 7 below). This can be seen most clearly in fracture and to a certain extent the degree of darkness depends upon the thickness of the bead itself. Ultimately, only chemical analysis can identify them correctly. Undecorated beads of very dark glass were certainly known in Britain as early as the Iron Age, though they were rare, and throughout most of the Roman period seem equally to have lacked popularity here (Guido 1978, 15 and 68: Group $6 \mathrm{ix})$. 
By the fourth century AD, however, large 'black' beads, often decorated with various colours, began to appear, in particular from the 'barbarian' world bordering the Roman Limes, but also further afield in Europe. They were presumably prized for their size and their gaudy exuberance, which stand out as a complete contrast with the decorous and rather uninteresting little beads of very standardized type then current in response to Roman taste. At this time, too (ie, the late third and fourth centuries), there was a fashion for small, roughly wound tapering beads in 'black' and other colours found at least on some sites in Roman Britain (for example, Cold Kitchen Hill, Wiltshire, and Lamyatt Beacon, Somerset). They may even have been made on the spot for cheap offerings at shrines or temples (Guido 1978,38 ). By the mid-fifth century, though, they had largely disappeared and were later replaced by segmented beads made using a different technique (Plate 1 and type ii on pp 20-1 below).

One of the earliest glass-houses known to have been producing 'black' beads (either large plain melon beads, or other forms with a variety of polychrome motifs marvered on as spots, waves, and so on) was at Trier. As discussed in the Introduction, Loeschcke (1925) drew attention to the discovery there of the beads in datable closed-find groups. They came from two superimposed, but separate, workshops, sealed by rubble. These belonged to the mid-third century and first half of the fifth century respectively. He argued that the late Roman industrial output had already been influenced by the various Germanic peoples living in the Rhine valley and its hinterland, whose tastes increasingly infiltrated those of the conservative Roman world.

The Migration period was a time of great change in Europe, and it is not yet possible to identify with any confidence the original makers of such 'black' beads. They could be any one of several barbarian peoples, perhaps even including Slavs, for there are certainly some 'black' decorated beads from central and eastern Europe. Quite apart from tribal and army movements, individual craftsmen could be surprisingly mobile, particularly in activities such as glass and stone-working, finding employment with rich patrons. In Trier, an outstandingly large number of inscriptions not only exhibit Greek and Latin names, but also suggest the presence of many craftsmen who might well have come from Syria. Syrian glass craftsmen seem also to be responsible for the output of glass vessels from Cologne and other, as yet unidentified, Rhineland glass-houses (Harden 1968b and 1969, 49-51, 55-9 and 64).

Contact between Whitby, York and Cologne also existed in the fourth century, when jet was imported into the Rhineland for the manufacture of finger-rings and other small objects. This gemstone can be found in underwater outcrops off the Yorkshire coast and was prized for its electrostatic properties. Whitby jet was mentioned by Solinus in the third century for the excellence of its quality (Mommsen 1895, 102). With the apparent interruption of this trade early in the fifth century, it seems likely that 'black' glass could have been adopted as a substitute material for jet. 
A number of colour-decorated 'black' beads reached lowland Britain before the abandonment of such major Roman urban and military sites as Cirencester, Silchester, Dorchester and Richborough. Much the same applies to continental Roman sites: for example, Vertillum (Vertaux), a Gallo-Roman town in the Côte d'Or, the finds from which are now in the museum at Châtillon-sur-Seine. On the lower Rhine at Krefeld-Gellep, beads of this type came from grave 333, which has been dated to the first half or middle of the fourth century (Pirling 1966, 116, Abb. 13.25, Taf. 27.6). In addition, two very early examples were found in Roman York (Sycamore Terrace, in 1901), associated with a bone artefact bearing an early Christian inscription (RCHM 1962, 73, fig 58; Guido 1978, 101-2 and 235) (see the schedule on pp 183-4).

Still earlier, glass-houses producing these beads may have existed in the Dniester and Danube regions, and at Theiss (Tibiscum) one was identified and partially excavated in 1976. As discussed in the Introduction, this factory was active between the second and fourth centuries, and among its products were 'black' crossed-wave and 'crumb' beads (Benea 1983, 116, Taf. 1). A short discussion of other possible factories has been provided by Tempelmann-Mączyńska (1985, 133-4), and the discovery and publication of more such sites is eagerly awaited.

It is interesting to find that at Schretzheim decorated beads on a 'black' ground appear in that cemetery's first two Stufen of $c .525 / 30-565 / 70$. Although this cemetery is to be found near Dilligen on a tributary of the Upper Danube in Alamannic south-west Germany, these ornamented 'black' beads may reflect the Thuringian and north German origins of the first small group of settlers, seemingly located at Schretzheim in the second quarter of the sixth century by their Frankish Merovingian overlords (Koch 1977, 187-90).

Decorated 'black' beads occur in both halves of the sixth century at Andover in Hampshire (Cook and Dacre 1985, 81-4, colour plate frontispiece). Association with two disc brooches in grave 32 more probably implies the first half of that century and the small-long brooch in grave 50 should be earlier rather than later. On the other hand, both graves 9 and 44 are attributed to the second half of the sixth century. A mid to second half sixth-century date for grave 23 at Alton (Hampshire) is attributed here on the basis of Tania Dickinson's 1976 dating of the seven-leg whirligig saucer brooches (Welch 1983, 50-1), despite Vera Evison placing this burial in her cemetery phase 2 of $c .475$ and 525 (Evison 1988a, 17, fig 4: D15). By contrast, the two decorated 'black' beads from graves 133 and 129 at Buckland, Dover (Kent), both come from phase 5 contexts of c.650-675 (Evison 1987, 76-7 and 82, text fig 12 and colour pls III and IV: D07 and D53), while plain 'black' beads are found in graves of phases 1 to 5 , extending from c.475 to 675 . Undecorated 'black' beads were almost always of annular form in England, like many in the Rhineland, and perhaps it was from there that they were imported. In contrast, the Schretzheim ones are more usually globular and these became particularly fashion- 
able in the early seventh-century Stufe 4, for example, grave 233 (Koch 1977, 54, Taf. 57.9).

It would seem that 'black' beads with polychrome patterns can be found either to be lingering on in England, or else reintroduced here between the seventh and tenth centuries. This was a time when glass workshops in Scandinavia, Ireland and Bohemia, for example, were making beads very similar to some of the types discussed in this section. These are liable to be discovered in Viking Age, Irish or Hiberno-Norse and late Germanic contexts (see Callmer 1977; Hencken 1950, 132-45; and Hruby 1955, 246-61, 354, Taf. 85-6).

It can be seen from the types listed below that 'black' beads were on the whole earlier than other coloured glass beads, and their frequent presence within former Roman provinces on the continent may well indicate the infiltration of barbarian peoples and armies into Roman territory between the third and fifth centuries. Among the earliest in lowland Britain were several 'black' cylinder beads from a late Roman multiple burial context at Cakebread Robey V in Canterbury (Kent), dated c.400 (see Introduction).

\section{MONOCHROME}

\section{i SMall 'Black' anNular and globular beads}

(Plate 1, Schedule 2, pp 170-3)

These are surprisingly almost invariably annular in form and can be seen to be largely confined to the south of England during the fifth and sixth centuries, but with some seventh-century contexts at Buckland, Dover. Their appearance in cemeteries in Kent, Sussex and Essex (for example, by the Thames estuary at Mucking and along the east coast at Springfield Lyons, on the outskirts of Chelmsford) suggests that they might be imports, perhaps from the Rhineland.

At Schretzheim 'black' annular beads cover the period c.525/30 to 590/600 (Stufen 1 to 3), for example, grave 36 (Koch 1977, 19, Taf. 13.1), and the globular ones a little later, c.565 to 620/30 (Stufen 3 and 4), for example, grave 20 (Koch 1977, 12, Taf. 1.10). By this stage it may be that the market for them was already on the wane in England. A date of $c .500$ for grave 4882 at Springfield Lyons, which contained a necklace of many variously shaped small 'black' beads, is based on an associated cruciform brooch with a developed form ( $\AA$ berg's Group II) and a pair of disc brooches of broadly similar date ( $c .450$ to 550 ). These little beads may have been among the first made to imitate jet beads.

\section{ii 'BLACK' SEGMENTED DOUBLE OR TRIPLE BEADS}

(Plate 1, Schedule 2, p 173)

This form of bead also occurs in opaque yellow glass and has been classified in that material as type vc (see Chapter 4, pp 39-40). Indeed, in England it is a much more common find in yellow than in 'black'. Examples come from sixth-century cemetery 
contexts from the Anglian sites of Fonaby (Lincolnshire) and Sewerby (Yorkshire) They have been noted also in the Low Countries, for example, Pry near Walcourt in Belgium (Namur Museum) as well as in south-west Germany at Marktoberdorf in Allgäu (triple) and at Schretzheim, where they are present in graves 146 and 543, dating respectively to Stufen $2(c .545 / 50-565 / 70)$ and 4 to 5 (ie, either side of 620/30) (Koch 1977, 35 and 116, Taf. 35.11 and 139.16).

Perhaps, like some other forms, these originated as imitations of jet beads. One example that is very similar to the Marktoberdorf find comes from Sarre in Kent (now in the British Museum). It is straight-sided and could indeed be made of jet, but it was not possible to examine it in person. These beads do not appear to have been made in the Trier workshops, though a somewhat similar double-segmented bead (possibly wound and so not identical) was illustrated by Böhner (1958, 76, Taf. 8.25) from the Trier region in Stufe III (525-600). As mentioned above, they are similar in form to the equivalent opaque yellow beads, and must have overlapped in date.

\section{iii 'BLACK' MELON, SUB-MELON OR LOBED BEADS}

(Plate 1, Map 2, Schedule 2, p 174)

It is known that large 'black' melon beads were being made at Trier as early as the late fourth century or even before then (Loeschcke 1925). Nevertheless, the types recovered from early Anglo-Saxon contexts, most of which are sub-melon beads, seem unlikely to pre-date the fifth century and are on the whole rather later, including a seventh-century context at Lechlade (Gloucestershire). Their distribution is limited to southern England and the south Midlands, and they are particularly found in counties to the west of the London region (Map 2). So far, the large versions from the Trier factory seem to be unrepresented in England. By contrast, the Anglo-Saxon sub-melons are smaller and are roughly made with nicks rather than gadroons. A later date also seems to apply to the continental sub-melon equivalents, for example, graves 509 and 425 at Schretzheim of later sixth to early seventh-century date: Stufen 3 and 4 (Koch 1977, 109 and 95, Taf. 132.10 and 114.12).

\section{POLYCHROME}

iv 'BLACK' ANNULAR BEADS WITH BROKEN WHITE GIRTH BAND

(Plate 1, Map 3, Schedule 2, pp 174-6)

The characteristic feature of these beads lies in the ornamentation: an irregular line of dot-and-drag in opaque white, around the centre of the bead. At first sight the aim might appear to be to create a white wave of common type or else a line of dots. It hardly seems conceivable that such a poorly executed decoration was deliberate and one even wonders if they were not made by inexperienced craftsmen. On the other hand, there are quite a number of them in England sharing precisely the same 
interrupted decoration. It is difficult, therefore, to dismiss this characteristic as anything other than intentional.

Although two came from the cemetery at Pry in Belgium on a late fifth to sixthcentury necklace (Bequet 1895), and another from Frisia in the Leeuwarden Museum, it has not been possible to find other continental examples. It may be that these beads reveal attempts by fifth-century craftsmen in lowland Britain to adapt their earlier Roman repertoire to wider tastes. Thus, like many other beads on a dark or 'black' ground (see type i, etc), these start at least as early as the fifth century in Anglo-Saxon England. Further, although they are sometimes found in late Roman contexts, they all seem to be essentially barbarian in taste.

For England, the schedules and Map 3 show that these beads were confined to the south and the south Midlands. Although they can occur very occasionally in cemetery contexts that continued into the seventh century, for example, Chariton (Wiltshire), they all come from sites where burial began within the fifth century. Still, it must be emphasized that the majority derive from old and poorly published excavations. It seems that the Franks and their subjects on the continent did not perpetuate these crudely made beads. Perhaps they were mostly made in England, as suggested above, and they disappeared once bead-craftsmanship improved. In many contexts they may only represent late survivals of an early tradition.

\section{v 'Black' ANNUlar BEADS: (a) With WHite OR YelLOW WAVE}

(SOME With GIRTH BAND) (Plate 2, Map 4, Schedule 2, pp 176-8)

This wave pattern is more flowing than the spikey zigzags, but both had an extremely long ancestry on the continent. In 'black' glass, they were almost certainly barbarian or, more specifically, 'Germanic' beads, and were largely confined to the late fifth and sixth centuries, though they probably began to reach the south of England even earlier. Occasionally they were still current in seventh-century contexts (for example, Buckland, Dover), most likely retained as heirlooms. Their distribution occurs across most areas of Anglo-Saxon settlement (Map 4).

\section{v 'Black' anNular beads: (b) With blue wave}

(Plate 2, Map 4, Schedule 2, p 178)

These are apparently earlier and were current from the fifth on into the sixth century. They should not be confused with beads where the blue is more powder blue and less azure. Their distribution is restricted to southern and eastern England (Map 4).

\section{vi 'Black' anNular beads With White or COLOURED Zigzags}

(Plate 2, Map 5, Schedule 2, pp 178-80)

These are all discussed together, although their schedules are kept separate. They clearly represent a group that is both chronologically and culturally very similar.

The bead type most frequently found is that decorated with white zigzags (a). These were almost certainly imported into lowland Britain and later England. For 
dating purposes, the Trier factory, already mentioned above and in the Introduction, is of paramount importance. Another significant site is that of the late Roman and Frankish cemeteries at Krefeld-Gellep on the lower Rhine, which demonstrates the overlap of the two material cultures (Pirling 1966, 1974, 1978 and 1989). Several beads of this variety came from datable contexts there: for example, grave 530 in Stufe I (400-450), grave 9 at the beginning of Stufe II (c.450 or slightly later) and grave 253 in Stufe III (525-600) (Pirling 1966, 159, Taf. 46.20, 8.18 and 24.1). From the Trier region, several were attributed by Böhner to his Stufe II (450-525) (Böhner 1958, 79-80).

Others were recorded from the Pry cemetery in Belgium attributable to the fifth or sixth centuries (Bequet 1895). At a settlement on the Gelbe Bürg near Dittenheim (Mittelfranken) in Germany, they were datable broadly between the third and fifth centuries (Dannheimer 1962, 29, 172, Taf. 18). Doubtless there are many more such beads awaiting publication or discovery, particularly to the east of the Roman Limes and along the rivers northwards and westwards from 'Slavonic' territories (see Tempelmann-Mączyńska 1985, 20, 52-8, Taf. 6: type 263, with white and red zigzags).

The schedules for England reveal the same date range. A bead from Nettleton, Wiltshire (Guido 1978, 64, 137: Group 5.D.iii; Wedlake 1982, 153-4), may be the earliest stratified example, and others are recorded from major Roman sites at Richborough (Kent), Silchester (Hampshire) and Caernarvon (Segontium) in north Wales. Perhaps these had been brought in as personal possessions, gifts or amulets by troops and their families. As such, they reflected the bead fashions of the regions in which these troops were recruited. Their precise background eludes us at present, but a non-Roman barbarian origin, perhaps Germanic, seems clear. Though largely discontinued by the Franks, they never entirely died out during the Anglo-Saxon period in England, but increasingly ceased to be acquired when 'black' glass was mostly replaced by other colours and notably by terracotta-coloured glass (see Chapter 8: type xvi). Their distribution covers most of Anglo-Saxon England (Map 5).

\section{vii LARGE 'BLACK' DECORATED PLANO-CONVEX OR BICONVEX BEADS (POSSIBLY SWORD-BEADS)}

(Plate 2, Maps 6 and 7, Schedule 2, pp 181-3)

Among the largest and most conspicuous items from the glass-bead repertoire in England are two closely related subtypes. The first, (a), is plano-convex or bunshaped. Its flat base is normally undecorated, while the convex side has a contrasting band in lighter colour around the hole and often also below the sharp zizags, which are the main decoration. No two are identical. The variant (b) is biconvex and sometimes decorated on the underside. Both subtypes measure about $30 \mathrm{~mm}$ in diameter and both are made of 'dark' glass, though a few, if held to the light, become pale translucent. It would also seem that both were imported from the continent. 
The schedules for England suggest that these beads were often worn attached to sword pommels and were associated with the scabbard and sword-strap fittings. They also had a possible secondary function as amulets with magical power to ward off injuries or death (Evison 1967 and 1975). This need not invariably have been their function, however, for some have been used in necklace bead festoons and others as belt-fasteners or spindle whorls, for which two latter purposes they are often so similar as to be readily confused. Unless the use is clear from the context, it is best to keep an open mind about their original function. According to Lindenschmit (1880-9, 420, figs 1-3), however, some examples from the Rhineland still retained their bone or bronze spindles in place (see also Werner 1956, 36, Taf. 21).

The two subtypes (a) and (b), in various colours, are discussed by TempelmannMączyńska (1985, 22, 62-3, Taf. 13) as her types 381 and 380 from central Europe, while Vera Evison (1967, 64-6 and 81) pointed out that the custom of wearing sword-beads was probably introduced into south-east England via the Franks in the second half of the fifth century. The information in the schedules supports this view and it is noticeable that the beads rarely spread very far afield from their presumed regions of entry of Kent, Sussex and the Thames estuary (Map 6). Subtype (b), though, reached as far north as some of the Anglian burial sites in Yorkshire (Map 7). As far as the continental source of origin is concerned, as mentioned above and in the Introduction, there is the glass-house for bead-making at Trier and operating as early as the third century, in which beads of this type were made (Loeschcke 1925).

Evison (1955, 171, fig 4.15) has noted that the petal-like design on the Rainham (Essex) bead had affinities with a similar design on fifth-century glass bowls from northern France, and the same applies to a 'sword-bead' possibly used as a spindle whorl from Long Wittenham (Berkshire), grave 35 (Meaney 1981, 201, fig VI.k.1). Unfortunately, the various beads of this general type illustrated by TempelmannMączyńska from central Europe were nearly all stray finds, but further to the north west there are three plano-convex ones from Pry (Bequet 1895) and another from Bioul, both in Belgium (Anon 1870-1, 354-8; Anon 1895, 374; Anon 1900, 467). Then at Drenske, in the Netherlands, two of these beads are shown in a painting made in the field of a burial with a fifth-century equal-arm brooch (Assen Museum). Others came from sites in Germany, such as Alternerding in Bavaria (Sage 1984); Gelbe Bürg (Dannheimer 1962, 29, 172, Taf. 18.12 and 13), with both subtypes (a) and (b); and Rittersdorf grave 95 (Böhner 1958, 218-19, Taf. 61.5). A fifth-century date would be appropriate for these and also for several found with late Roman military equipment in the Thorsberg ritual deposit (Raddatz 1958).

The same design also occurs on a bronze pendant from the seventh-century cemetery at Shudy Camps, Cambridgeshire (Lethbridge 1936, 12, fig 4.G.1), and two related designs can be seen on glass 'beads' from the same county (Ashmolean, Evans Collection, nos 1909.224 and 309). Both subtypes (a) and (b) are recorded 
from the Merovingian Frankish cemetery at Junkersdorf near Cologne (La Baume 1967, 104-5, Taf. 43.1-6), whose earliest burials date to $c .500$. In all probability, most of these beads were barbarian work dating to soon after, or, in some cases, even shortly before, the break-up of the western Roman empire. Those which arrived in England seem likely to have originated in or near Trier.

\section{viii 'Black' (OR DARK) GLOBUlar beads With COMPLEX CROSSED WAVES AND WITH EYES OR SPOTS (AND RELATED BEADS)}

(Plate 2, Map 8, Schedule 2, pp 183-4)

Like so many other beads on a 'black' ground, these too appear to be barbarian products. They fall into two subtypes. Beads (a) are globular, with a diameter usually of about $15 \mathrm{~mm}$, and are decorated with crossed waves in differing colours, with an extra finer line following one of the waves. They have randomly placed eyes, generally in yellow glass. Those of subtype (b) are very similar in decoration, but their form is noticeably pear-shaped. Both were current among barbarian peoples in central and eastern Europe such as the Dacians and the Sarmatians, but they occur only rarely on Roman and early Anglo-Saxon sites in lowland Britain.

The schedule for England shows that several of the subtype (a) beads came from late Roman contexts: for example, villas at Colliton Park, Dorchester (Dorset), and Barnsley Park (Gloucestershire). A closely related bead from a burial at Sycamore Terrace in York formed part of an important group dated to the third to fourth centuries, being found together with a bone artefact bearing a Christian inscription (RCHM 1962, 73, fig 58). The remaining beads listed in the schedule from cemeteries in Norfolk, Northamptonshire and Wiltshire (Map 8) may be even earlier than their contexts suggest. There is also a likelihood that native peoples in Britain may have imitated these beads, using different colours, instead of 'black', while their presence in north Wales may reflect the work of Irish craftsmen who were able to copy in their own idiom.

On the continent they do not appear to be common, though their dating supports an early origin. For instance, one came from a late Gallo-Roman grave at Ville-surRetourne in Champagne (information from Dr I Stead), others from an early sixthcentury grave (M12/B4) at Liebenau near Hanover (Hässler 1983b, 34, 60, Taf. 11.12 and 13); and from several graves at Krefeld-Gellep, including grave 192 with a cone beaker dated to Stufe II (450-525) and grave 9 placed early in Stufe II, c.450 or slightly later (Pirling 1966, 160 and 159, Taf. 18.3 and 8.18). A further example from grave 1500 apparently belongs to the middle third of the fourth century (Pirling 1974, 83-4, Abb. 4.3, Taf. 34.12).

From Trier, where such beads are known to have been made (though no doubt they were also produced in other, as yet unrecorded, bead factories), they could have found their way around the western Empire. Their vogue lasted only until the Franks developed a repertoire of their own, perhaps in the fifth to early sixth centuries. 
Subtype (b) beads (not scheduled or illustrated here) also occur in late RomanoBritish contexts at Barnsley Park (Gloucestershire) Roman villa (Webster and Smith 1982), Lansdown in Bath (noted by Guido in about 1948, but now lost) and the late Roman cemetery at Lankhills, Winchester, where a necklace in grave 323 was dated c.350-370 (Guido 1979, 293 and 300, fig 86.436f, colour plate Ia).

Among examples known from the continent, some came from Krefeld-Gellep (Pirling 1966), and others from Schretzheim (Koch 1977, 206, Farb. Taf. 3.33.17), as well as from Mogoşani in Dacia dated to the late fourth century (Diaconu 1969, 389-92, Abb. 17.1). It is surprising to find what appear to be identical beads from the Viking site at Birka on Lake Mälaren in Sweden (Arbman 1940-3). Grave 557 on this site produced one example, and others could be survivals from a nearby site occupied c.400 to 550 .

\section{ix 'Black' globular BeAdS With White or COLOURED CROSSED WAVES, WITH OR WITHOUT EYES OR SPOTS}

(Plate 2, Schedule 2, pp 184-6)

The schedules for England indicate that, like most other 'black' beads, these entered this country as early as the fifth century (or still earlier in the south) and became more widespread and popular during the sixth century.

Perhaps of Rhenish origin, they are well dated at Krefeld-Gellep: for example, grave 530, c.400-450 with other 'black' beads; grave 9, c.450; and grave 1818, c.525 to 600 (Pirling 1966, Taf. 46.20 and 8.18; 1974, Taf. 58.7). The last date range might be corrected to $c .530 / 40$ to 590 , if one follows a revision proposed by Ament (1976) to the absolute date framework of Böhner's 1958 seriation scheme. The implications of this have been discussed for Krefeld-Gellep by Dickinson (1980), while Siegmund (1982) has offered a rephasing of the Merovingian sequence here. An overall date range of $c .400$ to 600 also applies in Frisia: for example, Wetsinge (in Groningen Museum) and Zweeloo in Drenthe (van Es and Ypey 1977, 116-20, Abb. 5, 9 and 10, colour plate on $\mathrm{p}$ 105). It is around this time that they also appear at Pry in Belgium (Namur Museum) and in Anglo-Saxon England. They seem to occur rather later on Alamannic sites in Württemberg, where Veeck (1931, 51-2, Taf. 32.2 and 34.2) recorded them, while at Schretzheim, Koch (1977, 204 and 207, Farb. Taf. 2.25.3 and 4 and 3.34.22) placed them as early as her Stufe $2(545 / 50$ to 565/70) in grave $583 \mathrm{~b}$. At Marktoberdorf, in grave 167, they were placed in a context of around 580 to 640 (Christlein 1966, 71-3, 147-8, Taf. 41A and 64.1). On the other hand, a somewhat earlier period may account for the beads from the Gelbe Bürg (Dannheimer $1962,29,172$, Taf. 18.9, 11 and 12). Although the crossed-wave motif in many colours and forms lasted at least from the fifth century into the Viking period, in all probability the majority of those on a 'black' ground can be assigned to the sixth century, with a few continuing to be buried in the seventh century. 
$\mathrm{x}$ 'Black' beads With a fEW spots (MOstly anNular beads)

(Plate 2, Schedule 2, pp 186-7)

These were never popular in England and often they are too rare to allow hazarding a closer date range beyond a general 'safe' ascription to the sixth century.

xi 'Black' globular beads with Coloured specks ('Crumb'

BEADS) (Plate 2, Map 9, Schedule 2, pp 187-8)

The schedule of these 'crumb' beads clearly reflects a chronological horizon in England centred within the sixth century, though the Mucking cemetery does provide the first half of the fifth-century context of grave 989 (Evison 1981, 138-9, figs 4 and 5). Their distribution is concentrated in south-east England, East Anglia, the Midlands and as far north as Yorkshire, with outliers in Cornwall, Wales and Scotland (Map 9). It is not easy to find a source for them on the continent. The Franks never favoured them, nor, it seems, did the generality of northern peoples, in contrast to the Anglo-Saxons in England. One must bear in mind, however, the relatively small amount of study so far given to continental beads of the date under discussion. We are also hampered by not yet having the precise location and output of the factories, though perhaps some were made in England.

Certainly there were factories near the Danube and Tempelmann-Mạczyạska (1985, 47-8, Taf. 4) has grouped these beads as her type 198. They are not densely concentrated, but instead widely scattered, mostly in eastern Europe, and are possibly of Slavonic origin. They were commonest in the Roman Iron Age (the earliest she cites being from a context of AD 70 to the mid-second century), and they were still continuing to be buried in the early fifth century at the point where her study stops. A dated example was found in a late fourth-century Gallo-Roman grave at Ville-sur-Retourne in the Ardennes region (information from Dr I Stead). They were certainly being made at Tibiscum (Theiss) near the Danube in Romania (Benea 1983 and 1997). Possibly Roman soldiers or camp followers and their families from Dacian or Sarmatian areas introduced them into Roman Britain. They could then have been copied by the native craftsmen here, for of all the decorated glass beads, it seems that these 'crumb' beads would have been the easiest to make.

(For a variant version made in the same way on a blue ground, see Chapter 6: type xi.)

\section{xii 'BLACK' DECORATED LONG CYLINDER BEADS}

(Plate 3, Schedule 2, pp 188-9)

In shape, these are cylindrical, about $22 \mathrm{~mm}$ long and with a slight medial swelling. The ground colour varies: it may be very dark blue or olive green, but the effect is certainly 'black'. The decoration in a contrasting colour consists of a band round each end, and a zigzag or irregular lines in between.

The British examples seem to begin c.AD 400 and they were present at Richborough (Kent) and Cirencester (Gloucestershire), both Roman sites with a 
relatively late military occupation. By the time of the sixth-century Anglo-Saxon cemetery contexts, perhaps we should regard them as heirloom items, for example, at Petersfinger (Wiltshire) and Risby near Bury St Edmunds (Suffolk).

A continental origin is clear, for they were made around the fourth century at Trier (Loeschcke 1925). One bead, probably from a seventh-century context at Camerton (Somerset), has a wire-drawn scalloped or ogee design, which stands out from the rest. Beck (1933) suggested a possible Syrian origin for it, for the type continued to be made in Syria into the Roman period and they are fairly common in the sand-dunes of the Palestinian coast. Certainly this is not impossible and, as already mentioned ( $p 18$ ), there is evidence to suggest that Syrian craftsmen were employed at both Cologne and Trier.

\section{xiii LoNG fLUted BeAds Banded IN 'BLACK' AND WHite}

(Plate 3, but not scheduled)

One of these, though atypical in being two colours of fawn, was found at Sewerby (Yorkshire) and, categorized as type $\mathrm{C} 1 \mathrm{~b}$, is dated broadly to the sixth century (Hirst 1985, 66, fig 23). Another was recovered at Richborough (Kent), but without a recorded context (Richborough Museum, no. 575), while a third has been reported from East Anglia. Only a few comparable beads have been noted from the continent. The author (Guido) saw one of late sixth to early seventh-century date from Müngersdorf (Fremersdorf 1955, 80-90), but they also occur in the excavated late Roman Trier glass workshops (Loeschcke 1925). A further bead, in the collection of the French National Antiquities Museum, Saint Germain-en-Laye (no. 40621), but without context or provenance, is labelled as Frankish and attributed to the sixth to seventh centuries.

xiv SMALl CYLINDRICAL DARK BEADS With UNMARVERED Yellow ENDS AND CENTRAL Band (Plate 3, but not scheduled)

The colour may possibly be a very dark green, but only two beads are known to come from England: a stray find from Probus, Cornwall, and an identical find from a high-status Middle Saxon period settlement (c.650-850) at Staunch Meadow, Brandon (Suffolk). On the continent, close parallels can be observed from Avar cemeteries in central Europe (for example, Kiss 1975) and in Scandinavia from sites of the seventh to eighth centuries such as Helgö in Sweden and Ribe in Jutland (Näsman 1984 and refs).

\section{Large Dark globular beads With SEVERal lines of zigzags} IN OTHER Colours (Plate 3, Schedule 2, pp 189-90)

Although beads of this type are generically the same, in fact no two are quite identical. The earliest examples the author (Guido) found on the continent were made in the Barbaricum during the later Roman period, while many on a 'black' (or dark green) ground were made in Trier (Loeschcke 1925). In the Tempelmann- 
Mạczyńska classification (1985, 52-8, Taf. 7 to 8), they are types 292 to 303 and were made in various colours, sometimes being light and translucent. On very rare occasions they found their way to England, to be buried between $c .450$ and 600 . One from Abingdon, Berkshire, was found with a pair of small gilt square-headed brooches datable to the first half of the sixth century.

XVi TALL DRUM-SHAPED DARK BEADS WITH SEVERAL LINES OF COLOURED Zigzags (Schedule 2, p 190)

This is a more drum-shaped version of the type $\mathrm{xv}$ beads, and was also being made in Trier as early as the fourth century. One example, from Porchten, was illustrated by Böhner (1958, 80, Taf. 9.20) and dated by him as late as the seventh century (Stufe IV). A similar late date seems to apply to an example from Buire-sur-l'Ancre in Picardy (Boulanger 1902-5, 148, plate 40.7). Others are known from grave $91 \mathrm{~b}$, of sixth to seventh-century date at Müngersdorf (Fremersdorf 1955, 80-90, Taf. 16.9, $110.1,117.7$ and 133.6), and from Alternerding (Upper Bavaria), again attributed to the sixth and seventh century (Sage 1984). Earlier examples are known from Drenske, found with a fifth-century equal-arm brooch (Assen Museum) and one from Dębczyno, Poland, which could have been made in Trier (Stawiarska 1984). The most accurately dated comes from grave 426 at Schretzheim (Koch 1977, 205, Farb. Taf. 3.29.7), placed in Stufe 2 (545/50 to 565/70). Clearly, these beads enjoyed a long period of popularity. 


\title{
Chapter 3
}

\section{White (OR BLUE-WHITE) \\ GLASS BEADS}

\author{
MONOCHROME \\ i Opaque white annular, globular and short barrel-shaped beads: singles or \\ doubles \\ ii Opaque white cylinder, round and polygonal short beads: singles or doubles
}

\section{Polychrome}

iii Opaque white beads: (a) with light blue or turquoise crossed waves without dots, singles or doubles

(b) with light blue crossed waves and blue dots

(c) with light blue crossed waves and red dots

(d) with green or terracotta crossed waves, with or without dots

iv Miscellaneous opaque white beads with spots, single waves, etc (not scheduled)

$\mathrm{v} \quad$ Opaque white globular or biconical beads with red or blue spots

vi Opaque white cylinder beads with coloured wound bands

Although known to the Celtic peoples, and even much earlier, from the tenthcentury BC site of Frattesina, near Rovigo, in the Adige valley (Henderson 1988, 440-1), white opaque glass beads did not attain real popularity until after the western Roman empire had collapsed. Even then, they only seem to have been made in any quantity in the sixth century. Unfortunately, the bulk of opaque white glass in Britain comes from old and ill-documented excavations, so the white glass considered here may occasionally turn out, on closer inspection, to be chalk, meerschaum or bone. Of course, the effect given by the presence of white beads in a strung necklace was very much the same whichever material was used. A little opaque white glass was used in the late La Tène period in Britain for decorating beads and armlets (Guido 1978, 8).

Other than for such decoration, its use in the repertoire of northern Europe was limited in the Roman period as well. A few instances of the appearance of white glass may be noted though; for example, some beads have been recorded at Esch (North Brabant) and published by L J A M van den Hurk (1980, 374-8, fig $6 \mathrm{j}$ and 1$)$. They 
were small, globular and long oval in type and came from a necklace attributed to the second or third centuries on the basis of datable vessels. These are atypical beads, however, in terms of both form and dating.

A little later, between the second and fourth centuries, white beads have been recorded from Roman and post-Roman Tibiscum at Theiss on the Danube, a site important for its trade contacts with the peoples of central and eastern Europe. Here, as mentioned in the Introduction, evidence for their manufacture has recently been discovered in typical Roman forms (see Guido 1978, fig 37), in white and other colours (Benea 1983 and 1997). It may be suggested that some of these were traded up-river to reach the Alamannic territories in south-west Germany around Württemberg (Veeck 1931, 51-2, Taf. 33 and 34). From there, trade in these beads may have begun down the Rhine, for beads of annular and globular form with crossed waves appear in areas further north, around the lower Rhine, Frisia and the Netherlands during the sixth century. The introduction of the crossed-wave motif, so common in various colours in northern countries in the sixth century, may have come from Slavonic lands. It was a design that influenced the bead-makers at Trier as early as the third to fourth centuries, for new fashions evidently began to reach that area by the late Roman period (Loeschcke 1915).

It was probably from Frisian and Frankish territories in the Low Countries that monochrome white beads reached the south of England. As already discussed in the Introduction, a factory that evidently produced them around AD 600 has been identified at Rothulfuashem (Bloemers et al 1981, 132), near Leiden, in the Netherlands. Though not yet fully published, the workshop appears to be contemporary with many datable cemetery finds in England. In the cemetery at Buckland, Dover, such white beads (sometimes very slightly tinted light blue) first appear in Evison's phase 3 (575-625) and perhaps continue into the early eighth century of phase 7 (700-750). Some of these Dover beads may even have been made at Rothulfuashem or another related production centre operating in the Low Countries. There is also a strong possibility that certain decorated white beads may have been made in England as well as on the continent (see type iiid on p 33 below). It should be noted that glass-bead production seems to take place in separate glass-houses from those which manufactured glass vessels.

\section{MONOCHROME}

i Opaque white anNular, gLOBUlar and SHORT BarRel-SHaped BEADS: SINGLes OR DOUbLes (Plate 3, Schedule 3, pp 191-5)

Mostly quite small, and occasionally slightly barrel-shaped (owing to pressure, perhaps, while the glass was still viscous), all these bead types were common in England, as on the continent, between the fifth and seventh centuries. There is a later fifth-century context at Mucking (Essex) in grave 397, and late fifth to sixthcentury contexts at graves 351 and 845 there and again at Long Wittenham 
(Berkshire) in graves 52 and 96. Firmly sixth-century contexts occur at Alfriston (East Sussex) in grave 28 and still later at Prittlewell (Essex).

The distribution reveals concentrations in both Kent and Lincolnshire, and from the Thames estuary upstream as far west as Lechlade (Gloucestershire). Some at least reached Wiltshire (for example, Blackpatch, Pewsey) as early as c.500, but very few occur in Yorkshire or north-east England.

\section{ii Opaque White CYLINDER, ROUND AND POLYGONAL, SHORT BEADS: SINGLES OR DOUBLES}

(Plate 3, Map 10, Schedule 3, pp 195-7)

Nearly all of these are likely to be marginally later than type i beads and they seem to have reached England from the Rhineland and the Netherlands. Ultimately, many may have originated from south-west Germany much further upstream. Large numbers of cylinder beads were found at Schretzheim, reaching a maximum in Stufe 4 (590/600-620/30): for example, grave 149 (Koch 1977, 36, Taf. 34.1). Among the earliest dated beads is one from Krefeld-Gellep: grave 2111 (Pirling 1974, 119-20, Taf. 81.3), dated to the end of Stufe III and the beginning of Stufe IV, ie, c.590-610, or perhaps a little earlier.

The forms are late Roman ones, with the exception of the double-short cylinders, which may have originated in the Württemberg area. Their relatively late date, which appears to be limited to $c .590-700$, hardly suggests a combination with Roman types, unless they were inspired from some area where Roman material culture lingered very late.

In England there is the later fifth-century context of Mucking grave 334, but the majority were probably imported into Kent between the sixth and seventh centuries; a few reached East Anglian cemeteries and Anglian sites as far north as Yorkshire (Map 10). At Buckland, Dover, short cylinders fall within an overall date range of 575 to 725 (phases 3 to 7 ).

\section{POLYCHROME}

iii Opaque white beads: (a) With light blue or tURquolse CROSSED WAVES, WITHOUT DOTS, SINGLES OR DOUBLES

(Plate 3, Map 11, Schedule 3, pp 197-200)

As with many white beads with coloured decoration, crossed waves, both with or without dots in blue or red, were particularly favoured, especially in the Rhineland, the Netherlands and northern France, during the period from the mid-sixth up to at least the mid-seventh century. They were among the beads known to have been made $c .600$ in a factory at Rothulfuashem near Leiden (see above, pp 7 and 31) and thence they reached England, where most are concentrated in the southern counties, the Midlands, East Anglia and as far north as Yorkshire (Map 11) in contexts dated to the sixth and seventh centuries. There are a few examples from Belgium possibly 
as early as the late fifth century, and the same date may apply to a few from south Germany. Quite possibly they were manufactured at a number of centres, but they appear to have had a limited period of popularity.

iii Opaque white beads: (b) With Light blue crossed waves AND BLUe Dots (Plate 3, Map 12, Schedule 3, pp 200-2)

These are not significantly different in date or distribution from the iiia type beads, though Mucking grave 989 is a context datable as early as the first half of the fifth century. There seem to be significantly fewer in Kent than in East Anglia (see Map 12), so in England, perhaps, they may have come from a more easterly factory than that known to have produced the iiia type.

iii Opaque white beads: (c) with light blue crossed waves AND RED DOTs (Plate 3, Map 13, Schedule 3, pp 202-6)

Again, the earliest beads noted on the continent seem to come from Bavaria and Württemberg in south-west Germany, and in grave 59 at Marktoberdorf in Allgäu, such beads came from a late sixth or early seventh-century context (Christlein 1966, $71-3,120-1$, Taf. $15 \mathrm{~A}$ and 61.2 ), and by that time they had also become common in the Frankish lands to the north. Sonia Hawkes refers to them as having existed at Schretzheim and in the Trier region 'only during the first three or four decades of the seventh century' (Matthews and Hawkes 1985, 97), but some that were certainly earlier were found at Zemmer (Böhner 1958, 80, Taf. 9.21) in a context belonging to Böhner's Stufe III (525-600).

Generally speaking, both in the northern lands and in England (see the schedule), their chronological position agrees with that postulated for other crossed waves on a white ground, ie, $c .500-650$, though a significantly earlier fifth-century context does occur at Mucking, in grave 989 . There are many in Kent and it was probably via that kingdom that they were imported and distributed across most of Anglo-Saxon England (Map 13).

iii Opaque white beads: (d) with green or terracotta CROSSED WAVES, WITH OR WITHOUT DOTS

(Plate 3, Map 14, Schedule 3, pp 206-8)

Earlier remarks about dating for other beads of type iiia-c may hold good for these as well. The only outstanding difference is in terms of their distribution. This is more East Anglian than Kentish (see Map 14) and it is quite possible that they represent local variants made in Norfolk and Suffolk.

iv Miscellaneous opaque white beads with spots, single WAVES, ETC (Plate 3 but not scheduled)

These are suggested here to be contemporary with beads of type iiia-d above. 


\section{v OPAQUe WHITE GLOBULAR OR BICONICAL BEADS WITH RED OR BLUE SPOTS (Plate 3, Schedule 3, pp 208-9)}

The spots on these beads are larger than the dots described above for beads of types iiia-d and normally are red. These beads are relatively few in number and they vary considerably in size and shape. For this reason, it cannot be claimed that individual examples are necessarily of a similar date.

On the continent they appear to be relatively unusual. Dated examples may be cited from Schretzheim (Koch 1977, 198-9, Farb. Taf. 1), both (her types 3 and 4) from graves dated to Stufe 1 (525/30-545/50); several in the Rhineland came from Junkersdorf near Cologne from both sixth-century graves (246, 344 and probably 373 ) and the seventh-century grave 128 (La Baume 1967, 46-9, Taf. 15.246.3; 22.344.1; 24.373.1; and 6.128.1). All these are slightly biconical in form, as might be expected in the seventh century and shortly before. What may be a rather earlier one, and very close to the Chamberlain's Barn I example, came from Liebenau near Hannover (Hässler 1983b, 42, Taf. 87: type 30.6). Another was recorded from the Trier region at Zemmer, in grave 37/1 from Böhner's Stufe III, AD 525-600 (Böhner 1958,81 , Taf. 9.24).

It seems likely that they were Frankish in origin, and they may have been inspired by a kind of Gallo-Roman bead (Boulanger 1902-5, 16, plate 6.7) found in northeastern France. In these, however, the spots were painted instead of being applied in opaque glass. The vogue for coloured spots on a white ground lasted into the seventh century and probably later, and an example from the seventh to eighth century occurs in the museum at Laon (France).

As far as England is concerned, the best dated beads of the type come from Puddlehill, Dunstable (two beads in grave 10 , dated $c .600$ by a saucer brooch pair), and two from Leighton Buzzard of approximately the same date, but unusually large and biconical. Three very small examples were recently found at Wasperton (Warwickshire) (Crawford 1981, 1982 and 1983; Esmonde Cleary 1989, 201, fig 48), in a grave with saucer brooches of mid-sixth-century date, but it is possible that the brooches may have been made some years earlier than their deposition.

On the available evidence, then, it seems likely that these spotted beads came from the Rhineland in the sixth to seventh centuries, and in England date likewise between approximately 500 and 650 . They are not sufficiently common for any deductions to be drawn from their distribution.

\section{vi Opaque White CYLINDER BeAdS With COLOURED WOUND BANDS (Plate 3, Schedule 3, pp 209-10)}

These beads were present at Schretzheim (Koch 1977, 208, Farb. Taf. 4) being types 42.2-42.4, all with light blue bands on white, and dating from the sixth to the seventh centuries. At Krefeld-Gellep (Pirling 1966, 160, Taf. 21.5e), one with green bands from grave 224 is dated to Stufen III/IV (c.600), and they are attributed to the seventh century in the Burg Linn Museum at Krefeld, as they occur on a necklace 
with opaque orange beads of this date. Although probably Frankish, these beads were never very much in demand, and seldom occur in Holland or Belgium, though one with sky-blue bands was found at Honnay Revogne in a context of the fifth to sixth century and is now in Namur Museum.

In England, the schedule points to a sixth-century date, perhaps slightly overlapping with both the fifth and seventh centuries. Most of them have light blue bands. 


\title{
Chapter 4
}

\section{OPAQUE YELLOW GLASS BEADS}

\author{
MoNochrome \\ i Opaque yellow annular, globular and barrel-shaped beads \\ ii Opaque yellow cylinder beads: (a) round \\ (b) polygonal or square-sectioned \\ (c) short cylinder, singles or doubles \\ iii Opaque yellow biconical beads \\ iv Opaque yellow melon, sub-melon and 'nicked' beads \\ v Opaque yellow wound beads, or joined globular beads \\ Polychrome \\ vi Opaque yellow beads with coloured crossed waves, with or without dots, singles \\ or doubles \\ vii Miscellaneous opaque yellow decorated beads
}

Opaque yellow glass was popular in lowland Britain in two distinct periods, with a hiatus during the Roman occupation. Visually, the Iron Age and Anglo-Saxon beads of this glass can be easily confused in the absence of a distinguishing archaeological context. Fortunately, though, they can be distinguished analytically with more accuracy. During the last four centuries BC yellow beads, particularly annular ones, were made in large numbers at Meare in Somerset (Guido 1978, 16-17, 33, 73-6, plate II; Henderson 1987b), but during the Roman occupation they were no longer popular and only a few survived into the first century AD. The production of similar beads recommenced in the Anglo-Saxon period, however, and they gradually reached the peak of their popularity in the sixth century or thereabouts. We know that some of these were still being made around AD 600 at Rothulfuashem (Bloemers et al 1981, 132) near Leiden in the Netherlands, a workshop site discussed in the Introduction. As yet, we have no direct information of any other workshops making them either on the continent or in England.

Antimony was widely used in the late Iron Age yellow glass beads, but in a discussion of the mainly sixth-century Anglian beads from Sewerby (Yorkshire), Leo Biek has observed that the yellow opaque beads from this site, whether monochrome or polychrome, were coloured by lead-tin oxide (Biek et al 1985, 82). This suggests that none were residual Roman (period) beads, as these would have been 
made using antimony. Rooksby (1964) thought that lead-tin yellow did not seem to have appeared before c.500. More recent analyses, especially by Henderson and Warren $(1983)$, have led Biek $(1983,309)$ to propose that we need, rather, to think in terms of a central European (tin) tradition, now recognized as early as the second century BC, as distinct from a 'Mediterranean' (antimony) tradition. This proposition may well prove to be equally oversimplistic, but it reflects the present evidence.

Though extremely rare in Britain during the Roman period, opaque yellow beads do occasionally occur by the end of the fourth century. For instance, just one small bead was found among the masses discovered with late Roman burials at Lankhills, Winchester (Guido 1979, 298, fig 80.215), which dates from c.300-350 (grave 199), but there is no analytical pointer. Several others were found with a fourth-century necklace at Verulamium (Wheeler and Wheeler 1936, 214, fig 47.67.1). All of these were presumably continental imports. Many small annular and globular beads have recently been excavated from a late Roman and early Germanic Iron Age cemetery at Sejlflod near Aalborg in north-eastern Jutland, Denmark (Nielsen and Rasmussen 1986). These are thought to have been brought there either by immigrants from the Rhineland (where such types are known from Krefeld-Gellep in the first half of the fifth century), or through international trade and exchange, around AD 400 (information from the excavator, Jens Nielsen).

The forms of the great majority of opaque yellow beads are conspicuously Roman in tradition: ie, polygonal, hexagonal, cylindrical and biconical shapes. Theoretically, one might suppose that they originated somewhere within the former bounds of the Roman empire. Imports from the Rothulfuashem factory and other contemporary workshops in the Low Countries may account for the large numbers in England, with over 300 beads in various forms having been recovered from graves at Buckland, Dover (Kent). Those recognized so far at Rothulfuashem include annular and triple forms (types $\mathrm{i}$ and $\mathrm{v}$ below). Most of the Anglo-Saxon yellow opaque beads belong to sixth-century contexts, with a concentration in phase $3(575-625)$ at Buckland (Evison 1987, 61-82). They continued in use right through the seventh century, even when burial with glass beads was becoming much rarer in the so-called Final Phase cemeteries (Hyslop 1963, 173, 191, 198-9, fig 9.9c).

Relatively few Middle Saxon settlement sites have been excavated as yet and it is too early to claim that when yellow beads were no longer appearing in grave contexts they had also gone out of fashion in everyday dress. In fact, visually comparable beads were produced in Scandinavia and exported all over the Baltic region with a date range of the seventh to eighth centuries (Lundström 1976 and 1981; Näsman 1984). On the other hand, their occurrence in Rhenish sites from the Netherlands, upstream through Germany and across to Alamannic sites in the upper Danube region, tends to be earlier, as in England.

A notable feature of beads with a yellow ground colour is a frequent combination with green (see $\mathrm{p} 40$ ). This combination was particularly favoured in East Anglia, yet on the continent this particular combination is unusual. 


\section{MONOCHROME}

\section{i Opaque Yellow anNular, globular and barRel-shaped} BEADS (Plate 4, Map 15, Schedule 4, pp 211-18)

These forms have been considered together, since it is often almost impossible to distinguish clearly between them. This is particularly the case with badly made beads, or those which are not easily recognizable from old illustrations or descriptions. The majority are globular, about $10 \mathrm{~mm}$ or less in diameter, and some are rather barrel-shaped owing to a slight flattening of the upper and lower surfaces of the bead.

On the continent they may be found as early as around 400 in the Barbaricum (Tempelmann-Mączyńska 1985, 18, 27-31, Taf. 1), and we know that c.600 they were among the types being produced at Rothulfuashem (see p 36 above). At Krefeld-Gellep, in the lower Rhineland, they can be dated broadly to the sixth century. Most examples have been attributed there to graves in Stufe III (c.525-600, perhaps correctable to $c .530 / 40-590$ ): for example, graves 442 and 810 , while grave 189 belongs to the overlap between Stufen II and III (c.520-40) (Pirling 1966, 157-8, Taf. 19.5, 36.6, 70.11) and grave 2111 to the overlap between Stufen III and IV (c.590-600) (Pirling 1974, 119-20, Taf. 81.3). They are also very common in south Germany in that same century.

In England, the small globular beads have a particularly Kentish distribution near the probable trading beach markets. They are also known from cemeteries throughout southern England, East Anglia, the Midlands and as far north as the Tyne valley (Map 15). Eight rather similar beads from Wallingford may have come from a slightly earlier context (later fifth century), but in these particular cases the bead core was of translucent glass. On the whole, the majority of examples in England belong to the later fifth and sixth centuries, with quite a few also in seventh-century contexts.

\section{ii Opaque yellow cylinder beads: Round, Polygonal or SQUARE-SECTIONED, AND SHORT CYLINDERS, SINGLES OR DOUBLES \\ (Plate 4, Schedule 4, pp 218-21)}

The earliest examples found on the continent come from Württemberg, in late fifth-century contexts, but these are shaped wider in relation to their length than their equivalents in England. Still in south-west Germany, at Schretzheim, they began in a small way in Stufe 2 (545/50-565/70) (eg, grave 258 (Koch 1977, 60, Taf. 68.6)) and reached their greatest popularity in Stufe 4 (590/600-620/30) (eg, grave 149 (Koch 1977, 36, Taf. 34.1)). Of course, this chronology need not be strictly applicable to England, though the dating seems to be similar, but a rather earlier context no later than the middle of the fifth century is supplied by Mucking (Essex), in grave 989 .

Since these beads have been recorded from the Low Countries, where they are 
rare, and also from Junkersdorf near Cologne in the sixth century, a reasonable hypothesis is that most of them came into England from Frankish sources in the sixth century.

\section{iii Opaque yellow biconical beads}

(Plate 4, Schedule 4, pp 221-2)

Less common than the types discussed above, these seem to have been current exclusively in the sixth to seventh centuries. A few are known from the Trier district and some from Belgian cemeteries at Lessive and Pry, now in the Namur Museum. On the other hand, they do not seem to be represented either at Krefeld-Gellep or at Schretzheim in Germany. Little biconical yellow beads of much smaller size are known from the late Roman period, but should not be confused with those under discussion since they were made of translucent glass (Guido 1978, 97-8, fig 37.12 and 13).

iv Opaque yellow small melon, sub-melon and 'Nicked' beads

(Plate 4, Schedule 4, pp 222-3)

These beads are roughly 'nicked' rather than being accurately gadrooned, and the nicks only form a band round the centre of the bead. With the exception of those from Alamannic areas around Zürich and at Schretzheim, few have been found on the continent. Two points are worthy of mention: firstly, Spong Hill in Norfolk (Hills et al 1984) produced them both in opaque yellow and in other colours, and they may have been made in that district. Secondly, there is no concentration in Kent to suggest that they were imports from the continent. Further finds are needed to elucidate their origins and development. Again, their date is likely to fall in the sixth century and their origin may ultimately have been Alamannic.

\section{$v$ OPAQUe yellow WOUND BEADS, OR JOINED GLOBULAR BEADS}

(Plate 4, Schedule 4, pp 223-4)

There are four different kinds of segmented beads: (a) those produced by the crimped method, as used in the well-known Bronze Age segmented beads, and the gold-in-glass beads (discussed in Chapter 13); and (b) a late Roman variety, in which an unperforated rod was wound round a horizontal wire. The bead tapered as the rod was drawn thinner before being removed when cool. Neither of these first two variants was common in England in the post-Roman period, after c.450.

In the Anglo-Saxon period, the commonest method of manufacturing segmented beads is represented by (c): beads with larger double or multiple globules. These were also known in other colours as well as opaque yellow. Largely found in the Rhineland and the Netherlands, and also in north Germany at least as far east as the Elbe, they are dated to Stufe III (c.525-600, perhaps correctable to c.530/40-590) at Krefeld-Gellep in graves 267, 1953 and 1996 (Pirling 1966, 158, Taf. 25.4; 1974, 119-20, Taf. 72.16 and 76.1). Further south, at Schretzheim, these beads are slightly 
later, placed in the first half of the seventh century (Stufen 4 to 5): for example, graves 347 and 393 (Koch 1977, 80 and 90, Taf. 88.7 and 104.9). They are also known from the Viking period (see Chapter 6: type vi). In England, though, they seem to be broadly contemporary with those from Krefeld-Gellep, but continue into the seventh century. Both areas may have imported the beads from the Rothulfuashem factory $c .600$ (see $\mathrm{p} \mathrm{36}$ ).

The last type, (d), seem to have been made by winding a rod round an upright. This method resulted in very irregular beads, for although the coils are approximately the same size, some toppled over the ones below in the course of manufacture. Such beads are very rarely recorded in England, though they are not uncommon in northern Europe. Böhner (1958, 71 and 76, Taf. 8.22), referring to the Trier region, pointed out that they were limited there to the seventh century.

\section{POLYCHROME}

\section{vi Opaque yellow beads With COlOURED CROSSED WaVes, WITH OR WITHOUT DOTS, SINGLES OR DOUBLES \\ (Plate 4, Map 16, Schedule 4, pp 224-7)}

On the continent, particularly in the Rhineland and the Netherlands, these annular beads with a crossed-wave decoration, generally in terracotta red, are of sixthcentury date, as are most of the English examples, though grave 334 from Mucking provides a later fifth-century context. There is one possibly significant difference, however, between these and the English ones, for whereas terracotta waves are also common in England, there is a group, mostly from East Anglia, which has green waves instead. This latter group is not so commonly represented on the continent, so once again we have the suggestion of a specifically East Anglian fashion for combining green and yellow on glass beads. Possibly they were made here (see $p$ 37) and it seems probable that they reached Kent from East Anglia. The beads from Kent seem to be marginally later in date, but the sixth to early seventh centuries appear to have been the period of their greatest popularity. They occur as far west as Gloucestershire and Wiltshire and as far north as Yorkshire (Map 16).

\section{vii Miscellaneous opaQue yellow DeCorated beads}

(Schedule 4, pp 227-9)

Here the beads listed in the schedules must speak for themselves, as they do not represent a specific class. Most probably, they belong largely to the sixth and seventh centuries. As mentioned above, there is the combination of green with yellow, which again points to a possibility that these beads were made in East Anglia (see p 37). Among the earliest are some from Wallingford (Berkshire) - grave 15 - together with applied saucer brooches of Böhme's Great Chesterford type, dated by both Welch (1975) and Böhme (1986, 545-7, Abb. 62) to the second half of the fifth century. 


\section{Chapter 5}

\section{GREEN AND TURQUOISE GLASS BEADS}

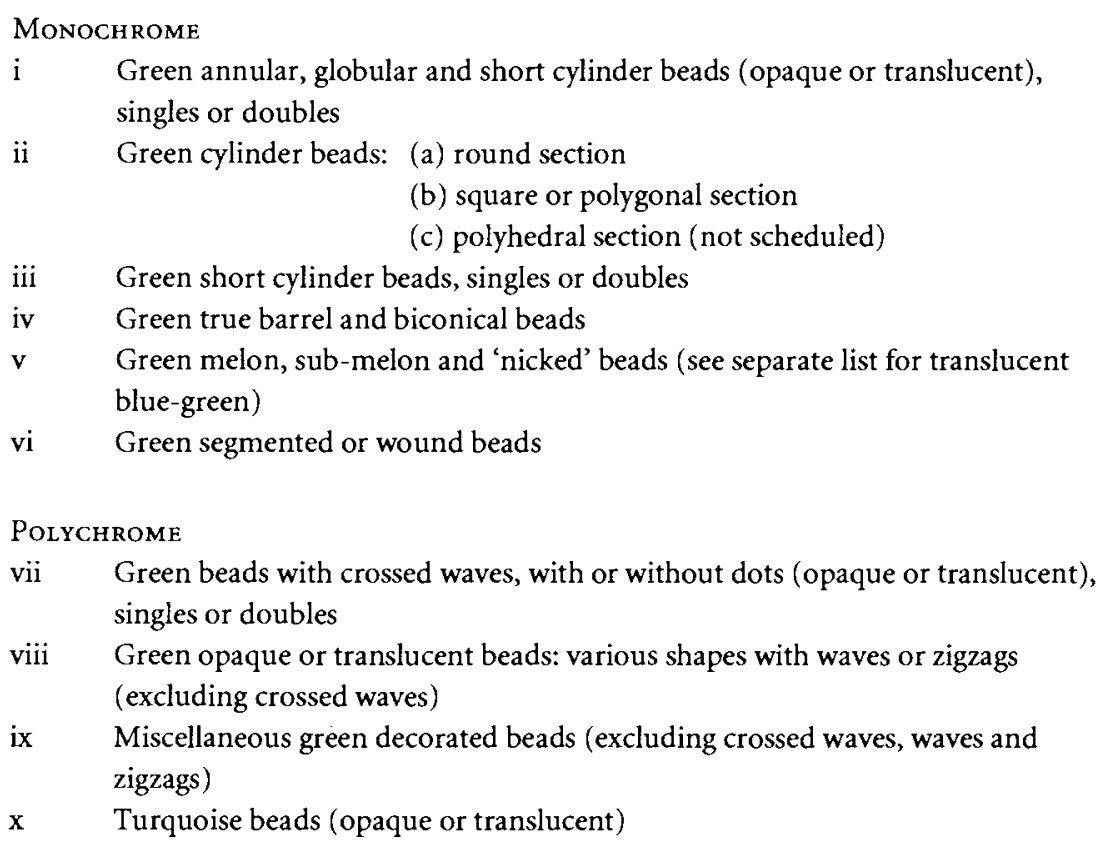

In his discussion of the beads from Sewerby (Yorkshire), Biek (et al 1985, 83) noted that there was an underlying similarity that allows turquoise and green examples to be considered together. All turquoise and all dark green beads were really translucent though not appearing to be so - opacity being apparent rather than real. The darker colours are clearly due to cupric copper (in lead glass) whereas this is absent in pale tints, which are, presumably, caused by (manganese and) iron. The gradual distinction between green and turquoise will often be a subjective one. The schedules show that turquoise was not restricted to one or two forms, but was widely used.

Significantly different in both visual and analytical terms, however, are the beads listed in the present study as 'black' (Chapter 2), many of which are an extremely dark olive green. These represent a different tradition, which it seems belong to the barbarian-made glass, as distinct from that produced within the Roman empire. This fact has recently been observed by Henderson $(1990,156-7)$ in his report on 
the beads from Apple Down in West Sussex. One bead of dark green (analysis 26) possessed 'barbaric' affinities and produced a significantly different analysis result from the rest of the sixth-century beads in the Anglo-Saxon cemetery there. It contained mixed alkalis $\left(\mathrm{Na}_{2} \mathrm{O}\right.$ and $\left.\mathrm{K}_{2} \mathrm{O}\right)$ instead of the usual single alkali.

The majority of the undecorated opaque green beads follow a very notable Roman tradition, in both form and colour. This applies not only to Britain, but also to extensive regions on the fringes of the empire. It has been claimed that many such Roman beads from the third and fourth centuries may represent cheap substitutes for emeralds, which also occasionally reached Britain. These beads did not die out with the end of Roman rule, but appear to have continued until the mid-fifth century, when they became more popular again. Even then, they never gained real favour with the Anglo-Saxon population, which seems to have preferred some decorated types not previously recorded. A late Roman context for plain green beads is provided by the multiple burial at Canterbury of c.400. For the second half of the fifth century, there is grave 15 at Wallingford (Berkshire) with its Great Chesterford type applied saucer brooches (Welch 1975; Böhme 1986, 545-7, Abb. 62). Both of these contexts also contained 'black' beads of 'Germanic' types. At Buckland, Dover (Evison 1987, 73-4 and 80), plain green beads are found in graves for every phase of the cemetery (c.475-750), though the few polychrome beads (Evison 1987, 77-8 and 82 ) are predominantly from phase 3 graves (c.575-625), with just one from phase 5 (c.650-675).

On the continent, the earliest post-Roman opaque green beads identified by the author are some cylinders from Holzgerlingen in Alamannic Württemberg (c.450-500) (Veeck 1931, 51-2, 190-205, Taf. 33). Some with a similar date range come from the Trier area in Stufe II (c.450-525) (Böhner 1958, 75 and 81-2), and also from Krefeld-Gellep in the lower Rhine region (Pirling 1966, 1974, 1978 and 1989). The Schretzheim examples date to Stufen 3 and 4 (c.565-620/30), for example, graves 22 and 149 respectively (Koch 1977, 13 and 36, Taf. 8.5 and 34.1).

For the dark olive green beads referred to above, it is necessary to consult the 'black' type and schedules (Chapter 2 and Schedule 2). Grass-green is a conspicuously common tint in East Anglia for various kinds of beads, but particularly when used on a terracotta-coloured background. These are discussed in Chapter 8 and they are thought to have been made locally in that region. Finally, for translucent green glass in various tints, see Chapter 1.

\section{MONOCHROME}

i Green anNular, GLOBUlar AND SHORT CYLINDER BEADS

(OPAQUE OR TRANSLUCENT), SINGLES OR DOUBLES

(Plate 4, Schedule 5, pp 230-7)

Very common in the Roman period (see Guido 1978, 8-13), the Anglo-Saxon beads are only occasionally found as early as the first half of the fifth century (for example, 
Mucking grave 989) on present-day evidence for dating. There is no reason to believe that all the bead factories responsible for them went out of production, though, so we must await further evidence. They would now seem to have been most popular during the sixth and early seventh centuries. (Those described as short cylinder beads may simply reflect the result of slight pressure being put on a globular bead while the glass was still viscous.)

\section{ii GREen CYLINDER BEADS: (a) ROUND SECTION}

(Plate 4, Schedule 5, pp 237-9)

The opaque beads of this form in particular probably continued without a break from the mid-fifth century. There is a late Roman example from the Canterbury multiple burial of $c .400$ and several have been found at South Shields, which are broadly attributable to the fourth or fifth centuries. One in grave 42 at Spong Hill (Norfolk) belongs rather later within the sixth century (Hills et al 1984, 95-7, fig 98) and the majority are from sixth-century contexts. It was made by pinching off to give tapered or pointed ends: a characteristic Anglo-Saxon form, which contrasts with the straight-cut ends of the Roman form. (See also blue cylinders on pp 48-50.) At Schretzheim, they range almost exclusively from Stufen 3 and 4 (c.565-620/30): for example, grave 22 (Koch 1977, 13, Taf. 8.5). The earliest is from Holzgerlingen in Württemberg (fifth to sixth centuries) (Veeck 1931, 51-2, 190-205, Taf. 33).

\section{ii Green Cylinder beAds: (b) SQUARE OR POlygonal SECTION}

(Plate 4, Schedule 5, pp 239-40)

These may also share the same history as the round cylinders, but are far less common. It seems that most hexagonal and pentagonal beads are of sixth to seventh-century date, but they still represent a continuation of a traditional late Roman form and are common in late Gaulish graves of the fourth century in the Aisne valley (Pilloy 1895 , plate 20).

\section{ii Green Cylinder beads: (c) polyhedral Section}

(Plate 4, but not scheduled)

These beads are similar in type to those of $6 \mathrm{iv}$ (see p 50), but green polyhedral beads occur less commonly than the blue type. The provenance of the example illustrated in Plate 4 is uncertain.

\section{iii GREen SHORT CYLINDER BEADS, SINGLES OR DOUBLES}

(Plate 4, Schedule 5, pp 240-2)

From the example illustrated in Plate 4, short cylinder beads can be seen to be both much wider and shorter than the true cylinder beads. They may have developed from the cut-cylinder beads of the Roman period, which, in green glass, were very popular between the third and fifth centuries (Guido 1978, 95). The double beads also became fairly common in terracotta-coloured glass as well as in opaque yellow. 
The majority were made and buried in the sixth to seventh centuries and they are sufficiently concentrated in Kent to suggest that some at least of them were imports.

\section{iv Green true barrel and biconical beads}

(Plate 4, Schedule 5, pp 242-3)

With the exception of an unusually early group of biconicals from the late Roman multiple burial at Canterbury of c.400, these barrel and biconical beads appear to occur consistently in contexts of the sixth to seventh century. Examples of small biconical beads are known from the fourth century in Picardy (France) and Belgium. These are probably still Roman products. True barrel beads seem to belong almost exclusively to the seventh century.

\section{v Green melon, SUb-MELON AND 'NICKed' beads \\ (Plate 4, Schedule 5, pp 243-5)}

With very rare exceptions, the Anglo-Saxon variety is made in a clumsier and often smaller form than the melon beads current in the Roman period. In place of carefully curved gadroons reaching from the top hole to the bottom, the sides of the bead are merely roughly nicked. On the continent, they are most common in the upper Rhine and Danube regions - for example, Schretzheim between the mid-sixth and seventh centuries - and much the same dating is likely for examples from the Württemberg cemeteries. At Krefeld-Gellep some are as early as the mid-fifth century (Stufen I/II), and they continue into the early eighth century (Stufe V).

The best-dated English examples are the type B43 beads from graves 129 and 141 in phases 5 and 6 at Buckland, Dover (c.650-700) (Evison 1987, 74 and 80, fig 11). As at Krefeld-Gellep, there are beads here in contexts datable as early as $c .400$ and the later fifth to sixth century. At present we can only record these facts and stress that the majority seem best placed in a bracket of $c .500$ to 650 . They occurred in a number of graves at Mucking and also at Morning Thorpe and at Spong Hill, both Norfolk cemeteries. It would seem, at this stage of the enquiry, that they did not arrive as cross-Channel imports, since it can be seen that they were favoured in East Anglia, but are rare in Kent.

\section{vi Green segmented or WOUnd beads \\ (Plate 4, Map 17, Schedule 5, pp 245-7)}

These beads, which may be opaque or translucent, vary in size and method of manufacture (see Chapter 4: type v). They are likely to have outlasted the early to midfifth century and continued into the seventh century and possibly beyond at Brandon (Suffolk). The Roman examples (see Guido 1978, 95) are rather bigger and better made. They seem to have dwindled in numbers after the second century, before being reintroduced in larger numbers in the sixth century.

Anglo-Saxon segmented beads are made in several differing techniques. There are some very small ones in green glass, both translucent and opaque, and side by side 
with these are many with larger globules, in double, triple or longer units. Of the small variety, generally drawn and crimped, several sites, in Kent particularly, produced quite a number: for example, Faversham (with more than forty), Buckland, Dover (type C04: seventy-six beads), and, in the West Sussex coastal plain, at Highdown (with thirty or more). Some globules are rather angular, but this does not seem to have any chronological significance. The Buckland ones, which seem to be characteristic, appear in one grave in phase 1 (c.475-525), but four graves in phase 3 (c.575-625) and just one in phase 5 (c.650-675). This small type almost surely came across the Channel from Frisia, for there are many of them in the museum at S'Herzogenbosch and elsewhere, attributed to a similar date to those from Buckland.

The larger, sometimes perhaps blown, beads also show the same range in date, and were evidently contemporary with the yellow and blue beads in the same form. They clearly lasted into the seventh century, but were much less common than the blue segmented beads.

Overall, the distribution shows concentrations in the south east, in Wiltshire and the upper Thames region, with outliers in Lincolnshire, Yorkshire and Northumberland (Map 17).

\section{POLYCHROME}

vii Green beads with Crossed-Waves, With or without dots (OPAQUE OR TRANSLUCENT), SINGLES OR DOUBLES

(Plate 4, Schedule 5, pp 247-8)

Though a very commonly used motif on a terracotta-coloured or opaque white glass background, they were made only very occasionally on a green background and these never gained popularity. The crossed waves may be white or terracottacoloured. The sixth century probably saw their introduction.

\section{viii GREEN OPAQUE OR TRANSLUCENT BEADS: VARIOUS SHAPES WITH WAVES OR ZIGZAGS (EXCLUDING CROSSED WAVES) \\ (Plate 4, Schedule 5, pp 248-9)}

A very few examples of this form, annular translucent green glass beads ornamented with a simple wave, found their way to Britain in Roman times: for example, Farley Heath, Albury (Akerman 1852, 48, plate V.11), and Newstead (Roxburghshire) in Scotland (Curle 1911, 336-7, plate XCI). These are sufficiently rare to be regarded as imports, probably from Dalmatia on the Adriatic, where they are known in the early centuries AD. There are, however, few examples of waves or zigzags in the AngloSaxon repertoire of the post-Roman period, though they are also found in both the Rhineland and Württemberg.

Attention has already been drawn to the very dark green beads, which appear black with a white or yellow wave or zigzag and sometimes unmarvered (types $2 \mathrm{v}$ and 2vi, see pp 22-3). An early (ie, fourth to fifth centuries) date has been proposed 
for them. It is therefore hardly unexpected to find that the earliest bead in the schedule here is a dark green example from Harwell (Berkshire) dated around 500 (see also 'black'-bead schedule 2, types $2 \mathrm{v}$ and $2 \mathrm{vi}$, pp 176-80).

The green-bead schedule makes it clear that many varied shapes were used that were decorated in this way, which one cannot attempt to classify. Nearly all come from contexts of the sixth to seventh century, and they may be regarded as imports.

ix Miscellaneous green decorated beads (excluding CROSSED WAVES, Waves and Zigzags) (Schedule 5, pp 249-52)

These beads are too individual for any conclusions to be drawn with certainty about their date or culture. Here, the combination of green and yellow have already been discussed (Chapter 4: types vi and vii). The majority of them seem to be largely sixth to seventh century in date, though fifth-century contexts are recorded at Mucking and Spong Hill, and they may have been imported from the Frankish territories.

$x$ Turquoise beads (opaque or translucent)

(Plate 4, Schedule 5, pp 252-4)

Various shapes are listed here, but they probably have little significance, since it is unlikely that turquoise-coloured beads are distinct from other green ones (see p 41 above and Chapter 14). 


\section{Chapter 6}

\section{BLUE GLASS BEADS}

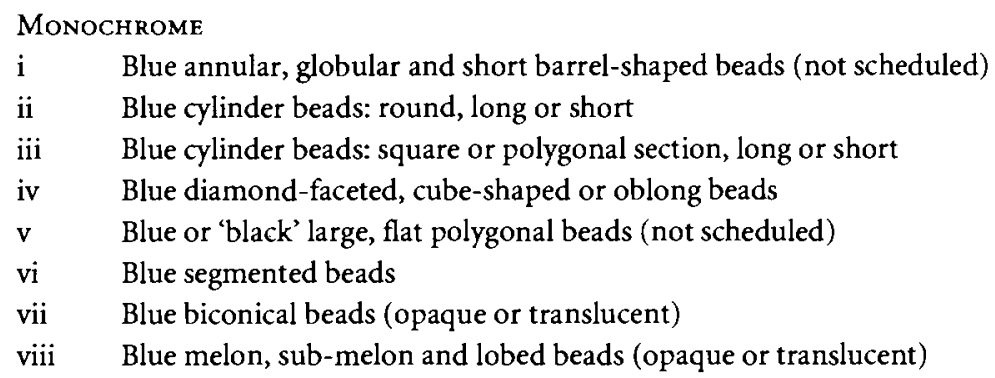

\section{POLYCHROME}

ix Blue beads with white or cream waves, or zigzags

$\mathrm{x} \quad$ Blue beads with crossed waves, with or without dots

xi Blue beads with white and coloured specks

xii Blue beads with red spots

xiii Blue beads with red-centred white eyes

xiv Blue beads with white or yellow spots

xv Miscellaneous blue decorated beads

(For beads with surface spirals on a blue ground, see Chapter 11.)

Blue beads were made over a very long period and the tints cover an extremely wide range. For these reasons, the majority cannot be definitely assigned a close date range in the absence of either associated finds from a closed archaeological context or chemical analysis. Some of the undecorated beads - the globular, annular, polygonal and square-sectioned, segmented or polyhedral forms - can be found on Iron Age sites in Britain and on the continent (Guido 1978, 13-14 and 17-18). They continued to be made throughout the Roman period and seem to have enjoyed a renewed surge of popularity in the Anglo-Saxon world. Their relative rarity in the Roman imperial period beyond the Limes is conspicuous. They are thinly scattered at best in the Barbaricum. Few can therefore be assigned to a specific date from their own characteristics alone.

For the fifth century, it is uncertain which beads might represent Roman survivals and which might have been brought to England by Anglo-Saxon settlers. While 
small biconical beads were very common between the third and fifth centuries, the larger biconical forms and some true barrel-shaped beads increased in numbers in the late sixth and seventh centuries. With the arrival of the Anglo-Saxons in the fifth century, there was a renewed interest in, and production of, decorated blue ground beads, which went beyond the range of Roman taste. Perhaps they carried a connotation of being 'barbarous'.

\section{MONOCHROME}

\section{i BLUE ANNULAR, GLOBULAR AND SHORT BARREL-SHAPED BEADS}

(Plate 5, but not scheduled)

The beads in this group are nearly all small and, without chemical analysis, the majority of them cannot be distinguished from Iron Age or Roman ones. They may be translucent or opaque and even in one necklace their tints of blue may vary considerably. We do not know whether the beads were made locally ab initio or from recycled glass. At least one factory producing them has been identified in the Netherlands, at Rothulfuashem, which apparently dates to around AD 600 and is discussed in the Introduction and several subsequent chapters (Bloemers et al 1981, 132).

Annular beads were discussed in an unpublished undergraduate dissertation available for consultation in the Institute of Archaeology Library at University College London (Sherlock 1977). Although the research was relatively superficial, its distribution map shows that they were very common throughout the areas of Anglo-Saxon settlement in England. The suggestion was made that these simple types may reflect status rather than date, for they were especially worn by people of fairly humble position to judge from their grave assemblages. It is doubtful if a more thorough study would yield conflicting views, but any theories offered there must be regarded as only provisional.

They were certainly worn over a very long period. For example, beads from continental Europe, as in Alamannic Württemberg (Veeck 1931, 51-2, Taf. 32-4), continued on into the seventh century and in Hungary into the eighth century at least.

Short barrel-shaped beads in this group are likely to be the result of slight pressure from above and below in the course of making globular beads. The 'true' barrel forms are discussed below (type vii).

\section{ii BLUE CYLINDER BEADS: ROUND, LONG OR SHORT \\ (Plate 5, Schedule 6, pp 255-60)}

Judging on visual examination only, and lacking a large sample of complementary chemical analyses, it would be premature to imagine that we can arrive at a very accurate chronological assessment for these beads. Although in various sizes they had a long life, it may be of some value to draw attention to what we can deduce so far. 
The earliest beads of this type in Britain and many provinces of the empire were Roman in origin. They were made by cutting drawn tubes of glass into short lengths. Unlike some of the later versions, these beads were generally polished. The length is normally between $6 \mathrm{~mm}$ and $20 \mathrm{~mm}$, and the cut ends are straight cut at right angles to the length of the bead. The blue colour is variable and quite frequently is translucent. Although occasionally found in early imperial contexts, these beads only attained considerable popularity later, notably between the late third and early fifth centuries (for Britain, see Guido 1978, 94-5).

It is doubtful if they ever completely ceased to be made before the apparent sudden expansion of demand for them in the later fifth and sixth centuries. The post-Roman cylinder beads were not necessarily made by the same technique. Many are 'stringy' in appearance and unpolished. From early illustrations made before exactitude was attained or even recognized as desirable, the individual methods of manufacture were not observed. We can recognize now that some were wound and then smoothed, while others show pointed ends indicating in all probability that they were pinched off a long rod. At the same time, and side by side with these, we can still find Roman-type cut-off beads persisting or still being made. Nevertheless, attention to the rounded-end feature can be noted as almost invariably indicating a post-Roman date and in particular a sixth-century context, though this proposal cannot be regarded as totally dependable as a chronological indicator.

While very common in England - for example, at Wasperton (Warwickshire) and Spong Hill (Norfolk) and other East Anglian sites in particular - their distribution on the continent is also worthy of discussion. They occur in the Low Countries, at Pry in Belgium (Namur Museum), for example, and Utrecht in the Netherlands, perhaps datable to the seventh century. North-east France also produces them in numbers: several are illustrated from Picardy and Artois, at Monceau-le-Neuf in the fourth to fifth centuries (Boulanger 1902-5, 22, plate 9.5), and at Achery-Maillot with six beads of the fifth to sixth centuries (Boulanger 1902-5, 83, plate 27.4 and 5). They have also been noted at Nogent-sur-l'Aube (one double bead on a necklace in the Hunterian Museum, Glasgow), while several occur at Vaux Donjon and also at the 'Frankish' row-grave cemetery of Herpes, Charente, in south-west France (Delamain 1892, 11-12, plate XII; Haith 1988). Their frequency in north-east France hints at the possibility of a Gallo-Roman origin before this region was fully absorbed into a Frankish kingdom by Childeric and Clovis in the later fifth and early sixth centuries (James 1988, esp 64-77).

As mentioned above, the plain-cut variety also persisted and can be found in Belgium and the Netherlands and, less commonly, in the upper Rhineland. In the Trier region, on the Mosel and around Cologne (for example, the Müngersdorf cemetery), they appear to have continued into the mid-seventh century and possibly even later (Böhner 1958, 71 and 75; Fremersdorf 1955, 81-90, Taf. 133.4 and 7).

Both the cut and the rounded varieties are common in England. At present, all that can be said is that the latter appear to be characteristic of the sixth century and 
that they may have been brought over from north-east France and the Low Countries. Of course, they may also have been made here, but a Gallo-Roman/Frankish origin for them is disputable.

Beads of a third variety are much rarer. They were made by winding, and though similar in appearance to common cylinder beads, they bear traces of parallel diagonal lines indicating the method of their manufacture. In some instances, these lines have been smoothed over and almost obliterated, so they have often passed unnnoticed by early excavators. Some gold-in-glass were sometimes made in the same way (see p 78). One blue cylinder bead of this type (type C01) was buried in several graves at Buckland, Dover, in phases 1, 2 and 3 (c.475-625) (Evison 1987, 75 and 81 ).

iii Blue Cylinder beads: SQuare or Polygonal Section, LONG OR SHORT (Plate 5, Schedule 6, pp 260-1)

These beads similarly continue a long Roman tradition. They appear to have the same history and dating as the round-sectioned cylinders of type ii discussed above.

\section{iv BLUE DIAMOND-FACETED, CUBE-SHAPED OR OBLONG BEADS}

(Plate 5, Schedule 6, pp 261-2)

These beads are generally, but not invariably, made of translucent blue glass. The examples found in Anglo-Saxon graves were evidently brought into this country before the end of the Roman period. Originally they seem to have come from central and eastern Europe, including Dacia and the Danube region, occupied by east Germanic peoples, Dacians and Sarmatians among others in cemeteries of the Sintana de Mureş-Cerneachov type (Heather and Matthews 1991, 81, fig 10.10). They were also found in a workshop site at Tibiscum (Theiss) in the third to fourth centuries (Benea 1983 and 1997) (discussed in the Introduction).

Perhaps the earliest group in Britain comes from Roman York and is probably datable to the third or fourth centuries (RCHM 1962, 73, fig 58). They were also present in the late Roman cemetery at Lankhills, Winchester (Guido 1979, 292-4 and 297-300). Across the Channel on the continent, they occur, for example, in the coastal Roman fort cemetery at Oudenburg in Belgium, in grave 67 (Böhme 1974, Taf. 95.10; Mertens and van Impe 1971, 95-6, Afb. 34, plate XXI.7), and there are others in the Boulogne Museum, again from sites by the Channel coast. Rather later, in the fifth to sixth centuries, they are found in the Alamannic territory of Württemberg (Veeck 1931, 51-2, Taf. 32.1) and at Schretzheim, mostly in Stufen 3 and 4 (c.565-620/30), for example, grave 173 (Koch 1977, 40, Taf. 38.11). Though occasionally present in late Roman Britain, most of those found in Anglo-Saxon burials in England belong in contexts of the sixth century. These had probably been imported from the Rhineland, where the same form of bead was made in jet. 


\section{$v$ Blue or 'BlaCK' LARGE, FLAT POLYGonAl BEADS}

(Plate 5, but not scheduled)

Resembling two flat superimposed polygons, the shape of these beads is clearly derived from rock-crystal forms, which are also known in early Anglo-Saxon cemetery contexts. So far, very few examples of the glass version have been recorded from England. Two of these come from Sussex cemeteries. One is from Alfriston (grave 92) and the other from Highdown (grave 58), a child's burial (Worthing Museum 3543). The Highdown context might be attributed a fifth-century date (Welch 1983, 83). Another belonging to $c .500$, of dark glass with red streaks, from Cleatham (Lincolnshire), is in Scunthorpe Museum. It is not yet clear whether these objects in fact functioned as beads, amulets, spindle-whorls or even sword-beads, but at least the Highdown example is unlikely to have had either of the last two uses. Comparable rock-crystal beads can be cited from burials at Upton Snodsbury near Worcester of the late sixth century and Linton Heath (Cambridgeshire) of the fifth to sixth centuries.

A Frankish origin seems probable for these glass beads. On the continent, at least one is recorded from Entringen in Württemberg (Veeck 1931, 254, Taf. K.7) and another from Herpes, Charente (Delamain 1892, 11-12), in south-west France. No beads of this type are recorded in the Barbaricum (Tempelmann-Mạczyńska 1985), so they seem likely to post-date the mid-fifth century. A dark example from grave 339 at Junkersdorf near Cologne (La Baume 1967, 46-9, Taf. 22.339.2) dates to the sixth century.

vi Blue Segmented beads (Plate 5, Schedule 6, pp 262-4)

There are three main types of segmented beads that differ in method of manufacture as well as in colour and, more importantly, in date. The earliest can be detected easily since it is generally small and tapering, a viscous glass rod wound round a wire. The glass became finer as it was pulled out and the resulting bead is wider at one end than the other. This bead type was very popular in the third to fourth centuries and may have lasted on into the fifth century. It is not normally found in any Anglo-Saxon context and is only mentioned here to be dismissed (see Guido 1978, 91-3, for further information).

The second type, and the one that is most usual in Anglo-Saxon contexts, is fundamentally different. It is made by pinching a long tube of glass to form segments at regularly spaced intervals. Sometimes it seems probable that these globular segments may have been blown. The beads with two well-finished segments seem to have been a form produced deliberately. So the ends must be examined with care to ensure that they do not simply represent two broken-off segments from an originally longer bead. These double-segmented beads, as well as larger multiple forms, were also made in certain other colours (for example, yellow, pink, green and black) and all were current both here in England and on the continent in the sixth to seventh centuries. 
There are also beads of this type from the Viking period, though these mostly have a wider 'collar' between each globule. Some tenth-century examples have been found at Kneep on the Isle of Lewis (Welander et al 1987, 155, 163-5, figs 6 and 9), and they were widespread in Sweden, at Birka (Arbman 1940-3) as well as at Helgö and Paviken, and also in Denmark, at Ribe (Lundström 1976; Näsman 1984). Those found in early Anglo-Saxon cemeteries are likely to have arrived here from the Frankish Rhineland or neighbouring regions.

Two other rare types of segmented beads should also be mentioned. One was perhaps made by coiling a rod round an upright in such a way that the coils do not accurately fit one upon the other, but topple over. Very few of these beads reached this country and they seem to come from Belgium and Frisia - for example, Bruges and Emelgem (Matthys 1975, 10, fig 3.13) and at S'Herzogenbosch, in the museum there, with other mixed beads of seventh-century date. They have also been reported from north-east France, at Achery-Maillot, attributed to the fifth to sixth centuries (Boulanger 1902-5, 83, plate 27.4).

Finally, it should be noted that two finely made segmented hexagonal beads were recovered from graves 7 and 77 at Alfriston (East Sussex), datable, presumably, between the mid-fifth and the later sixth century (Welch 1983, 83). These are quite exceptional, however, and the fact that they seem to have two segments may simply result from an accident in the course of manufacture. They have not been included in the schedule here.

\section{vii Blue biconical beads (opaque or translucent)}

(Plate 5, Schedule 6, pp 264-5)

Very small, generally translucent biconical beads were very common in the late Roman period (Guido 1978, 97) and the schedule implies that they continued into and beyond the later fifth century. There is the late Roman context of the multiple burial at Canterbury, of c.400, and two examples were found with disc brooches broadly datable to $c .450-550$ in grave 4382 at Springfield Lyons, Essex. Nevertheless, the majority in the schedule clearly belong to contexts of the sixth century or later (for example, Winchester grave 23, dated to the late seventh century), and their continental counterparts also have a date range of the sixth to the seventh century. At Schretzheim, they belong to Stufen 3 and 4 (between c.565-620/30): graves 22 and 233 (Koch 1977, 13 and 54, Taf. 8.7 and 57.9). With such a long-lived type, it would be unwise to suggest a close date for examples found without an archaeological setting.

viii Blue melon, SUb-Melon AND lobed beads (OPAQUe or TRANSLUCENT) (Plate 5, Map 18, Schedule 6, pp 265-7)

A very common type in the post-Roman period, they are more commonly translucent than opaque and are more carelessly made in comparison with Roman examples. They occur from the later fifth century in England and continued through the 
sixth and quite possibly beyond the seventh century. Their distribution covers most of Anglo-Saxon England, as far north as County Durham (Map 18). Continental beads come from graves of the fifth to sixth century and later in cemeteries in Belgium, north-east France and the Trier region. They were also popular among the Alamanni in Württemberg (Veeck 1931, 51-2, Taf. 33 and 34).

\section{POLYCHROME}

\section{ix Blue BeAdS With White OR CREAM WAVES, OR Zigzags}

(Plate 5, Schedule 6, pp 267-9)

These generally annular and translucent beads had a very long life, which makes it impossible to date them by visual means alone. They began in the Iron Age, continued through the Roman period (Guido 1978, 63-4, fig 21.1, colour plate I.10d) and on through much of the early Anglo-Saxon period. A translucent example from grave 404 was dated to Stufe II (450-525) at Krefeld-Gellep (Pirling 1966, 157, Taf. 33.13b), another from Marktoberdorf to $c .580$ to 640 , and others came from later phases there (Christlein 1966, 71-3). They span too broad a territory and time-span for conclusions to be reached about the origins and dating of examples from Anglo-Saxon contexts without chemical analysis results.

\section{$x$ Blue beads With CROSSEd Waves, With or Without dots}

(Plate 5, Schedule 6, p 269)

The crossed waves are mostly white and the dots when present are of white, red or yellow. These beads are not found very often on the continent and it is not surprising to find them to be equally rare in England. Evidently this motif, which was also used on other colour backgrounds, never became popular and beads of this group can seldom be closely dated. Some examples from Buckland, Dover, suggest an overall date range of $c .550$ to 700 .

\section{xi Blue beADS WITH WHite AND COLOURED SPECKS}

(Plate 5, Map 19, Schedule 6, pp 269-71)

Usually the ground of these beads is translucent and the specks are multi-coloured, while the beads themselves are more often globular than annular. The earliest example known to the author comes from the Danube region in eastern Europe, among the distinctive types made at Tibiscum (Theiss) in Romania (Benea 1983 and 1997) in around the third and fourth centuries (see Introduction). From there, they spread in small numbers to the west and north, reaching the Low Countries in the sixth and seventh centuries (examples are found at at Roguée and Biesmes, in the Namur Museum in Belgium, and at S'Herzogenbosch in the Netherlands). This type also seems to have crossed the Baltic to Sweden, and it is possible that they were made at Eketorp on Öland in the later sixth to seventh centuries (Näsman 1984). Their distribution in Anglo-Saxon England occurs predominantly in the eastern 
counties, as far north as Yorkshire, but with outliers further west (Map 19). This might suggest either Sweden or, rather closer to home, the Low Countries, as the origin for them here. As the Anglo-Saxon examples date mainly to the fifth and sixth centuries, this may help rule out Vendel period Sweden as an origin.

xii Blue beAds With Red SPOTS

(Plate 5, Map 20, Schedule 6, pp 272-3)

The distribution of these beads in Anglo-Saxon England (Map 20) points to crossChannel trade or contacts in the sixth and seventh centuries. Continental examples are rare and widely distributed at present. The Franks seem to have collected bead types from far afield before passing on some of their stock in commercial trade.

\section{Xiii BLUE BEADS WITH RED-CENTRED WHITE EYES}

(Plate 5, Schedule 6, p 273)

The tradition of making three equidistant eyes goes back into the Iron Age, in lowland Britain at least, with the so-called South Harting type (Guido 1978, 49-50, colour plate 1.3). There are few continental analogies and once again these were probably made in the sixth to seventh centuries. In grave 156 at Schretzheim they date to phases 4 to 5 in the first half of the seventh century (Koch 1977, 200, Farb. Taf. 1: type 8.5). The Brandon (Suffolk) example comes from a high-status Middle Saxon settlement, possibly a monastery, whose occupation spans the period from the seventh to the ninth centuries (Carr et al 1988).

\section{xiv BLUE BEADS WITH WHITE OR YELLOW SPOTS (Plate 5, Map 20, Schedule 6, pp 273-4)}

The search for a continental origin for these beads has proved elusive. The AngloSaxon examples include examples of the late fifth to sixth centuries from Alfriston (East Sussex) and Wasperton (Warwickshire). The others appear to belong to contexts of the sixth to seventh centuries, including that from Castle Bytham (Lincolnshire). Their distribution is scattered through much of Anglo-Saxon England (Map 20).

\section{xv Miscellaneous blue decorated beads}

(Plate 5, Schedule 6, pp 274-6)

Almost all of these beads appear to date to the sixth and seventh centuries in AngloSaxon contexts and their region of origin cannot yet be determined.

For beads with surface spirals on a blue ground, see Chapter 11. 


\section{CHAPTER 7}

\section{CRIMSON OR PINK GLASS BEADS}

Glass coloured with manganese to produce some tones of brown, or a tint variously described as mauve, purple, pink, violet, lilac or crimson by different individual writers, occurred extensively among the products of Celtic Iron Age bead-makers before the Roman period (Venclová 1981). It was particularly favoured for large 'whirl' or 'ray' beads made at some of the central European oppida, for example, Manching and Stradonitz. These beads (either made from or decorated with this coloured glass) found their way over almost all of Europe, including Britain (Guido 1978 , 57-9, figs 15 and 16, colour plate I.7). It is thought that large annular beads of simple 'pink' glass were also being made at Mathay, Doubs, near France's modern eastern frontier with Switzerland. (This material is currently being prepared for publication in the National Museum of Antiquities, Saint Germain-en-Laye.) Thanks to the kindness of Madame Willaume, the author was able to examine a large number of smaller, mostly annular, beads from a presumed factory inside the oppidum at Entremont near Aix-en-Provence (c.second century BC).

During the Roman period this colour was no longer favoured, except for balsamaries and glass vessels, which were made in large quantities in the Cologne-Trier region until the early fifth century. Some glass beads may have been made from recycled glass (Harden 1969; for the colour, see Goethert-Polaschek 1980, Farb. Taf. $\mathrm{B}$ and $\mathrm{C}$ ).

It is perhaps significant that a very similar colour was used for glass beads, whether annular or globular and sometimes bipartite, in the fifth century and later. Some of the glass was almost opaque. This seems to depend largely on the depth of the bead, for thick glass might give an almost 'black' appearance. These beads have been shown to have been concentrated in the lower Elbe region and in Jutland. They seem to end soon after the fifth century, according to Tempelmann-Mączyńska (1985, 18, 29-31, Taf. 1: type 41). Inevitably, recent recognition of this bead type appearing in lowland Britain with the onset of Anglo-Saxon settlement strongly suggests that their introduction might relate to the Anglo-Saxon migration around the North Sea littoral. The sudden introduction of 'pink' glass beads here is hardly likely to be purely coincidental. It may also be significant that they occur at several 
sites along the east coast - for example, at Mucking on the Thames estuary near Tilbury and Springfield Lyons near Chelmsford (both in Essex) - though all the graves containing them at the latter site can be dated to the sixth century (Mrs S Tyler, pers comm). Conceivably, some of these might relate to landfalls of settlers from the lower Elbe basin or Jutland.

Further, it would appear that this colour glass is not found to any noticeable extent in the Rhineland. It is almost absent from both the Krefeld-Gellep cemetery (Pirling 1966, 1974, 1978 and 1989) and from the cemetery founded around the end of the fifth century at Junkersdorf near Cologne (La Baume 1967, 46-9). Still further south, in Alamannic territory, only one or two examples were found at Marktoberdorf in Allgäu (in grave 79, dated c.540 to 580: Christlein 1966, 71-3, 126, Taf. 21A and 62.2). Some of these may have been made from scrap vessel glass recovered from Roman rubbish dumps.

As already mentioned, these beads do not seem to continue for long after the mid-fifth century in their continental region of origin, and their first deposition in Anglo-Saxon cemeteries may belong within that century, though clearly they continue on rather later. The abandonment of settlements and cemeteries in the western half of the Weser-Elbe estuary region around or even before the middle of the fifth century is well documented (Ament 1980; Böhme 1976 and 1986). Similarly, Bede's record of continental Anglian territory being left depopulated by the migration (Historia Ecclesiastica, i.15) seems to be confirmed by modern fieldwork in the region of Angeln near Schleswig (Müller-Wille et al 1988).

Nearly all the English beads are annular and it is less common to find globular or bipartite ones. The annular beads may often have been casually dismissed as dark blue, unless held up to a strong light. This would be particularly the case when they were found with a group of blue glass annular beads. In some instances, the globular beads have been broken in such a way that a jagged 'collar' reveals where a second element was originally present. Though less common, the bipartite beads are distinctive. They have recently been discovered in a cemetery of the fourth to fifth century at Sejlflod, near Aalborg in north-eastern Jutland (Nielsen and Rasmussen 1986). At Krefeld-Gellep, graves 1274 and 1123 both yielded bipartite pink beads of fourth-century date (Pirling 1974, 83-4, Taf. 8.9; 1966, 116, Taf. 93.4), but Rhenish examples are mostly conspicuous by their absence. The Anglo-Saxon examples listed in the schedule have a clear distribution in southern and eastern counties of England as far north as Yorkshire, but also occur in the west Midlands, Gloucestershire and Wiltshire (Map 21).

Once these attractive beads had been brought over by the Anglo-Saxons, production of them may have continued here. Several beads may be cited in support of this hypothesis. One is a roughly made unique pink melon bead from Kenninghall (Norfolk). Another, which also looks very home-made, is a pink annular bead with a white zigzag from Howletts (Kent). There may be others like these awaiting examination. 
Beads of this glass have a date of grave deposition from c.500 to 650 in England, though some early imported examples may have been manufactured a few decades earlier and still within the fifth century. Some lumps of raw glass found between Rottingdean and Kemptown to the east of Brighton (East Sussex) in 1848 may be relevant. The lumps were described as 'double the size of a man's fist' and were made in various colours, including 'amethyst, amber, emerald, green, and deep marone'. As Jewitt reported, they were evidently 'part of the massae made in the manner described by Pliny, and ready to be sent to the different glass-workers in other parts of the kingdom' (Jewitt 1884, 186-7). Although he regarded this glass as Roman, we cannot be sure of its date. The colours mentioned, particularly the maroon and amethyst, suggest they are rather more likely to be of early Anglo-Saxon origin, though whether intended for the manufacture of vessels or beads, it is impossible to know.

The beads discussed in this chapter are illustrated on Plate 6 and appear in Schedule 7, pp 277-82. Their distribution in Anglo-Saxon England is shown on Map 21. 


\title{
Chapter 8
}

\section{TERRACOTTA OR BRICK-RED \\ GLASS BEADS}

\author{
Monochrome \\ i Terracotta annular, globular and short cylinder beads, singles or doubles \\ ii Terracotta small biconical and true barrel-shaped beads \\ iii Terracotta cylinder beads \\ iv Terracotta short cylinder beads, singles or doubles \\ v Terracotta cylinder beads with square or panelled polygonal sections \\ vi Terracotta melon and sub-melon beads
}

\section{Polychrome}

vii Terracotta cylinder beads with coloured combing (including green and yellow)

viii Terracotta cylinder beads with square or polygonal sections and with coloured combing (including green and yellow)

ix Terracotta cylinder beads with wound bands

$\mathrm{x}$ Terracotta globular beads with white or yellow wound lines or bands

xi Terracotta tall biconical beads with random yellow lines

xii Terracotta beads with specks or spots

xiii Miscellaneous terracotta beads with eyes

xiv Terracotta beads: (a) with white or yellow crossed waves, without dots

(b) with white or yellow crossed waves and dots

(c) doubles with white or yellow crossed waves, with or without dots

xv Terracotta oval beads, some barrel-shaped, with white or yellow crossed waves, with or without dots

xvi Terracotta beads with a single wave or zigzag

xvii Terracotta drum-shaped and related beads:

(a) with herringbone and fine twist ornament (including green and yellow)

(b) related decoration, some with plain zones and some with imitation twists

xviii Terracotta, mainly bun-shaped, beads with herringbone and related decoration:

(a) with twisted decoration ( reticella) and related (excluding green and yellow)

(b) with green and yellow twisted bands

(c) with green and yellow marbling

xix Terracotta cylinder beads, round, square or polygonal sections:
(a) with green and yellow panels or stripes
(b) with green and yellow marbling 
Terracotta-coloured glass is the preferred term here for a colour also described in various reports and records as rust red, brick red or just red. It is not easy to explain why terracotta glass suddenly irrupted into the repertoire of post-Roman beads in the sixth century. There is no obvious ancestry in the Roman or immediately postRoman glasswork of northern Europe. It is not as if there were any technological difficulties which prevented such opaque glass being made earlier, if it had been so desired. Then, suddenly, in the sixth and seventh centuries, it was in demand in large quantities. Analytically, these beads appear to be similar to the composition of the orange opaque beads (Chapter 9 below). A few were made of fired clay, though the majority are produced in an opaque reddish-brown glass.

Of course, we may be witnessing no more than a change of fashion, but the change might have been induced by some economic or political factor at present obscure to us. The author has already tentatively suggested that 'black' glass replaced the dwindling trade in jet during the economic collapse of the western Roman empire (Chapter 2, p 18). It is possible that other comparable economic changes took place between the early sixth and seventh centuries. A similar relationship with dark red amber hitherto imported from the Baltic might be proposed, but against this it must be noted that terracotta-coloured glass would make a dull substitute for brilliant amber. Also, the large strings of amber beads are principally a feature of sixth-century rather than earlier or later female burials in England (Evison 1987, 57-60, fig 89).

The first six classes listed above and discussed below are all undecorated forms with a long tradition among Roman beads. By contrast, the decorated beads listed here as types vii to xix are not ornamented in Roman taste, but appear to represent 'barbarian' and possibly Germanic types. Many of these motifs may have had their inspiration from central European origins, particularly from Slavonic and Alamannic regions (see also under other colours).

Alongside these are more, complex and novel shapes and decoration, often cabled or twisted ornament in so-called 'reticella' glass, for which an obvious source of inspiration still remains to be discussed (see below, pp 64-6). The last groups were often decorated in this manner. It is interesting that some of these latter groups selected for discussion, ie, those ornamented in green and yellow, show a marked concentration in East Anglia. The author suggests that many of these were made in that region as clumsy imitations of 'reticella' beads for people wanting to obtain something similar, but whose craftsmen lacked the technical skill required for true 'reticella' glass (for reticella, see also under millefiori in Chapter 10). They seem to be a purely Anglo-Saxon phenomenon, limited to England. 


\section{MONOCHROME}

i Terracotta anNular, globular and SHORt Cylinder beads, SINGLES OR DOUBLES (Plate 6, Schedule 8, pp 284-90)

As might be expected, these simple forms are likely to have been amongst the earliest in this colour, though they do not seem to achieve true popularity until the early sixth century. A few seem to be assignable to an earlier date, notably Mucking grave 989, in the first half of the fifth century, while Harwell, Wallingford and Alfriston contain graves that might date to the later fifth century. Still, the majority fall within the sixth century and they continue right through the seventh into the eighth century, for example, grave 75 (c.700-750) from Buckland, Dover.

The schedule reveals a heavy concentration in Kent. A few double beads can be referred to the late sixth and seventh centuries, and similar beads have also been noted in other colours (possibly classified as segmented). One of the factories where thick annular or globular beads are known to have been made around AD 600 is at Rothulfuashem (Bloemers et al 1981, 132) in the Netherlands (discussed in the Introduction). They are also found distributed up the Rhine and across to the upper Danube at Schretzheim and other Alamannic cemeteries in south-west Germany. Annular beads in this glass are unusual from northern Europe, but some are known from southern Denmark.

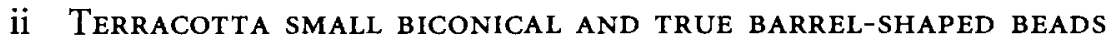
(Plate 6, Schedule 8, pp 290-1)

Once again, these belong essentially to the sixth and seventh centuries. The number found in Kent points to a cross-Channel trade in these beads.

iii Terracotta cylinder beads (Plate 6, Schedule 8, p 291) Cylinder beads, so popular in blue glass, never caught on in terracotta glass and they had a tendency to be thicker. The earliest recorded context is Mucking, grave 989, in the first half of the fifth century, and at Morning Thorpe they probably belong to the late fifth or early sixth centuries, so they may have been first imported or made in the fifth century. Their counterparts are equally rare on the continent. The earliest noted comes from S'Herzogenbosch, in the Netherlands, and the Württemberg region (Veeck 1931, 51-2, Taf. 33) in contexts of $c .400$ to 700 and the late fifth to mid-sixth century respectively. These dates not only agree with those of other beads of this type on the continent, which mainly belong to contexts of the sixth to seventh centuries, but also with most of those from England. An overall date bracket from the fifth to late seventh centuries is proposed here, after which they appear to die out. At the Buckland, Dover, cemetery, they were limited to one grave in phase 5 (c.650-675). They could have originated in Alamannia, or at least the production centres that supplied the Alamannic cemeteries of south-west Germany. 
iv TERRACOTTA SHORT CYLINDER BEADS, SINGLES OR DOUBLES (Plate 6, Schedule 8, pp 292-4)

The short cylinder beads are wider and shorter than the longer terracotta cylinders of type iii above and are commoner too. They appear to last into the seventh century, if not still later, with examples from Chamberlain's Barn II (grave 8: mid to late seventh century) and Buckland, Dover (phases 3, 4 and 6). The schedule contains others, both single and double, from similar though less precisely dated contexts. One bead from Holborough, Snodland (Kent), can perhaps be attributed to the late seventh century at the earliest (Evison 1956, 92, 109, 126 and fig 20.3m). The two varieties, singles and doubles, seem to have been contemporary and the schedule clearly shows a frequent occurrence in Kent. They have been noted in the Low Countries and Scandinavia, but were not very popular in the Rhineland. Unfortunately, the type is not easy to trace from old publications, either continental or English.

v TerRacotTa CYLINDER BEADS WITH SQUARE OR PANELLED POLygonal SECTIONS (Plate 6, Schedule 8, p 295)

Once again concentrated in Kent more than elsewhere, these can be considered together with beads of type iii: though they were less common, they were clearly contemporary with type iii beads in the sixth and seventh centuries.

\section{vi TERRACOTTA MELON AND SUB-MELON BEADS \\ (Plate 6, Map 22, Schedule 8, pp 295-6)}

When they occur in terracotta-coloured glass, these are normally not true melon beads, but what the author has termed sub-melons. These have rough nicks rather than carefully made gadroons. They were not popular in opaque glass and had a more varied cultural and chronological existence in translucent glass. Unlike many beads in terracotta-coloured glass, the sub-melons show a clear East Anglian-based distribution (Map 22). Their dating, centred on the sixth century, cannot be refined at present. Nor can their origin be defined. It may have been Frisian or Scandinavian. On the other hand, the type is well represented at Marktoberdorf and other Alamannic cemeteries in south-west Germany.

\section{POLYCHROME}

vii TERRACOTTA CYLINDER BEADS WITH COLOURED COMBING

(INCLUDING GREEN AND YELLOW)

(Plate 6, Map 23, Schedule 8, pp 296-7)

Combed or feathered decoration was common on beads in the Alamannic cemeteries of south-west Germany and may have travelled north down the Rhine valley in the sixth to seventh centuries. These beads appear to be roughly contemporary with millefiori glass beads and both may have reached north-west Europe together. 
In England, they occur in contexts of the sixth to seventh centuries. The most closely dated come from the Buckland, Dover, cemetery in Evison's phases 3 and 4 (c.575-650). Much of the combing was in white or yellow, and there are a number of other examples from sites in Kent. Interestingly, the only one noted by the author from Suffolk, at Lakenheath, has the characteristic green and yellow colouring so common in that region, but more frequently seen on cylinder beads of polygonal section (type viii) (see below). Their distribution extends as far west as Hampshire and as far north as Yorkshire (Map 23).

\section{viii Terracotta cylinder beads with sQuare or polygonal SECTIONS AND WITH COLOURED COMBING (INCLUDING GREEN AND Yellow) (Plate 6, Map 24, Schedule 8, pp 297-8)}

These beads must have been contemporary with the round-sectioned cylinder beads of type vii, but no very closely dated context has been identified so far. Green and yellow combed decoration shows the usual markedly East Anglian distribution (Map 24): Barrington (Cambridgeshire), Little Wilbraham (Cambridgeshire), South Willingham (Lincolnshire), Morning Thorpe (Norfolk) and West Stow (Suffolk). The absence of the green and yellow combination from Kent and rarity on the continent suggest that these varieties may have been a local product.

\section{ix Terracotta Cylinder beads With Wound bands (related TO TYPE XIX BEADS) (Plate 6, Schedule 8, pp 298-9)}

These beads have bands wound round them, generally in white or yellow, and are usually somewhat larger in dimensionthan normal round cylinders. They have been noted in south-west Germany (at Schretzheim and Marktoberdorf, for example) and in the lower Rhineland (for example, Junkersdorf and Krefeld-Gellep). The earliest examples occur in a late fifth-century context at Wallingford (Berkshire) and they are noted at Buckland, Dover, in graves dated to phases 3 and 4 (c.575-650), a period when quite a number of terracotta glass beads were reaching England and particularly the kingdom of Kent. A factory at Rothulfuashem in the Netherlands (Bloemers et al 1981,132) is known to have made this bead type c.600 (see also type xix beads on $\mathrm{p} 67$ and discussion on $\mathrm{p} 7$ ).

\section{$\mathrm{X}$ Terracotta globular beads with white or yellow wound LINES OR BANDS (Plate 6, Schedule 8 , pp 300-1)}

Some of these beads tend to be more biconical than true globular forms. They are small and the spiral wound bands on them were generally carefully made. These beads are common in northern Europe in the sixth to seventh centuries and seem to have been imported into Kent and the southern counties of England during that period, but their presence in fifth-century contexts at Mucking (Essex) in graves 989 and $924 \mathrm{~B}$ should also be noted. 


\section{xi Terracotta tall biconical beads with random yellow} LiNES (Plate 6, Schedule 8, p 301)

Never a very popular variety, most of these belong to the late sixth or seventh century both in England and in the case of the few rare examples found on the continent.

\section{xii TERRACOTTA BEADS WITH SPECKS OR SPOTS}

(Plate 6, Schedule 8, pp 301-2)

These beads are mostly globular or annular and not very large. They were current between the fifth (eg, grave 924B at Mucking) and seventh centuries and their distribution in England was fairly widespread for that period. It is almost certain that they originated from Alamannia (see beads from Marktoberdorf, Schretzheim and Württemberg), and may thus mark one of the Alamannic contributions to the bead repertoire of this country (see also Chapters 2 and 6 on 'black' and blue glass beads respectively).

\section{xiii Miscellaneous terracotta beads with eyes}

(Plate 6, Schedule 8, pp 303-2)

This is not a true class: most of these beads fall within a date bracket of the late fifth to seventh century.

\section{xiv Terracotta beads: (a) With White or yellow CROSSED WAVES, WITHOUT DOTs (see also types xivb and xivc below)}

(Plate 7, Schedule 8, pp 304-6)

Generally oval and small, these beads are widespread on the continent and came into England via Kent and the Thames estuary in the sixth to seventh centuries. Koch (1977, 207, Farb. Taf. 3) suggested that they were made in large quantities on the continent in the late sixth century for use as small change for trade between the Mediterranean region and the barbarians, but this hypothesis does not seem particularly convincing. They make their first appearance at Schretzheim, around $\mathrm{AD} 600$. Some may be double or triple forms (see type xivc below).

Certain of the English beads, whose sixth to seventh-century date range is broadly in agreement with those across the Channel, have a central line. Several examples from Buckland, Dover, were dated to phase 3 (c.575-625), which is broadly contemporary with the majority of Anglo-Saxon examples. One bead at the Middle Saxon trading and manufacturing settlement of Hamwic (also known as Hamwih or Saxon Southampton) has an atypical form, being more drum-shaped. Their number suggests that they were certainly made in many centres, including Rothulfuashem (Netherlands) around AD 600 (Bloemers et al 1981, 132), a workshop site already discussed in the Introduction. 
xiv TERRACOTTA BEADS: (b) WITH WHITE OR YELLOW CROSSED WAVES AND DOTS (Plate 7, Schedule 8, pp 307-9)

These seem to be only artificially separated from type xiva above and their cultural and chronological position appears to be similar, though the existence of fifthcentury contexts at Mucking should be noted. Predominantly Kentish in distribution, they were presumably imported from Francia and there is no strong presence in East Anglian regions.

\section{xiv TerRacotTa BEADS: (c) DOUbles WITH WHITE OR YELLOW CROSSED WAVES, WITH OR WITHOUT DOTS}

(Plate 7, Schedule 8, pp 309-11)

It is tempting to regard these beads as a stage in the manufacture of single beads, but this is improbable, except where a 'collar' or break can be seen. They are almost entirely confined to the south of England, particularly Kent. Once again, they belong to the sixth and seventh centuries. The two elements in a double band may be decorated in a different way.

\section{xv TERRACOTTA OVAL BEADS, SOME BARREL-SHAPED, WITH WHITE OR YELLOW CROSSED WAVES, WITH OR WITHOUT DOTS}

(Plate 7, Schedule 8, pp 311-12)

It is doubtful whether these beads can be usefully separated from the beads of type xiva to xivc listed above. In terms of both date range and distribution, they can all be considered as one type.

\section{xvi Terracotta beads With a SINGLE WAVE or zigzag}

(Plate 7, Schedule 8, pp 312-13)

There are few beads of this type: the most closely dated example is from Buckland, Dover, attributed to phase 3 (c.575-625), while the earliest may be from Alfriston in a grave of the late fifth or early sixth century.

\section{xvii TerRacotTa DRUM-SHAPED BEADS WITH HERRINGBONE AND} RELATED DECoration (Plate 7, Schedule 8, pp 313-17)

With only a few exceptions, the use of twisted or herringbone ornament on beads in England was almost exclusively confined to drum-shaped or bun-shaped forms on a terracotta-coloured ground.

Reticella is a term that has been widely and incorrectly used when describing beads decorated with fine twists or cables, often in a herringbone design and generally in two colours. The author has abandoned its usage in the present study, as the term is only correctly applicable to glass vessels and was used to describe a particular technique used by Venetian glassworkers in the seventeenth century.

The following terms are used here in place of 'reticella': single twist or double twist, or herringbone when the entire surface of the bead is covered. The author has 
preferred 'twist' to 'cable' in order to avoid confusion with the common cable design often used on beads of Romano-British date, although both these and earlier versions found in the east Mediterranean are generally much coarser (see Guido 1978, 77-9, colour plate II.14: Class 9).

The technique used to make herringbone glass beads involves two rods of contrasting colour being laid side by side, with one in a $\mathrm{Z}$ twist and the other in an $\mathrm{S}$ twist. The composite rod was then spiralled several times round a plain glass bead to produce zones or register. The whole bead was then marvered, giving an all-over herringbone effect. Variations of the technique would account for both forms of bead discussed here (Justine Bayley, pers comm). Both herringbone and millefiori beads made use of fine rods of glass. Owing to the seemingly complex nature of the technique, Evison linked the two and argued that the beads must have emanated from a few centres of skilled glass workmanship (Evison 1983, 92).

Although the technique, which requires stretching the glass rods, while twisting them and then reheating before applying the composite rod to the bead surface, appears to need considerable skill, Näsman (1984, 76-82 and 91-6) has pointed out that only the last stage needs particular dexterity. He has stressed that there is no necessity to visualize a limited number of production centres. Although both millefiori and herringbone beads overlap in date, there is no particular reason, therefore, to believe that they were necessarily made together.

Herringbone glass appears as early as the sixteenth century BC at Tell-el-Amarna. We have no clear evidence of its use in Europe before the sixth century AD, when it became popular in northern Europe and Ireland. Näsman envisaged an origin in Ireland for this technique and a great many examples (albeit in blue and white, or yellow) have been found on early Christian period sites there. The earliest dates for these sites cannot be accurately assessed as yet. In supporting an Irish attribution, Näsman followed the publication of possibly sixth-century beads from Lagore (Hencken 1950, 132-45). Later stages of this use of blue and white beads continued into the Hiberno-Norse period and they are sometimes called 'string' beads (Guido 1985, 101, fig $2 \mathrm{a}$ and b, where they are listed; see also Callmer 1977, 86, plate 9 and colour plate II: Group Bj).

Outside Ireland, there is a second concentration of sites with herringbone glass beads in the Alamannic cemeteries of south-west Germany, for example, at Schretzheim. They also occur in cemeteries down the length of the Rhine to the Low Countries and north-east France, and then on to northern Germany and Scandinavia. A plain yellow collar at top and bottom is characteristic of many of these continental varieties. All appear to date from the sixth century onwards and their origin is not easy to discover.

Näsman has argued for an Irish origin, which then spread to Anglo-Saxon England and from there via Frisia to Scandinavia. This seems a more convincing model than the often-repeated theory that herringbone survived in the Mediterranean region and then reached Ireland by a direct sea route associated with 
trade in luxuries and early Christian contacts. Yet until more and better dated Irish examples are discovered, it is impossible to assess the role played by Ireland in the dissemination of either the herringbone or the millefiori techniques. Nevertheless, the long history of Irish glass-working skills strongly supports a local origin.

xviia beads with herringbone and fine twist ornament (including green and yellow) This group began in the sixth century, for example, at Krefeld-Gellep in Stufe III (525-600), and continued into the seventh century and perhaps rarely into the eighth century. The earliest well-dated example from England comes from the middle decades of the sixth century at Buckland, Dover, grave 92 (Evison 1987, 65, 78 , 82, fig 42.3d, colour plate IV.D67). They are more common in the southern counties, but are also found in the Thames valley, East Anglia and as far north as Lincolnshire and Yorkshire. Though not frequently found on the continent, as with some other types of bead, the combination of green and yellow is more common in East Anglia (see types xviiib and xviiic below). A bead which probably came from a Frankish source was found at the Dalriadic fortified site of Dunadd in western Scotland. Though Irish occupation is supported there by written sources, the fort had at least two periods of occupation. Unfortunately, clear stratigraphic separation could not be obtained owing to the shallowness of the soil overlying bedrock there.

xviib beads with related decoration, some with plain zones and some with imitation twists

Some examples in this group seem to be imitations of 'reticella' beads with several plain zones for which no special skills were needed. One from Buckland, Dover (grave 76) is dated to the first half of the eighth century (Evison 1987, 76, 82, fig. 39.76.1b, colour plate III.D13).

\section{xviii TerRacotta, MaINLY BUN-SHaped, BEADS With HERRINGBONE AND RELATED DECORATION}

(Plate 7, Maps 25 to 27, Schedule 8, pp 317-23)

Bun-shaped beads with herringbone ornament are closely related to the drum beads of type xviia above. On the continent, such beads are sometimes slightly more biconical in shape than the English examples. These suggest that the type was introduced into England via Kent. Though sometimes made in the green and yellow combination typical of East Anglia, there is nothing to suggest that they were concentrated in that region.

xviiia beads with twisted decoration (reticella) and related (excluding green and yellow)

Clearly, these are closely related to the drum beads of type xviia above. On the continent they are sometimes slightly more biconical than English beads, which suggests that they entered the country via Kent. Though similar beads are sometimes made in 
the green and yellow combination, there is nothing to suggest that they were especially concentrated in East Anglia (Map 25). In terms of date, most belong to the sixth century, but they do continue into the seventh century, as seen by the surface find from mound 7 at Sutton Hoo (Suffolk).

xviiib beads with green and yellow twisted bands

These beads are occasionally found in Kent and other southern counties, but are markedly more common in East Anglia (Cambridgeshire, Norfolk and Suffolk), and also reached Lincolnshire (Map 26). Once again, in the majority of cases they belong to the sixth century, but probably continue into the seventh century.

xviiic beads with green and yellow marbling

In terms of both date and distribution, these beads are similar to beads in type xviiib described above, though they were also present at sites in Essex (Map 27).

xix Terracotta cylinder beads, With round, SQUARE OR POLYGONAL SECTIONS

(a) beads with green and yellow panels or stripes (Plate 7, Map 28, Schedule 8, pp 323-6) (see also 'fine twists' in Chapter 12)

The terms 'twists' and 'stripes' have been used here, which require some explanation. They describe the following techniques. 'Twists' refers simply to a marvered coarse twist in two colours: the marvering flattens the twists, which still retain a twisted appearance. 'Stripes' are more thoroughly marvered and the twist is completely lost: the result is a ladder pattern. How much the effect of these two results were intertwined and how much due to incompetence we cannot be sure.

These rather large and clumsy cylinder beads with green and yellow stripes and marvered twists, cables or panels all belong to contexts of the later fifth to seventh centuries. Once again, they are concentrated in East Anglia, including Essex (Map 28). The author has not been able to find prototypes on the continent, though some in different colours from Ketzendorf near Hamburg (Ahrens 1983, Farb. Taf. A to $D)$ may be relevant here.

(b) beads with green and yellow marbling (Plate 7, Map 28, Schedule 8, pp 326-7) Though rarer than type xixa, the date and distribution (Map 28) of these beads suggest a similar history. None has been closely dated, though the examples from fifth to sixth-century contexts at Great Chesterford (Essex) and Spong Hill (Norfolk) seem to be among the earliest known. 


\section{ChAPTER 9}

\section{OPAQUe ORANGE GLASS BEADS}

These orange beads are generally about $9 \mathrm{~mm}$ in diameter and globular, barrelshaped or biconical in form. Nearly always of a matt texture, they are often referred to as 'paste'. Analytically, they are very similar to beads of terracotta-coloured glass and cannot be distinguished, though visually they are distinct from the rust-red of terracotta glass. Although not very common in England, they are known from at least seventeen sites. Over twenty examples came from the rich cemetery complex at Faversham in Kent, the contents of which are now dispersed in a number of museums (Meaney 1964, 118-19), including a large collection in the Ashmolean Museum.

The first scholar to draw attention to examples in England was Sonia Hawkes (in Hawkes and Philp 1973, 192-3), who assigned them to the seventh century, though she noted that they may have lingered on in Germany into the eighth century. Further study reveals that they reached Kent in particular, but sporadically other areas as well (Map 29). Their almost exclusive attribution to the seventh century is fully supported by more recently published contexts from Buckland, Dover (Evison $1987,61-2)$, with two graves in phase $4(c .625-650)$ and six graves in phase 5 (c.650-675), though one atypical smooth-surfaced bead from Grave 1 probably belongs to the beginning of that century in phase 3 (c.575-625).

As far as their continental origins are concerned, the distribution indicates a certain concentration along the North Sea coastline, mostly in Belgium, the Netherlands and north-west Germany, as well as in the Rhineland and Scandinavia. The seventh-century cemeteries include Rosée, Lessive and Jamiolle (the finds from which are in Namur Museum), Emelgem (Bruges Museum: Matthys 1975, 10, fig 3.8), Godlinze, Massum and Groningen (Groningen Museum), Drenske and Hijken (Assen Museum), and, in the Trier region on the Mosel, in grave 58 at Newel (Gollub 1970, 85-8, 101-2, Abb. 9, Abb. H.58). In addition, in the lower Rhineland the cemetery at Krefeld-Gellep produced a few well-dated examples; grave 254, for example, has four beads (Pirling 1966, 158, Taf. 23.11) and belongs in Böhner's Stufe IV, now redated by Ament to c.600-670/80 (Ament 1976). Many beads of this type were found also in the Ketzendorf cemetery near Hamburg, for example, grave 187 (Ahrens 1983, 34, Farb. Taf. B). 
In south-west Germany, at Schretzheim, the earliest belongs in Stufe 1 (c.525/30-545/50), for example, grave 36 (Koch 1977, 19, Taf. 13.1), with a few in Stufe 2 (c.545/50-565/70), for example, grave 258 (Koch 1977, 60, Taf. 68.6), and the majority in Stufen 3 to 5 (c.565/70-650/60), after which their numbers quickly dwindle, for example, grave 615 in Stufe 6 (Koch 1977, 130, Taf. 161.9). Other beads of this sort are reported from Kirchheim-am-Ries (mid-seventh century) in the collections of the Stuttgart Museum. Grave 79 at Marktoberdorf in Allgäu belongs c.540-580, in contrast to the mid-seventh-century contexts of graves 152 and 216 there (Christlein 1966, 71-3, 108, 120-1, 126, 143, 164-5, Taf. 2E, 15A, 21A, 35D, 39E, 57B, 61.2, 62.5, 64.4 and 5).

A few beads are also recorded from Poland (Antoniewiez 1963, 174-6, 191, fig 3). In Sweden, they were present in the seventh-century ring-fort of Eketorp II, Öland (Iverson and Näsman 1977). Professor Ulla Lund-Hansen informs the author that she has been finding large numbers on Bornholm. Most of this island's cultural connections were with the Slavonic regions, perhaps via the Oder, rather than using the more westerly trans-Alpine routes and the Rhine. These beads on Bornholm continued up to the beginning of the Viking period, when they suddenly disappeared.

In her 1973 discussion of these orange beads, Sonia Hawkes suggested that they may have been imported into the Rhineland and thence to Kent and other parts of England, along with amethysts, millefiori beads and other exotica with which they are often associated in grave contexts. She cited a rich grave at Sarre on Thanet, which contained a bronze bowl, a Kentish composite disc brooch and Provençal gold solidi coins reused as pendants, which have a terminus post quem of $c .615$ and probably imply burial a decade or two later. Perhaps different types of exotica reached Kent through a variety of routes rather than as a single package, much as seems to have been the case with the Sutton Hoo mound 1 assemblage in Suffolk. Though many orange beads are regarded as items traded across the Alpine passes, we should allow for the possibility of a route to the south east via the Balkans or the Danube, which has not yet been recognized. Cemeteries in the Alamannic region of south-west Germany, such as Schretzheim might have received bead types from the central Danube region, the south coast of the Black Sea or elsewhere, as well as from trans-Alpine trade with Italy and the Adriatic.

The beads discussed in this chapter are illustrated on Plate 7 and listed in Schedule 9 , pp 328-9. Their distribution in Anglo-Saxon England is shown on Map 29. 


\section{Chapter 10}

\section{MILLEFIORI AND MOSAIC GLASS BEADS}

(a) MILLEFIORI BEADS (Plate 8, Map 30, Schedule 10, pp 330-1)

These elaborately decorated beads have either globular or cylindrical forms. The difference between mosaic and millefiori glass was best defined by Harden, who wrote: "I use the term "mosaic" to cover the whole range of glasses with polychrome patterns made by fusing together different-coloured pieces of glass, preferring, as is etymologically more correct, to confine the term "millefiori" to glasses built up of cane-sections bearing floral or related patterns' (Harden 1968a, 21, note 1a). In a more detailed description, Moorey cited Goldstein (1979, 30-1, figs 5 to 7), who explained 'how pre-formed canes of glass would be cut into the required lengths for the mosaic pattern. The cane-sections would then be assembled over a solid core, presumably with a binder of adhesive, and then enveloped in an outer mould. The mould would then have been heated slowly to fuse the canes without allowing them to become so hot that the design was lost. This would have required a sophisticated knowledge of the properties of various glasses. Once the canes had been fused, the mould would be removed, the glass surface cleaned and maybe polished' (Moorey 1985, 215).

It is hardly surprising that beads made by this technique were greatly prized and were rare in Anglo-Saxon England. They could have reached here from one of three sources: the Rhineland and Frisia; Ireland; or Scandinavia. Since those found in England, whether long globulars or polygonal-sectioned cylinder beads, have analogies with beads found in the first two stages of the Schretzheim cemetery in the sixth century (Koch 1977, 218, Taf. 240, Farb. Taf. 6), it seems probable that they were introduced from Frankish sources into the south and east of England (see p 72 below). The large numbers in the Helms Museum, Hamburg-Harburg (now awaiting publication), point to another possible export centre at the mouth of the Elbe.

It is thought that the technique of millefiori may have originated in Alexandria and been carried thence to Italy and over the Alps in the later sixth century. While 
discussing some examples found with Frankish types of bead in Finland, Ursula Koch (1974, 511-13) suggested that they were brought by fur-traders. They may have reached regions north of the Alps with Frankish troops returning home from the 'Gothic wars' of Italy in the second half of the sixth century, probably at the time of the Lombard invasion of 568. She also followed Werner (1953, 12-13, notes 39 and 40) in his hypothesis that there had been some, as yet undiscovered, bead factories in northern Italy (Koch 1974, 503, note 59). A distribution map of these millefiori beads showed them to be found mostly west of the Elbe, but also revealed some findspots in Sweden and on the Baltic island of Gotland (Koch 1974, 515-19, Abb. 3 ). This pattern suggests that they were transported down the major rivers north of the Alps.

Unfortunately, the situation in Ireland is unclear. There are many canes which may have been intended for millefiori beads or for beads with fine twists (see Chapter 8: terracotta types xvii and xviii on pp 64-7), generally in blue and white wound round a core. On the other hand, these may equally well have been used to make decorative plaques for metal objects (Henry 1938; Hencken 1950, 132, fig 64.277; Youngs 1989, 202-3). For Scandinavia, millefiori beads have been discussed by Näsman (1984, 76-82 and 91-2) in a study of the Vendel period glass from Eketorp II on the island of Oland (Sweden) between the later sixth and eighth centuries.

In considering the millefiori inlays set in metalwork from the Sutton Hoo ship burial, Mavis Bimson (1983) emphasized the presence of two different traditions. In the gold cloisonne fittings, the millefiori is cut and shaped from larger glass sheets, whereas the millefiori glass embedded in glass enamel in the 'Celtic' hanging-bowl escutcheons and mounts consisted of complete sections from single millefiori rods. There were also chemical differences in the glass, for the millefiori in the cloisonné shoulder clasps, buckles and other gold mounts contained an antimony compound as its opacifer-colourant, whereas the hanging-bowl millefiori glass used compounds of tin. 'Thus we find those working in the older, Celtic tradition employing the more recently introduced materials. The fact that Saxon millefiori contains an opacifier characteristic of the classical civilizations suggests the possibility that it may have been made with re-used Roman glass' (Bimson 1983, 944).

We should allow for the strong evidence from Anglo-Saxon grave contexts that the Celtic hanging-bowls found in Britain were not being manufactured in the fifth and early sixth centuries, but belong to a relatively brief production period between c.550 and 650 (Brenan 1991). Nevertheless, it now seems clear that there was no general change-over in the opacifiers used around AD 450 (Rooksby 1964). Instead, we perhaps need to think here of a 'central European' (tin) tradition as distinct from a 'Mediterranean' (antimony) tradition. In view of the widespread movement and mobility of craftsmen, and the enormous complexity of the archaeological scene, however, the analytical problems may prove to be still more formidable than they seem at present. 
The schedule (see also Map 30) shows the dating of Anglo-Saxon contexts containing these beads to belong in the sixth to early seventh centuries, which is in harmony with the dating proposed at Schretzheim by Koch $(1977,218)$. In private correspondence with the author, David Brown made an interesting suggestion that the Anglo-Saxon green and yellow decorated beads, both in millefiori and herringbone beads (see Chapter 8: terracotta types xvii to xix on pp 64-7), may have been an insular attempt to imitate imported beads in ignorance of the actual method of manufacture.

\section{(b) MOSAIC BEADS (Plate 8, not scheduled)}

This attractive bead group seems to have originated in the early centuries $\mathrm{AD}$ in the Mediterranean area. Frequently, the Roman-period versions are square in section or made of irregular chips. This variety reached a certain popularity in the late Roman period (Guido 1978, 98). During the Anglo-Saxon period at least, they were often long and slightly biconical in form, occasionally squared, often with a red stripe inside white central bands on a blue or other coloured background. Even rarer are the polygonal-section multi-striped beads. Such beads were made by folding longitudinally and the resulting overlap is clearly visible in many cases as the two ends of the central band meet irregularly.

Almost certainly, all these beads in Anglo-Saxon England were imported, and examples may be listed from the following sites: Canterbury, Kent (Ashmolean Museum 1909.215); grave 64 at Welbeck Hill, Lincolnshire: four beads (Scunthorpe Museum) in a context of the later fifth or sixth centuries; West Stow cemetery, Suffolk (Moyses Hall Museum, Bury St Edmunds: West 1985, 71-5); Stowting, Kent, perhaps sixth-century in date (Beck Collection, Cambridge); Faversham, Kent (Smith 1860, plate V.8); and, finally, types D57 and 58 at Buckland, Dover, Kent, in grave 30 (British Museum), attributed to phase 3 (c.575-625) there, an assemblage which produced four examples, mostly on a dark purple ground, but including one with a dark green colour (Evison 1987, 63-5, 77, 82, fig 18.4s and $t$, colour plate IV). Five examples of the multi-striped polygonal-section beads were found in grave 35 at Sewerby, Yorkshire (Hirst 1985, type C6, fig 23 and colour microfiche), dated to the first half of the sixth century by Åberg Group III cruciform brooches.

Continental bead dating seems to agree with the Anglo-Saxon chronology. Very few come from the Barbaricum before the fifth century (Tempelmann-Mączyńska 1985, 21, 52-8, Taf. 9: type 323). They are more frequently found in Alamannic south Germany, both in Württemberg and in Bavaria, notably at Marktoberdorf, and, in particular, in graves 103 and 106 there, dated to c.540-580 (Christlein 1966, Taf. 62.4 and 7). As at Buckland, Dover, some of the Marktoberdorf beads occur in more subdued colours. They are types M67 and M72 in the Schretzheim classification (Koch 1977, 217-18, Farb. Taf. 6), datable between the sixth and seventh centuries. These are not very common there, and once again sometimes occur in muted 
colours. In the lower Rhineland, one came from Krefeld-Gellep, grave 189, dated to the first half of the sixth century on the overlap between Stufen II and III (Pirling 1966, 159, Taf. 19.5c). Another probably sixth-century bead from Pry in Belgium possesses a rather wider band than usual.

The impression is left that this bead type was manufactured first in the Roman Mediterranean around the first and second centuries $\mathrm{AD}$ and spread from there throughout the Roman empire and the margins beyond the Limes. Subsequently, they were particularly favoured by the Alamanni around the headwaters of both the Danube and the Rhine. Presumably it was from south-west Germany that they travelled, either directly or indirectly, along the well-established Rhine trade route to Anglo-Saxon England in the sixth to seventh centuries. 


\section{Chapter 11}

\section{ANNULAR GLASS BEADS WITH COLOURED SURFACE SPIRALS}

\section{(a) WHITE GROUND}

These annular, generally well-made beads are decorated with a thin spiral line on the whole bead surface starting from the hole. This is a common type in England. The author noted two on the continent, which came respectively from Jamiolle in Belgium (Bequet 1886) and from grave 233 at Schretzheim in south-west Germany (Koch 1977, 54-5, 215, Taf. 57.7, Farb. Taf. 5.R1). In England, they have a wide distribution from Kent to Northumberland (Map 31). At first sight, one might think that the relatively large number from Kent indicated them to be imports from the Frankish continent. This seems improbable, however, since the Kentish examples appear from relatively late cemetery contexts of the sixth to seventh centuries. The two earliest known beads from an Anglo-Saxon grave were from Colchester (Essex), associated with a pair of early cruciform brooches of the Witmarsum Type dated to c.425-450 by Sonia Hawkes (1981, fig 13). Other beads from contexts of the fifth to sixth century are known from Spong Hill (Norfolk) and Holywell Row (Suffolk).

Over thirty examples of these white beads, nearly all with light blue or turquoise spirals, have been found in England and it is interesting to note that at Kempston (Bedfordshire), grave 16 produced two matching beads of this type apparently fused together. Perhaps this suggests a nearby workshop where they were made. Certainly, it seems valid to regard these not so much as the result of an accident or jeu d'ésprit, but rather as wasters which, even if accidentally marred in the process of manufacture, were still considered worth using as a single bead. Though its excavation was not well recorded, the Kempston cemetery is known to have been in use relatively early in the fifth century (for example, Kennett 1983; Böhme 1986, Abb. 52.2 and 11). In view of the apparent rarity of these spiral beads on the continent, the relatively large number of English examples seems to hint at a centre of production in this country. Beginning as we have seen in the fifth century, these beads became rather commoner in the sixth century and then subsequently rarer in the seventh century. 


\section{(b) VARIOUS COLOURED GROUNDS AND SPIRALS \\ (Plate 8, Map 31)}

The origin of this group of beads may go back to Roman-Alexandrian beads of the first century, though these tend to be more bun-shaped than annular. Clearly, they were current in northern Europe at the same time as type (a) above (white ground beads with surface spirals). Though most commonly blue, the background colours vary considerably, as can be seen in the schedule, and may be of translucent or opaque glass. The spirals also vary in colour. They were a long-lived type and in all probability lasted into the seventh century and beyond, as, for example, at Brandon (Suffolk).

The beads discussed in this chapter are illustrated on Plate 8 and listed in Schedule 11, pp 332-7. Their distribution in Anglo-Saxon England is shown on Map 31. 


\section{ChaPTER 12}

\section{ANNULAR GLASS BEADS WITH FINE TWIST DECORATION}

This is a rare and interesting type of bead, to which attention was first drawn in the publication of a rich woman's bed burial of the later seventh century under a barrow on Swallowcliffe Down, Wiltshire (see Guido 1989, fig 46). All the beads in this group are annular, about $20 \mathrm{~mm}$ in diameter, and apparently of translucent glass. The decoration is particularly skilled, being produced by twisting two fine rods of differing colours and so applying them as to make a pattern of opposing swags on the surfaces of the bead. The most skilfully made beads have the tips of the swags almost meeting. Occasionally, a girth cable may be applied in the same manner. The author has suggested that these rare beads may have been worn as amulets by wealthy women in the second half of the seventh century. By this date we would expect all these individuals to be baptized Christians. The decoration of these beads might represent a skeuomorph of a wire filigree loosely encasing the bead to make a bead-pendant.

Similar beads do not appear on the continent, and although twisted decoration on glass was being used and probably made in Ireland around this date, the author does not believe that they are Irish. As can be seen from the schedule and Map 32, they are to be found particularly in Kent (with four from Sibertswold/Barfreston) and in East Anglia, which shared certain influences and cultural similarities with Kent during this period, as Lethbridge (1926a, 122-3, and 1936, 27-9) pointed out long ago. In any case, the fine craftsmanship as well as their rarity point towards their production in an individual workshop by a skilled glass-worker, quite probably in Kent, which was notable for the richness and variety of its invention both in glass and in metalwork in the seventh century.

As a design carried out in marvered swags rather than twists, it appears to have had a long life. One bead from Drenske in the Netherlands was found with an equal-arm brooch of the fifth century (Assen Museum). In England, a comparable bead comes from a sixth-century context (grave 20) at Petersfinger, Wiltshire (Leeds and Shortt 1953, 16,44, plate I.XX.58). The origin of the pattern was almost surely Germanic, but the craftsmen who made the English version could equally well have been Anglo-Saxon, British or Irish by birth. 
The beads discussed in this chapter are illustrated on Plate 8 and listed in Schedule 12, pp 338-9. Their distribution in Anglo-Saxon England is shown on Map 32. 


\section{Chapter 13}

\section{GOLD AND SILVER-IN-GLASS BEADS}

Gold-in-glass beads have a very long history in the Mediterranean lands, where they date from at least Ptolemaic times. A Hellenistic factory for them has been identified in Rhodes (Weinberg 1969). Here, the technique used was closely similar to that for glass vessels such as the famous Canosa glasses (Harden 1968a).

Boon has described the method of making beads of this type (Boon 1966, 104; see also Boon 1977):

The working procedure was evidently to draw out a tube of glass over a wire core; metal foil was wrapped round this tube and coated with a further thin protective layer of glass. The completed ensemble was then threaded upon a narrower wire, to be crimped at intervals into the segments which could be broken apart for use as single or multiple units.

When designed as individual segments, these beads are neatly finished at each end and when accidentally broken they show ragged 'collars' where they have snapped off. This feature is frequently noted among the huge numbers made in the Roman period - and even more in the post-Roman period. Many, too, have lost the metal filling and the outer casing of thin glass and survive as fragile colourless 'beads'. It is unlikely that these were made deliberately as such.

It may be noted that while single segments of metal-in-glass beads have sometimes been reported from old excavations, they have normally been regarded as broken pieces of longer segmented beads, the rest of whose parts have been dispersed or not observed in the excavation. This cannot now be accepted, for it is common in present-day excavations, for example, Empingham II (Rutland), to find that only one segment was buried in the grave (for example, grave 81: see Timby 1996, 48 and 49, figs 130 and 131). The implication is that some special significance was attached even to fragments. Perhaps they were held in some way to be magical or reflected the wealth of the dead person.

These beads were often acquired through trade or troop movements and are not uncommon finds on Roman sites throughout the imperial period. The majority of these Roman period beads have been listed elsewhere (Guido 1978, 93-4, fig 37.3). 
Most of those in Britain are late Roman, but a fine necklace of forty-four gold-inglass beads from Baldock burial group 89 is rather earlier. It is dated by associated pottery to the second century (Westell 1931, 272). At Newstead, several broken segments were discovered in the Roman fort occupied between $c .80$ and 200 (Curle 1911, 336-7, plate XCI.24), and one bead was stratified to c.130 to 230 at Caerleon (listed by Boon 1966). On the whole, however, they tend to be far commoner in the fourth to early fifth centuries. They next seem to have flooded over northern Europe, particularly in the sixth century, though they can also occur later.

It is to be expected that once the manufacturing method had been developed in Egypt and elsewhere in the east Mediterranean region, local factories might have been set up in continental Europe. In the first instance these would probably have been located near the trade routes from the Mediterranean via the Black Sea to the Dnieper and Vistula rivers and thus to the Baltic. This would help to account for the large concentration around Gdansk between the first and fifth centuries (Tempelmann-Mączyńska 1985, 64-5, Taf. 14.387: group XXIX with map Taf. 57). Analyses have shown that during this period (and indeed later), silver was used as commonly as gold (Stawiarska 1984, 155; see also the valuable remarks by Biek in Biek et al 1985, 77-9).

Expansion after the fourth century drew many of the Slavic peoples into the orbit of the Roman provinces in south-east Europe. By the mid sixth century, the Slavs had spread south of the Danube and had access to the Mediterranean. In the sixth to seventh centuries, they also moved west to settle Poland and northern Germany as far as the lower Elbe region (Herrmann 1980).

The traditional trade routes of the earlier Roman Iron Age had been much disrupted in the process. By around AD 700 new ethnic groups had established themselves in all regions south of the Baltic and an economic boom had begun.

A suggested pattern for the distribution of metal-in-glass beads might have operated as follows. From a Hellenistic starting point, the type was scattered in the Roman imperial period throughout the empire and beyond the Limes into Germanic Europe. At the same time, or else soon after, the trade route linking the Dnieper and Vistula rivers carried larger quantities of these beads northwards to export centres such as Gdansk. As the Slavs spread southwards across the Danube, a massive trade grew up and new factories seem to have started production. One such workshop has already been identified at Roman Tibiscum in south-west Dacia (Benea 1983 and 1997). Unfortunately, archaeological investigation there is still in its very early stages. Bead-making near Schretzheim in south-west Germany may have started around AD 550 and production attained very considerable numbers by the last third of the sixth century, before it dwindled in the seventh century.

As mentioned above, the sporadic import trade in gold-in-glass beads to Britain may not have died out completely during the fifth century: it was suddenly augmented in the sixth century, as the schedule shows clearly enough. Many of these beads were recovered from old and poorly dated excavations, but grave 989 from 
Mucking (Essex) is a context with four beads dated to the first half of the fifth century, while another example comes from a recently excavated late fifth-century burial context at Wasperton (Warwickshire). Others from graves at Buckland, Dover (Evison 1987, 62-3, 75 and 81, text fig 11, class C), may also belong within the later fifth century in phase 1 (c.475-525). Since few others can be dated so accurately, it would be premature to claim a definite sixth-century date for any other burial containing one of these beads in the schedule. Nevertheless, the likelihood of a sixth-century date is clear and has been pointed out by Tania Dickinson (1976). Two recent excavations in north Germany support her argument. The earlier at Liebenau near Hannover produced more of these beads (Siegmann 1997, 136; Hässler 1983b, 41, Taf. 86, types 20.2 and 20.3). Slightly later, at Hamburg-Harburg, they had become very common and may even have been shipped northwards from the Elbe estuary. The same area also produced brown glass beads enclosing foil.

The beads discussed in this chapter are illustrated on Plate 8 and listed in Schedule 13 , pp 340-4. It should be noted that the schedule is partial only, owing to the difficulty of identifying single colourless globules from broken-off segments that have lost their foil. 


\title{
Chapter 14
}

\section{TECHNOLOGICAL ASPECTS OF ANGLO-SAXON GLASS BEADS}

\author{
Julian Henderson
}

\section{INTRODUCTION}

The investigation of the technology of Anglo-Saxon glass beads can potentially provide a wide range of information about their production and use. Broad archaeological questions can be addressed using scientific analysis such as the question of where the beads were made based on the detection of a specific combination of raw materials and impurities in the glass revealing specific 'workshop' technologies or production zones. Chemically diagnostic combinations of raw materials could, in turn, be correlated to the composition of specific bead types, and there is the possibility that the same bead type produced in different zones would have had different chemical compositions. There is also the question of whether there was a change in the chemical composition of glass moving from the late Roman into the Anglo-Saxon bead-making tradition (reflecting a change in the raw materials used) and whether there was a change at the end of the Anglo-Saxon period. Many Anglo-Saxon beads are multi-coloured and involve both translucent glass, which sometimes forms the matrix of the bead, and opaque glass, which is used both for the bead matrix and for the decoration.

Opaque white, yellow and red glasses were probably manufactured in smaller quantities than translucent glasses. It is likely that the production of opaque glass occurred under more tightly controlled conditions using more unusual raw materials than was the case for some translucent glass. Therefore, if one is attempting to reconstruct production zones based on glass chemical compositions, the use of relatively exotic raw materials for the manufacture of opaque glasses must enhance the possibility of success. The other significant question that must be broached in the investigation of ancient glass technology is the extent to which the glass has been recycled. It is less likely that specialized opaque glasses would have been recycled than translucent glass, because the effect of mixing opaque glass with translucent glass would be to introduce specific suites of impurities, which in turn would affect the overall colour achieved (and would be recognizable analytically). It is also more 
likely that exotic highly coloured glasses would have been traded in the form of ingots (Charleston 1963). Both recycling and the trade of raw glass would tend to make the identification of production centres more difficult. This is why the excavation of primary industrial evidence, if it can be found, is so important to the interpretation of ancient industry in general and Anglo-Saxon glass-bead production in particular.

In the investigation of glass production, a range of evidence for glass-working should be sought. With the current lack of evidence for the manufacture of AngloSaxon beads in Britain, it is necessary to cite the evidence from contemporary nonBritish sites and evidence for bead-making from other periods. The evidence ranges from globules of glass to partly formed beads, crucibles and hearths (see below, pp 85-6). On a glass-making site there might be evidence for the use of primary raw materials, such as sand, the presence of crucibles and furnace remains and possibly the manufacture and use of frit, which is the first stage in glass production and is a partially fused material consisting of sand or quartz and an alkali. There would also have been the use of mineralogically based glass colorants, such as those rich in cobalt and copper (Turner 1956; Henderson 1985 and 1989, 33-6). If opaque glass was being manufactured, then the use of substances such as lead antimonate (which produces an opaque yellow colour in glass) might be found (Turner and Rooksby 1961; Rooksby 1962) on either glass-making or glass-working sites. Occasionally, evidence for the manufacture of yellow glass is found, or the modification of translucent glass to make an opaque glass in crucibles from contexts in which glass beads were made (see below, pp 85-6). A careful evaluation of the full range of evidence should be included in the investigation of glass-working, glass-making and bead manufacture, so that the technology can be reconstructed as fully as possible.

\section{RAW MATERIALS AND COLOUR PRODUCTION}

In any discussion of glass production, a distinction should be made between glassmaking (the fusing of primary raw materials or partial fusion, ie, fritting) and glassworking (activities involving only the softening of glass to make the bead shapes or the application of decoration to beads). Lower temperatures would normally be involved in the working of the glass into beads, and for fritting. The addition of scrap glass to the melt could also help to reduce the overall melting temperature. Anglo-Saxon glass beads were often made from a combination of opaque and translucent glass. Almost all Anglo-Saxon translucent glass, when chemically analysed, has turned out to be of a soda-lime-silica composition and is therefore regarded by many as belonging to the 'Roman' tradition of glass production. There are many technical differences between Roman and Anglo-Saxon vessels, however (Henderson and Holand 1992), and the same is true of glass beads. Indeed, the technical finesse achieved with Anglo-Saxon glass vessels is of the highest standard and characteristically different from Roman vessels. Anglo-Saxon glass beads display a 
far wider range of types and are generally of a more flamboyant appearance with more exotic raw materials involved. Nevertheless, in spite of the observed differences between Roman and Anglo-Saxon glass technologies, the basic principles of glass manufacture would have been the same.

The glass-melting process could have been carried out in furnaces, which may have had three superimposed chambers of the so-called 'southern' furnace type (Tabacynska 1968; Gasparetto 1965 and 1967). The fuel was inserted into the lowest one to heat up the furnace; the middle one held the crucibles ('pots') in which the glass raw materials were melted and may have included scrap glass and/or frit (see above, $\mathrm{p} 82$ ); while the top chamber was that in which glass vessels in particular were annealed, leading to a relaxation of the strains which developed in the glass during its manufacture. Alternatively, annealing could have been carried out in a separate smaller and lower temperature installation in the same workshop as a twochambered ('northern' type of) furnace, as described by the medieval (twelfthcentury) monk Theophilus Presbiter (Hawthorne and Smith 1963). It is presently unclear what kind of apparatus was used for heating up the glass for the manufacture of 'Anglo-Saxon' glass beads, however, and indeed, in view of the lack of industrial evidence, whether glass beads were manufactured in England at all.

The primary raw materials used in the manufacture of soda-lime-silica glass (whether fritted or not) are an alkali, silica and a lime-rich material, as well as other raw materials used for modifying the appearance of the glass. The alkali (soda, $\mathrm{Na}_{2} \mathrm{O}$ ) used in Anglo-Saxon glass is thought to have been of a mineral origin, judging from the impurities that are introduced with it. An example of such a mineral is a sodium sequicarbonate, natron (Turner 1956; Henderson 1985). The second primary raw material would have been calcium-rich and shell fragments, which occur naturally in sand, are considered to provide one such source. The third raw material would have been silica $\left(\mathrm{SiO}_{2}\right)$, which occurs naturally as sand and would introduce a range of mineralogical impurities such as titanite and sphene (Henderson 1985). An alternative silica source is quartz, which is usually purer than sand, but can introduce other impurities. The chemical analysis of Anglo-Saxon glass beads supports the contention that a natron-like soda-rich mineral has been utilized as the alkali, together with a silica sand source, while lime $(\mathrm{CaO})$ was probably introduced in the form of shell fragments in the sand used, though there are other possible calcium sources. The use of this mineral alkali source results in low impurity levels of magnesia and potassium oxide (Henderson 1989, 38-9). If a plant ash had been used, the level of these two oxides would have been significantly elevated (Brill 1970).

Lead-rich opaque glass was often used for bead decoration, because it has a lower softening temperature and a longer working period than low-lead translucent bead matrix glasses. It will, therefore, not melt the bead when applied, allowing the overall bead shape to be retained. In many cases, the bead was marvered after the trails or 
spots were applied, pressing the decoration into the bead, and showing that the whole object had been softened.

\section{Colorants}

The colorants found in Anglo-Saxon translucent glass beads rely heavily on the introduction of a minute amount of a mineral-rich material to the glass melt. This would be achieved by adding a small quantity of a frit rich in the colorant mineral, perhaps in a ground-up form (Henderson 1985). Only a minute proportion of the colorant oxide is necessary to give a deep colour to the glass. For example, only about 0.1 per cent cobalt oxide is necessary to produce a deep cobalt blue colour in soda-lime-silica glass; more than this and the glass can be rendered apparently 'black' (a deep translucent colour). The basic glass composition is also important in determining the final glass colour (see the Appendix to this chapter, pp 89-93).

The compounds used to produce opaque colours in glasses are different in nature from those used for translucent glasses. Biek discusses the subtle degrees of opacification achievable in Anglo-Saxon beads (Biek et al 1985), from glass with masses of dense crystals, which prevent the glass from transmitting light, to semi-opaque glass, sometimes with crystalline impurities in it such as quartz and with a less dense distribution of bubbles. Bubbled white Anglo-Saxon glasses have been examined both at Sewerby (Biek et al 1985) and amongst the beads qualitatively analysed by Bayley and Wilthew (1986).

Some crystalline opacifiers occur as natural mineralogical substances, such as tin oxide in the form of cassiterite, and these can be added directly to the glass and render it opaque. Other opacifiers must be developed by heat-treating glass that already contains the elements dissolved in the glass and which make up the crystals to be formed out of the glass. For fuller details of the colouring and opacifying effects of different additions, see the Appendix, pp 89-93 below.

\section{SAMPLING STRATEGIES}

In order to maximize the potential results of scientific investigations, glass beads should be sampled with a range of archaeological questions in mind. An integrated research strategy in which quite specific samples are chosen to test archaeological hypotheses is desirable. The chemical compositions of glass obtained from a range of archaeological contexts, producing beads with different social values and potentially articulating with different production and exchange systems, should be tested (Henderson 1991). Where possible, the investigation of production sites must be included. Naturally, such tests should ultimately aim to relate their technological implications to a broad economic and social context, although this will only be possible once a statistically significant population of analyses have been carried out. 


\section{THE ARCHAEOLOGICAL EVIDENCE FOR BEAD PRODUCTION}

There is evidence for bead production contemporary with the Anglo-Saxon period or slightly later on the continent and in Ireland. This takes the form of glass globules, partly formed beads, metal armatures on which glass beads were gathered or wound, rods of coloured glass, glass bead moulds, surfaces on which glass has fallen, hearths associated with glass-working and crucibles with melted glass on their inner faces. Yet even where a range of this evidence is found, it is normally only found in pits containing by-products from bead production and none of the structures that might have been used to heat the glass in has survived. It is possible that only hearths with bellows were used for bead production, as the lead-rich glass often used for bead decoration and some bead matrices would only require temperatures of $550^{\circ} \mathrm{C}$ to $700^{\circ} \mathrm{C}$ ( Gam 1990). This is well within the temperature range that can be achieved in a bonfire, so perhaps we should not expect to find a furnace associated with leadrich bead production. In other contexts, glass-vessel and glass-bead production could have occurred in the same workshop, with the heat from the furnace being used to soften or make the glass for bead production, and some of the raw materials were no doubt shared.

The published evidence for non-British bead production, when considered together, is helpful in the reconstruction of bead-making processes. Both Sarfatij (1977) and van Klaveren (1988) make reference to the context for bead production from seventh-century Rothulfuashem, the modern Rijnsburg near Leiden in the Netherlands (see also p 7 above). The beads made there are types that are found in Anglo-Saxon contexts in England, and the evidence includes crucible fragments bearing opaque yellow glass. The evidence for bead manufacture in early Christian Ireland is summarized by Henderson and Ivens (1992). Of particular significance is the occurrence of crucibles bearing opaque yellow glass on their inner faces from Dunmisk, Co. Tyrone (Henderson 1988; Henderson and Ivens 1992), with the evidence for adding an opacifier to a translucent glass and possibly the manufacture of yellow glass using other primary raw materials.

This production occurred in an industrial zone at Dunmisk in association with copper alloy working. In contexts of the sixth to seventh century in Maastricht (the Netherlands), amongst the evidence for bead production, there are crucible fragments apparently bearing colourless, translucent green and opaque yellow glass (Sablerolles et al 1997). Here, the crucibles had almost certainly been used during the bead-manufacturing process, as they were associated with glass rods, both melted and broken beads, complete beads, glass drops and ends of threads with tong marks. Some of these artefacts have the same composition as the glass in the crucibles. A frit-like material of this date has also been found at Maastricht, so it is possible that glass was being made there from primary raw materials (see also $p$ 7).

It would seem that Gam's point, that crucibles were not necessarily always used for bead production (Gam 1990), could be valid, though they certainly were used in 
early medieval Ireland, Holland and Denmark (for example). It cannot be a coincidence that of the evidence for glass found in crucibles, the majority is opaque yellow. This has a high lead content and its production/melting was apparently one specialization in early medieval Europe. Perhaps, as at Dunmisk, this glass colour was manufactured in these contexts using hearths. Reviews of the evidence for the manufacture of glass beads from Viking-Age Scandinavian sites by Lundström (1976) and Bencard with others (1978) do not refer to evidence for crucibles. Instead, they list failed beads, glass rods, glass tesserae, glass vessel fragments, dribbles of glass, some with tong marks, and chunks of raw glass and bichrome cables.

In a forthcoming publication, Callmer, with others, will discuss the Scandinavian evidence in more detail and will include crucible fragments found at Ribe in Denmark. Biek and Bayley (1979) record the evidence for high-lead glass-bead manufacture in early medieval (Viking-period) contexts and Henderson (1986) describes a range of by-products and their chemical compositions from the manufacture of high lead oxide (lead oxide-silica) beads in twelfth-century York. In much earlier contexts of around the third century BC, there is evidence for the technologically sophisticated manufacture of glass beads in moulds (Henderson 1987b), and the use of open moulds for Anglo-Saxon bead production is certainly a possibility, though to the author's knowledge none has been found.

\section{CASE STUDIES}

Two reports have been published on the scientific analysis of Anglo-Saxon beads from excavated cemeteries using quantitative analysis. They are for beads from Sewerby on the east coast of Yorkshire (Biek et al 1985) and Apple Down in West Sussex (Henderson 1990). Discussions of the colorants used for specific bead types are presented in a slightly different way in these reports. Unfortunately, it is too early to be able to state whether or not beads of the same type found in different regions, such as Yorkshire and Sussex, had different compositions or to suggest that they were made in different places. It is worth presenting, however, some examples of the range of chemical compositions that have been found in specific Anglo-Saxon bead types, from which we can suggest the probable raw materials used. The beads sampled and analysed from Apple Down included annular, globular, melon, barrel-shaped, cross-swag decorated globular, biconical, cross-swag decorated double globular, zigzag decorated annular, and globular with banded and zigzag decoration. Apart from just one exception (a globular bead with banded and zigzag decoration), all of these bead types analysed fell within the tight compositional range of soda-lime-silica (SLS) translucent glasses. The silica ranged from 62.9 per cent to 70.5 per cent, the soda levels 16.1 per cent to 20.3 per cent and the calcium oxide levels 5 per cent to 8.3 per cent. Variations of this composition linked directly to glass colour are SLS with high tin oxide in opaque white glasses, SLS with elevated tin oxide levels in opaque green glasses, SLS with high lead oxide and tin oxide in 
opaque yellow glasses and SLS with high iron and copper levels plus elevated lead oxide levels in red glasses. So, in spite of quite a wide range of glass bead types analysed at Apple Down, there was a consistent use of a well-defined range of glass chemical compositions.

One difference between the ways in which glass colours were achieved in the beads analysed from Sewerby and Apple Down is that many of the 'opaque' white glasses from Sewerby were made of a bubbly clear glass (Biek et al 1985). Occasionally, beads found in Anglo-Saxon contexts exhibit unusual compositions, such as the one mentioned above from the Apple Down cemetery. The dark green translucent matrix was found to have a mixed-alkali (potassium and sodium oxide) composition (Henderson 1990, table 2.1, analysis 26, plate 41). The technological implication here is that at some stage in the glass manufacture a plant ash must have been used as an alkali source. It would be interesting to find out whether such a radically different glass composition is characteristic of a specific production centre or zone, but only further detailed chemical analyses of excavated beads will provide the answer.

A further group of beads, from the Buckland, Dover cemetery, have also been analysed, though only semi-quantitatively (Bayley 1987). As well as identifying the colorants used, a more general consideration of the compositional data suggested origins in multiple manufacturing centres. Comparison of the Dover data with that from Portway, Andover and Sewerby (Bayley 1987, 186-7) highlighted compositional differences, which may be reflections of differences in place or date of manufacture, although only true quantitative determinations will eventually provide the necessary scientific rigour to be sure of these interpretations.

\section{ANGLO-SAXON BEADS AND GLASS TECHNOLOGY}

Clearly, Anglo-Saxon glass beads can be characterized as being a modification of the late Roman standardized technology, in which glass compositions are almost always a predictable soda-lime-silica composition, often with relatively high levels of manganese and iron oxides (at between 0.5 per cent and 1 per cent for both oxides). Similarly, Anglo-Saxon vessel glass tends to be soda-lime-silica in composition and one should not forget that glass-bead technology represents just one branch of a wider vitreous technology, which includes vessel glass, window glass and enamel. Indeed, enamel compositions are frequently very similar to the opaque glasses used in bead making, so the crafts are likely to have been linked at the primary production stage. In spite of this, there are some signs that Anglo-Saxon and other early medieval vessel glass contains impurities, which indicates a distinction in the manufacture between Roman and Anglo-Saxon glass (Henderson and Holand 1992).

Whilst it can be suggested that some Roman glass was recycled for the manufacture of beads and other glass objects (as mentioned above), there is evidence from early Christian Ireland that opaque yellow glass was manufactured by modifying 
translucent glass with the addition of opacifiers, or just possibly that it was fused from raw materials (Henderson and Ivens 1992). For the late Roman period, there is evidence for the melting of opaque yellow glass in crucibles at Catsgore in Somerset (Biek and Kay 1982) and similar glass, as well as opaque white glass, was present in some crucibles of early Saxon date from Buckden in Huntingdonshire/Cambridgeshire (Biek and Bayley 1979). In other parts of early medieval Europe, opaque yellow glass was prepared/heated up in crucibles associated with bead making (ie, Rijnsburg, Maastricht and Wynaldum in the Netherlands, and Ribe in Denmark). Overall, the use of colouring materials in glass shows nothing particularly new to be occurring in the Anglo-Saxon period. Opacifiers had already been exploited for thousands of years. Thus, white crystals of calcium antimonate in glass occur from at least the fourteenth century BC (Turner and Rooksby 1961) and tin oxide is first found in the second century BC (Henderson and Warren 1983) in late Iron Age glass from Europe, specifically associated with the appearance there of large protourban centres, the oppida.

The introduction of glass-blowing in the first century $\mathrm{BC}$ obviously made a vast difference to the supply of glass, in that glass vessels were now mass-produced. In turn, this had the effect of introducing much larger volumes of glass into the system (and increased the likelihood of recycled glass being used in manufacture in both the Roman and Anglo-Saxon periods).

Towards the end of the first millennium AD, we are able to observe gradual changes in glass technology in the Viking Age, for example, at Peel on the Isle of Man (Henderson 1993). Although soda-lime-silica glass was still in use in the ninth and tenth centuries, transitional glasses of a mixed-alkali (soda and potassium oxide) and other new compositions are also present, reflecting a glass technology in a state of 'flux'. Eventually, these changes led to the use of potassium-rich glass in the high medieval period for cathedral and church windows. 


\title{
ApPEndix \\ Notes ON THE COMPOSITION \\ OF COLOURED GLASSES
}

\author{
JUSTINE BAYLEY
}

Coloured glass is a complex subject as many different factors affect the final appearance of the glass. Among the most important are the impurities it contains (which may have been added accidentally or deliberately), the furnace conditions under which it was founded (such as the time for which it was heated, the temperature it reached and the furnace atmosphere, which can be oxidising or reducing) and its bulk composition.

Some of the minor elements in glass are accidentally incorporated since they are present as impurities in one of the major glass-forming ingredients. An example of this is iron, which is an almost ubiquitous component of ancient glass. Other minor elements are deliberate additions, usually intended to produce a change in the colour or the opacity of the glass. These effects are described in detail below.

Furnace conditions can affect the appearance of glass. If it has not been raised to a high enough temperature for long enough, the gases it contains cannot escape and the resulting glass is 'seedy' (full of bubbles), which can make a transparent glass appear opaque, as mentioned above ( $\mathrm{p} 84$ ). Some truly opaque colours, produced by precipitating a crystalline compound from the glass melt, are unstable when heated. One example is the yellow of lead-tin oxide (see below, $\mathrm{p} 91$ ).

The most important facet of the furnace conditions is the atmosphere, ie, whether it is oxidising or reducing. For instance, a glass containing iron will appear blue if melted in strongly reducing conditions, green in less strongly reducing conditions and yellow or brown in oxidising conditions. These reduction/oxidation (redox) effects are often complicated by the presence of more than one colouring impurity in the glass, as there will be a redox equilibrium between the two colourants as well as with the external atmosphere. In glasses containing both iron and manganese, this can (with time as an added variable) produce a whole range of colours and even colourless glass (Sellner et al 1979; Green and Hart 1987).

\section{COLORANTS}

The elements found in ancient glass that affect its appearance are mainly manganese, iron, cobalt, copper, tin and antimony. The presence or absence of lead is also important, but selenium, chromium and uranium, though added as colorants in modern glass, were not used in antiquity.

The colorants can be either metals in solution in the glass or precipitated 
compounds, which are usually crystalline. The first type produces transparent colours, while the second is normally opaque. With transparent glasses, the depth of colour is related to the amount of colorant present (other things being equal), but with precipitated colorants an increase in concentration above a certain minimum level tends merely to increase the opacity of the glass.

\section{MANGANESE AND IRON}

The range of manganese and iron colours has already been mentioned (see above, p 89), although it should be noted that conditions in antiquity were rarely sufficiently reducing to give a true blue. In practice, the reduced iron colour was a green or bluish-green. The decolorizing effect of manganese on iron is a complementary colour effect, the purple produced by the manganese balancing the iron yellow and giving a pale neutral grey, which appears colourless in thin pieces. The use of manganese as a decolorizer appears in the second century BC (Sayre 1963; Sayre and Smith 1967, 300). A glass containing large amounts of iron, with or without manganese, appears opaque black. In fact, the glass is neither opaque nor black. If a thin enough piece is examined, it is usually seen to be transparent dark olive, the apparent opacity being due to the depth of colour rather than the presence of opacifying agents (Bayley 1987, 185).

\section{Cobalt}

Cobalt is a very intense colorant; even very small amounts produce a deep cobalt blue colour in glass. Phillips (1941) states that 10ppm (ie, 0.001 per cent) of cobalt gives a distinct colour, while $100 \mathrm{ppm}$ ( 0.01 per cent) gives an 'intense' blue, though Biek et al $(1980,73)$ have detected as much as 2 per cent in some Roman enamels, albeit in those described as 'very dark blue'. Sometimes blue cobalt-coloured glass contains similar amounts of iron to that in the iron-coloured glasses, but the effect of the iron is totally masked by that of the cobalt.

\section{COPPER}

Copper produces a range of colours. In oxidising conditions it dissolves in the glass to give clear turquoise blues in alkali glass and greens in high-lead glass (Sayre and Smith 1974). Under reducing conditions, it is precipitated from lead glass either as cuprous oxide $\left(\mathrm{Cu}_{2} \mathrm{O}\right)$, or as finely divided metallic copper, which can produce a translucent 'ruby' red (Weyl 1962), though this is not usually found in glass beads, or a totally opaque red colour, often referred to as 'sealing-wax red' (Biek et al 1980, 73-4; Henderson 1993). The presence of iron in red glass helps to retain the reducing conditions. A red colour can also develop in glasses which contain little or no lead, varying in colour from a bright true red through a dull brick red to a chestnut brown, or even black if heated in contact with air (Hughes 1972; Brill 1970, 119-28). Glass with the same composition can also appear a bright or dull orange (Biek et al 1980, 74). 
The factors which govern the appearance of one rather than another of these reduced copper colours are incompletely understood, but the total amounts of copper and lead and the particle size of the precipitate both appear to be significant. The brown colours may be associated with insufficiently reducing conditions. Often glass which appears 'red' in the hand can be seen under the low-power microscope to be red with brown or black streaks, and orange glass often has red streaks. One tessera from early Viking-period levels in Ribe (Denmark) was a block with layers of red and orange glass with over twenty bands in under a centimetre.

TiN

Although small amounts can dissolve in glass and have little, if any, effect on its appearance, tin is normally associated with opaque glasses. Tin oxide $\left(\mathrm{SnO}_{2}\right)$ is a white crystalline material that gives glass an opaque white colour (Turner and Rooksby 1959,25$)$. If the glass is coloured, then an opaque colour will be produced; for example, cobalt and tin will give an opaque blue glass.

Tin in lead glasses can produce an opaque yellow colour by precipitating lead-tin oxide ' $\mathrm{PbSnO}_{3}$ ', but this pigment is unstable on heating and above $900^{\circ} \mathrm{C}$ begins to give tin oxide and so becomes white (Rooksby 1964, 21). This change is irreversible; reheating below $900^{\circ} \mathrm{C}$ will not restore the yellow colour.

These tin-containing opaque pigments are relatively late introductions to glass technology. They only become common in late Iron Age Europe after the second century BC (Henderson and Warren 1983), but are almost completely absent from opaque Roman glass. Recent analyses have found lead-tin oxide in all the yellow beads from Sewerby (Hirst and Biek 1981, 141; Biek et al 1985), and both yellow and white tin pigments were identified in glass in early Saxon crucibles from Buckden in Huntingdonshire/Cambridgeshire. There is a yellow bead from a woman's grave in Jutland dating to around AD 400 (Brinch Madsen 1975), which has significant amounts of tin and very little antimony, and presumably this is lead-tin coloured and opacified (information from G Gilmore).

\section{ANTIMONY}

Antimony is the other major opacifier, but it is also found in similar concentrations in transparent glass (Sayre and Smith 1974). In a reduced state antimony acts as a decolorizer for transparent iron-containing glass producing an '. . exceptionally colourless limpid glass ...' (Sayre and Smith 1967, 300) as it also acts as a clarifier, aiding the removal of bubbles from the glass. Sayre (1963) states that antimony was not used as a decolorizer from the fifth century AD onwards.

In an oxidized state antimony forms compounds, which precipitate in glass, producing opacity. Calcium antimonates $\left(\mathrm{Ca}_{2} \mathrm{Sb}_{2} \mathrm{O}_{7}\right.$ and $\left.\mathrm{CaSb}_{2} \mathrm{O}_{6}\right)$ are white and lead antimonate $\left(\mathrm{Pb}_{2} \mathrm{Sb}_{2} \mathrm{O}_{7}\right)$ yellow (Turner and Rooksby 1959, 21-8). These opaque colorants are used from antiquity down into the Roman period, but are only exceptionally found in later periods (Biek and Bayley 1979, 10), though 
antimony was detected in some of the opaque polychrome beads from the Buckland, Dover cemetery (Bayley 1987, 186). Interestingly though, the yellow colour on tin-opacified lead-glazed pottery (maiolica) is due to lead antimonate (Gillies 1982).

\section{LEAD}

Lead in glass does not itself produce colour (except in the form of lead-tin and leadantimony oxides), but can change the hue produced by colorants in solution. Its more important role is, however, in the opaque glasses. Most of the crystalline colorants are soluble in molten lead glass, but precipitate out of solution as the glass cools and solidifies. This precipitation happens gradually as the temperature falls, so the crystalline colorants form in a controlled way and are uniformly distributed through the glass giving a uniform colour and opacity.

\section{OPACITY}

Opacity is not an absolute, but a matter of degree. It varies from total opacity (not allowing passage of light) through partial opacity to translucence (where light is transmitted) and transparency (where the background can be distinctly seen through the object).

Opacity in glass is due to a number of factors, most of which have already been mentioned in passing. It can be due to intensity of colour (eg, black, iron-containing glass), to bubbles in the glass, to decay of the glass or to the inclusion of opacifying agents (eg, tin oxide).

The 'colour intensity' type of opacity also affects more transparent glasses. Most ancient 'transparent' coloured glass is in fact translucent as the intensity of the coloration affects the transmission of light. There can be no hard and fast division, however, as a thick piece of glass will appear less transparent than an otherwise identical thin piece.

The bubbles and other inhomogeneities in a glass are a measure of how well made it is. Sometimes unreacted quartz (part of the original glass-making raw materials) is present. Other crystalline components are also found, ie, those that can be formed by partial devitrification at the time of manufacture, if the glass cools too slowly from its liquid state. Even well-made glass can be poorly worked, producing striations in the finished objects.

The changes that have taken place in the glass since it was fashioned into an object have to be taken into account in arriving at a description of how the object would originally have appeared. The glass itself can decay in any number of ways. Its surface may have become crusted, pitted or fissured, or have developed a thin irridescent coating, or even a far thicker layer, which may have fallen off. The whole thickness of the glass may have decayed or oxidized, changing its colour. The commonest change is purely physical abrasion, however. Thus, an originally transparent piece of glass will now appear partly opaque, because its once smooth shiny surface has become roughened. 
Even the presence of opacifying agents need not produce a totally opaque glass. Small amounts of opacifying agents produce a milky effect and inspection under a low-power microscope shows that opaque particles are distributed through a translucent or transparent matrix. Even in totally opaque glass, more intensely coloured patches can be seen, where there is a larger than usual crystalline particle.

One further facet of opacity and colour should be borne in mind. Opaque blues and greens are often not very opaque. If they were, most of what would be seen would be the opaque particles with little surrounding clear blue or green glass. Conversely, the colour of the transparent base glass of a totally opaque white should not matter much, as little or none of it will be visible between the precipitated white particles. 


\title{
ConClusion
}

\section{A future for Anglo-Saxon GLASS BEAD STUDIES?}

\author{
Martin Welch
}

This chapter was begun after the death of Margaret Guido, who sadly did not live to see her study of Anglo-Saxon glass beads in print. Her contribution in two major published studies has been to offer us a national picture for glass beads in Britain, from its prehistory to the Anglo-Saxon period, placed within a broader context provided by the Irish, Scandinavian and European evidence. She has offered us a framework from which to build, though not every scholar who has constructed a glass-bead report for an Anglo-Saxon site in recent years will necessarily agree with her choice of terminology or all of her opinions. Nevertheless, her present study gives us a starting point and, it is to be hoped, will prove a useful first point of reference for many finds officers processing material from future Anglo-Saxon cemetery and settlement excavations. Detailed excavation recording of beads should be combined with efforts to recover the fullest possible evidence and ideally this will allow us to reconstruct the original order of bead strings. Such work will also include the study and identification of preserved threads from the strings, as well as their conservation.

In one sense, this study represented a disappointment to its author, for she had hoped to be able to define fairly tightly the date range of individual glass-bead types and colours on the basis of their associated grave assemblages. In practice, at best only very broad groupings have emerged, with some relatively early beads from burial contexts of the fifth and sixth centuries overlapping with others rather later, belonging to assemblages datable to the sixth and seventh centuries, or even to the seventh and eighth centuries. It seems that changes in glass-bead fashions were spread over rather longer time spans than the relatively short archaeological phase during which fully dressed deposition on the funeral pyre or in the trenched grave was a feature of Anglo-Saxon cemeteries in eastern, northern and southern England, ie, the fifth to early eighth centuries. Excavation and, more importantly, the publication of wealthy Middle Saxon settlements such as Barking (Essex), Brandon (Suffolk), Flixborough (Lincolnshire/Humberside) and Fishergate, York (Rogers 1993, 1378-82 and 1456-8), are beginning to throw new light on bead fashions in 
the succeeding period and will provide a thread of continuity seen in the artefact studies of bone combs using the Hamwic (Southampton) evidence and dress pins, as will be revealed in the forthcoming study by Dr S Ross.

Of course, within the early Anglo-Saxon period, it is clear that glass beads should never be studied in isolation and we still need an overall synthesis of all beads and pendants, whether made of natural materials, glass or metal, which formed the festooned strings, necklaces and armlets on the necks, chests, wrists and waists of women and some children. The contrast observed at Sewerby between bead strings dominated by the presence of either translucent blue annular glass beads or amber beads (Hirst 1985) has made finds researchers in England aware that the overall patterning of the strings is a significant factor. Bead assemblages from continental and Scandinavian burials need to be analysed in the same manner, so that we can compare and contrast not just the individual bead types, but also the patterning of their combinations. It is through the statistical analysis of such comparisons that a fuller understanding of the fashions and distribution patterns of glass beads will emerge. A computer program has been developed recently for this purpose at the Römisch-Germanische Kommission at Frankfurt-am-Main, and information about this project and the English-language version of this program can be obtained from the Department of Medieval and Later Antiquities at the British Museum.

It is linked to initiatives associated with the international conference on beads held in Mannheim in 1994, whose recent publication provides a valuable summary of the present state of research across Europe (von Freeden and Wieczorek 1997). Many of the continental comparisons cited by Margaret Guido reflected the availability to her of accessible publications, as well as the museum collections she was able to visit in person, and it may well be that, for example, she exaggerated the relevance of the rich cemetery evidence from south-west Germany to the development of Anglo-Saxon glass beads. Still, any such imbalance should be rectified by the further research of others.

For the future, real advances in our understanding of glass beads will only be made when we have located and excavated workshop sites for their manufacture and perhaps also identified the sites of the fairs and markets where they changed hands. Workshops have been located, for example, in Ireland, the Netherlands and southern Scandinavia, and it should be a national research priority to identify and excavate a wide range of industrial craft sites in post-Roman Britain and not merely those relevant to glass products. The graves or hoards of smiths, such as that recently excavated by chance at Tattershall Thorpe (Hinton 1993), may provide additional insights, but the opportunity to analyse the glass from crucibles and rejected beads dumped when manufacturing sites were cleared at the end of each season's activity will prove a major advance, assisting us in identifying which bead types were fashioned here in England.

In the meantime, a major element of current research involves the use of sampling techniques for the application of scientific analysis to glass beads, as 
described by Julian Henderson and Justine Bayley in the previous chapter. Teamwork and co-operation will be essential, if archaeologists, conservators and archaeological scientists are to work together to prioritize the research questions that need to be addressed and construct a sampling strategy to match. The publication in recent years of so many early Anglo-Saxon sites excavated since the 1950s, and the promised appearance in print of many more before the century is out, means that we will not be short of fresh and well-recorded raw data to test theories and develop research strategies. Equally, we must not be afraid to propose new research excavations where these can advance studies further. Much will depend on what stance English Heritage and other national bodies in the field of archaeology take to the funding of future research in what is left of this decade and into the next millennium. 


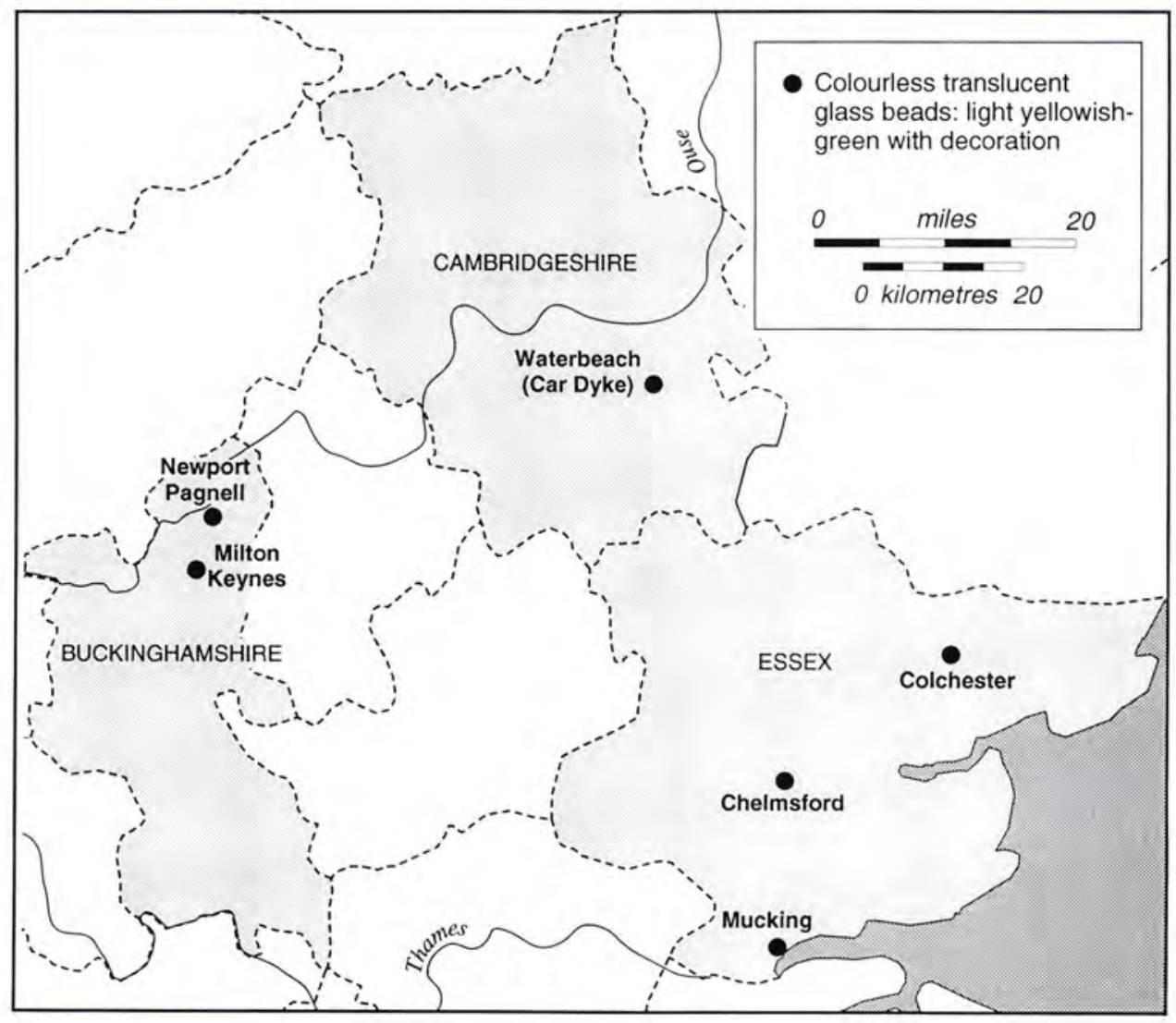

Map 1 Distribution of type 1viii beads 


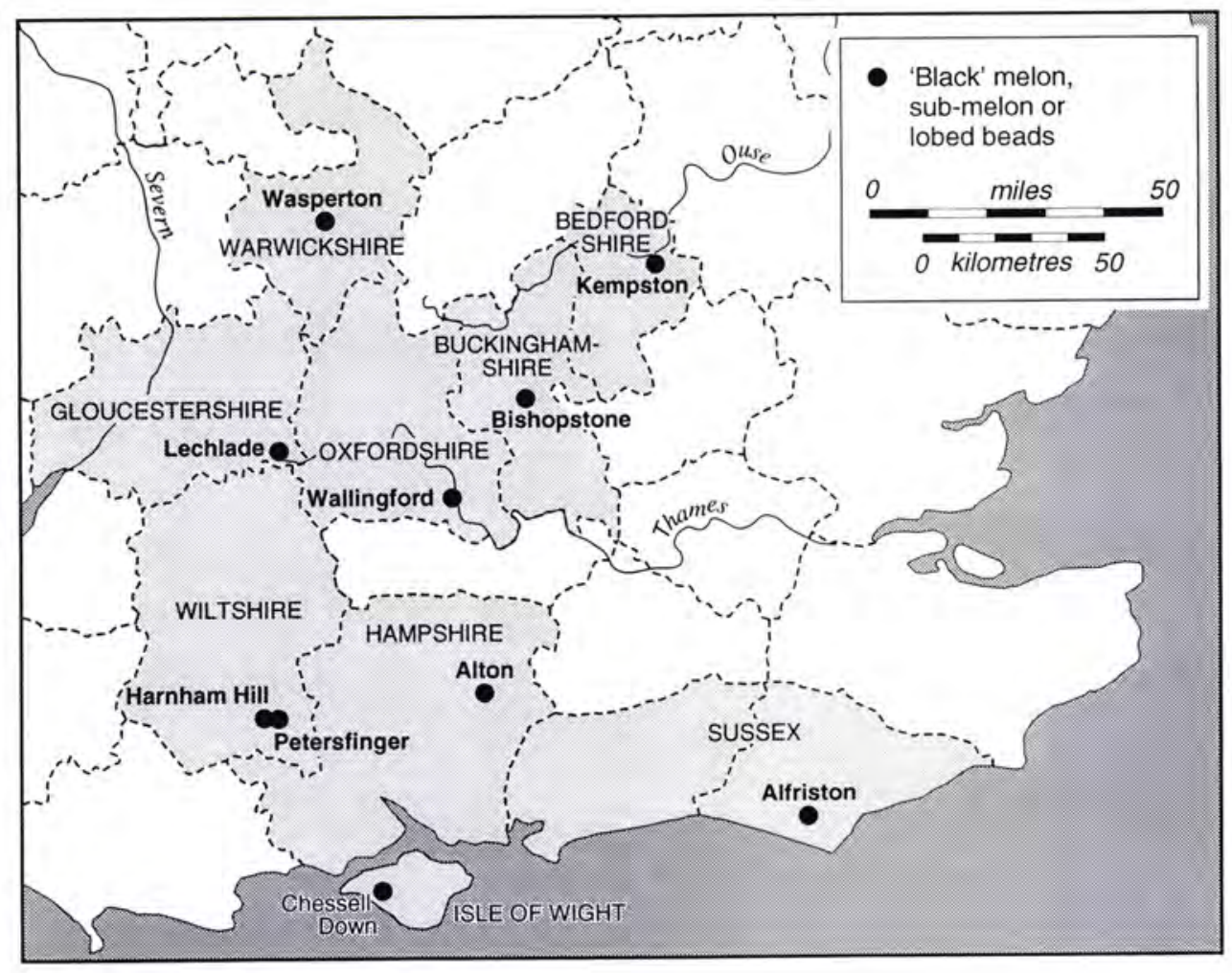

Map 2 Distribution of type 2 iii beads 


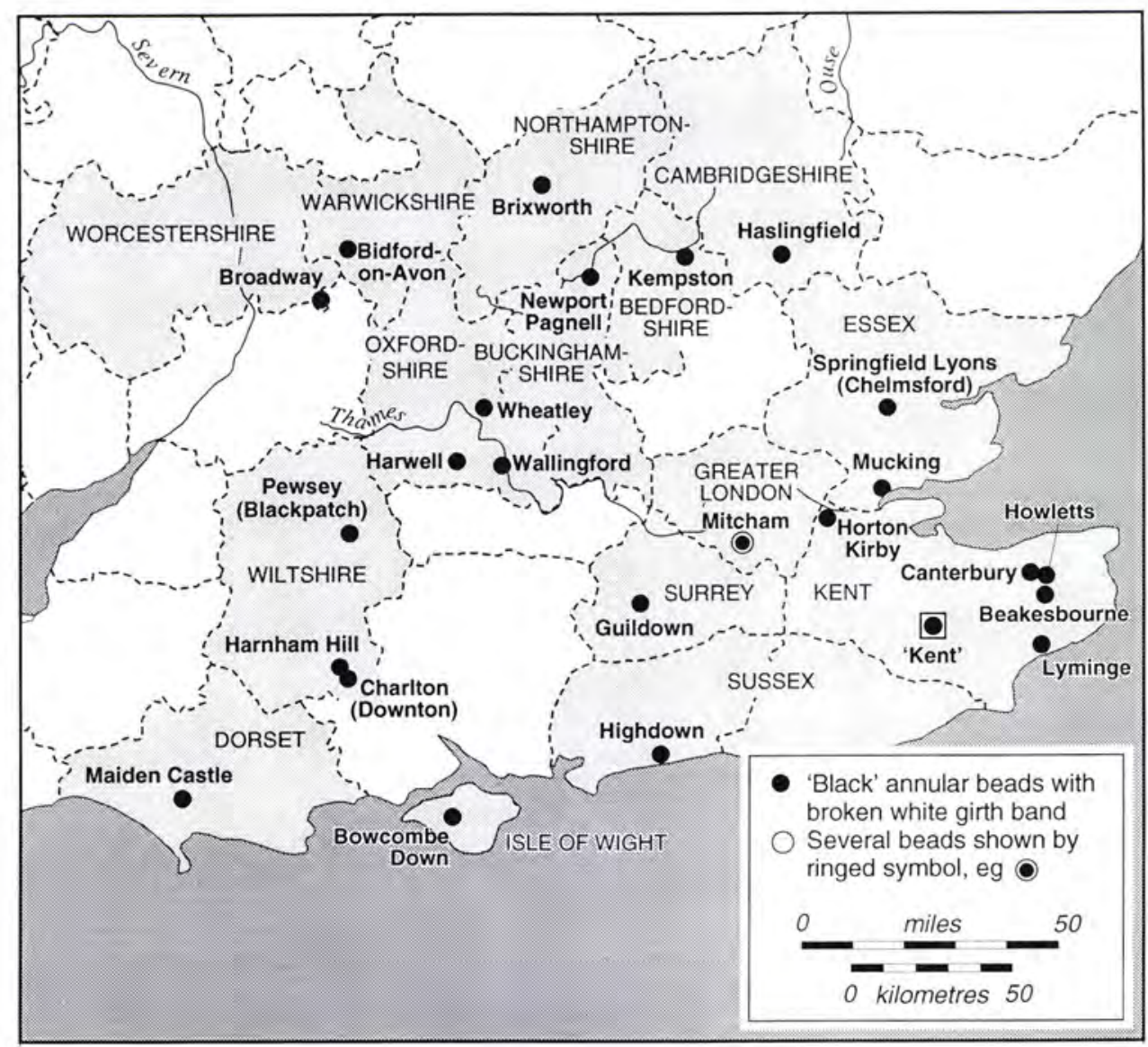

Map 3 Distribution of type 2iv beads 


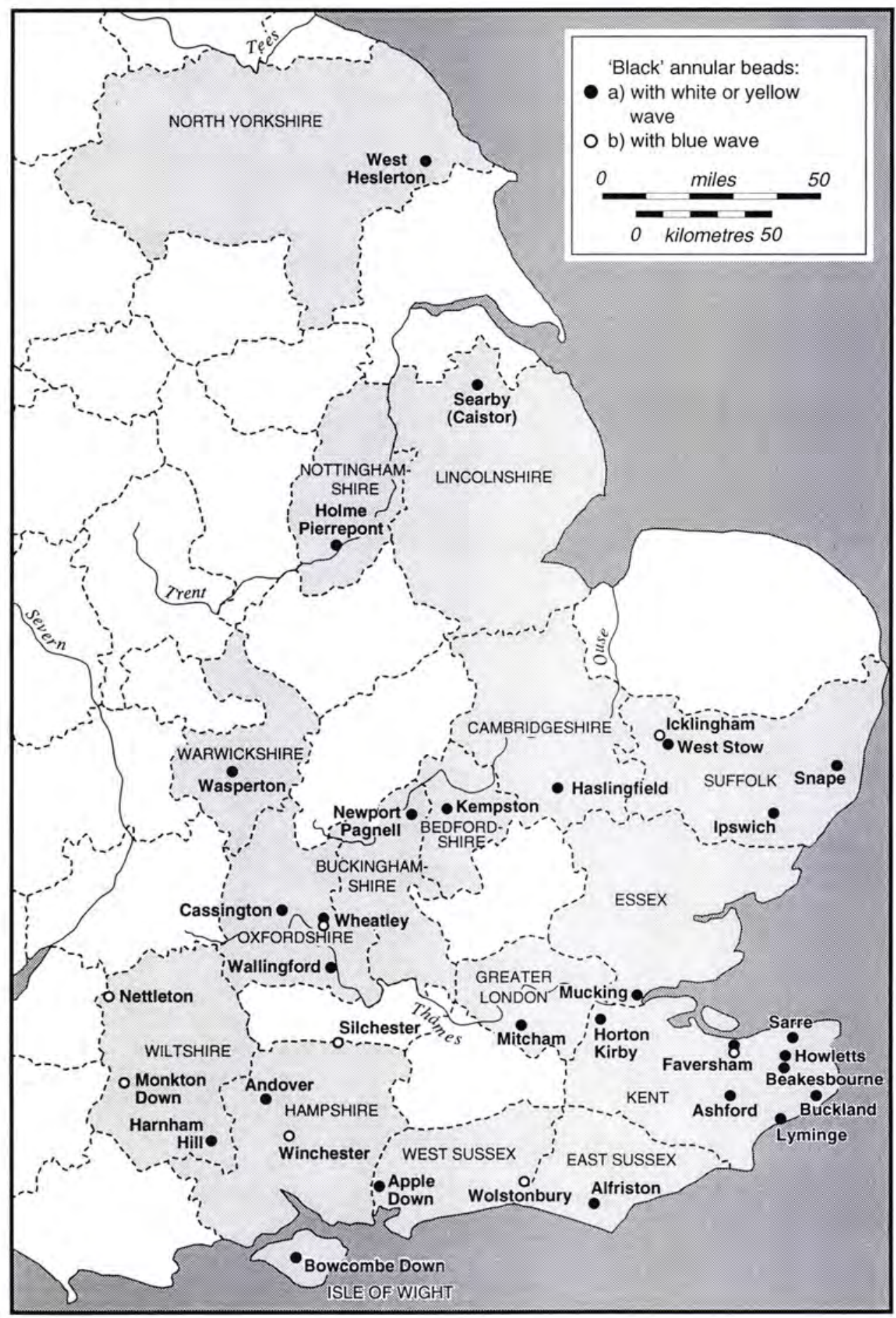

Map 4 Distribution of type $2 v$ beads 


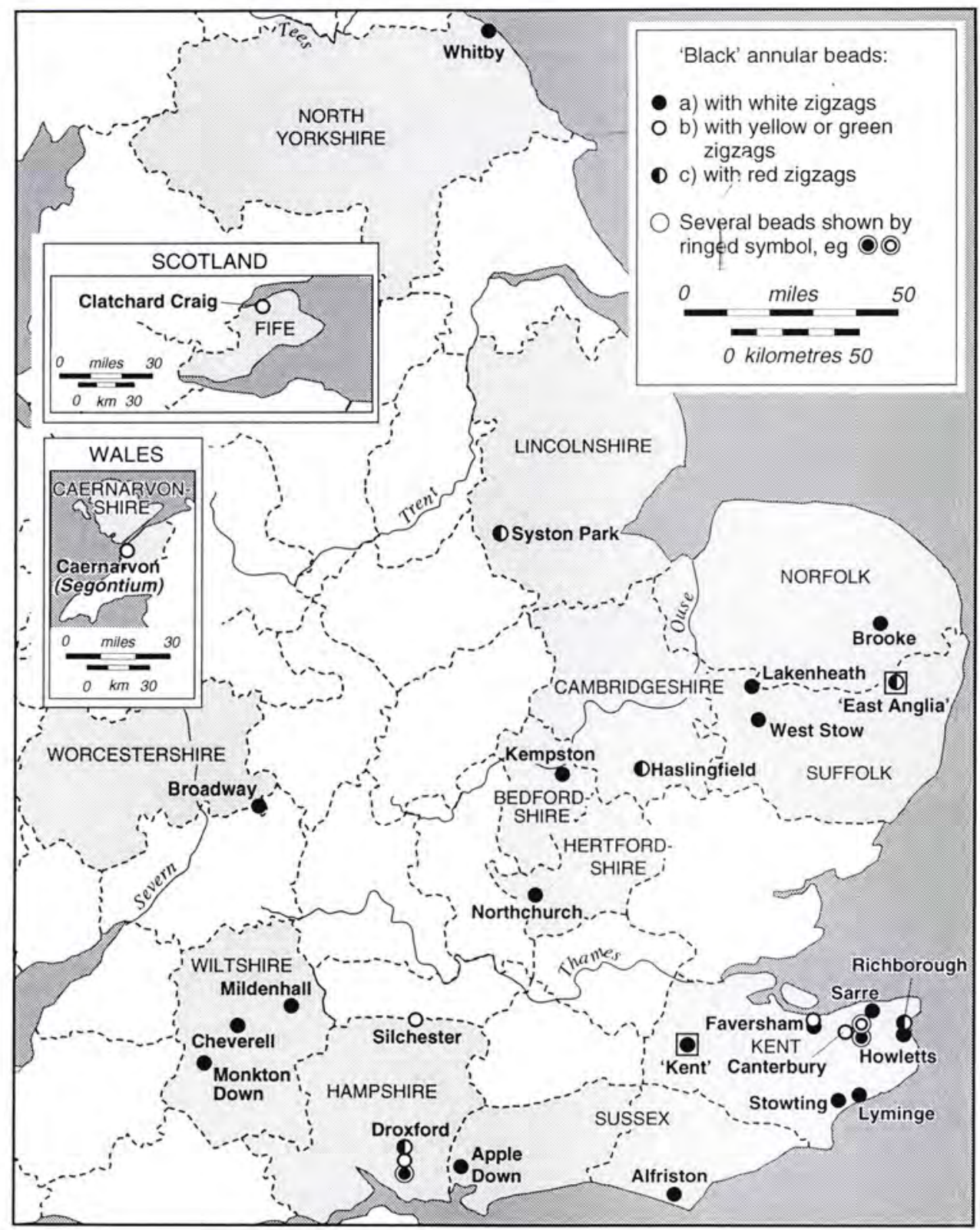

Map 5 Distribution of type 2vi beads 


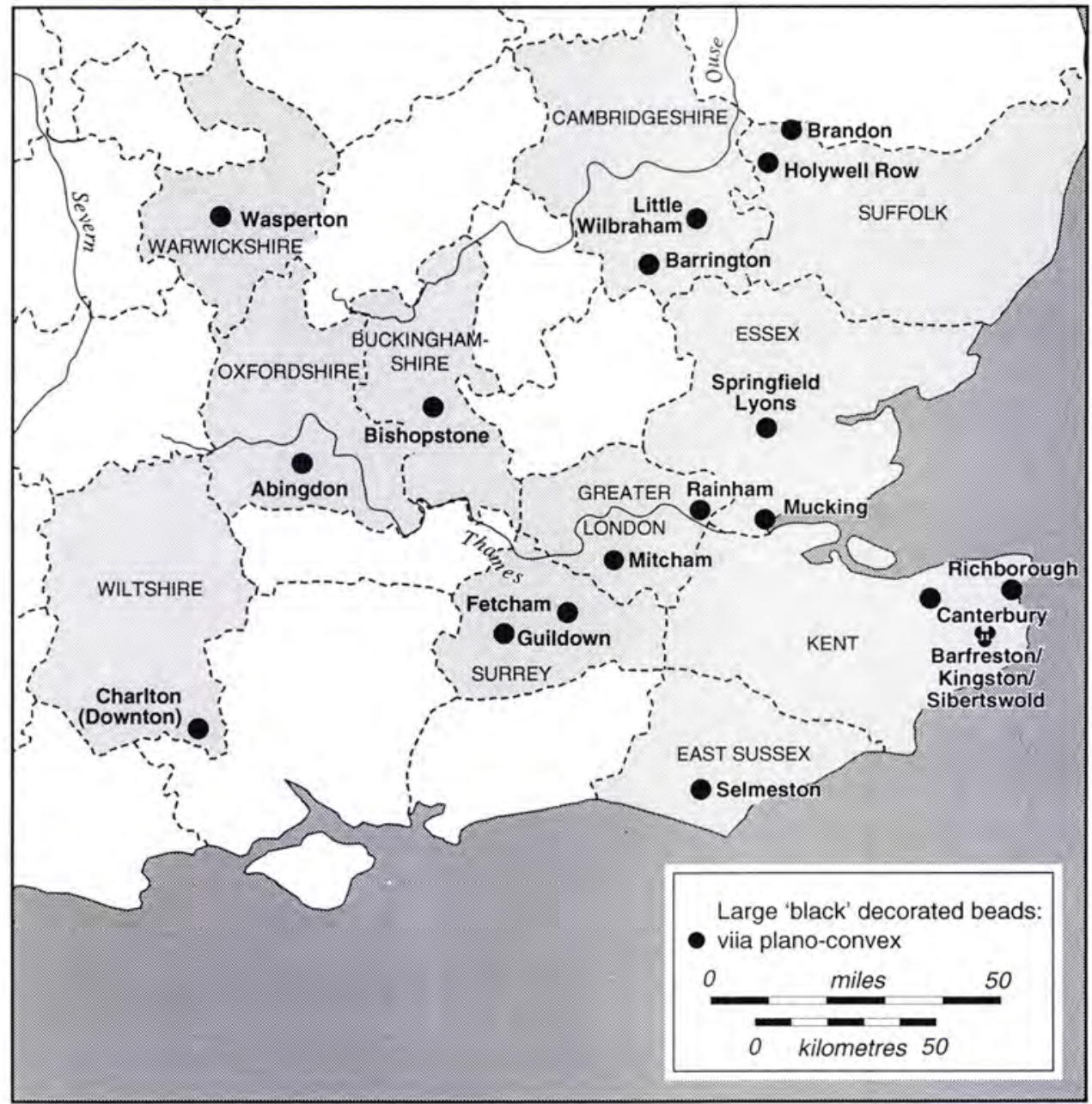

Map 6 Distribution of type 2viia beads 


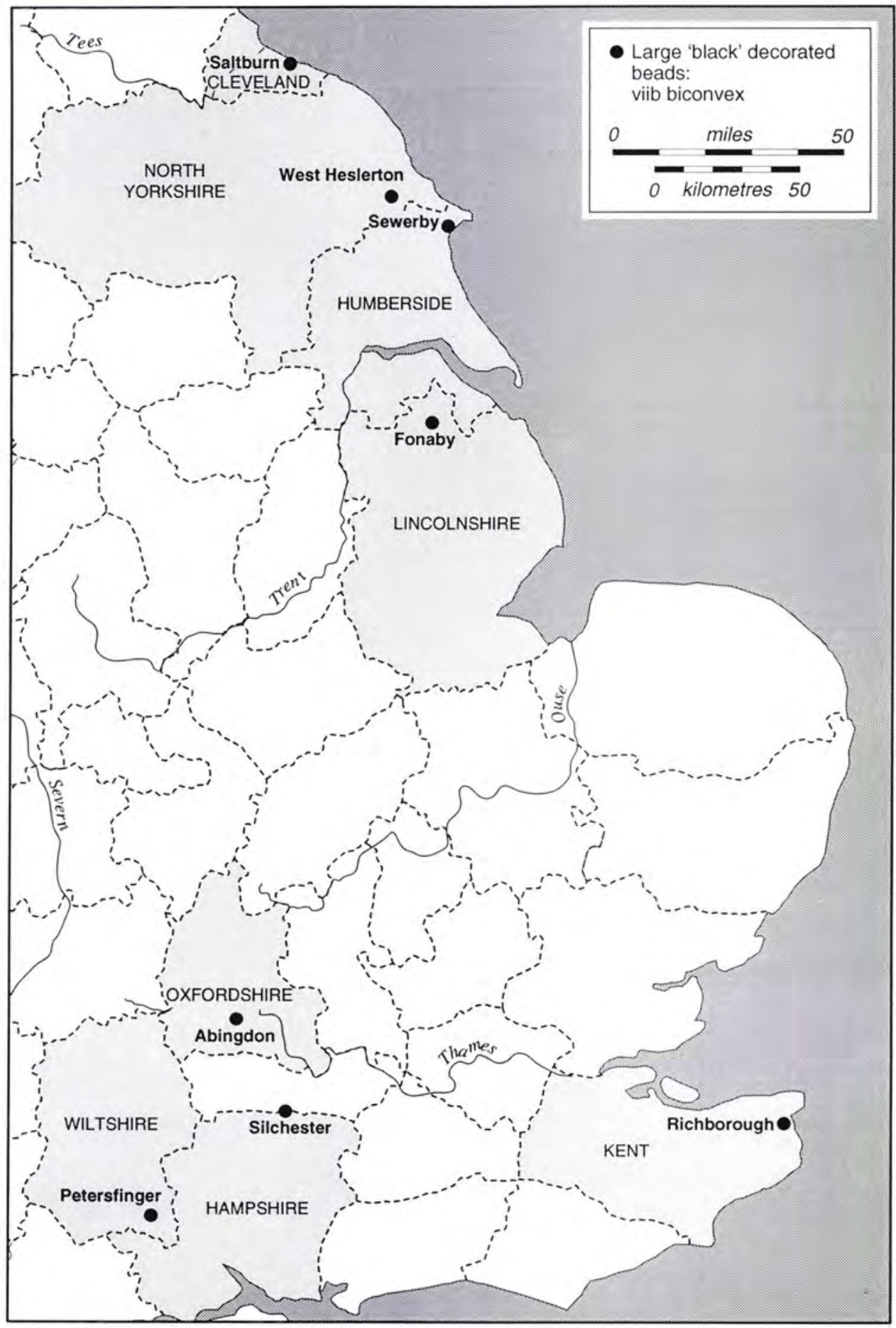

Map 7 Distribution of type 2viib beads 


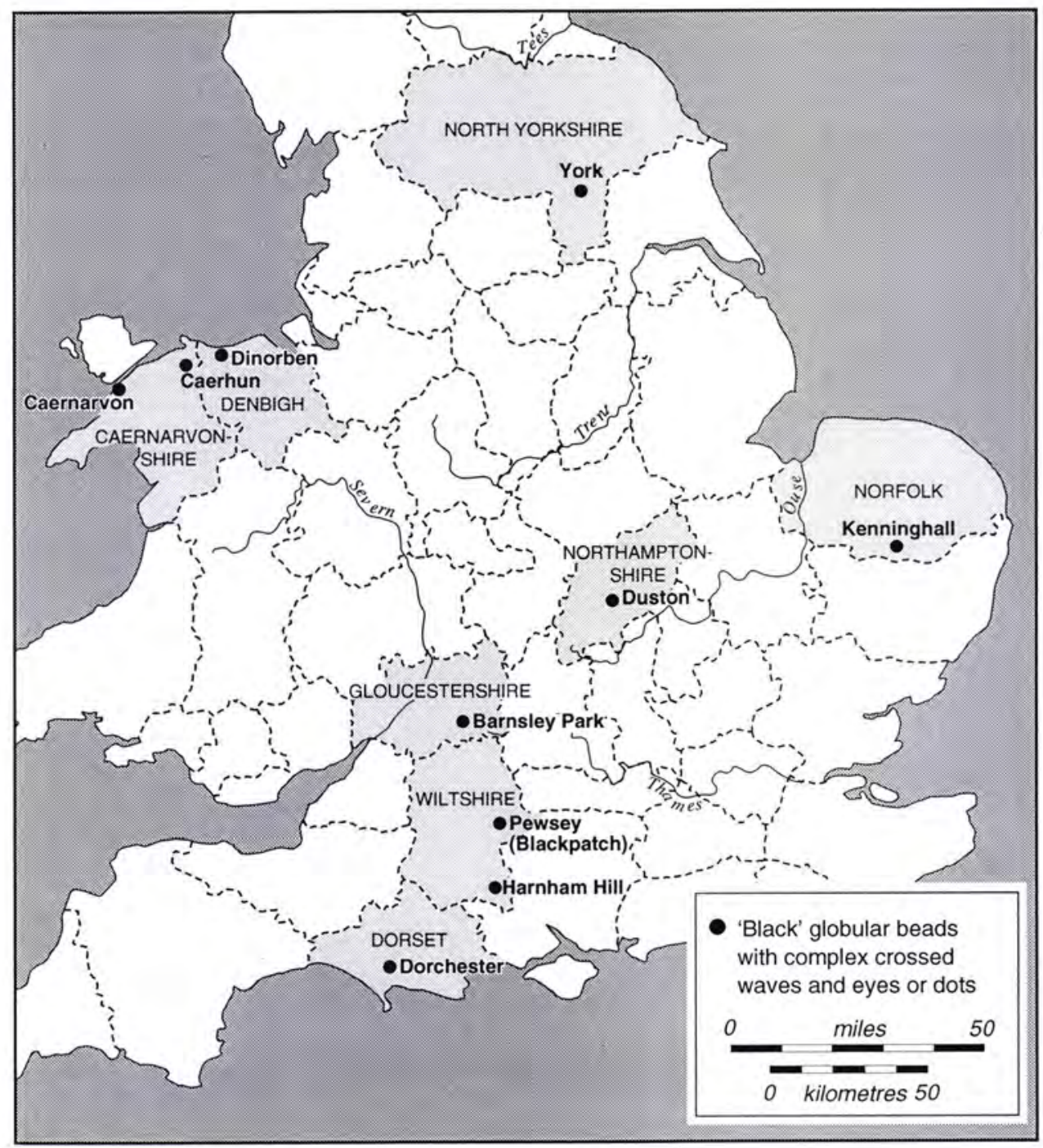

Map 8 Distribution of type 2viii beads 


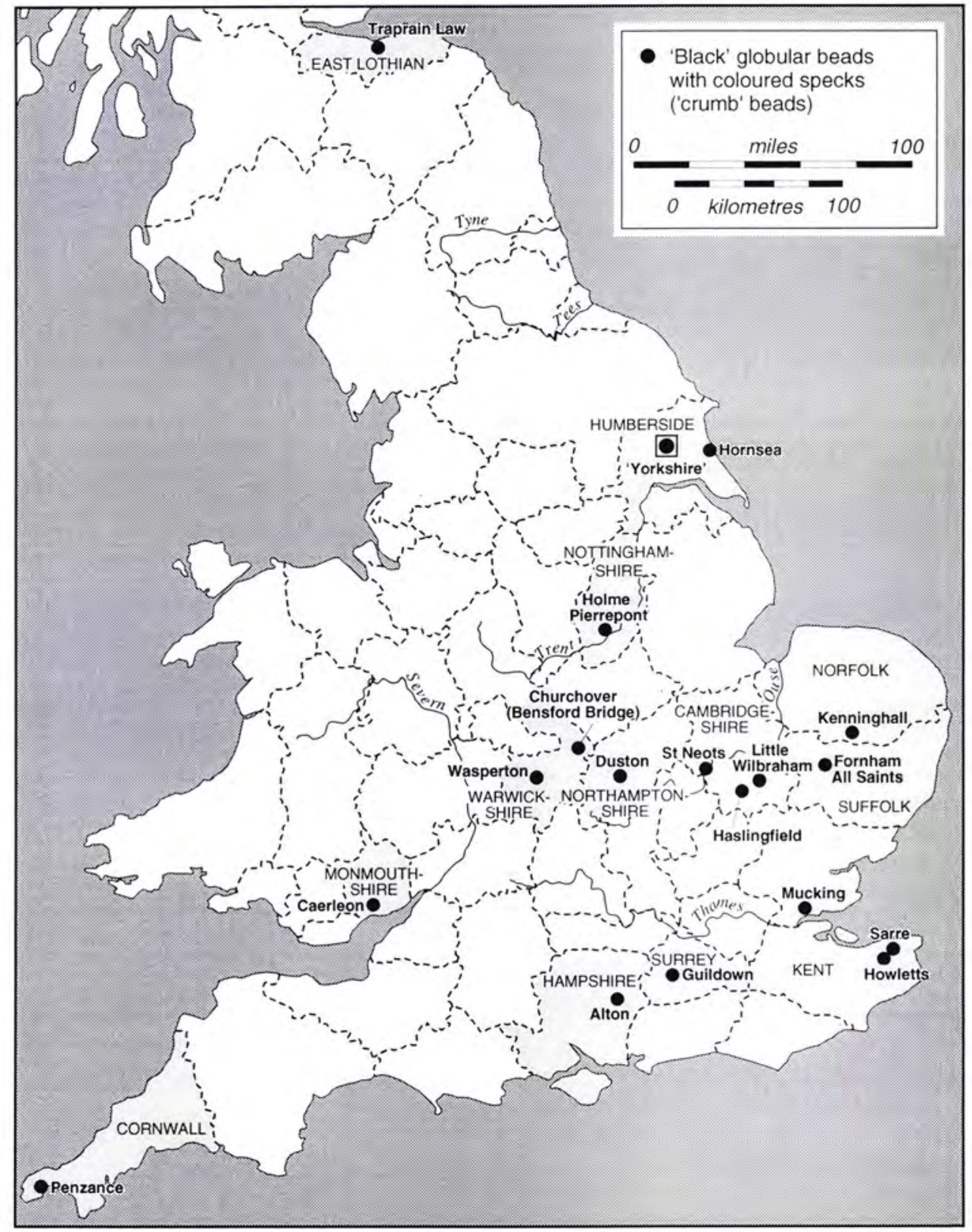

Map 9 Distribution of type 2xi beads 


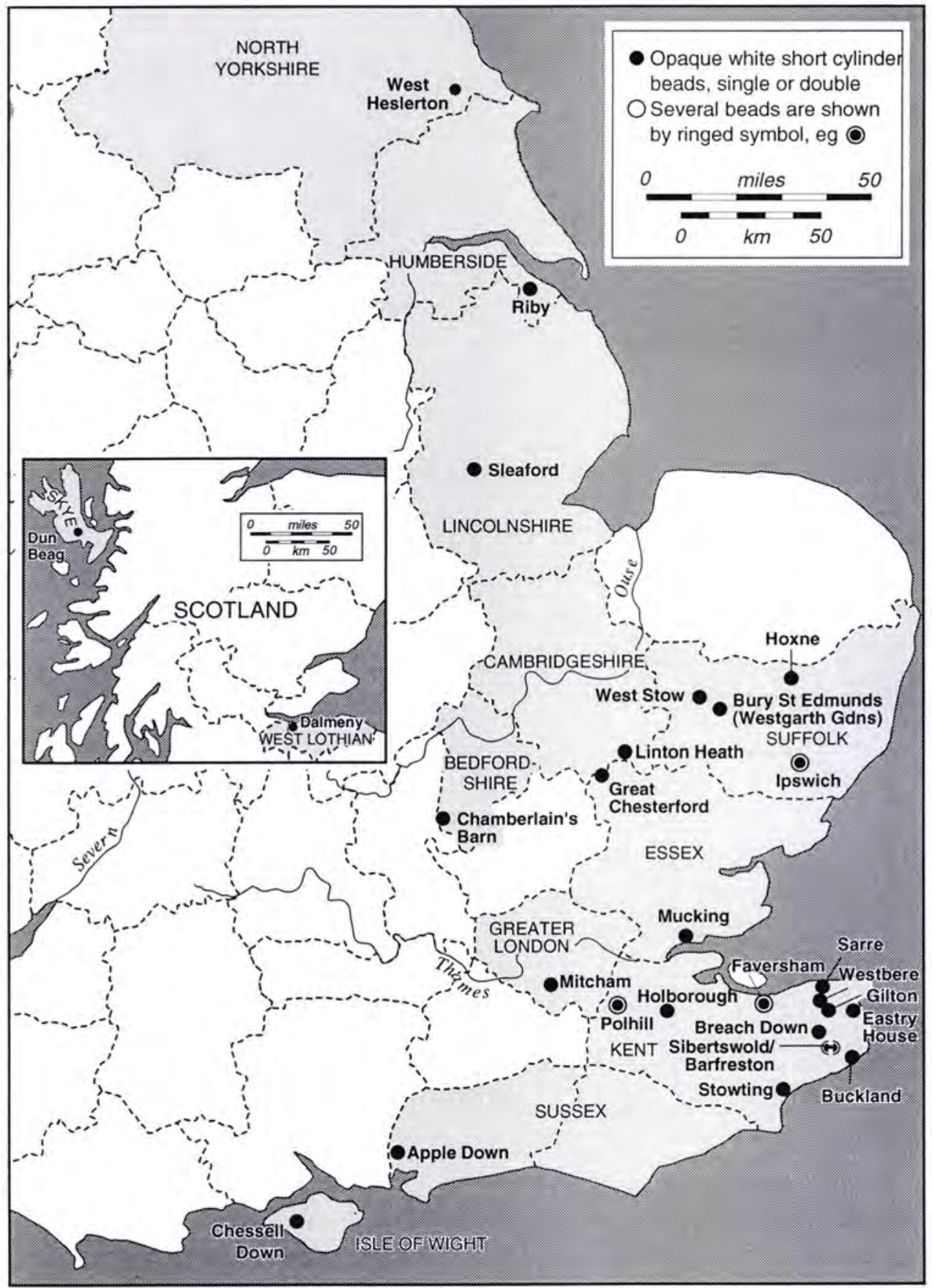

Map 10 Distribution of type 3 ii beads 


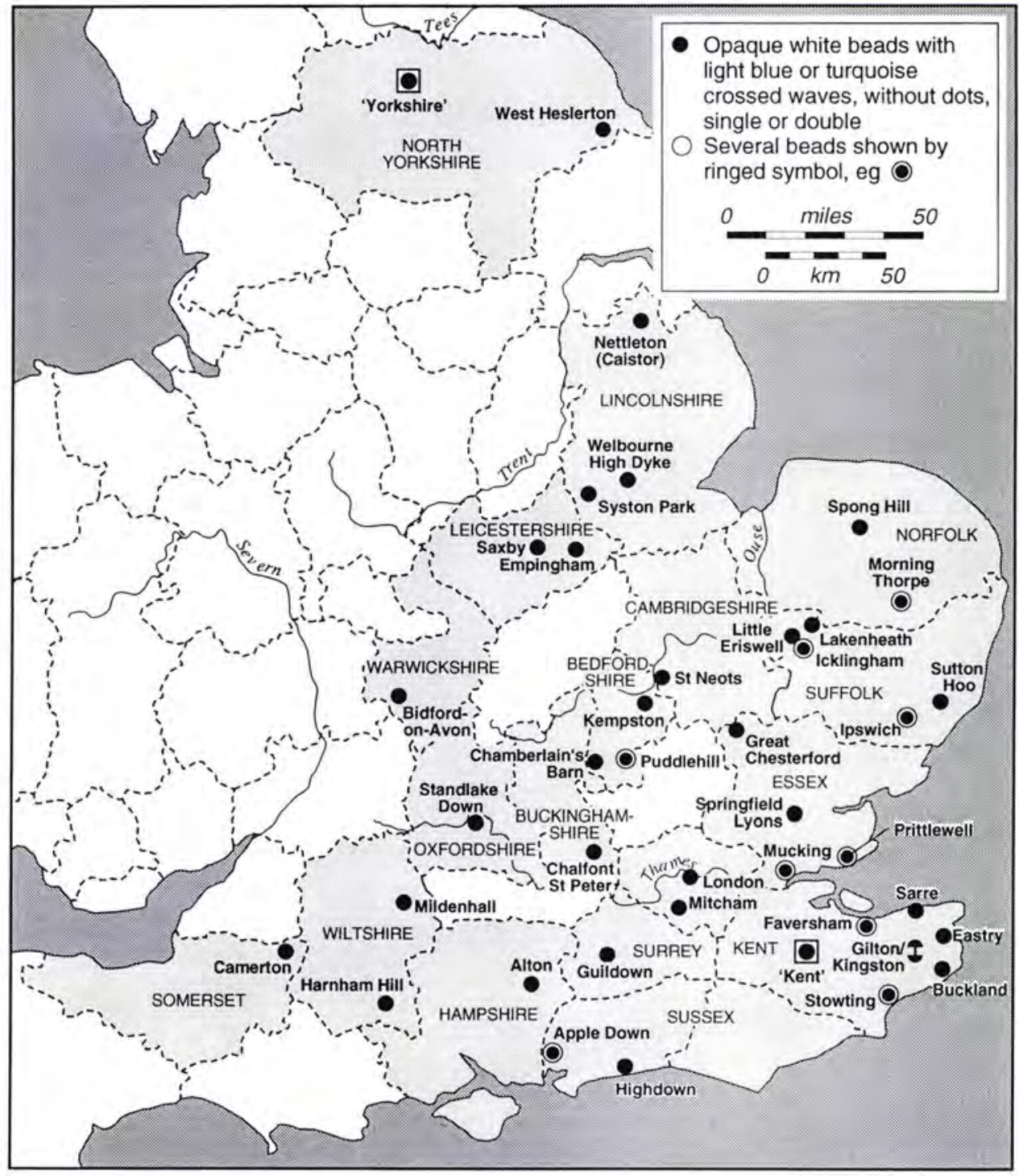

Map 11 Distribution of type 3iiia beads 


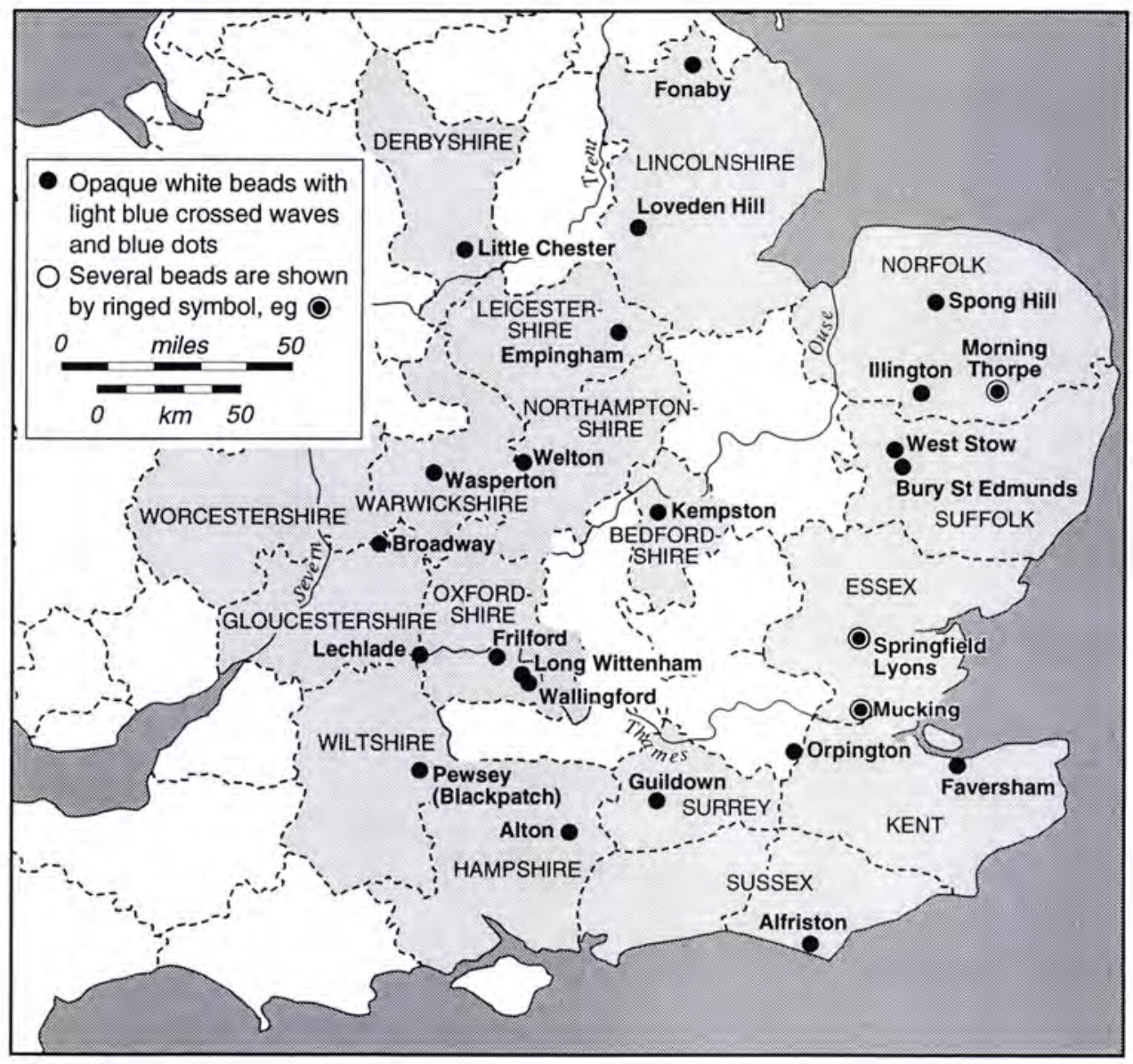

Map 12 Distribution of type 3 iiib beads 


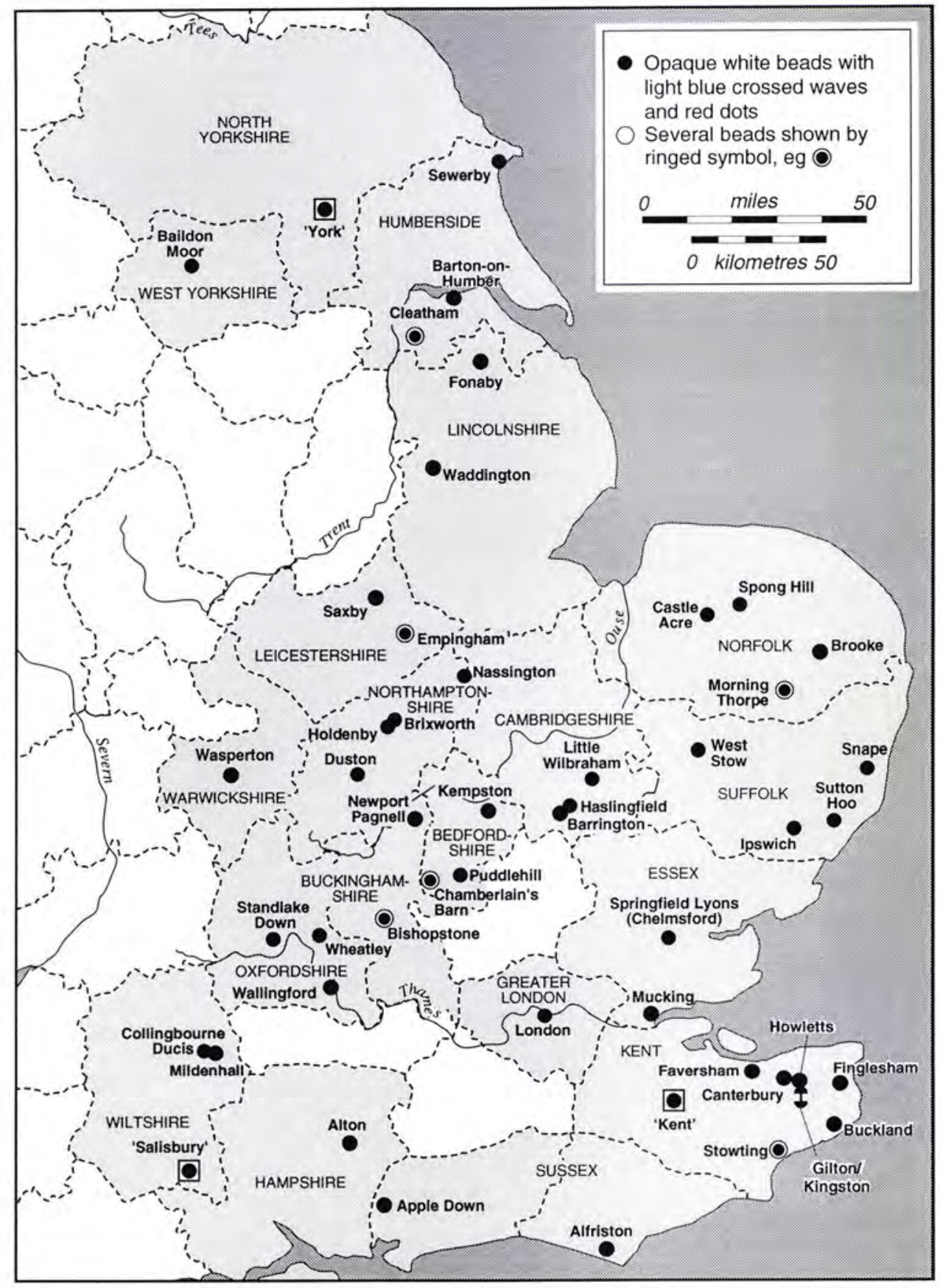

Map 13 Distribution of type 3iiic beads 


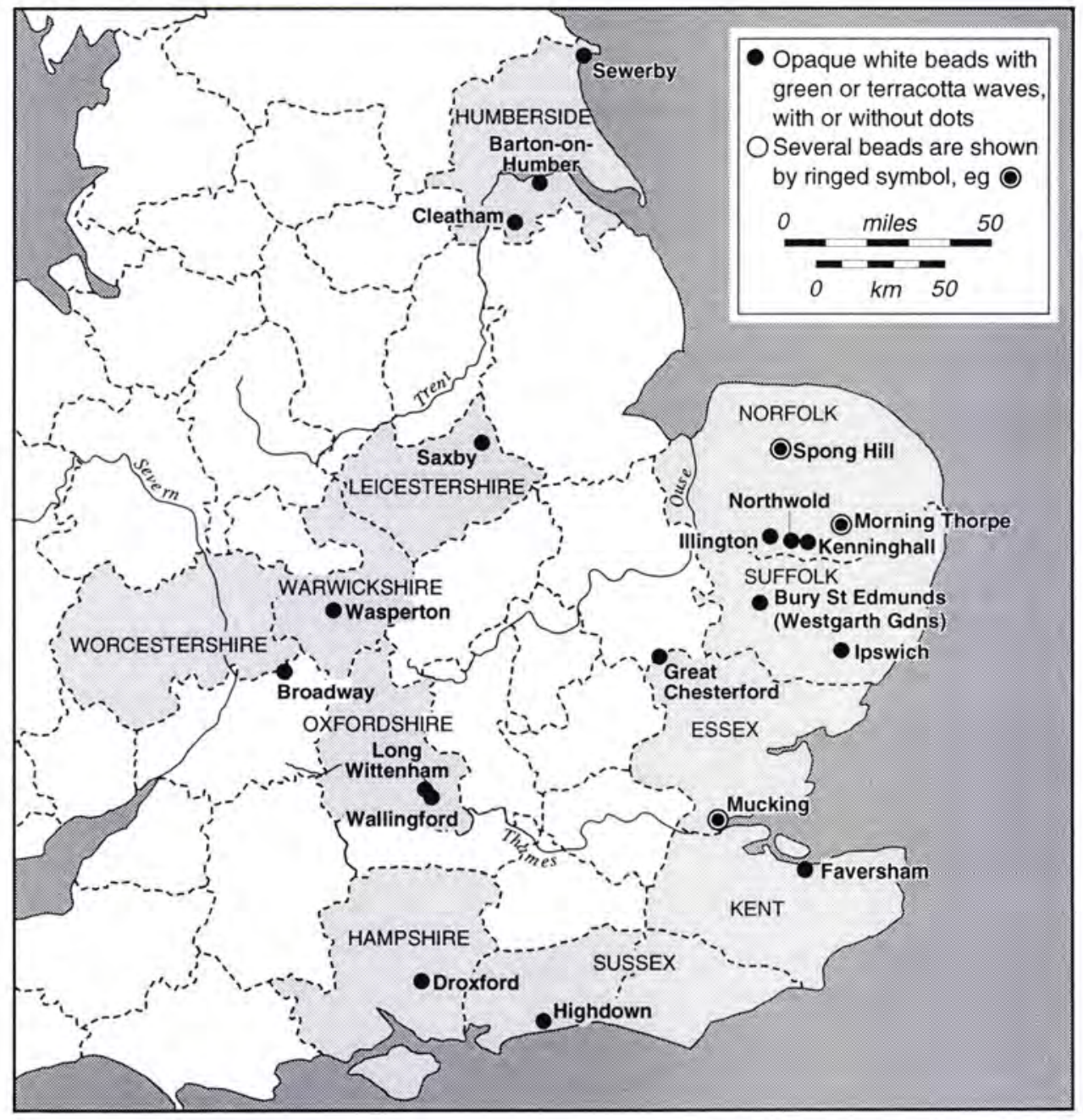

Map 14 Distribution of type 3iiid beads 


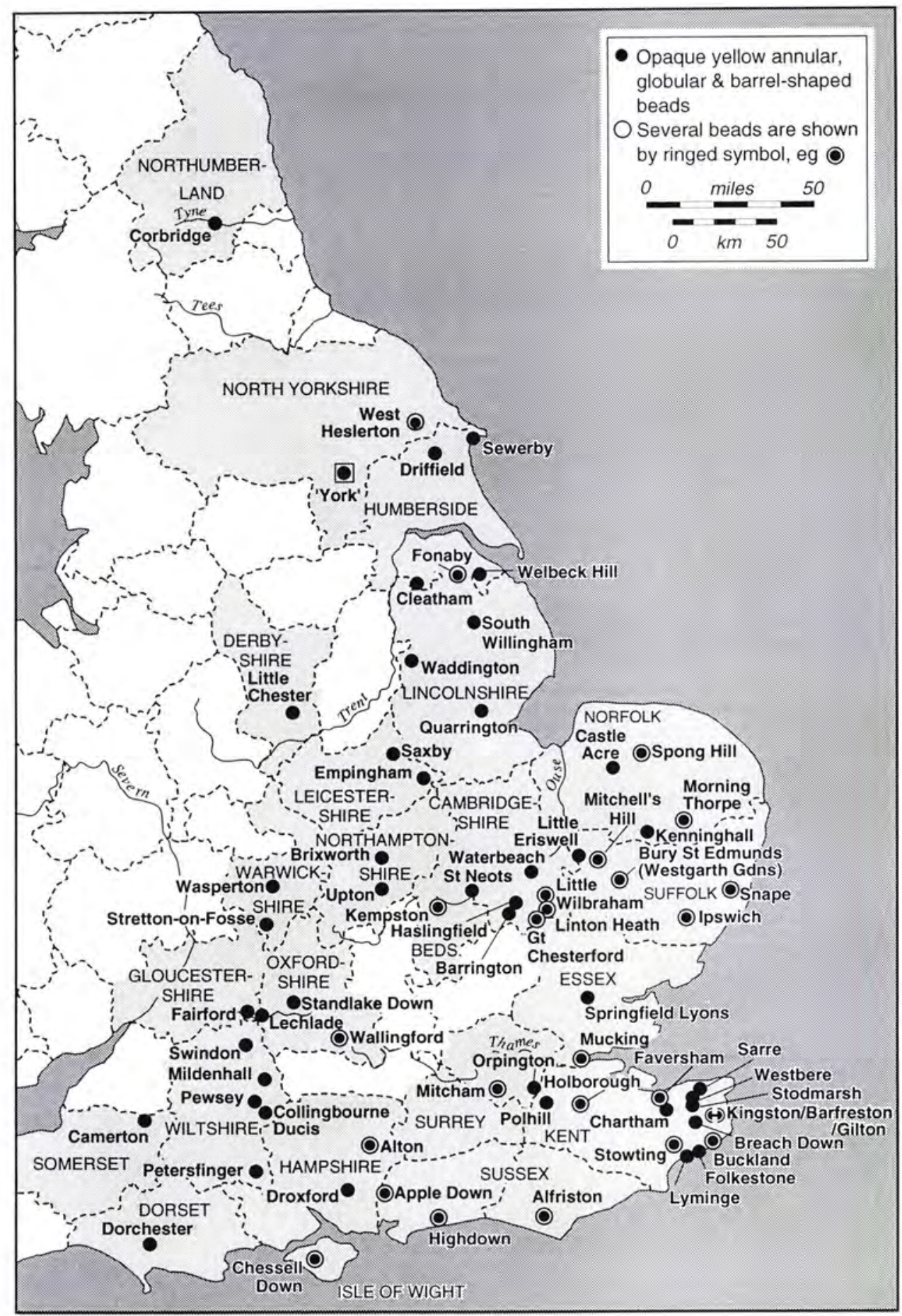

Map 15 Distribution of type $4 i$ beads 


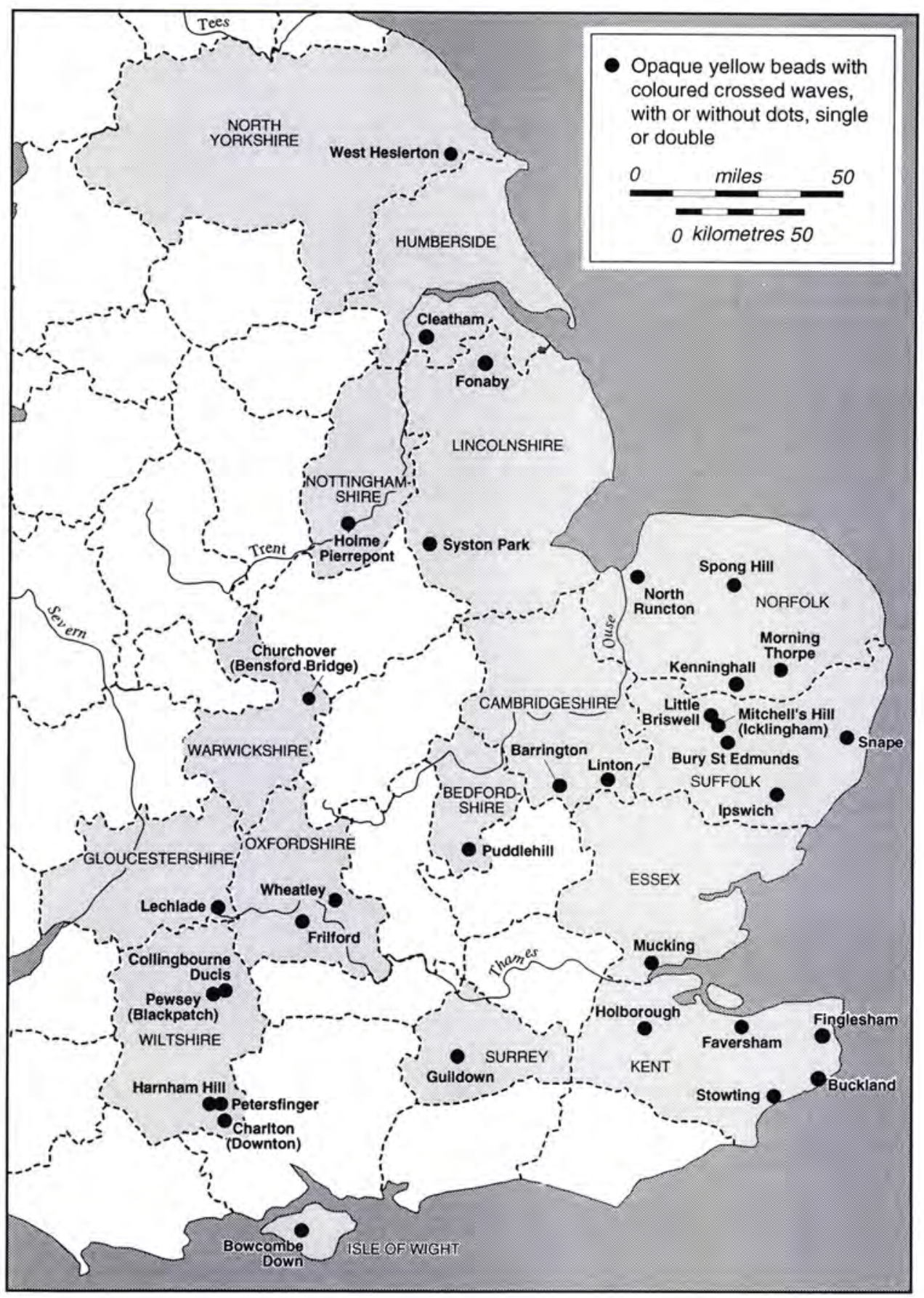

Map 16 Distribution of type 4vi beads 


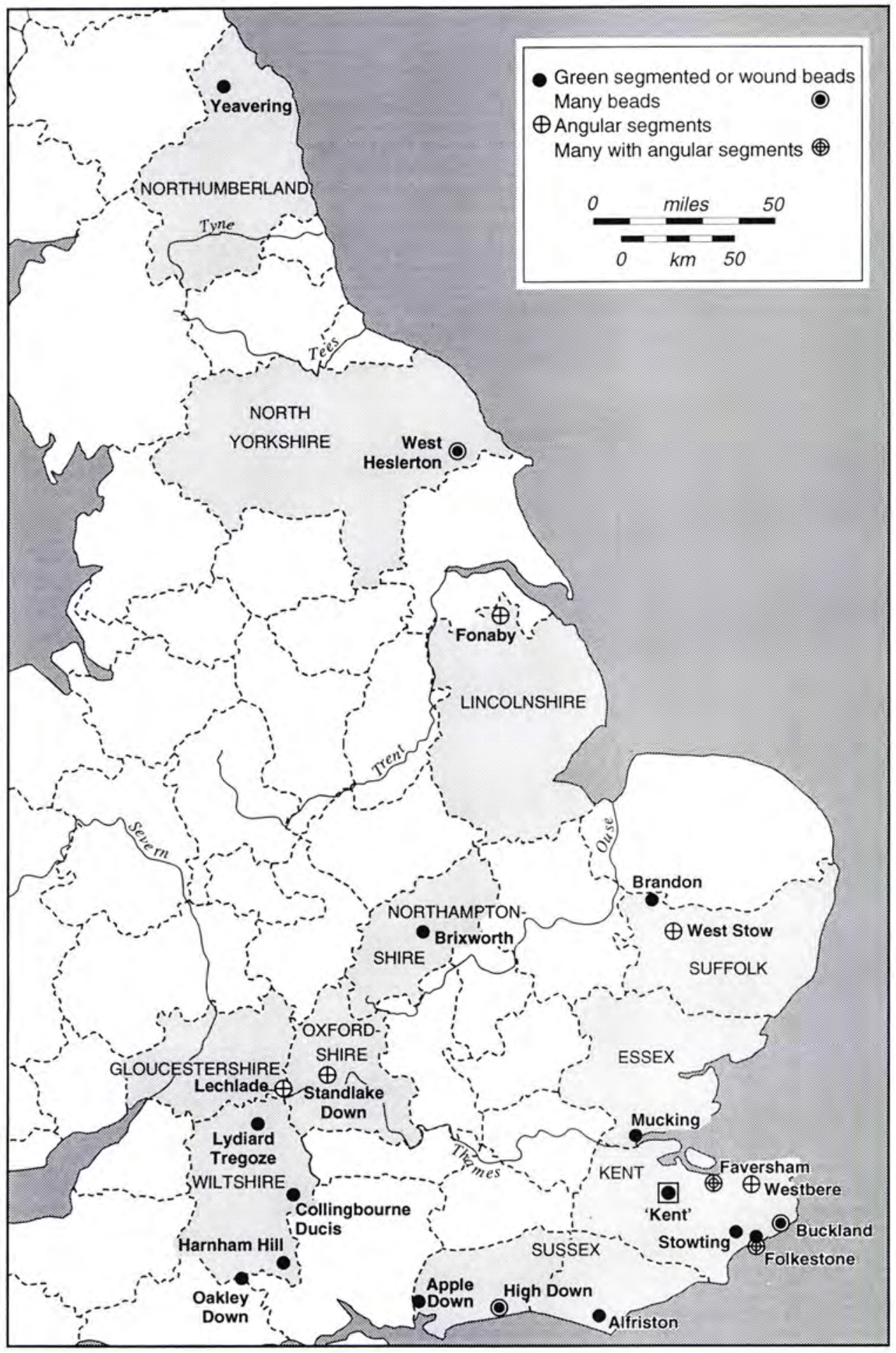

Map 17 Distribution of type 5vi beads 


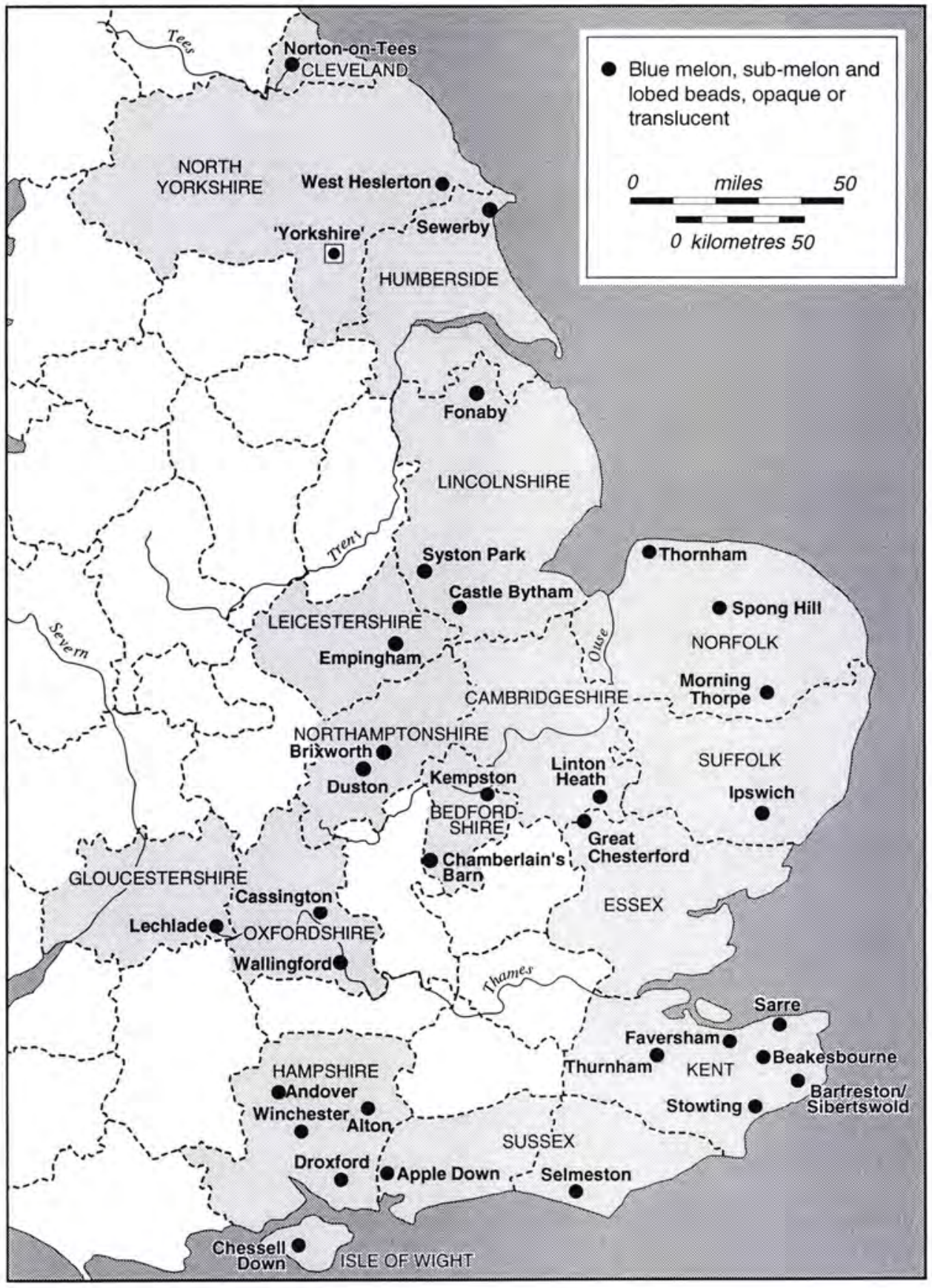

Map 18 Distribution of type 6 viii beads 


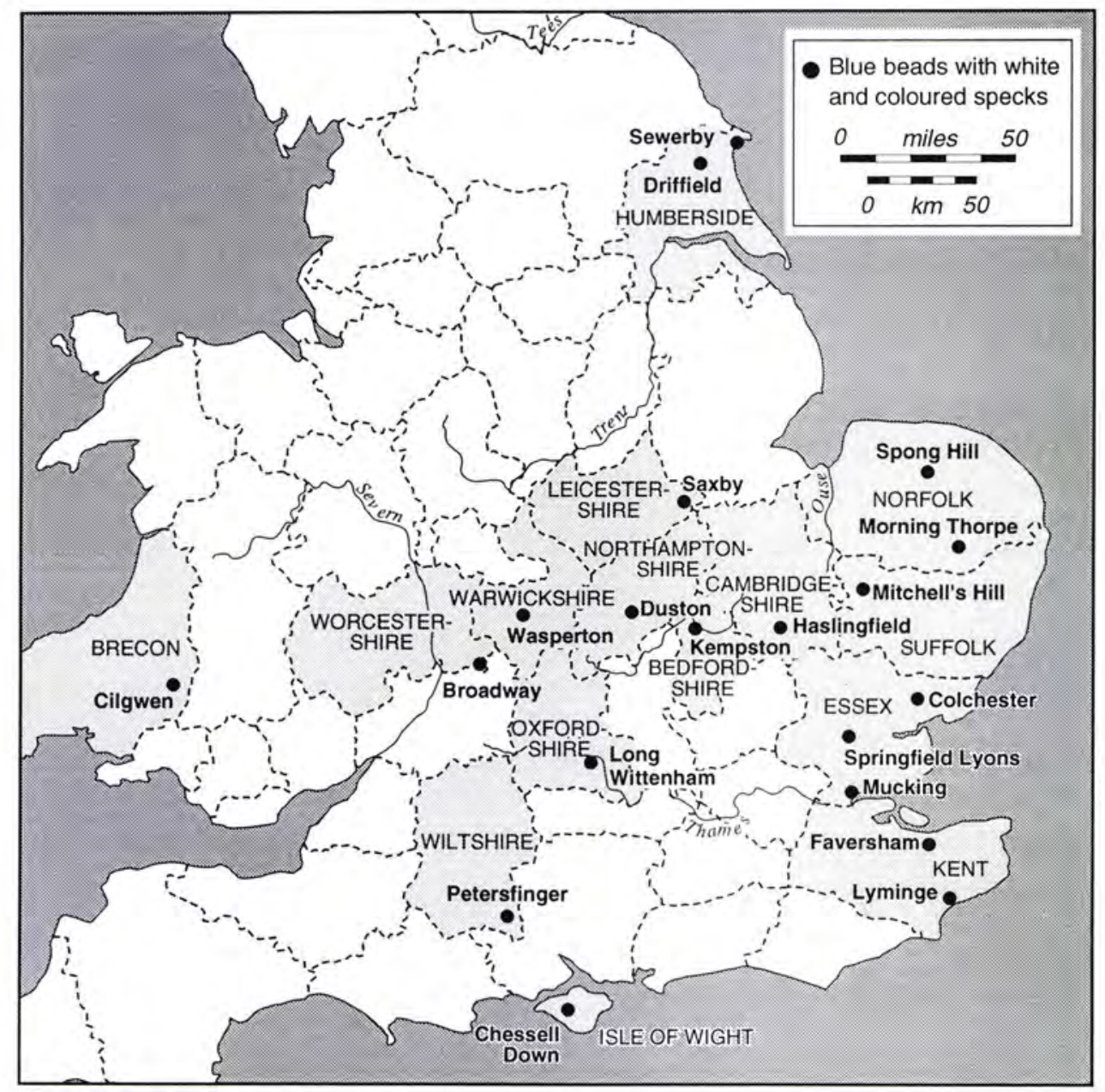

Map 19 Distribution of type 6xi beads 


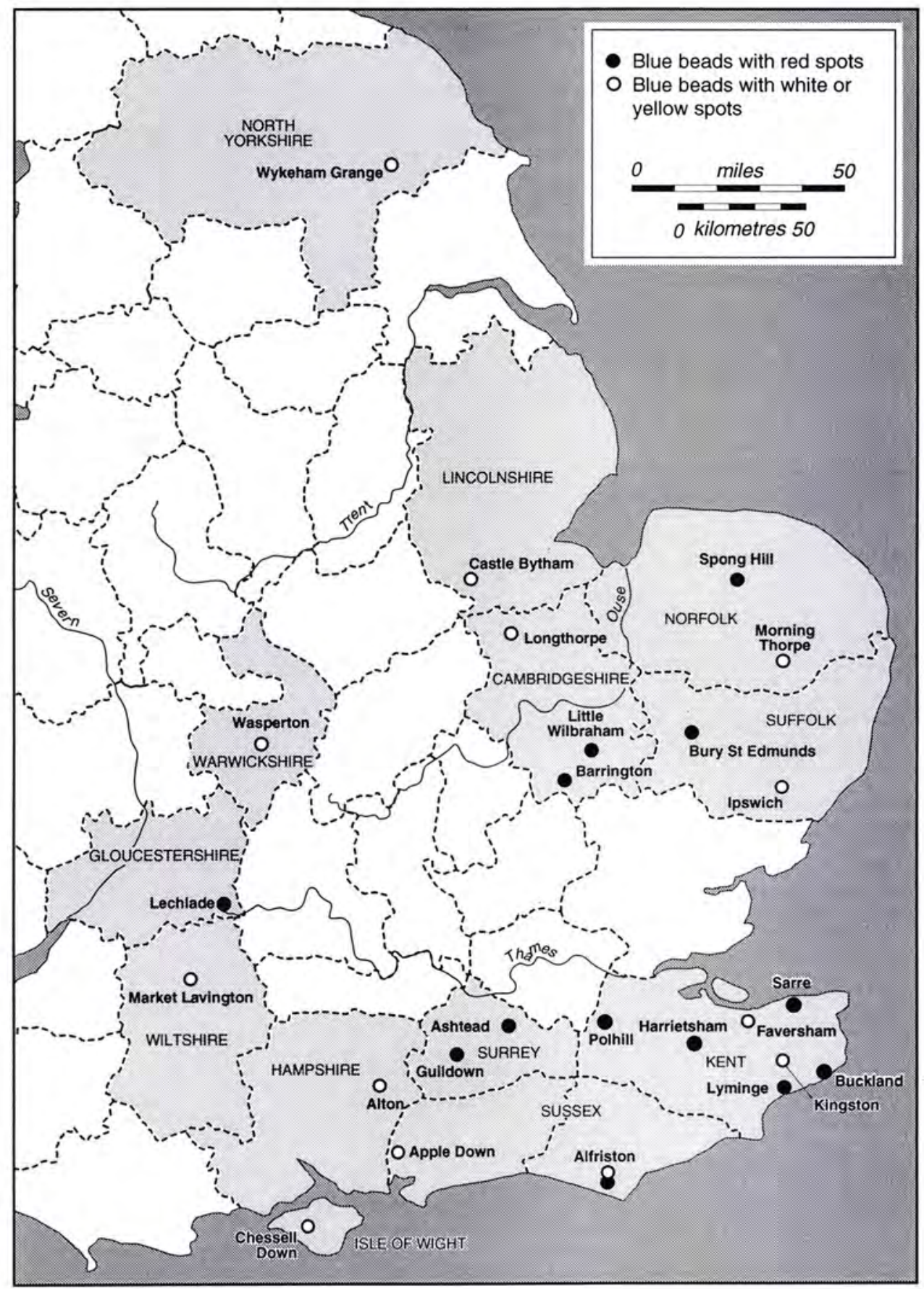

Map 20 Distribution of type 6xii and 6xiv beads 


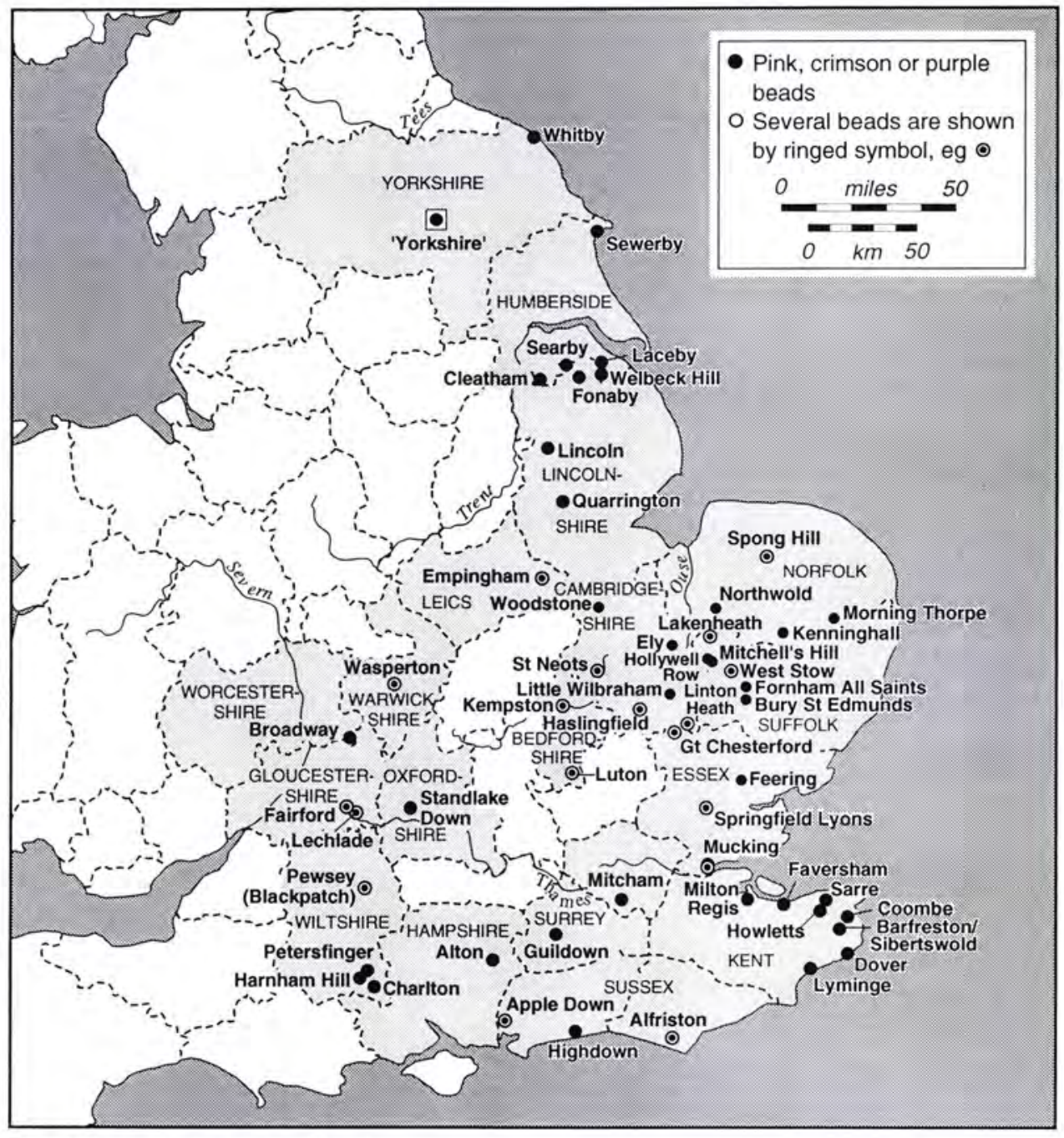

Map 21 Distribution of type 7 beads 


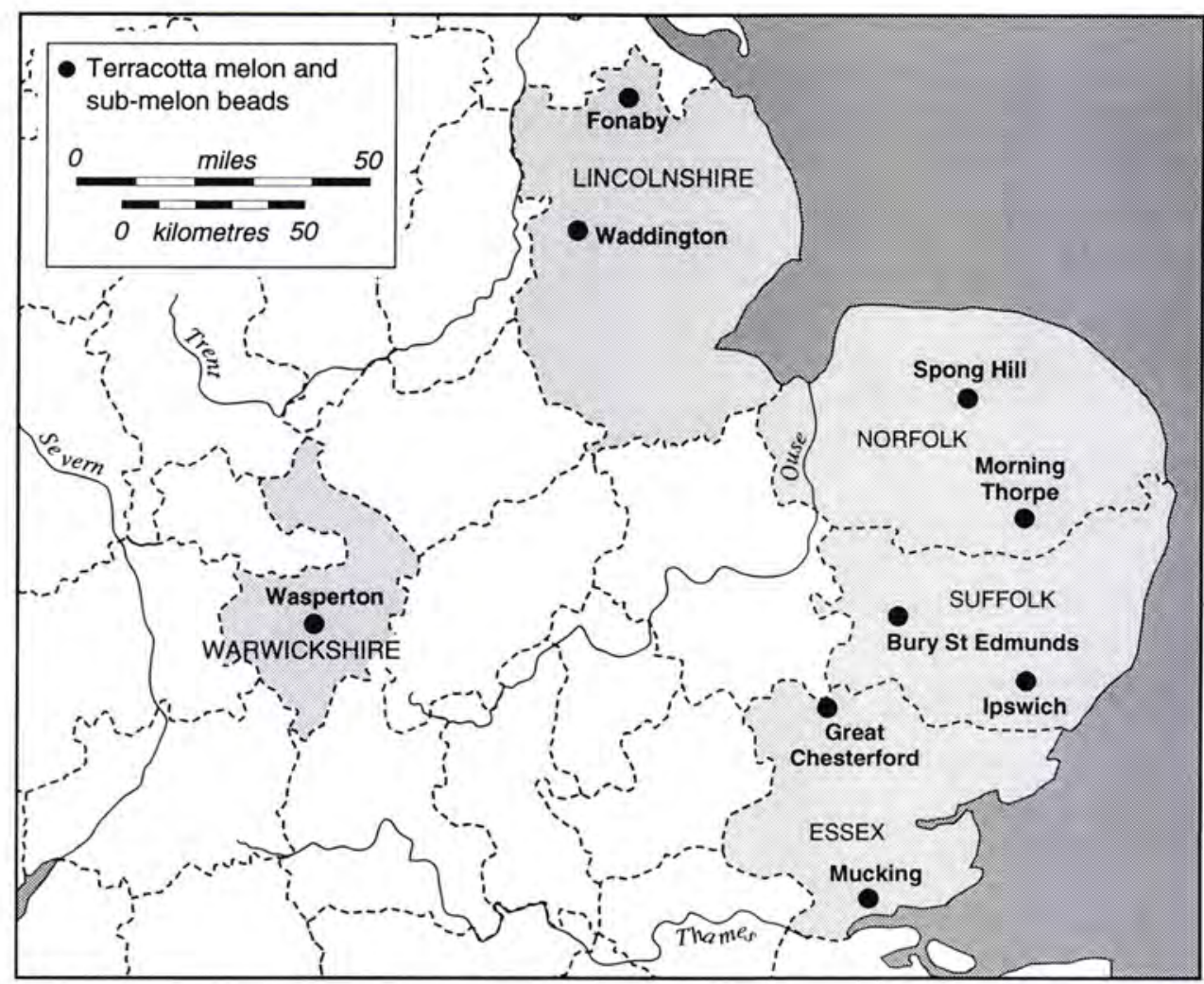

Map 22 Distribution of type $8 v i$ beads 


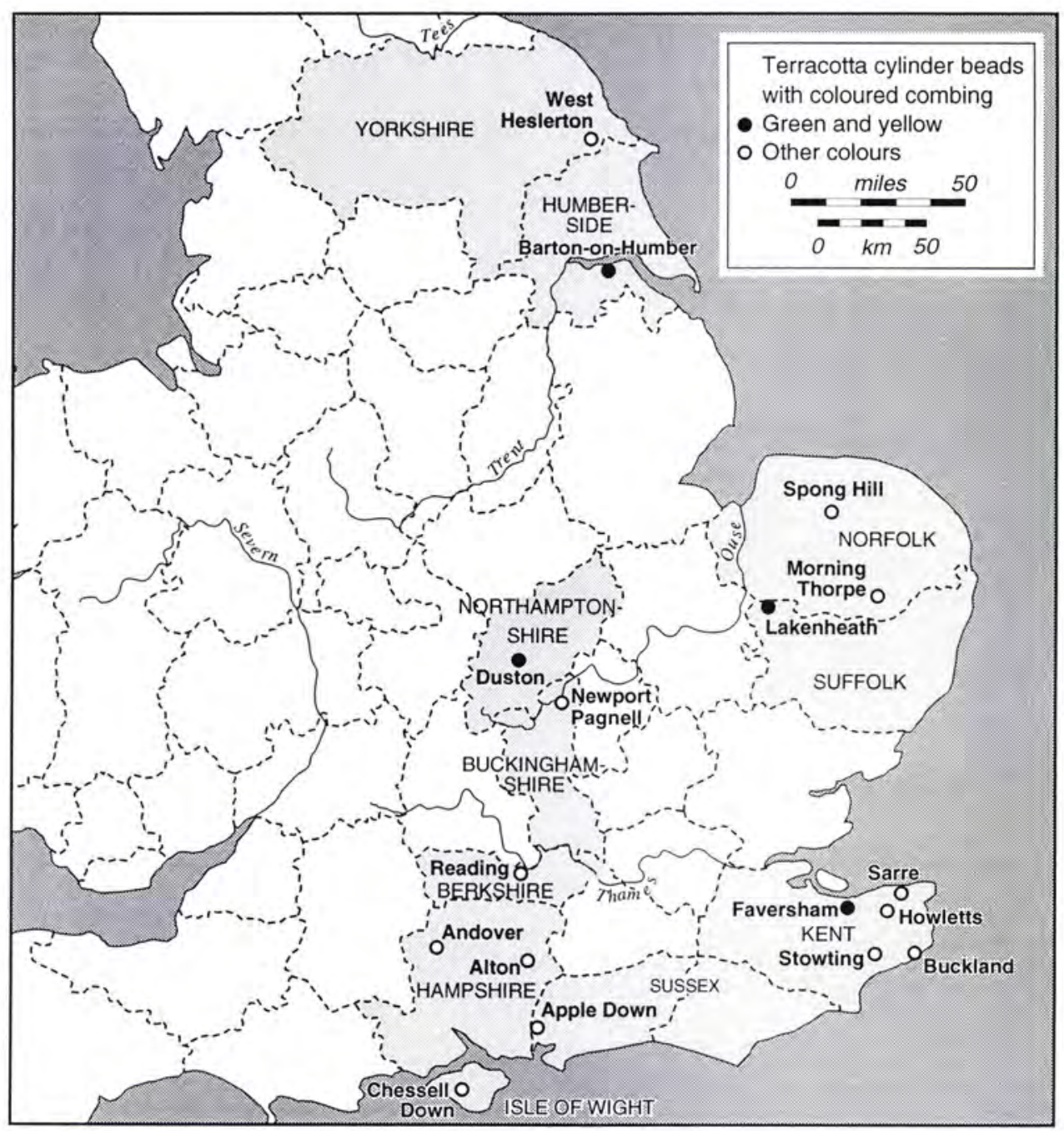

Map 23 Distribution of type 8vii beads 


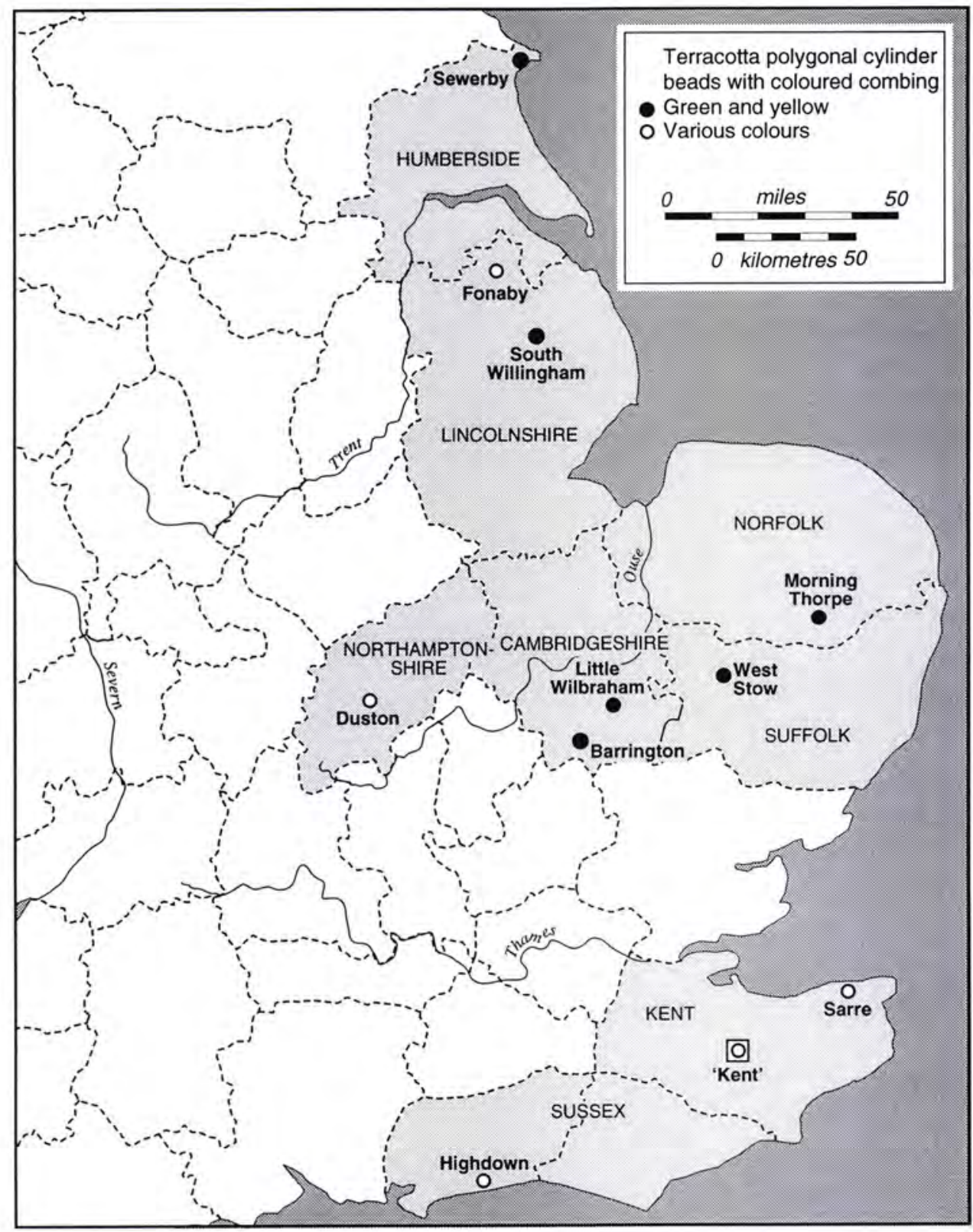

Map 24 Distribution of type 8viii beads 


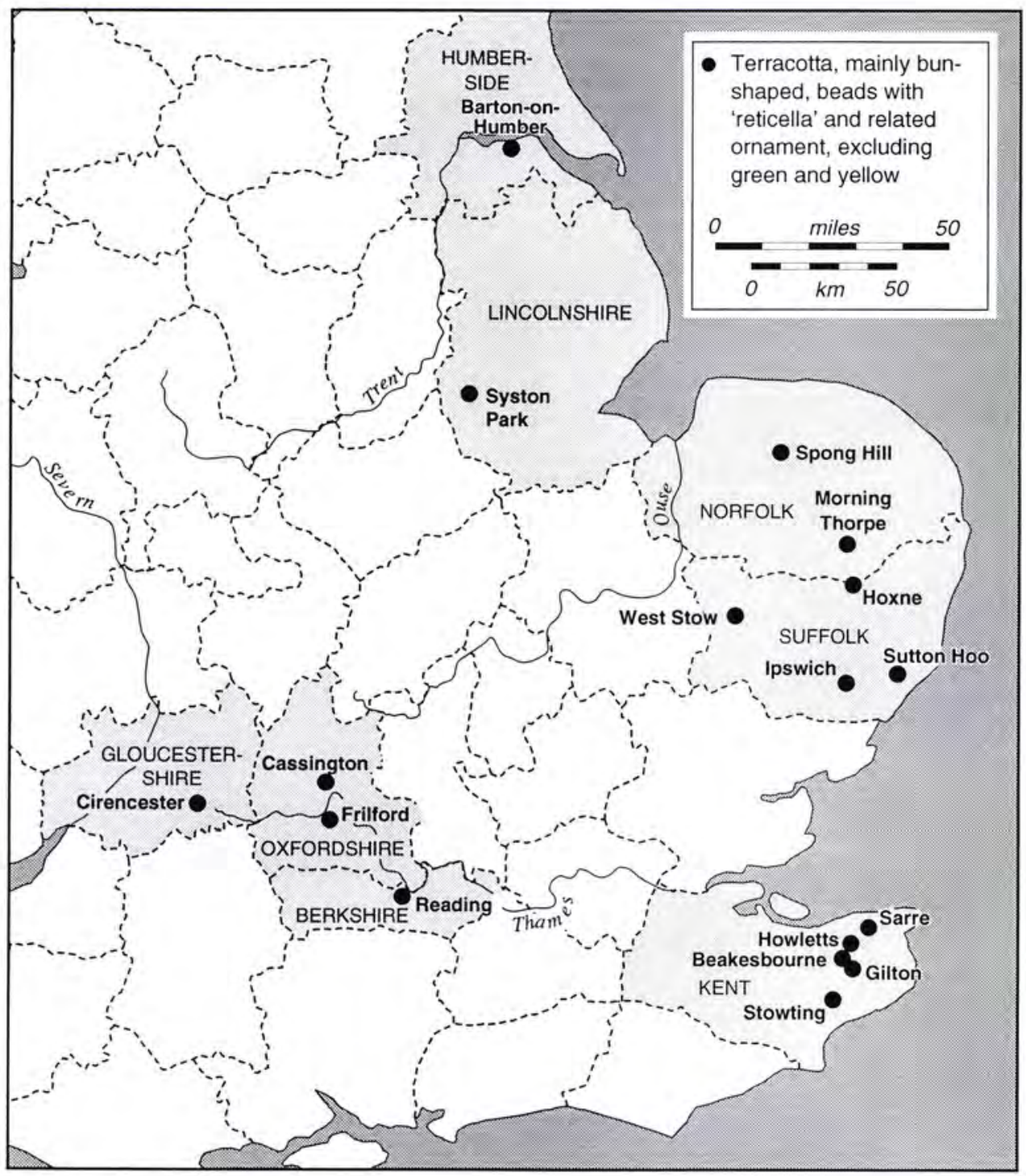

Map 25 Distribution of type 8xviiia beads 


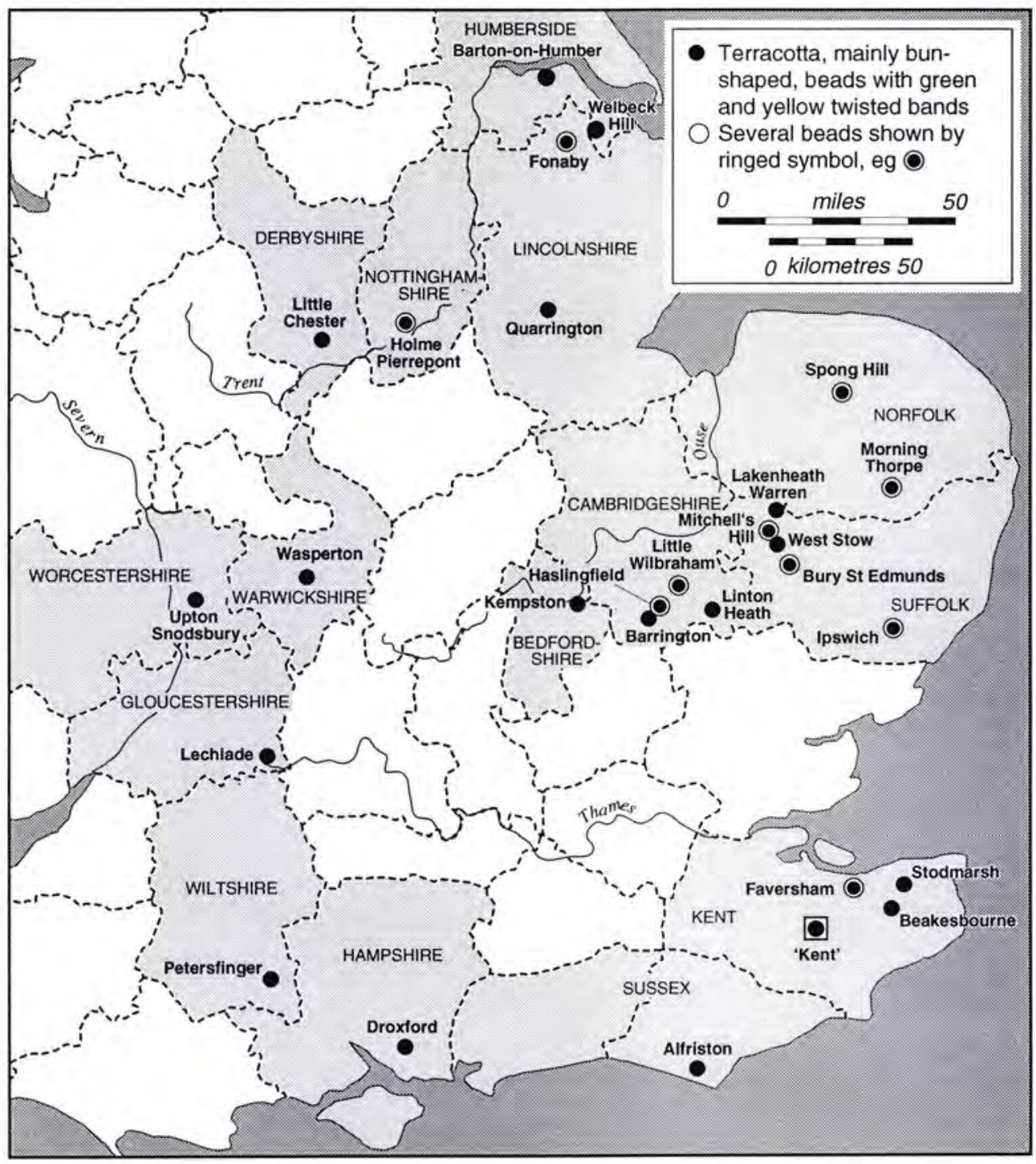

Map 26 Distribution of type 8xviiib beads 


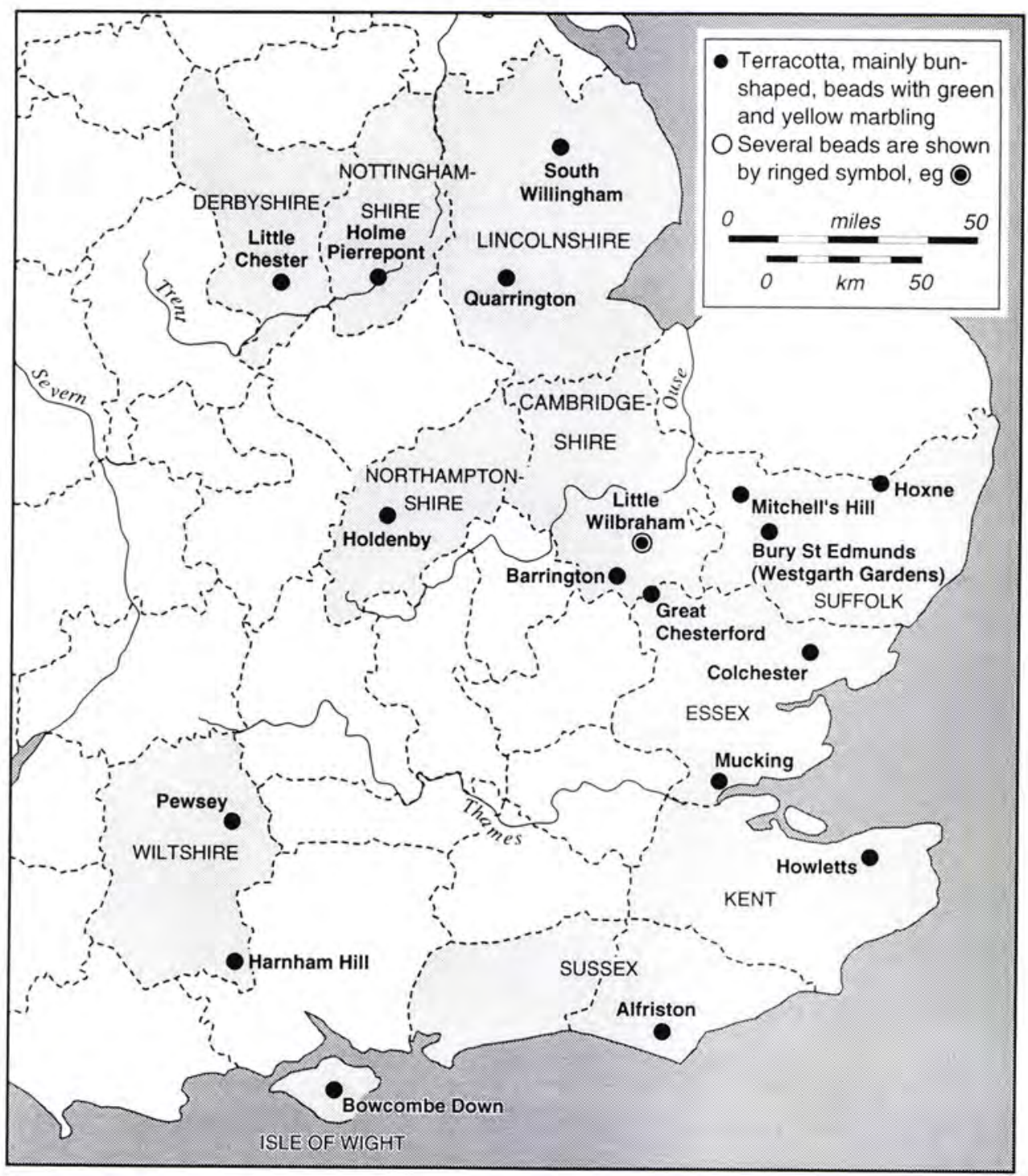

Map 27 Distribution of type 8xviiic beads 


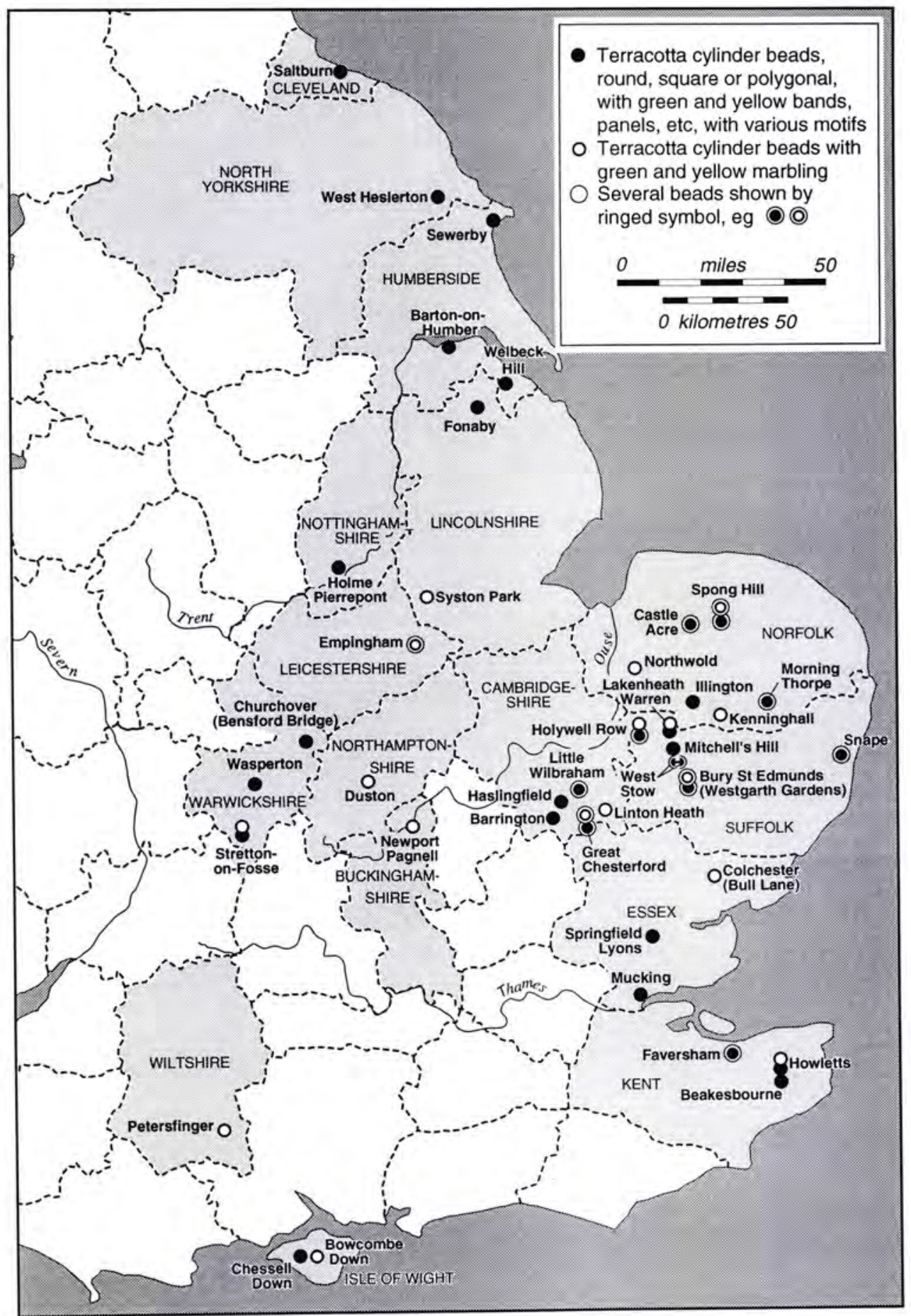

Map 28 Distribution of type 8xixa and 8xixb beads 


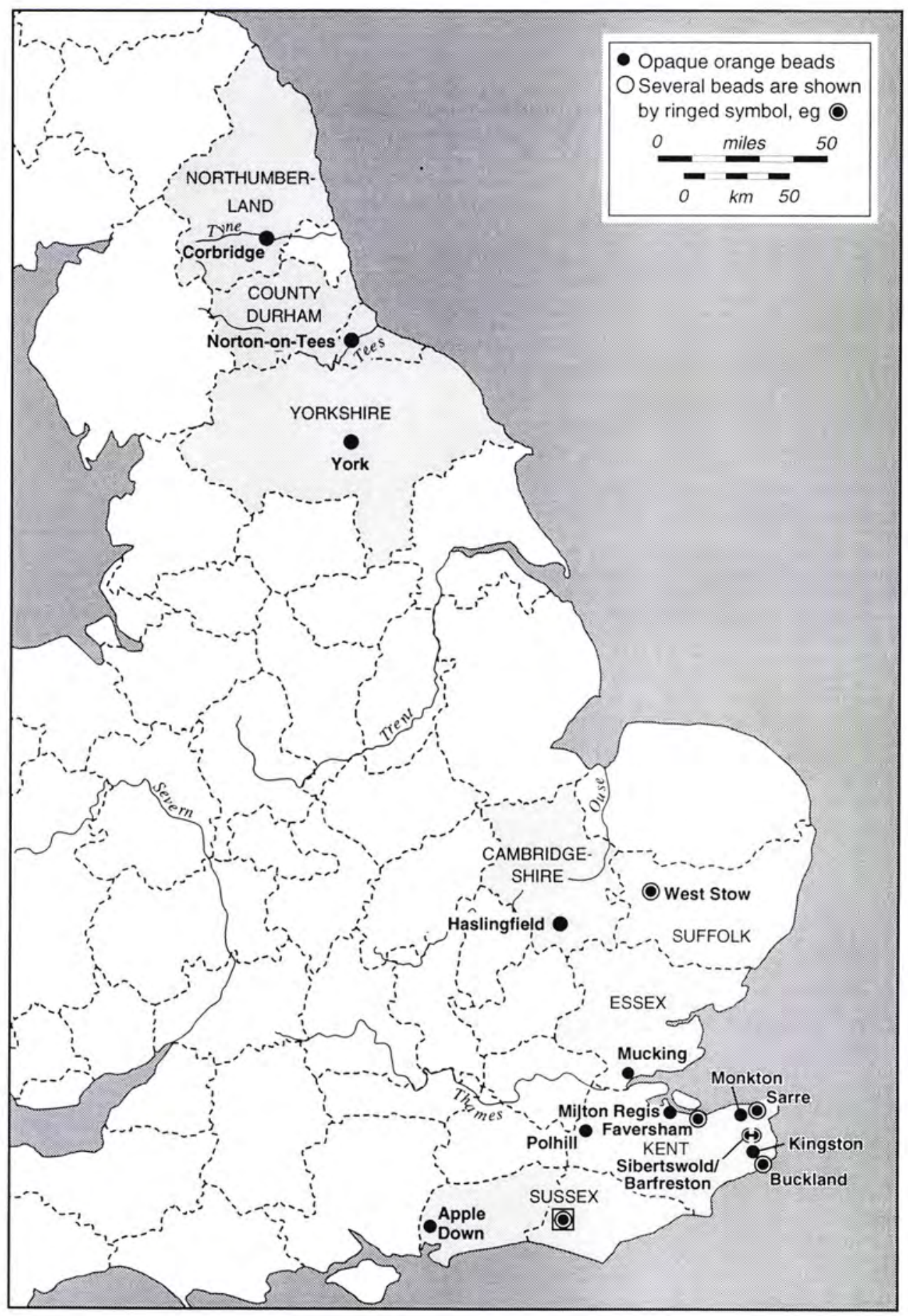

Map 29 Distribution of type 9 beads 


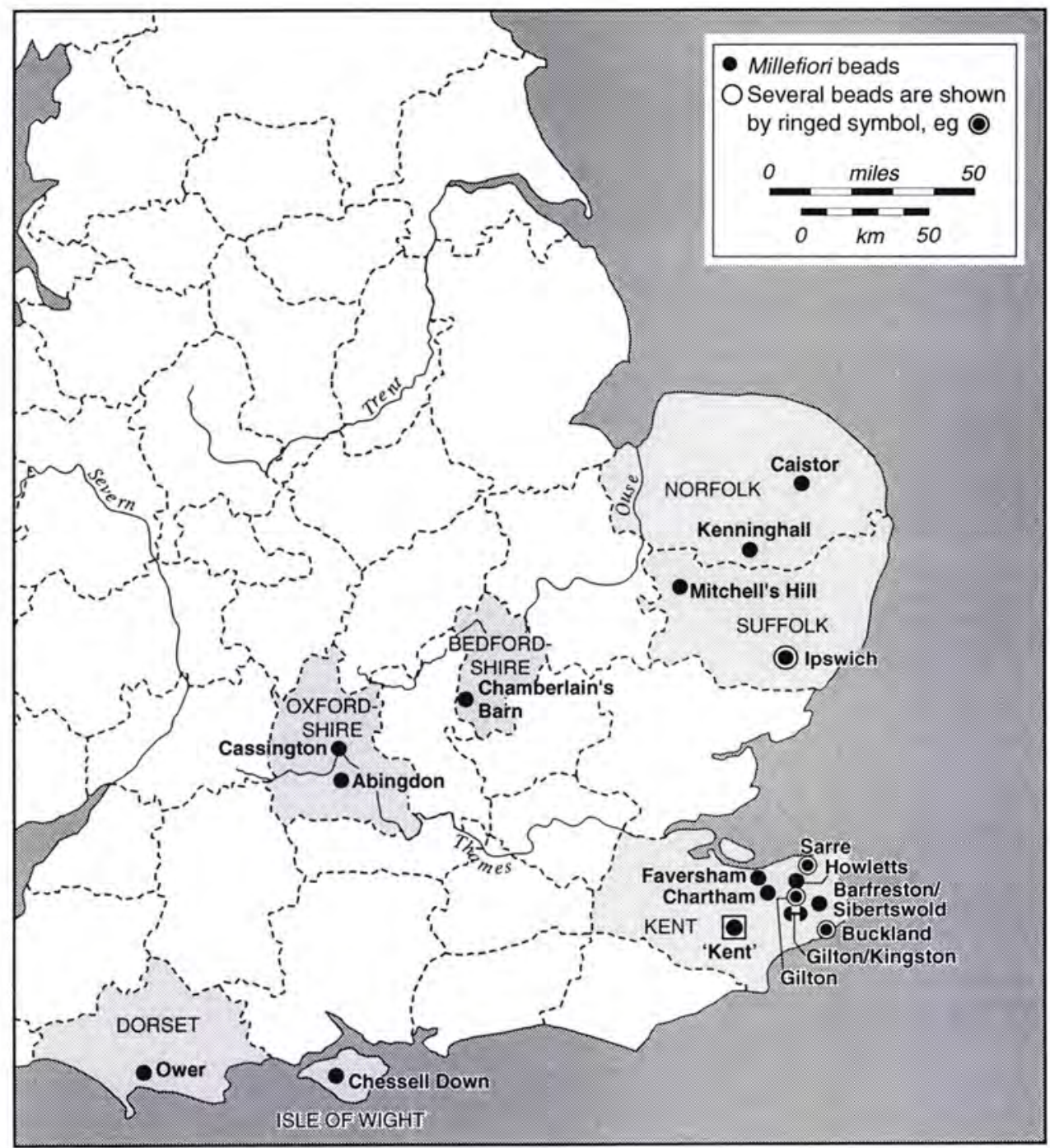

Map 30 Distribution of type $10 a$ beads 


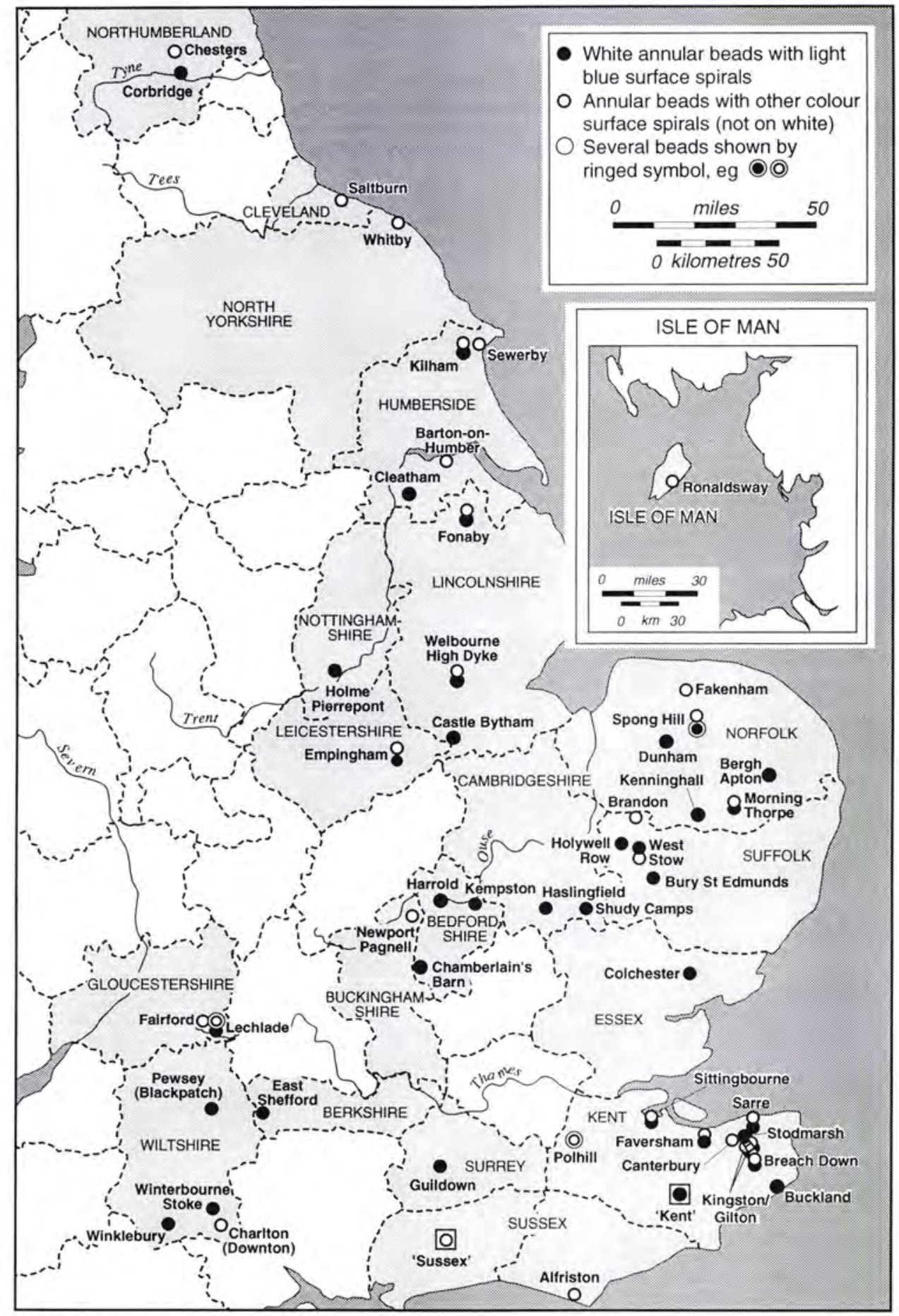

Map 31 Distribution of type 11 beads 


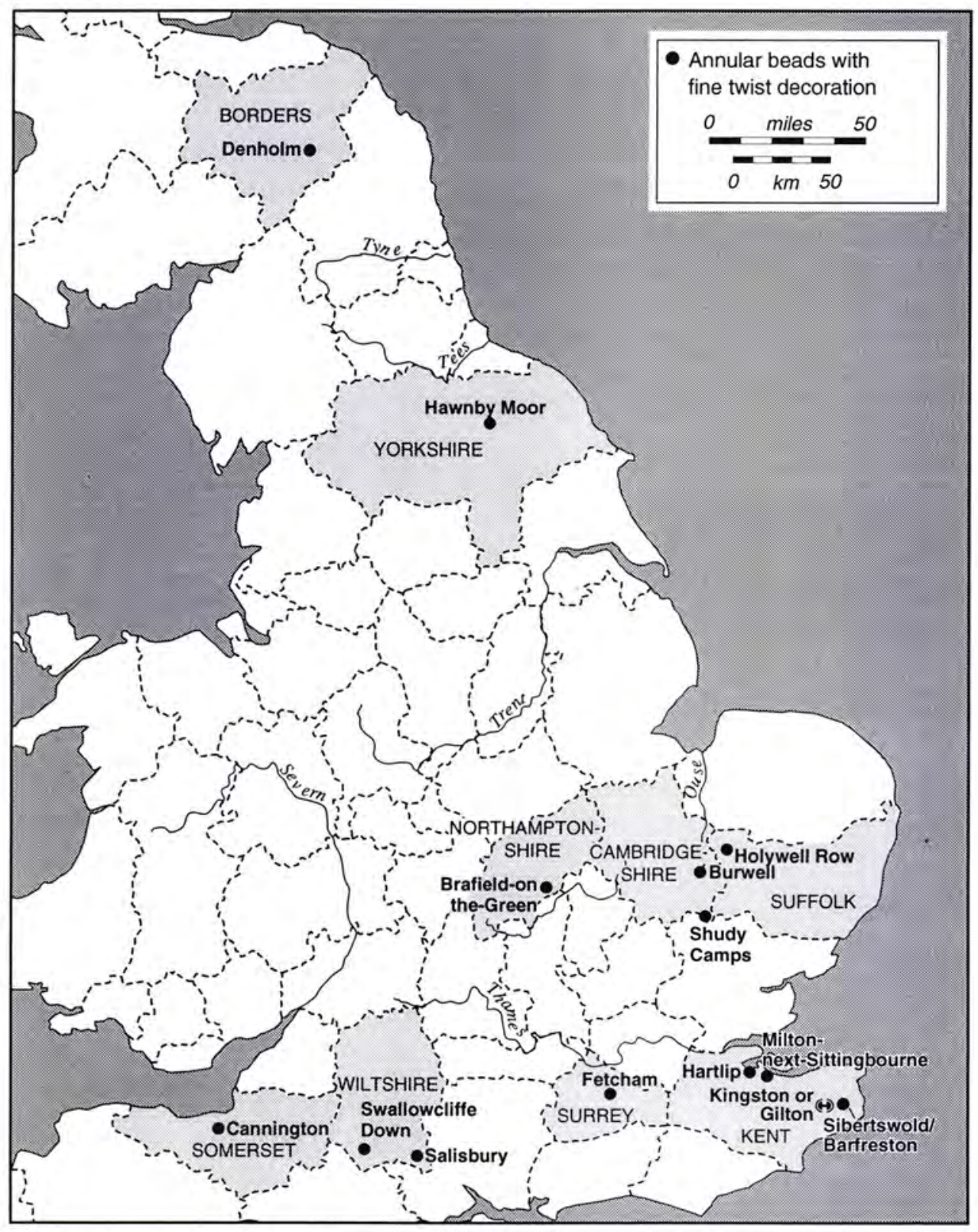

Map 32 Distribution of type 12 beads 


\section{PART II \\ The Schedules}





\section{INTRODUCTION}

The schedules of beads are organized in the same order as the chapters they support. In the first column on the left is the provenance, usually the name by which the site is best known and not necessarily the parish name. For a fuller version of each site name, and cross-references to alternative names, see the topographical index given below. The county name appears (often abbreviated) in the second column and sites are ordered alphabetically within each county. The counties used in the schedules and in the main text are those in existence up to 1974, rather than the present-day (post-1996 reorganization) county, district and unitary authority administrative regions, although the county boundaries shown on the maps post-date1974, with the counties shaded wherever they contain a site. While most of the sites are in England, a number of sites in Wales, Scotland and the Isle of Man are also listed.

The third column provides an estimated date range for the archaeological context. This may be quite precise in the case of a fully recorded burial (eg, a quarter or a half century), but may be the overall range of the site as a whole, whether a cemetery or a settlement.

A fourth column gives the finds context where known (eg, the number of the grave, cremation or building) or states that the find is unstratified. Where appropriate, this column will include a brief record of the form and/or decoration of the bead(s) and will list a selection of associated material that permits the definition of the date range given in column three.

The fifth and final column provides one or more published references to the site and the site context using an author/date format (the full reference being given in the Bibliography), together with the name of the museum or other organization holding the material (where known). 


\title{
ABBREVIATIONS OF ENGLISH COUNTY NAMES IN COLUMN TWO
}

$\begin{array}{llllll}\text { Beds } & \text { Bedfordshire } & \text { Hunts } & \text { Huntingdonshire } & \text { Somerset } & \text { Somerset } \\ \text { Berks } & \text { Berkshire } & \text { Kent } & \text { Kent } & \text { Suffolk } & \text { Suffolk } \\ \text { Bucks } & \text { Buckinghamshire } & \text { Leics } & \text { Leicestershire } & \text { Surrey } & \text { Surrey } \\ \text { Cambs } & \text { Cambridgeshire } & \text { Lincs } & \text { Lincolnshire } & \text { Sussex } & \text { East Sussex and } \\ \text { Derbys } & \text { Derbyshire } & \text { London } & \text { City of London } & & \text { West Sussex } \\ \text { Durham } & \text { County Durham } & \text { Mddx } & \text { Middlesex } & \text { Warks } & \text { Warwickshire } \\ \text { Essex } & \text { Essex } & \text { Norfolk } & \text { Norfolk } & \text { Wilts } & \text { Wiltshire } \\ \text { Glos } & \text { Gloucestershire } & \text { Northants } & \text { Northampton- } & \text { Worcs } & \text { Worcestershire } \\ \text { Hants } & \text { Hampshire } & & \text { shire } & \text { Yorks } & \text { Yorkshire (East, } \\ \text { Isle of } & \text { Isle of Wight } & \text { Oxon } & \text { Oxfordshire } & & \text { North and } \\ \text { Wight } & \text { (Hampshire) } & \text { Rutland } & \text { Rutland } & & \text { West Ridings) }\end{array}$

\section{ABBREVIATIONS OF MUSEUMS AND UNITS WITH THEIR FULL TITLES AND ADDRESSES IN COLUMN FIVE}

\author{
Alnwick Museum Museum of Antiquities, Alnwick Castle, Alnwick, Northumberland NE66 \\ INQ \\ Ashmolean Museum Department of Antiquities, Ashmolean Museum, Beaumont Street, Oxford \\ OX1 2PH \\ Aylesbury Museum Buckinghamshire County Museum, Church Street, Aylesbury, \\ Buckinghamshire HP20 2QP \\ Banbury Museum Banbury Museum, 8 Horsefair, Banbury, Oxfordshire OX16 0AA \\ Bedford Museum Bedford Museum, Castle Lane, Bedford MK40 3XD \\ BM Department of Medieval and Later Antiquities, British Museum, Great \\ Russell Street, London WC1B 3DG \\ Birmingham Museum Birmingham Museum and Art Gallery, Chamberlain Square, Birmingham B3 \\ $3 \mathrm{DH}$ \\ Bristol Museum City of Bristol Museum and Art Gallery, Queen's Road, Bristol BS8 1RL \\ Bury St Edmunds $\quad$ Moyse's Hall Museum, Cornhill, Bury St Edmunds, Suffolk IP33 IDX \\ Museum \\ Caerleon Museum \\ The Roman Legionary Museum, High Street, Caerleon, Gwent NP6 1AE \\ (correspondence to Cardiff Museum, address given below) \\ Cambridge Museum Cambridge University Museum of Archaeology and Anthropology, Downing \\ Street, Cambridge CB2 3DZ \\ Canterbury Archaeo- Canterbury Archaeological Trust Ltd, 92a Broad Street, Canterbury, Kent \\ logical Trust CT12LU \\ Canterbury Museum Canterbury City Museums, The Royal Museum and Art Gallery, High Street, \\ Canterbury, Kent CT1 2JE \\ Cardiff Museum National Museum of Wales, Cathays Park, Cardiff CF1 3NP \\ Carisbrooke Museum Carisbrooke Castle Museum, Carisbrooke Castle, Newport, Isle of Wight \\ PO30 1XY \\ Cheltenham Museum Cheltenham Art Gallery and Museum, Clarence Street, Cheltenham, \\ Gloucestershire GL50 3JT \\ Chesters Museum Chesters Fort and Museum, Chollerford, Humshaugh, Hexham, \\ Northumberland NE46 4EP
}


Chichester Museum

Cirencester Museum

Colchester Museum

Corbridge Museum

Dartford Museum

Deal Museum

Devizes Museum

Dorchester Museum

Dover Museum

Edinburgh Museum

Folkestone Museum

Guildford Museum

Hampshire Museums

Hereford Museum

Hull Museum

Ipswich Museum

Keighley Museum

Lewes Museum

Lincoln Museum

Liverpool Museum

Luton Museum

Maidstone Museum

Middlesbrough

Museum

Museum of London

Newbury Museum

Newcastle Museum

Norfolk Arch Unit

Northampton

Museum

Norwich Museum
Chichester District Museum, 29 Little London, Chichester, West Sussex PO19 1PB

Corinium Museum, Park Street, Cirencester, Gloucestershire GL7 2BX

Castle Museum, Castle Park, Colchester, Essex CO1 1TJ

Corbridge Roman Site, Corbridge, Northumberland NE45 5NT

Dartford Borough Museum, Market Street, Dartford, Kent DAl 1EU

Deal Archaeological Collection (correspondence to Dover Museum, address given below)

Devizes Museum, Wiltshire Archaeological and Natural History Society, 41

Long Street, Devizes, Wiltshire SN10 1NS

Dorset County Museum, High West Street, Dorchester, Dorset DT1 1XA

Dover Museum, Market Square, Dover, Kent CT16 1PB

National Museums of Scotland, Queen Street, Edinburgh EH2 1JD

(correspondence to National Museums of Scotland, Chambers Street,

Edinburgh EH1 1JF)

Folkestone Museum, Grace Hill, Folkestone, Kent CT20 1HD

Guildford Museum, Castle Arch, Quarry Street, Guildford, Surrey GU1 3SX

Hampshire County Museums Service, Chilcomb House, Chilcomb Lane,

Winchester, Hampshire SO23 8RD: responsible for Andover Museum, 6 Church Close, Andover, Hampshire SP10 1DP; and the Curtis Museum,

High Street, Alton, Hampshire GU34 1BA

Hereford City Museum and Art Gallery, Broad Street, Hereford HR4 9AU

Hull and East Riding Museum, 36 High Street, Kingston-upon-Hull, North

Humberside (correspondence to Hull City Museums, Art Galleries and

Archives, Monument Buildings, Ferens Art Gallery, Queen Victoria Square,

Kingston-upon-Hull HU1 3RA)

Ipswich Museum, High Street, Ipswich, Suffolk IP1 3QH

Cliffe Castle Art Gallery and Museum, Spring Gardens Lane, Keighley, West

Yorkshire BD20 6LH

Museum of Sussex Archaeology, Barbican House, 169 High Street, Lewes,

East Sussex BN7 1YE

City and County Museum, 12 Friars Lane, Lincoln, Lincolnshire LN2 5AL

Liverpool Museum, National Museums and Galleries on Merseyside, William Brown Street, Liverpool L3 8EN

Luton Museum and Art Gallery, Wardown Park, Old Bedford Road, Luton, Bedfordshire LU2 7HA

Maidstone Museum and Art Gallery, St Faith's Street, Maidstone, Kent ME14 $1 \mathrm{LH}$

Dorman Museum, Linthorpe Road, Middlesbrough, Cleveland TS5 6LA

Museum of London, London Wall, London EC2Y 5HN

Newbury District Museum, The Wharf, Newbury, Berkshire RG14 5AS

Museum of Antiquities, The Quadrangle, The University, Newcastle upon

Tyne NE1 7RU

Field Archaeology Section, Norfolk Museums Service, Union House,

Gressenhall, Dereham, Norfolk NR20 4DR

Northampton Museums and Art Gallery, Central Museum and Art Gallery,

Guildhall Road, Northampton NN1 1DP

Norwich Castle Museum, Norwich, Norfolk NR1 4JU 
Oakham Museum
Orpington Museum
Oxfordshire Museum

Peterborough Museum City Museum and Art Gallery, Priestgate, Peterborough, Cambridgeshire PE1

1LF

Portsmouth Museum

Powell-Cotton

Museum

Reading Museum

Richborough Museum

Rochester Museum

Saffron Walden

Museum

Salisbury Museum

Scunthorpe Museum

Segontium Museum

Sheffield Museum

Skipton Museum

Southampton

Museum

Southend Museum

South Shields

Museum

Stratford Museum

Sunderland Museum

Swindon Museum

Taunton Museum

Thetford Museum Warwick Museum Whitby Museum

Rutland County Museum, Catmos Street, Oakham, Rutland LE15 6HW Bromley Museum, The Priory, Church Hill, Orpington, London BR6 OHH Oxfordshire County Museum, Fletcher's House, Park Street, Woodstock, Oxfordshire OX20 ISN

Portsmouth City Museums and Records Service, City Museum and Records Office, Museum Road, Portsmouth PO1 2LJ

Powell-Cotton Museum and Quex House, Quex Park, Birchington, Kent CT7 0BH

Museum of Reading, Town Hall, Blagrave Street, Reading, Berkshire RG1 $1 \mathrm{QH}$

Richborough Castle, Richborough, Sandwich, Kent CT13 9JW

Guildhall Museum, High Street, Rochester, Kent NE1 1PY

Saffron Walden Museum, Museum Street, Saffron Walden, Essex CB10 1JL

Salisbury and South Wiltshire Museum, The King's House, 65 The Close, Salisbury, Wiltshire SP1 2EN

Scunthorpe Museum and Art Gallery, Oswald Road, Scunthorpe, South Humberside DN15 7BD

Segontium Roman Museum, Beddgelert Road, Caernarfon, Gwynedd (correspondence to Cardiff Museum above)

Sheffield City Museum and Mappin Art Gallery, Weston Park, Sheffield S10 2TP

The Craven Museum, Town Hall, High Street, Skipton, North Yorkshire $\mathrm{BD} 231 \mathrm{AH}$

Museum of Archaeology, God's House Tower, Winkle Street, Southampton, Hampshire SO14 2NY (correspondence to City Heritage Services, Civic Centre, Southampton SO14 7LP)

Southend Museums Service, Central Museum, Victoria Avenue, Southend-on-Sea, Essex SS2 6EW

Arbeia Roman Fort and Museum, Barling Street, South Shields, Tyne and Wear NE33 2BB (correspondence to Tyne and Wear Museums, Newcastle Discovery Museum, Blandford Square, Newcastle upon Tyne NE1 4JA) New Place and Nash's House, Chapel Street, Stratford-upon-Avon, Warwickshire (correspondence to The Shakespeare Birthplace Trust, The Shakespeare Centre, Henley Street, Stratford-upon-Avon, Warwickshire CV37 6QW)

Sunderland Museum and Art Gallery, Borough Road, Sunderland, Tyne and Wear SR1 1PP (correspondence to Tyne and Wear Museums, Newcastle Discovery Museum, Blandford Square, Newcastle upon Tyne NE1 4JA) Swindon Museum and Art Gallery, Bath Road, Swindon, Wiltshire SN1 4BA Somerset County Museum, The Castle, Castle Green, Taunton, Somerset TA1 4AA Ancient House Museum, White Hart Street, Thetford, Norfolk IP24 1AA Warwickshire Museum, Market Place, Warwick CV34 4SA Whitby Museum, Pannett Park, Whitby, North Yorkshire Y021 IRE 
Winchester Museum Winchester City Museum, The Square, Winchester, Hampshire (correspondence to Winchester Museums Service, Hyde Historic Resources Centre, 75 Hyde Street, Winchester, Hampshire SO23 7DW)

Worcester Museum Worcester City Museum and Art Gallery, Foregate Street, Worcester WR1 1DT

Worthing Museum Worthing Museum and Art Gallery, Chapel Road, Worthing, West Sussex BN11 1HP

Yorkshire Museum Yorkshire Museum, Museum Gardens, York, North Yorkshire YOl 2DR 


\section{TOPOGRAPHICAL INDEX}

ABINGDON Berkshire (now Oxfordshire)

SU 490963

Inhumation and cremation cemetery

Ashmolean Museum

Leeds and Harden 1936; Leeds and Bradford

1942; Meaney 1964, 43; Härke 1992, 244-5, Abb. 42

Aerodrome see BEAKESBOURNE II below

ALFRISTON Sussex (East) TQ 516037

Inhumation cemetery

Lewes Museum

Griffith and Salzmann 1914; Griffith 1915;

Meaney 1964, 246; Welch 1983, 347-87; Härke

1992, 245-6, Abb. 443

All Saints Green see NORWICH below

ALTON Hampshire SU 718388

Inhumation and cremation cemetery

Hampshire Museums

Meaney 1964, 94; Evison 1988a

ANDOVER (Portway) Hampshire SU 343462

Inhumation and cremation cemetery

Hampshire Museums

Cook and Dacre 1985; Härke 1992, 246-7, Abb. 44

Ansty see SWALLOWCLIFFE DOWN below

APPLE DOWN (Compton) Sussex (West)

SU 795152

Inhumation and cremation cemetery

Chichester Museum

Down and Welch 1990

Argyll Avenue see LUTON below

Ash see GILTON below

ASHFORD Kent

Salisbury Museum

ASHTEAD Surtey

Poulton 1989
AVEBURY Wiltshire

Settlement: Grubenhaus

BAILDON MOOR Yorkshire

Keighley Museum

Barfreston see SIBERTSWOLD below

Bargate Hill see LONDON below

Barham see BREACH DOWN below

BARNSLEY PARK (Cirencester) Gloucestershire Roman villa

Cirencester Museum

Webster and Smith 1982, 109, fig 28.64

BARRINGTON (A: Orwell, Malton, Edix Hill)

Cambridgeshire TL 375496

Inhumation cemetery

Ashmolean Museum; BM; Cambridge Museum Smith 1868a; Fox 1923, 250-2; Meaney 1964, $60-1$; publication in preparation (T Malim)

BARRINGTON (B: Hooper's Field)

Cambridgeshire TL 387497

Inhumation cemetery

Ashmolean Museum; Cambridge Museum

Foster 1880; Fox 1923, 250, 252-5; Meaney 1964, 61

Barton see CIRENCESTER and HASLINGFIELD below

BARTON-ON-HUMBER (Castledyke South)

Lincolnshire TA 031217

Inhumation cemetery (with one cremation)

Hull Museum; Scunthorpe Museum

Meaney 1964, 151; Leahy 1993, 39; Drinkall and

Foreman forthcoming

Bassett Down see LYDLARD TREGOZE below

BEAKESBOURNE (I, Cowslip Wood) Kent

TR 207548

Inhumation barrow cemetery

Liverpool Museum

Faussett 1856, 144-59; Meaney 1964, 108-9 
BEAKESBOURNE (II, Aerodrome) Kent

TR 200555

Inhumation cemetery

Canterbury Museum

Meaney 1964, 109; Härke 1992, 247-8, Abb. 46

Bensford Bridge see CHURCHOVER below

BERGH APTON Norfolk TG 306002

Inhumation cemetery

Norwich Museum

Green and Rogerson 1978; Härke 1992, 248-51,

Abb. 47

Berwick St John see WINKELBURY below

BIDFORD-ON-AVON Warwickshire

SP 099518

Inhumation and cremation cemetery

Stratford Museum

Humphreys et al 1923; Humphreys et al 1924;

Meaney 1964, 258; Härke 1992, 251, Abb. 49

BIFRONS (Patrixbourne) Kent TR 186552 Inhumation cemetery

Maidstone Museum

Meaney 1964, 109-10

Biscot Mill see LUTON below

BISHOPSTONE (Stone, Cursley Hill)

Buckinghamshire SP 799110

Inhumation cemetery

Aylesbury Museum

Lowndes 1870; Lowndes 1878; Meaney 1964, 56

Blacknall see PEWSEY below

Blackpatch see PEWSEY below

BLAKENEY Gloucestershire

Roman context

BOUGHTON Norfolk

Inhumation burial

Norwich Museum

BOURTON-ON-THE-WATER (Salmonsbury)

Gloucestershire SP 177204

Inhumation cemetery

Cheltenham Museum

Meaney 1964, 93
BOWCOMBE DOWN (Carisbrooke) Isle of

Wight (Hampshire) SZ 462872

Inhumation and cremation cemetery

Carisbrooke Museum

Meaney 1964, 94-5; Arnold 1982

Bradford Platt see WROTHAM below

BRADWELL (Milton Keynes) Buckinghamshire

Roman villa

Aylesbury Museum

Green 1975

BRAFIELD-ON-THE-GREEN Northamptonshire SP 818582

Northampton Museum

BRANDON (Staunch Meadow) Suffolk

TL 770860

Settlement (Middle Saxon period)

Carr et al 1988; publication in preparation (R D Carr)

BREACH DOWN (Barham) Kent TR 205485 Inhumation barrow cemetery

$\mathrm{BM}$

Conyngham and Akerman 1844; Meaney 1964, 111

BRIXWORTH (I or II) Northamptonshire

SP 747720 or 744715

Inhumation and cremation cemeteries

Northampton Museum

Smith 1902, 245-6; Meaney 1964, 187

BROADSTAIRS Kent

Hurd 1913

BROADWAY HILL (Broadway) Worcestershire SP 118369

Inhumation cemetery

Cook 1958; Meaney 1964, 280-1; Härke 1992, 253-4, Abb. 51

BROOKE (Howe) Norfolk TM 280998

Cremation and inhumation cemetery

BM

Meaney 1964, 170

BRUNDALL (II) Norfolk TG 330079

Cremation and inhumation cemetery

Norwich Museum

Johnson 1926; Meaney 1964, 170-1 
Buckland see DOVER II below

Burton Road see LINCOLN below

BURWELL Cambridgeshire TL 590665

Inhumation cemetery

Cambridge Museum

Lethbridge 1925 and 1926a; Meaney 1964, 61-2

BURY ST EDMUNDS (Westgarth Gardens)

Suffolk TL 843634

Inhumation and cremation cemetery

Bury St Edmunds Museum

West 1988; Härke 1992, 284, Abb. 79

Bushy Close see WESTBERE below

Butler's Field see LECHLADE below

Butt Lane see COLCHESTER below

Buttsole see EASTRY I below

Cadbury Congresbury see CONGRESBURY below

CAERHUN (Kanovium) Caernarvonshire

(Wales)

Segontium Museum

Reynolds 1938

CAERLEON Monmouthshire (Wales)

Caerleon Museum

Isca Silurum Cat 1862, plate XXVIII.6; Alcock

1963 (Dinas Powys)

CAERNARVON (Segontium) Caernarvonshire

(Wales)

Segontium Museum

Wheeler 1924

Caistor see FONABY and NETTLETON below

CAISTOR ST EDMUNDS (Caistor-by-Norwich) Norfolk TG 235032

Cremation and inhumation cemetery

Meaney 1964, 171-2

Cakebread Robey V see CANTERBURY below

CAMERTON Somerset ST 686566

Inhumation cemetery

Taunton Museum
Horne 1928; Horne 1933; Beck 1933; Meaney 1964,218

CANNINGTON Somerset ST 255405

Post-Roman inhumation cemetery

Meaney 1964, 218-19; Rahtz et al forthcoming

CANTERBURY (Cakebread Robey V) Kent

Late Roman multiple inhumation burial pit

Canterbury Archaeological Unit

Bennett 1980; Guido forthcoming (a)

CANTERBURY (Mint Yard) Kent

Canterbury Archaeological Unit

Bennett 1980; Guido forthcoming (b)

Car Dyke see WATERBEACH below

Carisbrooke see BOWCOMBE DOWN above

CASSINGTON (I, Purwell Farm) Oxfordshire SP 443117

Inhumation cemetery

Ashmolean Museum

Leeds and Riley 1942; Meaney 1964, 205

CASTLE ACRE Norfolk TF 797156

Cremation cemetery

Meaney 1964, 172-3

CASTLE BROMWICH Warwickshire SE 137893

Information from $\mathrm{N}$ Thomas

Birmingham Museum

CASTLE BYTHAM Lincolnshire SK 991185

Inhumation burial

Cambridge Museum

Akerman 1855a, 26, plate XII; Meaney 1964, 153

Castledyke South see BARTON-ON-HUMBER above

Castle Island see MOCHRUM below

Cathedral Green see WINCHESTER below

Cavers see DENHOLM HILL below

Cesterover see CHURCHOVER below 
CHALFONT ST PETER Buckinghamshire

Aylesbury Museum

Rouse 1931; Meaney 1964, 56

CHAMBERLAIN'S BARN I and II (Leighton

Buzzard II) Bedfordshire SP 926264

Two inhumation cemeteries

Luton Museum

Hyslop 1963; Meaney 1964, 37-8; Härke 1992, 264-5, Abb. 61-2

CHARLTON (Charlton Plantation, Downton)

Wiltshire SU 167249

Inhumation cemetery

Salisbury Museum

Davies 1985; Härke 1992, 254, Abb. 52

CHARTHAM DOWN (Swadling Down) Kent TR 108542

Inhumation barrow cemetery

Liverpool Museum

Faussett 1856, 160-76; Meaney 1964, 114

CHELMSFORD Essex

Information from P J Drury

CHESSELL DOWN (Shalfleet) Isle of Wight

(Hampshire) SZ 401854

Inhumation and cremation cemetery

BM

Meaney 1964, 95-7; Arnold 1982

Chester, Little see LITTLE CHESTER below

Chesterford, Great see GREAT CHESTERFORD below

CHESTERS Northumberland NY 912704

Roman fort

Chesters Museum

Miket 1980, 293

CHEVERELL (Great Cheverell) Wiltshire

ST 980544

Devizes Museum

Meaney 1964, 267

CHURCHOVER (Bensford Bridge, Cesterover, Gibbet Hill) Warwickshire

SP 526 812-524 816

Inhumation and cremation cemetery

Warwick Museum

Bloxam 1884; Meaney 1964, 259-60
CIRENCESTER (The Barton) Gloucestershire SP 016023

Inhumation graves

Cirencester Museum

Meaney 1964, 90-1; Brown 1976

CIRENCESTER Gloucestershire

Cirencester Museum

Brown 1976

Cirencester see also BARNSLEY PARK above

Clarendon see PETERSFINGER below

CLATCHARD CRAIG (Newburgh) Fife

(Scotland)

Hillfort excavation

Edinburgh Museum

Close-Brooks 1986

CLEATHAM (Manton, Kirton-in-Lindsey I)

Lincolnshire SE 936008

Cremation and inhumation cemetery

Scunthorpe Museum

Meaney 1964, 156-7; Leahy 1993, 39-40

Cleavel Point see OWER below

COLCHESTER (Butt Lane) Essex

Colchester Museum

Akerman 1852, plate V.5

COLCHESTER (Guildford Road, Site K) Essex TM 000256

Inhumation cemetery

Colchester Museum

Crummy 1981, 10-12, fig 13

COLCHESTER (Mersea Road, Sites N and O)

Essex TL 999 473-999 453

Inhumation cemetery

Colchester Museum

Crummy 1981, 14-17, fig 19

COLCHESTER EsSeX

Colchester Museum

Crummy 1983, 35, fig 37.1504

College Green see WINCHESTER below 
COLLINGBOURNE DUCIS Wiltshire

SU 246542

Inhumation cemetery

Devizes Museum

Gingell 1975; Härke 1992, 255, Abb. 53

Compton see APPLE DOWN above

CONGRESBURY (Cadbury Congresbury)

Somerset

Settlement

Rahtz et al 1992, 143-4, fig 99

COOMBE (Woodnesborough) Kent

TR 298575

Cremation(s) in barrow

BM; Saffron Walden Museum

Meaney 1964, 115; Davidson and Webster 1967;

Evison 1967

Coppergate see YORK below

CORBRIDGE Northumberland NY 982648

Inhumation burial

Corbridge Museum

Knowles and Forster 1909, 406-8, fig 25; Meaney

1964, 198; Miket 1980, 293

COW LOW (Green Fairfield) Derbyshire

SK 102730

Inhumation in barrow

Sheffield Museum

Meaney 1964, 74-5

Cowslip Wood see BEAKESBOURNE I above

Cratendune see ELY below

CRUNDALE DOWN Kent TR 062494

Inhumation cemetery

BM; Liverpool Museum

Faussett 1856, 177-98; Meaney 1964, 116

Cursley Hill see BISHOPSTONE above

DALMENY (Hound Point) Fife/West Lothian

(Scotland) NT 157796

Cist burial

Edinburgh Museum

Brown 1915a (IV), 812-13, fig 29; Brown 1915b
DENHOLM HILL (Cavers) Roxburghshire

(Scotland)

Edinburgh Museum

Deodora see HARRIETSHAM below

DINAS POWYS Glamorganshire (Wales)

Hillfort: unstratified finds

Cardiff Museum

Alcock 1963, 185-6, fig 41

DINORBEN Denbighshire/Clwyd (Wales)

Cardiff Museum

Gardner and Savory 1964

DORCHESTER (Trumpet Major Public House)

Dorset SY 703900

Inhumation cemetery

Sparey-Green 1984

DORCHESTER Dorset

Roman villa

Dorchester Museum

DOVER (I, Priory Hill) Kent TR 319414

Inhumation cemetery

BM; Dover Museum

Meaney 1964, 117

DOVER (II, Buckland, Old Park) Kent

TR 310430

Inhumation cemetery

BM; Dover Museum

Meaney 1964, 117; Evison 1987

Dover Hill see FOLKESTONE III below

DOWALTON LOCH Wigtonshire (Scotland)

Dowgate Hill see LONDON below

Downton see CHARLTON above

DRAGONBY Lincolnshire

Lincoln Museum

DRIFFIELD Yorkshire

Inhumation burials

Hull Museum

Mortimer 1905, 297, plate CII.809-13, 824-6, 880-3; Meaney 1964, 286-7 
DROXFORD Hampshire SU 612184

Inhumation cemetery

BM; Winchester Museum

Meaney 1964, 97; Aldsworth 1978; Härke 1992,

256-7, Abb. 54

DUNADD Argyllshire (Scotland)

Craw 1930

DUN BEAG Skye (Scotland)

Callander 1921, 126

DUNHAM Norfolk

Norwich Museum

DUNSTABLE (Totternhoe, Marina Drive)

Bedfordshire TL 000213

Inhumation cemetery

Luton Museum

Meaney 1964, 41-2; Härke 1992, 257-8, Abb. 55

Dunton Green see POLHILL below

DUSTON Northamptonshire SP 726602

Inhumation and cremation cemetery

Northampton Museum

George 1903; Meaney 1964, 189

Earley see READING below

Easden see WESTBERE below

East Harnham see HARNHAM HILL below

EASTRY (I: Buttsole) Kent TR 311545

Inhumation cemetery

Canterbury Museum; Maidstone Museum

Meaney 1964, 113; Hawkes 1979

EASTRY (II: Eastry House) Kent TR 309549

Inhumation burial

Deal Museum

Hawkes 1979

EAST SHEFFORD Berkshire SU 389749

Inhumation cemetery

Newbury Museum; Reading Museum

Peake and Hooton 1915; Meaney 1964, 50-1

Edix Hill see BARRINGTON A above

Elkington, South see SOUTH ELKINGTON below
ELY (Ely Fields Farm, Cratendune)

Cambridgeshire TL 522777

Inhumation cemetery

Cambridge Museum

Fowler 1948; Meaney 1964, 64

EMPINGHAM (II) Rutland SK 935082

Inhumation cemetery

Oakham Museum

Clough et al 1975, 79-80; Härke 1992, 258, Abb.

56; Timby 1996

Eriswell see LITTLE ERISWELL below

ESCOMB County Durham

Parish church foundations

Pocock and Wheeler 1971, 23

EVERTHORPE HALL (Everthorpe) Yorkshire SE 900310

Inhumation cemetery

Hull Museum

Meaney 1964, 288

FAIRFORD (Tanner's Field, Waterslade)

Gloucestershire SP 145015

Inhumation cemetery

Ashmolean Museum; BM

Smith 1852b; Wylie 1852; Meaney 1964, 91

FAKENHAM Norfolk

Norwich Museum

FARNHAM (near) Surrey

Information from W F Rankine, Farnham

Huband 1925; Morris 1959, 140

FAVERSHAM (II, King's Field) Kent

TR 013609

Inhumation cemetery

Ashmolean Museum; BM; Cambridge Museum

(Beck Collection); Rochester Museum; Salisbury

Museum

Smith 1860; Meaney 1964, 118-19

Felixstowe see TRIMLEY ST MARY below

FEERING (Feering-Kelvedon) Essex

TL 868192

Inhumation cemetery

Colchester Museum

Anon 1888, 124; Meaney 1964, 86-7 
Ferring see HIGHDOWN below

FETCHAM (Hawks Hill) Surrey

TQ $155554-160561$

Inhumation cemetery

Guildford Museum

Smith 1901b, 254; Smith 1907; Cotton 1933;

Meaney 1964, 242-3

FETCHAM Surrey TQ 160550

Possible settlement

BM

Cotton 1933; Meaney 1964, 241

FINGLESHAM (Northbourne) Kent TR 326 535

Inhumation cemetery

Deal Museum

Chadwick 1958; Meaney 1964, 119-20; Hawkes

and Pollard 1981; Härke 1992, 258-60, Abb. 57

FINGRINGHOE (Frog Hill Farm) Essex

Information from Philip Crummy

FOLKESTONE (III, Dover Hill) Kent

TR 238376

Inhumation cemetery

Folkestone Museum; Maidstone Museum

Meaney 1964, 120-1

FONABY (Caistor-on-the-Wolds) Lincolnshire TA 110032

Inhumation and cremation cemetery

Scunthorpe Museum

Meaney 1964, 155; Cook 1981; Härke 1992,

260-1, Abb. 58; Leahy 1993, 40

Fordcroft see ORPINGTON below

FORNHAM ALL SAINTS Suffolk

$\mathrm{BM}$

FRILFORD (I) Berkshire (now Oxfordshire)

SU 437964

Inhumation cemetery

Ashmolean Museum; BM

Akerman 1860; Rolleston 1869; Rolleston 1880;

Bradford and Goodchild 1939; Meaney 1964, $46-7$

Frog Hill Farm see FINGRINGHOE above

Gibbet Hill see CHURCHOVER above
GILTON (Ash) Kent TR 282582

Inhumation cemetery

Liverpool Museum

Faussett 1856, 1-34; Meaney 1964, 121-2

GREAT CHESTERFORD Essex TL 501435

Inhumation and cremation cemetery

$\mathrm{BM}$

Meaney 1964, 85-6; Evison 1994

Great Cheverell see CHEVERELL above

Green Fairfield see COW LOW above

GRUNDISBURGH Suffolk

Norwich Museum

Guildford see GUILDOWN below

Guildford Road see COLCHESTER above

GUILDOWN (Guildford) Surrey SU 988488

Inhumation and cremation cemetery

Guildford Museum

Lowther 1931; Lowther 1933; Meaney 1964, 241-2

Hadleigh Road see IPSWICH below

Hamwic see SOUTHAMPTON below

Harlton see HASLINGFIELD below

HARNHAM HILL (East Harnham) Wiltshire SU 137387

Inhumation cemetery

BM

Akerman 1853; Jackson 1854; Meaney 1964, 268-9; Härke 1992, 261

HARRIETSHAM (Deodora) Kent

Inhumation burials

Maidstone Museum

Meaney 1964, 123

HARROLD Bedfordshire SP 953527

Inhumation cemetery

Bedford Museum

Meaney 1964, 36; Eagles and Evison 1970

HARTLIP Kent

Roman villa

Maidstone Museum

Smith 1846b; Smith 1852c 
HARWELL Berkshire (now Oxfordshire)

SU 489882

Inhumation cemetery

Ashmolean Museum

Kirk and Marshall 1956; Meaney 1964, 47

HASLINGFIELD (Harlton, Barton)

Cambridgeshire TL 413520

Inhumation and cremation cemetery

Ashmolean Museum; BM; Cambridge Museum;

Hull Museum

Fox 1923, 255-9; Meaney 1964, 66-7

Hawks Hill see FETCHAM above

HAWNBY MOOR (Sunny Bank, Hawnby)

Yorkshire, North Riding

(now North Yorkshire) SE 526893

Inhumation barrow burial

BM

Denny 1866; Smith 1912, 96, figs 16 and 17;

Brown 1915a (IV), 809; Meaney 1964, 290-1

HEPPLE Northumberland NY 983025

Inhumation burials

Miket 1974; Miket 1980, 295; Cramp and Miket

1982, 4-5, fig 3.2; Meaney 1964, 199

Hersden see WESTBERE below

Heslerton see WEST HESLERTON below

HESSLE (II) Yorkshire TA 021265

Inhumation burials

Hull Museum

Meaney 1964, 291

HIGHDOWN (Ferring) Sussex (West)

TQ 092043

Inhumation and cremation cemetery

Worthing Museum

Read 1895; Read 1896; Meaney 1964, 250-1;

Welch 1983

High Dyke see WELBOURNE below

HOB HILL (Saltburn) Yorkshire, North Riding

(now Cleveland) NZ 651205

Inhumation and cremation cemetery

Middlesbrough Museum

Hornsby 1912; Meaney 1964, 297-8; Gallagher 1987
HOLBOROUGH (Snodland) Kent TQ 698626 Inhumation cemetery

BM; Maidstone Museum

Evison 1956; Meaney 1964, 123-4; Härke 1992, 262, Abb. 59

HOLDENBY Northamptonshire SP 695671

Inhumation and cremation cemetery

Northampton Museum

Anon 1901; Leeds 1909; Meaney 1964, 190

HOLME PIERREPONT Nottinghamshire SK 625391

Inhumation and cremation cemetery

$\mathrm{BM}$

Meaney 1964, 200; Myres 1977, 315, fig 303.2093

HOLYWELL ROW (Mildenhall) Suffolk

TL 714765

Inhumation cemetery

Cambridge Museum

Lethbridge 1931; Meaney 1964, 228; Härke 1992, 262, Abb. 60

Hooper's Field see BARRINGTON B above

HORNDEAN (Snell's Corner) Hampshire

SU 707153

Inhumation cemetery

Portsmouth Museums

Knocker 1957; Meaney 1964, 100; Härke 1992, 279, Abb. 74

HORNSEA Yorkshire TA 207484

Inhumation cemetery

Yorkshire Museum

Meaney 1964, 291

HORNTON Oxfordshire SP 392450

Inhumation burials

BM

Meaney 1964, 209

HORTON KIRBY (I) Kent TQ 564694

Inhumation cemetery

Maidstone Museum

Meaney 1964, 124

HORTON KIRBY (II, Riseley) Kent

TQ 562675

Inhumation and cremation cemetery

Dartford Museum

Meaney 1964, 133-4 
Hough-on-the-Hill see LOVEDEN HILL below

Houghton Regis see PUDDLEHILL below

Hound Point see DALMENY above

Howe see BROOKE above

HOWICK HEUGH Northumberland

NU 260173

Inhumation cemetery

Newcastle Museum

Keeney 1939; Meaney 1964, 199; Miket 1980, 295; Cramp and Miket 1982, 5-6, fig 4

HOWLETTS (Littlebourne) Kent TR 200568 Inhumation cemetery

BM

Smith 1918; Meaney 1964, 125

HOXNE (Old Newton) Suffolk TM 180775

Inhumation burials

BM

Meaney 1964, 228

Huggins Fields see

MILTON-NEXT-SITTINGBOURNE (I) below

HUNSTANTON (Hunstanton Park) Norfolk

TF 696411

Inhumation cemetery

Norwich Museum

Hughes 1901, 310-21; Meaney 1964, 176

ICKLINGHAM Suffolk

Warwick Museum

Meaney 1964, 231

Icklingham All Saints see MITCHELL'S HILL below

ILLINGTON (Wretham) Norfolk TL 948898 Cremation and inhumation cemetery

Norwich Museum

Meaney 1964, 176; Davison et al 1993

IPSWICH (Hadleigh Road) Suffolk

TM 146445

Inhumation and cremation cemetery

Ipswich Museum

Layard 1907; Layard 1909; Meaney 1964, 228

Irby-on-Humber see WELBECK HILL below
Kanovium see CAERHUN above

Kelvedon see FEERING above

KEMPSTON Bedfordshire TL 031476

Inhumation and cremation cemetery

BM

Fitch 1864; Smith 1868a, 166-72; Smith 1868b;

Smith 1904, 176-84; Meaney 1964, 36-7

Kemsing see POLHILL below

KENNINGHALL (I) Norfolk TM 034861

Inhumation cemetery

BM

Manning 1872a, 292; Manning 1872b; Meaney 1964, 176-7

KILHAM Yorkshire TA 079659

Inhumation cemetery

York Museum

Brown 1915a (IV), 806-8; Meaney 1964, 292

King's Field see FAVERSHAM above

KINGSTON (Kingston Down) Kent

TR 202519

Inhumation barrow cemetery

Liverpool Museum

Faussett 1856, 35-94; Meaney 1964, 125-6

KINGSTON-BY-LEWES Sussex (East)

TQ 407095

Inhumation cemetery

Lewes Museum

Meaney 1964, 254; Craddock 1979; Welch 1983, 409-18

Kirton-in-Lindsey I see CLEATHAM above

LACEBY Lincolnshire TA 203066

Inhumation cemetery

Lincoln Museum

Phillips 1934; Myres 1951, 89 and 98; Thompson

1956; Meaney 1964, 157; Leahy 1993, 40

LAKENHEATH Suffolk TL 729830

Inhumation and cremation cemetery

BM

Meaney 1964, 230

Leatherhead also see FETCHAM 
LECHLADE (Butler's Field) Gloucestershire SP 2100

Inhumation and cremation cemetery

Cirencester Museum

Miles and Palmer 1986; Boyle et al forthcoming

Leighton Buzzard see CHAMBERLAIN'S BARN

I and II above

LINCOLN (Burton Road) Lincolnshire

Lincoln Museum

Ling Hill see WHITBY below

LINTON HEATH (B) Cambridgeshire

TL 583486

Inhumation cemetery

Cambridge Museum

Neville 1854; Meaney 1964, 67-8

Littlebourne see HOWLETTS above

LITTLE CHESTER Derbyshire SK 355375

Inhumation cemetery

Wheeler 1985, 304; publication in preparation (C Sparey-Green)

\section{LITTLE ERISWELL (Eriswell) Suffolk}

TL 731802

Inhumation cemetery

Bury St Edmunds Museum

Hutchinson 1966; Härke 1992, 266, Abb. 63

LITTLE WILBRAHAM Cambridgeshire

TL 560577

Inhumation and cremation cemetery

Ashmolean Museum; Cambridge Museum

Neville 1852; Fox 1923, 260-2; Lethbridge and

Carter 1926; Meaney 1964, 70-1

\section{LLANIGON Herefordshire}

Neolithic barrow finds

Morgan and Marshall 1921

LONDESBOROUGH Yorkshire SE 871462

Inhumation cemetery

Newcastle Museum

Meaney 1964, 294-5; Swanton 1964; Cramp and

Miket 1982, 6-8, fig 5

LONDON (Bargate Hill)

Museum of London
LONDON (Dowgate Hill)

Museum of London

LONDON (Milk Street)

Museum of London

LONDON (Wandsworth)

River Thames

Museum of London

LONGTHORPE Northamptonshire TL 158977

Cremation and inhumation cemetery

Frere and St Joseph 1974, 112-20

LONG WITTENHAM (I) Berkshire (now

Oxfordshire) SU 545937

Inhumation and cremation cemetery

Ashmolean Museum; BM

Akerman 1860; Meaney 1964, 53-4; Härke 1992, $266-7$

Lord of the Manor see OZINGELL below

Lords Bridge see ST NEOTS below

LOVEDEN HILL (Hough-on-the-Hill)

Lincolnshire SK 908458

Cremation and inhumation cemetery

Lincoln Museum

Fennell 1964; Meaney 1964, 158-9; Leahy 1993, 40; Fennell forthcoming

Lower Brook Street see WINCHESTER below

LUTON (I: Peddar's Way, Argyll Avenue, Biscot Mill) Bedfordshire TL 081229

Inhumation and cremation cemetery

Luton Museum

Austin 1928; Meaney 1964, 38-9

LYDIARD TREGOZE (Bassett Down) Wiltshire SU 115799

Inhumation cemetery

Devizes Museum

Meaney 1964, 265

LYMINGE (II) Kent TQ 163416

Inhumation cemetery

Maidstone Museum

Warhurst 1955; Meaney 1964, 127; Härke 1992, 268-9, Abb. 64 
MAIDENHEAD (Somerlease Road) Berkshire Reading Museum

Malton see BARRINGTON A above

Manton see CLEATHAM above

Marina Drive see DUNSTABLE above

MARKET LAVINGTON Wiltshire

Inhumation cemetery

Information from Wessex Trust for Archaeology

MARSTON ST LAWRENCE Northamptonshire SP 542439

Inhumation cemetery

Dryden 1849; Dryden 1885; Meaney 1964, 192

\section{MELTON CONSTABLE Norfolk}

Norwich Museum

Mersea Road see COLCHESTER above

MILDENHALL (Poulton Down) Wiltshire SU 204715

Inhumation burial

Devizes Museum

Meaney 1964, 271-2

Mildenhall also see HOLYWELL ROW above

Milk Street see LONDON above

Milton Keynes see BRADWELL above

MILTON REGIS Kent

Inhumation cemetery

BM

Hawkes and Grove 1963; Rigold and Webster 1970

Milton Regis see

MILTON-NEXT-SITTINGBOURNE below

MILTON-NEXT-SITTINGBOURNE (I:

Huggins Fields) Kent TQ 906640

Inhumation cemetery

BM; Maidstone Museum

Vallance 1848; Meaney 1964, 128-9; Hawkes and Grove 1963, 36-8, fig 3 (sites 2 and 3)

MILTON-NEXT-SITTINGBOURNE (II:

Rondeau Estate) Kent TQ 900638
Inhumation cemetery

BM

Meaney 1964, 129; Hawkes and Grove 1963, 36-8, fig 3 (site 4)

Mint Yard see CANTERBURY above

MITCHAM Surrey TQ 270681

Inhumation cemetery

Cambridge Museum; Museum of London

Bidder and Duckworth 1906; Bidder and Morris

1959; Meaney 1964, 243-4

MITCHELL'S HILL (Icklingham All Saints)

Suffolk TL 779722

Inhumation cemetery

Bury St Edmunds Museum

Meaney 1964, 231; West 1985, 157

MOCHRUM (Castle Island) Wigtonshire

(Scotland)

Radford 1950

MONKTON (Thanet) Kent TR 290658

Inhumation cemetery

Ashmolean Museum

Hawkes and Hogarth 1974

MONKTON DOWN Wiltshire

Devizes Museum

MORNING THORPE Norfolk TM 221944

Inhumation cemetery

Norwich Museum

Green et al 1987

MUCKING Essex TQ 673803

Inhumation cemetery (I); inhumation and cremation cemetery (II) BM

Härke 1992, 269, Abb. 65-6; Evison 1981; Hirst and Clark forthcoming

NASSINGTON Northamptonshire TL 071956 Inhumation and cremation cemetery

Peterborough Museum

Leeds and Atkinson 1944; Meaney 1964, 192-3;

Härke 1992, 271-2, Abb. 67

Netheravon Avenue see SALISBURY below 
NETTLETON (Caistor) Lincolnshire

TA 111007

Inhumation cemetery

Lincoln Museum

Meaney 1964, 160; Leahy 1993, 41

\section{NETTLETON SHRUB Wiltshire}

Roman settlement

Information from W J Wedlake

Newburgh see CLATCHARD CRAIG above

NEWPORT PAGNELL Buckinghamshire SP 887433

Inhumation cemetery

Aylesbury Museum

Meaney 1964, 58

Northbourne see FINGLESHAM above

\section{NORTHCHURCH Hertfordshire}

$\mathrm{BM}$

NORTH EARLSTON Berwickshire (Scotland)

Edinburgh Museum

North Elmham see SPONG HILL below

NORTH RUNCTON Norfolk TL 646159

Inhumation and cremation cemetery

Norwich Museum

Meaney 1964, 180-1

NORTHWOLD Norfolk TL 770961

Inhumation cemetery

Akerman 1852, plate V.1-4; Meaney 1964,

$179-80$

NORTON-ON-TEES County Durham (now

Cleveland) NZ 449226

Inhumation cemetery

Vyner 1984; Sherlock and Welch 1992

NORWICH (All Saints Green) Norfolk

Norwich Museum

Oakley Down see WOODYATES below

Old Newton see HOXNE above

Old Park see DOVER II above
ORPINGTON (Fordcroft) Kent (London

Borough) TQ 468676

Inhumation and cremation cemetery

Orpington Museum

Tester 1968; Tester 1969; Härke 1992, 272-3,

Abb. 68

Orwell see BARRINGTON A above

Osengal see OZINGELL below

OWER (Cleavel Point) Dorset SY 998866

Woodward 1987

OZINGELL (Osengal, Lord of the Manor) Kent TR 357652

Inhumation cemetery

Liverpool Museum; Powell-Cotton Museum

Smith 1854; Meaney 1964, 131; Millard et al 1969

PAINSTHORPE WOLD I Yorkshire SE 829585 Inhumation barrow cemetery

Hull Museum

Mortimer 1905, plate XXXIV.278; Meaney 1964, 295-6

PANGBOURNE Berkshire

Cambridge Museum (Beck Collection)

Patrixbourne see BIFRONS above

Peddar's Way see LUTON above

PENZANCE (Trencom Fort) Cornwall

PETERSFINGER (Clarendon) Wiltshire

SU 163293

Inhumation cemetery

Salisbury Museum

Leeds and Shortt 1953; Meaney 1964, 271; Härke 1992, 273, Abb. 69

PEWSEY (Blacknall/Blackpatch) Wiltshire

SU 156581

Inhumation cemetery

Devizes Museum

Härke 1992, 275, Abb. 70; publication in preparation (K Annable)

Plasworth Square see SUNDERLAND below

Old Yeavering see YEAVERING below 
POLHILL (Dunton Green, Kemsing) Kent

TQ 550159

Inhumation cemetery

Hawkes and Philp 1973; Meaney 1964, 132;

Härke 1992, 275, Abb. 71

Portway see ANDOVER above

Poulton Down see MILDENHALL above

Priory Hill see DOVER I above

PRITTLEWELL Essex TQ 878873

Inhumation cemetery

BM; Prittlewell Priory

Meaney 1964, 87-8; Tyler 1988

PUDDLEHILL (Houghton Regis Cemetery 2)

Bedfordshire TQ 004234

Inhumation cemetery

Matthews and Hawkes 1985; Meaney 1964, 39

Purwell Farm see CASSINGTON above

QUARRINGTON Lincolnshire TF 043447

Cremation and inhumation cemetery

Alnwick Museum

Bruce 1880, 69-77; Meaney 1964, 160-1; Leahy 1993, 41

RAINHAM Essex TQ 554840

Inhumation cemetery

BM

Evison 1955; Meaney 1964, 88

RATLEY Warwickshire

Information from $F$ Radcliffe, Leamington Spa

READING (I: Earley) Berkshire SU 741739

Inhumation cemetery

Reading Museum

Stevens 1894; Meaney 1964, 50

RIBY (Riby Park) Lincolnshire TA 186078

Inhumation cemetery

Lincoln Museum

Phillips 1934, 148, 154 and 177; Myres 1951, 88,

fig 9; Meaney 1964, 161; Leahy 1993, 41

RICHBOROUGH Kent

Roman fort

Richborough Museum

Bushe-Fox 1949, 148-50, plate L.V
RISBY (Risby Heath) Suffolk TL 777679

Barrow burial

Bury St Edmunds Museum

Meaney 1964, 232

Riseley see HORTON KIRBY (II) above

ROCHE COURT DOWN (Winterslow)

Wiltshire SU 250357,252357 or 251357

Inhumations

Salisbury Museum

Stone and Tildesley 1932; Meaney 1964, 272-3

ROCHESTER (II: Watts Avenue) Kent

TQ 740680

Inhumation cemetery

Skipton Museum

Meaney 1964, 134

ROLLRIGHT Oxfordshire/Warwickshire

SP 295309

Information from Oxford Archaeological Unit

RONALDSWAY Isle of Man

Anon 1937, fig 3

Rondeau Estate see

MILTON-NEXT-SITTINGBOURNE (II) above

RUSKINGTON Lincolnshire TF 076514

Inhumation cemetery

Lincoln Museum

Meaney 1964, 161-2; Leahy 1993, 41

St Mary's see SOUTHAMPTON below

ST NEOTS (Lords Bridge) Huntingdonshire Inhumation grave: 1892

$\mathrm{BM}$

SALISBURY (3 Netheravon Avenue) Wiltshire

Salisbury Museum

SALISBURY Wiltshire

Unprovenanced find

Salisbury Museum

Salmonsbury see

BOURTON-ON-THE-WATER above

Saltburn see HOB HILL above 
SANCTON Yorkshire (now Humberside)

SE 903402

Cremation and inhumation cemetery

Hull Museum

Meaney 1964, 298-9; Myres and Southern 1973

SARRE (Thanet) Kent TR 261650

Inhumation cemetery

BM; Canterbury Museum; Liverpool Museum

Smith 1860; Brent 1863; Brent 1866; Brent 1868;

Meaney 1964, 135-6; Härke 1992, 277

\section{SAXBY Leicestershire}

Information from Jane Timby

\section{SEARBY Lincolnshire TA 075060}

Inhumation cemetery

BM; Lincoln Museum

Smith 1852a, 234; Smith 1861, 137-40; Meaney

1964, 162; Leahy 1993, 41

Segontium see CAERNARVON above

SELMESTON Sussex (East) TQ 510070

Inhumation cemetery

Lewes Museum

Meaney 1964, 255; Welch 1983

SEWERBY Yorkshire TA 205691

Inhumation cemetery

Meaney 1964, 300-1; Hirst 1985; Härke 1992, 278, Abb. 73

Shalfleet see CHESSELL DOWN above

Shefford, East see EAST SHEFFORD above

SHUDY CAMPS Cambridgeshire TL 604444

Inhumation cemetery

Cambridge Museum

Lethbridge 1936; Meaney 1964, 69

SIBERTSWOLD (Sibertswold-Barfreston) Kent

TR 266488

Inhumation barrow cemetery

Liverpool Museum

Faussett 1856, 101-43; Meaney 1964, 136

SILCHESTER Hampshire SU 640625

Reading Museum

Boon 1959
Sittingbourne see MILTON REGIS and

MILTON-NEXT-SITTINGBOURNE above

SLEAFORD Lincolnshire TF 066454

Inhumation and cremation cemetery

BM

Thomas 1887; Meaney 1964, 162-3; Leahy 1993, 41

SNAPE Suffolk TM 402593

Inhumation and cremation cemetery

Meaney 1964, 232-3; Filmer-Sankey 1990;

Filmer-Sankey and Pestell forthcoming

Snell's Corner see HORNDEAN above

Snodland see HOLBOROUGH above

Somerlease Road see MAIDENHEAD above

SOUTHAMPTON (St Mary's) Hampshire

Excavated find: 1988

Southampton Museum

Publication in preparation

SOUTH ELKINGTON Lincolnshire TF 312883

Cremation and inhumation cemetery

Webster 1951; Meaney 1964, 154; Leahy 1993, 41

SOUTH SHIELDS Northumberland

Group of beads (information from R Miket)

South Shields Museum

\section{SOUTH WILLINGHAM Lincolnshire}

TF 200830

Cremation cemetery

$\mathrm{BM}$

Meaney 1964, 165; Leahy 1993, 41

SPONG HILL (North Elmham) Norfolk

TF 981195

Cremation and inhumation cemetery

Norfolk Archaeological Unit

Meaney 1964, 173-5; Hills 1977; Hills and Penn

1981; Hills et al 1984; Hills et al 1987; Härke

1992, 280, Abb. 75; Hills et al 1994

SPRINGFIELD LYONS Essex TL 736082

Inhumation and cremation cemetery

BM

Buckley and Hedges 1987; publication in preparation (S Tyler) 
STANDLAKE DOWN (I) Oxfordshire

SP 387044

Inhumation cemetery

Ashmolean Museum

Meaney 1964, 212-13; Dickinson 1973

Staunch Meadow see BRANDON above

STODMARSH Kent TR 215603

Inhumation barrow burials

BM

Akerman 1855b, 179-81, plate I; Meaney 1964, 137

Stone see BISHOPSTONE above

STOWTING Kent TR 123423

Inhumation cemetery

Cambridge Museum (Beck Collection);

Maidstone Museum

Smith 1846a; Brent 1867; Brown 1915a (IV),

712-15; Meaney 1964, 137-8

STRETTON-ON-FOSSE Warwickshire

SP $216283 / 218383$

Inhumation cemeteries and settlement

Warwick Museum

Gelling 1992, 40-1, fig 21; Härke 1992, 281, Abb. 76

Sturry see WESTBERE below

SUNDERLAND (Plasworth Square) County

Durham NZ397 584

Sunderland Museum

Miket 1980, 296

Sunny Bank see HAWNBY MOOR above

SUTTON HOO Suffolk TM 288487

Cremation and inhumation barrow cemetery

BM; Ipswich Museum

Meaney 1964, 233-5; Longworth and Kinnes 1980; Selkirk 1990, 357; Carver 1992

Swadling Down see CHARTHAM DOWN above

SWALLOWCLIFFE DOWN (Ansty) Wiltshire ST 967254

Inhumation barrow burial

Salisbury Museum

Speake 1989
SWINDON (Swindon Hill) Wiltshire

SU 157836

Settlement: Grubenhaus 4

Swindon Museum

Information from Caroline Washbourne,

Swindon

Sycamore Terrace see YORK below

SYSTON (Syston Park) Lincolnshire

SK 941405

BM

Akerman 1855a; Meaney 1964, 164

Tanner's Field see FAIRFORD above

Thanet see MONKTON and SARRE above

THORNHAM Norfolk TF 725425

Inhumation cemetery

Norwich Museum

Meaney 1964, 183

THURNHAM (Thurnham Friars) Kent

TQ 806578

Inhumation cemetery

Beck 1940; Meaney 1964, 139

Totternhoe see DUNSTABLE above

TRAPRAIN LAW East Lothian (Scotland)

Edinburgh Museum

Trencom Fort see PENZANCE above

TRIMLEY ST MARY (near Felixstowe) Suffolk

Information from $T$ Sapwell, Norwich

Trumpet Major Public House see

DORCHESTER above

UPTON Northamptonshire SP 713603

Settlement: Grubenhaus

Jackson et al 1969

UPTON SNODSBURY Worcestershire SO 944544

Inhumation cemetery

Worcester Museum

Ponting 1867; Meaney 1964, 281 
WADDINGTON Lincolnshire SK 976640

Inhumation cemetery

Lincoln Museum

Petch 1957; Meaney 1964, 165; Leahy 1993, 41

WALLINGFORD Berkshire (now Oxfordshire) SU 604890

Inhumation and cremation cemetery

Ashmolean Museum

Leeds 1938; Harden 1940; Meaney 1964, 52-3

Wandsworth see LONDON above

WASPERTON Warwickshire SP 261581

Inhumation and cremation cemetery

Warwick Museum

Crawford 1981; Crawford 1982; Crawford 1983;

Esmonde Cleary 1989; publication in

preparation (M Carver)

WATCHFIELD Berkshire (now Oxfordshire)

SU 249907

Inhumation cemetery

Oxfordshire Museum

Scull 1992

WATERBEACH (Car Dyke) Cambridgeshire Settlement: Grubenhaus

Lethbridge 1927

Waterslade see FAIRFORD above

Watts Avenue see ROCHESTER above

WELBECK HILL (Irby-on-Humber)

Lincolnshire (Humberside) TA 217042

Inhumation and cremation cemetery

Scunthorpe Museum

Leahy 1993, 40; publication in preparation (G Taylor)

WELBOURNE (High Dyke) Lincolnshire

SK 980540

Inhumation burial

Alnwick Museum

Bruce 1880, 75-6; Meaney 1964, 165; Leahy

1993, 41

WELTON (Welton-by-Lincoln) Lincolnshire TF 008798

Inhumation cemetery

Lincoln Museum

Leahy 1993, 41
WELTON Northamptonshire SF 570664

Inhumation cemetery

Northampton Museum

Dryden 1885, 337; Meaney 1964, 196-7

WESTBERE (Hersden, Easden, Bushy Close, Sturry) Kent TR 199615

Inhumation and cremation cemetery

Canterbury Museum

Jessup 1946; Meaney 1964, 140

Westgarth Gardens see BURY ST EDMUNDS above

WEST HESLERTON Yorkshire (now North Yorkshire) SE 917765

Inhumation cemetery

Yorkshire Museum

Publication in preparation (D Powlesland)

WEST STOW Suffolk TL 797713

Inhumation cemetery and settlement

Ashmolean Museum; Bury St Edmunds

Museum; Thetford Museum

Meaney 1964, 233; West 1985

WHEATLEY Oxfordshire SP 602046

Inhumation cemetery

Ashmolean Museum

Leeds 1916; Meaney 1964, 213-14

WHITBY (Ling Hill) Yorkshire (now North

Yorkshire)

Whitby Museum

WHITBY Yorkshire (now North Yorkshire)

Beck 1943

Wilbraham, Little see LITTLE WILBRAHAM above

WILLINGTON Derbyshire

Settlement: Grubenhäuser

Wheeler 1979

WINCHESTER (Cathedral Green) Hampshire Excavated find

Publication in preparation (M Biddle)

WINCHESTER (College Green) Hampshire

Excavated find

Publication in preparation (M Biddle) 
WINCHESTER (Lower Brook Street) Hampshire Inhumation grave

Hawkes 1990b

WINCHESTER (Wolvesey Palace) Hampshire

Excavated finds

Publication in preparation (M Biddle)

WINGHAM (Witherden Farm) Kent

TR 249569

Inhumation cemetery

BM

Meaney 1964, 140-1

WINKELBURY (II, Berwick St John) Wiltshire ST 951212

Burial

Salisbury Museum

Pitt-Rivers 1888, 266, plate CL.29

WINTERBOURNE GUNNER Wiltshire

SU 182352

Inhumation cemetery

Salisbury Museum

Meaney 1964, 278; Musty and Stratton 1964;

Härke 1992, 286, Abb. 81

WINTERBOURNE STOKE (II) Wiltshire SU 104422

Inhumation barrow burial

Devizes Museum

Hoare 1812, 119; Meaney 1964, 278

Winterslow see ROCHE COURT DOWN above

Witherden Farm see WINGHAM above

Wittenham, Long see LONG WITTENHAM above

WOLSTONBURY (Wolstonbury Hill) Sussex (East)

Roman settlement

Lewes Museum

Holleyman 1935, 38, fig 1

Wolvesey Palace see WINCHESTER above
Woodnesborough see COOMBE above

WOODSTONE (Woodston) Huntingdonshire (now Cambridgeshire)

TL 177976 or 185975

Barrow excavated 1827 (part of inhumation cemetery)

BM

Artis 1828, plate LV; Walker 1899; Meaney 1964, 107

WOODYATES (Oakley Down Barrow I)

Wiltshire

Barrow burial

Devizes Museum (Catalogue 199/200/222b)

Wretham see ILLINGTON above

WROTHAM (II, Bradford Platt) Kent

TQ 615598

Inhumation burials

BM

Anon 1920; Meaney 1964, 141-2

WYKEHAM GRANGE Yorkshire, North Riding (now North Yorkshire)

$\mathrm{BM}$

YEAVERING (Old Yeavering) Northumberland NY 925305

Settlement and cemetery contexts

Meaney 1964, 199; Hope-Taylor 1977; Miket

$1980,296-7$

YORK (Coppergate) Yorkshire (now North

Yorkshire)

Excavated context

YORK (Sycamore Terrace) Yorkshire (now North Yorkshire)

Roman burial

Yorkshire Museum

RCHM 1962, 73, fig 58

YORK Yorkshire (now North Yorkshire)

$\mathrm{BM}$; Yorkshire Museum 


\title{
SCHEdULe 1
}

\section{Colourless AND PALE TRANSLUCENT GLASS BEADS}

\author{
MONOCHROME \\ i Colourless translucent beads in various forms \\ ii Light-coloured annular, globular, biconical and double beads (excluding \\ smokey yellow forms) \\ iii Light blue-green translucent melon and sub-melon beads \\ iv Smokey yellow beads (excluding melon and sub-melon forms) \\ $\mathrm{v} \quad$ Smokey yellow melon and sub-melon beads \\ Polychrome \\ vi Decorated translucent beads: (a) on a light blue-green ground \\ (b) on a smokey yellow ground \\ vii Light-coloured translucent annular or globular beads with coloured parallel \\ bands (including lobed forms) \\ viii Light yellowish-green beads with decoration (mostly square-sectioned form) \\ (see Map 1) \\ ix Light-coloured decorated lobed or horned beads
}

\section{MONOCHROME}

\begin{tabular}{|c|c|c|c|c|}
\hline & Col & RLESS & TRANSLUCENT BEADS & IN VARIOUS FORMS \\
\hline Frilford & Berks & 5 th $\mathrm{c}$ & $\begin{array}{l}\text { Grave 196: } 1 \text { shape- } \\
\text { less blob, ?reused } \\
\text { piece of Roman glass } \\
\text { vessel, with applied } \\
\text { saucer brooch }\end{array}$ & $\begin{array}{l}\text { Rolleston 1880, 264; } \\
\text { Brown 1975, 293; } \\
\text { Evison 1978, pl LVd; } \\
\text { Böhme 1986, 531, } \\
\text { Abb. 53.2; } \\
\text { Ashmolean } \\
\text { Museum 1869.12.3 }\end{array}$ \\
\hline Wallingford & Berks & $\begin{array}{l}\text { 5th- } \\
\text { late } \\
6 \text { th c }\end{array}$ & $\begin{array}{l}\text { Grave 13: thick } \\
\text { annular about } 10 \mathrm{~mm} \\
\text { diameter }\end{array}$ & $\begin{array}{l}\text { Leeds } 1938,98 ; \\
\text { Ashmolean } \\
\text { Museum } 1938.1222\end{array}$ \\
\hline $\begin{array}{l}\text { Linton } \\
\text { Heath }\end{array}$ & Cambs & $\begin{array}{l}\text { 5th- } \\
\text { 6th } c\end{array}$ & $\begin{array}{l}\text { One annular, rather } \\
\text { large }\end{array}$ & $\begin{array}{l}\text { Neville 1854; } \\
\text { Cambridge Museum }\end{array}$ \\
\hline
\end{tabular}




\begin{tabular}{|c|c|c|c|}
\hline $\begin{array}{l}\text { Little } \\
\text { Wilbraham }\end{array}$ & Cambs & $\begin{array}{l}5 \text { th- } \\
7 \text { th } c\end{array}$ & $\begin{array}{l}\text { Unassociated group: } \\
\text { small annular form }\end{array}$ \\
\hline $\begin{array}{l}\text { Great } \\
\text { Chesterford }\end{array}$ & Essex & $\begin{array}{l}\text { 2nd } \\
\text { half } \\
5 \text { th c }\end{array}$ & $\begin{array}{l}\text { Grave 113: } 1 \\
\text { roughly square- } \\
\text { sectioned bead (B56) } \\
\text { with floriate } \\
\text { cross applied saucer } \\
\text { brooch pair (Great } \\
\text { Chesterford type) }\end{array}$ \\
\hline Mucking II & Essex & $\begin{array}{l}\text { late } \\
5 \text { th- } \\
6 \text { th c }\end{array}$ & $\begin{array}{l}\text { Grave } 874: 1 \\
\text { flattened coiled } \\
\text { bead with biconical } \\
\text { sides in slightly } \\
\text { gold-tinged colourles } \\
\text { glass (Plate } 1 \text { ) }\end{array}$ \\
\hline Droxford & Hants & $\begin{array}{l}\text { late } \\
\text { 5th- } \\
\text { 6th c }\end{array}$ & $\begin{array}{l}\text { Grave 21: annular } \\
\text { (class XIV) }\end{array}$ \\
\hline Faversham & Kent & $\begin{array}{l}5 \text { th- } \\
7 \text { th c }\end{array}$ & Annular \\
\hline $\begin{array}{l}\text { Milton } \\
\text { Regis }\end{array}$ & Kent & $\begin{array}{l}\text { prob } \\
\text { 6th- } \\
\text { 7th c }\end{array}$ & $\begin{array}{l}\text { Large bead or } \\
\text { amulet with opaque } \\
\text { white irregular trail }\end{array}$ \\
\hline Monkton & Kent & $\begin{array}{l}\text { 6th- } \\
7 \text { th } c\end{array}$ & One thick annular \\
\hline
\end{tabular}

Fonaby Lincs 6th c Grave 32: very small cylindrical bead, with Åberg Group III cruciform brooch

\begin{tabular}{|c|c|c|c|}
\hline Ruskington & Lincs & $\begin{array}{l}\text { prob } \\
\text { 6th } c\end{array}$ & $\begin{array}{l}\text { Group 2: long square- } \\
\text { section (2) }\end{array}$ \\
\hline Brooke & Norfolk & $\begin{array}{l}\text { prob } \\
\text { 6th c }\end{array}$ & Irregular-shaped bead \\
\hline Brundall & Norfolk & & One globular bead \\
\hline $\begin{array}{l}\text { Morning } \\
\text { Thorpe }\end{array}$ & Norfolk & $\begin{array}{l}5 \text { th- } \\
6 \text { th } c\end{array}$ & Several \\
\hline $\begin{array}{l}\text { Empingham } \\
\text { II }\end{array}$ & Rutland & $\begin{array}{l}\text { 5th- } \\
7 \text { th c }\end{array}$ & $\begin{array}{l}\text { Grave } 37: \text { short square- } \\
\text { section cylinder; } \\
\text { Grave } 69: 1 \text { cylinder; } \\
\text { Grave } 98: 2 \text { annulars }\end{array}$ \\
\hline
\end{tabular}

Fox 1923, 260-3;

Neville 1852, illustrated;

Ashmolean Museum

Evison 1994, 12,

15,46 and 106 ,

figs 5 and 43.113.3a;

Böhme 1986, 545-7,

Abb. 62.1;

BM 1964 7-2 342

Hirst and Clark forthcoming; BM

Aldsworth 1978, 126 and 173, fig 25.21;

Winchester Museum

Meaney 1964, 118-19;

Ashmolean Museum 1909.155

Vallance 1848,100 , pl XXXVII.3

Hawkes and Hogarth 1974; Perkins and Hawkes 1984; Ashmolean Museum 1972.1427

Cook 1981, 32, fig 11.32.2; Scunthorpe Museum

Meaney 1964, 161-2;

Lincoln Museum

Meaney 1964, 170;

BM 186669

Norwich Museum

Green et al 1987;

Norwich Museum

Timby 1996;

Oakham Museum 


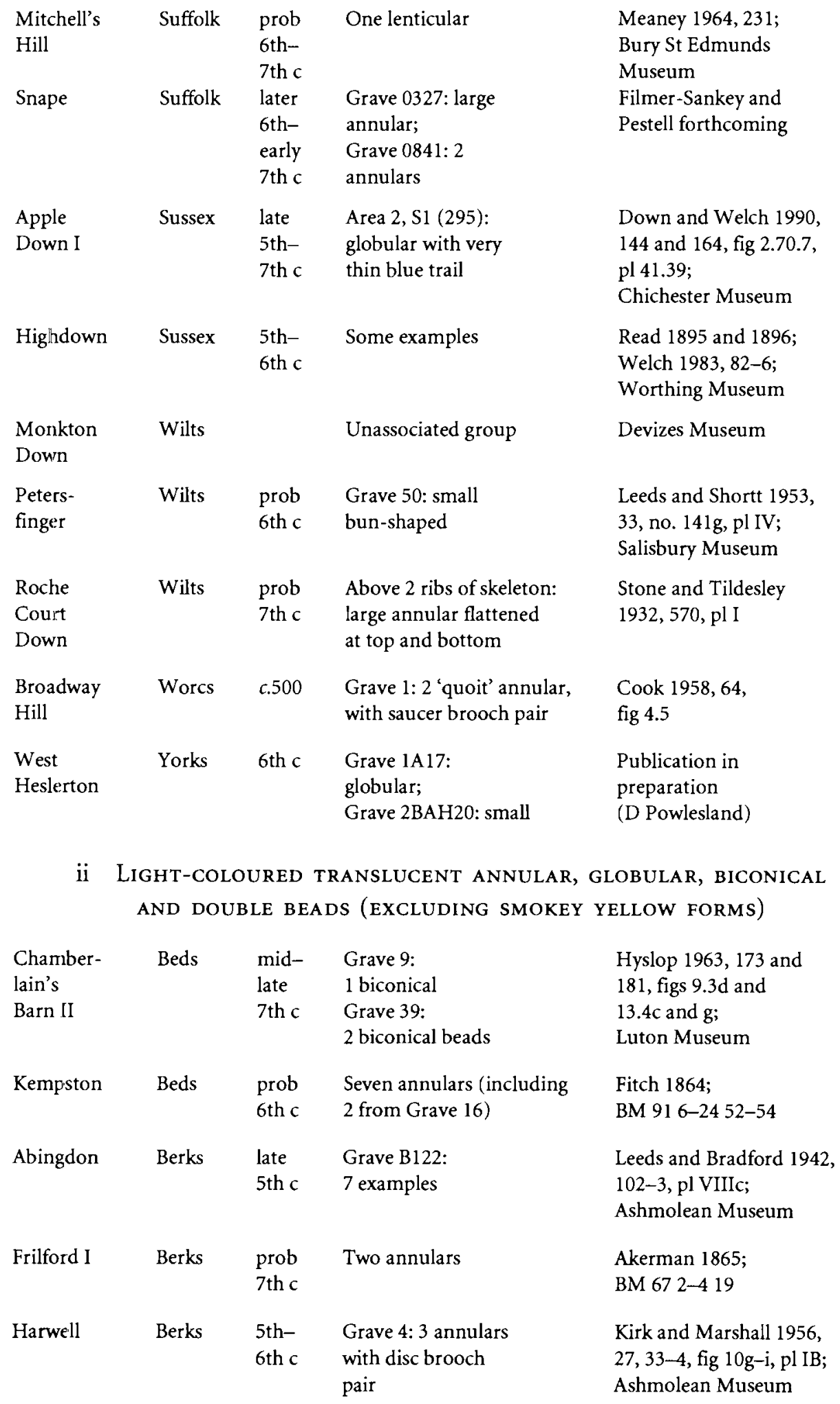




\begin{tabular}{|c|c|c|c|c|}
\hline Wallingford & Berks & $\begin{array}{l}\text { 5th- } \\
\text { 6th c }\end{array}$ & $\begin{array}{l}\text { Grave } 11: 16 \text { small } \\
\text { annulars with } 2 \text { small-long } \\
\text { brooches and pot }\end{array}$ & $\begin{array}{l}\text { Leeds } 1938,97, \mathrm{pl} \mathrm{III} ; \\
\text { Ashmolean Museum }\end{array}$ \\
\hline $\begin{array}{l}\text { Great } \\
\text { Chesterford }\end{array}$ & Essex & $\begin{array}{l}\text { 5th- } \\
\text { 6th c }\end{array}$ & $\begin{array}{l}\text { Grave 79: } 1 \text { globular } \\
\text { (B18) with broken girdle- } \\
\text { hanger, etc; } \\
\text { Grave 103: } 1 \text { annular } \\
\text { (B18) with applied saucer } \\
\text { brooch pair, etc; } \\
\text { Grave 127: } 2 \text { annulars } \\
\text { (B34) with disc brooch } \\
\text { pair, etc }\end{array}$ & $\begin{array}{l}\text { Evison } 1994,14-15,46 \\
103,105 \text { and } 109, \text { figs } 5 \text {, } \\
37.79 .2 \mathrm{c}, 42103.3 \mathrm{~d} \text { and } \\
49.127 .5 \mathrm{a} \text {; } \\
\text { BM } 19647-2\end{array}$ \\
\hline Mucking II & Essex & $\begin{array}{l}\text { 5th- } \\
6 \text { th c }\end{array}$ & $\begin{array}{l}\text { Grave } 334: 5 \text { pale } \\
\text { green discs; } \\
\text { Grave 548: } 1 \text { pale } \\
\text { blue-green and } 4 \\
\text { pale blue discs; } \\
\text { Grave 550: } 1 \text { pale green } \\
\text { disc; } \\
\text { Grave 648: } 1 \text { pale green } \\
\text { globular; } \\
\text { Grave 649: } 2 \text { pale green-blue } \\
\text { discs (Plate 1); } \\
\text { Grave 860: 1 pale green } \\
\text { globular, } 3 \text { small pale blue- } \\
\text { green double globular, } \\
1 \text { pale green-blue disc; } \\
\text { Grave 874: } 1 \text { pale blue-green } \\
\text { Grave 924B: } 1 \text { pale green-blu } \\
\text { Grave 960: } 3 \text { small pale green } \\
\text { Grave } 989: 3 \text { pale blue-green }\end{array}$ & $\begin{array}{l}\text { Hirst and Clark forthcoming; } \\
\text { BM } \\
\\
\text { nnular; } \\
\text { disc; } \\
\text { lue globular; } \\
\text { iscs }\end{array}$ \\
\hline Lechlade & Glos & $\begin{array}{l}\text { 6th } \mathrm{c} \\
\text { 6th } \mathrm{c}\end{array}$ & $\begin{array}{l}\text { Grave } 41: 1 \text { pale blue- } \\
\text { green disc and } 2 \text { pale green- } \\
\text { blue annulars with disc } \\
\text { brooch pair, etc; } \\
\text { Grave } 45: 1 \text { pale green- } \\
\text { blue annular with cast } \\
\text { saucer brooch pair, etc; } \\
\text { Grave 53: } 1 \text { pale green- } \\
\text { blue annular; } \\
\text { Grave 176: } 1 \text { pale green- } \\
\text { yellow large annular with } \\
\text { annular or penannular } \\
\text { brooches; } \\
\text { Grave 14: } 1 \text { pale blue- } \\
\text { green large annular with } \\
\text { workbox, cowrie, etc; } \\
\text { Grave 138: } 2 \text { pale blue- } \\
\text { green discs with linked } \\
\text { pins, etc; }\end{array}$ & $\begin{array}{l}\text { Boyle et al forthcoming; } \\
\text { Cirencester Museum }\end{array}$ \\
\hline
\end{tabular}




\begin{tabular}{|c|c|c|c|c|}
\hline & & 7 th $\mathrm{c}$ & $\begin{array}{l}\text { Grave } 148: 1 \text { pale blue- } \\
\text { green annular with lattice- } \\
\text { decorated glass pendant, } \\
\text { bell, etc; } \\
\text { Grave } 172 / 2: 1 \text { pale green- } \\
\text { blue barrel }\end{array}$ & \\
\hline Andover & Hants & 6th $c$ & $\begin{array}{l}\text { Grave 50: } 1 \text { annular, } \\
2 \text { barrels with small-long } \\
\text { brooch and Quoit Brooch } \\
\text { Style buckle }\end{array}$ & $\begin{array}{l}\text { Cook and Dacre } 1985,38, \\
\text { fig 63.10, } 14 \text { and } 27 ; \\
\text { Hampshire Museums }\end{array}$ \\
\hline $\begin{array}{l}\text { Dover, } \\
\text { Buckland }\end{array}$ & Kent & $\begin{array}{l}650- \\
675 \\
700- \\
750\end{array}$ & $\begin{array}{l}\text { Grave 6: } 1 \text { 'disc' (B26); } \\
\text { Grave 134: annular (B23); } \\
\text { Grave 157: annular (B23); } \\
\text { Grave 83: 1 'disc' (B23) }\end{array}$ & $\begin{array}{l}\text { Evison } 1987,73,80,217, \\
236,245 \text { and } 250 \text {, } \\
\text { figs } 7.10 \mathrm{~d}, 39.83 .1 \text {, } \\
55.134 .2 \mathrm{c}, 62.157 .1 \mathrm{c} \text {, } \\
\text { colour pl III (B23 and B26); } \\
\text { BM }\end{array}$ \\
\hline Faversham & Kent & $\begin{array}{l}\text { prob } \\
\text { 6th- } \\
\text { 7th c }\end{array}$ & Three annulars & $\begin{array}{l}\text { Meaney } 1964,118-19 ; \\
\text { Ashmolean Museum } \\
1909.143 \text { and } 145\end{array}$ \\
\hline $\begin{array}{l}\text { Horton } \\
\text { Kirby I }\end{array}$ & Kent & $\begin{array}{l}\text { prob } \\
\text { 6th c }\end{array}$ & $\begin{array}{l}\text { One annular and } \\
1 \text { slightly faceted }\end{array}$ & $\begin{array}{l}\text { Meaney } 1964,124 \\
\text { Maidstone Museum } \\
\text { AS } 200\end{array}$ \\
\hline Fonaby & Lincs & $\begin{array}{l}\text { 6th- } \\
7 \text { th } c\end{array}$ & $\begin{array}{l}\text { Several annular and } \\
\text { globular }\end{array}$ & $\begin{array}{l}\text { Cook 1981; } \\
\text { Scunthorpe Museum }\end{array}$ \\
\hline Laceby & Lincs & $\begin{array}{l}\text { prob } \\
\text { 6th- } \\
7 \text { th c }\end{array}$ & Two annulars & $\begin{array}{l}\text { Myres } 1951,89 \text { and } 98 ; \\
\text { Thompson 1956; } \\
\text { Lincoln Museum }\end{array}$ \\
\hline 'Lincs' & Lincs & & One drop & Scunthorpe Museum \\
\hline Riby Park & Lincs & $\begin{array}{l}\text { prob } \\
\text { 7th c }\end{array}$ & Two small and 2 large & $\begin{array}{l}\text { Meaney } 1964,161 ; \\
\text { Lincoln Museum }\end{array}$ \\
\hline Ruskington & Lincs & $\begin{array}{l}\text { prob } \\
\text { 6th } c\end{array}$ & $\begin{array}{l}\text { One annular, } \\
1 \text { irregular drop }\end{array}$ & $\begin{array}{l}\text { Meaney 1964, 161-2; } \\
\text { Lincoln Museum }\end{array}$ \\
\hline Searby & Lincs & $\begin{array}{l}\text { late } \\
\text { 5th- } \\
\text { 6th c }\end{array}$ & One annular & $\begin{array}{l}\text { Smith 1852a and 1861; } \\
\text { BM } 936-1824\end{array}$ \\
\hline $\begin{array}{l}\text { South } \\
\text { Willingham }\end{array}$ & Lincs & & One annular & BM OA 5057 \\
\hline Waddington & Lincs & & Three annulars & $\begin{array}{l}\text { Meaney } 1964,165 \\
\text { Lincoln Museum }\end{array}$ \\
\hline $\begin{array}{l}\text { Welton- } \\
\text { by-Lincoln }\end{array}$ & Lincs & & $\begin{array}{l}\text { Findspot not known: } \\
\text { flattened annular with } \\
\text { amber and other glass beads }\end{array}$ & Lincoln Museum \\
\hline Boughton & Norfolk & & $\begin{array}{l}\text { One greenish yellow } \\
\text { globular }\end{array}$ & $\begin{array}{l}\text { Fitch Collection, } \\
\text { Norwich Museum } 371\end{array}$ \\
\hline
\end{tabular}




\begin{tabular}{|c|c|c|c|c|}
\hline Brooke & Norfolk & $\begin{array}{l}\text { prob } \\
\text { 6th c }\end{array}$ & One annular & $\begin{array}{l}\text { Meaney 1964, 170; } \\
\text { BM } 186669\end{array}$ \\
\hline Brundall & Norfolk & $\begin{array}{l}5 \text { th- } \\
6 \text { th } c\end{array}$ & & $\begin{array}{l}\text { Johnson 1926, 195-6; } \\
\text { Norwich Museum 68/13 }\end{array}$ \\
\hline Illington & Norfolk & $\begin{array}{l}\text { prob } \\
6 \text { th } c\end{array}$ & $\begin{array}{l}\text { One yellow green } \\
\text { melted ?slab }\end{array}$ & $\begin{array}{l}\text { Davison et al 1993; } \\
\text { Norwich Museum }\end{array}$ \\
\hline Spong Hill & Norfolk & $\begin{array}{l}\text { late } \\
\text { 5th- } \\
6 \text { th c }\end{array}$ & $\begin{array}{l}\text { Graves } 22,26,38 \text { and } \\
\text { 39: several annulars with } \\
\text { cruciform brooches, etc }\end{array}$ & $\begin{array}{l}\text { Hills et al } 1984 \text {, } \\
70,74,89-90,90-1 \text {, } \\
\text { figs } 79,83.1,92 ; \\
\text { Norfolk Arch Unit }\end{array}$ \\
\hline $\begin{array}{l}\text { Bury St } \\
\text { Edmunds, } \\
\text { Westgarth } \\
\text { Gardens }\end{array}$ & Suffolk & 6th $c$ & $\begin{array}{l}\text { Grave 31: small globular } \\
\text { with stamp-ornamented } \\
\text { pot }\end{array}$ & $\begin{array}{l}\text { West } 1988,28 \text {, fig } 29 . A 1 \text {; } \\
\text { Bury St Edmunds Museum }\end{array}$ \\
\hline Icklingham & Suffolk & $\begin{array}{l}\text { prob } \\
6 \text { th- } \\
7 \text { th c }\end{array}$ & One annular & $\begin{array}{l}\text { Meaney } 1964,231 ; \\
\text { Warwick Museum }\end{array}$ \\
\hline Lakenheath & Suffolk & $\begin{array}{l}\text { 6th- } \\
7 \text { th } c\end{array}$ & One small globular & $\begin{array}{l}\text { Meaney 1964, 230; } \\
\text { BM 1910 12-22 11 }\end{array}$ \\
\hline $\begin{array}{l}\text { Little } \\
\text { Eriswell }\end{array}$ & Suffolk & $\begin{array}{l}6 \text { th- } \\
7 \text { th } c\end{array}$ & One globular & $\begin{array}{l}\text { Hutchinson 1966; } \\
\text { Bury St Edmunds Museum }\end{array}$ \\
\hline $\begin{array}{l}\text { Mitchell's } \\
\text { Hill }\end{array}$ & Suffolk & $\begin{array}{l}\text { prob } \\
6 \text { th- } \\
7 \text { th } c\end{array}$ & $\begin{array}{l}\text { One annular and } \\
1 \text { ?globular }\end{array}$ & $\begin{array}{l}\text { Meaney 1964, 231; } \\
\text { Bury St Edmunds Museum }\end{array}$ \\
\hline West Stow & Suffolk & $\begin{array}{l}5 \text { th- } \\
7 \text { th c }\end{array}$ & $\begin{array}{l}\text { Cemetery: double } \\
\text { globular; } \\
\text { Cemetery: } 3 \text { irregular } \\
\text { globulars, } 1 \text { annular, } \\
1 \text { double }\end{array}$ & $\begin{array}{l}\text { West } 1985,74 ; \\
\text { Bury St Edmunds Museum; } \\
\text { Ashmolean Museum } \\
1909.424\end{array}$ \\
\hline Guildown & Surrey & $\begin{array}{l}5 \text { th- } \\
6 \text { th c }\end{array}$ & $\begin{array}{l}\text { Grave } 78: 3 \text { annular and } \\
\text { globular with small-long } \\
\text { brooch pair, etc }\end{array}$ & $\begin{array}{l}\text { Lowther } 1931,12,36 \text {, } \\
\text { pl IX.7; Guildford Museum } \\
\text { A/S } 7333\end{array}$ \\
\hline Alfriston & Sussex & $\begin{array}{l}5 \text { th- } \\
6 \text { th } c\end{array}$ & $\begin{array}{l}\text { Grave } 47: 1 \text { annular with } \\
\text { small square-headed } \\
\text { brooch pair, etc; } \\
\text { Grave } 68: 2 \text { annulars } \\
\text { with quoit brooch }\end{array}$ & $\begin{array}{l}\text { Griffith and Salzmann } \\
\text { 1914,41 and 47, pl I.1; } \\
\text { Welch 1983, 82-6; } \\
\text { Lewes Museum }\end{array}$ \\
\hline Highdown & Sussex & $\begin{array}{l}5 \text { th- } \\
6 \text { th } c\end{array}$ & Some examples & $\begin{array}{l}\text { Read } 1895 \text { and } 1896 \text {; } \\
\text { Welch 1983, 82-6; } \\
\text { Worthing Museum }\end{array}$ \\
\hline $\begin{array}{l}\text { Peters- } \\
\text { finger }\end{array}$ & Wilts & $\begin{array}{l}\text { prob } \\
\text { 6th c }\end{array}$ & Grave 2: 1 small annular & $\begin{array}{l}\text { Leeds and Shortt 1953, } \\
6 \text { (no. 5), pl III; } \\
\text { Salisbury Museum }\end{array}$ \\
\hline $\begin{array}{l}\text { Saltburn, } \\
\text { Hob Hill }\end{array}$ & Yorks & $\begin{array}{l}\text { 6th- } \\
7 \text { th } c\end{array}$ & One small annular & $\begin{array}{l}\text { Gallagher 1987, 19, } 25 \text {, } \\
\text { fig 5.53; } \\
\text { Middlesbrough Museum }\end{array}$ \\
\hline
\end{tabular}




\begin{tabular}{|c|c|c|}
\hline $\begin{array}{l}\text { 'near } \\
\text { York' }\end{array}$ & Yorks & One annular \\
\hline
\end{tabular}

\section{iii Light BLUE-GREEN TRANSLUCENT MELON AND SUB-MELON BEADS}

\begin{tabular}{|c|c|c|c|c|}
\hline $\begin{array}{l}\text { Chamber- } \\
\text { lain's } \\
\text { Barn II }\end{array}$ & Beds & 7th $\mathrm{c}$ & $\begin{array}{l}\text { Grave 9: 6-lobed } \\
\text { flattish; } \\
\text { Grave 39: 5-lobed; } \\
\text { Grave 57: 4-lobed }\end{array}$ & $\begin{array}{l}\text { Hyslop } 1963,173,181 \text {, } \\
\text { and } 185-7, \text { figs } 9 \mathrm{~h}, \\
13 \mathrm{e} \text { and } 17 \mathrm{~g} ; \\
\text { Luton Museum }\end{array}$ \\
\hline Frilford & Berks & $\begin{array}{l}\text { prob } \\
6 \text { th- } \\
7 \text { th c }\end{array}$ & $\begin{array}{l}\text { Two beads (similar to } \\
\text { Chamberlain's Barn } \\
\text { above, but larger) }\end{array}$ & $\begin{array}{l}\text { Meaney 1964, 46-7; } \\
\text { BM } 67 \text { 2-4 } 19\end{array}$ \\
\hline Wallingford & Berks & $\begin{array}{l}\text { 2nd } \\
\text { half } \\
5 \text { th c }\end{array}$ & $\begin{array}{l}\text { Grave 15: with applied } \\
\text { saucer brooch pair } \\
\text { (floriate/anchor cross } \\
\text { design) and } 2 \text { quoit } \\
\text { brooches }\end{array}$ & $\begin{array}{l}\text { Leeds 1938, 98-9, pl VII; } \\
\text { Welch 1975; } \\
\text { Evison 1978; Böhme 1986, } \\
\text { 545-7 and 571; } \\
\text { Ashmolean Museum }\end{array}$ \\
\hline $\begin{array}{l}\text { Linton } \\
\text { Heath }\end{array}$ & Cambs & $\begin{array}{l}\text { prob } \\
5 \text { th- } \\
7 \text { th } c\end{array}$ & $\begin{array}{l}\text { Small globular, } \\
\text { ?sub-melon; } \\
\text { another larger and flatter }\end{array}$ & $\begin{array}{l}\text { Neville 1854; } \\
\text { Cambridge Museum }\end{array}$ \\
\hline Mucking II & Essex & $\begin{array}{l}\text { 5th- } \\
6 \text { th } c\end{array}$ & $\begin{array}{l}\text { Grave } 845: 5 \text {-lobed } \\
\text { with disc brooch pair; } \\
\text { Grave } 860: 8 \text { ribs } \\
\text { with small-long brooch } \\
\text { pair and penannular brooch; } \\
\text { Grave } 989: 1 \text { pale } \\
\text { blue-green }\end{array}$ & $\begin{array}{l}\text { Hirst and Clark forthcoming; } \\
\text { BM }\end{array}$ \\
\hline $\begin{array}{l}\text { Springfield } \\
\text { Lyons }\end{array}$ & Essex & 6th $c$ & $\begin{array}{l}\text { Grave 4758: atypical } \\
\text { with red tips } \\
\text { on } 5 \text { projections }\end{array}$ & $\begin{array}{l}\text { Publication in } \\
\text { preparation (S Tyler); } \\
\text { BM }\end{array}$ \\
\hline Lechlade & Glos & 6th $c$ & $\begin{array}{l}\text { Grave } 45 \text { : light green, } \\
\text { with saucer brooch pair, } \\
\text { etc; } \\
\text { Grave 160: pale blue-green } \\
\text { lobed, with disc brooch, } \\
\text { dress pin, etc }\end{array}$ & $\begin{array}{l}\text { Boyle et al forthcoming; } \\
\text { Cirencester Museum }\end{array}$ \\
\hline Droxford & Hants & 6th $c$ & $\begin{array}{l}\text { One flat } 5 \text {-lobed and } 1 \text { tall, } \\
\text { narrow with lobes (class } \\
\text { IIIa) }\end{array}$ & $\begin{array}{l}\text { Aldsworth } 1978,138 \text {, } \\
172 \text {, fig } 36.71 \text { and } 62 ; \\
\text { BM }\end{array}$ \\
\hline $\begin{array}{l}\text { Chessell } \\
\text { Down }\end{array}$ & $\begin{array}{l}\text { Isle of } \\
\text { Wight }\end{array}$ & 6th $c$ & $\begin{array}{l}\text { Several light colours } \\
\text { (type 11.1-5) }\end{array}$ & $\begin{array}{l}\text { Arnold } 1982 ; 49-50, \\
121 \text {, colour frontispiece; } \\
\text { BM }\end{array}$ \\
\hline $\begin{array}{l}\text { Canterbury, } \\
\text { Cakebread } \\
\text { Robey V }\end{array}$ & Kent & $c .400$ & $\begin{array}{l}\text { Multiple burial: } \\
5 \text { lobes }\end{array}$ & $\begin{array}{l}\text { Information from } \\
\text { T Tatton-Brown and } \\
\text { P Garrard }\end{array}$ \\
\hline Faversham & Kent & $\begin{array}{l}\text { prob } \\
\text { 6th- } \\
7 \text { th c }\end{array}$ & Greenish & $\begin{array}{l}\text { Meaney 1964, 118-19; } \\
\text { Ashmolean Museum }\end{array}$ \\
\hline
\end{tabular}




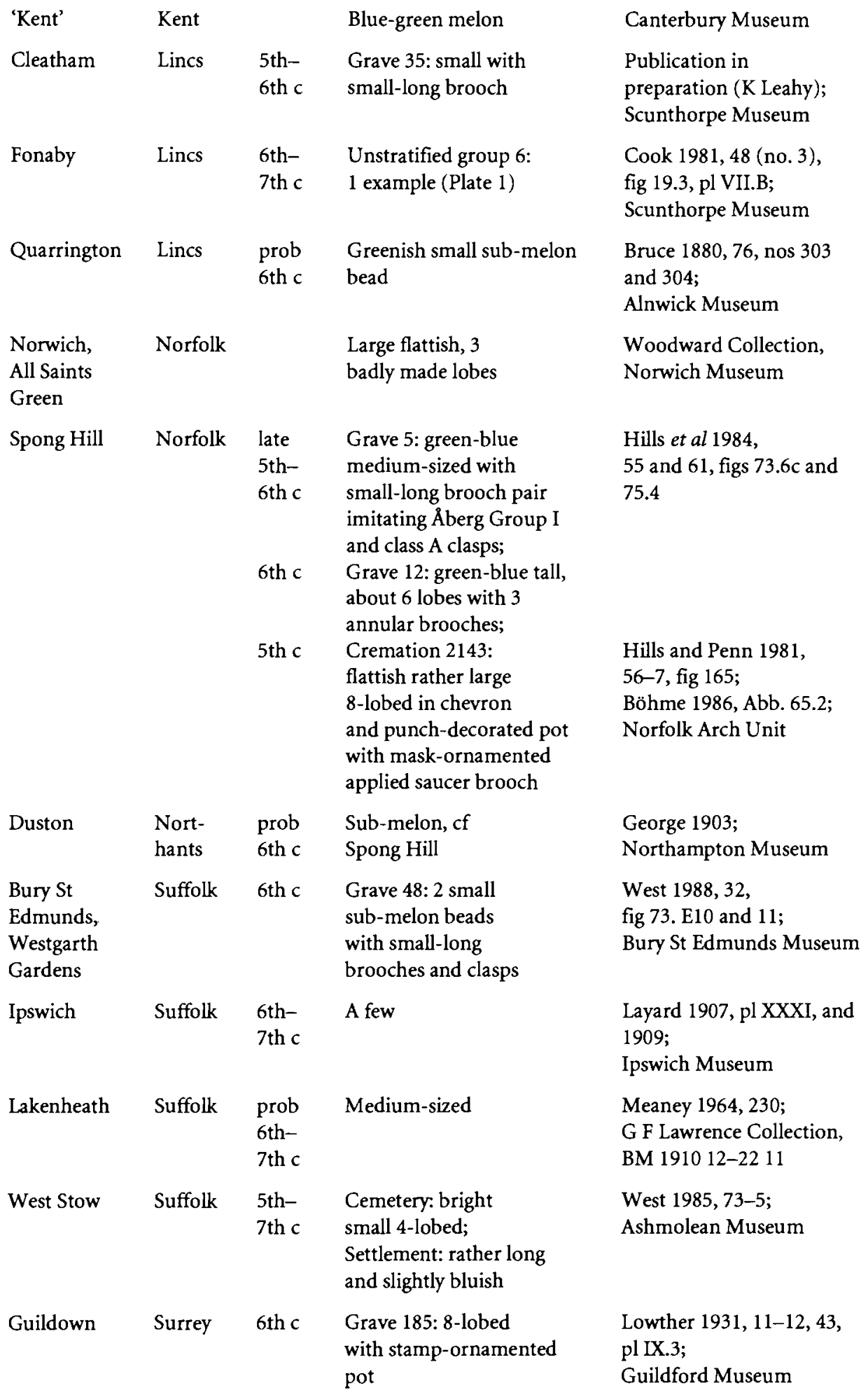




\begin{tabular}{|c|c|c|c|c|}
\hline Alfriston & Sussex & $\begin{array}{l}\text { first } \\
\text { half } \\
6 \text { th c }\end{array}$ & $\begin{array}{l}\text { Grave } 62: 1 \text { sub-melon } \\
\text { and probably another } \\
\text { with dress pin, saucer } \\
\text { brooch pair and } 5 \text { button } \\
\text { brooches, etc }\end{array}$ & $\begin{array}{l}\text { Griffith and Salzmann } 1914 \text {, } \\
44 ; \\
\text { Welch 1983, 82-6; } \\
\text { Lewes Museum }\end{array}$ \\
\hline $\begin{array}{l}\text { Apple } \\
\text { Down I }\end{array}$ & Sussex & $\begin{array}{l}\text { prob } \\
\text { 6th c }\end{array}$ & $\begin{array}{l}\text { Grave 10: large melon } \\
\text { with Style I cast saucer } \\
\text { brooch pair; } \\
\text { Grave } 38 \text { : blue melon } \\
\text { with amber beads }\end{array}$ & $\begin{array}{l}\text { Down and Welch } 1990,35 \\
\text { and } 40,102 \text {, } \\
\text { figs } 2.17 .3 \text { and } 2.24 .1 \text {, } \\
\text { pls } 41.31 \text { and } 40.6 \text {; } \\
\text { Chichester Museum }\end{array}$ \\
\hline Charlton & Wilts & 7th $\mathrm{c}$ & Grave 37: 1 sub-melon & $\begin{array}{l}\text { Davies } 1984,120,139-40 \text {, } \\
\text { fig 7P; } \\
\text { Salisbury Museum }\end{array}$ \\
\hline $\begin{array}{l}\text { Harnham } \\
\text { Hill }\end{array}$ & Wilts & 6th $\mathrm{c}$ & $\begin{array}{l}\text { One large pale melon and } \\
2 \text { smaller darker }\end{array}$ & $\begin{array}{l}\text { Meaney 1964, 268-9; } \\
\text { BM } 53 \text { 12-14 }\end{array}$ \\
\hline $\begin{array}{l}\text { Peters- } \\
\text { finger }\end{array}$ & Wilts & 6th $\mathrm{c}$ & $\begin{array}{l}\text { Grave } 57: \text { large light } \\
\text { greenish melon bead }\end{array}$ & $\begin{array}{l}\text { Leeds and Shortt } 1953 \text {, } \\
37, \text { no. } 150 \mathrm{~g}, \mathrm{pl} \text { IV; } \\
\text { Salisbury Museum }\end{array}$ \\
\hline $\begin{array}{l}\text { Winter- } \\
\text { bourne } \\
\text { Gunner }\end{array}$ & Wilts & 6th $\mathrm{c}$ & $\begin{array}{l}\text { Grave 9: large with } \\
\text { Style I applied saucer } \\
\text { brooch pair }\end{array}$ & $\begin{array}{l}\text { Musty and Stratton 1964, } \\
\text { 93, fig 8.IX.e; } \\
\text { Salisbury Museum }\end{array}$ \\
\hline $\begin{array}{l}\text { Broadway } \\
\text { Hill }\end{array}$ & Worcs & $c .500$ & $\begin{array}{l}\text { Grave 1: flat greenish } \\
\text { 6-lobed 'melon' with cast } \\
\text { saucer brooch pair }\end{array}$ & Cook 1958,84 , fig 4.7 \\
\hline \multirow[t]{2}{*}{$\begin{array}{l}\text { West } \\
\text { Heslerton }\end{array}$} & Yorks & $\begin{array}{l}\text { prob } \\
\text { 6th c }\end{array}$ & Grave 503: 1 bead & $\begin{array}{l}\text { Publication in } \\
\text { preparation (D Powlesland) }\end{array}$ \\
\hline & i & Tral & $\begin{array}{l}\text { LUCENT SMOKEY YELLOW } \\
\text { MELON AND SUB-MELON }\end{array}$ & $\begin{array}{l}\text { BEADS } \\
\text { FORMS) }\end{array}$ \\
\hline Kempston & Beds & 6th c & $\begin{array}{l}\text { Necklace: several small } \\
\text { annular beads }\end{array}$ & $\begin{array}{l}\text { Fitch 1864; } \\
\text { BM } 916-2454\end{array}$ \\
\hline $\begin{array}{l}\text { Little } \\
\text { Wilbraham }\end{array}$ & Cambs & $\begin{array}{l}\text { prob } \\
\text { 6th c }\end{array}$ & $\begin{array}{l}\text { Unassociated group: } \\
1 \text { medium annular; } \\
\text { annular with pair of } \\
\text { gilt brooches }\end{array}$ & $\begin{array}{l}\text { Neville 1852, } \\
\text { illustrated; } \\
\text { Lethbridge and Carter 1926; } \\
\text { Cambridge Museum }\end{array}$ \\
\hline Mucking II & Essex & $\begin{array}{l}6 \text { th } \mathrm{c} \\
5 \text { th } \mathrm{c}\end{array}$ & $\begin{array}{l}\text { Grave 878: } 1 \text { large } \\
\text { annular; } \\
\text { Grave 989: } 1 \text { large } \\
\text { annular }\end{array}$ & $\begin{array}{l}\text { Hirst and Clark forthcoming; } \\
\text { BM }\end{array}$ \\
\hline $\begin{array}{l}\text { Bourton- } \\
\text { on-the- } \\
\text { Water }\end{array}$ & Glos & & $\begin{array}{l}\text { Unstratified medium } \\
\text { annular }\end{array}$ & $\begin{array}{l}\text { Meaney 1964, 93; } \\
\text { Cheltenham Museum }\end{array}$ \\
\hline
\end{tabular}




\begin{tabular}{|c|c|c|c|c|}
\hline Andover & Hants & 6th $c$ & $\begin{array}{l}\text { Grave 50: } 2 \text { very pale olive } \\
\text { brown, } 1 \text { barrel and } 1 \\
\text { annular with small-long } \\
\text { brooch and Quoit Brooch } \\
\text { Style buckle }\end{array}$ & $\begin{array}{l}\text { Cook and Dacre } 1985,38, \\
\text { fig } 63.9 \text { and } 21 ; \\
\text { Hampshire Museums }\end{array}$ \\
\hline Droxford & Hants & $\begin{array}{l}\text { late } \\
5 \text { th- } \\
6 \text { th c }\end{array}$ & $\begin{array}{l}\text { One medium annular } \\
\text { (class XIII) }\end{array}$ & $\begin{array}{l}\text { Aldsworth } 1978,138 \text { and } \\
173 \text {, fig } 36.70 ; \\
\text { BM }\end{array}$ \\
\hline $\begin{array}{l}\text { Canterbury, } \\
\text { Cakebread } \\
\text { Robey V }\end{array}$ & Kent & $c .400$ & $\begin{array}{l}\text { Multiple burial: } \\
\text { thick annular }\end{array}$ & $\begin{array}{l}\text { Information } T \text { Tatton-Brown } \\
\text { and } P \text { Garrard }\end{array}$ \\
\hline $\begin{array}{l}\text { Dover, } \\
\text { Buckland }\end{array}$ & Kent & $\begin{array}{l}650- \\
675\end{array}$ & Grave 157: disc (B22) & $\begin{array}{l}\text { Evison 1987, 73, 80, 250; } \\
\text { fig } 61.157 .1 \mathrm{~b} ; \\
\text { colour pl III (B22); } \\
\text { BM }\end{array}$ \\
\hline $\begin{array}{l}\text { Folkestone } \\
\text { III }\end{array}$ & Kent & $\begin{array}{l}\text { prob } \\
\text { 6th c }\end{array}$ & No. 27: cylinder & $\begin{array}{l}\text { Meaney } 1964,120-1 \\
\text { Folkestone Museum }\end{array}$ \\
\hline Kingston & Kent & $\begin{array}{l}\text { 6th- } \\
7 \text { th c }\end{array}$ & Small annular & $\begin{array}{l}\text { Faussett } 1856,35-94 \\
\text { Liverpool Museum }\end{array}$ \\
\hline $\begin{array}{l}\text { Lyminge } \\
\text { II }\end{array}$ & Kent & $\begin{array}{l}\text { first } \\
\text { half } \\
\text { 6th c }\end{array}$ & $\begin{array}{l}\text { Grave 16: small annulars } \\
\text { with Hahnheim-type radiate } \\
\text { brooch, button brooch, } \\
\text { gold D bracteate, etc; } \\
\text { Grave } 39: \text { small annulars } \\
\text { with small square-headed } \\
\text { and cast saucer brooch pairs }\end{array}$ & $\begin{array}{l}\text { Warhurst } 1955,15 \text { and } 27 \text {, } \\
\text { pl VII.b.2; } \\
\text { Maidstone Museum }\end{array}$ \\
\hline Stowting & Kent & $\begin{array}{l}\text { prob } \\
\text { 6th c }\end{array}$ & $\begin{array}{l}\text { One medium and } 1 \text { small } \\
\text { annular }\end{array}$ & $\begin{array}{l}\text { Meaney } 1964,137-8 \\
\text { Maidstone Museum }\end{array}$ \\
\hline Laceby & Lincs & $\begin{array}{l}\text { prob } \\
\text { 6th- } \\
7 \text { th c }\end{array}$ & $\begin{array}{l}\text { Medium and large } \\
\text { annulars }\end{array}$ & $\begin{array}{l}\text { Meaney 1964, 157; } \\
\text { Lincoln Museum }\end{array}$ \\
\hline Ruskington & Lincs & $\begin{array}{l}\text { prob } \\
\text { 6th- } \\
7 \text { th c }\end{array}$ & One cylinder & $\begin{array}{l}\text { Meaney } 1964,161-2 \\
\text { Lincoln Museum }\end{array}$ \\
\hline Brooke & Norfolk & $\begin{array}{l}\text { prob } \\
\text { 6th } c\end{array}$ & One cylinder & $\begin{array}{l}\text { Meaney 1964, 170; } \\
\text { BM } 186669\end{array}$ \\
\hline Kenninghall & Norfolk & $\begin{array}{l}\text { prob } \\
6 \text { th- } \\
7 \text { th c }\end{array}$ & $\begin{array}{l}\text { Four annulars and } \\
1 \text { cylinder }\end{array}$ & $\begin{array}{l}\text { Manning } 1872 a, 292 \text {, and } \\
1872 b ; \\
\text { BM } 837-231\end{array}$ \\
\hline Camerton & Somerset & $\begin{array}{l}\text { prob } \\
7 \text { th } c\end{array}$ & Grave 79: small annular & $\begin{array}{l}\text { Horne } 1933,46 \text { and } 57 ; \\
\text { Taunton Museum }\end{array}$ \\
\hline $\begin{array}{l}\text { Bury St } \\
\text { Edmunds, } \\
\text { Westgarth }\end{array}$ & Suffolk & $\begin{array}{l}\text { prob } \\
\text { 6th c }\end{array}$ & $\begin{array}{l}\text { Grave 44: slightly } \\
\text { pentagonal cylinder }\end{array}$ & $\begin{array}{l}\text { West } 1988,31 \text {, } \\
\text { fig } 72 \mathrm{Al} \text {; } \\
\text { Bury St Edmunds Museum }\end{array}$ \\
\hline
\end{tabular}




\begin{tabular}{|c|c|c|c|c|}
\hline $\begin{array}{l}\text { Holywell } \\
\text { Row }\end{array}$ & Suffolk & $\begin{array}{l}5 \text { th- } \\
7 \text { th c }\end{array}$ & One globular & $\begin{array}{l}\text { Lethbridge 1931, 1-46; } \\
\text { Cambridge Museum }\end{array}$ \\
\hline $\begin{array}{l}\text { Mitchell's } \\
\text { Hill }\end{array}$ & Suffolk & $\begin{array}{l}\text { prob } \\
\text { 6th } c\end{array}$ & $\begin{array}{l}\text { One globular, } 1 \text { annular } \\
\text { and } 1 \text { square-sectioned } \\
\text { cylinder }\end{array}$ & $\begin{array}{l}\text { Meaney 1964,231; } \\
\text { Bury St Edmunds Museum }\end{array}$ \\
\hline Guildown & Surrey & $\begin{array}{l}\text { 5th- } \\
\text { 6th c }\end{array}$ & $\begin{array}{l}\text { Grave 77: } 1 \text { annular; } \\
\text { Grave 78: } 1 \text { annular; } \\
\text { Grave 206: } 1 \text { annular }\end{array}$ & $\begin{array}{l}\text { Lowther 1931, 11-12,35, } \\
36 \text { and 44, pl IX.2, } 7 \text { and 1; } \\
\text { Guildford Museum }\end{array}$ \\
\hline $\begin{array}{l}\text { Stretton- } \\
\text { on-Fosse }\end{array}$ & Warks & & Large annular & $\begin{array}{l}\text { Gelling 1992, 32, 40-1, } \\
\text { fig } 21 \text {; } \\
\text { Warwick Museum }\end{array}$ \\
\hline Wasperton & Warks & 6th $c$ & $\begin{array}{l}\text { With saucer brooches; } \\
\text { and possibly others }\end{array}$ & $\begin{array}{l}\text { Publication in } \\
\text { preparation (M Carver); } \\
\text { Warwick Museum }\end{array}$ \\
\hline Charlton & Wilts & 6th $c$ & Burial 16: 1 annular & $\begin{array}{l}\text { Davies } 1984,120,139-40 \text {, } \\
\text { fig } 8 \text { B; } \\
\text { Salisbury Museum }\end{array}$ \\
\hline $\begin{array}{l}\text { Harnham } \\
\text { Hill }\end{array}$ & Wilts & $\begin{array}{l}\text { prob } \\
6 \text { th- } \\
7 \text { th c }\end{array}$ & $\begin{array}{l}\text { Annulars of } 5 \text { different } \\
\text { sizes }\end{array}$ & $\begin{array}{l}\text { Akerman 1853; } \\
\text { Jackson 1854; } \\
\text { BM } 5312-1495\end{array}$ \\
\hline $\begin{array}{l}\text { Peters- } \\
\text { finger }\end{array}$ & Wilts & $\begin{array}{l}\text { prob } \\
6 \text { th c }\end{array}$ & $\begin{array}{l}\text { Graves } 50 \text { and } 57: \\
\text { small and medium } \\
\text { annulars }\end{array}$ & $\begin{array}{l}\text { Leeds and Shortt 1953, } \\
33 \text { (141b) and } 37(150 j), \\
\text { pl IV; } \\
\text { Salisbury Museum }\end{array}$ \\
\hline $\begin{array}{l}\text { Winter- } \\
\text { bourne } \\
\text { Gunner }\end{array}$ & Wilts & 6th $c$ & $\begin{array}{l}\text { Grave 7: } 1 \text { annular } \\
\text { with small-long brooch } \\
\text { and perforated spoon, etc }\end{array}$ & $\begin{array}{l}\text { Musty and Stratton 1964, } \\
\text { 93, fig 6.VII.c.3; } \\
\text { Salisbury Museum }\end{array}$ \\
\hline $\begin{array}{l}\text { Upton } \\
\text { Snodsbury }\end{array}$ & Worcs & 6th $c$ & Cylinder (Plate 1) & $\begin{array}{l}\text { Meaney 1964,281; } \\
\text { Worcester Museum }\end{array}$ \\
\hline $\mathrm{v}$ & SMOKEY & YELLOW & TRANSLUCENT MELON AND & SUB-MELON BEADS \\
\hline $\begin{array}{l}\text { Linton } \\
\text { Heath }\end{array}$ & Cambs & $\begin{array}{l}\text { 6th- } \\
7 \text { th } c\end{array}$ & Two examples & $\begin{array}{l}\text { Neville 1854; } \\
\text { Cambridge Museum } \\
481598\end{array}$ \\
\hline Mucking II & Essex & 6th $c$ & Grave 845: 1 example & $\begin{array}{l}\text { Hirst and Clark forthcoming; } \\
\text { BM }\end{array}$ \\
\hline Lechlade & Glos & 6th $c$ & $\begin{array}{l}\text { Grave } 180: 5 \text { brown-yellow } \\
\text { beads with Style I mount } \\
\text { (Plate 1) }\end{array}$ & $\begin{array}{l}\text { Boyle et al forthcoming; } \\
\text { Cirencester Museum }\end{array}$ \\
\hline Droxford & Hants & $\begin{array}{l}\text { 5th- } \\
\text { 6th c }\end{array}$ & $\begin{array}{l}\text { Graves } 20,21 \text { and } 32 \text { : } \\
\text { (class IIIa) }\end{array}$ & $\begin{array}{l}\text { Aldsworth } 1978,124 \text { and } \\
132 \text {, figs } 24.9,25.12 \text { and } 28.8 \text {; } \\
\text { Winchester Museum }\end{array}$ \\
\hline Sarre & Kent & $\begin{array}{l}\text { 5th- } \\
7 \text { th } c\end{array}$ & $\begin{array}{l}\text { Marked 'string } \\
\text { of } 92 \text { beads' }\end{array}$ & $\begin{array}{l}\text { Meaney 1964, 135-6; } \\
\text { BM 93 6-1 } 218\end{array}$ \\
\hline
\end{tabular}




\begin{tabular}{|c|c|c|c|c|}
\hline Kenninghall & Norfolk & $\begin{array}{l}5 \text { th- } \\
7 \text { th } c\end{array}$ & Two examples & $\begin{array}{l}\text { Manning 1872a, 292, and } \\
1872 \mathrm{~b} \text {; } \\
\text { BM }\end{array}$ \\
\hline Duston & Northants & $\begin{array}{l}\text { 5th- } \\
7 \text { th c }\end{array}$ & $\begin{array}{l}\text { One with wide flattish } \\
\text { lobes }\end{array}$ & $\begin{array}{l}\text { George 1903; } \\
\text { Northampton Museum }\end{array}$ \\
\hline $\begin{array}{l}\text { Holme } \\
\text { Pierrepont }\end{array}$ & Notts & 6th $c$ & $\begin{array}{l}\text { One from a chevron- and } \\
\text { stamp-ornamented pot }\end{array}$ & $\begin{array}{l}\text { Myres 1977, } 315 \\
\text { (Corpus no. 2093), fig 303; } \\
\text { BM 1931 3-13 }\end{array}$ \\
\hline $\begin{array}{l}\text { Holywell } \\
\text { Row }\end{array}$ & Suffolk & $\begin{array}{l}\text { 5th- } \\
\text { 7th c }\end{array}$ & & $\begin{array}{l}\text { Lethbridge 1931, 1-46; } \\
\text { Cambridge Museum }\end{array}$ \\
\hline Ipswich & Suffolk & $\begin{array}{l}\text { 6th-. } \\
7 \text { th c }\end{array}$ & Several & $\begin{array}{l}\text { Layard 1907, pl XXXI, and } \\
\text { 1909; } \\
\text { Ipswich Museum }\end{array}$ \\
\hline West Stow & Suffolk & $\begin{array}{l}5 \text { th- } \\
7 \text { th c }\end{array}$ & Cemetery & $\begin{array}{l}\text { West 1985, 74; } \\
\text { Ashmolean Museum } \\
\text { 1909.424 }\end{array}$ \\
\hline Guildown & Surrey & 6th $c$ & $\begin{array}{l}\text { Grave } 78: 2 \text { beads; } \\
\text { Grave } 185\end{array}$ & $\begin{array}{l}\text { Lowther 1931, 11-12, } \\
36 \text { and } 43, \mathrm{pl} \text { IX.3 and 7; } \\
\text { Guildford Museum }\end{array}$ \\
\hline Highdown & Sussex & $\begin{array}{l}\text { 5th- } \\
\text { 6th c }\end{array}$ & Bracelet: 2 examples & $\begin{array}{l}\text { Read } 1895 \text { and } 1896 ; \\
\text { Welch 1983, 82-6; } \\
\text { Worthing Museum }\end{array}$ \\
\hline Wasperton & Warks & 6th $c$ & At least two & $\begin{array}{l}\text { Publication in } \\
\text { preparation (M Carver); } \\
\text { Warwick Museum }\end{array}$ \\
\hline Avebury & Wilts & $\begin{array}{l}\text { prob } \\
\text { 6th c }\end{array}$ & Grubenhaus & $\begin{array}{l}\text { Publication in } \\
\text { preparation }\end{array}$ \\
\hline $\begin{array}{l}\text { Harnham } \\
\text { Hill }\end{array}$ & Wilts & $\begin{array}{l}\text { 6th- } \\
7 \text { th } c\end{array}$ & $\begin{array}{l}\text { One large badly made } \\
\text { and } 1 \text { smaller }\end{array}$ & $\begin{array}{l}\text { Akerman 1853; } \\
\text { Jackson 1854; } \\
\text { BM } 53 \text { 12-14 } 95\end{array}$ \\
\hline $\begin{array}{l}\text { Peters- } \\
\text { finger }\end{array}$ & Wilts & $\begin{array}{l}\text { prob } \\
\text { 6th c }\end{array}$ & Grave $57: 2$ beads & $\begin{array}{l}\text { Leeds and Shortt 1953, } \\
37 \text { (150f), pl IV; } \\
\text { Salisbury Museum }\end{array}$ \\
\hline $\begin{array}{l}\text { Londes- } \\
\text { borough }\end{array}$ & Yorks & $\begin{array}{l}\text { mid- } \\
\text { 6th c }\end{array}$ & $\begin{array}{l}\text { Grave 7: with } \\
\text { Áberg Group IVa } \\
\text { cruciform brooch, etc }\end{array}$ & $\begin{array}{l}\text { Swanton 1964, 275, fig 8.6; } \\
\text { Cramp and Miket 1982, } \\
\text { fig 5.4.2; } \\
\text { Newcastle Museum }\end{array}$ \\
\hline
\end{tabular}

\section{POLYCHROME}

\section{vi Decorated translucent beads:}

(a) ON A LIGHT BLUE-GREEN GROUND

$\begin{array}{lllll}\text { Chamber- } & \text { Beds } & \text { mid- } & \text { Grave 8: light olive green } & \text { Hyslop 1963, 173, } \\ \text { lain's } & & \text { late } & \text { annular with angular wave } & \text { fig 8c; } \\ \text { Barn II } & & \text { 7th c } & & \text { Luton Museum }\end{array}$




\begin{tabular}{|c|c|c|c|c|}
\hline Wallingford & Berks & $\begin{array}{l}\text { 5th- } \\
6 \text { th c }\end{array}$ & $\begin{array}{l}\text { Grave 11: small translucent } \\
\text { annular with } 2 \text { red bands } \\
\text { around, perhaps related to } \\
\text { group vii below, with } \\
2 \text { small-long brooches }\end{array}$ & $\begin{array}{l}\text { Leeds 1938, } 97, \\
\text { pl III; } \\
\text { Ashmolean Museum }\end{array}$ \\
\hline $\begin{array}{l}\text { Newport } \\
\text { Pagnell }\end{array}$ & Bucks & $\begin{array}{l}\text { 6th- } \\
7 \text { th c }\end{array}$ & $\begin{array}{l}\text { Several annulars decorated } \\
\text { with terracotta or yellow }\end{array}$ & $\begin{array}{l}\text { Meaney } 1964,58 \\
\text { Aylesbury Museum }\end{array}$ \\
\hline $\begin{array}{l}\text { Hasling- } \\
\text { field }\end{array}$ & Cambs & $\begin{array}{l}\text { prob } \\
6 \text { th- } \\
7 \text { th c }\end{array}$ & $\begin{array}{l}\text { One annular with crossed } \\
\text { waves and dots in } \\
\text { yellow and another } \\
\text { similar, but filling lost }\end{array}$ & $\begin{array}{l}\text { Fox 1923, 255-9; } \\
\text { Ashmolean Museum }\end{array}$ \\
\hline $\begin{array}{l}\text { Linton } \\
\text { Heath }\end{array}$ & Cambs & $\begin{array}{l}\text { prob } \\
\text { 6th c }\end{array}$ & $\begin{array}{l}\text { One green with yellow } \\
\text { waves, } 2 \text { beads or ?double } \\
\text { with } 2 \text { small-long brooches }\end{array}$ & $\begin{array}{l}\text { Neville 1854; } \\
\text { Cambridge Museum }\end{array}$ \\
\hline $\begin{array}{l}\text { Great } \\
\text { Chesterford }\end{array}$ & Essex & $\begin{array}{l}\text { late } \\
5 \text { th- } \\
6 \text { th c }\end{array}$ & $\begin{array}{l}\text { Grave } 45: 2 \text { with single } \\
\text { yellow wave (D52) with } \\
\text { with small-long brooch } \\
\text { pair }\end{array}$ & $\begin{array}{l}\text { Evison } 1994,6,12, \\
18,46 \text { and } 98, \\
\text { figs } 6 \text { and } 29.45 .31 \\
\text { BM }\end{array}$ \\
\hline Mucking II & Essex & 6th c & $\begin{array}{l}\text { Grave } 615: 2 \text {-lobed beads } \\
\text { with } 5 \text { ribs and } 6 \text { ribs } \\
\text { respectively in translucent } \\
\text { pale green glass with red and } \\
\text { yellow spirals with cast } \\
\text { saucer brooch pair and } \\
\text { amber beads, etc; } \\
\text { Grave } 845 \text { (Plate 1) with } \\
\text { disc brooches, etc }\end{array}$ & $\begin{array}{l}\text { Hirst and Clark forthcoming; } \\
\text { BM }\end{array}$ \\
\hline $\begin{array}{l}\text { Milton } \\
\text { Regis }\end{array}$ & Kent & 7th $\mathrm{c}$ & $\begin{array}{l}\text { Large with white vague } \\
\text { trails, used as pendant }\end{array}$ & Rigold and Webster 1970,4 \\
\hline Cleatham & Lincs & $\begin{array}{l}\text { 5th- } \\
\text { 6th c }\end{array}$ & $\begin{array}{l}\text { Grave } 34 \text { : annular } \\
\text { with white wave }\end{array}$ & $\begin{array}{l}\text { Publication in } \\
\text { preparation (K Leahy); } \\
\text { Scunthorpe Museum }\end{array}$ \\
\hline Fonaby & Lincs & $\begin{array}{l}\text { 6th- } \\
7 \text { th c }\end{array}$ & $\begin{array}{l}\text { Unstratified group } 6(7) \text { : } \\
\text { rough globular with white } \\
\text { crossed waves and central } \\
\text { band }\end{array}$ & $\begin{array}{l}\text { Cook 1981, 48, fig 19.7; } \\
\text { Scunthorpe Museum }\end{array}$ \\
\hline Brooke & Norfolk & $\begin{array}{l}\text { prob } \\
\text { 6th c }\end{array}$ & $\begin{array}{l}\text { Small cylinder with } \\
\text { egg-shaped yellow blobs } \\
\text { and small yellow-green } \\
\text { globular with red crossed wave }\end{array}$ & $\begin{array}{l}\text { Meaney 1964, 170; } \\
\text { BM } 186669\end{array}$ \\
\hline $\begin{array}{l}\text { East } \\
\text { Anglia }\end{array}$ & $\begin{array}{l}\text { prob } \\
\text { Norfolk }\end{array}$ & & $\begin{array}{l}\text { Small tall bead with } \\
\text { angular yellow wave }\end{array}$ & $\begin{array}{l}\text { Fitch Collection, } \\
\text { Norwich Museum }\end{array}$ \\
\hline $\begin{array}{l}\text { Cadbury- } \\
\text { Congresbury }\end{array}$ & Somerset & $\begin{array}{l}5 \text { th- } \\
6 \text { th c }\end{array}$ & $\begin{array}{l}\text { Two thick annulars, } 1 \text { with } \\
\text { yellow wave overlying } \\
\text { white girth bands and } 1 \text { with } \\
\text { colour lost (GO123 and } \\
\text { PO297) }\end{array}$ & $\begin{array}{l}\text { Guido 1992, } \\
\text { fig } 99 . \mathrm{GO} 123\end{array}$ \\
\hline
\end{tabular}




\begin{tabular}{|c|c|c|c|c|}
\hline $\begin{array}{l}\text { Mitchell's } \\
\text { Hill }\end{array}$ & Suffolk & $\begin{array}{l}\text { prob } \\
\text { 6th- } \\
7 \text { th c }\end{array}$ & $\begin{array}{l}\text { Atypical big biconical with } \\
\text { central white band over } \\
\text { large herringbone design } \\
\text { in opaque yellow }\end{array}$ & $\begin{array}{l}\text { Meaney } 1964,231 \\
\text { Bury St Edmunds Museum }\end{array}$ \\
\hline West Stow & Suffolk & $\begin{array}{l}5 \text { th- } \\
7 \text { th c }\end{array}$ & $\begin{array}{l}\text { Cemetery: } 8 \text { white flower- } \\
\text { like trails on } 1 \text { side and } \\
6 \text { on the other, ?sword- } \\
\text { bead or spindle whorl }\end{array}$ & $\begin{array}{l}\text { West 1985, } 75(014) \text {, } \\
\text { fig 276.26; } \\
\text { Ashmolean Museum } \\
1909.419\end{array}$ \\
\hline $\begin{array}{l}\text { Castle } \\
\text { Bromwich }\end{array}$ & Warks & & $\begin{array}{l}\text { Large annular with } \\
\text { opaque yellow random } \\
\text { lines and spiral }\end{array}$ & Birmingham Museum \\
\hline $\begin{array}{l}\text { Harnham } \\
\text { Hill }\end{array}$ & Wilts & $\begin{array}{l}\text { prob } \\
\text { 6th } c\end{array}$ & $\begin{array}{l}\text { Annular with crossed red } \\
\text { and yellow waves }\end{array}$ & $\begin{array}{l}\text { Akerman 1853; } \\
\text { Jackson 1854; } \\
\text { Salisbury Museum } \\
\text { Catalogue 1864, p } 69\end{array}$ \\
\hline Pewsey & Wilts & $\begin{array}{l}\text { prob } \\
\text { 6th c }\end{array}$ & $\begin{array}{l}\text { Grave 85: annular with } \\
\text { reddish crossed waves and } \\
\text { spots }\end{array}$ & $\begin{array}{l}\text { Publication in } \\
\text { preparation (K Annable); } \\
\text { Devizes Museum }\end{array}$ \\
\hline
\end{tabular}

\begin{tabular}{|c|c|c|c|c|}
\hline $\begin{array}{l}\text { Colchester, } \\
\text { Guildford } \\
\text { Road (Site K) }\end{array}$ & Essex & $\begin{array}{l}\text { first } \\
\text { half } \\
\text { 5th c }\end{array}$ & $\begin{array}{l}\text { Atypical thick annular with } \\
\text { white bands round top and } \\
\text { bottom and angular surface } \\
\text { wave encloses terracotta } \\
\text { spots (Plate 1) with Witmarsun } \\
\text { Type cruciform brooch, etc }\end{array}$ & $\begin{array}{l}\text { Crummy } 1981,12 \text {, } \\
\text { fig } 13.8 ; \\
\text { Colchester Museum }\end{array}$ \\
\hline Ozingell & Kent & $\begin{array}{l}\text { prob } \\
\text { 6th- } \\
7 \text { th c }\end{array}$ & $\begin{array}{l}\text { One annular with sharp } \\
\text { yellow waves and } \\
1 \text { tall ?biconical with } \\
\text { opaque yellow feathering }\end{array}$ & $\begin{array}{l}\text { Smith } 1854,5 \text {, } \\
\text { pl V.6 and 8; ex Rolfe } \\
\text { Collection, Liverpool } \\
\text { Museum M7281 }\end{array}$ \\
\hline $\begin{array}{l}\text { Holywell } \\
\text { Row }\end{array}$ & Suffolk & $\begin{array}{l}\text { mid- } \\
\text { 6th } c\end{array}$ & $\begin{array}{l}\text { Grave 58: double bead with } \\
\text { opaque yellow waves on } \\
\text { each part (Plate 1) with } \\
\text { Aberg Group IV cruciform } \\
\text { brooch }\end{array}$ & $\begin{array}{l}\text { Lethbridge 1931, 32, } \\
\text { fig 15; } \\
\text { Cambridge Museum }\end{array}$ \\
\hline Guildown & Surrey & $\begin{array}{l}5 \text { th- } \\
6 \text { th } \mathrm{c}\end{array}$ & $\begin{array}{l}\text { Grave } 78 \text { necklace: } \\
\text { annular with blue lines } \\
\text { round hole at one end }\end{array}$ & $\begin{array}{l}\text { Lowther 1931, 12, 36, } \\
\text { pl IX.7; } \\
\text { Guildford Museum }\end{array}$ \\
\hline Wasperton & Warks & 6th $c$ & $\begin{array}{l}\text { Annular with white } \\
\text { dots and ?some lines } \\
\text { with cruciform brooch }\end{array}$ & $\begin{array}{l}\text { Publication in } \\
\text { preparation (M Carver); } \\
\text { Warwick Museum }\end{array}$ \\
\hline
\end{tabular}




\section{vii LIGHT-COLOURED TRANSLUCENT ANNULAR OR GLOBULAR BEADS \\ WITH COLOURED PARALLEL OR SPIRAL BANDS \\ (EXCLUDING LOBED FORMS)}

$\begin{array}{lllll}\begin{array}{l}\text { Milton } \\ \text { Keynes }\end{array} & \text { Bucks } & \begin{array}{l}\text { prob } \\ \text { 5th c }\end{array} & \begin{array}{l}\text { Bradwell Roman villa: } \\ \text { blue-green annular with } \\ \text { opaque white and terracotta } \\ \text { bands (Plate 1) (see } \\ \text { group viii below) }\end{array} & \begin{array}{l}\text { Price 1975 in Green 1975; } \\ \text { information from }\end{array} \\ \text { Dr J Price }\end{array}$

$\begin{array}{lllll}\text { Highdown } & \text { Sussex } & \begin{array}{l}\text { 5th- } \\ \text { 6th c }\end{array} & \begin{array}{l}\text { Pale smokey yellow } \\ \text { with white bands }\end{array} & \begin{array}{l}\text { Read 1895 and 1896; } \\ \text { Welch 1983, 82-6; } \\ \text { Worthing Museum }\end{array} \\ \text { Ratley } & \text { Warks } & & \begin{array}{l}\text { Unstratified: annular } \\ \text { translucent glass with } \\ \text { opaque white waves } \\ \text { overlaid by red bands }\end{array} & \begin{array}{l}\text { Information from } \\ \text { F Radcliffe }\end{array} \\ \text { Wasperton } & \text { Warks } & \text { 6th c } & \begin{array}{l}\text { One globular translucent } \\ \text { yellow with yellowish lines } \\ \text { with cruciform brooch; }\end{array} & \begin{array}{l}\text { Publication in } \\ \text { preparation (M Carver); } \\ \text { Warwick Museum }\end{array}\end{array}$


6th c One annular yellowish-green

semi-translucent with 3

white lines round, with

cruciform brooch

Charlton Wilts 6th c $\begin{aligned} & \begin{array}{l}\text { Burial 24: blue-green with } \\ \text { white wave overlaid by } \\ 3 \text { dark crimson bands }\end{array} \\ & \text { viii LIGHT YELLOWISH-GREEN BEADS WITH DECORATION }\end{aligned}$
$\begin{aligned} & \text { (MOSTLY SQUARE-SECTIONED FORM) (see Map 1) } \\ & \text { Salisbury Museum }\end{aligned}$

Milton

Bucks 4th-

Bradwell Roman villa:

Price 1975, 14, fig 33.19,

Keynes

5th $\mathrm{c}$

square section with red

lines and yellow dots;

in Green 1975;

broken square section

information from

with red lines; folded

Dr J Price

annular translucent with

white and terracotta bands

(see group vii above)

$\begin{array}{lll}\text { Newport } & \text { Bucks } & \text { 6th- } \\ \text { Pagnell } & & \text { 7th c } \\ \text { Waterbeach } & \text { Cambs } & \text { 5th c }\end{array}$

Grubenhaus with some

Roman finds as well: 2 long square-sectioned with red and green streaks

$\begin{array}{llll}\text { Chelmsford } & \text { Essex } & \text { 4th c } & \begin{array}{l}\text { Very similar to Milton } \\ \text { Keynes: Bradwell villa } \\ \text { bead (group viii) above }\end{array} \\ \text { Colchester } & \text { Essex } & \begin{array}{l}\text { early } \\ \text { 5th c }\end{array} & \begin{array}{l}\text { Thick annular white opaque } \\ \text { bands round top and bottom } \\ \text { and angular wave enclosing } \\ \text { terracotta spots, with } \\ \text { brooches, etc }\end{array} \\ \text { Mucking II } & \text { Essex } & \begin{array}{l}\text { 5th- } \\ \text { 6th c }\end{array} & \begin{array}{l}\text { Grave 845: large disc bead } \\ \text { in pale green with broad } \\ \text { opaque white band round } \\ \text { centre overlaid by opaque } \\ \text { red crossing trail; with disc } \\ \text { brooch pair (Plate 1) }\end{array}\end{array}$

Meaney 1964, 58;

Aylesbury Museum

Lethbridge 1927

Information from

P J Drury

Information from

P Crummy

Hirst and Clark forthcoming; BM

\section{ix LIGHT-COLOURED DECORATED LOBED OR HORNED BEADS}

$\begin{array}{llll}\text { Abingdon } & \text { Berks } & \begin{array}{l}\text { ?late } \\ \text { 5th } \mathrm{c}\end{array} & \begin{array}{l}\text { Grave B122: white ?trans- } \\ \text { lucent with 4 lobes each } \\ \text { with 3 reddish bands }\end{array} \\ \text { Harwell } & \text { Berks } & \begin{array}{l}\text { 5th- } \\ \text { 6th } \mathrm{c}\end{array} & \begin{array}{l}\text { Grave 4: as Abingdon } \\ \text { above, 3 greenish-blue } \\ \text { (2 with red and 1 with } \\ \text { ?dark green) (Plate 1) with } \\ \text { disc brooch pair }\end{array} \\ & & \end{array}$

Leeds and Bradford 1942, 102-3, pl VIIIC;

Ashmolean Museum

Kirk and Marshall 1956, 27, 33-4, fig 10g-i, pl IB;

Ashmolean Museum 


\begin{tabular}{|c|c|c|c|c|}
\hline Wallingford & Berks & $\begin{array}{l}5 \text { th- } \\
\text { 6th c }\end{array}$ & $\begin{array}{l}\text { Grave 15: blue-green } \\
\text { translucent } 4 \text { lobes with red } \\
\text { tips (Plate 1) (see Guildown } \\
\text { below) with applied saucer } \\
\text { brooch (floriate/anchor cross) } \\
\text { pair and } 2 \text { quoit brooches }\end{array}$ & $\begin{array}{l}\text { Leeds 1938, 98-9, pl VII; } \\
\text { Welch 1975; } \\
\text { Evison 1978; Böhme } \\
\text { 1986, 545-7 and 571; } \\
\text { Ashmolean Museum }\end{array}$ \\
\hline \multirow[t]{2}{*}{ Mucking II } & Essex & $\begin{array}{l}\text { first } \\
\text { half } \\
5 \text { th c }\end{array}$ & $\begin{array}{l}\text { Grave 989: green-yellow } \\
\text { sub-melon with } 2 \text { or } 3 \text { red } \\
\text { spiral trails overlaid } \\
\text { by yellow spot with a } \\
\text { Glaston-Mucking bow } \\
\text { brooch, etc; }\end{array}$ & $\begin{array}{l}\text { Hirst and Clark forthcoming; } \\
\text { Evison 1981, 138-9, } \\
\text { figs } 4 \text { and 5; } \\
\text { BM }\end{array}$ \\
\hline & & $\begin{array}{l}\text { 2nd } \\
\text { half } \\
5 \text { th c }\end{array}$ & $\begin{array}{l}\text { Grave 334: green-yellow } \\
\text { translucent } 6 \text {-lobed with } \\
\text { opaque red spiral, white } \\
\text { wave and yellow spots, } \\
\text { with disc brooch pair } \\
\text { and inlaid buckle }\end{array}$ & $\begin{array}{l}\text { Hirst and Clark forthcoming; } \\
\text { BM }\end{array}$ \\
\hline $\begin{array}{l}\text { Springfield } \\
\text { Lyons }\end{array}$ & Essex & $\begin{array}{l}\text { mid- } \\
\text { 6th c }\end{array}$ & $\begin{array}{l}\text { Grave 4578: light blue- } \\
\text { green, red tip to each } \\
\text { lobe }\end{array}$ & $\begin{array}{l}\text { Publication in } \\
\text { preparation (S Tyler); } \\
\text { BM }\end{array}$ \\
\hline Lechlade & Glos & 6th $c$ & $\begin{array}{l}\text { Grave } 142: 4 \text {-lobed blue- } \\
\text { green translucent with } 4 \\
\text { red bands }\end{array}$ & $\begin{array}{l}\text { Boyle et al forthcoming; } \\
\text { Cirencester Museum }\end{array}$ \\
\hline $\begin{array}{l}\text { Loveden } \\
\text { Hill }\end{array}$ & Lincs & $\begin{array}{l}\text { prob } \\
\text { 6th- } \\
7 \text { th c }\end{array}$ & $\begin{array}{l}\text { Mixed cemetery: lobed with } \\
\text { bands, very burnt }\end{array}$ & $\begin{array}{l}\text { Fennell 1964; } \\
\text { Meaney 1964, 158-9; } \\
\text { Lincoln Museum }\end{array}$ \\
\hline Guildown & Surrey & $\begin{array}{l}\text { 2nd } \\
\text { half } \\
\text { 5th c }\end{array}$ & $\begin{array}{l}\text { Grave 123: } 6 \text { lobes } \\
\text { each with red tips } \\
\text { with } 2 \text { floriate cross } \\
\text { applied saucer brooches }\end{array}$ & $\begin{array}{l}\text { Lowther 1931, 11, 39, } \\
\text { pl VIII.3; Welch 1975; } \\
\text { Guildford Museum }\end{array}$ \\
\hline Mitcham & Surrey & $\begin{array}{l}\text { prob } \\
\text { 6th c }\end{array}$ & $\begin{array}{l}\text { Grave 197: } 6 \text { lobes with } 3 \\
\text { bands }\end{array}$ & $\begin{array}{l}\text { Bidder and Morris 1959, } \\
\text { 73, 112, pl XVI.197; } \\
\text { Cambridge Museum }\end{array}$ \\
\hline
\end{tabular}




\title{
SCHEDUle 2
}

\section{'BLACK' GLASS BEADS}

\author{
MONOCHROME \\ i Small 'black' annular and globular beads \\ ii 'Black' segmented double or triple beads \\ iii 'Black' melon, sub-melon or lobed beads (see Map 2) \\ Polychrome \\ iv 'Black' annular beads with broken white girth band (see Map 3) \\ v 'Black' annular beads (see Map 4): (a) with white or yellow wave (some with \\ girth band) \\ (b) with blue wave \\ vi 'Black' annular beads (see Map 5): (a) with white zigzags \\ (b) with yellow or green zigzags \\ (c) with red zigzags \\ vii Large 'black' decorated beads: (a) plano-convex (see Map 6) \\ (b) biconvex (see Map 7) \\ viii 'Black' (or dark) globular beads with complex crossed waves, and spots or eyes \\ (and related beads) (see Map 8) \\ ix 'Black' globular beads with white or coloured crossed waves, with or without \\ spots or eyes \\ $\mathrm{x} \quad$ 'Black' beads with a few spots (mostly annular beads) \\ xi 'Black' globular beads with coloured specks ('crumb' beads) (see Map 9) \\ xii 'Black' decorated long cylinder beads \\ xiii Long fluted beads banded in 'black' and white: no schedule \\ xiv Small cylindrical dark beads with unmarvered yellow ends and central band: no \\ schedule \\ xv Large globular dark beads with several lines of coloured zigzags \\ xvi Tall drum-shaped dark beads with several lines of coloured zigzags
}

\section{MONOCHROME}

\section{i SMall 'Black' anNular and globular beads}

\begin{tabular}{lllll} 
Kempston & Beds & 6th c & $\begin{array}{l}\text { Grave 16: several annulars } \\
\text { seeming black }\end{array}$ & $\begin{array}{l}\text { Fitch 1864; } \\
\text { BM 91 6-24 53 }\end{array}$ \\
$\begin{array}{l}\text { Linton } \\
\text { Heath }\end{array}$ & Cambs & & Larger globular & Neville 1854; \\
\hline
\end{tabular}




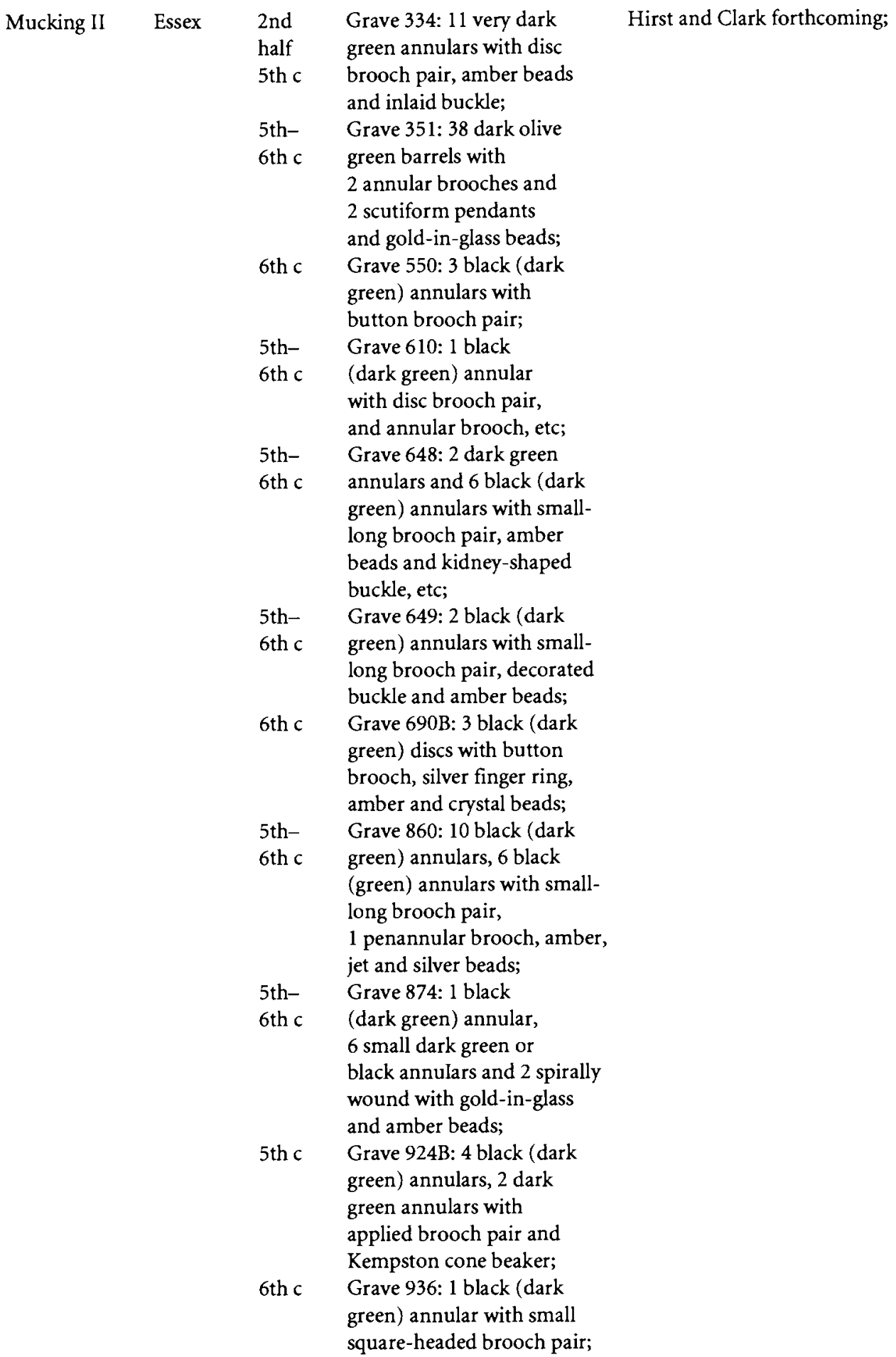




\begin{tabular}{|c|c|c|c|c|}
\hline & & $\begin{array}{l}\text { first } \\
\text { half } \\
5 \text { th c }\end{array}$ & $\begin{array}{l}\text { Grave 989: } 2 \text { black (dark } \\
\text { green) discs with Glaston- } \\
\text { Mucking and supporting- } \\
\text { arm brooches, dolphin } \\
\text { buckle loop, finger ring and } \\
\text { gold-in-glass beads }\end{array}$ & $\begin{array}{l}\text { Evison 1981, 138-9, } \\
\text { figs } 4 \text { and } 5 \text {; } \\
\text { BM }\end{array}$ \\
\hline $\begin{array}{l}\text { Springfield } \\
\text { Lyons }\end{array}$ & Essex & $\begin{array}{l}\text { 5th- } \\
6 \text { th } c\end{array}$ & $\begin{array}{l}\text { Grave 4882: numbers } \\
\text { of tiny annulars with } \\
\text { Áberg Group II cruciform } \\
\text { brooch, disc brooch pair } \\
\text { and carinated bossed pot }\end{array}$ & $\begin{array}{l}\text { Publication in } \\
\text { preparation (S Tyler); } \\
\text { BM }\end{array}$ \\
\hline Alton & Hants & 6th $c$ & Grave 9: 3 annulars (discs) & $\begin{array}{l}\text { Evison } 1988 \mathrm{a}, 73 \text {, fig } \\
25.9 .1 \mathrm{~g} ; \\
\text { Hampshire Museums }\end{array}$ \\
\hline Droxford & Hants & 6th $c$ & Rather large (class Id) & $\begin{array}{l}\text { Aldsworth } 1978,138,172 \text {, } \\
\text { fig } 36.65 ; \mathrm{BM}\end{array}$ \\
\hline $\begin{array}{l}\text { Dover, } \\
\text { Buckland }\end{array}$ & Kent & $\begin{array}{l}475- \\
525 \\
575- \\
625 \\
625- \\
650 \\
650- \\
675\end{array}$ & $\begin{array}{l}\text { Grave 20: } 2 \text { (discs) (B61); } \\
\text { Grave 30: } 1 \text { (disc) (B61); } \\
\text { Grave 60: } 2 \text { (discs) (B61); } \\
\text { Grave 62: } 1 \text { (disc) (B61); } \\
\text { Grave 157: } 1 \text { (disc) (B61) }\end{array}$ & $\begin{array}{l}\text { Evison } 1987,74 \text { and } 81 \text {, } \\
\text { figs } 12.5 \mathrm{e}, 18.4 \mathrm{~g} \text {, } \\
34.3 \mathrm{e}, 35.62 .4 \mathrm{~d} \text {, } \\
61.157 .1 \mathrm{~d} \text {, colour pl } \\
\text { III (B61); BM }\end{array}$ \\
\hline Faversham & Kent & $\begin{array}{l}\text { prob } \\
6 \text { th- } \\
7 \text { th c }\end{array}$ & Several annulars & $\begin{array}{l}\text { Meaney } 1964,118-19 \\
\text { Ashmolean Museum } \\
1909.142,143 \text { and } 155\end{array}$ \\
\hline Polhill & Kent & 7th $\mathrm{c}$ & $\begin{array}{l}\text { Grave } 102: 1 \text { medium } \\
\text { to large flat-surfaced } \\
\text { 'quoit-shaped' annular }\end{array}$ & $\begin{array}{l}\text { Hawkes and Philp 1973, } \\
\text { 207, fig 55.528 }\end{array}$ \\
\hline Brooke & Norfolk & $\begin{array}{l}\text { prob } \\
\text { 6th c }\end{array}$ & & $\begin{array}{l}\text { Meaney 1964, 170; } \\
\text { BM } 186669\end{array}$ \\
\hline Illington & Norfolk & $\begin{array}{l}\text { prob } \\
\text { 6th c }\end{array}$ & Several & $\begin{array}{l}\text { Davison et al 1993, fig 47; } \\
\text { Norwich Museum }\end{array}$ \\
\hline $\begin{array}{l}\text { Morning } \\
\text { Thorpe }\end{array}$ & Norfolk & $\begin{array}{l}\text { late } \\
5 \text { th- } \\
6 \text { th c }\end{array}$ & $\begin{array}{l}\text { Grave } 30: 1 \text { annular } \\
\text { with } 1 \text { \&berg Group III, } \\
2 \text { Group II cruciform } \\
\text { brooches, etc; } \\
\text { Grave } 90: 2 \text { annulars } \\
\text { with } 3 \text { \& berg Group II } \\
\text { cruciform brooches, etc }\end{array}$ & $\begin{array}{l}\text { Green et al } 1987, \\
42 \text { and } 60 \text {, figs } 303 . \text { Biii } \\
\text { and } 324 . \text { Axiii; } \\
\text { Norwich Museum }\end{array}$ \\
\hline Spong Hill & Norfolk & $\begin{array}{l}\text { late } \\
\text { 5th- } \\
6 \text { th c }\end{array}$ & $\begin{array}{l}\text { Grave 26: } 4 \text { 'globular' } \\
\text { with Åberg Group I } \\
\text { cruciform brooch, etc }\end{array}$ & $\begin{array}{l}\text { Hills et al } 1984 \text {, } \\
74 \text {, fig } 83.1 \text {; } \\
\text { Norfolk Arch Unit }\end{array}$ \\
\hline Guildown & Surrey & 6th $c$ & $\begin{array}{l}\text { Grave 77: } 2 \text { annulars; } \\
\text { Grave 78: } 2 \text { globulars }\end{array}$ & $\begin{array}{l}\text { Lowther 1931, 11-12, } \\
\text { 35, 36, pl IX.2 and 7; } \\
\text { Guildford Museum }\end{array}$ \\
\hline
\end{tabular}




\begin{tabular}{|c|c|c|c|c|}
\hline Alfriston & Sussex & $\begin{array}{l}\text { 5th- } \\
\text { 7th c }\end{array}$ & $\begin{array}{l}\text { Graves } 47,77 \text { and } 94: \\
\text { a few annulars with } \\
\text { small square-headed } \\
\text { brooch pair (Gr } 47 \text { ) }\end{array}$ & $\begin{array}{l}\text { Griffith and Salzmann } \\
1914,41 ; \text { Griffith } 1915 \text {, } \\
203 \text { and } 206 ; \\
\text { Welch 1983, 84; } \\
\text { Lewes Museum }\end{array}$ \\
\hline $\begin{array}{l}\text { Apple } \\
\text { Down I }\end{array}$ & Sussex & $\begin{array}{l}\text { prob } \\
\text { 6th c } \\
\text { 6th c }\end{array}$ & $\begin{array}{l}\text { Grave 90: annular } \\
\text { (?dark green); } \\
\text { Grave 128: } 1 \text { very } \\
\text { small annular with } \\
\text { button brooch, etc }\end{array}$ & $\begin{array}{l}\text { Down and Welch } 1990 \text {, } \\
46 \text { and } 51,162 \text { and } 163 \text {, } \\
\text { figs } 2.32 .90 .1 \\
\text { and } 2.41 ; \\
\text { Chichester Museum }\end{array}$ \\
\hline Highdown & Sussex & $\begin{array}{l}5 \text { th- } \\
6 \text { th c }\end{array}$ & $\begin{array}{l}\text { Grave 10: annular; } \\
\text { Grave 31: globular }\end{array}$ & $\begin{array}{l}\text { Read } 1895,373-4 \text { and } 378 \\
\text { Welch } 1983,84 ; \\
\text { Worthing Museum } \\
3488 \text { and } 3480\end{array}$ \\
\hline $\begin{array}{l}\text { Harnham } \\
\text { Hill }\end{array}$ & Wilts & $\begin{array}{l}\text { 6th- } \\
7 \text { th c }\end{array}$ & Several annulars & $\begin{array}{l}\text { Akerman } 1853 \\
\text { Jackson } 1854 ; \\
\text { BM } 5312-14\end{array}$ \\
\hline $\begin{array}{l}\text { Peters- } \\
\text { finger }\end{array}$ & Wilts & 6th $c$ & $\begin{array}{l}\text { Grave 50: annular; signs } \\
\text { of red mixture in glass }\end{array}$ & $\begin{array}{l}\text { Leeds and Shortt 1953, } \\
33-4 \text {, pl IV; } \\
\text { Salisbury Museum }\end{array}$ \\
\hline $\begin{array}{l}\text { Broadway } \\
\text { Hill }\end{array}$ & Worcs & $c .500$ & $\begin{array}{l}\text { Grave } 1: 5 \text { small 'quoit' } \\
\text { annulars with cast } \\
\text { saucer brooch pair }\end{array}$ & $\begin{array}{l}\text { Cook 1958, 64, } \\
\text { fig } 4.6\end{array}$ \\
\hline $\begin{array}{l}\text { West } \\
\text { Heslerton }\end{array}$ & Yorks & & $\begin{array}{l}\text { Some globulars at } \\
\text { least }\end{array}$ & $\begin{array}{l}\text { Publication in } \\
\text { preparation } \\
\text { (D Powlesland) }\end{array}$ \\
\hline & $\mathrm{ii}$ & \multicolumn{3}{|c|}{ 'BLACK' SEGMENTED DOUBLE OR TRIPLE BEADS } \\
\hline $\begin{array}{l}\text { Springfield } \\
\text { Lyons }\end{array}$ & Essex & $\begin{array}{l}\text { mid- } \\
\text { 6th c }\end{array}$ & Grave 4899: 2 segments & $\begin{array}{l}\text { Publication in } \\
\text { preparation (S Tyler); } \\
\text { BM }\end{array}$ \\
\hline Faversham & Kent & $\begin{array}{l}\text { prob } \\
6 \text { th- } \\
7 \text { th c }\end{array}$ & $\begin{array}{l}\text { Two double beads } \\
\text { (Plate 1) }\end{array}$ & $\begin{array}{l}\text { Meaney 1964, 118-19; } \\
\text { Ashmolean Museum } \\
1909.142\end{array}$ \\
\hline Sarre & Kent & $\begin{array}{l}5 \text { th- } \\
7 \text { th c }\end{array}$ & $\begin{array}{l}\text { Triplex straight-sided and } \\
\text { possibly made of jet }\end{array}$ & $\begin{array}{l}\text { Meaney 1964, 135-6; } \\
\text { BM 93 6-1 } 218\end{array}$ \\
\hline Westbere & Kent & $\begin{array}{l}\text { prob } \\
7 \text { th c }\end{array}$ & $\begin{array}{l}\text { With metal-in-glass } \\
\text { beads, etc }\end{array}$ & $\begin{array}{l}\text { Jessup 1946; } \\
\text { Canterbury Museum } \\
\text { RM 6422, } 6425 \text { and } 6426\end{array}$ \\
\hline Fonaby & Lincs & 6th $c$ & $\begin{array}{l}\text { Unstratified group 5: } \\
\text { double (Plate 1) }\end{array}$ & $\begin{array}{l}\text { Cook 1981, 45, } \\
\text { fig 18.5.6, pl VIIC; } \\
\text { Scunthorpe Museum }\end{array}$ \\
\hline Sewerby & Yorks & $\begin{array}{l}\text { later } \\
\text { 6th } c\end{array}$ & $\begin{array}{l}\text { Grave 49: double (A2d) } \\
\text { with Leeds Class C2 } \\
\text { square-headed cruciform } \\
\text { brooch, etc }\end{array}$ & $\begin{array}{l}\text { Hirst 1985, 66, fig 52, } \\
\text { String II.a }\end{array}$ \\
\hline
\end{tabular}




\begin{tabular}{|c|c|c|c|c|}
\hline iii & 'BLACK' & MELON, & SUB-MELON OR LOBED & BEADS (see Map 2) \\
\hline Kempston & Beds & $\begin{array}{l}5 \text { th- } \\
6 \text { th } c\end{array}$ & $\begin{array}{l}\text { Dark colour uncertain, } \\
\text { 4-lobed sub-melon }\end{array}$ & $\begin{array}{l}\text { Fitch 1864; } \\
\text { BM 91 6-24 } 54\end{array}$ \\
\hline Wallingford & Berks & $\begin{array}{l}\text { 2nd } \\
\text { half } \\
5 \text { th c }\end{array}$ & $\begin{array}{l}\text { Grave 15: with applied } \\
\text { saucer brooch pair } \\
\text { (floriate/anchor cross) } \\
\text { and } 2 \text { quoit brooches, etc }\end{array}$ & $\begin{array}{l}\text { Leeds } 1938,98-9, \text { pl VII; } \\
\text { Welch 1975; } \\
\text { Evison 1978; } \\
\text { Böhme 1986, 545-7 and 571; } \\
\text { Ashmolean Museum }\end{array}$ \\
\hline Bishopstone & Bucks & & $\begin{array}{l}\text { One sub-melon: colour } \\
\text { uncertain }\end{array}$ & $\begin{array}{l}\text { Meaney } 1964,56 \\
\text { Aylesbury Museum }\end{array}$ \\
\hline Lechlade & Glos & 7 th $\mathrm{c}$ & Grave 172/2: 5-lobed & $\begin{array}{l}\text { Boyle et al forthcoming; } \\
\text { Cirencester Museum }\end{array}$ \\
\hline Alton & Hants & 6th $\mathrm{c}$ & Grave 9: 1 sub-melon & $\begin{array}{l}\text { Evison 1988a, } 73 \text {, } \\
\text { fig 25.9.1k; } \\
\text { Hampshire Museums }\end{array}$ \\
\hline $\begin{array}{l}\text { Chessell } \\
\text { Down }\end{array}$ & $\begin{array}{l}\text { Isle of } \\
\text { Wight }\end{array}$ & 6th $\mathrm{c}$ & Two sub-melons (type 11.6) & $\begin{array}{l}\text { Arnold } 1982,49-50 \text { and } 121 \text {, } \\
\text { colour frontispiece; BM }\end{array}$ \\
\hline Alfriston & Sussex & $\begin{array}{l}\text { 5th- } \\
7 \text { th } c\end{array}$ & Unstratified: 3 flat beads & $\begin{array}{l}\text { Griffith and Salzmann } \\
\text { 1914, 51, pl I.4-6; } \\
\text { Welch 1983, 84, fig 45a; } \\
\text { Lewes Museum }\end{array}$ \\
\hline Wasperton & Warks & 6th $c$ & $\begin{array}{l}\text { Six-lobed sub-melon } \\
\text { with small-long and } \\
\text { cruciform brooches }\end{array}$ & $\begin{array}{l}\text { Publication in } \\
\text { preparation } \\
\text { (M Carver); } \\
\text { Warwick Museum }\end{array}$ \\
\hline $\begin{array}{l}\text { Harnham } \\
\text { Hill }\end{array}$ & Wilts & $\begin{array}{l}\text { prob } \\
\text { 6th- } \\
7 \text { th c }\end{array}$ & $\begin{array}{l}\text { One 5-lobed sub-melon } \\
\text { (Plate 1) }\end{array}$ & $\begin{array}{l}\text { Akerman 1853; } \\
\text { Jackson 1854; } \\
\text { Salisbury Museum }\end{array}$ \\
\hline $\begin{array}{l}\text { Peters- } \\
\text { finger }\end{array}$ & Wilts & $\begin{array}{l}\text { late } \\
5 \text { th- } \\
\text { 6th c }\end{array}$ & $\begin{array}{l}\text { Grave 29: sub-melon } \\
\text { with applied saucer } \\
\text { brooch pair, etc }\end{array}$ & $\begin{array}{l}\text { Leeds and Shortt } 1953 \text {, } \\
25(104 \mathrm{f}), \mathrm{pl} \text { III; } \\
\text { Salisbury Museum }\end{array}$ \\
\hline
\end{tabular}

\section{POLYCHROME}

iv 'BLACK' ANNULAR BEADS WITH BROKEN WHITE GIRTH BAND (see Map 3)

$\begin{array}{lllll}\text { Kempstion } & \text { Beds } & \begin{array}{l}\text { 5th- } \\ \text { 6th c }\end{array} & \text { Meaney 1964, 36-7; } \\ \text { Harwell } & \text { Berks } & \begin{array}{l}\text { 5th- } \\ \text { 6th c }\end{array} & \begin{array}{l}\text { Grave 4: with disc brooch } \\ \text { pair }\end{array} & \begin{array}{l}\text { Kirk and Marshall 1956, } \\ \text { 27, 33-4, fig 10h.3-6; } \\ \text { Ashmolean Museum }\end{array} \\ \text { Wallingford } & \text { Berks } & \begin{array}{l}\text { 5th- } \\ \text { late } \\ \text { 6th c }\end{array} & \text { Grave 13 } & \begin{array}{l}\text { Leeds 1938, 98; } \\ \text { Ashmolean Museum }\end{array}\end{array}$




\begin{tabular}{|c|c|c|c|c|}
\hline $\begin{array}{l}\text { Newport } \\
\text { Pagnell }\end{array}$ & Bucks & $\begin{array}{l}\text { prob } \\
\text { 6th c }\end{array}$ & 'Armlet of beads' & $\begin{array}{l}\text { Meaney 1964, 58; } \\
\text { Aylesbury Museum }\end{array}$ \\
\hline $\begin{array}{l}\text { Hasling- } \\
\text { field }\end{array}$ & Cambs & $\begin{array}{l}5 \text { th- } \\
\text { 6th c }\end{array}$ & & Fox $1923,255-9$ \\
\hline $\begin{array}{l}\text { Maiden } \\
\text { Castle }\end{array}$ & Dorset & & Stray find & Wheeler 1943 \\
\hline Mucking II & Essex & $\begin{array}{l}\text { 5th- } \\
6 \text { th c }\end{array}$ & $\begin{array}{l}\text { Grave 649: } 1 \text { dark green } \\
\text { with small-long brooch } \\
\text { pair, decorated buckle, etc }\end{array}$ & $\begin{array}{l}\text { Hirst and Clark forthcoming; } \\
\text { BM }\end{array}$ \\
\hline $\begin{array}{l}\text { Springfield } \\
\text { Lyons }\end{array}$ & Essex & $\begin{array}{l}\text { mid- } \\
\text { 6th c }\end{array}$ & Grave 4899 & $\begin{array}{l}\text { Publication in } \\
\text { preparation (S Tyler); } \\
\text { BM }\end{array}$ \\
\hline $\begin{array}{l}\text { Bowcombe } \\
\text { Down }\end{array}$ & $\begin{array}{l}\text { Isle of } \\
\text { Wight }\end{array}$ & $\begin{array}{l}\text { late } \\
5 \text { th- } \\
6 \text { th c }\end{array}$ & & $\begin{array}{l}\text { Arnold 1982, 89-96, } \\
\text { figs } 62-66\end{array}$ \\
\hline $\begin{array}{l}\text { Beakes- } \\
\text { bourne } \\
\text { I and II }\end{array}$ & Kent & $\begin{array}{l}\text { early } \\
6 \text { th- } \\
7 \text { th c }\end{array}$ & & $\begin{array}{l}\text { Faussett 1856, 144-59; } \\
\text { Meaney 1964, 108-9 }\end{array}$ \\
\hline $\begin{array}{l}\text { Canterbury, } \\
\text { Cakebread } \\
\text { Robey V }\end{array}$ & Kent & $c .400$ & From multiple burial & $\begin{array}{l}\text { Information T Tatton- } \\
\text { Brown and } \mathrm{P} \text { Garrard }\end{array}$ \\
\hline $\begin{array}{l}\text { Horton } \\
\text { Kirby I }\end{array}$ & Kent & $\begin{array}{l}\text { 5th- } \\
\text { 6th } c\end{array}$ & & $\begin{array}{l}\text { Meaney } 1964,124 \\
\text { Maidstone Museum }\end{array}$ \\
\hline Howletts & Kent & $\begin{array}{l}\text { late } \\
5 \text { th- } \\
6 \text { th c }\end{array}$ & & $\begin{array}{l}\text { Smith 1918; } \\
\text { BM 1936 5-11 } 143\end{array}$ \\
\hline 'Kent' & Kent & & & Canterbury Museum \\
\hline $\begin{array}{l}\text { Lyminge } \\
\text { II }\end{array}$ & Kent & $\begin{array}{l}\text { first } \\
\text { half } \\
6 \text { th c }\end{array}$ & $\begin{array}{l}\text { Grave 39: with small } \\
\text { square-headed and cast } \\
\text { saucer brooch pairs }\end{array}$ & $\begin{array}{l}\text { Warhurst } 1955,27, \\
\text { pl VIIb.2; } \\
\text { Maidstone Museum }\end{array}$ \\
\hline Brixworth & Northants & & & $\begin{array}{l}\text { Meaney 1964, 187-8; } \\
\text { Northampton Museum }\end{array}$ \\
\hline Wheatley & Oxon & 6th $c$ & $\begin{array}{l}\text { Grave 27: with amber } \\
\text { beads }\end{array}$ & $\begin{array}{l}\text { Leeds } 1916,54-5 \text {, fig 6; } \\
\text { Ashmolean Museum }\end{array}$ \\
\hline Guildown & Surrey & 6th $c$ & $\begin{array}{l}\text { Grave } 206: 2 \text { examples } \\
\text { with square-headed } \\
\text { brooch pair }\end{array}$ & $\begin{array}{l}\text { Lowther 1931, 11, 44, } \\
\text { pl IX.1; } \\
\text { Guildford Museum }\end{array}$ \\
\hline Mitcham & Surrey & $\begin{array}{l}\text { prob } \\
\text { 6th c }\end{array}$ & $\begin{array}{l}\text { Grave 197: } 4 \text { beads annular } \\
\text { pinkish black }\end{array}$ & $\begin{array}{l}\text { Bidder and Morris } 1959 \text {, } \\
73,112 \text {, pl XVI.197; } \\
\text { Cambridge Museum }\end{array}$ \\
\hline Highdown & Sussex & $\begin{array}{l}5 \text { th- } \\
6 \text { th c }\end{array}$ & $\begin{array}{l}\text { Graves } 10 \text { and } 85 \text { and one } \\
\text { eg from an unrecorded } \\
\text { grave }\end{array}$ & $\begin{array}{l}\text { Read } 1895,373-4 \text {, and } \\
1896,214 ; \\
\text { Welch } 1983,84 \text {, figs } 92 \mathrm{i} \\
\text { and } 108 \mathrm{c} ; \text { Worthing Museum } \\
3488,3478 \text { and } 3484\end{array}$ \\
\hline
\end{tabular}




\begin{tabular}{|c|c|c|c|c|}
\hline $\begin{array}{l}\text { Bidford- } \\
\text { on-Avon }\end{array}$ & Warks & 6th c & $\begin{array}{l}\text { From necklace: } \\
\text { atypical bead with } \\
\text { more regular dots }\end{array}$ & $\begin{array}{l}\text { Humphreys et al 1923, } \\
\text { 105, pl XVII.1-3; } \\
\text { Humphreys et al 1924, } \\
\text { 279-80, pl LVIII.1 and 2; } \\
\text { Stratford Museum }\end{array}$ \\
\hline Charlton & Wilts & 7th $\mathrm{c}$ & $\begin{array}{l}\text { Burial 37: dark green } \\
\text { (Plate 1) }\end{array}$ & $\begin{array}{l}\text { Davies } 1984,120 \text {, fig } 7 P \text {; } \\
\text { Salisbury Museum }\end{array}$ \\
\hline $\begin{array}{l}\text { Harnham } \\
\text { Hill }\end{array}$ & Wilts & $\begin{array}{l}\text { 6th- } \\
7 \text { th } c\end{array}$ & & $\begin{array}{l}\text { Akerman 1853; } \\
\text { Jackson 1854; } \\
\text { BM } 53 \text { 12-14.196 and 204-95 }\end{array}$ \\
\hline $\begin{array}{l}\text { Pewsey } \\
\text { Blackpatch }\end{array}$ & Wilts & $\begin{array}{l}\text { early, } \\
\text { 6th c }\end{array}$ & Grave 15: with brooches & $\begin{array}{l}\text { Publication in } \\
\text { preparation (K Annable); } \\
\text { Devizes Museum }\end{array}$ \\
\hline $\begin{array}{l}\text { Broadway } \\
\text { Hill }\end{array}$ & Worcs & $\begin{array}{l}\text { late } \\
5 \text { th- } \\
6 \text { th c }\end{array}$ & $\begin{array}{l}\text { Disturbed find from } \\
\text { an inhumation grave }\end{array}$ & $\begin{array}{l}\text { Cook 1958, } 70 \text {, } \\
\text { fig } 9.10\end{array}$ \\
\hline $\mathbf{v}$ & 'BLACK' & $\begin{array}{l}\text { ANNULAR } \\
\text { (SOME WI }\end{array}$ & $\begin{array}{l}\text { BEADS: (a) WITH WHITE } \\
\text { ITH GIRTH BAND) (see N }\end{array}$ & $\begin{array}{l}\text { OR YELLOW WAVE } \\
\text { Iap } 4)\end{array}$ \\
\hline Kempston & Beds & $\begin{array}{l}\text { prob } \\
\text { 6th } \mathrm{c}\end{array}$ & Four examples & $\begin{array}{l}\text { Fitch 1864; } \\
\text { BM 91 6-24 } 52\end{array}$ \\
\hline Wallingford & Berks & $\begin{array}{l}\text { 5th- } \\
\text { late } \\
\text { 6th c }\end{array}$ & Near Grave 13 & $\begin{array}{l}\text { Leeds 1938, } 98 ; \\
\text { Ashmolean Museum } \\
\text { 1938.1223 }\end{array}$ \\
\hline $\begin{array}{l}\text { Newport } \\
\text { Pagnell }\end{array}$ & Bucks & $\begin{array}{l}\text { prob } \\
\text { 6th } c\end{array}$ & $\begin{array}{l}\text { Necklace with one } \\
\text { wave bead }\end{array}$ & $\begin{array}{l}\text { Meaney } 1964,58 \\
\text { Aylesbury CAS } 1373\end{array}$ \\
\hline $\begin{array}{l}\text { Hasling- } \\
\text { field }\end{array}$ & Cambs & $\begin{array}{l}\text { prob } \\
\text { 6th c }\end{array}$ & $\begin{array}{l}\text { Strung on a group, } \\
\text { but not necessarily } \\
\text { an associated group }\end{array}$ & Fox $1923,255-9$ \\
\hline Mucking II & Essex & $\begin{array}{l}\text { 5th- } \\
\text { 6th c }\end{array}$ & $\begin{array}{l}\text { Grave } 860: 9 \text { dark-green } \\
\text { ( } 5 \text { annular and } 4 \text { barrels) } \\
\text { with white waves with } \\
\text { small-long brooch pair, } \\
\text { penannular brooch, etc }\end{array}$ & $\begin{array}{l}\text { Hirst and Clark forthcoming; } \\
\text { BM }\end{array}$ \\
\hline Andover & Hants & 6th $c$ & $\begin{array}{l}\text { Grave 44: } 7 \text { examples } \\
\text { (Plate 2) on string with } \\
\text { purse-mount and toilet } \\
\text { set, etc }\end{array}$ & $\begin{array}{l}\text { Cook and Dacre } 1985,35, \\
\text { fig } 59.1,5,7,9,15, \\
17 \text { and } 38 ; \\
\text { Hampshire Museums }\end{array}$ \\
\hline $\begin{array}{l}\text { Bowcombe } \\
\text { Down }\end{array}$ & $\begin{array}{l}\text { Isle of } \\
\text { Wight }\end{array}$ & $\begin{array}{l}\text { prob } \\
\text { 6th } c\end{array}$ & Type 6.22 & $\begin{array}{l}\text { Arnold } 1982,89-96,120 \text {, } \\
\text { colour frontispiece }\end{array}$ \\
\hline Ashford & Kent & & & $\begin{array}{l}\text { Pitt-Rivers Collection, } \\
\text { Salisbury Museum }\end{array}$ \\
\hline $\begin{array}{l}\text { Beakes- } \\
\text { bourne II }\end{array}$ & Kent & $\begin{array}{l}\text { prob } \\
\text { 6th } c\end{array}$ & $\begin{array}{l}\text { Burial with brooches } \\
\text { and string of beads }\end{array}$ & $\begin{array}{l}\text { Meaney 1964, 109; } \\
\text { Canterbury RM 7515-6 }\end{array}$ \\
\hline
\end{tabular}




\begin{tabular}{|c|c|c|c|c|}
\hline $\begin{array}{l}\text { Dover, } \\
\text { Buckland }\end{array}$ & Kent & $\begin{array}{l}650- \\
675\end{array}$ & Grave 133: 1 bead (D07) & $\begin{array}{l}\text { Evison 1987, 245, } \\
\text { fig 55.133.2e, } \\
\text { colour pl III (D07); BM }\end{array}$ \\
\hline Faversham & Kent & $\begin{array}{l}\text { prob } \\
6 \text { th- } \\
7 \text { th c }\end{array}$ & $\begin{array}{l}\text { Annular with yellow } \\
\text { wave }\end{array}$ & $\begin{array}{l}\text { Meaney } 1964,118-19 ; \\
\text { Beck Collection, } \\
\text { Cambridge } 47.1830 \text { a and b }\end{array}$ \\
\hline $\begin{array}{l}\text { Horton } \\
\text { Kirby I }\end{array}$ & Kent & $\begin{array}{l}\text { prob } \\
\text { 6th c }\end{array}$ & On necklace & $\begin{array}{l}\text { Meaney } 1964,124 \\
\text { Maidstone A/S } 200\end{array}$ \\
\hline Howletts & Kent & $\begin{array}{l}\text { late } \\
5 \text { th- } \\
6 \text { th c }\end{array}$ & $\begin{array}{l}\text { Grave 142: several } \\
\text { black with white }\end{array}$ & $\begin{array}{l}\text { Smith 1918; } \\
\text { BM 1936 5-11 } 143\end{array}$ \\
\hline $\begin{array}{l}\text { Lyminge } \\
\text { II }\end{array}$ & Kent & $\begin{array}{l}\text { first } \\
\text { half } \\
\text { 6th c }\end{array}$ & $\begin{array}{l}\text { Grave 39: possibly } \\
\text { anklets with cast saucer } \\
\text { and square-headed brooch } \\
\text { pairs }\end{array}$ & $\begin{array}{l}\text { Warhurst 1955, 27, } \\
\text { pl VIIb.2; } \\
\text { Maidstone Museum }\end{array}$ \\
\hline Sarre & Kent & $\begin{array}{l}5 \text { th- } \\
7 \text { th c }\end{array}$ & & $\begin{array}{l}\text { Meaney } 1964,135-6 \text {; } \\
\text { BM OA } 4904\end{array}$ \\
\hline Searby & Lincs & $\begin{array}{l}\text { late } \\
5 \text { th- } \\
\text { early } \\
\text { 6th c }\end{array}$ & $\begin{array}{l}\text { Several atypical } \\
\text { wave beads, rather } \\
\text { cylindrical }\end{array}$ & $\begin{array}{l}\text { Smith } 1852 \mathrm{a} \text { and } \\
1861 \text {; } \\
\text { BM } 936-1824\end{array}$ \\
\hline $\begin{array}{l}\text { Holme } \\
\text { Pierrepont }\end{array}$ & Notts & 6th $c$ & $\begin{array}{l}\text { Two rather cylindrical } \\
\text { dated by brooches }\end{array}$ & $\begin{array}{l}\text { Meaney 1964, 200; } \\
\text { BM } 1931 \text { 3-13 }\end{array}$ \\
\hline $\begin{array}{l}\text { Cassington, } \\
\text { Purwell } \\
\text { Farm }\end{array}$ & Oxon & 6th $c$ & Slightly pinkish wave & $\begin{array}{l}\text { Leeds and Riley } 1942, \\
64, \mathrm{pl} \text { VIB; } \\
\text { Ashmolean } 1942.157\end{array}$ \\
\hline Wheatley & Oxon & 6th $c$ & $\begin{array}{l}\text { Grave 27: with amber and } \\
\text { gold-in-glass beads, etc }\end{array}$ & $\begin{array}{l}\text { Leeds } 1916,54-5 \text {, fig } 6 \text {; } \\
\text { Ashmolean } \\
\text { Museum } 1883.69\end{array}$ \\
\hline Ipswich & Suffolk & $\begin{array}{l}\text { 6th- } \\
7 \text { th c }\end{array}$ & & $\begin{array}{l}\text { Layard } 1907 \text { and 1909; } \\
\text { Ipswich Museum }\end{array}$ \\
\hline Snape & Suffolk & $\begin{array}{l}\text { prob } \\
\text { 6th c }\end{array}$ & Grave 0841 & $\begin{array}{l}\text { Filmer-Sankey and } \\
\text { Pestell forthcoming }\end{array}$ \\
\hline West Stow & Suffolk & 6th $c$ & $\begin{array}{l}\text { Settlement SFB47: } \\
\text { 1 dark blue SF1336 }\end{array}$ & $\begin{array}{l}\text { West } 1985,73 \text {, fig } 161.7 \text {; } \\
\text { Bury St Edmunds Museum }\end{array}$ \\
\hline Mitcham & Surrey & $\begin{array}{l}\text { prob } \\
\text { 6th c }\end{array}$ & $\begin{array}{l}\text { Grave 62: large globular } \\
\text { with white wave }\end{array}$ & $\begin{array}{l}\text { Bidder and Duckworth 1906; } \\
\text { Bidder and Morris 1959, 64, } \\
\text { 110, pl XVI.62; } \\
\text { Cambridge Museum }\end{array}$ \\
\hline Alfriston & Sussex & $\begin{array}{l}\text { 6th c } \\
\text { first } \\
\text { half } \\
6 \text { th c } \\
5 \text { th- } \\
7 \text { th c }\end{array}$ & $\begin{array}{l}\text { Grave } 43: 2 \text { with great } \\
\text { square-headed brooch, etc; } \\
\text { Grave } 47: 6 \text { with small } \\
\text { square-headed brooch } \\
\text { pair; } \\
\text { Grave } 51 \text { : very dark green } \\
\text { seeming black; } \\
\text { Grave } 94: 2 \text { examples }\end{array}$ & $\begin{array}{l}\text { Griffith and Salzmann } \\
\text { 1914, 40,41 and 42, pl 1.9; } \\
\text { Griffith 1915, 206; } \\
\text { Welch 1983, 84; } \\
\text { Lewes Museum }\end{array}$ \\
\hline
\end{tabular}




\begin{tabular}{|c|c|c|c|c|}
\hline $\begin{array}{l}\text { Apple } \\
\text { Down I }\end{array}$ & Sussex & $\begin{array}{l}\text { first } \\
\text { half } \\
\text { 6th c }\end{array}$ & $\begin{array}{l}\text { Grave 128: white girth } \\
\text { band and single white } \\
\text { wave with button brooch }\end{array}$ & $\begin{array}{l}\text { Down and Welch } 1990,51 \text {, } \\
163(402 / 2) \text {, fig } 2.41 \text {; } \\
\text { Chichester Museum }\end{array}$ \\
\hline Wasperton & Warks & $\begin{array}{l}\text { late } \\
5 \text { th- } \\
\text { early } \\
6 \text { th c }\end{array}$ & $\begin{array}{l}\text { Two on necklace with cast } \\
\text { saucer brooches }\end{array}$ & $\begin{array}{l}\text { Publication in preparation } \\
\text { (M Carver); } \\
\text { Warwick Museum }\end{array}$ \\
\hline $\begin{array}{l}\text { Harnham } \\
\text { Hill }\end{array}$ & Wilts & $\begin{array}{l}\text { prob } \\
\text { 6th c }\end{array}$ & One example & $\begin{array}{l}\text { Akerman 1853; } \\
\text { Jackson 1854; } \\
\text { BM } 53 \text { 12-14 }\end{array}$ \\
\hline $\begin{array}{l}\text { West } \\
\text { Heslerton }\end{array}$ & Yorks & & $\begin{array}{l}\text { Grave } 467 \text { : small with } \\
\text { yellow wave }\end{array}$ & $\begin{array}{l}\text { Publication in } \\
\text { preparation } \\
\text { (D Powlesland) }\end{array}$ \\
\hline
\end{tabular}

\begin{tabular}{|c|c|c|c|c|}
\hline $\mathbf{v}$ & 'BLACK' & ANNULAR & BEADS: (b) WITH BLUE & wave (see Map 4) \\
\hline Silchester & Hants & & $\begin{array}{l}\text { Occupation of Roman } \\
\text { town ends early in } 5 \text { th } c\end{array}$ & $\begin{array}{l}\text { Boon 1959; } \\
\text { Reading Museum }\end{array}$ \\
\hline $\begin{array}{l}\text { Winchester, } \\
\text { Wolvesley } \\
\text { Palace }\end{array}$ & Hants & $\begin{array}{l}\text { poss } \\
5 \text { th c }\end{array}$ & $\begin{array}{l}\text { Two-colour scrabble } \\
\text { in blue and yellow }\end{array}$ & $\begin{array}{l}\text { Publication in } \\
\text { preparation } \\
\text { (M Biddle) }\end{array}$ \\
\hline Faversham & Kent & $\begin{array}{l}\text { 5th- } \\
7 \text { th c }\end{array}$ & & $\begin{array}{l}\text { Meaney } 1964,118-19 \\
\text { Ashmolean Museum } \\
1909.143 \text { and } 144\end{array}$ \\
\hline Wheatley & Oxon & $\begin{array}{l}\text { 6th- } \\
7 \text { th c }\end{array}$ & Grave 18 & $\begin{array}{l}\text { Leeds } 1916,53 \text {, fig } 2.18 \text {; } \\
\text { Ashmolean } \\
\text { Museum } 1883.60\end{array}$ \\
\hline Icklingham & Suffolk & $\begin{array}{l}\text { ?late } \\
\text { 4th c }\end{array}$ & $\begin{array}{l}\text { Site with Roman to } \\
\text { Anglo-Saxon overlap }\end{array}$ & \\
\hline Wolstonbury & Sussex & $c .400$ & $\begin{array}{l}\text { (Plate 2) Dated typologically } \\
\text { by Kendrick }\end{array}$ & $\begin{array}{l}\text { Holleyman 1935, 38, } \\
\text { fig } 1\end{array}$ \\
\hline $\begin{array}{l}\text { Monkton } \\
\text { Down }\end{array}$ & Wilts & & $\begin{array}{l}\text { Several beads, not } \\
\text { necessarily associated }\end{array}$ & Devizes Museum 131 \\
\hline $\begin{array}{l}\text { Nettleton } \\
\text { Shrub }\end{array}$ & Wilts & $\begin{array}{l}\text { late } \\
\text { 4th c }\end{array}$ & $\begin{array}{l}\text { Roman settlement: } \\
\text { building XVIII }\end{array}$ & $\begin{array}{l}\text { Information from W J } \\
\text { Wedlake; } \\
\text { Wedlake } 1982\end{array}$ \\
\hline
\end{tabular}

It should be noted that there are a number of black beads with blue waves or scrabbles ffom Northern Ireland. Their characteristic blue is different from these British and Anglo-Saxon beads, being a powder blue rather than sky blue.

\begin{tabular}{|c|c|c|c|c|}
\hline vi & 'BLACK' & ANNULAR & BEADS: (a) WITH WHITE & zIGZAGS (see Map 5 \\
\hline Kempston & Beds & $\begin{array}{l}\text { prob } \\
5 \text { th c }\end{array}$ & Grave 39 & $\begin{array}{l}\text { Fitch 1864; } \\
\text { BM 91 6-24 } 52\end{array}$ \\
\hline Droxford & Hants & $\begin{array}{l}\text { late } \\
5 \text { th- } \\
6 \text { th } c\end{array}$ & Grave 20: 7 beads (class Ia) & $\begin{array}{l}\text { Aldsworth } 1978,124 \text {, } \\
\text { 172, fig 24.3-5; } \\
\text { Winchester Museum }\end{array}$ \\
\hline
\end{tabular}




\begin{tabular}{|c|c|c|c|c|}
\hline Northchurch & Herts & & With brooch, etc & BM 93 4-9 8 \\
\hline Faversham & Kent & $\begin{array}{l}5 \text { th- } \\
7 \text { th c }\end{array}$ & & $\begin{array}{l}\text { Meaney 1964, 118-19; } \\
\text { Beck Collection, } \\
\text { Cambridge Museum } 1677\end{array}$ \\
\hline Howletts & Kent & $\begin{array}{l}\text { 5th- } \\
\text { 6th } c\end{array}$ & Several & $\begin{array}{l}\text { Smith 1918; } \\
\text { BM } 1936 \text { 5-11 } 143 \mathrm{ff}\end{array}$ \\
\hline 'Kent' & Kent & & $\begin{array}{l}\text { Necklace: with white } \\
\text { zigzag }\end{array}$ & $\begin{array}{l}\text { Canterbury Museum } \\
\text { RM } 2620\end{array}$ \\
\hline $\begin{array}{l}\text { Lyminge } \\
\text { II }\end{array}$ & Kent & $\begin{array}{l}\text { first } \\
\text { half } \\
\text { 6th c }\end{array}$ & $\begin{array}{l}\text { Grave 39: with cast saucer } \\
\text { and square-headed brooch } \\
\text { pairs }\end{array}$ & $\begin{array}{l}\text { Warhurst 1955, } 27, \\
\text { pl VIIb.2; } \\
\text { Maidstone Museum }\end{array}$ \\
\hline Richborough & Kent & & $\begin{array}{l}\text { Roman fort: } 2 \text { beads, } \\
\text { one is very dark green }\end{array}$ & $\begin{array}{l}\text { Richborough Museum, } \\
\text { nos d and } 492\end{array}$ \\
\hline Sarre & Kent & $\begin{array}{l}\text { 5th- } \\
7 \text { th c }\end{array}$ & & $\begin{array}{l}\text { Meaney 1964, 135-6; } \\
\text { BM 93 6-1 } 218\end{array}$ \\
\hline Stowting & Kent & $\begin{array}{l}\text { prob } \\
\text { early } \\
\text { 6th c }\end{array}$ & Necklace: 2 & $\begin{array}{l}\text { Meaney 1964, 137-8; } \\
\text { Maidstone Museum }\end{array}$ \\
\hline Brooke & Norfolk & $\begin{array}{l}\text { prob } \\
\text { 6th c }\end{array}$ & & $\begin{array}{l}\text { Meaney 1964, 170; } \\
\text { BM } 186669\end{array}$ \\
\hline Lakenheath & Suffolk & & & Meaney 1964,230 \\
\hline West Stow & Suffolk & $\begin{array}{l}5 \text { th- } \\
7 \text { th c }\end{array}$ & $\begin{array}{l}\text { Cemetery: } \\
1 \text { bead (02I) }\end{array}$ & $\begin{array}{l}\text { West } 1985,74 \text {, fig } 276.10 \text {; } \\
\text { Ashmolean Museum } 1909.424\end{array}$ \\
\hline Alfriston & Sussex & 6th $c$ & $\begin{array}{l}\text { Grave } 43 \text { with great } \\
\text { square-headed brooch, } \\
\text { etc }\end{array}$ & $\begin{array}{l}\text { Griffith and Salzmann } \\
\text { 1914, 40, pl 1.9; } \\
\text { Welch 1983,84; } \\
\text { Lewes Museum }\end{array}$ \\
\hline $\begin{array}{l}\text { Apple } \\
\text { Down I }\end{array}$ & Sussex & $\begin{array}{l}\text { prob } \\
\text { 6th c }\end{array}$ & Grave 117: white zigzag & $\begin{array}{l}\text { Down and Welch } 1990,49, \\
163(380 \mathrm{~B} / 1) \text {, fig } 2.37, \\
\text { pl 41.34; } \\
\text { Chichester Museum }\end{array}$ \\
\hline Cheverell & Wilts & $\begin{array}{l}5 \text { th- } \\
6 \text { th } c\end{array}$ & $\begin{array}{l}\text { (Plate 2) With saucer } \\
\text { brooches, etc }\end{array}$ & $\begin{array}{l}\text { Meaney } 1964,267 \\
\text { Devizes Museum }\end{array}$ \\
\hline Mildenhall & Wilts & $\begin{array}{l}\text { prob } \\
6 \text { th c }\end{array}$ & $\begin{array}{l}\text { Two beads, one from } \\
\text { necklace with saucer } \\
\text { brooches }\end{array}$ & $\begin{array}{l}\text { Meaney 1964, 271-2; } \\
\text { Devizes Museum S.30 }\end{array}$ \\
\hline $\begin{array}{l}\text { Monkton } \\
\text { Down }\end{array}$ & Wilts & & & Devizes Museum 131 \\
\hline $\begin{array}{l}\text { Broadway } \\
\text { Hill }\end{array}$ & Worcs & $c .500$ & $\begin{array}{l}\text { Grave 1: with cast saucer } \\
\text { brooch pair }\end{array}$ & Cook 1958,64 , fig 4.8 \\
\hline $\begin{array}{l}\text { Whitby, } \\
\text { Ling Hill }\end{array}$ & Yorks & & Stray find $c .1856$ & Whitby Museum \\
\hline
\end{tabular}


vi 'BLACK' ANNULAR BEADS: (b) WITH YELLOW OR GREEN ZIGZAGS (see Map 5)

\begin{tabular}{|c|c|c|c|c|}
\hline Droxford & Hants & $\begin{array}{l}\text { late } \\
5 \text { th- } \\
6 \text { th c }\end{array}$ & Grave 20: 1 bead (class Ib) & $\begin{array}{l}\text { Aldsworth } 1978,124 \text {, } \\
\text { 172, fig 24.3-5; } \\
\text { Winchester Museum }\end{array}$ \\
\hline Silchester & Hants & & $\begin{array}{l}\text { Occupation of Roman } \\
\text { town ends in early } 5 \text { th } \mathrm{c} \text { : } \\
\text { opaque yellow scrabble }\end{array}$ & $\begin{array}{l}\text { Boon 1959; } \\
\text { Reading Museum }\end{array}$ \\
\hline $\begin{array}{l}\text { Canterbury, } \\
\text { Cakebread } \\
\text { Robey V }\end{array}$ & Kent & $c .400$ & $\begin{array}{l}\text { From multiple burial: } \\
\text { straight-sided annular } \\
\text { with unmarvered zigzag } \\
\text { and another green zigzag }\end{array}$ & $\begin{array}{l}\text { Information T Tatton- } \\
\text { Brown and } \mathrm{P} \text { Garrard }\end{array}$ \\
\hline Faversham & Kent & $\begin{array}{l}5 \text { th- } \\
7 \text { th } \mathrm{c}\end{array}$ & One with green zigzags & $\begin{array}{l}\text { Meaney } 1964,118-19 \\
\text { Ashmolean Museum }\end{array}$ \\
\hline Howletts & Kent & $\begin{array}{l}\text { late } \\
5 \text { th- } \\
6 \text { th c }\end{array}$ & Several beads & $\begin{array}{l}\text { Smith 1918; } \\
\text { Meaney 1964, 125; } \\
\text { BM 1936 5-11 } 143\end{array}$ \\
\hline $\begin{array}{l}\text { Clatchard } \\
\text { Craig }\end{array}$ & $\begin{array}{l}\text { Scotland: } \\
\text { Fife }\end{array}$ & $\begin{array}{l}\text { prob } \\
5 \text { th- } \\
6 \text { th c }\end{array}$ & $\begin{array}{l}\text { Post-Roman occupation } \\
\text { of } 6 \text { th or } 7 \text { th centuries: } \\
\text { yellow zigzag }\end{array}$ & $\begin{array}{l}\text { Guido 1986, 167, } \\
\text { illus 28.120; } \\
\text { Close-Brooks 1986, 146-9; } \\
\text { Edinburgh Museum }\end{array}$ \\
\hline Caernarvon & $\begin{array}{l}\text { Wales: } \\
\text { Caer- } \\
\text { narvon- } \\
\text { shire }\end{array}$ & & $\begin{array}{l}\text { Unstratified find from } \\
\text { Segontium Roman fort: } \\
\text { could be late Roman or } \\
\text { ?Irish: irregular wave }\end{array}$ & Wheeler 1924 \\
\hline vi & 'BLACK' & ANNULAR & BEADS: (c) WITH RED & ZIGZAGS (see Map 5) \\
\hline $\begin{array}{l}\text { Hasling- } \\
\text { field }\end{array}$ & Cambs & $\begin{array}{l}5 \text { th- } \\
7 \text { th } \mathrm{c}\end{array}$ & $\begin{array}{l}\text { With unassociated } \\
\text { collection on a string }\end{array}$ & $\begin{array}{l}\text { Fox 1923, 255-9; } \\
\text { Ashmolean Museum } \\
1909.233\end{array}$ \\
\hline Droxford & Hants & $\begin{array}{l}\text { late } \\
5 \text { th- } \\
6 \text { th c }\end{array}$ & Grave 20: 1 bead (class Ic) & $\begin{array}{l}\text { Aldsworth } 1978,124 \text {, } \\
\text { 172, fig 24.3-5; } \\
\text { Winchester Museum }\end{array}$ \\
\hline Richborough & Kent & & $\begin{array}{l}\text { Surface find } 238 \\
\text { and another without } \\
\text { a number (Plate 2) }\end{array}$ & $\begin{array}{l}\text { Bushe-Fox } 1949,149, \\
\text { pl LV.238; } \\
\text { Richborough Museum }\end{array}$ \\
\hline $\begin{array}{l}\text { Syston } \\
\text { Park }\end{array}$ & Lincs & $\begin{array}{l}\text { prob } \\
\text { 6th } c\end{array}$ & $\begin{array}{l}\text { On an unassociated } \\
\text { string }\end{array}$ & $\begin{array}{l}\text { Akerman 1855a, 41-2, } \\
\text { pl XXI; } \\
\text { BM (18) } 1111-92\end{array}$ \\
\hline 'E Anglia' & $\begin{array}{l}\text { Norfolk/ } \\
\text { Suffolk }\end{array}$ & & Unassociated & $\begin{array}{l}\text { Fitch Collection, } \\
\text { Norwich Museum } \\
427641-264 \\
\text { and } 6497694\end{array}$ \\
\hline
\end{tabular}


vii LARGE 'BLACK' DECORATED BEADS: (a) PLANO-CONVEX

$$
\text { (see Map 6) }
$$

\begin{tabular}{|c|c|c|c|c|}
\hline Abingdon & Berks & $\begin{array}{l}\text { first } \\
\text { half } \\
\text { 6th } c\end{array}$ & $\begin{array}{l}\text { Grave B117: dark green } \\
\text { with blue zigzag between } \\
2 \text { white waves on edge }\end{array}$ & $\begin{array}{l}\text { Leeds and Harden } 1936 \text {, } \\
\text { pl XVI; } \\
\text { Ashmolean Museum }\end{array}$ \\
\hline Bishopstone & Bucks & $\begin{array}{l}\text { prob } \\
\text { early } \\
\text { 6th } c\end{array}$ & $\begin{array}{l}\text { 'Sword-bead' with sword } \\
\text { and spearhead: white on } \\
\text { dark glass }\end{array}$ & Meaney 1981,96 and 196 \\
\hline Barrington & Cambs & $\begin{array}{l}\text { 5th } \\
\text { or } \\
\text { early } \\
\text { 6th } \mathrm{c}\end{array}$ & $\begin{array}{l}\text { Green band round top } \\
\text { and yellow zigzag (Plate 2); } \\
\text { ditto with yellow } \\
\text { zigzags }\end{array}$ & $\begin{array}{l}\text { Fox 1923, 250-2; } \\
\text { Ashmolean Museum; } \\
\text { Beck Collection, } \\
\text { Cambridge } 47.1830 \mathrm{c}\end{array}$ \\
\hline $\begin{array}{l}\text { Little } \\
\text { Wilbraham }\end{array}$ & Cambs & $\begin{array}{l}\text { 5th- } \\
\text { 6th } c\end{array}$ & $\begin{array}{l}\text { Grave 96: 'sword-bead' } \\
\text { with white star pattern; } \\
\text { Grave 44: another }\end{array}$ & $\begin{array}{l}\text { Evison 1967, 83, figs } 2 \mathrm{c} \\
\text { and d, pl VIIIc and d }\end{array}$ \\
\hline Mucking II & Essex & $\begin{array}{l}5 \text { th- } \\
6 \text { th c }\end{array}$ & $\begin{array}{l}\text { Grave } 351 \text { : wire-drawn } \\
\text { spindle-whorl/bead (Plate 2) } \\
\text { with } 2 \text { annular brooches and } \\
2 \text { scutiform pendants, etc; } \\
\text { Grave } 924 \mathrm{~B} \text { : wire-drawn } \\
\text { dark green/black plano- } \\
\text { convex spindle-whorl/bead } \\
\text { with applied brooch pair, } \\
\text { Kempston cone beaker, etc }\end{array}$ & $\begin{array}{l}\text { Hirst and Clark forthcoming; } \\
\text { BM }\end{array}$ \\
\hline Rainham & Essex & $\begin{array}{l}\text { prob } \\
\text { early } \\
\text { 6th c }\end{array}$ & $\begin{array}{l}\text { Very dark blue with petal- } \\
\text { like design, ?spindle-whorl } \\
\text { white design }\end{array}$ & $\begin{array}{l}\text { Evison } 1955,171 \text {, fig } 4.15 \text {; } \\
\text { BM }\end{array}$ \\
\hline $\begin{array}{l}\text { Springfield } \\
\text { Lyons }\end{array}$ & Essex & 6th $c$ & Grave $4735:$ white pattern & $\begin{array}{l}\text { Publication in } \\
\text { preparation (S Tyler); } \\
\text { BM }\end{array}$ \\
\hline $\begin{array}{l}\text { Canterbury, } \\
\text { Cakebread } \\
\text { Robey V }\end{array}$ & Kent & $c .400$ & $\begin{array}{l}\text { From multiple burial: } \\
\text { pendant, yellow scrawls } \\
\text { and green round hole and } \\
\text { edge }\end{array}$ & $\begin{array}{l}\text { Information } \mathrm{T} \text { Tatton- } \\
\text { Brown and } \mathrm{P} \text { Garrard }\end{array}$ \\
\hline $\begin{array}{l}\text { Kingston } \\
\text { or Barfeston } \\
\text { or } \\
\text { Sibertswold }\end{array}$ & Kent & & $\begin{array}{l}\text { Graves } 30-31 \text { : variants } \\
\text { with whitish design and } \\
\text { another yellow or } \\
\text { brownish }\end{array}$ & $\begin{array}{l}\text { Faussett } 1856 \text {, fig on p } 108 \text {; } \\
\text { Liverpool Museum: } \\
\text { only one eg found }\end{array}$ \\
\hline Richborough & Kent & $\begin{array}{l}\text { prob } \\
\text { late } \\
\text { 4th- } \\
\text { 5th c }\end{array}$ & $\begin{array}{l}\text { Stray find from Roman } \\
\text { fort: very dark green } \\
\text { with light green band and } \\
\text { yellowish zigzag }\end{array}$ & $\begin{array}{l}\text { Bushe-Fox } 1949,149 ; \\
\text { Richborough Museum, } \\
\text { no. c }\end{array}$ \\
\hline Brandon & Suffolk & $\begin{array}{l}\text { 7th- } \\
9 \text { th c }\end{array}$ & $\begin{array}{l}\text { Middle Saxon settlement: } \\
\text { variant with yellow on } \\
\text { dark glass }\end{array}$ & $\begin{array}{l}\text { Carr et al 1988; } \\
\text { publication in } \\
\text { preparation (R D Carr) }\end{array}$ \\
\hline
\end{tabular}




\begin{tabular}{|c|c|c|c|c|}
\hline $\begin{array}{l}\text { Holywell } \\
\text { Row }\end{array}$ & Suffolk & 6th $\mathrm{c}$ & $\begin{array}{l}\text { Grave 39: 'blue' and white } \\
\text { with } 2 \text { small-long brooches, } \\
\text { etc }\end{array}$ & $\begin{array}{l}\text { Lethbridge } 1931,21 \text {, } \\
\text { fig } 10.7 ; \\
\text { Cambridge Museum }\end{array}$ \\
\hline Fetcham & Surrey & $\begin{array}{l}\text { prob } \\
\text { early } \\
\text { 6th c }\end{array}$ & $\begin{array}{l}\text { 'Large glass bead of } \\
\text { clear green with wavy } \\
\text { line of white' (perhaps } \\
\text { not this type) }\end{array}$ & $\begin{array}{l}\text { Cotton 1933; } \\
\text { Guildford Museum }\end{array}$ \\
\hline Guildown & Surrey & $\begin{array}{l}\text { prob } \\
\text { early } \\
\text { 6th c }\end{array}$ & $\begin{array}{l}\text { Grave } 215 \text { 'sword-bead': } \\
\text { very dark blue }\end{array}$ & $\begin{array}{l}\text { Lowther 1931, 10-11, } \\
\text { 45, pl VIII.2; } \\
\text { Guildford Museum } 2369\end{array}$ \\
\hline Mitcham & Surrey & $\begin{array}{l}5 \text { th- } \\
\text { 6th c }\end{array}$ & $\begin{array}{l}\text { Grave } 223 \text { 'sword-bead': } \\
\text { found left of waist with } \\
\text { spearhead, knife and } \\
\text { tweezers }\end{array}$ & $\begin{array}{l}\text { Bidder and Morris 1959, } 74 \\
\text { 112, pl XVII.223; } \\
\text { Cambridge Museum }\end{array}$ \\
\hline Selmeston & Sussex & $\begin{array}{l}\text { prob } \\
\text { 6th } c\end{array}$ & Grave 11 & $\begin{array}{l}\text { Evison 1967, 84; } \\
\text { Meaney 1981, 96; } \\
\text { Welch 1983, 84; } \\
\text { Lewes Museum }\end{array}$ \\
\hline Wasperton & Warks & $\begin{array}{l}\text { first } \\
\text { half } \\
\text { 6th c }\end{array}$ & $\begin{array}{l}\text { Dated grave: large, } \\
\text { light translucent } \\
\text { green, looking dark }\end{array}$ & $\begin{array}{l}\text { Publication in } \\
\text { preparation (M Carver); } \\
\text { Warwick Museum }\end{array}$ \\
\hline Charlton & Wilts & & $\begin{array}{l}\text { Unprovenanced find: } \\
\text { atypical, perhaps } \\
\text { a whorl }\end{array}$ & $\begin{array}{l}\text { Davies } 1984,136,140 \text {, } \\
\text { fig 20.SF136; } \\
\text { Salisbury Museum }\end{array}$ \\
\hline
\end{tabular}

vii Large 'Black' Decorated Beads: (b) Biconvex (see Map 7)

\begin{tabular}{|c|c|c|c|c|}
\hline Abingdon & Berks & $\begin{array}{l}5 \text { th- } \\
7 \text { th } c\end{array}$ & $\begin{array}{l}\text { Stray find from } \\
\text { cemetery area }\end{array}$ & $\begin{array}{l}\text { Leeds and Harden 1936; } \\
\text { Ashmolean Museum } \\
1935.582\end{array}$ \\
\hline Silchester & Hants & $\begin{array}{l}\text { prob } \\
5 \text { th c }\end{array}$ & $\begin{array}{l}\text { (Plate 2) Occupation of } \\
\text { Roman town ends in early } \\
\text { 5th c; compare with } \\
\text { 'Frankish' beads of this date }\end{array}$ & $\begin{array}{l}\text { Guido } 1978,186 \text { and } \\
\text { pl II.10f; Boon } 1959 ; \\
\text { Reading Museum }\end{array}$ \\
\hline Richborough & Kent & & $\begin{array}{l}\text { Unstratified from Roman } \\
\text { fort: yellow waves, green } \\
\text { round edges (Plate } 2 \text { ) }\end{array}$ & $\begin{array}{l}\text { Bushe-Fox 1949, 149, } \\
\text { pl LV; } \\
\text { Richborough Museum }\end{array}$ \\
\hline Fonaby & Lincs & 6th c & $\begin{array}{l}\text { Grave 32: with Aberg } \\
\text { Group III cruciform brooch } \\
\text { and } 2 \text { small-long brooches }\end{array}$ & $\begin{array}{l}\text { Cook 1981,34, } \\
\text { fig 11.32.3; } \\
\text { Scunthorpe Museum }\end{array}$ \\
\hline $\begin{array}{l}\text { Peters- } \\
\text { finger }\end{array}$ & Wilts & $\begin{array}{l}\text { mid- } \\
6 \text { th c }\end{array}$ & $\begin{array}{l}\text { Grave } 20 \text { 'sword-bead': } \\
\text { yellow waves divided } \\
\text { by band with signs of } \\
\text { red with Style I buckle } \\
\text { set with garnet }\end{array}$ & $\begin{array}{l}\text { Leeds and Shortt 1953, } \\
\text { 16, pl I.58; } \\
\text { Salisbury Museum }\end{array}$ \\
\hline $\begin{array}{l}\text { Saltburn, } \\
\text { Hob Hill }\end{array}$ & Yorks & $\begin{array}{l}5 \text { th- } \\
7 \text { th c }\end{array}$ & Dark with white zigzags & $\begin{array}{l}\text { Gallagher } 1987,19,25 \text {, } \\
\text { fig 5.33; } \\
\text { Middlesbrough Museum }\end{array}$ \\
\hline
\end{tabular}




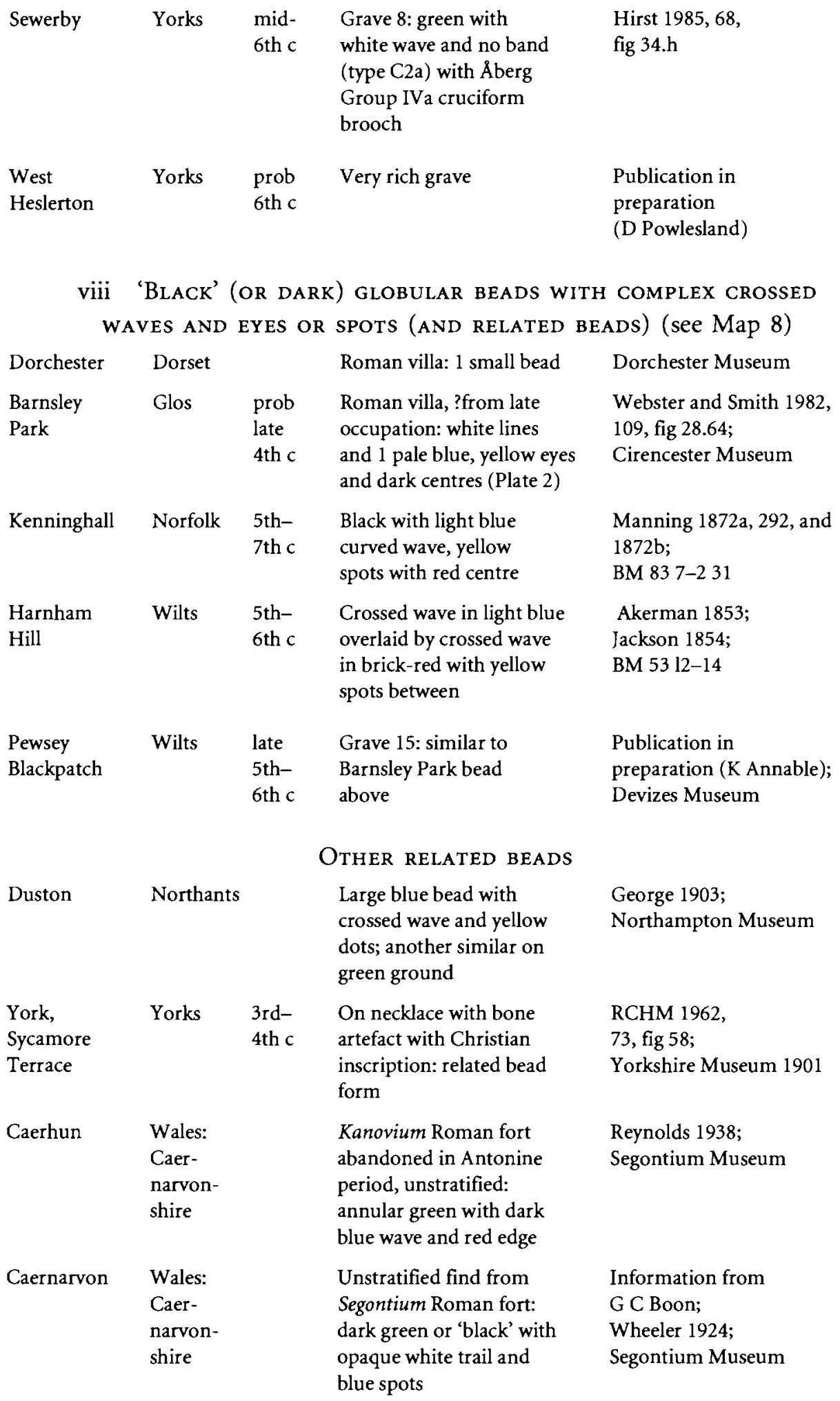




$\begin{array}{lllll}\text { Dinorben } & \text { Wales: } & \text { post- } & \text { Translucent cobalt blue } & \text { Gardner and Savory } \\ \text { Denbigh } & \begin{array}{l}\text { Rom- } \\ \text { an }\end{array} & \begin{array}{l}\text { with white crossed waves } \\ \text { and 1 white line, yellow eyes } \\ \text { with green centres }\end{array} & \begin{array}{l}\text { 1964; } \\ \text { Cardiff Museum }\end{array}\end{array}$

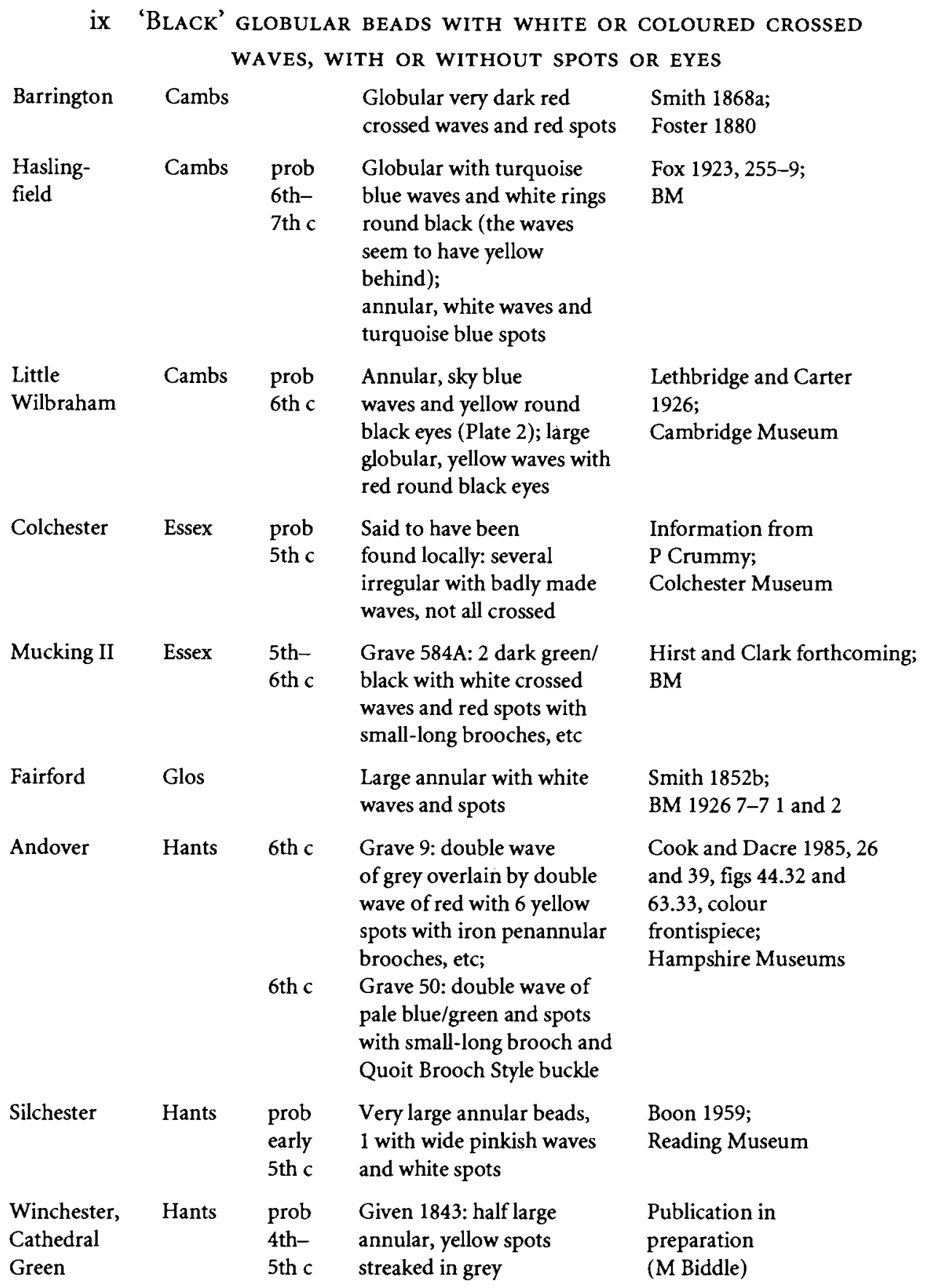




\begin{tabular}{|c|c|c|c|c|}
\hline $\begin{array}{l}\text { Winchester, } \\
\text { Wolvesley } \\
\text { Palace }\end{array}$ & Hants & $\begin{array}{l}\text { prob } \\
4 \text { th- } \\
5 \text { th c }\end{array}$ & $\begin{array}{l}\text { Globular, sky blue } \\
\text { loop and wave }\end{array}$ & $\begin{array}{l}\text { Publication in } \\
\text { preparation } \\
\text { (M Biddle) }\end{array}$ \\
\hline Winchester & Hants & & $\begin{array}{l}\text { Very irregular scrabble } \\
\text { with blue on yellow } \\
\text { waves }\end{array}$ & $\begin{array}{l}\text { Publication in } \\
\text { preparation } \\
\text { (M Biddle) }\end{array}$ \\
\hline $\begin{array}{l}\text { Bowcombe } \\
\text { Down }\end{array}$ & $\begin{array}{l}\text { Isle of } \\
\text { Wight }\end{array}$ & 6th $\mathrm{c}$ & $\begin{array}{l}\text { Grave 18: annular with } \\
\text { white crossed waves } \\
\text { (type } 6.21 \text { ) with button } \\
\text { brooch, etc }\end{array}$ & $\begin{array}{l}\text { Arnold } 1982,93 \text { and } 120, \\
\text { colour frontispiece; } \\
\text { Carisbrooke Museum }\end{array}$ \\
\hline $\begin{array}{l}\text { Canterbury, } \\
\text { Cakebread } \\
\text { Robey V }\end{array}$ & Kent & $c .400$ & $\begin{array}{l}\text { From multiple burial: } \\
\text { annular with yellow } \\
\text { curved waves and } \\
\text { turquoise spots }\end{array}$ & $\begin{array}{l}\text { Information T Tatton- } \\
\text { Brown and } \mathrm{P} \text { Garrard }\end{array}$ \\
\hline $\begin{array}{l}\text { Dover, } \\
\text { Buckland }\end{array}$ & Kent & $\begin{array}{l}650- \\
675\end{array}$ & $\begin{array}{l}\text { Grave 129: } 1 \text { barrel with } \\
\text { white trails and terracotta } \\
\text { dots (D53) }\end{array}$ & $\begin{array}{l}\text { Evison } 1987,74 \text { and } 244, \\
\text { fig 53.5h, colour } \\
\text { pl IV (D53); BM }\end{array}$ \\
\hline $\begin{array}{l}\text { Eastry, } \\
\text { Eastry House }\end{array}$ & Kent & $\begin{array}{l}\text { late } \\
\text { 6th } c\end{array}$ & $\begin{array}{l}\text { Biconical with } \\
\text { Gotlandic bow brooch, etc }\end{array}$ & $\begin{array}{l}\text { Hawkes } 1979,86,93 \\
\text { fig 4.4.20; Deal Museum }\end{array}$ \\
\hline Faversham & Kent & $\begin{array}{l}5 \text { th- } \\
7 \text { th } c\end{array}$ & $\begin{array}{l}\text { One large annular and } 1 \\
\text { smaller, yellow crossed } \\
\text { waves and spots; } \\
1 \text { large globular, blue } \\
\text { crossed waves and yellow } \\
\text { spots }\end{array}$ & $\begin{array}{l}\text { Meaney } 1964,118-19 ; \\
\text { Beck Collection, } \\
\text { Cambridge } 47.1822\end{array}$ \\
\hline Finglesham & Kent & $\begin{array}{l}\text { prob } \\
\text { 6th- } \\
7 \text { th c }\end{array}$ & $\begin{array}{l}\text { Small bead, yellow } \\
\text { waves and red spots; } \\
\text { cf Howletts below }\end{array}$ & $\begin{array}{l}\text { Chadwick 1958, 1-7; } \\
\text { Deal Museum }\end{array}$ \\
\hline Howletts & Kent & $\begin{array}{l}\text { late } \\
5 \text { th- } \\
6 \text { th c } \\
5 \text { th- } \\
6 \text { th c }\end{array}$ & $\begin{array}{l}\text { Several hardly } \\
\text { recognizable examples; } \\
\text { Approx } 8 \text { very dark beads, } \\
\text { some with crossed waves; } \\
\text { barrel-shaped, yellow wave } \\
\text { with red spots }\end{array}$ & $\begin{array}{l}\text { Smith 1918; } \\
\text { BM } 1936 \text { 5-11 } 143\end{array}$ \\
\hline Richborough & Kent & $\begin{array}{l}\text { prob } \\
5 \text { th c }\end{array}$ & $\begin{array}{l}\text { Annular with white } \\
\text { waves and blue spots }\end{array}$ & $\begin{array}{l}\text { Bushe-Fox 1949, 149, } \\
\text { no. } 237, \text { pl LV; } \\
\text { Richborough Museum }\end{array}$ \\
\hline Stowting & Kent & $\begin{array}{l}\text { prob } \\
6 \text { th- } \\
7 \text { th c } \\
\text { prob } \\
6 \text { th c }\end{array}$ & $\begin{array}{l}\text { Grave 15: barrel-shaped, } \\
\text { yellow top and bottom, } \\
\text { yellow zigzag related } \\
\text { form only; } \\
\text { small globular, white } \\
\text { crossed waves and spots }\end{array}$ & $\begin{array}{l}\text { Beck Collection, } \\
\text { Cambridge Museum } 47.1800\end{array}$ \\
\hline $\begin{array}{l}\text { London, } \\
\text { Milk Street }\end{array}$ & London & & $\begin{array}{l}\text { Two annular with cream } \\
\text { crossed waves }\end{array}$ & Museum of London \\
\hline
\end{tabular}




\begin{tabular}{|c|c|c|c|c|}
\hline Brooke & Norfolk & $\begin{array}{l}\text { prob } \\
\text { 6th } c\end{array}$ & $\begin{array}{l}\text { Small annular, light } \\
\text { blue crossed waves }\end{array}$ & $\begin{array}{l}\text { Meaney } 1964,170 \text {; } \\
\text { BM } 186669\end{array}$ \\
\hline $\begin{array}{l}\text { Caistor } \\
\text { by Norwich }\end{array}$ & Norfolk & $\begin{array}{l}5 \text { th- } \\
7 \text { th c }\end{array}$ & $\begin{array}{l}\text { Red crossed waves and } \\
\text { white spots }\end{array}$ & $\begin{array}{l}\text { Myres and Green } 1973 \text {; } \\
\text { Norwich Museum }\end{array}$ \\
\hline Illington & Norfolk & 6th $\mathrm{c}$ & $\begin{array}{l}\text { With urn no. } 173 \mathrm{a}: \\
\text { white crossed waves }\end{array}$ & $\begin{array}{l}\text { Davison et al 1993, 35, } \\
\text { fig 20.173a; } \\
\text { Norwich Museum }\end{array}$ \\
\hline Ipswich & Suffolk & $\begin{array}{l}6 \text { th- } \\
7 \text { th } \mathrm{c}\end{array}$ & $\begin{array}{l}\text { Globular, white waves } \\
\text { and spots }\end{array}$ & $\begin{array}{l}\text { Layard } 1907 \text { and 1909; } \\
\text { Ipswich Museum }\end{array}$ \\
\hline Guildown & Surrey & $\begin{array}{l}\text { 5th- } \\
6 \text { th } c\end{array}$ & $\begin{array}{l}\text { Grave } 78 \text { : white waves and } \\
\text { spots with small-long } \\
\text { brooch pair, etc; } \\
\text { another with red waves } \\
\text { and spots }\end{array}$ & $\begin{array}{l}\text { Lowther } 1931,12,36 \text {, } \\
\text { pl IX.7; } \\
\text { Guildford Museum }\end{array}$ \\
\hline Alfriston & Sussex & 6th $c$ & $\begin{array}{l}\text { Grave 43: large annular, } \\
\text { crossed waves and turquoise } \\
\text { spots with great square- } \\
\text { headed brooch, etc }\end{array}$ & $\begin{array}{l}\text { Griffith and Salzmann } \\
\text { 1914, 40, pl I.9; } \\
\text { Welch 1983,84; } \\
\text { Lewes Museum }\end{array}$ \\
\hline $\begin{array}{l}\text { Stretton- } \\
\text { on-Fosse }\end{array}$ & Warks & & $\begin{array}{l}\text { Large annular, cream } \\
\text { waves and turquoise spots } \\
\text { (Plate 2); another badly } \\
\text { made }\end{array}$ & $\begin{array}{l}\text { Gelling 1992, 31, 40-1, } \\
\text { fig 21; } \\
\text { Warwick Museum }\end{array}$ \\
\hline Wasperton & Warks & $\begin{array}{l}\text { first } \\
\text { half } \\
\text { 6th c } \\
\text { 6th c }\end{array}$ & $\begin{array}{l}\text { White waves and spots } \\
\text { with cruciform brooch; } \\
\text { F365: globular, white } \\
\text { waves and spots }\end{array}$ & $\begin{array}{l}\text { Publication in } \\
\text { preparation (M Carver); } \\
\text { Warwick Museum }\end{array}$ \\
\hline Sewerby & Yorks & 6th $\mathrm{c}$ & $\begin{array}{l}\text { Grave } 12: 1 \text { annular with } \\
\text { white waves, bright red } \\
\text { spots, small hole (type C3) } \\
\text { with A Aberg Group IVb } \\
\text { and Group II cruciform } \\
\text { brooches, small-long brooch }\end{array}$ & Hirst 1985,68 , fig 36d \\
\hline
\end{tabular}

\section{$X$ 'Black' BEADS WITH A FEW SPOTS (MOSTLY ANNULAR BEADS)}

$\begin{array}{lllll}\text { Lechlade } & \text { Glos } & \text { 6th c } & \begin{array}{l}\text { Grave 25: dark blue } \\ \text { biconical scatter of } \\ \text { white spots (Plate 2) with } \\ \text { cast saucer brooch, etc; } \\ \text { Unstratified: but similar }\end{array} & \begin{array}{l}\text { Boyle } \text { et al forthcoming; } \\ \text { Cirencester Museum }\end{array} \\ \text { Droxford } & \text { Hants } & \begin{array}{l}\text { 5th- } \\ \text { 6th c }\end{array} & \begin{array}{l}\text { Unstratified: globular } \\ \text { with white spots (class Ie) }\end{array} & \begin{array}{l}\text { Aldsworth 1978, 172, } \\ \text { fig 36.69; BM }\end{array} \\ \begin{array}{l}\text { Winchester, } \\ \text { Cathedral } \\ \text { Green }\end{array} & \text { Hants } & & \begin{array}{l}\text { Very dark brown, large } \\ \text { opaque, few big blobs } \\ \text { in yellow or grey with } \\ \text { bluish bands }\end{array} & \begin{array}{l}\text { Publication in } \\ \text { preparation } \\ \text { (M Biddle) }\end{array} \\ & & & & \end{array}$




\begin{tabular}{|c|c|c|c|c|}
\hline $\begin{array}{l}\text { Chessell } \\
\text { Down }\end{array}$ & $\begin{array}{l}\text { Isle of } \\
\text { Wight }\end{array}$ & 6th $c$ & $\begin{array}{l}\text { Annular, white spots } \\
\text { (type } 6.18 \text { ) }\end{array}$ & $\begin{array}{l}\text { Arnold } 1982,50 \text { and } 120 \text {, } \\
\text { colour frontispiece; } \mathrm{BM}\end{array}$ \\
\hline $\begin{array}{l}\text { Dover, } \\
\text { Priory Hill }\end{array}$ & Kent & $\begin{array}{l}\text { late } \\
6 \text { th c } \\
\text { spots }\end{array}$ & $\begin{array}{l}\text { Long, oval slightly } \\
\text { cylindrical, red and white }\end{array}$ & $\begin{array}{l}\text { Meaney } 1964,117 \\
\text { Dover Museum }\end{array}$ \\
\hline Faversham & Kent & $\begin{array}{l}5 \text { th- } \\
7 \text { th } \mathrm{c}\end{array}$ & $\begin{array}{l}\text { Small with greyish } \\
\text { white spots }\end{array}$ & $\begin{array}{l}\text { Meaney } 1964,118-19 ; \\
\text { Ashmolean } \\
\text { Museum } 1909.142\end{array}$ \\
\hline Guildown & Surrey & 6 th $\mathrm{c}$ & $\begin{array}{l}\text { Grave } 78 \text { : annular, } \\
\text { white spots round } \\
\text { circumference }\end{array}$ & $\begin{array}{l}\text { Lowther } 1931,12,36 \text {, } \\
\text { pl IX.7; } \\
\text { Guildford Museum }\end{array}$ \\
\hline \multirow[t]{2}{*}{ Kilham } & Yorks & & $\begin{array}{l}\text { 'Black' with white blobs, } \\
\text { but this may be a later } \\
\text { bead }\end{array}$ & $\begin{array}{l}\text { Meaney } 1964,292 ; \\
\text { Yorkshire Museum }\end{array}$ \\
\hline & xi 'BLA & \multicolumn{3}{|c|}{$\begin{array}{l}\text { K' GLOBULAR BEADS WITH COLOURED SPECKS } \\
\text { ('CRUMB' BEADS) (see Map 9) }\end{array}$} \\
\hline $\begin{array}{l}\text { Hasling- } \\
\text { field }\end{array}$ & Cambs & $\begin{array}{l}\text { prob } \\
6 \text { th } c\end{array}$ & $\begin{array}{l}\text { Globular with white } \\
\text { and red specks }\end{array}$ & Fox $1923,255-9$ \\
\hline $\begin{array}{l}\text { Little } \\
\text { Wilbraham }\end{array}$ & Cambs & $\begin{array}{l}\text { prob } \\
6 \text { th c }\end{array}$ & $\begin{array}{l}\text { Globular with white } \\
\text { and red specks }\end{array}$ & $\begin{array}{l}\text { Lethbridge and Carter } \\
\text { 1926; Cambridge Museum }\end{array}$ \\
\hline Penzance & Cornwall & & $\begin{array}{l}\text { Surface find from } \\
\text { Trencrom Fort: annular } \\
\text { red and green specks }\end{array}$ & $\begin{array}{l}\text { Information from Miss } \\
\text { D Dudley }\end{array}$ \\
\hline Mucking II & Essex & $\begin{array}{l}\text { first } \\
\text { half } \\
5 \text { th } c \\
\text { etc }\end{array}$ & $\begin{array}{l}\text { Grave } 989: 1 \text { bead with } \\
\text { Glaston-Mucking and } \\
\text { supporting-arm brooches, }\end{array}$ & $\begin{array}{l}\text { Hirst and Clark forthcoming; } \\
\text { Evison 1981, 138-9, } \\
\text { figs } 4 \text { and } 5 ; \\
\text { BM }\end{array}$ \\
\hline Alton & Hants & 6 th $c$ & $\begin{array}{l}\text { Grave } 23: 2 \text { black } \\
\text { annulars with white } \\
\text { and terracotta specks } \\
\text { (D15) with saucer } \\
\text { brooches, etc }\end{array}$ & $\begin{array}{l}\text { Evison 1988a, } 17 \text { and } 77 \text {, } \\
\text { fig 29.4if; } \\
\text { Hampshire Museums }\end{array}$ \\
\hline $\begin{array}{l}\text { St Neots, } \\
\text { Lords } \\
\text { Bridge }\end{array}$ & Hunts & & $\begin{array}{l}\text { Two globulars with } \\
\text { varied coloured specks }\end{array}$ & BM $195610-2,1$ and 2 \\
\hline Howletts & Kent & & $\begin{array}{l}\text { Annular with white } \\
\text { specks }\end{array}$ & $\begin{array}{l}\text { Smith 1918; } \\
\text { BM 1918 7-8 } 47\end{array}$ \\
\hline Sarre & Kent & $\begin{array}{l}\text { prob } \\
6 \text { th } c\end{array}$ & $\begin{array}{l}\text { Globular with white } \\
\text { and red specks }\end{array}$ & $\begin{array}{l}\text { Meaney 1964, 135-6; } \\
\text { BM OA } 4905\end{array}$ \\
\hline Kenninghall & Norfolk & $\begin{array}{l}5 \text { th- } \\
7 \text { th c }\end{array}$ & $\begin{array}{l}\text { Globular with red and } \\
\text { yellow specks (Plate } 2 \text { ) }\end{array}$ & $\begin{array}{l}\text { Manning } 1872 \mathrm{a}, 292 \text {, and } \\
1872 \mathrm{~b} ; \mathrm{BM}\end{array}$ \\
\hline Duston & Northants & $\begin{array}{l}\text { prob } \\
6 \text { th } c\end{array}$ & $\begin{array}{l}\text { Two globular with } \\
\text { yellow and reddish specks }\end{array}$ & $\begin{array}{l}\text { George 1903; } \\
\text { Northampton Museum }\end{array}$ \\
\hline
\end{tabular}




\begin{tabular}{|c|c|c|c|c|}
\hline $\begin{array}{l}\text { Holme } \\
\text { Pierrepont }\end{array}$ & Notts & $\begin{array}{l}\text { prob } \\
\text { 6th c }\end{array}$ & $\begin{array}{l}\text { Globular with white } \\
\text { and red specks with } \\
\text { dated brooches }\end{array}$ & $\begin{array}{l}\text { Myres 1977, Corpus } \\
\text { no. 209, fig 303; } \\
\text { BM 1931 3-13 }\end{array}$ \\
\hline $\begin{array}{l}\text { Fornham } \\
\text { All Saints }\end{array}$ & Suffolk & $\begin{array}{l}\text { prob } \\
\text { 6th c }\end{array}$ & $\begin{array}{l}\text { Globular with yellow and } \\
\text { red specks }\end{array}$ & BM $19584-61$ \\
\hline Guildown & Surrey & 6th $c$ & $\begin{array}{l}\text { Grave } 185: 2 \text { with red } \\
\text { annular with stamp- } \\
\text { ornamented pot; } \\
\text { Grave 206: } 2 \text { globular } \\
\text { with square-headed } \\
\text { brooch pair, etc }\end{array}$ & $\begin{array}{l}\text { Lowther } 1931,11,43 \text {, } \\
\text { 44, pl IX.1; } \\
\text { Guildford Museum }\end{array}$ \\
\hline Churchover & Warks & $\begin{array}{l}\text { 6th- } \\
7 \text { th c }\end{array}$ & $\begin{array}{l}\text { Globular with white and } \\
\text { red specks }\end{array}$ & $\begin{array}{l}\text { Bloxam 1884; } \\
\text { Warwick Museum A.1430 }\end{array}$ \\
\hline Wasperton & Warks & $\begin{array}{l}\text { prob } \\
\text { 6th c }\end{array}$ & $\begin{array}{l}\text { Group } 1267 / 1 \text { and F365: } \\
\text { globular with red } \\
\text { and white specks }\end{array}$ & $\begin{array}{l}\text { Publication in } \\
\text { preparation (M Carver); } \\
\text { Warwick Museum }\end{array}$ \\
\hline 'E.Yorks' & Yorks & 7th $c$ & $\begin{array}{l}\text { Globular with red and white } \\
\text { specks, dated by other. } \\
\text { beads }\end{array}$ & Hull Museum \\
\hline Hornsea & Yorks & 6th $c$ & $\begin{array}{l}\text { Two with multicoloured } \\
\text { specks }\end{array}$ & $\begin{array}{l}\text { Meaney 1964, 291; } \\
\text { Yorkshire Museum }\end{array}$ \\
\hline $\begin{array}{l}\text { Traprain } \\
\text { Law }\end{array}$ & $\begin{array}{l}\text { Scotland: } \\
\text { East } \\
\text { Lothian }\end{array}$ & & $\begin{array}{l}\text { Post-Roman occupation } \\
\text { of native stronghold: } \\
\text { annular with red, green and } \\
\text { yellow specks }\end{array}$ & $\begin{array}{l}\text { Edinburgh Museum } \\
1932.101\end{array}$ \\
\hline \multirow[t]{2}{*}{ Caerleon } & \multicolumn{2}{|c|}{$\begin{array}{l}\text { Wales: } \\
\text { Monmouths }\end{array}$} & $\begin{array}{l}\text { Roman ?fort site: } \\
\text { unstratified find }\end{array}$ & \\
\hline & xii & 'BLACK' & \multicolumn{2}{|c|}{ DECORATED LONG CYLINDER BEADS } \\
\hline Pangbourne & Berks & & $\begin{array}{l}\text { Almost opaque ?dark } \\
\text { blue, zigzag round } \\
\text { centre and band each end }\end{array}$ & $\begin{array}{l}\text { Beck Collection, } \\
\text { Cambridge Museum } \\
1696\end{array}$ \\
\hline Colchester & Essex & $\begin{array}{l}\text { late } \\
\text { Roman }\end{array}$ & $\begin{array}{l}\text { Grave: exotic, black with } \\
\text { yellow bands each } \\
\text { end and suspension loop }\end{array}$ & $\begin{array}{l}\text { Crummy 1983, } 35 \text {, } \\
\text { fig } 37.1504 \text {; } \\
\text { Colchester Museum, } \\
\text { no. } 169\end{array}$ \\
\hline Blakeney & Glos & $\begin{array}{l}\text { late } \\
\text { Roman }\end{array}$ & $\begin{array}{l}\text { Central zigzag in white } \\
\text { overlaid by yellow }\end{array}$ & $\begin{array}{l}\text { Information from } \\
\text { M Johns }\end{array}$ \\
\hline Cirencester & Glos & $\begin{array}{l}\text { late } \\
\text { Roman }\end{array}$ & $\begin{array}{l}\text { Grave: } 2 \text { beads, } 1 \text { shiny } \\
\text { black with yellow end } \\
\text { bands and central scrawl } \\
\text { (Plate } 3 \text { ); another (half only) } \\
\text { with white end bands } \\
\text { and zigzag }\end{array}$ & Cirencester Museum \\
\hline
\end{tabular}




\begin{tabular}{|c|c|c|c|c|}
\hline Richborough & Kent & $\begin{array}{l}\text { prob } \\
\text { late } \\
\text { Roman }\end{array}$ & $\begin{array}{l}\text { Dark olive green, } \\
\text { very burnt and distorted }\end{array}$ & $\begin{array}{l}\text { Information from } \\
\text { G C Dunning; } \\
\text { Richborough Museum, no.e }\end{array}$ \\
\hline Camerton & Somerset & 7th $\mathrm{c}$ & $\begin{array}{l}\text { Dark olive green, } \\
\text { yellowish wire-drawn } \\
\text { wave round centre and } \\
\text { band each end, perhaps } \\
\text { originally white }\end{array}$ & $\begin{array}{l}\text { Beck 1933; } \\
\text { Taunton Museum }\end{array}$ \\
\hline $\begin{array}{l}\text { near } \\
\text { Risby }\end{array}$ & Suffolk & 6 th $c$ & $\begin{array}{l}\text { Found } 1869 \text { by Canon } \\
\text { Greenwell: 'black' with } \\
\text { turquoise blue zigzag } \\
\text { and end bands }\end{array}$ & $\begin{array}{l}\text { Meaney 1964, 232; } \\
\text { Bury St Edmunds } \\
\text { Museum }\end{array}$ \\
\hline Alfriston & Sussex & $\begin{array}{l}\text { later } \\
5 \text { th } c\end{array}$ & $\begin{array}{l}\text { Grave 103: with Quoit } \\
\text { Brooch Style buckle }\end{array}$ & $\begin{array}{l}\text { Griffith } 1915,207 \text {, } \\
\text { pl XXII.3; Evison 1965; } \\
\text { Welch 1983, 84, fig 40c; } \\
\text { Lewes Museum }\end{array}$ \\
\hline \multirow[t]{4}{*}{$\begin{array}{l}\text { Peters- } \\
\text { finger }\end{array}$} & Wilts & $\begin{array}{l}5 \text { th- } \\
\text { 6th c }\end{array}$ & $\begin{array}{l}\text { Grave 29: like the } \\
\text { Cirencester bead above, } \\
\text { but red instead of } \\
\text { yellow (Plate } 3 \text { ), with } \\
2 \text { applied saucer brooch } \\
\text { bases, iron inlaid buckle, etc }\end{array}$ & $\begin{array}{l}\text { Leeds and Shortt 1953, } \\
25 \text { (104.j), } \\
\text { pl III.104; } \\
\text { Salisbury Museum }\end{array}$ \\
\hline & xiii LONG & FLUTED & $\begin{array}{l}\text { BEADS BANDED IN 'BLAC } \\
\text { no schedule (Plate } 3 \text { ) }\end{array}$ & CK' AND WHITE: \\
\hline & \multicolumn{4}{|c|}{$\begin{array}{r}\text { SMALL CYLINDRICAL DARK BEADS WITH UN } \\
\text { ENDS AND CENTRAL BAND: } \\
\text { no schedule (Plate } 3 \text { ) }\end{array}$} \\
\hline & XV LARGE & \multicolumn{3}{|c|}{$\begin{array}{c}\text { SE GLOBULAR DARK BEADS WITH SE } \\
\text { OF COLOURED ZIGZAGS }\end{array}$} \\
\hline Abingdon & Berks & 6th $\mathrm{c}$ & $\begin{array}{l}\text { Grave B117: dark brown/ } \\
\text { black with sky blue zigzag } \\
\text { between } 2 \text { white ones } \\
\text { (Plate 3), found with small } \\
\text { square-headed brooch }\end{array}$ & $\begin{array}{l}\text { Leeds and Harden } 1936 \text {, } \\
54, \mathrm{pl} \text { XVI.117; } \\
\text { Ashmolean Museum } \\
\text { 1935.61b }\end{array}$ \\
\hline Mucking II & Essex & $\begin{array}{l}\text { prob } \\
\text { 6th c }\end{array}$ & $\begin{array}{l}\text { Grave 944: very large } \\
\text { barrel, dark green/black, } \\
2 \text { white waves and } 1 \text { green/ } \\
\text { blue in between (Plate } 3 \text { ) } \\
\text { with } 2 \text { annular brooches }\end{array}$ & $\begin{array}{l}\text { Hirst and Clark forthcoming; } \\
\text { BM }\end{array}$ \\
\hline Cleatham & Lincs & $\begin{array}{l}5 \text { th- } \\
\text { 6th c }\end{array}$ & $\begin{array}{l}\text { Very burnt black green } \\
\text { with } 1 \text { remaining chevron } \\
\text { in white; possibly more } \\
\text { originally }\end{array}$ & $\begin{array}{l}\text { Publication in } \\
\text { preparation } \\
\text { (K Leahy); } \\
\text { Scunthorpe Museum }\end{array}$ \\
\hline
\end{tabular}




\begin{tabular}{|c|c|c|c|c|}
\hline Highdown & Sussex & $\begin{array}{l}\text { late } \\
5 \text { th- } \\
\text { early } \\
\text { 6th c }\end{array}$ & $\begin{array}{l}\text { Grave } 31 \text { : greenish black } \\
\text { with } 3 \text { white zigzags } \\
\text { and a band at top and } \\
\text { bottom }\end{array}$ & $\begin{array}{l}\text { Read } 1895, \text { pl } 31.10 ; \\
\text { Welch } 1983,84 \text {, fig } 99 \mathrm{~b} ; \\
\text { Worthing Museum }\end{array}$ \\
\hline Sewerby & Yorks & 6th $c$ & $\begin{array}{l}\text { Grave } 41 \text { : small 'black' } \\
\text { (blue) rather biconical, } \\
2 \text { rows of white zigzags } \\
\text { (C2b) with annular brooch } \\
\text { pair }\end{array}$ & Hirst 1985,68 , fig $48 d$ \\
\hline & i TALL & \multicolumn{2}{|c|}{$\begin{array}{c}\text { DRUM-SHAPED DARK BEADS WITH } \\
\text { OF COLOURED ZIGZAGS }\end{array}$} & SEVERAL LINES \\
\hline Mucking II & Essex & $\begin{array}{l}\text { 2nd } \\
\text { half } \\
5 \text { th c }\end{array}$ & $\begin{array}{l}\text { Grave } 334: 2 \text { cylinders } \\
\text { opaque green with red } \\
\text { band above and below } \\
\text { yellow zigzag with disc } \\
\text { brooch pair, inlaid buckle, } \\
\text { etc }\end{array}$ & $\begin{array}{l}\text { Hirst and Clark forthcoming; } \\
\text { BM }\end{array}$ \\
\hline $\begin{array}{l}\text { Castle } \\
\text { Bytham }\end{array}$ & Lincs & 7th $c$ & $\begin{array}{l}\text { Dark (?blue) with } 2 \text { white } \\
\text { zigzags, quoit brooch, etc }\end{array}$ & $\begin{array}{l}\text { Akerman 1855a, 26-7, } \\
\text { pl XII.4 }\end{array}$ \\
\hline $\begin{array}{l}\text { near } \\
\text { Farnham }\end{array}$ & Surrey & & $\begin{array}{l}\text { Dark ground with } 2 \\
\text { yellow zigzags and } \\
\text { creamy central band, } \\
\text { with knife, clasp, etc }\end{array}$ & Morris 1959,140 \\
\hline Churchover & Warks & & $\begin{array}{l}\text { Dark rather drum-shaped, } \\
\text { central longwise zigzag } \\
\text { and white band at top and } \\
\text { bottom }\end{array}$ & $\begin{array}{l}\text { Bloxam 1884; } \\
\text { Warwick Museum }\end{array}$ \\
\hline
\end{tabular}




\title{
SCHEDUle 3
}

\section{White (OR BLUE-WHITE) GLASS BEADS}

\author{
MONOCHROME \\ i Opaque white annular, globular and short barrel-shaped single or double beads \\ ii Opaque white cylinder beads: round and polygonal, short single or double \\ beads (see Map 10)
}

Polychrome

iii Opaque white beads: (a) with light blue crossed waves without dots, singles or doubles (see Map 11)

(b) with light blue crossed waves with blue dots (see Map 12)

(c) with light blue crossed waves with red dots (see Map 13)

(d) with green or terracotta-coloured crossed waves, with or without dots (see Map 14)

iv Miscellaneous opaque white beads with spots, single waves, etc: no schedule

v Opaque white globular or biconical beads with red or blue spots

vi Opaque white cylinder beads with coloured wound bands

\section{MONOCHROME}

\section{i OPAQUe WHite ANNULAR, GLOBULAR AND SHORT BARREL-SHAPED} SINGLE OR DOUBLE BEADS

\begin{tabular}{|c|c|c|c|c|}
\hline $\begin{array}{l}\text { Long } \\
\text { Wittenham }\end{array}$ & Berks & $\begin{array}{l}5 \text { th- } \\
6 \text { th c } \\
5 \text { th- } \\
6 \text { th c }\end{array}$ & $\begin{array}{l}\text { Grave } 52: 3 \text { annulars } \\
\text { with disc brooches; } \\
\text { Grave } 96: \text { with small- } \\
\text { long brooch }\end{array}$ & Akerman 1860 \\
\hline Bishopstone & Bucks & $\begin{array}{l}\text { prob } \\
6 \text { th } c\end{array}$ & $\begin{array}{l}\text { Several annular with } \\
\text { saucer brooches, etc }\end{array}$ & $\begin{array}{l}\text { Meaney 1964, 56; } \\
\text { Aylesbury Museum }\end{array}$ \\
\hline $\begin{array}{l}\text { Newport } \\
\text { Pagnell }\end{array}$ & Bucks & $\begin{array}{l}\text { prob } \\
6 \text { th c }\end{array}$ & Necklace with 2 annulars & $\begin{array}{l}\text { Meaney 1964, } 58 \\
\text { Aylesbury Museum }\end{array}$ \\
\hline $\begin{array}{l}\text { Linton } \\
\text { Heath }\end{array}$ & Cambs & & Few annulars or globulars & $\begin{array}{l}\text { Neville 1854; } \\
\text { Cambridge Museum }\end{array}$ \\
\hline
\end{tabular}




\begin{tabular}{|c|c|c|c|}
\hline $\begin{array}{l}\text { Norton-on- } \\
\text { Tees }\end{array}$ & Durham & 6th $c$ & $\begin{array}{l}\text { Grave 7: } 1 \text { bead (Ala) } \\
\text { (possibly modern) }\end{array}$ \\
\hline Mucking II & Essex & $\begin{array}{l}\text { 2nd } \\
\text { half } \\
5 \text { th c } \\
5 \text { th- } \\
6 \text { th c } \\
\text { 2nd } \\
\text { half } \\
\text { 5th c } \\
\text { 2nd } \\
\text { half } \\
\text { 5th c } \\
\text { 7th c } \\
\text { 6th c }\end{array}$ & $\begin{array}{l}\text { Grave 334: } 17 \text { beads with } \\
\text { disc brooch pair, amber } \\
\text { beads, inlaid buckle, etc; } \\
\text { Grave } 351: 1 \text { disc bead } \\
\text { with } 2 \text { annular brooches and } \\
2 \text { scutiform pendants, etc; } \\
\text { Grave } 397: 1 \text { globular with } \\
\text { small-long and annular } \\
\text { brooches; } \\
\text { Grave } 548: 1 \text { bead with } \\
\text { pennanular quoit brooch, } \\
\text { etc; } \\
\text { Grave } 608: 1 \text { double } \\
\text { annular; } \\
\text { Grave } 615: 1 \text { annular with } \\
\text { cast saucer brooch pair, } \\
\text { etc; } \\
\text { Grave } 845: 15 \text { discs (Plate } 3 \text { ) } \\
\text { with disc brooch pair, etc; } \\
\text { Grave } 936: 2 \text { annulars with } \\
\text { small square-headed } \\
\text { brooch pair, etc }\end{array}$ \\
\hline
\end{tabular}

Prittlewell Essex c.600 Grave-group 32: with cast saucer brooch pair

\begin{tabular}{|c|c|c|c|}
\hline $\begin{array}{l}\text { Springfield } \\
\text { Lyons }\end{array}$ & Essex & $\begin{array}{l}\text { prob } \\
\text { mid- } \\
\text { 6th c }\end{array}$ & Grave 4578: globular \\
\hline Lechlade & Glos & 6th $c$ & $\begin{array}{l}\text { Grave 48: 'disc' with } \\
\text { buckle }\end{array}$ \\
\hline Andover & Hants & $\begin{array}{l}\text { late } \\
\text { 5th- } \\
\text { 6th c }\end{array}$ & $\begin{array}{l}\text { Grave 19: two ( } 15 \text { and } 21 \text { ) } \\
\text { with small-long brooches; } \\
\text { Grave 50: one ( } 32 \text { ) with } \\
\text { small-long brooch, late } \\
\text { Roman buckle, etc }\end{array}$ \\
\hline Droxford & Hants & $\begin{array}{l}\text { late } \\
5 \text { th- } \\
\text { 6th c }\end{array}$ & $\begin{array}{l}\text { Unstratified: } 1 \text { globular } \\
\text { (class XVIa) }\end{array}$ \\
\hline $\begin{array}{l}\text { Chessell } \\
\text { Down }\end{array}$ & $\begin{array}{l}\text { Isle of } \\
\text { Wight }\end{array}$ & $\begin{array}{l}\text { prob } \\
\text { 6th c }\end{array}$ & $\begin{array}{l}\text { Fourteen annulars } \\
\text { (type } 6.8 \text { ) }\end{array}$ \\
\hline
\end{tabular}

$\begin{array}{llll}\text { Breach } & \text { Kent } & \text { prob } & \begin{array}{l}\text { Necklace including } \\ \text { Down }\end{array} \\ \text { 7th } \mathrm{c} & \text { several }\end{array}$

Sherlock and Welch 1992, 125, fig 34.7.1

Hirst and Clark forthcoming; BM

Tyler 1988, 108, 113, fig 15;

Southend Museum

Publication in preparation (S Tyler); BM

Boyle et al forthcoming; Cirencester Museum

Cook and Dacre 1985, 28, 38, 81-7, figs 47 and 63.32, Table X, colour pl frontispiece; Hampshire Museums

Aldsworth 1978, 138 and 173, fig 36.63;

BM

Arnold 1982, 49 and 120, colour pl frontispiece; BM 67 7-29 146 and 148, $6910-114$

Conyngham and Akerman 1844, pl 1;

BM 79 5-24 29 and 89 


\begin{tabular}{|c|c|c|c|c|}
\hline $\begin{array}{l}\text { Dover, } \\
\text { Buckland }\end{array}$ & Kent & $\begin{array}{l}625- \\
650 \\
650- \\
675\end{array}$ & $\begin{array}{l}\text { Grave 1: } 1 \text { 'disc' (B57); } \\
\text { Grave 30: } 6 \text { 'discs' (B52); } \\
\text { Grave 35: } 1 \text { 'disc', (B57); } \\
\text { Grave 59: } 3 \text { 'discs' (B52); } \\
\text { Grave 60: } 1 \text { 'disc' (B52); } \\
\text { Grave 62: } 1 \text { 'disc' (B52); } \\
\text { Grave 6: } 1 \text { 'disc' (B57); } \\
\text { Grave 129: } 1 \text { 'disc' and } \\
1 \text { biconical (B52 and B53) }\end{array}$ & $\begin{array}{l}\text { Evison } 1987,74,80-1,216 \text {, } \\
217,224-5,226,232-3 \text {, } \\
233,234,244, \text { figs } 5.4 d \text {, } \\
7.10 \mathrm{e}, 18.4 \mathrm{f}, 21.5 \mathrm{e} \text {, } \\
33.3 \mathrm{f}, 34.3 \mathrm{~d}, 35.62 .4 \mathrm{c}, \\
53.5 \mathrm{c} \text { and g, colour pl III } \\
\text { (B52); BM }\end{array}$ \\
\hline Faversham & Kent & $\begin{array}{l}\text { 5th- } \\
\text { 7th c }\end{array}$ & $\begin{array}{l}\text { Globulars, } 1 \text { wound } \\
\text { annular, etc (Plate 3) }\end{array}$ & $\begin{array}{l}\text { Meaney 1964, 118-19; } \\
\text { Ashmolean Museum; } \\
\text { Beck Collection, } \\
\text { Cambridge Museum }\end{array}$ \\
\hline $\begin{array}{l}\text { Gilton or } \\
\text { Kingston }\end{array}$ & Kent & $\begin{array}{l}\text { 6th- } \\
7 \text { th c }\end{array}$ & & $\begin{array}{l}\text { Faussett } 1856 \text {, pls } \mathrm{V} \text { and } \mathrm{VI} \\
\text { Liverpool Museum }\end{array}$ \\
\hline Holborough & Kent & $\begin{array}{l}\text { late } \\
7 \text { th c } \\
\text { or later }\end{array}$ & Necklace: 1 globular & $\begin{array}{l}\text { Evison 1956, 126, fig 20; } \\
\text { BM } 19475-2358\end{array}$ \\
\hline $\begin{array}{l}\text { Lyminge } \\
\text { II }\end{array}$ & Kent & 6th $c$ & $\begin{array}{l}\text { Grave } 24: 1 \text { white with } \\
\text { equal-arm brooch pair } \\
\text { and pot, etc }\end{array}$ & $\begin{array}{l}\text { Warhurst } 1955,18 \text {, } \\
\text { fig } 12.1, \text { pl VIIb.1; } \\
\text { Maidstone Museum }\end{array}$ \\
\hline Polhill & Kent & 7th $c$ & $\begin{array}{l}\text { Grave } 41: 2 \text { short cylinder- } \\
\text { shaped with } 2 \text { wire rings, } \\
\text { etc }\end{array}$ & $\begin{array}{l}\text { Hawkes and Philp 1973, } \\
177,193 \text { and } 207, \\
\text { fig } 55.520\end{array}$ \\
\hline Sarre & Kent & 7th $c$ & $\begin{array}{l}\text { Necklace: many globulars } \\
\text { and annulars with gold coins, } \\
\text { millefiori pendant and } \\
\text { amethysts, etc }\end{array}$ & Smith 1860, pl II \\
\hline $\begin{array}{l}\text { Sitting- } \\
\text { Bourne, } \\
\text { Rondeau } \\
\text { Estate }\end{array}$ & Kent & & One whitish short cylinder & $\begin{array}{l}\text { Payne } 1880 \text { and } 1881 \text {; } \\
\text { BM } 8312-13644\end{array}$ \\
\hline Stowting & Kent & $\begin{array}{l}\text { prob } \\
6 \text { th- } \\
7 \text { th c }\end{array}$ & $\begin{array}{l}\text { Several globulars and } \\
\text { annulars }\end{array}$ & $\begin{array}{l}\text { Smith 1846a; Brent 1867; } \\
\text { Beck Collection, } \\
\text { Cambridge Museum }\end{array}$ \\
\hline Wingham & Kent & 7th $c$ & $\begin{array}{l}\text { From robbed grave } \\
\text { with amethysts, etc }\end{array}$ & $\begin{array}{l}\text { Meaney } 1964,140-1 ; \\
\text { BM } 5412-218 \text { and } 20\end{array}$ \\
\hline $\begin{array}{l}\text { Castle } \\
\text { Bytham }\end{array}$ & Lincs & $\begin{array}{l}\text { prob } \\
7 \text { th c }\end{array}$ & $\begin{array}{l}\text { Grave: one with quoit } \\
\text { brooch and beads }\end{array}$ & $\begin{array}{l}\text { Akerman 1855a, 26, } \\
\text { pl XII.4 }\end{array}$ \\
\hline Cleatham & Lincs & $\begin{array}{l}\text { prob } \\
\text { 6th c }\end{array}$ & $\begin{array}{l}\text { Grave 9; } \\
\text { Grave } 34\end{array}$ & $\begin{array}{l}\text { Publication in } \\
\text { preparation (K Leahy); } \\
\text { Scunthorpe Museum }\end{array}$ \\
\hline Laceby & Lincs & $\begin{array}{l}\text { 6th- } \\
7 \text { th } c\end{array}$ & Group: 1 annular & $\begin{array}{l}\text { Myres } 1951,85 \text { and } 98 \text {; } \\
\text { Thompson 1956, 184-9; } \\
\text { Lincoln Museum }\end{array}$ \\
\hline
\end{tabular}




\begin{tabular}{|c|c|c|c|c|}
\hline $\begin{array}{l}\text { Nettleton } \\
\text { Caistor }\end{array}$ & Lincs & & $\begin{array}{l}\text { One bead with others } \\
\text { on bronze needle }\end{array}$ & $\begin{array}{l}\text { Meaney 1964, 160; } \\
\text { Lincoln Museum }\end{array}$ \\
\hline Ruskington & Lincs & & Group I: 1 annular & $\begin{array}{l}\text { Meaney 1964, 161-2; } \\
\text { Lincoln Museum }\end{array}$ \\
\hline $\begin{array}{l}\text { South } \\
\text { Elkington }\end{array}$ & Lincs & & Cremation: part of a bead & Webster 1951 \\
\hline $\begin{array}{l}\text { Morning } \\
\text { Thorpe }\end{array}$ & Norfolk & $\begin{array}{l}\text { later } \\
6 \text { th c } \\
\text { mid- } \\
\text { 6th c } \\
\text { prob } \\
\text { 6th c }\end{array}$ & $\begin{array}{l}\text { Grave } 353: 2 \text { beads } \\
\text { with florid cruciform } \\
\text { brooch, Style I clasps, etc; } \\
\text { Grave } 358: 1 \text { annular } \\
\text { with Åberg Group IVa } \\
\text { cruciform brooch, etc; } \\
\text { and a number of others }\end{array}$ & $\begin{array}{l}\text { Green et al 1987, } \\
136 \text { and } 138, \text { figs } 414 . \text { Miii } \\
\text { and } 417 . \text { Div; } \\
\text { Norwich Museum }\end{array}$ \\
\hline Spong Hill & Norfolk & $\begin{array}{l}\text { late } \\
5 \text { th- } \\
6 \text { th c } \\
\text { mid- } \\
6 \text { th c } \\
\text { late } \\
\text { 5th- } \\
6 \text { th c } \\
\text { 6th c } \\
\text { 6th c }\end{array}$ & $\begin{array}{l}\text { Grave } 5: 2 \text { globulars } \\
\text { and } 1 \text { annular with } 2 \text { small- } \\
\text { long/cruciform brooches, } \\
\text { etc; } \\
\text { Grave } 22: 4 \text { annulars } \\
\text { with Åberg Group IVa } \\
\text { cruciform brooch, etc; } \\
\text { Grave } 26: 2 \text { barrels and } \\
1 \text { fluted with Åberg } \\
\text { Group I cruciform brooch, } \\
\text { etc; } \\
\text { Grave } 37: 2 \text { annulars with } \\
\text { annular brooch pair and } \\
\text { B clasps; } \\
\text { Grave } 42: 1 \text { annular with } \\
\text { small-long brooch pair, } \\
\text { B clasps, etc }\end{array}$ & $\begin{array}{l}\text { Hills et al } 1984 \text {, } \\
55,70,74,86 \text { and } 95-6 \text {, } \\
\text { figs } 73.3 \text { b and } 6 c \text {, } \\
79.5 .1,18,25 \text { and } 26 \text {, } \\
83.1,90.6 \text { and } \\
98.42 .3 ; \\
\text { Norfolk Arch Unit }\end{array}$ \\
\hline $\begin{array}{l}\text { Mitchell's } \\
\text { Hill }\end{array}$ & Suffolk & $\begin{array}{l}\text { prob } \\
6 \text { th } c\end{array}$ & Several small & $\begin{array}{l}\text { Meaney 1964, 231; } \\
\text { Bury St Edmunds } \\
\text { Museum }\end{array}$ \\
\hline West Stow & Suffolk & $\begin{array}{l}5 \text { th- } \\
7 \text { th c }\end{array}$ & $\begin{array}{l}\text { Cemetery: } 4 \text { 'discs' } \\
\text { (O2 and T7) }\end{array}$ & $\begin{array}{l}\text { West } 1985,74(\mathrm{O} 2, \mathrm{~T} 7) \text {, } \\
\text { figs } 275.12 \text { and } 13 ; \\
\text { Ashmolean Museum; } \\
\text { Thetford Museum }\end{array}$ \\
\hline Guildown & Surrey & 6th c & $\begin{array}{l}\text { Several annulars and } \\
\text { globulars }\end{array}$ & $\begin{array}{l}\text { Lowther 1931, 10-13, } \\
\text { pls VIII and IX; } \\
\text { Guildford Museum }\end{array}$ \\
\hline Alfriston & Sussex & $\begin{array}{l}\text { first } \\
\text { half } \\
6 \text { th c }\end{array}$ & $\begin{array}{l}\text { Grave } 28: 3 \text { bluish with } \\
\text { great square-headed } \\
\text { brooch, etc }\end{array}$ & $\begin{array}{l}\text { Griffiths and Salzmann } \\
1914,36 ; \\
\text { Welch 1983, 85; } \\
\text { Lewes Museum }\end{array}$ \\
\hline $\begin{array}{l}\text { Apple } \\
\text { Down I }\end{array}$ & Sussex & $\begin{array}{l}6 \text { th- } \\
7 \text { th } c\end{array}$ & $\begin{array}{l}\text { Grave } 55: 1 \text { globular } \\
\text { with buckle and knife, } \\
\text { etc }\end{array}$ & $\begin{array}{l}\text { Down and Welch } 1990,42 \\
\text { and } 162, \text { fig } 2.26 .55 .5 ; \\
\text { Chichester Museum }\end{array}$ \\
\hline
\end{tabular}




\begin{tabular}{|c|c|c|c|c|}
\hline Highdown & Sussex & $\begin{array}{l}\text { prob } \\
\text { 6th } c\end{array}$ & $\begin{array}{l}\text { Several whitish } \\
\text { annulars }\end{array}$ & $\begin{array}{l}\text { Read } 1895 \text { and } 1896 ; \\
\text { Welch } 1983,85 ; \\
\text { Worthing Museum }\end{array}$ \\
\hline ‘Sussex’ & Sussex & $\begin{array}{l}\text { prob } \\
7 \text { th c }\end{array}$ & $\begin{array}{l}\text { String with amethysts: } \\
\text { several }\end{array}$ & $\begin{array}{l}\text { Welch 1983, 85; } \\
\text { Lewes Museum }\end{array}$ \\
\hline Wasperton & Warks & 6th $c$ & $\begin{array}{l}\text { Find no. F365 126-7/1: } \\
1 \text { annular }\end{array}$ & $\begin{array}{l}\text { Publication in } \\
\text { preparation (M Carver); } \\
\text { Warwick Museum }\end{array}$ \\
\hline $\begin{array}{l}\text { Pewsey } \\
\text { Blackpatch }\end{array}$ & Wilts & $\begin{array}{l}\text { late } \\
5 \text { th- } \\
\text { early } \\
6 \text { th } c\end{array}$ & Grave 38: group B & $\begin{array}{l}\text { Publication in } \\
\text { preparation } \\
\text { (K Annable); } \\
\text { Devizes Museum }\end{array}$ \\
\hline $\begin{array}{l}\text { Winter- } \\
\text { bourne } \\
\text { Gunner }\end{array}$ & Wilts & $\begin{array}{l}\text { prob } \\
\text { 6th } c\end{array}$ & $\begin{array}{l}\text { Grave 10: } 1 \text { greenish-white } \\
\text { small annular }\end{array}$ & $\begin{array}{l}\text { Musty and Stratton } 1964 \\
93 \text { and } 102 \text {, fig } 8 . X . a 2 ; \\
\text { Salisbury Museum }\end{array}$ \\
\hline $\begin{array}{l}\text { West } \\
\text { Heslerton }\end{array}$ & Yorks & 6th $c$ & A number & $\begin{array}{l}\text { Publication in } \\
\text { preparation } \\
\text { (D Powlesland) }\end{array}$ \\
\hline
\end{tabular}

ii Opaque white cylinder beads: round and polygonal, SHORT SINGLE OR DOUBLE BEADS (see Map 10)

\begin{tabular}{|c|c|c|c|c|}
\hline $\begin{array}{l}\text { Chamber- } \\
\text { lain's } \\
\text { Barn II }\end{array}$ & Beds & $\begin{array}{l}\text { mid- } \\
\text { late } \\
7 \text { th c }\end{array}$ & $\begin{array}{l}\text { Grave } 32: 1 \text { 'ring-shaped' } \\
\text { short cylinder on silver } \\
\text { wire ring, with quoit brooch }\end{array}$ & $\begin{array}{l}\text { Hyslop 1963, } 179 \text {, } \\
\text { fig } 12 \mathrm{e} ; \\
\text { Luton Museum }\end{array}$ \\
\hline $\begin{array}{l}\text { Linton } \\
\text { Heath }\end{array}$ & Cambs & & $\begin{array}{l}\text { One short cylinder } \\
\text { (Plate 3) }\end{array}$ & $\begin{array}{l}\text { Neville 1854; } \\
\text { Cambridge Museum }\end{array}$ \\
\hline $\begin{array}{l}\text { Great } \\
\text { Chesterford }\end{array}$ & Essex & 6th $c$ & $\begin{array}{l}\text { Grave 103: } 1 \text { short cylinder } \\
\text { (B58) with Style I applied } \\
\text { saucer brooch pair }\end{array}$ & $\begin{array}{l}\text { Evison } 1994,6,12,15,46 \\
\text { 105-6, fig 42.103.3p; } \\
\text { BM } 19647-2328\end{array}$ \\
\hline Mucking II & Essex & $\begin{array}{l}\text { 2nd } \\
\text { half } \\
5 \text { th c }\end{array}$ & $\begin{array}{l}\text { Grave 334: } 1 \text { bead with } \\
\text { disc brooch pair, amber } \\
\text { beads, inlaid buckle, etc }\end{array}$ & $\begin{array}{l}\text { Hirst and Clark forthcoming; } \\
\text { BM }\end{array}$ \\
\hline $\begin{array}{l}\text { Chessell } \\
\text { Down }\end{array}$ & $\begin{array}{l}\text { Isle of } \\
\text { Wight }\end{array}$ & 6th $c$ & $\begin{array}{l}\text { Long cylinder on } \\
\text { string (type } 3.2 \text { ) }\end{array}$ & $\begin{array}{l}\text { Arnold } 1982,50 \text { and } 120 \text {, } \\
\text { colour frontispiece; BM }\end{array}$ \\
\hline $\begin{array}{l}\text { Breach } \\
\text { Down }\end{array}$ & Kent & $\begin{array}{l}\text { late } \\
6 \text { th- } \\
\text { early } \\
7 \text { th c }\end{array}$ & Necklace: 1 short cylinder & $\begin{array}{l}\text { Conyngham and Akerman } \\
\text { 1844; } \\
\text { BM 79 5-24 } 89\end{array}$ \\
\hline $\begin{array}{l}\text { Dover, } \\
\text { Buckland }\end{array}$ & Kent & $\begin{array}{l}575- \\
625\end{array}$ & $\begin{array}{l}\text { Grave 1: } 5 \text { short (B54); } \\
\text { Grave 29: } 1 \text { short (B58); } \\
\text { Grave 35: } 3 \text { short (B54 and } \\
\text { B58); } \\
\text { Grave 60: } 3 \text { short (B54); } \\
\text { Grave 42: } 1 \text { short (B58); } \\
\text { Grave 62: } 1 \text { (B56) and } \\
2 \text { pentagonal (B60); }\end{array}$ & $\begin{array}{l}\text { Evison } 1987,74 \text { and } 81 \text {, } \\
216,224,226,228,233 \text {, } \\
234,235,241,243,251 \text {, } \\
\text { figs } 5.4 \mathrm{o}, 17.21 \text {, } \\
21.5 \mathrm{~g} \text { and } \mathrm{h}, 34.3 \mathrm{j} \text {, } \\
24.42 .1 \mathrm{~d} \text {, } \\
35.62 .4 \mathrm{~g} \text { and } \mathrm{k} \text {, } \\
37.67 .1 \mathrm{~g}, 49.110 .12 \mathrm{~d} \text {, }\end{array}$ \\
\hline
\end{tabular}




\begin{tabular}{|c|c|c|c|c|}
\hline & & $\begin{array}{l}650- \\
675 \\
675- \\
700 \\
700- \\
725\end{array}$ & $\begin{array}{l}\text { Grave 67: } 1 \text { short (B54); } \\
\text { Grave 110: } 3 \text { short (B55); } \\
\text { Grave 160: } 2 \text { short (B59); } \\
\text { Grave 127: } 1 \text { short (B55) }\end{array}$ & $\begin{array}{l}62.160 .2 \mathrm{c}, 52.127 .1 \mathrm{e} \\
\mathrm{BM}\end{array}$ \\
\hline $\begin{array}{l}\text { Eastry, } \\
\text { Eastry House }\end{array}$ & Kent & $\begin{array}{l}\text { late } \\
\text { 6th } c\end{array}$ & $\begin{array}{l}\text { Grave 1: five short } \\
\text { cylinders, with Gotlandic } \\
\text { bow brooch }\end{array}$ & $\begin{array}{l}\text { Hawkes } 1979,86 \text {, } \\
\text { fig } 4.4(13,16,30-32) \text {; } \\
\text { Deal Museum }\end{array}$ \\
\hline Faversham & Kent & $\begin{array}{l}5 \text { th- } \\
7 \text { th c }\end{array}$ & $\begin{array}{l}\text { and double cylinders } \\
\text { (Plate } 3 \text { ); and } c .10 \text { short } \\
\text { cylinders } 1 \text { cube and } \\
1 \text { double cylinder }\end{array}$ & $\begin{array}{l}\text { Meaney 1964, 118-19; } \\
\text { Ashmolean Museum } \\
1909.140(2), 1909.171(2), \\
1909.168(1), 1909.169(1), \\
1909.149(1), 1909.161(2), \\
1909.140(1), 1909.155(2) ; \\
\text { Beck Collection, } \\
\text { Cambridge Museum }\end{array}$ \\
\hline $\begin{array}{l}\text { 'mainly } \\
\text { Gilton' }\end{array}$ & Kent & $\begin{array}{l}\text { prob } \\
7 \text { th c }\end{array}$ & $\begin{array}{l}\text { One short cylinder, } \\
1 \text { long cylinder and } \\
1 \text { double }\end{array}$ & $\begin{array}{l}\text { Faussett } 1856, \text { pls V and VI; } \\
\text { Liverpool Museum }\end{array}$ \\
\hline Holborough & Kent & $\begin{array}{l}\text { late } \\
7 \text { th } c \\
\text { or } \\
\text { later }\end{array}$ & $\begin{array}{l}\text { Unstratified string: } \\
1 \text { badly made short } \\
\text { cylinder }\end{array}$ & $\begin{array}{l}\text { Evison } 1956,126 \text {, } \\
\text { fig } 20 \text {; } \\
\text { BM } 19475-2358\end{array}$ \\
\hline Polhill & Kent & 7th $\mathrm{c}$ & $\begin{array}{l}\text { Grave } 37: 2 \text { 'grey' short } \\
\text { cylinders with jewelled } \\
\text { disc brooch, etc; } \\
\text { Grave } 41: 1 \text { 'grey' short } \\
\text { cylinder with } 2 \text { wire rings, } \\
\text { etc }\end{array}$ & $\begin{array}{l}\text { Hawkes and Philp 1973, } \\
176,177,193 \text { and } 207 \text {, } \\
\text { fig } 55.517 \text { and } 520\end{array}$ \\
\hline Sarre & Kent & $\begin{array}{l}\text { 5th- } \\
\text { 7th c }\end{array}$ & $\begin{array}{l}\text { Now on miscellaneous } \\
\text { string: } 1 \text { short cylinder }\end{array}$ & $\begin{array}{l}\text { Meaney 1964, 135-6; } \\
\text { BM 93 6-1 } 218\end{array}$ \\
\hline $\begin{array}{l}\text { Sibertswold } \\
\text { or } \\
\text { Barfreston }\end{array}$ & Kent & $\begin{array}{l}\text { 6th- } \\
7 \text { th c }\end{array}$ & $\begin{array}{l}\text { c. } 30 \text { cylinders and } \\
4 \text { doubles }\end{array}$ & Liverpool Museum \\
\hline Stowting & Kent & $\begin{array}{l}\text { 6th- } \\
\text { 7th } c\end{array}$ & $\begin{array}{l}\text { Two short cylinders, } \\
1 \text { with square section }\end{array}$ & $\begin{array}{l}\text { Meaney 1964, 137-8; } \\
\text { Beck Collection, } \\
\text { Cambridge Museum }\end{array}$ \\
\hline Westbere & Kent & $\begin{array}{l}\text { prob } \\
\text { 7th c }\end{array}$ & $\begin{array}{l}\text { One short cylinder } \\
\text { with amethysts }\end{array}$ & $\begin{array}{l}\text { Jessup 1946; } \\
\text { Canterbury Museum } 6422\end{array}$ \\
\hline Riby Park & Lincs & 7th $\mathrm{c}$ & $\begin{array}{l}\text { Burial: } 1 \text { short cylinder } \\
\text { with amethyst bead }\end{array}$ & $\begin{array}{l}\text { Phillips } 1934,148,154 \text { and } \\
\text { 177; Myres 1951, 88, fig 9; } \\
\text { Lincoln Museum }\end{array}$ \\
\hline Sleaford & Lincs & 6th $c$ & Two with bronze clasps & Thomas 1887; BM \\
\hline
\end{tabular}




\begin{tabular}{|c|c|c|c|c|}
\hline $\begin{array}{l}\text { Bury St } \\
\text { Edmunds, } \\
\text { Westgarth } \\
\text { Gardens }\end{array}$ & Suffolk & $\begin{array}{l}\text { prob } \\
\text { 6th c }\end{array}$ & Grave 57: 1 double & $\begin{array}{l}\text { West } 1988,35 \text {, } \\
\text { fig } 79.57 . \mathrm{Cl} ; \\
\text { Bury St Edmunds } \\
\text { Museum }\end{array}$ \\
\hline Hoxne & Suffolk & & One long cylinder & $\begin{array}{l}\text { Meaney 1964, 228; } \\
\text { BM } 1912 \text { 5-28 } 36\end{array}$ \\
\hline Ipswich & Suffolk & $\begin{array}{l}6 \text { th- } \\
7 \text { th } c\end{array}$ & Many short cylinders & $\begin{array}{l}\text { Layard 1907, pls XXXI and } \\
\text { XXXII, and 1909, pl VII; } \\
\text { Ipswich Museum }\end{array}$ \\
\hline West Stow & Suffolk & $\begin{array}{l}\text { 5th c } \\
\text { 5th- } \\
\text { 7th c }\end{array}$ & $\begin{array}{l}\text { Settlement SFB27: } \\
1 \text { double (SF688); } \\
\text { Unstratified } 1 \text { five-sided } \\
\text { (SF1493) }\end{array}$ & $\begin{array}{l}\text { West } 1985,73 \text {, } \\
\text { figs } 108.4,275.29 \text { and } 33 \text {; } \\
\text { Bury St Edmunds } \\
\text { Museum }\end{array}$ \\
\hline Mitcham & Surrey & $\begin{array}{l}\text { prob } \\
\text { 6th c }\end{array}$ & $\begin{array}{l}\text { Grave 109: } 1 \text { short } \\
\text { cylinder; } \\
\text { Grave 129: } 1 \text { polygonal }\end{array}$ & $\begin{array}{l}\text { Bidder and Morris } 1959,67, \\
69,112 \text {, pl XVI.109 and 129; } \\
\text { Cambridge Museum }\end{array}$ \\
\hline $\begin{array}{l}\text { Apple } \\
\text { Down I }\end{array}$ & Sussex & $\begin{array}{l}\text { prob } \\
\text { 6th c }\end{array}$ & $\begin{array}{l}\text { Grave } 117: 2 \text { short } \\
\text { cylinders; } \\
\text { Grave 134: } 1 \text { short cylinder }\end{array}$ & $\begin{array}{l}\text { Down and Welch } 1990 \text {, } \\
49,51,162 \text { and } 164 \text {, } \\
\text { figs } 2.37 .3 \text { and } 2.43 .3 \text {, } \\
\text { pl } 40.4 \text {; } \\
\text { Chichester Museum }\end{array}$ \\
\hline $\begin{array}{l}\text { West } \\
\text { Heslerton }\end{array}$ & Yorks & $\begin{array}{l}\text { prob } \\
\text { 6th c }\end{array}$ & $\begin{array}{l}\text { One square-sectioned and } \\
\text { l pentagonal }\end{array}$ & $\begin{array}{l}\text { Publication in preparation } \\
\text { preparation } \\
\text { (D Powlesland) }\end{array}$ \\
\hline Dalmeny & $\begin{array}{l}\text { Scotland: } \\
\text { West } \\
\text { Lothian }\end{array}$ & $\begin{array}{l}\text { prob } \\
\text { 7th c }\end{array}$ & $\begin{array}{l}\text { Cist burial: } 1 \text { short cylinder } \\
\text { with biconical beads }\end{array}$ & $\begin{array}{l}\text { Brown 1915a (IV), } \\
812-13 \text {, fig 29; } \\
\text { Brown 1915b; } \\
\text { Edinburgh Museum }\end{array}$ \\
\hline Dun Beag & $\begin{array}{l}\text { Scotland: } \\
\text { Skye }\end{array}$ & & $\begin{array}{l}\text { Broch, secondary } \\
\text { occupation: } 1 \text { short cylinder }\end{array}$ & Callander 1921, 126 \\
\hline
\end{tabular}

iii Opaque white beads: (a) With light blue or tUrquoise CROSSED WAVES Without Dots, SINGLes OR DOUbles (see Map 11)

\begin{tabular}{|c|c|c|c|c|}
\hline $\begin{array}{l}\text { Chamber- } \\
\text { lain's } \\
\text { Barn I }\end{array}$ & Beds & $\begin{array}{l}6 \text { th- } \\
\text { early } \\
7 \text { th } c\end{array}$ & Grave 3: 2 'ring-shaped' & $\begin{array}{l}\text { Hyslop } 1963,167 \text {, } \\
\text { fig } 4 \mathrm{a} \text { and } \mathrm{b} \text {; } \\
\text { Luton Museum }\end{array}$ \\
\hline Kempston & Beds & $\begin{array}{l}\text { 6th- } \\
7 \text { th c }\end{array}$ & Grave 16 & $\begin{array}{l}\text { Fitch 1864; } \\
\text { BM 91 6-24 } 53\end{array}$ \\
\hline Puddlehill & Beds & c. 600 & $\begin{array}{l}\text { Grave } 10: 7 \text { with cast } \\
\text { saucer brooch pair, etc }\end{array}$ & $\begin{array}{l}\text { Matthews and Hawkes } \\
1985,91 \text { and } 97-8 \text {, } \\
\text { fig } 40.19-28\end{array}$ \\
\hline $\begin{array}{l}\text { Chalfont } \\
\text { St Peter }\end{array}$ & Bucks & & $\begin{array}{l}\text { Single find: large } \\
\text { with angular waves }\end{array}$ & $\begin{array}{l}\text { Rouse 1931; } \\
\text { Aylesbury Museum }\end{array}$ \\
\hline
\end{tabular}




\begin{tabular}{|c|c|c|c|c|}
\hline $\begin{array}{l}\text { Great } \\
\text { Chesterford }\end{array}$ & Essex & $\begin{array}{l}\text { prob } \\
\text { 6th c }\end{array}$ & Grave $48: 2$ beads (D27) & $\begin{array}{l}\text { Evison } 1994,12,17 \text {, } \\
98, \text { figs } 6 \text { and } 30.48 .1 \text {; } \\
\text { BM } 19647-2\end{array}$ \\
\hline Mucking II & Essex & $\begin{array}{l}\text { 6th } c \\
6 \text { th- } \\
7 \text { th } c \\
6 \text { th- } \\
7 \text { th } c \\
6 \text { th- } \\
7 \text { th } c\end{array}$ & $\begin{array}{l}\text { Grave 608: } 3 \text { annulars } \\
\text { with amber beads; } \\
\text { Grave } 648: 1 \text { disc bead and } \\
1 \text { fragmentary with small- } \\
\text { long brooch pair, amber } \\
\text { beads and kidney-shaped } \\
\text { buckle; } \\
\text { Grave } 690 \mathrm{~B}: 2 \text { perhaps } \\
3 \text { with button brooch, etc; } \\
\text { Grave } 914: 1 \text { barrel; } \\
\text { Grave } 962: 8 \text { barrels with } \\
\text { knife (Evison type } 5 \text { ); } \\
\text { Grave } 995: 1 \text { annular with } \\
\text { single amber bead, etc }\end{array}$ & $\begin{array}{l}\text { Hirst and Clark forthcoming; } \\
\text { BM }\end{array}$ \\
\hline Prittlewell & Essex & $c .600$ & $\begin{array}{l}\text { Grave-group 32: several } \\
\text { with cast saucer brooch } \\
\text { pair }\end{array}$ & $\begin{array}{l}\text { Tyler 1988, 108, } 113 \text {, } \\
\text { fig 15; } \\
\text { Southend Museum }\end{array}$ \\
\hline $\begin{array}{l}\text { Springfield } \\
\text { Lyons }\end{array}$ & Essex & $\begin{array}{l}\text { prob } \\
\text { 6th c }\end{array}$ & & $\begin{array}{l}\text { Publication in } \\
\text { preparation (S Tyler); BM }\end{array}$ \\
\hline Alton & Hants & 7th $c$ & $\begin{array}{l}\text { Grave 39: } 1 \text { double and } \\
1 \text { single with buckle and } \\
\text { knife (partially excavated) }\end{array}$ & $\begin{array}{l}\text { Evison 1988a, 81, } \\
\text { fig 33.39.1i and j; } \\
\text { Hampshire Museums }\end{array}$ \\
\hline $\begin{array}{l}\text { St Neots, } \\
\text { Lords Bridge }\end{array}$ & Hunts & & Random collection & BM $195610-2,1$ and 2 \\
\hline $\begin{array}{l}\text { Dover, } \\
\text { Buckland }\end{array}$ & Kent & $\begin{array}{l}650- \\
675\end{array}$ & $\begin{array}{l}\text { Grave 32: disc (D25), } \\
4 \text { discs (D26) and 2 } \\
\text { short cylinders (D36); } \\
\text { Grave 60: } 3 \text { discs (D24) } \\
\text { and disc (D26); } \\
\text { Grave 134: disc (D26) }\end{array}$ & $\begin{array}{l}\text { Evison } 1987,76-7,82 \text {, } \\
225,233,245 \text {, } \\
\text { figs } 19.41, \mathrm{~m} \text { and } \mathrm{o} \text {, } \\
34.3 \mathrm{p} \text { and } \mathrm{q}, 55.134 .2 \mathrm{f} \\
\text { colour pl IV; } \mathrm{BM}\end{array}$ \\
\hline $\begin{array}{l}\text { Eastry, } \\
\text { Eastry House }\end{array}$ & Kent & $\begin{array}{l}\text { late } \\
\text { 6th } c\end{array}$ & $\begin{array}{l}\text { Grave 1: with Gotlandic } \\
\text { bow brooch }\end{array}$ & $\begin{array}{l}\text { Hawkes } 1979,86 \text {, fig } 4.4 \\
\text { (12); Deal Museum }\end{array}$ \\
\hline Faversham & Kent & $\begin{array}{l}5 \text { th- } \\
7 \text { th } c\end{array}$ & Three beads and 1 bead & $\begin{array}{l}\text { Meaney 1964, 118-19; } \\
\text { Ashmolean Museum } \\
\text { 1909.144-46; } \\
\text { Beck Collection, } \\
\text { Cambridge Museum }\end{array}$ \\
\hline $\begin{array}{l}\text { Gilton or } \\
\text { Kingston }\end{array}$ & Kent & $\begin{array}{l}\text { prob } \\
6 \text { th- } \\
7 \text { th c }\end{array}$ & Several & $\begin{array}{l}\text { Faussett } 1856 \text {, pls V and VI; } \\
\text { Liverpool Museum }\end{array}$ \\
\hline 'Kent' & Kent & & $\begin{array}{l}\text { One double bead, both } \\
\text { elements similar, others }\end{array}$ & Canterbury Museum \\
\hline
\end{tabular}




\begin{tabular}{|c|c|c|c|c|}
\hline Sarre & Kent & $\begin{array}{l}\text { 6th- } \\
7 \text { th c }\end{array}$ & & Meaney $1964,135-6$ \\
\hline Stowting & Kent & $\begin{array}{l}\text { prob } \\
\text { 6th c }\end{array}$ & $\begin{array}{l}\text { Three doubles and } 1 \text { triple } \\
\text { and some singles }\end{array}$ & $\begin{array}{l}\text { Smith 1846a; Brent } 1867 \text {; } \\
\text { Maidstone Museum; } \\
\text { Beck Collection, } \\
\text { Cambridge Museum }\end{array}$ \\
\hline Saxby & Leics & & & Information from J Timby \\
\hline $\begin{array}{l}\text { Nettleton } \\
\text { Caistor }\end{array}$ & Lincs & & One badly made example & $\begin{array}{l}\text { Meaney } 1964,160 \\
\text { Lincoln Museum }\end{array}$ \\
\hline Syston Park & Lincs & $\begin{array}{l}\text { prob } \\
\text { 6th c }\end{array}$ & String with 2 beads & $\begin{array}{l}\text { Akerman } 1855 a_{3} 41-2 \text {, } \\
\text { pl XXI; } \\
\text { BM (18) } 1111-92\end{array}$ \\
\hline $\begin{array}{l}\text { Welbourn, } \\
\text { High Dyke }\end{array}$ & Lincs & & Two small beads & $\begin{array}{l}\text { Bruce } 1880,76, \text { no. } 305 \text {; } \\
\text { Alnwick Museum }\end{array}$ \\
\hline $\begin{array}{l}\text { London, } \\
\text { Dowgate } \\
\text { Hill }\end{array}$ & London & & No information & $\begin{array}{l}\text { Museum of London } \\
3064\end{array}$ \\
\hline $\begin{array}{l}\text { Morning } \\
\text { Thorpe }\end{array}$ & Norfolk & 6th $c$ & $\begin{array}{l}\text { Grave } 371: 2 \text { beads with } \\
\text { great square-headed } \\
\text { brooch, etc; and other } \\
\text { examples (Plate } 3 \text { ) }\end{array}$ & $\begin{array}{l}\text { Green et al } 1987, \\
145, \text { fig } 431 . H x i ; \\
\text { Norwich Museum }\end{array}$ \\
\hline Spong Hill & Norfolk & 6th $c$ & $\begin{array}{l}\text { Grave } 42: 1 \text { badly made } \\
\text { with small-long brooch, } \\
\text { B clasps and stamped pot }\end{array}$ & $\begin{array}{l}\text { Hills et al } 1984 \text {, } \\
96 \text {, fig } 98.42 .3 ; \\
\text { Norfolk Arch Unit }\end{array}$ \\
\hline $\begin{array}{l}\text { Standlake } \\
\text { Down }\end{array}$ & Oxon & $\begin{array}{l}\text { early } \\
\text { 7th c }\end{array}$ & Grave $3(24)$ & $\begin{array}{l}\text { Dickinson } 1973,245 \text {, } \\
\text { fig 5h; } \\
\text { Ashmolean Museum }\end{array}$ \\
\hline $\begin{array}{l}\text { Empingham } \\
\text { II }\end{array}$ & Rutland & $\begin{array}{l}5 \text { th- } \\
\text { 7th c }\end{array}$ & Grave 98B & $\begin{array}{l}\text { Timby 1996; } \\
\text { Oakham Museum }\end{array}$ \\
\hline Camerton & Somerset & $\begin{array}{l}\text { prob } \\
\text { 7th c }\end{array}$ & One biconical & $\begin{array}{l}\text { Horne } 1928 \text { and 1933; } \\
\text { Taunton Museum }\end{array}$ \\
\hline Icklingham & Suffolk & & $\begin{array}{l}\text { Three triple, } 3 \text { double } \\
\text { and } 3 \text { single beads }\end{array}$ & $\begin{array}{l}\text { Bury St Edmunds } \\
\text { Museum }\end{array}$ \\
\hline Ipswich & Suffolk & $\begin{array}{l}\text { 6th- } \\
7 \text { th c }\end{array}$ & $\begin{array}{l}\text { Many examples on } \\
\text { various necklaces }\end{array}$ & $\begin{array}{l}\text { Layard 1907, pl XXXII, and } \\
\text { 1909, pl VII; } \\
\text { Ipswich Museum }\end{array}$ \\
\hline Lakenheath & Suffolk & $\begin{array}{l}\text { 6th- } \\
7 \text { th c }\end{array}$ & Cemetery and 'nearby' & $\begin{array}{l}\text { Meaney 1964, 230; } \\
\text { BM } 1910 \text { 12-22 } 11\end{array}$ \\
\hline $\begin{array}{l}\text { Little } \\
\text { Eriswell }\end{array}$ & Suffolk & $\begin{array}{l}\text { 6th- } \\
7 \text { th c }\end{array}$ & One globular & $\begin{array}{l}\text { Hutchinson 1966; } \\
\text { Bury St Edmunds } \\
\text { Museum }\end{array}$ \\
\hline
\end{tabular}




\begin{tabular}{|c|c|c|c|c|}
\hline Sutton Hoo & Suffolk & $\begin{array}{l}\text { prob } \\
\text { 7th c }\end{array}$ & $\begin{array}{l}\text { Area A Cutting VIIIA } \\
\text { Grave Pit } 1 \text { (mole-run): } \\
\text { large globular bead with } \\
\text { turquoise roughly } \\
\text { crossed waves }\end{array}$ & $\begin{array}{l}\text { Longworth and Kinnes } \\
\text { 1980, 29, fig 19a; } \\
\text { BM }\end{array}$ \\
\hline Guildown & Surrey & $\begin{array}{l}\text { 5th- } \\
\text { 6th } c \\
6 \text { th } c\end{array}$ & $\begin{array}{l}\text { Grave } 75 \text { : with } 2 \text { applied } \\
\text { saucer brooches; } \\
\text { Grave } 206 \text { : with square- } \\
\text { headed brooch pair }\end{array}$ & $\begin{array}{l}\text { Lowther } 1931,11,12,35 \text {, } \\
\text { 44, pl IX.7 and 1; } \\
\text { Guildford Museum }\end{array}$ \\
\hline Mitcham & Surrey & $\begin{array}{l}\text { prob } \\
\text { 6th c }\end{array}$ & $\begin{array}{l}\text { Grave 62: large; } \\
\text { Grave 177: } 4 \text { doubles; } \\
\text { Grave 129: } 1 \text { globular }\end{array}$ & $\begin{array}{l}\text { Bidder and Morris } 1959,64 \text {, } \\
69,70,110,112 \text {, pl XVI.62 } \\
\text { and 109; Cambridge Museum }\end{array}$ \\
\hline $\begin{array}{l}\text { Apple } \\
\text { Down I }\end{array}$ & Sussex & $\begin{array}{l}\text { prob } \\
\text { 6th c }\end{array}$ & $\begin{array}{l}\text { Grave } 117 \text { : double bead, } \\
\text { crossed waves without } \\
\text { dots with amber beads, etc; } \\
\text { Grave } 134: 2 \text { globular and } \\
1 \text { annular with amber } \\
\text { beads, etc }\end{array}$ & $\begin{array}{l}\text { Down and Welch } 1990 \text {, } \\
162,164, \text { figs } 2.37 \\
2.43, \text { pl } 41.25 \text { and } 28 ; \\
\text { Chichester Museum }\end{array}$ \\
\hline Highdown & Sussex & $\begin{array}{l}5 \text { th- } \\
\text { 6th c }\end{array}$ & $\begin{array}{l}\text { On bracelet: } \\
\text { uncertain example }\end{array}$ & $\begin{array}{l}\text { Read } 1895 \text { and } 1896 \text {; } \\
\text { Welch } 1983,85 ; \\
\text { Worthing Museum } 3482\end{array}$ \\
\hline $\begin{array}{l}\text { Bidford-on- } \\
\text { Avon }\end{array}$ & Warks & 6th $c$ & $\begin{array}{l}\text { Necklace with } \\
\text { saucer brooches }\end{array}$ & $\begin{array}{l}\text { Humphreys et al 1923; } \\
\text { Stratford Museum }\end{array}$ \\
\hline $\begin{array}{l}\text { Harnham } \\
\text { Hill }\end{array}$ & Wilts & $\begin{array}{l}\text { prob } \\
\text { 6th- } \\
\text { 7th c }\end{array}$ & & $\begin{array}{l}\text { Akerman 1853; } \\
\text { Jackson 1854; } \\
\text { BM } 53 \text { 12-14 } 95\end{array}$ \\
\hline Mildenhall & Wilts & $\begin{array}{l}\text { 5th- } \\
\text { 6th c }\end{array}$ & $\begin{array}{l}\text { With saucer brooches, } \\
\text { bronze needles, etc }\end{array}$ & $\begin{array}{l}\text { Meaney 1964, 270; } \\
\text { Devizes Museum }\end{array}$ \\
\hline $\begin{array}{l}\text { West } \\
\text { Heslerton }\end{array}$ & Yorks & 6th $c$ & No. 620: 1 double & $\begin{array}{l}\text { Publication in } \\
\text { preparation } \\
\text { (D Powlesland) }\end{array}$ \\
\hline 'Yorkshire' & Yorks & $\begin{array}{l}\text { prob } \\
\text { 6th c }\end{array}$ & Necklace: 2 & Hull Museum \\
\hline
\end{tabular}

iii Opaque White beads: (b) With light blue Crossed WAVES AND BLUE DOTS (see Map 12)

\begin{tabular}{|c|c|c|c|c|}
\hline Kempston & Beds & $\begin{array}{l}\text { 6th- } \\
7 \text { th c }\end{array}$ & Grave 16 & $\begin{array}{l}\text { Fitch 1864; } \\
\text { BM 91 6-24 } 53\end{array}$ \\
\hline Frilford & Berks & $\begin{array}{l}\text { prob } \\
\text { 6th c }\end{array}$ & $\begin{array}{l}\text { Said to have been found } \\
\text { with saucer brooches }\end{array}$ & $\begin{array}{l}\text { Akerman 1865; } \\
\text { BM } 672-419\end{array}$ \\
\hline $\begin{array}{l}\text { Long } \\
\text { Wittenham }\end{array}$ & Berks & $\begin{array}{l}\text { 5th- } \\
6 \text { th } c\end{array}$ & $\begin{array}{l}\text { Grave } 96: 2 \text { beads } \\
\text { with small-long brooches }\end{array}$ & $\begin{array}{l}\text { Akerman 1860; } \\
\text { BM }\end{array}$ \\
\hline Wallingford & Berks & $\begin{array}{l}5 \text { th- } \\
\text { late } \\
6 \text { th } c\end{array}$ & Near Grave 13 & $\begin{array}{l}\text { Leeds 1938, } 98 ; \\
\text { Ashmolean Museum }\end{array}$ \\
\hline
\end{tabular}




\begin{tabular}{|c|c|c|c|c|}
\hline $\begin{array}{l}\text { Little } \\
\text { Chester }\end{array}$ & Derbys & $\begin{array}{l}\text { prob } \\
6 \text { th- } \\
7 \text { th c }\end{array}$ & $\begin{array}{l}\text { No. } 776187 \text { : among beads } \\
\text { from several graves }\end{array}$ & $\begin{array}{l}\text { Wheeler 1985, 304; } \\
\text { publication in } \\
\text { preparation } \\
\text { (C Sparey-Green) }\end{array}$ \\
\hline Mucking II & Essex & $\begin{array}{l}\text { 5th- } \\
\text { 6th c } \\
5 \text { th- } \\
6 \text { th c } \\
\text { first } \\
\text { half } \\
\text { 5th c }\end{array}$ & $\begin{array}{l}\text { Grave } 649: 2 \text { globulars } \\
\text { with small-long brooch } \\
\text { pair, decorated buckle, etc; } \\
\text { Grave } 860: 2 \text { annulars with } \\
\text { small-long brooch pair, } \\
\text { penannular brooch, etc; } \\
\text { Grave 989: } 1 \text { barrel with } \\
\text { Glaston-Mucking type and } \\
\text { supporting-arm brooches }\end{array}$ & $\begin{array}{l}\text { Evison 1981, 138-9, } \\
\text { figs } 4 \text { and 5; } \\
\text { BM }\end{array}$ \\
\hline $\begin{array}{l}\text { Springfield } \\
\text { Lyons }\end{array}$ & Essex & $\begin{array}{l}\text { prob } \\
\text { 6th c }\end{array}$ & $\begin{array}{l}\text { No. } 4758 \text {; } \\
\text { No. } 4882 \text {; } \\
\text { No. } 4988 ; \\
\text { No. } 6033(2)\end{array}$ & $\begin{array}{l}\text { Publication in } \\
\text { preparation (S Tyler); } \\
\text { BM }\end{array}$ \\
\hline Lechlade & Glos & 6th $c$ & $\begin{array}{l}\text { Grave } 78: 1 \text { thick annular } \\
\text { with cast saucer brooch } \\
\text { pair, etc }\end{array}$ & $\begin{array}{l}\text { Boyle et al forthcoming; } \\
\text { Cirencester Museum }\end{array}$ \\
\hline Alton & Hants & $\begin{array}{l}\text { first } \\
\text { half } \\
6 \text { th c }\end{array}$ & $\begin{array}{l}\text { Grave } 37: 2 \text { discs (D09) } \\
\text { with } 2 \text { button brooches }\end{array}$ & $\begin{array}{l}\text { Evison } 1988 \text { a, } 10-11 \text {, } \\
13,17,80, \\
\text { figs } 4 \text { and } 33.37 .4 \mathrm{i} \text {; } \\
\text { Hampshire Museums }\end{array}$ \\
\hline Faversham & Kent & $\begin{array}{l}\text { 5th- } \\
7 \text { th c }\end{array}$ & (Plate 3) & $\begin{array}{l}\text { Meaney 1964, 118-19; } \\
\text { Ashmolean Museum } \\
1909.143\end{array}$ \\
\hline Orpington & Kent & $\begin{array}{l}\text { 5th- } \\
6 \text { th c }\end{array}$ & $\begin{array}{l}\text { Grave } 58: 2 \text { beads with disc } \\
\text { brooch pair }\end{array}$ & $\begin{array}{l}\text { Tester 1969, 44-6, } \\
\text { fig 4.58b; } \\
\text { Orpington Museum }\end{array}$ \\
\hline Fonaby & Lincs & 6th $c$ & $\begin{array}{l}\text { Grave 3: } 2 \text { 'barrels' with } \\
2 \text { annular brooches, etc }\end{array}$ & $\begin{array}{l}\text { Cook 1981, 18-20, } \\
\text { fig 4.1.7 and 29; } \\
\text { Scunthorpe Museum }\end{array}$ \\
\hline $\begin{array}{l}\text { Loveden } \\
\text { Hill }\end{array}$ & Lincs & $\begin{array}{l}\text { late } \\
5 \text { th- } \\
7 \text { th c }\end{array}$ & Mixed rite cemetery & $\begin{array}{l}\text { Fennell 1964; } \\
\text { Meaney 1964, 158-9 }\end{array}$ \\
\hline Illington & Norfolk & 6th $c$ & Possibly with plain urn 167 & $\begin{array}{l}\text { Davison et al } 1993,34 \text {, } \\
\text { fig } 47.167 \mathrm{a} \text {; } \\
\text { Norwich Museum }\end{array}$ \\
\hline $\begin{array}{l}\text { Morning } \\
\text { Thorpe }\end{array}$ & Norfolk & 6th $c$ & $\begin{array}{l}\text { Several beads, eg, } \\
\text { Grave 90: } 13 \text { beads with } \\
3 \text { Åberg Group II } \\
\text { cruciform brooches, } \\
\text { B clasps, etc }\end{array}$ & $\begin{array}{l}\text { Green et al 1987, } \\
60-1 \text {, fig 324.Avii; } \\
\text { Norwich Museum }\end{array}$ \\
\hline Spong Hill & Norfolk & $\begin{array}{l}\text { late } \\
5 \text { th- } \\
6 \text { th c }\end{array}$ & $\begin{array}{l}\text { Grave } 5: 2 \text { beads with } \\
\text { small-long brooch pair, } \\
\text { A clasps, etc; }\end{array}$ & $\begin{array}{l}\text { Hills et al 1984, } \\
54-5,74-5 \text {, } \\
\text { figs } 73.3 \mathrm{~b} \text { and } 83.1 \text {; }\end{array}$ \\
\hline
\end{tabular}




\begin{tabular}{|c|c|c|c|c|}
\hline & & & $\begin{array}{l}\text { Grave } 26: 1 \text { bead with } \\
\text { Åberg Group I cruciform } \\
\text { brooch, etc }\end{array}$ & Norwich Museum \\
\hline Welton & \multicolumn{2}{|c|}{ Northants } & & $\begin{array}{l}\text { Meaney } 1964,196-7 ; \\
\text { Dryden } 1885,337 ; \\
\text { Northampton Museum }\end{array}$ \\
\hline $\begin{array}{l}\text { Empingham } \\
\text { II }\end{array}$ & Rutland & $\begin{array}{l}5 \text { th- } \\
7 \text { th c }\end{array}$ & Grave $67: 1$ small annular & $\begin{array}{l}\text { Timby 1996; } \\
\text { Oakham Museum }\end{array}$ \\
\hline $\begin{array}{l}\text { Bury St } \\
\text { Edmunds, } \\
\text { Westgarth } \\
\text { Gardens }\end{array}$ & Suffolk & 6th $c$ & $\begin{array}{l}\text { Grave } 48: 1 \text { globular with } \\
2 \text { small-long brooches, } \\
\text { B clasps, etc }\end{array}$ & $\begin{array}{l}\text { West } 1988,32 \text {, } \\
\text { fig } 73 . E 17 ; \\
\text { Bury St Edmunds } \\
\text { Museum }\end{array}$ \\
\hline West Stow & Suffolk & $\begin{array}{l}5 \text { th- } \\
7 \text { th c }\end{array}$ & Cemetery: (C12 and O29) & $\begin{array}{l}\text { West } 1985,72 \text { and } 74 \text {, } \\
\text { fig } 276.18 \text {; } \\
\text { Ashmolean Museum; } \\
\text { Cambridge Museum }\end{array}$ \\
\hline Guildown & Surrey & $\begin{array}{l}\text { 5th- } \\
\text { 6th c }\end{array}$ & Grave 78 & $\begin{array}{l}\text { Lowther 1931, 12, 36, } \\
\text { pl IX.7; Guildford Museum }\end{array}$ \\
\hline Alfriston & Sussex & $\begin{array}{l}\text { 5th- } \\
\text { early } \\
\text { 6th c }\end{array}$ & $\begin{array}{l}\text { Grave } 68 \text { : with quoit } \\
\text { brooch }\end{array}$ & $\begin{array}{l}\text { Griffith and Salzmann } \\
\text { 1914, 46-7, pl 1.1; } \\
\text { Welch 1983, 85; } \\
\text { Lewes Museum AJ/68 }\end{array}$ \\
\hline Wasperton & Warks & $\begin{array}{l}\text { mid- } \\
\text { 6th c }\end{array}$ & $\begin{array}{l}1081.60 \text { : with saucer } \\
\text { brooches }\end{array}$ & $\begin{array}{l}\text { Publication in } \\
\text { preparation (M Carver); } \\
\text { Warwick Museum }\end{array}$ \\
\hline $\begin{array}{l}\text { Pewsey } \\
\text { Blackpatch }\end{array}$ & Wilts & $\begin{array}{l}\text { late } \\
\text { 5th- } \\
\text { early } \\
\text { 6th c }\end{array}$ & Grave 38 & $\begin{array}{l}\text { Publication in } \\
\text { preparation } \\
\text { (K Annable); } \\
\text { Devizes Museum }\end{array}$ \\
\hline $\begin{array}{l}\text { Broadway } \\
\text { Hill }\end{array}$ & Worcs & $c .500$ & $\begin{array}{l}\text { Grave } 1 \text { : } 1 \text { bead with } \\
\text { cast saucer brooch pair }\end{array}$ & Cook 1958,64 , fig 4.10 \\
\hline
\end{tabular}

iii Opaque white beads: (c) With light blue crossed waves AND RED DOTS (see Map 13)

\begin{tabular}{|c|c|c|c|c|}
\hline $\begin{array}{l}\text { Chamber- } \\
\text { lain's } \\
\text { Barn I }\end{array}$ & Beds & $\begin{array}{l}\text { late } \\
6 \text { th- } \\
\text { early } \\
\text { 7th c }\end{array}$ & $\begin{array}{l}\text { Grave 3: } 5 \text { biconical } \\
\text { beads with iron keys } \\
\text { and knife, etc }\end{array}$ & $\begin{array}{l}\text { Hyslop 1963, 167, } \\
\text { fig 4.3.c-g; } \\
\text { Luton Museum }\end{array}$ \\
\hline Kempston & Beds & 6th $c$ & Grave 16 & $\begin{array}{l}\text { Fitch 1864; } \\
\text { BM 91 6-24 } 53\end{array}$ \\
\hline Puddlehill & Beds & $c .600$ & $\begin{array}{l}\text { Grave } 10: 3 \text { beads with cast } \\
\text { saucer brooch pair, etc }\end{array}$ & $\begin{array}{l}\text { Matthews and Hawkes } 1985 \text {, } \\
91-9 \text {, fig } 40.29,32 \text { and } 33\end{array}$ \\
\hline Bishopstone & Bucks & $\begin{array}{l}\text { prob } \\
\text { 6th c }\end{array}$ & $\begin{array}{l}\text { Necklace: several beads } \\
\text { with saucer brooches, etc }\end{array}$ & $\begin{array}{l}\text { Meaney 1964, 56; } \\
\text { Aylesbury Museum }\end{array}$ \\
\hline
\end{tabular}




\begin{tabular}{|c|c|c|c|c|}
\hline $\begin{array}{l}\text { Newport } \\
\text { Pagnell }\end{array}$ & Bucks & $\begin{array}{l}\text { prob } \\
\text { 6th c }\end{array}$ & Necklace & $\begin{array}{l}\text { Meaney 1964, 58; } \\
\text { Aylesbury Museum }\end{array}$ \\
\hline Barrington & Cambs & $\begin{array}{l}\text { 5th- } \\
7 \text { th } c\end{array}$ & One example & $\begin{array}{l}\text { Fox 1923, 250-5; } \\
\text { Cambridge Museum }\end{array}$ \\
\hline $\begin{array}{l}\text { Hasling- } \\
\text { field }\end{array}$ & Cambs & $\begin{array}{l}\text { prob } \\
\text { 6th c }\end{array}$ & $\begin{array}{l}\text { One small annular with } \\
\text { Upper Thames type brooch }\end{array}$ & $\begin{array}{l}\text { Fox 1923, 255-9; } \\
\text { Cambridge Museum }\end{array}$ \\
\hline $\begin{array}{l}\text { Little } \\
\text { Wilbraham }\end{array}$ & Cambs & $\begin{array}{l}\text { late } \\
5 \text { th- } \\
\text { early } \\
6 \text { th } c\end{array}$ & Near a grave: 2 examples & $\begin{array}{l}\text { Neville 1852; } \\
\text { Lethbridge and Carter 1926; } \\
\text { Cambridge Museum }\end{array}$ \\
\hline Mucking II & Essex & $\begin{array}{l}\text { 5th- } \\
\text { 6th c } \\
\text { first } \\
\text { half } \\
\text { 5th c }\end{array}$ & $\begin{array}{l}\text { Grave 648: } 1 \text { disc with } \\
\text { small-long brooch pair, } \\
\text { etc; } \\
\text { Grave } 846: 1 \text { bicone with } \\
2 \text { spots between wave and } \\
2 \text { barrels/bicones with } 1 \\
\text { spot between waves with } \\
\text { silver ring and amber beads; } \\
\text { Grave 975: } 1 \text { bead with } \\
\text { 5-star applied saucer } \\
\text { brooch pair, plain } \\
\text { carinated pot, etc; } \\
\text { Grave } 989: 2 \text { beads with } \\
\text { Glaston-Mucking and } \\
\text { supporting-arm brooches, } \\
\text { etc }\end{array}$ & $\begin{array}{l}\text { Hirst and Clark forthcoming; } \\
\text { BM }\end{array}$ \\
\hline $\begin{array}{l}\text { Springfield } \\
\text { Lyons }\end{array}$ & Essex & $\begin{array}{l}\text { prob } \\
\text { 6th c }\end{array}$ & Grave 4758 & $\begin{array}{l}\text { Publication in } \\
\text { preparation (S Tyler); } \\
\text { BM }\end{array}$ \\
\hline Alton & Hants & 6th $c$ & $\begin{array}{l}\text { Grave 23: (D08) with 7-leg } \\
\text { whirligig cast saucer } \\
\text { brooch pair, etc }\end{array}$ & $\begin{array}{l}\text { Evison } 1988 \text { a, } 9,13 \text {, } \\
15,17,41-4,77, \\
\text { figs } 4 \text { and } 29.4 \text { i.e; } \\
\text { Hampshire Museums }\end{array}$ \\
\hline $\begin{array}{l}\text { Canterbury, } \\
\text { Mint Yard }\end{array}$ & Kent & $\begin{array}{l}\text { prob } \\
5 \text { th- } \\
7 \text { th c }\end{array}$ & One example & $\begin{array}{l}\text { Information } \mathrm{T} \text { Tatton- } \\
\text { Brown and } \mathrm{P} \text { Garrard }\end{array}$ \\
\hline $\begin{array}{l}\text { Dover, } \\
\text { Buckland }\end{array}$ & Kent & $\begin{array}{l}475- \\
525 \\
575- \\
625 \\
625- \\
650\end{array}$ & $\begin{array}{l}\text { Grave 13: } 1 \text { (D43); } \\
\text { Grave 29: } 1 \text { (D44); } \\
\text { Grave 132: } 1 \text { (D45) }\end{array}$ & $\begin{array}{l}\text { Evison } 1987,77,82, \\
219,224,245, \\
\text { figs } 9.3 \mathrm{~h}, 17.2 \mathrm{q} \text { and } \\
54.132 .2 \mathrm{~d}, \text { colour pl IV } \\
\text { (D43); BM }\end{array}$ \\
\hline Faversham & Kent & $\begin{array}{l}5 \text { th- } \\
7 \text { th } c\end{array}$ & One double & $\begin{array}{l}\text { Beck Collection, } \\
\text { Cambridge Museum }\end{array}$ \\
\hline Finglesham & Kent & $\begin{array}{l}\text { first } \\
\text { half } \\
\text { 6th c }\end{array}$ & $\begin{array}{l}\text { Grave D3: } 1 \text { bead with } \\
\text { square-headed brooch, } \\
\text { radiate-headed brooches, } \\
\text { etc }\end{array}$ & $\begin{array}{l}\text { Chadwick } 1958,11-18 \text {, } \\
\text { fig } 5 \text { h; Deal Museum }\end{array}$ \\
\hline
\end{tabular}




\begin{tabular}{|c|c|c|c|c|}
\hline $\begin{array}{l}\text { Gilton or } \\
\text { Kingston }\end{array}$ & Kent & $\begin{array}{l}6 \text { th- } \\
7 \text { th } c\end{array}$ & & $\begin{array}{l}\text { Faussett 1856, pl VI; } \\
\text { Liverpool Museum }\end{array}$ \\
\hline Howletts & Kent & 6th $c$ & One bead & Smith 1918; BM \\
\hline ‘Kent' & Kent & & Three and one on necklace & Canterbury Museum \\
\hline Stowting & Kent & $\begin{array}{l}\text { 6th- } \\
7 \text { th } c\end{array}$ & $\begin{array}{l}\text { Several including cylinder, } \\
\text { atypical }\end{array}$ & $\begin{array}{l}\text { Smith 1846a; Brent 1867; } \\
\text { Cambridge Museum }\end{array}$ \\
\hline Saxby & Leics & & & $\begin{array}{l}\text { Publication in } \\
\text { preparation (J Timby) }\end{array}$ \\
\hline $\begin{array}{l}\text { Barton- } \\
\text { on-Humber }\end{array}$ & Lincs & 6th $c$ & $\begin{array}{l}\text { Grave 61: with green } \\
\text { monochrome beads, } \\
\text { silver pendant, etc }\end{array}$ & $\begin{array}{l}\text { Drinkall and Foreman } \\
\text { forthcoming; } \\
\text { Scunthorpe Museum }\end{array}$ \\
\hline Cleatham & Lincs & 6th $c$ & Grave 34: 5 examples & $\begin{array}{l}\text { Publication in } \\
\text { preparation (K Leahy); } \\
\text { Scunthorpe Museum }\end{array}$ \\
\hline Fonaby & Lincs & $\begin{array}{l}\text { prob } \\
\text { 6th c }\end{array}$ & $\begin{array}{l}\text { Grave 39: } 1 \text { 'barrel' } \\
\text { with iron buckle, etc }\end{array}$ & $\begin{array}{l}\text { Cook 1981, } 38, \\
\text { fig 14.39.1.5; } \\
\text { Scunthorpe Museum }\end{array}$ \\
\hline Waddington & Lincs & & With inhumation & $\begin{array}{l}\text { Petch 1957, 19; } \\
\text { Lincoln Museum }\end{array}$ \\
\hline $\begin{array}{l}\text { London, } \\
\text { Dowgate Hill }\end{array}$ & London & & Stray find & Museum of London \\
\hline Brooke & Norfolk & $\begin{array}{l}\text { prob } \\
\text { 6th c }\end{array}$ & One bead & $\begin{array}{l}\text { Meaney 1964, 170; } \\
\text { BM 1866-69 }\end{array}$ \\
\hline $\begin{array}{l}\text { Castle } \\
\text { Acre }\end{array}$ & Norfolk & $\begin{array}{l}\text { prob } \\
6 \text { th } c\end{array}$ & One slightly cylindrical & $\begin{array}{l}\text { Meaney } 1964,172-3 ; \\
\text { Norwich Museum }\end{array}$ \\
\hline $\begin{array}{l}\text { Morning } \\
\text { Thorpe }\end{array}$ & Norfolk & $\begin{array}{l}\text { prob } \\
\text { 6th c }\end{array}$ & $\begin{array}{l}\text { Grave 177: } 1 \text { bead with } \\
\text { pot, etc; } \\
\text { Grave } 216: 2 \text { beads with } \\
\text { annular brooch pair, etc; } \\
\text { Grave } 325: 2 \text { beads with } \\
\text { bucket pendant, etc }\end{array}$ & $\begin{array}{l}\text { Green } \text { et al } 1987 \text {, } \\
83-5,93 \text { and } 126, \\
\text { figs } 354 . \text { Diii, } \\
\text { 366.Jiv and } 402.325 . \text { Biv; } \\
\text { Norwich Museum }\end{array}$ \\
\hline Spong Hill & Norfolk & $\begin{array}{l}\text { late } \\
5 \text { th- } \\
6 \text { th c }\end{array}$ & $\begin{array}{l}\text { Grave } 5: 2 \text { beads with } \\
\text { small-long brooch pair, } \\
\text { A clasps, etc }\end{array}$ & $\begin{array}{l}\text { Hills et al } 1984 \text {, } \\
54-5 \text {, fig } 73.3 b ; \\
\text { Norwich Museum }\end{array}$ \\
\hline $\begin{array}{l}\text { Brixworth } \\
\text { (prob II) }\end{array}$ & Northants & 6th $c$ & $\begin{array}{l}\text { Necklace of many } \\
\text { beads }\end{array}$ & $\begin{array}{l}\text { Meaney } 1964,187-8 ; \\
\text { Northampton Museum }\end{array}$ \\
\hline Duston & Northants & & & $\begin{array}{l}\text { George } 1903 ; \\
\text { Northampton Museum }\end{array}$ \\
\hline Holdenby & Northants & $\begin{array}{l}\text { prob } \\
\text { 6th- } \\
\text { 7th c }\end{array}$ & Many beads & $\begin{array}{l}\text { Anon 1901; } \\
\text { Leeds 1909; } \\
\text { Northampton Museum }\end{array}$ \\
\hline Nassington & Northants & $\begin{array}{l}\text { first } \\
\text { half } \\
\text { 6th c }\end{array}$ & $\begin{array}{l}\text { Grave 13: one with cast } \\
\text { saucer brooches, etc }\end{array}$ & $\begin{array}{l}\text { Leeds and Atkinson 1944, } \\
\text { 105-6, pl XXIIb; } \\
\text { Peterborough Museum }\end{array}$ \\
\hline
\end{tabular}




\begin{tabular}{|c|c|c|c|c|}
\hline $\begin{array}{l}\text { Standlake } \\
\text { Down }\end{array}$ & Oxon & $\begin{array}{l}\text { early } \\
7 \text { th c }\end{array}$ & Grave 24: 2 cylinders & $\begin{array}{l}\text { Dickinson } 1973,244-5 \text {, } \\
\text { fig } 5 f \text {; } \\
\text { Ashmolean Museum }\end{array}$ \\
\hline Wheatley & Oxon & $\begin{array}{l}6 \text { th- } \\
7 \text { th c } \\
7 \text { th c }\end{array}$ & $\begin{array}{l}\text { Grave 27: with coins, etc } \\
\text { (Plate 3) } \\
\text { Grave 14: } 2 \text { examples }\end{array}$ & $\begin{array}{l}\text { Leeds 1916, 51, fig 5; } \\
\text { Ashmolean Museum } \\
1883.69\end{array}$ \\
\hline $\begin{array}{l}\text { Empingham } \\
\text { II }\end{array}$ & Rutland & $\begin{array}{l}\text { 5th- } \\
7 \text { th c }\end{array}$ & Graves $40,83,85 \mathrm{~A}$ and 93 & $\begin{array}{l}\text { Timby 1996; } \\
\text { Oakham Museum }\end{array}$ \\
\hline Ipswich & Suffolk & $\begin{array}{l}6 \text { th- } \\
7 \text { th } c\end{array}$ & Several & $\begin{array}{l}\text { Layard } 1907 \text { and } 1909 ; \\
\text { Ipswich Museum }\end{array}$ \\
\hline Snape & Suffolk & $\begin{array}{l}\text { 2nd } \\
\text { half } \\
\text { 6th- } \\
\text { 7th c } \\
\text { prob } \\
\text { 6th c }\end{array}$ & $\begin{array}{l}\text { Grave } 0327: 2 \text { rough } \\
\text { barrel-shaped beads } \\
\text { with terracotta } \\
\text { crossed waves and dots; } \\
\text { Grave } 0421 \text { : with terracotta } \\
\text { crossed waves and dots }\end{array}$ & $\begin{array}{l}\text { Filmer-Sankey and } \\
\text { Pestell forthcoming }\end{array}$ \\
\hline Sutton Hoo & Suffolk & $\begin{array}{l}\text { prob } \\
\text { 7th } \mathrm{c}\end{array}$ & Grave fill: 1 bead & $\begin{array}{l}\text { Information from } \\
\text { Dr I Kinnes (1978); BM }\end{array}$ \\
\hline West Stow & Suffolk & $\begin{array}{l}5 \text { th- } \\
7 \text { th c }\end{array}$ & $\begin{array}{l}\text { Settlement: } 1 \text { bead } \\
\text { SF 1444, WE } 8, \text { L2 }\end{array}$ & $\begin{array}{l}\text { West } 1985,74 \text {; Bury St } \\
\text { Edmunds Museum }\end{array}$ \\
\hline Alfriston & Sussex & 6 th $\mathrm{c}$ & $\begin{array}{l}\text { Grave } 23 \text { : with amber } \\
\text { beads and buckle loop, } \\
\text { etc }\end{array}$ & $\begin{array}{l}\text { Griffith and Salzmann } \\
\text { 1914, 34, pl II.5; } \\
\text { Welch 1983, 85, fig 8b; } \\
\text { Lewes Museum }\end{array}$ \\
\hline $\begin{array}{l}\text { Apple } \\
\text { Down I }\end{array}$ & Sussex & $\begin{array}{l}\text { prob } \\
7 \text { th c }\end{array}$ & $\begin{array}{l}\text { Grave } 125: 1 \text { bead } \\
\text { with tweezers, knife, etc }\end{array}$ & $\begin{array}{l}\text { Down and Welch } 1990,50, \\
\text { 163, fig } 2.39 .125 .4 \\
\text { colour pl } 40.12 ; \\
\text { Chichester Museum }\end{array}$ \\
\hline Wasperton & Warks & $\begin{array}{l}\text { mid- } \\
6 \text { th } c \\
6 \text { th } c \\
\text { first } \\
\text { half } \\
\text { 6th } c\end{array}$ & $\begin{array}{l}\text { With saucer brooches; } \\
\text { and with saucer brooch; } \\
\text { another globular }\end{array}$ & $\begin{array}{l}\text { Publication in } \\
\text { preparation (M Carver); } \\
\text { Warwick Museum }\end{array}$ \\
\hline $\begin{array}{l}\text { Colling- } \\
\text { bourne } \\
\text { Ducis }\end{array}$ & Wilts & $\begin{array}{l}5 \text { th- } \\
6 \text { th c }\end{array}$ & $\begin{array}{l}\text { Grave } 27: 1 \text { bead with } \\
\text { small-long brooch pair }\end{array}$ & $\begin{array}{l}\text { Gingell } 1975,87 \text {, fig 20.4; } \\
\text { Devizes Museum }\end{array}$ \\
\hline Mildenhall & Wilts & $\begin{array}{l}\text { prob } \\
6 \text { th c }\end{array}$ & $\begin{array}{l}\text { Inhumation: with bronze } \\
\text { needle and iron knife }\end{array}$ & $\begin{array}{l}\text { Found by D Meyrick } \\
\text { in 1948; Devizes Museum }\end{array}$ \\
\hline $\begin{array}{l}\text { 'Salisbury } \\
\text { area' }\end{array}$ & Wilts & & Very faint decoration & $\begin{array}{l}\text { Old collection in } \\
\text { Salisbury Museum }\end{array}$ \\
\hline $\begin{array}{l}\text { Baildon } \\
\text { Moor }\end{array}$ & Yorks & & & Keighley Museum \\
\hline
\end{tabular}




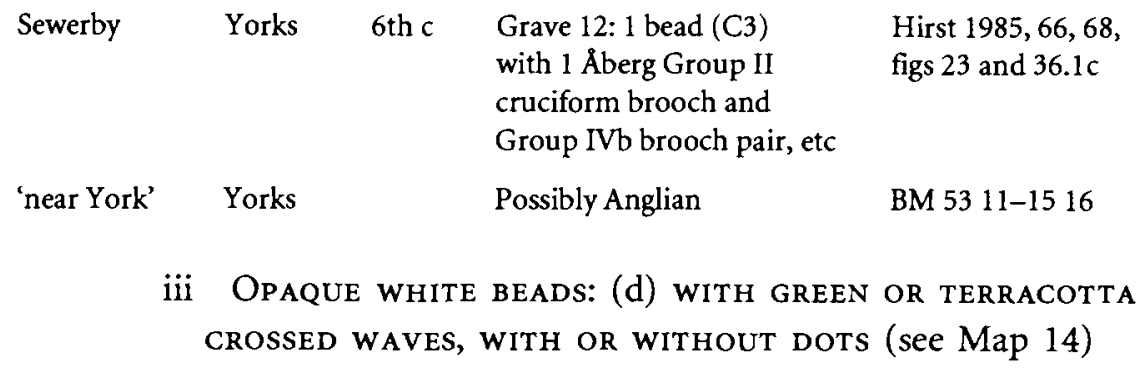

\begin{tabular}{|c|c|c|c|c|}
\hline $\begin{array}{l}\text { Long } \\
\text { Wittenham }\end{array}$ & Berks & $\begin{array}{l}\text { prob } \\
\text { 6th } c\end{array}$ & $\begin{array}{l}\text { Grave 142: with green } \\
\text { waves and terracotta dots, } \\
\text { with small-long brooches }\end{array}$ & Akerman 1860 \\
\hline Wallingford & Berks & $\begin{array}{l}5 \text { th- } \\
\text { late } \\
6 \text { th c }\end{array}$ & $\begin{array}{l}\text { One example with terracotta } \\
\text { crossed waves and central } \\
\text { band }\end{array}$ & $\begin{array}{l}\text { Leeds } 1938,101 \text {; } \\
\text { Reading Museum }\end{array}$ \\
\hline $\begin{array}{l}\text { Great } \\
\text { Chesterford }\end{array}$ & Essex & $\begin{array}{l}\text { mid- } \\
\text { 5th- } \\
\text { mid- } \\
\text { 6th c }\end{array}$ & $\begin{array}{l}\text { Grave } 127: 1 \text { 'barrel' with } \\
\text { dark crossed waves and } \\
\text { reddish blob }\end{array}$ & $\begin{array}{l}\text { Evison } 1994,6-7,12 \text {, } \\
18 \text { (D39), } 46 \text { and } 109 \text {, } \\
\text { fig } 49.127 .5 \mathrm{~d} \text {; } \\
\text { BM }\end{array}$ \\
\hline
\end{tabular}

\begin{tabular}{|c|c|c|c|c|}
\hline Mucking II & Essex & $\begin{array}{l}\text { 5th- } \\
6 \text { th c } \\
\text { 5th- } \\
6 \text { th c } \\
\text { 6th- } \\
7 \text { th c }\end{array}$ & $\begin{array}{l}\text { Grave } 550: 1 \text { barrel with } \\
\text { red crossed waves and blue- } \\
\text { green dots with button } \\
\text { brooch pair; } \\
\text { Grave } 608: 2 \text { annulars with } \\
\text { blue-green crossed waves } \\
\text { and red spots, with amber } \\
\text { beads; } \\
\text { Grave } 610: 2 \text { barrels with } \\
\text { blue-green crossed waves } \\
\text { and red spots with disc } \\
\text { brooch pair, } 1 \text { annular } \\
\text { brooch, etc; } \\
\text { Grave } 648: 2 \text { annulars with } \\
\text { terracotta crossed waves and } \\
\text { dots with small-long } \\
\text { brooch pair, etc; } \\
\text { Grave } 962: 8 \text { barrels with } \\
\text { green-blue crossed waves, } \\
\text { but no dots, with Evison } \\
\text { Type } 3 \text { knife }\end{array}$ & $\begin{array}{l}\text { Hirst and Clark forthcoming; } \\
\text { BM }\end{array}$ \\
\hline
\end{tabular}

$\begin{array}{lllll}\text { Droxford } & \text { Hants } & \begin{array}{l}\text { late } \\ \text { 5th- } \\ \text { 6th c }\end{array} & \begin{array}{l}\text { Grave 20: annular (class } \\ \text { IIb) with green waves and } \\ \text { red dots, with buckle and } \\ \text { knife }\end{array} & \begin{array}{l}\text { Aldsworth 1978, 124, } \\ 172, \text { fig 24.7; } \\ \text { Winchester Museum }\end{array} \\ \text { Faversham } \quad \text { Kent } & \begin{array}{l}\text { 5th- } \\ 7 \text { th c }\end{array} & \begin{array}{l}\text { Reddish crossed waves } \\ \text { and green dots (Plate 3); } \\ \text { another with green } \\ \text { crossed wave (Plate 3); } \\ 1 \text { with pink crossed waves } \\ \text { and dots }\end{array} & \begin{array}{l}\text { Meaney 1964, 118-19; } \\ \text { Ashmolean Museum }\end{array} \\ & & \end{array}$




\begin{tabular}{|c|c|c|c|c|}
\hline Saxby & Leics & & $\begin{array}{l}\text { Double short cylinder } \\
\text { with green crossed waves; } \\
\text { another with yellow } \\
\text { crossed waves }\end{array}$ & $\begin{array}{l}\text { Publication in } \\
\text { preparation (J Timby) }\end{array}$ \\
\hline $\begin{array}{l}\text { Barton- } \\
\text { on-Humber }\end{array}$ & Lincs & $\begin{array}{l}\text { mid- } \\
\text { 6th c }\end{array}$ & $\begin{array}{l}\text { Grave 115: several with } \\
\text { blue dots, with annular } \\
\text { brooch pair, Aberg Group } \\
\text { IVb cruciform brooch, etc }\end{array}$ & $\begin{array}{l}\text { Drinkall and Foreman } \\
\text { forthcoming; } \\
\text { Scunthorpe Museum }\end{array}$ \\
\hline Cleatham & Lincs & $\begin{array}{l}\text { prob } \\
6 \text { th c } \\
\text { prob } \\
6 \text { th c }\end{array}$ & $\begin{array}{l}\text { Grave 9: terracotta crossed } \\
\text { waves with cruciform } \\
\text { brooches; } \\
\text { Grave 30: terracotta crossed } \\
\text { waves and green dots; } \\
\text { Grave 35: terracotta crossed } \\
\text { waves with red dots }\end{array}$ & $\begin{array}{l}\text { Publication in } \\
\text { preparation (K Leahy); } \\
\text { Scunthorpe Museum }\end{array}$ \\
\hline Illington & Norfolk & $\begin{array}{l}5 \text { th- } \\
6 \text { th } \mathrm{c}\end{array}$ & $\begin{array}{l}\text { Cremation 11: red crossed } \\
\text { waves and green dots with } \\
\text { cruciform brooch half- } \\
\text { round knob }\end{array}$ & $\begin{array}{l}\text { Davison et al } 1993 \text {, } \\
23 \text {, fig 47.11a; } \\
\text { Norwich Museum }\end{array}$ \\
\hline Kenninghall & Norfolk & $\begin{array}{l}6 \text { th- } \\
\text { early } \\
7 \text { th c }\end{array}$ & Reddish crossed waves & $\begin{array}{l}\text { Manning 1872a, 292, and } \\
1872 \mathrm{~b} ; \\
\text { BM }\end{array}$ \\
\hline $\begin{array}{l}\text { Morning } \\
\text { Thorpe }\end{array}$ & Norfolk & $\begin{array}{l}\text { late } \\
5 \text { th- } \\
\text { early } \\
6 \text { th c }\end{array}$ & $\begin{array}{l}\text { Grave } 16: 1 \text { terracotta } \\
\text { crossed waves and green } \\
\text { dots with florid cruciform } \\
\text { brooch, etc; } \\
\text { Grave } 90 \text { : } 1 \text { terracotta } \\
\text { crossed waves and blue dots; } \\
1 \text { green crossed waves } \\
\text { and terracotta dots with } 3 \\
\text { Åberg Group II cruciform } \\
\text { brooches, B clasps, etc }\end{array}$ & $\begin{array}{l}\text { Green et al 1987, } \\
37-8,60-1 \text { and } 135-6, \\
\text { figs } 298 \text {. Evi and 324.Aix } \\
\text { and } x \text {; Norwich Museum }\end{array}$ \\
\hline Northwold & Norfolk & & $\begin{array}{l}\text { Reddish crossed waves and } \\
\text { blue dots }\end{array}$ & Akerman 1852 , pl V.1-4 \\
\hline Spong Hill & Norfolk & $\begin{array}{l}\text { late } \\
5 \text { th- } \\
\text { 6th c } \\
\text { 6th c }\end{array}$ & $\begin{array}{l}\text { Grave 5: } 1 \text { green crossed } \\
\text { waves and red dots with } \\
\text { small-long brooch pair, } \\
\text { A clasps, etc; } \\
\text { Grave } 18: 1 \text { terracotta } \\
\text { crossed waves and blue } \\
\text { dots, with } 1 \text { square-headed } \\
\text { brooch and } 1 \text { small-long } \\
\text { brooch, etc; } \\
\text { Grave } 26: 1 \text { green crossed } \\
\text { waves and dots, with } 1 \text { Aberg } \\
\text { Group I cruciform brooch, } \\
\text { etc; } \\
\text { Grave } 42: 1 \text { green crossed } \\
\text { waves and yellow dots with }\end{array}$ & $\begin{array}{l}\text { Hills et al } 1984 \text {, } \\
54-5,66-7,74-5 \text { and } \\
95-7, \text { figs } 73.3 b \text {, } \\
77.18 .4,83.1 \text { and } \\
98.42 .3 \text {; }\end{array}$ \\
\hline
\end{tabular}




\begin{tabular}{|c|c|c|c|c|}
\hline & & 5th $c$ & $\begin{array}{l}\text { small-long brooch pair, } \\
\text { B clasps, etc; } \\
\text { Cremation } 2143 \text { : terracotta } \\
\text { crossed waves and dots with } \\
\text { linear and punch-dot } \\
\text { ornamented urn and applied } \\
\text { saucer brooch with } 6 \text { masks }\end{array}$ & $\begin{array}{l}\text { Hills and Penn 1981, } \\
\text { 56-7, fig 165.2143; } \\
\text { Norfolk Arch Unit }\end{array}$ \\
\hline $\begin{array}{l}\text { Bury St } \\
\text { Edmunds, } \\
\text { Westgarth } \\
\text { Gardens }\end{array}$ & Suffolk & 6 th $c$ & $\begin{array}{l}\text { Grave } 27: \text { large annular } \\
\text { with red crossed waves, } \\
\text { with great square-headed } \\
\text { brooch etc; } \\
\text { Grave } 38 \text { : dark green } \\
\text { crossed waves and red dots }\end{array}$ & $\begin{array}{l}\text { West } 1988,27,30 \text {, } \\
\text { figs } 66.27 . \text { E and } 70.38 \text {; } \\
\text { Bury St Edmunds } \\
\text { Museum }\end{array}$ \\
\hline Ipswich & Suffolk & $\begin{array}{l}\text { 6th- } \\
7 \text { th } \mathrm{c}\end{array}$ & $\begin{array}{l}\text { Terracotta crossed waves and } \\
\text { dots; } \\
\text { another with yellow dots }\end{array}$ & $\begin{array}{l}\text { Layard 1907, pl XXXI, and } \\
\text { 1909; } \\
\text { Ipswich Museum }\end{array}$ \\
\hline Highdown & Sussex & $\begin{array}{l}\text { prob } \\
\text { 6th c }\end{array}$ & & $\begin{array}{l}\text { Read } 1895 \text { and } 1896 ; \\
\text { Welch 1983, 85; } \\
\text { Worthing Museum }\end{array}$ \\
\hline Wasperton & Warks & $\begin{array}{l}\text { mid- } \\
6 \text { th } c\end{array}$ & $\begin{array}{l}\text { Cube-shaped with green } \\
\text { crossed waves with saucer } \\
\text { brooches }\end{array}$ & $\begin{array}{l}\text { Publication in } \\
\text { preparation (M Carver); } \\
\text { Warwick Museum }\end{array}$ \\
\hline $\begin{array}{l}\text { Broadway } \\
\text { Hill }\end{array}$ & Worcs & $\begin{array}{l}\text { prob } \\
\text { 6th c }\end{array}$ & $\begin{array}{l}\text { Disturbed finds from } \\
\text { graves: terracotta crossed } \\
\text { waves and dots }\end{array}$ & Cook 1958,68 , fig 9.7 \\
\hline \multirow[t]{2}{*}{ Sewerby } & Yorks & 6th $c$ & $\begin{array}{l}\text { Grave 19: } 1 \text { biconical with } \\
\text { green crossed waves and } \\
\text { red dots (C3) with great } \\
\text { square-headed brooch, etc }\end{array}$ & $\begin{array}{l}\text { Hirst } 1985,59-60,68 \text {, } \\
\text { figs } 23 \text { and } 39.12\end{array}$ \\
\hline & \multicolumn{2}{|c|}{ Miscellane } & $\begin{array}{l}\text { US OPAQUE WHITE BEADS } \\
\text { SINGLE WAVES, ETC: } \\
\text { no schedule (Plate } 3 \text { ) }\end{array}$ & WITH SPOTS, \\
\hline
\end{tabular}

v Opaque white globular or biconical Beads with Red OR BLUE SPOTS

$\begin{array}{lllll}\begin{array}{l}\text { Chamber- } \\ \text { lain's } \\ \text { Barn I }\end{array} & \text { Beds } & \begin{array}{l}\text { late } \\ 6 \text { th- } \\ \text { early } \\ 7 \text { th } \mathrm{c}\end{array} & \begin{array}{l}\text { Grave 3: 2 large biconicals } \\ \text { with red spots (Plate 3) } \\ \text { with iron keys and } \\ \text { knife, etc }\end{array} & \begin{array}{l}\text { Hyslop 1963, 167, } \\ \text { fig 4n and o; } \\ \text { Luton Museum }\end{array} \\ \text { Puddlehill } & \text { Beds } & \text { c.600 } & \begin{array}{l}\text { Grave 10: 2 very slightly } \\ \text { biconical with red spots } \\ \text { (Plate 3) with saucer } \\ \text { brooch pair }\end{array} & \begin{array}{l}\text { Matthews and Hawkes } \\ 1985,91-9, \text { fig 40.30 } \\ \text { and 31 }\end{array} \\ \text { Alton } & \text { Hants } & \text { 7th c } & \begin{array}{l}\text { Grave 39: 1 'disc' with } \\ \text { blue spots (D12) with iron } \\ \text { knife, etc }\end{array} & \begin{array}{l}\text { Evison 1988a, 13, 17, 44 } \\ \text { and 80-1, fig 33.39.1n; } \\ \text { Hampshire Museums }\end{array}\end{array}$




\begin{tabular}{|c|c|c|c|c|}
\hline $\begin{array}{l}\text { Breach } \\
\text { Down }\end{array}$ & Kent & $\begin{array}{l}\text { 7th- } \\
\text { 8th c }\end{array}$ & $\begin{array}{l}\text { Terracotta spots on } \\
\text { globular }\end{array}$ & $\begin{array}{l}\text { Conyngham and Akerman } \\
1844 \text {, pl 1; BM } 79 \text { 5-24 } 29\end{array}$ \\
\hline $\begin{array}{l}\text { Gilton or } \\
\text { Kingston }\end{array}$ & Kent & $\begin{array}{l}\text { 6th- } \\
7 \text { th } c\end{array}$ & One with pairs of red spots & $\begin{array}{l}\text { Faussett 1856; } \\
\text { Liverpool Museum }\end{array}$ \\
\hline Ruskington & Lincs & $\begin{array}{l}\text { 6th- } \\
7 \text { th } \mathrm{c}\end{array}$ & $\begin{array}{l}\text { Large annular with a few } \\
\text { powder blue spots }\end{array}$ & $\begin{array}{l}\text { Meaney } 1964,161-2 ; \\
\text { Lincoln Museum 23/75 }\end{array}$ \\
\hline Brooke & Norfolk & $\begin{array}{l}\text { prob } \\
\text { 6th c }\end{array}$ & $\begin{array}{l}\text { One bluish white annular } \\
\text { with irregular reddish spots }\end{array}$ & $\begin{array}{l}\text { Meaney } 1964,170 \\
\text { BM 1866-69 }\end{array}$ \\
\hline Spong Hill & Norfolk & $\begin{array}{l}\text { mid- } \\
\text { 6th } c\end{array}$ & $\begin{array}{l}\text { Grave 22: } 1 \text { annular with } \\
\text { few terracotta spots with } \\
\text { Åberg Group IVa } \\
\text { cruciform brooch and } \\
\text { Group II brooch pair, etc }\end{array}$ & $\begin{array}{l}\text { Hills } \text { et al } 1984 \text {, } \\
69-70 \text {, fig } 79.5 .22 ; \\
\text { Norfolk Arch Unit }\end{array}$ \\
\hline Ipswich & Suffolk & $\begin{array}{l}\text { prob } \\
\text { 6th c }\end{array}$ & $\begin{array}{l}\text { Two globulars with large } \\
\text { blue spots; } \\
\text { large globular with red } \\
\text { spots }\end{array}$ & $\begin{array}{l}\text { Layard 1907, pl XXXI, } \\
\text { and 1909; } \\
\text { Ipswich Museum }\end{array}$ \\
\hline Snape & Suffolk & 6th $c$ & $\begin{array}{l}\text { Grave } 0421: 1 \text { rather } \\
\text { biconical with red spots }\end{array}$ & $\begin{array}{l}\text { Filmer-Sankey and } \\
\text { Pestell forthcoming }\end{array}$ \\
\hline Guildown & Surrey & 6th $c$ & $\begin{array}{l}\text { Grave 206: bluish-white } \\
\text { with red spots ( } 4 \text { ) with } \\
\text { square-headed brooch pair }\end{array}$ & $\begin{array}{l}\text { Lowther } 1931,11,44, \\
\text { pl IX.1; } \\
\text { Guildford Museum }\end{array}$ \\
\hline Mitcham & Surrey & $\begin{array}{l}\text { prob } \\
\text { 6th } c\end{array}$ & Grave 197: 1 with red spots & $\begin{array}{l}\text { Bidder and Morris 1959, } 73 \text {, } \\
\text { 112, pl XVI.197; } \\
\text { Cambridge Museum }\end{array}$ \\
\hline $\begin{array}{l}\text { Apple } \\
\text { Down I }\end{array}$ & Sussex & $\begin{array}{l}\text { prob } \\
\text { 6th c }\end{array}$ & $\begin{array}{l}\text { Grave 134: biconical with } \\
\text { red spots (cf } \\
\text { Chamberlain's Barn I } \\
\text { above) with amber beads }\end{array}$ & $\begin{array}{l}\text { Down and Welch } 1990,51 \text {, } \\
164, \text { fig } 2.43 \text {, } \\
\text { colour pl } 41.29 \text {; } \\
\text { Chichester Museum }\end{array}$ \\
\hline Wasperton & Warks & $\begin{array}{l}\text { late } \\
5 \text { th- } \\
6 \text { th c }\end{array}$ & $\begin{array}{l}\text { One small globular with red } \\
\text { spots with saucer brooches; } \\
2 \text { small globulars with } \\
\text { saucer brooch; } \\
\text { One annular with red spots, } \\
\text { with saucer brooch }\end{array}$ & $\begin{array}{l}\text { Publication in } \\
\text { preparation (M Carver); } \\
\text { Warwick Museum }\end{array}$ \\
\hline \multirow[t]{2}{*}{$\begin{array}{l}\text { Dowalton } \\
\text { Loch }\end{array}$} & $\begin{array}{l}\text { Scotland: } \\
\text { Wigton }\end{array}$ & & $\begin{array}{l}\text { Crannog site: } 3 \text { rows of } \\
\text { red spots }\end{array}$ & $\begin{array}{l}\text { Stuart 1865; } \\
\text { Edinburgh Museum } \\
\text { HLL } 31\end{array}$ \\
\hline & vi Opac & QUE WHIT & $\begin{array}{l}\text { TE CYLINDER BEADS WITH } \\
\text { WOUND BANDS }\end{array}$ & H COLOURED \\
\hline $\begin{array}{l}\text { Linton } \\
\text { Heath }\end{array}$ & Cambs & $\begin{array}{l}\text { prob } \\
\text { 6th } c\end{array}$ & $\begin{array}{l}\text { Small with yellowish green } \\
\text { bands }\end{array}$ & $\begin{array}{l}\text { Neville 1854; } \\
\text { Cambridge Museum }\end{array}$ \\
\hline
\end{tabular}




\begin{tabular}{|c|c|c|c|c|}
\hline Mucking II & Essex & $\begin{array}{l}\text { first } \\
\text { half } \\
5 \text { th c }\end{array}$ & $\begin{array}{l}\text { Grave 989: } 1 \text { short barrel } \\
\text { with translucent pale blue } \\
\text { spiral with Glaston- } \\
\text { Mucking type and } \\
\text { supporting-arm brooches }\end{array}$ & $\begin{array}{l}\text { Hirst and Clark forthcom } \\
\text { Evison 1981, 138-9, } \\
\text { figs } 4 \text { and 5; } \\
\text { BM }\end{array}$ \\
\hline $\begin{array}{l}\text { St Neots, } \\
\text { Lords Bridge }\end{array}$ & Hunts & $\begin{array}{l}\text { prob } \\
\text { 6th } c\end{array}$ & Light blue bands & BM $195610-2,1$ and 2 \\
\hline 'Kent' & Kent & & Blue bands & Canterbury Museum \\
\hline Orpington & Kent & $\begin{array}{l}\text { prob } \\
5 \text { th- } \\
6 \text { th c }\end{array}$ & $\begin{array}{l}\text { Grave } 32 \text { : necklace with } \\
\text { small bead with light blue } \\
\text { bands }\end{array}$ & $\begin{array}{l}\text { Tester 1968, 139-40, } \\
\text { fig 6.32a; } \\
\text { Hawkes 1969, 189; } \\
\text { Orpington Museum }\end{array}$ \\
\hline Sarre & Kent & $\begin{array}{l}6 \text { th- } \\
7 \text { th c }\end{array}$ & Green bands & $\begin{array}{l}\text { Meaney } 1964,135-6 \\
\text { BM OA } 4904\end{array}$ \\
\hline Wingham & Kent & 7th $c$ & $\begin{array}{l}\text { From robbed grave dug by } \\
\text { J Y Akerman: colour } \\
\text { missing }\end{array}$ & $\begin{array}{l}\text { Meaney } 1964,140-1 ; \\
\text { BM } 5412-218 \text { and } 20\end{array}$ \\
\hline $\begin{array}{l}\text { Morning } \\
\text { Thorpe }\end{array}$ & Norfolk & $\begin{array}{l}\text { first } \\
\text { half } \\
6 \text { th } c \\
\text { late } \\
5 \text { th- } \\
\text { early } \\
6 \text { th } c\end{array}$ & $\begin{array}{l}\text { Grave 30: blue bands with } \\
\text { Aberg Group III cruciform } \\
\text { brooch and Group II brooch } \\
\text { pair, B clasps, etc (Plate 3); } \\
\text { Grave 90: } 2 \text { beads with } \\
\text { blue bands with } 3 \text { Åberg } \\
\text { Group II cruciform } \\
\text { brooches, B clasps, etc }\end{array}$ & $\begin{array}{l}\text { Green et al } 1987 \text {, } \\
42-3 \text { and } 60-1 \text {, } \\
\text { figs } 303.30 . \text { Dv and } \\
\text { 324.Axxviii; } \\
\text { Norwich Museum }\end{array}$ \\
\hline Spong Hill & Norfolk & 5th $c$ & $\begin{array}{l}\text { Cremation 2143: } 1 \text { with } 3 \\
\text { pale blue bands with linear } \\
\text { and punch-dot decorated } \\
\text { urn and applied saucer } \\
\text { brooch with } 6 \text { masks }\end{array}$ & $\begin{array}{l}\text { Hills and Penn 1981, } \\
56-7 \text {, fig 165.2143; } \\
\text { Norfolk Arch Unit }\end{array}$ \\
\hline Duston & Northants & $\begin{array}{l}\text { prob } \\
\text { 6th } c\end{array}$ & Two beads, light blue bands & $\begin{array}{l}\text { George 1903; } \\
\text { Northampton Museum }\end{array}$ \\
\hline $\begin{array}{l}\text { Bury St } \\
\text { Edmunds, } \\
\text { Westgarth } \\
\text { Gardens }\end{array}$ & Suffolk & $\begin{array}{l}\text { prob } \\
\text { 6th } c\end{array}$ & $\begin{array}{l}\text { Grave 33: brownish bands } \\
\text { with amber and glass beads }\end{array}$ & $\begin{array}{l}\text { West } 1988,28 \text {, } \\
\text { fig } 68.33 .8 ; \\
\text { Bury St Edmunds } \\
\text { Museum }\end{array}$ \\
\hline Alfriston & Sussex & $\begin{array}{l}\text { later } \\
5 \text { th- } \\
\text { early } \\
\text { 6th c }\end{array}$ & $\begin{array}{l}\text { Grave } 87: 2 \text { with cobalt } \\
\text { blue bands with } 5 \text {-spiral } \\
\text { cast saucer brooch pair }\end{array}$ & $\begin{array}{l}\text { Griffith } 1915,204 \text {, } \\
\text { pl XXII.2; } \\
\text { Welch 1983, 85, fig 36c; } \\
\text { Lewes Museum }\end{array}$ \\
\hline $\begin{array}{l}\text { Pewsey } \\
\text { Blackpatch }\end{array}$ & Wilts & $\begin{array}{l}\text { prob } \\
5 \text { th- } \\
6 \text { th } c\end{array}$ & $\begin{array}{l}\text { Grave } 102: 2 \text { roughly made } \\
\text { blue bands, with } 2 \text { pen- } \\
\text { annular brooches of Fowler } \\
\text { Types D and F }\end{array}$ & $\begin{array}{l}\text { Publication in } \\
\text { preparation (K Annable); } \\
\text { Devizes Museum }\end{array}$ \\
\hline
\end{tabular}




\title{
SCHEDUle 4
}

\section{Opaque Yellow glass BeAdS}

\author{
Monochrome \\ i Opaque yellow annular, globular and barrel-shaped beads (see Map 15) \\ ii Opaque yellow cylinder beads: (a) round \\ (b) polygonal or square-sectioned \\ (c) short cylinders, singles or doubles \\ iii Opaque yellow biconical beads \\ iv Opaque yellow melon, sub-melon and 'nicked' beads \\ v Opaque yellow wound beads or joined globular beads \\ Polychrome \\ vi Opaque yellow beads with crossed waves, with or without dots, singles or \\ doubles (see Map 16) \\ vii Opaque yellow miscellaneous decorated beads (excluding crossed waves)
}

\section{MONOCHROME}

i Opaque yellow anNular, globular AND BARREL-SHAPED BEADS (see Map 15)

\begin{tabular}{|c|c|c|c|c|}
\hline Kempston & Beds & & Six beads & Fitch 1864; BM \\
\hline Wallingford & Berks & $\begin{array}{l}5 \text { th- } \\
\text { late } \\
6 \text { th c }\end{array}$ & $\begin{array}{l}\text { Grave 21: } 8 \text { beads with } \\
\text { clear glass core }\end{array}$ & $\begin{array}{l}\text { Harden 1940, 164; } \\
\text { Ashmolean Museum } \\
1939.454\end{array}$ \\
\hline Barrington & Cambs & $\begin{array}{l}5 \text { th- } \\
7 \text { th c }\end{array}$ & One bead & $\begin{array}{l}\text { Fox 1923, 250-2; } \\
\text { Ashmolean Museum }\end{array}$ \\
\hline Haslingfield & Cambs & & One bead & Fox $1923,255-9$ \\
\hline $\begin{array}{l}\text { Linton } \\
\text { Heath }\end{array}$ & Cambs & & $\begin{array}{l}\text { Approx } 10 \text { various sizes } \\
\text { and shapes }\end{array}$ & $\begin{array}{l}\text { Neville 1854; } \\
\text { Cambridge Museum }\end{array}$ \\
\hline $\begin{array}{l}\text { Little } \\
\text { Wilbraham }\end{array}$ & Cambs & $\begin{array}{l}5 \text { th- } \\
7 \text { th c }\end{array}$ & $\begin{array}{l}\text { Two beads; } \\
4 \text { beads }\end{array}$ & $\begin{array}{l}\text { Meaney 1964,70-1; } \\
\text { Cambridge Museum }\end{array}$ \\
\hline $\begin{array}{l}\text { Waterbeach, } \\
\text { Car Dyke }\end{array}$ & Cambs & $\begin{array}{l}\text { prob } \\
6 \text { th c }\end{array}$ & Grubenhaus & Lethbridge 1927 \\
\hline
\end{tabular}




\begin{tabular}{|c|c|c|c|c|}
\hline $\begin{array}{l}\text { Little } \\
\text { Chester }\end{array}$ & Derbys & $\begin{array}{l}5 \text { th- } \\
7 \text { th } c\end{array}$ & $\begin{array}{l}\text { Grave 320: rather barrel- } \\
\text { shaped and greenish }\end{array}$ & $\begin{array}{l}\text { Wheeler } 1985,304 ; \\
\text { publication in } \\
\text { preparation } \\
\text { (C Sparey-Green) }\end{array}$ \\
\hline $\begin{array}{l}\text { Dorchester, } \\
\text { Trumpet } \\
\text { Major }\end{array}$ & Dorset & $\begin{array}{l}\text { prob } \\
7 \text { th } c\end{array}$ & One bead & $\begin{array}{l}\text { Sparey-Green 1984, } \\
149 \text {, fig } 13.9\end{array}$ \\
\hline $\begin{array}{l}\text { Great } \\
\text { Chesterford }\end{array}$ & Essex & $\begin{array}{l}\text { mid- } \\
\text { 5th- } \\
6 \text { th c } \\
\text { mid- } \\
\text { 5th- } \\
\text { 6th c } \\
\text { late } \\
\text { 5th- } \\
\text { 6th c } \\
\text { 6th c }\end{array}$ & $\begin{array}{l}\text { Grave 2B: } 1 \text { disc (B09) } \\
\text { with great square-headed } \\
\text { brooch, etc; } \\
\text { Grave 31: } 2 \text { discs (B09) } \\
\text { with bracelet, etc; } \\
\text { Grave 56: } 1 \text { disc (B10); } \\
\text { Grave 79: } 1 \text { disc (B10) } \\
\text { with penannular brooch } \\
\text { etc; } \\
\text { Grave 103: } 7 \text { discs (B09, } \\
\text { B10 and B11) with Style I } \\
\text { applied saucer brooch } \\
\text { pair, etc; } \\
\text { Grave 126: } 1 \text { disc (B10) } \\
\text { with great square-headed } \\
\text { brooch, etc; } \\
\text { Cremation } 5: \text { in plain urn }\end{array}$ & $\begin{array}{l}\text { Evison } 1994,12,14, \\
46,90-1,96,100,102, \\
105-6,109 \text { and } 114-15 ; \\
\text { figs } 15.2 \mathrm{~B} .4 \mathrm{Nb} \text {, } \\
27.31 .4 \mathrm{~b}, 33.56 .2 \mathrm{~b} \text {, } \\
37.79 .2 \mathrm{~b}, \\
42.103 .3 \mathrm{~b}, \mathrm{c} \text { and } \mathrm{l}, \\
49.126 .5 \mathrm{~b} \text { and } 58 . \mathrm{C} 5.2 \mathrm{c} \text {; } \\
\text { BM } 19647-2\end{array}$ \\
\hline Mucking II & Essex & $\begin{array}{l}\text { 5th- } \\
6 \text { th } c \\
6 \text { th } c \\
7 \text { th } c\end{array}$ & $\begin{array}{l}\text { Fourteen various beads } \\
\text { (Plate 4) including: } \\
\text { Grave 874: } 1 \text { annular; } \\
\text { Grave 578: } 1 \text { globular; } \\
\text { Grave 621: } 4 \text { globulars with } \\
\text { wooden box, knife, etc }\end{array}$ & $\begin{array}{l}\text { Hirst and Clark forthcoming; } \\
\text { BM }\end{array}$ \\
\hline $\begin{array}{l}\text { Springfield } \\
\text { Lyons }\end{array}$ & Essex & $\begin{array}{l}\text { later } \\
5 \text { th- } \\
\text { 6th c }\end{array}$ & $\begin{array}{l}\text { Grave } 6033: 3 \text { beads barrel- } \\
\text { shaped with small-long } \\
\text { brooches }\end{array}$ & $\begin{array}{l}\text { Publication in } \\
\text { preparation (S Tyler); } \\
\text { BM }\end{array}$ \\
\hline Fairford & Glos & & Annular & $\begin{array}{l}\text { Meaney } 1964,91 \\
\text { BM } 19267-71 \text { and } 2\end{array}$ \\
\hline Lechlade & Glos & $\begin{array}{l}\text { late } \\
5 \text { th- } \\
\text { 6th } c \\
\text { 6th c }\end{array}$ & $\begin{array}{l}\text { Grave } 48 \text { : disc, } \\
\text { with buckle, etc; } \\
\text { Grave } 130 \text { : disc with cast } \\
\text { saucer brooch pair with } \\
\text { central star and Style I outer } \\
\text { border, etc }\end{array}$ & $\begin{array}{l}\text { Boyle et al forthcoming; } \\
\text { Cirencester Museum }\end{array}$ \\
\hline
\end{tabular}




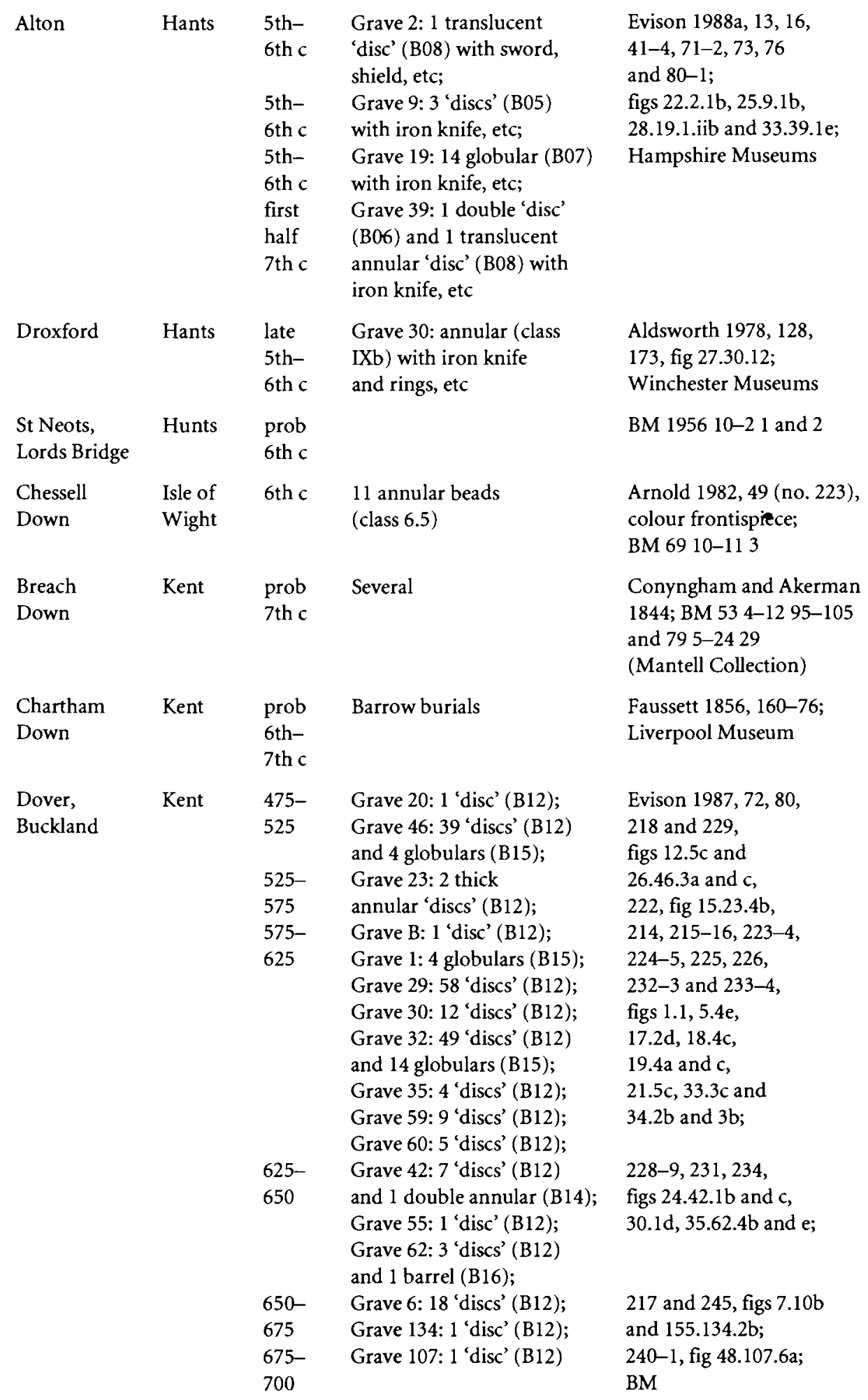




\begin{tabular}{|c|c|c|c|c|}
\hline Faversham & Kent & $\begin{array}{l}\text { prob } \\
\text { 6th- } \\
\text { 7th c }\end{array}$ & Many & $\begin{array}{l}\text { Meaney 1964, 118-19; } \\
\text { Ashmolean Museum; } \\
\text { Beck Collection, } \\
\text { Cambridge Museum }\end{array}$ \\
\hline Folkestone & Kent & $\begin{array}{l}\text { prob } \\
\text { 6th c }\end{array}$ & $\begin{array}{l}\text { No. 19: small annulars } \\
\text { of varying sizes }\end{array}$ & $\begin{array}{l}\text { Meaney } 1964,120-1 ; \\
\text { one each at Maidstone } \\
\text { and Folkestone Museums }\end{array}$ \\
\hline $\begin{array}{l}\text { Gilton or } \\
\text { Kingston }\end{array}$ & Kent & $\begin{array}{l}\text { 6th- } \\
\text { 7th c }\end{array}$ & A number & $\begin{array}{l}\text { Faussett 1856; } \\
\text { Liverpool Museum }\end{array}$ \\
\hline Holborough & Kent & $\begin{array}{l}\text { late } \\
7 \text { th or } \\
\text { later }\end{array}$ & Unstratified: 9 beads & $\begin{array}{l}\text { Evison } 1956,92 \text { and } 126 \text {, } \\
\text { fig 20.3a; } \\
\text { BM } 19475-2358\end{array}$ \\
\hline $\begin{array}{l}\text { Kingston or } \\
\text { Barfreston }\end{array}$ & Kent & $\begin{array}{l}\text { 6th- } \\
7 \text { th } c\end{array}$ & Necklace: several & $\begin{array}{l}\text { Faussett 1856; } \\
\text { Liverpool Museum }\end{array}$ \\
\hline Lyminge II & Kent & $\begin{array}{l}\text { first } \\
\text { half } \\
\text { 6th c }\end{array}$ & $\begin{array}{l}\text { Grave } 16: \text { with button } \\
\text { and radiate brooches and } \\
\text { single } D \text { bracteate }\end{array}$ & $\begin{array}{l}\text { Warhurst } 1955,13-15 \\
\text { Maidstone Museum }\end{array}$ \\
\hline Orpington & Kent & $\begin{array}{l}5 \text { th- } \\
6 \text { th c }\end{array}$ & $\begin{array}{l}\text { Grave } 32 \text {; } \\
\text { Grave } 58 \text { : with disc } \\
\text { brooch pair }\end{array}$ & $\begin{array}{l}\text { Tester } 1968,139-40 \text {, } \\
\text { fig } 6.32 \mathrm{a} ; \\
\text { Tester } 1969,44-6 \text {, } \\
\text { fig } 4.58 \mathrm{~b} ; \\
\text { Orpington Museum }\end{array}$ \\
\hline Polhill & Kent & 7th $\mathrm{c}$ & $\begin{array}{l}\text { Grave } 51: 1 \text { barrel (no. } 522 \text { ) } \\
\text { with } 2 \text { disc pendants, etc; } \\
\text { Grave } 95: 1 \text { barrel (no. } 526 \text { ) } \\
\text { with iron latch-lifter, etc }\end{array}$ & $\begin{array}{l}\text { Hawkes and Philp 1973, } \\
178,184 \text { and } 207, \\
\text { fig } 55.522 \text { and } 526\end{array}$ \\
\hline Sarre & Kent & $\begin{array}{l}5 \text { th- } \\
7 \text { th c }\end{array}$ & A number & $\begin{array}{l}\text { Meaney } 1964,135-6 ; \\
\text { BM OA } 4905 \\
\text { and } 936-1218\end{array}$ \\
\hline Stodmarsh & Kent & $\begin{array}{l}\text { prob } \\
\text { 6th c }\end{array}$ & & Akerman 1855b, 179-81 \\
\hline Stowting & Kent & $\begin{array}{l}\text { prob } \\
\text { 6th c }\end{array}$ & Many beads & $\begin{array}{l}\text { Smith 1846a; Brent } 1867 \text {; } \\
\text { Beck Collection, } \\
\text { Cambridge Museum; } \\
\text { Maidstone A/S } 300\end{array}$ \\
\hline Westbere & Kent & & Three short strings & $\begin{array}{l}\text { Jessup 1946; } \\
\text { Canterbury Museum } \\
6422,6245 \text { and } 6426\end{array}$ \\
\hline Saxby & Leics & & One globular & $\begin{array}{l}\text { Publication in } \\
\text { preparation ( } \mathrm{J} \text { Timby) }\end{array}$ \\
\hline Cleatham & Lincs & $\begin{array}{l}\text { prob } \\
5 \text { th- } \\
6 \text { th c }\end{array}$ & $\begin{array}{l}\text { Grave } 30 \\
\text { Grave } 34\end{array}$ & $\begin{array}{l}\text { Publication in } \\
\text { preparation (K Leahy); } \\
\text { Scunthorpe Museum }\end{array}$ \\
\hline Fonaby & Lincs & $\begin{array}{l}\text { prob } \\
\text { 6th c } \\
\text { first }\end{array}$ & $\begin{array}{l}\text { Grave } 25: 4 \text { barrels } \\
\text { with girdle hangers, keys; } \\
\text { Grave } 32: 5 \text { 'oblates' with }\end{array}$ & $\begin{array}{l}\text { Cook } 1981,28-30,32-4 \text {, } \\
\text { figs } 9.1 .3 \text { and } 11.32 .1 .4 ; \\
\text { Scunthorpe Museum }\end{array}$ \\
\hline
\end{tabular}




\begin{tabular}{|c|c|c|c|c|}
\hline & & $\begin{array}{l}\text { half } \\
\text { 6th } c\end{array}$ & $\begin{array}{l}\text { Åberg Group III cruciform } \\
\text { brooch and } 2 \text { small-long } \\
\text { brooches, etc }\end{array}$ & \\
\hline Quarrington & Lincs & $\begin{array}{l}\text { prob } \\
\text { 6th } c\end{array}$ & No details & $\begin{array}{l}\text { Bruce } 1880,76 \text {, nos } 303-4 \text {; } \\
\text { Alnwick Museum }\end{array}$ \\
\hline $\begin{array}{l}\text { South } \\
\text { Willingham }\end{array}$ & Lincs & $\begin{array}{l}\text { 6th- } \\
7 \text { th } c\end{array}$ & $\begin{array}{l}\text { Some unassociated and } \\
\text { others with } 6 \text { th to } 7 \text { th- } \\
\text { century beads }\end{array}$ & $\begin{array}{l}\text { Meaney } 1964,165 \\
\text { BM OA } 5057\end{array}$ \\
\hline Waddington & Lincs & $\begin{array}{l}\text { prob } \\
\text { 7th c }\end{array}$ & Two beads & $\begin{array}{l}\text { Meaney 1964, 165; } \\
\text { Lincoln Museum }\end{array}$ \\
\hline $\begin{array}{l}\text { Welbeck } \\
\text { Hill }\end{array}$ & Lincs & 6th $c$ & $\begin{array}{l}\text { With } 2 \text { small cruciform } \\
\text { brooches }\end{array}$ & $\begin{array}{l}\text { Publication in } \\
\text { preparation (G Taylor); } \\
\text { Scunthorpe Museum }\end{array}$ \\
\hline $\begin{array}{l}\text { Castle } \\
\text { Acre }\end{array}$ & Norfolk & & One barrel-shaped & $\begin{array}{l}\text { Meaney } 1964,172-3 ; \\
\text { Norwich Museum }\end{array}$ \\
\hline Kenninghall & Norfolk & $\begin{array}{l}5 \text { th- } \\
7 \text { th c }\end{array}$ & Three beads & $\begin{array}{l}\text { Manning } 1872 a, 292 \text {, and } \\
1872 b \text {; } \\
\text { BM } 837-231\end{array}$ \\
\hline $\begin{array}{l}\text { Morning } \\
\text { Thorpe }\end{array}$ & Norfolk & 6th $c$ & $\begin{array}{l}\text { Many from this cemetery; } \\
\text { eg, Grave 153: } 2 \text { beads } \\
\text { with Åberg Group III } \\
\text { cruciform brooch, etc }\end{array}$ & $\begin{array}{l}\text { Green } \text { et al } 1987,78 \text {, } \\
\text { fig } 347.153 \text {. Giv and Hiii; } \\
\text { Norwich Museum }\end{array}$ \\
\hline Spong Hill & Norfolk & $\begin{array}{l}\text { late } \\
5 \text { th- } \\
6 \text { th c } \\
\text { mid- } \\
\text { 6th c }\end{array}$ & $\begin{array}{l}\text { Grave } 5: 2 \text { globulars } \\
\text { with small-long brooch } \\
\text { pair, A clasps, etc; } \\
\text { Grave } 22: 7 \text { annulars } \\
\text { with Åberg Group IVa } \\
\text { cruciform brooch, } \\
\text { Group III brooch pair, etc; } \\
\text { Grave } 24: 3 \text { globulars and } \\
1 \text { annular with great } \\
\text { square-headed brooch, etc; } \\
\text { Grave } 26: 1 \text { barrel with } \\
\text { Aberg Group I cruciform } \\
\text { brooch, etc; } \\
\text { Grave } 42: 1 \text { globular with } \\
\text { small-long brooch pair, } \\
\text { C clasps, etc; } \\
\text { Grave } 57: 2 \text { globulars } \\
\text { with florid cruciform } \\
\text { brooch, B clasps, etc }\end{array}$ & $\begin{array}{l}\text { Hills et al } 1984 \text {, } \\
54-5,72-3,74-5, \\
95-7 \text { and } 110-12, \\
\text { figs } 73.3 b, 79.5 \text {, } \\
80.2 \text { and } 5 b, 83.1,98.3 \\
\text { and } 108.10 ; \\
\text { Norfolk Arch Unit }\end{array}$ \\
\hline $\begin{array}{l}\text { prob } \\
\text { Brixworth II }\end{array}$ & Northants & & Two beads & $\begin{array}{l}\text { Smith } 1902,245-6 \text {; } \\
\text { Northampton Museum }\end{array}$ \\
\hline Upton & Northants & 7 th $\mathrm{c}$ & Grubenhaus & $\begin{array}{l}\text { Jackson et al 1969; } \\
\text { Ashmolean Museum }\end{array}$ \\
\hline
\end{tabular}




\begin{tabular}{|c|c|c|c|c|}
\hline Corbridge & $\begin{array}{l}\text { Northum- } \\
\text { berland }\end{array}$ & $\begin{array}{l}\text { late } \\
5 \text { th- } \\
\text { early } \\
\text { 6th c }\end{array}$ & $\begin{array}{l}\text { Bead string with } 2 \\
\text { Åberg Group II } \\
\text { cruciform brooches }\end{array}$ & $\begin{array}{l}\text { Knowles and Forster } \\
\text { 1909, 406-8, fig 25; } \\
\text { Brown 1915a (IV), } \\
811-12 \text {, pl CIV.2; } \\
\text { Corbridge Museum }\end{array}$ \\
\hline $\begin{array}{l}\text { Standlake } \\
\text { Down }\end{array}$ & Oxon & $\begin{array}{l}\text { early } \\
7 \text { th c }\end{array}$ & Grave 24 & $\begin{array}{l}\text { Dickinson 1973, 244-5, } \\
\text { fig 5.1.k; } \\
\text { Ashmolean Museum }\end{array}$ \\
\hline $\begin{array}{l}\text { Empingham } \\
\text { II }\end{array}$ & Rutland & $\begin{array}{l}\text { 5th- } \\
7 \text { th c }\end{array}$ & Three annulars & $\begin{array}{l}\text { Timby 1996; } \\
\text { Oakham Museum }\end{array}$ \\
\hline Camerton & Somerset & $\begin{array}{l}\text { prob } \\
\text { 7th c }\end{array}$ & Grave 79: with iron pin & $\begin{array}{l}\text { Horne } 1933,46 \text { and } 57 \text {; } \\
\text { Taunton Museum }\end{array}$ \\
\hline $\begin{array}{l}\text { Bury St } \\
\text { Edmunds, } \\
\text { Westgarth } \\
\text { Gardens }\end{array}$ & Suffolk & $\begin{array}{l}\text { late } \\
5 \text { th- } \\
6 \text { th c } \\
\text { prob } \\
6 \text { th c }\end{array}$ & $\begin{array}{l}\text { Grave 13: } 5 \text { beads with } \\
\text { annular brooch pair, } \\
\text { stamped long-bossed } \\
\text { pot, etc; } \\
\text { Grave } 52: 1 \text { 'disc' } \\
\text { with A } 8 \text { berg Group II } \\
\text { cruciform brooch, etc; } \\
\text { Grave } 57: 5 \text { beads with } \\
\text { iron knife, etc }\end{array}$ & $\begin{array}{l}\text { West } 1988,23,33-4 \\
\text { and } 35 \text {, figs } 63 . D .7,11 \text {, } \\
17,20 \text { and } 24 \text {, figs } \\
76.52 . F .1 \text { and } \\
79.57 . C 2-7 \text {; } \\
\text { Bury St Edmunds } \\
\text { Museum }\end{array}$ \\
\hline Ipswich & Suffolk & $\begin{array}{l}\text { 6th- } \\
7 \text { th c }\end{array}$ & Many examples & $\begin{array}{l}\text { Layard } 1907 \text { and 1909; } \\
\text { Ipswich Museum }\end{array}$ \\
\hline $\begin{array}{l}\text { Little } \\
\text { Eriswell }\end{array}$ & Suffolk & 6th $c$ & Grave 27 & $\begin{array}{l}\text { Hutchinson 1966; } \\
\text { Bury St Edmunds } \\
\text { Museum }\end{array}$ \\
\hline $\begin{array}{l}\text { Mitchell's } \\
\text { Hill }\end{array}$ & Suffolk & $\begin{array}{l}\text { prob } \\
\text { 6th } c\end{array}$ & $\begin{array}{l}\text { Many small globulars and } \\
\text { annulars }\end{array}$ & $\begin{array}{l}\text { Meaney } 1964,231 ; \\
\text { Ashmolean Museum } \\
1909.477\end{array}$ \\
\hline Snape & Suffolk & $\begin{array}{l}\text { 6th- } \\
\text { 7th c }\end{array}$ & $\begin{array}{l}\text { Grave } 0420: 7 \text { annulars } \\
\text { or globulars }\end{array}$ & $\begin{array}{l}\text { Filmer-Sankey and } \\
\text { Pestell forthcoming }\end{array}$ \\
\hline Mitcham & Surrey & $\begin{array}{l}\text { prob } \\
\text { 6th c }\end{array}$ & $\begin{array}{l}\text { Grave 129: (7); } \\
\text { Grave 197: (9) }\end{array}$ & $\begin{array}{l}\text { Bidder and Morris } 1959 \text {, } \\
69,73,112, \\
\text { pl XVI.129 and 197; } \\
\text { Cambridge Museum }\end{array}$ \\
\hline Alfriston & Sussex & $\begin{array}{l}\text { 6th c } \\
\text { first } \\
\text { half } \\
6 \text { th c } \\
5 \text { th- } \\
6 \text { th c } \\
5 \text { th- } \\
7 \text { th c }\end{array}$ & $\begin{array}{l}\text { Grave } 23 \text { : with buckle, } \\
\text { amber beads, etc; } \\
\text { Grave } 43 \text { : on silver } \\
\text { wire ring with } 2 \text { other } \\
\text { glass beads with great } \\
\text { square-headed brooch, etc; } \\
\text { Grave } 68 \text { : with quoit } \\
\text { brooch, etc; } \\
\text { Grave } 77 \text { : with iron knife, } \\
\text { etc }\end{array}$ & $\begin{array}{l}\text { Griffith and Salzmann } \\
\text { 1914, 34, 39-41 and 46-7, } \\
\text { pls II.5 and I.1; } \\
\text { Griffith 1915, 202-3; } \\
\text { Welch } 1983,82 \text { and } 84-5 \text {, } \\
\text { figs } 8 \text { b, 20a and 32c; } \\
\text { Lewes Museum }\end{array}$ \\
\hline $\begin{array}{l}\text { Apple } \\
\text { Down I }\end{array}$ & Sussex & $\begin{array}{l}\text { first } \\
\text { half } \\
\text { 6th c }\end{array}$ & $\begin{array}{l}\text { Grave 13: } 2 \text { globulars; } \\
\text { with cast saucer brooch, } \\
\text { finger ring, etc; }\end{array}$ & $\begin{array}{l}\text { Down and Welch } 1990 \text {, } \\
162 \text { and } 164,36,46 \text { and } \\
51 \text {, figs } 2.19 .10 \text {, }\end{array}$ \\
\hline
\end{tabular}




\begin{tabular}{|c|c|c|c|c|}
\hline & & $\begin{array}{l}\text { prob } \\
\text { 6th c } \\
\text { prob } \\
\text { 6th c }\end{array}$ & $\begin{array}{l}\text { Grave } 90: 1 \text { very small } \\
\text { globular with amber } \\
\text { bead; } \\
\text { Grave } 134: \text { globular } \\
\text { with amber beads }\end{array}$ & $\begin{array}{l}2.32 .90 .1 \text { and } 2.43 .3 \text {, } \\
\text { colour pls } 40.2 \text { and } \\
41.27 \text { and } 32 ; \\
\text { Chichester Museum }\end{array}$ \\
\hline Highdown & Sussex & $\begin{array}{l}\text { prob } \\
\text { 6th c }\end{array}$ & $\begin{array}{l}\text { Grave 15: }(3489) ; \\
\text { Grave } 36:(3481) ; \\
\text { Grave 58: }(3548) ; \\
\text { Grave } 85:(3478) ; \\
\text { unassociated: } 3485 \text { and } \\
3490\end{array}$ & $\begin{array}{l}\text { Read } 1895 \text { and } \\
1896 ; \\
\text { Welch } 1983,85-6 \text {, } \\
\text { figs } 94 \mathrm{~d}, 101 \mathrm{~b}, 104 \mathrm{~d} \\
\text { and } 108 \mathrm{c} ; \\
\text { Worthing Museum }\end{array}$ \\
\hline $\begin{array}{l}\text { Stretton- } \\
\text { on-Fosse II }\end{array}$ & Warks & $\begin{array}{l}c .450- \\
550\end{array}$ & $\begin{array}{l}\text { Grave } 41 \text { : with silvered } \\
\text { bronze disc brooch }\end{array}$ & $\begin{array}{l}\text { Gelling } 1992,31,40-1 \text {, } \\
\text { fig } 21\end{array}$ \\
\hline Wasperton & Warks & $\begin{array}{l}\text { late } \\
5 \text { th- } \\
6 \text { th c }\end{array}$ & Some dated examples & $\begin{array}{l}\text { Publication in } \\
\text { preparation (M Carver); } \\
\text { Warwick Museum }\end{array}$ \\
\hline $\begin{array}{l}\text { Colling- } \\
\text { bourne } \\
\text { Ducis }\end{array}$ & Wilts & $\begin{array}{l}\text { late } \\
5 \text { th- } \\
6 \text { th c }\end{array}$ & $\begin{array}{l}\text { Grave } 20: 1 \text { with cast } \\
\text { saucer brooch }\end{array}$ & $\begin{array}{l}\text { Gingell } 1975,85, \\
\text { fig 18.10; } \\
\text { Devizes Museum }\end{array}$ \\
\hline Mildenhall & Wilts & 6th $c$ & $\begin{array}{l}\text { With applied saucer } \\
\text { brooch, etc }\end{array}$ & $\begin{array}{l}\text { Meaney } 1964,270 \\
\text { Devizes Museum }\end{array}$ \\
\hline $\begin{array}{l}\text { Peters- } \\
\text { finger }\end{array}$ & Wilts & $\begin{array}{l}\text { first } \\
\text { half } \\
6 \text { th c } \\
\text { late } \\
\text { 5th- } \\
6 \text { th c } \\
\text { late } \\
\text { 5th- } \\
\text { 6th c }\end{array}$ & $\begin{array}{l}\text { Grave 25: } 4 \text { beads with } \\
\text { small bow brooch and } 3 \\
\text { button brooches, etc; } \\
\text { Grave 29: } 1 \text { bead with } \\
\text { applied saucer brooch } \\
\text { base, etc; } \\
\text { Grave 50: } 2 \text { beads with } \\
\text { iron knife, etc }\end{array}$ & $\begin{array}{l}\text { Leeds and Shortt } 1953 \text {, } \\
21-2,24-6 \text { and } 33-4, \\
\text { pls III ( } 94 . X X V \text { and } \\
104 . X X I X) \text { and IV } \\
\text { (141.L); } \\
\text { Salisbury Museum }\end{array}$ \\
\hline $\begin{array}{l}\text { Pewsey } \\
\text { Blackpatch }\end{array}$ & Wilts & $\begin{array}{l}5 \text { th- } \\
6 \text { th c }\end{array}$ & Grave 48 & $\begin{array}{l}\text { Publication in } \\
\text { preparation (K Annable); } \\
\text { Devizes Museum }\end{array}$ \\
\hline Swindon & Wilts & 7th $\mathrm{c}$ & Grubenhaus & $\begin{array}{l}\text { Information from } \\
\text { Caroline Washbourne }\end{array}$ \\
\hline Driffield & Yorks & $\begin{array}{l}\text { prob } \\
6 \text { th c }\end{array}$ & $\begin{array}{l}\text { Barrow C38 Grave 21: } \\
\text { with amber beads, etc }\end{array}$ & $\begin{array}{l}\text { Mortimer } 1905,280 \text {, } \\
\text { pl C, no. } 795 ; \\
\text { Hull Museum }\end{array}$ \\
\hline Sewerby & Yorks & $\begin{array}{l}\text { mid- } \\
\text { 6th c } \\
\text { 6th c }\end{array}$ & $\begin{array}{l}\text { Grave 8: } 2 \text { annulars (A1a) } \\
\text { with Aberg Group IVa } \\
\text { cruciform brooch, etc; } \\
\text { Grave } 41 \text { : } 41 / 35 \text { (A2c) with } \\
\text { annular brooch pair, etc }\end{array}$ & $\begin{array}{l}\text { Hirst } 1985,62-4 \\
\text { (Ala) and } 66(\mathrm{~A} 2 \mathrm{c}) \text {, } \\
\text { figs } 22,34.6-65 \mathrm{~g} \\
\text { and } 48.11 \mathrm{a}\end{array}$ \\
\hline $\begin{array}{l}\text { West } \\
\text { Heslerton }\end{array}$ & Yorks & $\begin{array}{l}\text { prob } \\
\text { 6th c }\end{array}$ & A large number & $\begin{array}{l}\text { Publication in } \\
\text { preparation } \\
\text { (D Powlesland) }\end{array}$ \\
\hline
\end{tabular}




$\begin{array}{llll}\begin{array}{l}\text { near } \\ \text { York }\end{array} & \text { Yorks } & \begin{array}{l}\text { Group not necessarily } \\ \text { associated }\end{array} & \text { BM 53 11-15 } 16\end{array}$

\section{ii Opaque yellow cylinder beads: (a) Round}

$\begin{array}{llll}\text { Little } & \text { Derbys } & \text { 6th- } & \text { Grave 320 } \\ \text { Chesters } & & 7 \text { th c }\end{array}$

Mucking II Essex

6th-

7th c

first

half

5 th c

Folkestone Kent prob

6th $\mathrm{c}$

Gilton or Kent 6th-

Kingston

\begin{tabular}{|c|c|}
\hline $\begin{array}{l}\text { Horton } \\
\text { Kirby I }\end{array}$ & Kent \\
\hline Sarre & Kent \\
\hline Saxby & Leics \\
\hline $\begin{array}{l}\text { Welbeck } \\
\text { Hill }\end{array}$ & Lincs \\
\hline
\end{tabular}

Kenninghall Norfolk 5th-

7 th $\mathrm{c}$

Spong Hill Norfolk 6 th $\mathrm{c}$

West Stow

Suffolk

etc late Settlement SFB39:

5 th- 1 bead (SF930) with

6th c small-long brooch, etc;

5th- Cemetery: 1 example

7 th $\mathrm{c}$
Wheeler 1985, 304;

publication in

preparation

(C Sparey-Green)

Ten beads overall, including: Hirst and Clark forthcoming;

Grave 914: 2 beads;

Grave 962: 2 beads with

Evison Type $3 \mathrm{knife}$, etc;

Grave 989: 2 beads with Evison 1981, 138-9,

Glaston-Mucking and

supporting-arm brooches,

No. 38: broken bead

Some examples

One large thick cylinder

(an atypical bead)

One example

figs 4 and 5 ;

BM

Meaney 1964, 120-1;

Folkestone Museum

Faussett 1856;

Liverpool Museum

Meaney 1964, 124;

Maidstone Museum

Meaney 1964, 135-6;

BM 93 6-1 218

Publication in

preparation (J Timby)

Publication in preparation (G Taylor);

Scunthorpe Museum

Manning 1872a, 292, and

1872b;

BM 83 7-2 31

Hills et al 1984, 66-7, fig 77.18.4; Norfolk Arch Unit

West 1985, 34-5, 73, fig 135.9; Bury St

Edmunds Museum; West 1985, 74, fig 275.27;

Ashmolean Museum 
ii Opaque yellow cylinder beads: (b) polygonal OR SQUARE-SECTIONED

\begin{tabular}{|c|c|c|c|c|}
\hline $\begin{array}{l}\text { Linton } \\
\text { Heath B }\end{array}$ & Cambs & $\begin{array}{l}\text { late } \\
5 \text { th- } \\
6 \text { th c }\end{array}$ & $\begin{array}{l}\text { One square-sectioned } \\
\text { bead (Plate } 4 \text { ) with } 2 \text { small- } \\
\text { long brooches }\end{array}$ & $\begin{array}{l}\text { Neville 1854; } \\
\text { Cambridge Museum }\end{array}$ \\
\hline Mucking II & Essex & $\begin{array}{l}\text { 6th- } \\
7 \text { th } \mathrm{c}\end{array}$ & $\begin{array}{l}\text { Grave } 608: 1 \text { bead; } \\
\text { Grave } 962: 1 \text { bead with } \\
\text { Evison Type } 3 \text { knife; } \\
\text { both pentagonal }\end{array}$ & $\begin{array}{l}\text { Hirst and Clark forthcoming; } \\
\text { BM }\end{array}$ \\
\hline $\begin{array}{l}\text { Dover, } \\
\text { Buckland }\end{array}$ & Kent & $\begin{array}{l}625- \\
650\end{array}$ & $\begin{array}{l}\text { Grave } 62: 6 \text { pentagonal } \\
\text { beads }(B 21)\end{array}$ & $\begin{array}{l}\text { Evison } 1987,73,80, \\
\text { 234, fig } 35.62 .4 \mathrm{i}, \\
\text { colour pl III (B21); BM }\end{array}$ \\
\hline Faversham & Kent & $\begin{array}{l}5 \text { th- } \\
7 \text { th } c\end{array}$ & Square-sectioned & $\begin{array}{l}\text { Meaney 1964, 118-19; } \\
\text { Ashmolean Museum }\end{array}$ \\
\hline 'Kent' & Kent & & One pentagonal (Plate 4) & Canterbury Museum \\
\hline Stowting & Kent & $\begin{array}{l}\text { prob } \\
6 \text { th- } \\
7 \text { th c } \\
\text { prob } \\
6 \text { th } \mathrm{c}\end{array}$ & One pentagonal; & $\begin{array}{l}\text { Smith 1846a; Brent 1867; } \\
\text { Beck Collection, } \\
\text { Cambridge Museum; } \\
\text { Maidstone Museum }\end{array}$ \\
\hline Fonaby & Lincs & $\begin{array}{l}\text { prob } \\
6 \text { th c }\end{array}$ & $\begin{array}{l}\text { Group 6: } 1 \text { square- } \\
\text { sectioned with amber and } \\
\text { meerschaum beads, etc }\end{array}$ & $\begin{array}{l}\text { Cook 1981, 48, } \\
\text { fig 19.12, pl VIIB; } \\
\text { Scunthorpe Museum }\end{array}$ \\
\hline Brooke & Norfolk & $\begin{array}{l}\text { prob } \\
\text { 6th } c\end{array}$ & Pentagonal & $\begin{array}{l}\text { Meaney 1964, 170; } \\
\text { BM 1866-69 }\end{array}$ \\
\hline Kenninghall & Norfolk & $\begin{array}{l}\text { 5th- } \\
7 \text { th } c\end{array}$ & Pentagonal & $\begin{array}{l}\text { Manning 1872a, 292, and } \\
\text { 1872b; BM }\end{array}$ \\
\hline Spong Hill & Norfolk & $\begin{array}{l}5 \text { th- } \\
\text { 6th } c\end{array}$ & $\begin{array}{l}\text { Cremation 2213: } 1 \text { long } \\
\text { square-sectioned bead } \\
\text { in shoulder-bossed urn } \\
\text { with linear decoration }\end{array}$ & $\begin{array}{l}\text { Hills and Penn 1981, 61, } \\
\text { fig 166.2213; } \\
\text { Norfolk Arch Unit }\end{array}$ \\
\hline Wasperton & Warks & $\begin{array}{l}\text { mid- } \\
\text { 6th c }\end{array}$ & $\begin{array}{l}\text { Several pentagonal } \\
\text { with saucer brooches }\end{array}$ & $\begin{array}{l}\text { Publication in } \\
\text { preparation (M Carver); } \\
\text { Warwick Museum }\end{array}$ \\
\hline $\begin{array}{l}\text { Saltburn, } \\
\text { Hob Hill }\end{array}$ & Yorks & $\begin{array}{l}\text { 6th- } \\
7 \text { th } c\end{array}$ & $\begin{array}{l}\text { Long square-sectioned } \\
\text { bead }\end{array}$ & $\begin{array}{l}\text { Gallagher } 1987,19,25 \text {, } \\
\text { fig 5.43; } \\
\text { Middlesbrough Museum }\end{array}$ \\
\hline $\begin{array}{l}\text { West } \\
\text { Heslerton }\end{array}$ & Yorks & $\begin{array}{l}\text { prob } \\
\text { 6th c }\end{array}$ & $\begin{array}{l}\text { Grave 2B 58: square } \\
\text { section, with annular } \\
\text { brooch, etc }\end{array}$ & $\begin{array}{l}\text { Publication in } \\
\text { preparation } \\
\text { (D Powlesland) }\end{array}$ \\
\hline
\end{tabular}




\section{ii Opaque yellow Cylinder BeADS: (c) SHORT CYLINDERS, SINGLES OR DOUBLES}

\begin{tabular}{|c|c|c|c|c|}
\hline Mucking II & Essex & $\begin{array}{l}\text { prob } \\
\text { 6th c }\end{array}$ & Grave 578 & $\begin{array}{l}\text { Hirst and Clark forthco } \\
\text { BM }\end{array}$ \\
\hline $\begin{array}{l}\text { Dover, } \\
\text { Buckland }\end{array}$ & Kent & $\begin{array}{l}700- \\
750\end{array}$ & $\begin{array}{l}\text { Grave } 1: 19 \text { short } \\
\text { (B18 and B20); } \\
\text { Grave 29: } 4 \text { short (B18); } \\
\text { Grave 32: } 1 \text { short (B18); } \\
\text { Grave 60: } 4 \text { short (B18); } \\
\text { Grave } 76: 1 \text { short } \\
\text { (B19) }\end{array}$ & $\begin{array}{l}\text { Evison } 1987,73,80 \text {, } \\
215-16,223-4,225 \text {, } \\
233 \text { and } 236 \text {, } \\
\text { figs } 5.4 \mathrm{j} \text { and } \mathrm{p} \text {, } \\
17.2 \mathrm{~h}, 19.4 \mathrm{~d} \text {, } \\
34.3 \mathrm{~g} \text { and } 39.76 .1 \mathrm{a} \text {; } \\
\mathrm{BM}\end{array}$ \\
\hline $\begin{array}{l}\text { Eastry, } \\
\text { Eastry House }\end{array}$ & Kent & $\begin{array}{l}\text { late } \\
\text { 6th } c\end{array}$ & $\begin{array}{l}\text { Grave 1: several very } \\
\text { short or elements of } \\
\text { doubles (nos } 6 \text { and } 28 \text {, } \\
11,24 \text { and } 35 \text { ) with a } \\
\text { Gotlandic square-headed } \\
\text { bow brooch }\end{array}$ & $\begin{array}{l}\text { Hawkes } 1979,86,93 \text {, } \\
\text { fig 4.4.11, } 24,28 \text { and } 35 \\
\text { Deal Museum }\end{array}$ \\
\hline Faversham & Kent & $\begin{array}{l}5 \text { th- } \\
7 \text { th c }\end{array}$ & $\begin{array}{l}\text { Several singles and } \\
\text { doubles }\end{array}$ & $\begin{array}{l}\text { Meaney 1964, 118-19; } \\
\text { Ashmolean Museum } \\
1909.170\end{array}$ \\
\hline Folkestone & Kent & $\begin{array}{l}\text { prob } \\
\text { 6th c }\end{array}$ & One double and 1 short & $\begin{array}{l}\text { Meaney } 1964,120-1 \\
\text { Folkestone Museum }\end{array}$ \\
\hline Holborough & Kent & $\begin{array}{l}\text { late } \\
7 \text { th- } \\
8 \text { th c }\end{array}$ & Twelve doubles & $\begin{array}{l}\text { Evison } 1956,92,126 \text {, } \\
\text { fig } 20.3 \mathrm{a} \text {; } \\
\text { BM } 1947 \text { 5-12 } 358\end{array}$ \\
\hline 'Kent' & Kent & & One example & Maidstone Museum \\
\hline Stowting & Kent & $\begin{array}{l}\text { prob } \\
\text { 6th } c\end{array}$ & One example & $\begin{array}{l}\text { Meaney } 1964,137-8 \\
\text { Maidstone Museum }\end{array}$ \\
\hline Cleatham & Lincs & $\begin{array}{l}5 \text { th- } \\
7 \text { th } c\end{array}$ & Grave 30: 1 short & $\begin{array}{l}\text { Publication in } \\
\text { preparation (K Leahy); } \\
\text { Scunthorpe Museum }\end{array}$ \\
\hline Syston Park & Lincs & $\begin{array}{l}\text { prob } \\
\text { 6th } c\end{array}$ & One short & $\begin{array}{l}\text { Akerman 1855a, 41-2, } \\
\text { pl XXI; } \\
\text { BM (18) } 1111-92\end{array}$ \\
\hline Waddington & Lincs & & Two examples & $\begin{array}{l}\text { Meaney } 1964,165 \\
\text { Lincoln Museum }\end{array}$ \\
\hline $\begin{array}{l}\text { Morning } \\
\text { Thorpe }\end{array}$ & Norfolk & $\begin{array}{l}\text { late } \\
5 \text { th- } \\
6 \text { th c } \\
\text { late } \\
5 \text { th- } \\
6 \text { th c }\end{array}$ & $\begin{array}{l}\text { Grave 231: with small- } \\
\text { long brooch pair, etc; } \\
\text { Grave } 284: 1 \text { bead with } \\
2 \text { iron buckles, etc; }\end{array}$ & $\begin{array}{l}\text { Green } \text { et al } 1987, \\
98,113,115 \text { and } 142 \text {, } \\
\text { figs } 371 . D, 387.284 . \\
\text { Ciii, 389.288.G and } \\
\text { 425.Bi; } \\
\text { Norwich Museum }\end{array}$ \\
\hline
\end{tabular}


6th c

Grave 288: 1 bead with great square-headed brooch, etc; Grave 363: 1 bead with 1 green cylinder and 2 potsherds

\begin{tabular}{|c|c|c|c|c|}
\hline Standlake & Oxon & $\begin{array}{l}\text { early } \\
7 \text { th c }\end{array}$ & $\begin{array}{l}\text { Grave 24: double } \\
\text { (Plate 4) }\end{array}$ & $\begin{array}{l}\text { Dickinson 1973, 244-5, } \\
\text { fig 5.1.b; } \\
\text { Ashmolean Museum }\end{array}$ \\
\hline $\begin{array}{l}\text { Bury St } \\
\text { Edmunds, } \\
\text { Westgarth } \\
\text { Gardens }\end{array}$ & Suffolk & $\begin{array}{l}5 \text { th- } \\
7 \text { th } \mathrm{c}\end{array}$ & $\begin{array}{l}\text { Grave } 14: 2 \text { beads out } \\
\text { of a string of four }\end{array}$ & $\begin{array}{l}\text { West 1988, 24, } \\
\text { fig } 61.14 . A 1 \text { and 2; } \\
\text { Bury St Edmunds } \\
\text { Museum }\end{array}$ \\
\hline Ipswich & Suffolk & $\begin{array}{l}\text { 6th- } \\
7 \text { th } c\end{array}$ & Several & $\begin{array}{l}\text { Layard } 1907 \text { and 1909; } \\
\text { Ipswich Museum }\end{array}$ \\
\hline West Stow & Suffolk & $\begin{array}{l}\text { 5th- } \\
7 \text { th } c\end{array}$ & $\begin{array}{l}\text { Cemetery: small } \\
\text { singles and doubles }\end{array}$ & $\begin{array}{l}\text { West } 1985,74 \text {, fig 275.16; } \\
\text { Bury St Edmunds } \\
\text { Museum }\end{array}$ \\
\hline Mitcham & Surrey & 6th $c$ & Grave $117: 3$ beads & $\begin{array}{l}\text { Bidder and Morris 1959, } \\
68,112 \text {, pl XVI.117; } \\
\text { Cambridge Museum }\end{array}$ \\
\hline Wasperton & Warks & $\begin{array}{l}\text { 5th- } \\
6 \text { th } c\end{array}$ & One short & $\begin{array}{l}\text { Publication in } \\
\text { preparation (M Carver); } \\
\text { Warwick Museum }\end{array}$ \\
\hline
\end{tabular}

\begin{tabular}{|c|c|c|c|c|}
\hline $\begin{array}{l}\text { Chamber- } \\
\text { lain's } \\
\text { Barn II }\end{array}$ & Beds & $\begin{array}{l}\text { mid- } \\
\text { late } \\
\text { 7th c }\end{array}$ & $\begin{array}{l}\text { Grave 9: } 1 \text { example } \\
\text { (Plate 4) with silver } \\
\text { wire rings, etc }\end{array}$ & $\begin{array}{l}\text { Hyslop 1963, 173, } \\
\text { fig 9.9.c; } \\
\text { Luton Museum }\end{array}$ \\
\hline Kempston & Beds & & One example & $\begin{array}{l}\text { Fitch 1864; } \\
\text { BM 91 6-24 } 54\end{array}$ \\
\hline Frilford & Berks & $\begin{array}{l}\text { prob } \\
\text { 7th } c\end{array}$ & $\begin{array}{l}\text { String with } 2 \text { beads of } \\
\text { this type }\end{array}$ & $\begin{array}{l}\text { Meaney } 1964,46-7 \\
\text { BM OA } 4880\end{array}$ \\
\hline Lechlade & Glos & $\begin{array}{l}\text { 6th c } \\
\text { 7th c } \\
\text { 7th c } \\
\text { 7th c }\end{array}$ & $\begin{array}{l}\text { Grave } 17: 1 \text { bead with } \\
\text { Kentish disc brooch, etc; } \\
\text { Grave } 84: 2 \text { beads; } \\
\text { Grave } 145 / 2: 1 \text { bead with } \\
\text { silver wire rings, etc; } \\
\text { Grave } 177: 1 \text { bead with } \\
\text { silver wire rings }\end{array}$ & $\begin{array}{l}\text { Boyle et al forthcoming; } \\
\text { Cirencester Museum }\end{array}$ \\
\hline Horndean & Hants & $\begin{array}{l}\text { prob } \\
\text { 7th c }\end{array}$ & $\begin{array}{l}\text { Grave S. } 27 \text { : with other } \\
\text { beads and bronze wire ring }\end{array}$ & $\begin{array}{l}\text { Knocker 1957, 136, } \\
\text { fig 15.1; } \\
\text { Portsmouth Museums }\end{array}$ \\
\hline $\begin{array}{l}\text { Dover, } \\
\text { Buckland }\end{array}$ & Kent & $\begin{array}{l}575- \\
625\end{array}$ & Grave 1: 1 bead (B17) & $\begin{array}{l}\text { Evison } 1987,73,80 \text {, } \\
215-16 \text {, fig 5.4h; BM }\end{array}$ \\
\hline
\end{tabular}




\begin{tabular}{|c|c|c|c|c|}
\hline Howletts & Kent & 6th $c$ & One tall & $\begin{array}{l}\text { Smith 1918; } \\
\text { BM } 1936 \text { 5-11 } 144\end{array}$ \\
\hline Polhill & Kent & 7th $c$ & $\begin{array}{l}\text { Grave } 37: 1 \text { bead (no. } 517 \text { ) } \\
\text { with amethyst bead and } \\
\text { jewelled disc brooch, etc; } \\
\text { Grave } 51: 1 \text { bead (no. } 522 \text { ) } \\
\text { with } 2 \text { disc pendants, etc; } \\
\text { Grave } 55: 1 \text { biconical (Plate 4) }\end{array}$ & $\begin{array}{l}\text { Hawkes and Philp 1973, } \\
176,179 \text { and } 207, \\
\text { fig } 55.518 \text { and } 522\end{array}$ \\
\hline $\begin{array}{l}\text { Saltburn, } \\
\text { Hob Hill }\end{array}$ & Yorks & $\begin{array}{l}\text { 6th- } \\
7 \text { th } c\end{array}$ & Abnormally tall & $\begin{array}{l}\text { Gallagher } 1987,25 \text {, } \\
\text { fig } 6.67 \text {; } \\
\text { Middlesbrough Museum }\end{array}$ \\
\hline $\begin{array}{l}\text { Dalmeny } \\
\text { Hound Point }\end{array}$ & $\begin{array}{l}\text { Scotland: } \\
\text { West } \\
\text { Lothian }\end{array}$ & $\begin{array}{l}\text { prob } \\
7 \text { th } c\end{array}$ & $\begin{array}{l}\text { Cist burial necklace: } \\
\text { several }\end{array}$ & $\begin{array}{l}\text { Brown 1915b; } \\
\text { Brown 1915a (IV), } \\
\text { 812-13, fig 29; } \\
\text { Edinburgh Museum }\end{array}$ \\
\hline iv & Opaque & YELLOW & MELON, SUB-MELON AND & 'NICKED' BEADS \\
\hline Halton & Cambs & $\begin{array}{l}\text { prob } \\
6 \text { th c }\end{array}$ & $\begin{array}{l}\text { Four-lobed small sub-melon } \\
\text { with pink beads }\end{array}$ & $\begin{array}{l}\text { Meaney } 1964,66-7 \text {; } \\
\text { Birmingham Museum } \\
\text { A } 38673\end{array}$ \\
\hline Mucking II & Essex & $\begin{array}{l}5 \text { th- } \\
6 \text { th c } \\
5 \text { th- } \\
6 \text { th c }\end{array}$ & $\begin{array}{l}\text { Grave } 615: 2 \text { beads with } \\
\text { cast saucer brooch pair, } \\
\text { etc; } \\
\text { Grave } 860: 1 \text { bead with } \\
\text { small-long brooch pair, etc; } \\
\text { Grave } 874: 2 \text { beads with } \\
\text { gold-in-glass and amber } \\
\text { beads, etc }\end{array}$ & $\begin{array}{l}\text { Hirst and Clark forthcoming; } \\
\text { BM }\end{array}$ \\
\hline Stodmarsh & Kent & 6th c & One small example & $\begin{array}{l}\text { Akerman 1855b, 179-81, } \\
\text { pl I; BM }\end{array}$ \\
\hline Saxby & Leics & & & $\begin{array}{l}\text { Publication in } \\
\text { preparation ( } \mathrm{T} \text { Timby) }\end{array}$ \\
\hline Brooke & Norfolk & $\begin{array}{l}\text { prob } \\
\text { 6th c }\end{array}$ & One small bead & $\begin{array}{l}\text { Meaney 1964, 170; } \\
\text { BM 1866-69 }\end{array}$ \\
\hline $\begin{array}{l}\text { Castle } \\
\text { Acre }\end{array}$ & Norfolk & $\begin{array}{l}5 \text { th- } \\
6 \text { th } c\end{array}$ & One example & $\begin{array}{l}\text { Meaney } 1964,172-3 \text {; } \\
\text { Norwich Museum }\end{array}$ \\
\hline Spong Hill & Norfolk & $\begin{array}{l}\text { late } \\
5 \text { th- } \\
6 \text { th c } \\
\text { mid- } \\
6 \text { th c }\end{array}$ & $\begin{array}{l}\text { Grave 5: } 2 \text { abnormal } \\
\text { long melon-shaped with } \\
\text { small-long brooch } \\
\text { pair, A clasps, etc; } \\
\text { Grave 22: very small } \\
\text { with Aberg Group IVa } \\
\text { cruciform and Group III } \\
\text { brooch pair, etc; } \\
\text { Grave 37: } 1 \text { with annular } \\
\text { brooch pair, B clasps, etc; } \\
\text { Grave 42: } 1 \text { with small-long } \\
\text { brooch pair, C clasps, etc }\end{array}$ & $\begin{array}{l}\text { Hills et al } 1984 \text {, } \\
54-5,69-70,86-7 \text { and } \\
95-7, \text { figs } 73.3 b \text {, } \\
90.37 .6,79.5 .29 \\
\text { and } 98.42 .3 ; \\
\text { Norfolk Arch Unit }\end{array}$ \\
\hline
\end{tabular}




\begin{tabular}{|c|c|c|c|c|}
\hline Lakenheath & Suffolk & 6th $\mathrm{c}$ & $\begin{array}{l}\text { Necklace: } 2 \text { small } \\
\text { examples }\end{array}$ & $\begin{array}{l}\text { Meaney 1964, 230; } \\
\text { BM } 191012-229-11\end{array}$ \\
\hline $\begin{array}{l}\text { Mitchell's } \\
\text { Hill }\end{array}$ & Suffolk & $\begin{array}{l}5 \text { th- } \\
7 \text { th } \mathrm{c}\end{array}$ & Two sub-melons & $\begin{array}{l}\text { Meaney 1964, 231; } \\
\text { Ashmolean Museum } \\
1909.477\end{array}$ \\
\hline Snape & Suffolk & $\begin{array}{l}5 \text { th- } \\
7 \text { th c }\end{array}$ & & $\begin{array}{l}\text { Filmer-Sankey and } \\
\text { Pestell forthcoming }\end{array}$ \\
\hline $\begin{array}{l}\text { West } \\
\text { Heslerton }\end{array}$ & Yorks & $\begin{array}{l}\text { prob } \\
\text { 6th c }\end{array}$ & $\begin{array}{l}\text { No. } 917 \text { and possibly } \\
\text { others not seen }\end{array}$ & $\begin{array}{l}\text { Publication in } \\
\text { preparation } \\
\text { (D Powlesland) }\end{array}$ \\
\hline
\end{tabular}

$v$ Opaque yellow wound beads, or joINed globular beads

\begin{tabular}{|c|c|c|c|c|}
\hline Lechlade & Glos & 7th $c$ & $\begin{array}{l}\text { Grave } 145 / 2: 2 \text { wound } \\
\text { coiled cylinders with silver } \\
\text { wire rings, etc }\end{array}$ & $\begin{array}{l}\text { Boyle et al forthcoming; } \\
\text { Cirencester Museum }\end{array}$ \\
\hline Alton & Hants & 7th $\mathrm{c}$ & $\begin{array}{l}\text { Grave 39: } 1 \text { disc double } \\
\text { (B06) with iron knife, etc }\end{array}$ & $\begin{array}{l}\text { Evison 1988a, 16, 41-4, } \\
80-1 \text {, fig 33.39.1d; } \\
\text { Hampshire Museums }\end{array}$ \\
\hline $\begin{array}{l}\text { Breach } \\
\text { Down }\end{array}$ & Kent & $\begin{array}{l}700- \\
730\end{array}$ & One double & $\begin{array}{l}\text { Conyngham and Akerman } \\
1844 \text {, pl I; BM 79 5-29 }\end{array}$ \\
\hline $\begin{array}{l}\text { Dover, } \\
\text { Buckland }\end{array}$ & Kent & $\begin{array}{l}475- \\
525 \\
575- \\
625\end{array}$ & $\begin{array}{l}\text { Grave 46: } 10 \text { 'disc' } \\
\text { doubles (B13); } \\
\text { Grave 29:11 'disc' } \\
\text { doubles (B13); } \\
\text { Grave 32: 9 'disc' } \\
\text { doubles (B13): } \\
\text { Grave 35: 1 'disc' } \\
\text { double (B13); } \\
\text { Grave 6: } 5 \text { 'disc' } \\
\text { doubles (B13) }\end{array}$ & $\begin{array}{l}\text { Evison } 1987,72,80 \text {, } \\
217,223-4,225,226 \\
\text { and } 229 ; \text { figs } 7.10 c \text {, } \\
17.2 \mathrm{e}, 19.4 \mathrm{~b}, 21.5 \mathrm{~b} \\
\text { and } 26.46 .3 \mathrm{~b} \text {; } \\
\text { BM }\end{array}$ \\
\hline Faversham & Kent & $\begin{array}{l}5 \text { th- } \\
7 \text { th c }\end{array}$ & $\begin{array}{l}\text { One double and some } \\
\text { ?small doubles (broken) }\end{array}$ & $\begin{array}{l}\text { Meaney 1964, 118-19; } \\
\text { Ashmolean Museum }\end{array}$ \\
\hline Folkestone & Kent & $\begin{array}{l}\text { prob } \\
\text { 6th c }\end{array}$ & $\begin{array}{l}\text { No. 19: } 2 \text { doubles; } \\
\text { No. 28: } 4 \text { doubles }\end{array}$ & $\begin{array}{l}\text { Meaney } 1964,120-1 \\
\text { Maidstone Museum }\end{array}$ \\
\hline $\begin{array}{l}\text { Gilton or } \\
\text { Kingston }\end{array}$ & Kent & $\begin{array}{l}\text { prob } \\
\text { 6th- } \\
7 \text { th c }\end{array}$ & Several & $\begin{array}{l}\text { Faussett } 1856 \text {, pls V and VII } \\
\text { Liverpool Museum }\end{array}$ \\
\hline Holborough & Kent & $\begin{array}{l}\text { prob } \\
\text { 7th- } \\
\text { 8th c }\end{array}$ & At least 12 doubles & $\begin{array}{l}\text { Evison } 1956,92 \text { and } 126 \text {, } \\
\text { fig 20a; } \\
\text { BM 1947 5-2 } 358\end{array}$ \\
\hline Howletts & Kent & $\begin{array}{l}\text { prob } \\
\text { 6th c }\end{array}$ & $\begin{array}{l}\text { One segment of broken } \\
\text { double or triple bead }\end{array}$ & $\begin{array}{l}\text { Smith 1918; } \\
\text { BM 1936 5-11 } 144\end{array}$ \\
\hline ‘Kent' & Kent & & $\begin{array}{l}\text { One double and } \\
1 \text { quadruple (Plate 4); } \\
1 \text { coiled }\end{array}$ & $\begin{array}{l}\text { Canterbury } \\
\text { Museum; } \\
\text { Liverpool Museum }\end{array}$ \\
\hline
\end{tabular}




\begin{tabular}{|c|c|c|c|c|}
\hline Sarre & Kent & $\begin{array}{l}\text { prob } \\
\text { 6th- } \\
7 \text { th c }\end{array}$ & $\begin{array}{l}\text { Several doubles and } \\
1 \text { quadruple }\end{array}$ & Meaney $1964,135-6$ \\
\hline Stowting & Kent & $\begin{array}{l}\text { prob } \\
\text { 6th c }\end{array}$ & $\begin{array}{l}\text { Two doubles (Plate 4) and } \\
\text { some annulars that might } \\
\text { have been joined; } \\
\text { l double (wound) }\end{array}$ & $\begin{array}{l}\text { Meaney 1964, 137-8; } \\
\text { Maidstone Museum; } \\
\text { Beck Collection, } \\
\text { Cambridge Museum }\end{array}$ \\
\hline Westbere & Kent & $\begin{array}{l}\text { 6th- } \\
7 \text { th c }\end{array}$ & $\begin{array}{l}\text { ? } 14 \text { triple beads on } \\
\text { strings }\end{array}$ & $\begin{array}{l}\text { Jessup 1946; } \\
\text { Canterbury Museum }\end{array}$ \\
\hline Brooke & Norfolk & $\begin{array}{l}\text { prob } \\
\text { 6th } c\end{array}$ & One double & $\begin{array}{l}\text { Meaney 1964, 170; } \\
\text { BM 1866-69 }\end{array}$ \\
\hline Mitcham & Surrey & $\begin{array}{l}\text { prob } \\
\text { 6th c }\end{array}$ & $\begin{array}{l}\text { Grave 129: } 2 \text { double } \\
\text { and } 1 \text { triple }\end{array}$ & $\begin{array}{l}\text { Bidder and Morris } 1959 \text {, } \\
69,112 \text {, pl XVI.129; } \\
\text { Cambridge Museum }\end{array}$ \\
\hline $\begin{array}{l}\text { Bidford-on- } \\
\text { Avon }\end{array}$ & Warks & $\begin{array}{l}\text { prob } \\
\text { 6th c }\end{array}$ & Two ?doubles on necklace & $\begin{array}{l}\text { Humphreys et al } 1923 \text {, } \\
105, \text { pl XVII.1-3; } \\
\text { Humphreys et al } 1924 \text {, } \\
279-80 \text {, pl LVIII.1 and 2; } \\
\text { Stratford Museum }\end{array}$ \\
\hline 'Yorks' & Yorks & & One triple & Hull Museum \\
\hline
\end{tabular}

\section{POLYCHROME}

vi Opaque yellow Beads With CROSSED WaVes, With or WITHOUT DOTS, SINGLES OR DOUBLES (see Map 16)

\begin{tabular}{|c|c|c|c|c|}
\hline Puddlehill & Beds & $c .600$ & $\begin{array}{l}\text { Grave } 10: 3 \text { with } \\
\text { reddish waves with cast } \\
\text { saucer brooch pair, etc }\end{array}$ & $\begin{array}{l}\text { Matthews and Hawkes } \\
1985,91-9 \text {, } \\
\text { fig } 40.16-18\end{array}$ \\
\hline Frilford I & Berks & $\begin{array}{l}\text { prob } \\
\text { 6th c }\end{array}$ & White waves and green dots & $\begin{array}{l}\text { Meaney 1964, 46-7; } \\
\text { BM } 672-419 ; \\
\text { Ashmolean Museum }\end{array}$ \\
\hline Barrington & Cambs & $\begin{array}{l}5 \text { th- } \\
7 \text { th c }\end{array}$ & $\begin{array}{l}\text { Two beads bottle glass } \\
\text { waves and dots }\end{array}$ & $\begin{array}{l}\text { Fox 1923, 250-2; } \\
\text { Ashmolean Museum }\end{array}$ \\
\hline $\begin{array}{l}\text { Linton } \\
\text { Heath B }\end{array}$ & Cambs & & $\begin{array}{l}\text { Two annulars with green } \\
\text { crossed waves and dots }\end{array}$ & $\begin{array}{l}\text { Neville 1854; } \\
\text { Cambridge Museum }\end{array}$ \\
\hline Mucking II & Essex & $\begin{array}{l}\text { 2nd } \\
\text { half } \\
5 \text { th c } \\
7 \text { th c } \\
\text { 6th c }\end{array}$ & $\begin{array}{l}\text { Grave 334: annular and blue- } \\
\text { green crossed waves with } \\
\text { disc brooch pair, inlaid } \\
\text { buckle, etc; } \\
\text { Grave } 608 \text { : with crossed } \\
\text { waves in red; } \\
\text { Grave } 936: \text { yellow-brown } \\
\text { waves and dots with small } \\
\text { square-headed brooch pair }\end{array}$ & $\begin{array}{l}\text { Hirst and Clark forthcoming; } \\
\text { BM }\end{array}$ \\
\hline
\end{tabular}




\begin{tabular}{|c|c|c|c|c|}
\hline Lechlade & Glos & $\begin{array}{l}\text { mid- } \\
6 \text { th } c \\
5 \text { th- } \\
6 \text { th } c\end{array}$ & $\begin{array}{l}\text { Grave } 86 \text { : red and blue } \\
\text { waves with saucer } \\
\text { brooches, etc; } \\
\text { Grave } 77 \text { : bicone with } \\
\text { one green-blue wave and } \\
\text { red crossing trails } \\
\text { with disc brooch pair, etc }\end{array}$ & $\begin{array}{l}\text { Boyle et al forthcoming; } \\
\text { Cirencester Museum }\end{array}$ \\
\hline $\begin{array}{l}\text { Bowcombe } \\
\text { Down }\end{array}$ & $\begin{array}{l}\text { Isle of } \\
\text { Wight }\end{array}$ & $\begin{array}{l}5 \text { th- } \\
\text { 6th } c\end{array}$ & $\begin{array}{l}\text { Small with green waves } \\
\text { (?type 17.1) }\end{array}$ & $\begin{array}{l}\text { Arnold 1982, 92-3, } \\
120-1\end{array}$ \\
\hline $\begin{array}{l}\text { Dover, } \\
\text { Buckland }\end{array}$ & Kent & $\begin{array}{l}575- \\
625\end{array}$ & $\begin{array}{l}\text { Grave } 30: 1 \text { 'disc' with } \\
\text { rust red waves (D21); } \\
\text { Grave 38: } 1 \text { 'disc' with rust } \\
\text { red waves (D22); } \\
\text { Grave } 60: 1 \text { 'disc' with rust } \\
\text { red waves (D22) }\end{array}$ & $\begin{array}{l}\text { Evison } 1987,76,82, \\
224-5,226-8 \text { and } 233 \text {, } \\
\text { figs } 18.4 \mathrm{~m}, 22.4 \mathrm{f} \\
\text { and } 34.3 \mathrm{o} \text {, } \\
\text { colour pl IV (D21 and D22); } \\
\text { BM }\end{array}$ \\
\hline Faversham & Kent & $\begin{array}{l}\text { prob } \\
\text { 6th- } \\
7 \text { th c }\end{array}$ & $\begin{array}{l}\text { Approx } 6 \text { single and } 1 \\
\text { double with reddish waves; } \\
\text { red waves overlying } \\
\text { green on well-made bead; } \\
1 \text { with greenish crossed } \\
\text { wave; } \\
1 \text { with terracotta crossed } \\
\text { wave }\end{array}$ & $\begin{array}{l}\text { Beck Collection, } \\
\text { Cambridge Museum }\end{array}$ \\
\hline Finglesham & Kent & $\begin{array}{l}\text { first } \\
\text { half } \\
\text { 6th c }\end{array}$ & $\begin{array}{l}\text { Grave D3: } 1 \text { small with } \\
\text { green waves and red dots } \\
\text { with great square-headed } \\
\text { brooch, etc; } \\
1 \text { with green waves }\end{array}$ & $\begin{array}{l}\text { Chadwick 1958, 11-18, } \\
\text { fig } 5 \mathrm{~m}, \text { pl III; } \\
\text { Deal Museum }\end{array}$ \\
\hline Holborough & Kent & $\begin{array}{l}\text { 7th- } \\
\text { 8th c }\end{array}$ & $\begin{array}{l}\text { Two beads yellowish green } \\
\text { with light green crossed } \\
\text { waves }\end{array}$ & $\begin{array}{l}\text { Evison 1956, 92, 126, } \\
\text { fig 20.3b; } \\
\text { BM 1947 5-2 } 358\end{array}$ \\
\hline Stowting & Kent & $\begin{array}{l}\text { prob } \\
\text { 6th- } \\
7 \text { th c }\end{array}$ & $\begin{array}{l}\text { One with green waves and } \\
1 \text { with terracotta waves }\end{array}$ & $\begin{array}{l}\text { Smith 1846a; } \\
\text { Brent 1867; } \\
\text { Beck Collection, } \\
\text { Cambridge Museum }\end{array}$ \\
\hline Cleatham & Lincs & $\begin{array}{l}\text { late } \\
5 \text { th- } \\
6 \text { th c }\end{array}$ & $\begin{array}{l}\text { Grave 30: terracotta } \\
\text { waves }\end{array}$ & $\begin{array}{l}\text { Publication in } \\
\text { preparation (K Leahy); } \\
\text { Scunthorpe Museum }\end{array}$ \\
\hline Fonaby & Lincs & 6th $c$ & $\begin{array}{l}\text { Grave } 3: 1 \text { 'barrel' } \\
\text { reddish waves and dots } \\
\text { with } 2 \text { annular brooches, } \\
\text { etc }\end{array}$ & $\begin{array}{l}\text { Cook 1981, 18-20, } \\
\text { fig 4.3.1.44; } \\
\text { Scunthorpe Museum }\end{array}$ \\
\hline $\begin{array}{l}\text { Syston } \\
\text { Park }\end{array}$ & Lincs & $\begin{array}{l}\text { prob } \\
\text { 6th c }\end{array}$ & Green crossed waves & $\begin{array}{l}\text { Akerman 1855a, 41-2, } \\
\text { pl XXI; } \\
\text { BM (18) } 1111-92\end{array}$ \\
\hline Kenninghall & Norfolk & $\begin{array}{l}\text { 5th- } \\
\text { 7th c }\end{array}$ & $\begin{array}{l}\text { Double bead, each } \\
\text { with reddish waves }\end{array}$ & $\begin{array}{l}\text { Manning } 1872 a, 292 \text {, and } \\
1872 \mathrm{~b} ; \mathrm{BM}\end{array}$ \\
\hline
\end{tabular}




\begin{tabular}{|c|c|c|c|c|}
\hline $\begin{array}{l}\text { Morning } \\
\text { Thorpe }\end{array}$ & Norfolk & 6th $\mathrm{c}$ & $\begin{array}{l}\text { A number, eg, } \\
\text { Grave } 30: 2 \text { beads with } \\
\text { green waves and red dots } \\
\text { with Åberg Group III } \\
\text { cruciform brooch and } \\
\text { Group II pair, annular } \\
\text { brooch pair, B clasps, etc }\end{array}$ & $\begin{array}{l}\text { Green et al 1987, } \\
42-3 \text {, fig 304.D.xxxi; } \\
\text { Norwich Museum }\end{array}$ \\
\hline $\begin{array}{l}\text { North } \\
\text { Runcton }\end{array}$ & Norfolk & $\begin{array}{l}\text { 6th- } \\
7 \text { th } c\end{array}$ & Brown crossed waves & $\begin{array}{l}\text { Meaney 1964, 180-1; } \\
\text { Norwich Museum }\end{array}$ \\
\hline Spong Hill & Norfolk & $\begin{array}{l}\text { mid- } \\
6 \text { th } c \\
\text { 2nd } \\
\text { half } \\
5 \text { th } c\end{array}$ & $\begin{array}{l}\text { Grave 5: reddish waves } \\
\text { and green dots with } \\
\text { small-long brooch pair, } \\
\text { A clasps, etc; } \\
\text { Grave } 42 \text { : reddish waves } \\
\text { with small-long brooch } \\
\text { pair, C clasps, etc; } \\
\text { Grave } 45: 2 \text { with red } \\
\text { waves with Åberg Group } \\
\text { IVa cruciform brooch, etc; } \\
\text { Cremation } 2143 \text { : very burnt } \\
\text { reddish waves and ?green } \\
\text { dots with applied saucer } \\
\text { brooch with } 6 \text { face masks } \\
\text { in urn with punched dots } \\
\text { and linear ornament }\end{array}$ & $\begin{array}{l}\text { Hills et al } 1984 \text {, } \\
54-5,95-7 \text { and } 98-100 \text {, } \\
\text { figs } 73.3 b, 98.42 .3 \\
\text { and } 99.45 .3-4 ;\end{array}$ \\
\hline $\begin{array}{l}\text { Holme } \\
\text { Pierre- } \\
\text { pont }\end{array}$ & Notts & $\begin{array}{l}\text { prob } \\
\text { 6th } c\end{array}$ & $\begin{array}{l}\text { Red waves and dots with a } \\
\text { stamp in chevron- } \\
\text { ornamented pot }\end{array}$ & $\begin{array}{l}\text { Myres } 1977,51-2,315 \text {, } \\
\text { fig 303.2093; } \\
\text { BM 31 3-13 1 }\end{array}$ \\
\hline Wheatley & Oxon & $c .600$ & $\begin{array}{l}\text { Grave 14: red waves and } \\
\text { dots with cast saucer } \\
\text { brooch pair, etc }\end{array}$ & $\begin{array}{l}\text { Leeds 1916, 51-3, fig 5; } \\
\text { Ashmolean Museum }\end{array}$ \\
\hline $\begin{array}{l}\text { Bury St } \\
\text { Edmunds, } \\
\text { Westgarth } \\
\text { Gardens }\end{array}$ & Suffolk & $\begin{array}{l}\text { prob } \\
\text { 6th } c \\
\text { 6th } c\end{array}$ & $\begin{array}{l}\text { Grave 33: green waves } \\
\text { with amber beads; } \\
\text { Grave 52: with green } \\
\text { waves with Aberg Group } \\
\text { II cruciform brooch, } \\
\text { C clasps, etc }\end{array}$ & $\begin{array}{l}\text { West } 1988,28 \text { and } \\
33-4, \text { figs } 68.33 .7 \text { and } \\
76.52 . F .5 ; \\
\text { Bury St Edmunds } \\
\text { Museum }\end{array}$ \\
\hline Ipswich & Suffolk & $\begin{array}{l}\text { 6th- } \\
7 \text { th } c\end{array}$ & $\begin{array}{l}\text { Two with terracotta crossed } \\
\text { waves }\end{array}$ & $\begin{array}{l}\text { Layard } 1907 \text { and 1909; } \\
\text { Ipswich Museum }\end{array}$ \\
\hline $\begin{array}{l}\text { Little } \\
\text { Eriswell }\end{array}$ & Suffolk & $\begin{array}{l}\text { 6th- } \\
7 \text { th } c\end{array}$ & $\begin{array}{l}\text { Grave 11: terracotta } \\
\text { crossed wave and green } \\
\text { dots }\end{array}$ & $\begin{array}{l}\text { Hutchinson 1966; } \\
\text { Bury St Edmunds } \\
\text { Museum }\end{array}$ \\
\hline $\begin{array}{l}\text { Mitchell's } \\
\text { Hill }\end{array}$ & Suffolk & $\begin{array}{l}\text { 6th- } \\
7 \text { th } c\end{array}$ & $\begin{array}{l}\text { One terracotta wave and dark } \\
\text { dots; } 1 \text { terracotta wave } \\
\text { and terracotta dots; } \\
1 \text { longwise wave and red dots }\end{array}$ & $\begin{array}{l}\text { Meaney 1964, 231; } \\
\text { Bury St Edmunds } \\
\text { Museum }\end{array}$ \\
\hline
\end{tabular}




\begin{tabular}{|c|c|c|c|c|}
\hline Snape & Suffolk & $\begin{array}{l}5 \text { th- } \\
7 \text { th c }\end{array}$ & $\begin{array}{l}\text { Grave } 0421: 1 \text { with terra- } \\
\text { cotta waves } \\
\text { Grave } 0576: 1 \text { with terra- } \\
\text { cotta waves and } 1 \text { with } \\
\text { green waves }\end{array}$ & $\begin{array}{l}\text { Filmer-Sankey and } \\
\text { Pestell forthcoming }\end{array}$ \\
\hline Guildown & Surrey & 6th c & $\begin{array}{l}\text { Grave } 206 \text { : terracotta } \\
\text { crossed wave with square- } \\
\text { headed brooch pair }\end{array}$ & $\begin{array}{l}\text { Lowther } 1931,11,44 \text {, } \\
\text { pl IX.1; } \\
\text { Guildford Museum }\end{array}$ \\
\hline $\begin{array}{l}\text { Churchover, } \\
\text { Bensford } \\
\text { Bridge }\end{array}$ & Warks & 6th c & Terracotta waves and dots & $\begin{array}{l}\text { Bloxam 1884; } \\
\text { Meaney 1964, 259-60; } \\
\text { Warwick Museum }\end{array}$ \\
\hline Charlton & Wilts & $\begin{array}{l}5 \text { th- } \\
6 \text { th c }\end{array}$ & $\begin{array}{l}\text { Burial } 24: 4 \text { with terracotta } \\
\text { wave and } 1 \text { with turquoise } \\
\text { waves and red dots }\end{array}$ & $\begin{array}{l}\text { Davies } 1984,120, \\
139-40, \text { fig } 9 \mathrm{~N} ; \\
\text { Salisbury Museum }\end{array}$ \\
\hline $\begin{array}{l}\text { Colling- } \\
\text { bourne Ducis }\end{array}$ & Wilts & 6 th $\mathrm{c}$ & $\begin{array}{l}\text { Grave } 20 \text { : terracotta } \\
\text { crossed waves with } \\
\text { saucer brooch pair }\end{array}$ & $\begin{array}{l}\text { Gingell } 1975,85 \text {, } \\
\text { fig 18.7; } \\
\text { Devizes Museum }\end{array}$ \\
\hline $\begin{array}{l}\text { Harnham } \\
\text { Hill }\end{array}$ & Wilts & $\begin{array}{l}\text { prob } \\
\text { 6th c }\end{array}$ & $\begin{array}{l}\text { Terracotta crossed } \\
\text { waves }\end{array}$ & $\begin{array}{l}\text { Akerman 1853; } \\
\text { Jackson 1854; } \\
\text { BM 53-12 14 }\end{array}$ \\
\hline $\begin{array}{l}\text { Peters- } \\
\text { finger }\end{array}$ & Wilts & $\begin{array}{l}\text { late } \\
5 \text { th- } \\
6 \text { th c }\end{array}$ & $\begin{array}{l}\text { Grave } 25 \text { : possibly a single } \\
\text { rather than crossed } \\
\text { reddish wave with } 3 \\
\text { button brooches and small } \\
\text { bow brooch, etc }\end{array}$ & $\begin{array}{l}\text { Leeds and Shortt } 1953 \text {, } \\
21-2, \text { pl III.94.XXV; } \\
\text { Salisbury Museum }\end{array}$ \\
\hline $\begin{array}{l}\text { Pewsey } \\
\text { Blackpatch }\end{array}$ & Wilts & $\begin{array}{l}5 \text { th- } \\
6 \text { th } \mathrm{c}\end{array}$ & $\begin{array}{l}\text { Grave } 86: \text { terracotta waves } \\
\text { and dots }\end{array}$ & $\begin{array}{l}\text { Publication in } \\
\text { preparation (K Annable } \\
\text { Devizes Museum }\end{array}$ \\
\hline \multirow[t]{2}{*}{$\begin{array}{l}\text { West } \\
\text { Heslerton }\end{array}$} & Yorks & $\begin{array}{l}\text { prob } \\
\text { 6th } \mathrm{c}\end{array}$ & $\begin{array}{l}\text { One at least with green } \\
\text { waves }\end{array}$ & $\begin{array}{l}\text { Publication in } \\
\text { preparation } \\
\text { (D Powlesland) }\end{array}$ \\
\hline & \multicolumn{4}{|c|}{$\begin{array}{l}\text { vii Miscellaneous opaque Yellow DeCoF } \\
\text { (EXCLUDING CROSSED WAVES) }\end{array}$} \\
\hline Wallingford & Berks & $\begin{array}{l}\text { 2nd } \\
\text { half } \\
5 \text { th c }\end{array}$ & $\begin{array}{l}\text { Grave 15: globular with } \\
\text { green central band overlaid } \\
\text { by reddish wave, with } \\
\text { applied saucer brooch } \\
\text { (floriate/anchor cross) } \\
\text { pair and } 2 \text { quoit brooches, } \\
\text { etc; } \\
\text { Grave } 13 \text { : small annular } \\
\text { with random line in green }\end{array}$ & $\begin{array}{l}\text { Leeds 1938, 98-9, } \\
\text { pl VII; } \\
\text { Welch 1975, 92; } \\
\text { Evison 1978; } \\
\text { Böhme 1986; } \\
\text { Ashmolean Museum } \\
\text { 1988.1232; } \\
\text { Leeds 1938, 98; } \\
\text { Ashmolean Museum }\end{array}$ \\
\hline $\begin{array}{l}\text { Colchester, } \\
\text { Mersea Road }\end{array}$ & Essex & $\begin{array}{l}\text { prob } \\
5 \text { th- } \\
6 \text { th c }\end{array}$ & $\begin{array}{l}\text { Annular with } 3 \text { eyes in } \\
\text { white and pale blue } \\
\text { associated with amber } \\
\text { beads and a faceted } \\
\text { amethyst bead }\end{array}$ & $\begin{array}{l}\text { Crummy } 1981,15-17 \text {, } \\
\text { fig } 19.10 ; \\
\text { Colchester Museum } \\
5318.26\end{array}$ \\
\hline
\end{tabular}




\begin{tabular}{|c|c|c|c|c|}
\hline Mucking II & Essex & $\begin{array}{l}\text { 5th- } \\
\text { 6th } c\end{array}$ & $\begin{array}{l}\text { Grave } 860 \text { : with dark green } \\
\text { translucent trail with small- } \\
\text { long brooch pair, etc }\end{array}$ & $\begin{array}{l}\text { Hirst and Clark forthcomi } \\
\text { BM }\end{array}$ \\
\hline $\begin{array}{l}\text { Dover, } \\
\text { Buckland }\end{array}$ & Kent & $\begin{array}{l}525- \\
575\end{array}$ & $\begin{array}{l}\text { Grave 23: small 'disc' } \\
\text { with white decomposed } \\
\text { dots (D55) }\end{array}$ & $\begin{array}{l}\text { Evison } 1987,77,82 \text {, } \\
222, \text { fig } 15.23 .4 \mathrm{~d} ; \\
\text { BM }\end{array}$ \\
\hline Faversham & Kent & $\begin{array}{l}\text { prob } \\
\text { 6th } c\end{array}$ & $\begin{array}{l}\text { Large globular with red } \\
\text { rings around circumference }\end{array}$ & $\begin{array}{l}\text { Meaney 1964, 118-19; } \\
\text { Beck Collection, } \\
\text { Cambridge Museum }\end{array}$ \\
\hline Finglesham & Kent & $\begin{array}{l}\text { first } \\
\text { half } \\
\text { 6th c }\end{array}$ & $\begin{array}{l}\text { Grave D3: } 3 \text { small annulars } \\
\text { with green interlace with } \\
\text { great square-headed } \\
\text { brooch, D bracteates, etc }\end{array}$ & $\begin{array}{l}\text { Chadwick 1958, 11-18, } \\
\text { fig 5j, pl III; } \\
\text { Deal Museum }\end{array}$ \\
\hline $\begin{array}{l}\text { Gilton } \\
\text { (mainly) }\end{array}$ & Kent & $\begin{array}{l}\text { prob } \\
6 \text { th- } \\
7 \text { th c }\end{array}$ & $\begin{array}{l}\text { Cylindrical with terracotta } \\
\text { wave from top to bottom }\end{array}$ & $\begin{array}{l}\text { Faussett 1856; } \\
\text { Liverpool Museum }\end{array}$ \\
\hline $\begin{array}{l}\text { Lyminge } \\
\text { II }\end{array}$ & Kent & $\begin{array}{l}\text { first } \\
\text { half } \\
\text { 6th c }\end{array}$ & $\begin{array}{l}\text { Grave 39: irregular } \\
\text { globular with dark smudge, } \\
\text { with saucer and small square- } \\
\text { headed brooch pairs, etc }\end{array}$ & $\begin{array}{l}\text { Warhurst 1955, 27, } \\
\text { pl VIIb, no. 2; } \\
\text { Maidstone Museum }\end{array}$ \\
\hline Richborough & Kent & & $\begin{array}{l}\text { Roman fort stray find: } \\
\text { exotic biconical bead } \\
\text { with central blue band in } \\
\text { white and } 3 \text { spots of } \\
\text { turquoise and white }\end{array}$ & $\begin{array}{l}\text { Bushe-Fox } 1949,149 \\
\text { Richborough Museum }\end{array}$ \\
\hline Westbere & Kent & 7th $c$ & $\begin{array}{l}\text { Annular with random } \\
\text { reddish lines }\end{array}$ & $\begin{array}{l}\text { Jessup 1946, 16, pl II; } \\
\text { Canterbury Museum } \\
\text { RM } 6422\end{array}$ \\
\hline $\begin{array}{l}\text { Welbeck } \\
\text { Hill }\end{array}$ & Lincs & 6th $c$ & $\begin{array}{l}\text { Two with terracotta girth } \\
\text { bands }\end{array}$ & $\begin{array}{l}\text { Publication in } \\
\text { preparation (G Taylor); } \\
\text { Scunthorpe Museum }\end{array}$ \\
\hline Brooke & Norfolk & 6th $\mathrm{c}$ & $\begin{array}{l}\text { Small rather cylindrical } \\
\text { with central band and thin } \\
\text { reddish lines }\end{array}$ & $\begin{array}{l}\text { Meaney } 1964,170 \\
\text { BM } 1866-69\end{array}$ \\
\hline Kenninghall & Norfolk & $\begin{array}{l}5 \text { th- } \\
7 \text { th c }\end{array}$ & $\begin{array}{l}\text { Necklace (?): very small } \\
\text { with red spot, with } \\
\text { millefiori bead on same } \\
\text { string }\end{array}$ & $\begin{array}{l}\text { Manning 1872a, 292, and } \\
\text { 1872b; } \\
\text { BM } 83 \text { 7-2 } 31\end{array}$ \\
\hline $\begin{array}{l}\text { Morning } \\
\text { Thorpe }\end{array}$ & Norfolk & $\begin{array}{l}\text { mid- } \\
\text { 6th } c\end{array}$ & $\begin{array}{l}\text { Grave } 16: 1 \text { rather } \\
\text { cylindrical with terracotta } \\
\text { tall waves and green central } \\
\text { band with florid cruciform } \\
\text { brooch, etc; } \\
\text { Grave } 96: 1 \text { short cylinder } \\
\text { with wound green band } \\
\text { with Aberg Group IVa } \\
\text { cruciform brooch, etc; }\end{array}$ & $\begin{array}{l}\text { Green et al 1987, } \\
37-8,62-3,82-3,90, \\
\text { 122-3, figs 298.Ev, } \\
\text { 327.Hv, 352.173.Dvii, } \\
\text { 362.Dviii, } 399.316 \text { and } \\
\text { 321.Svii; } \\
\text { Norwich Museum }\end{array}$ \\
\hline
\end{tabular}




\begin{tabular}{|c|c|c|c|c|}
\hline & & $\begin{array}{l}\text { mid- } \\
\text { 6th } \mathrm{c} \\
\text { 6th } \mathrm{C}\end{array}$ & $\begin{array}{l}\text { Grave 173: globular with } \\
\text { green lines round and green } \\
\text { central band with } 3 \text { annular } \\
\text { brooches, B clasps, etc; } \\
\text { Grave } 209: 2 \text { cylinders with } \\
\text { wound green band with } \\
\text { Åberg Group IVa } \\
\text { cruciform brooch, etc; } \\
\text { Graves } 316 \text { and } 321 \text { : } \\
3 \text { cylinders with wound } \\
\text { green band with annular } \\
\text { brooch, etc }\end{array}$ & \\
\hline $\begin{array}{l}\text { Cassington, } \\
\text { Purwell } \\
\text { Farm }\end{array}$ & Oxon & 6th $\mathrm{c}$ & $\begin{array}{l}\text { Unusually large with } \\
\text { rather cylindrical dark } \\
\text { parallel swags alternating } \\
\text { with light swags, with } \\
\text { millefiori and other beads }\end{array}$ & $\begin{array}{l}\text { Leeds and Riley 1942, 64, } \\
\text { pl VIB; } \\
\text { Ashmolean Museum } \\
1942.157\end{array}$ \\
\hline $\begin{array}{l}\text { Bury St } \\
\text { Edmunds, } \\
\text { Westgarth } \\
\text { Gardens }\end{array}$ & Suffolk & $\begin{array}{l}\text { late } \\
5 \text { th- } \\
\text { early } \\
6 \text { th } \mathrm{c}\end{array}$ & $\begin{array}{l}\text { Grave 52: globular with } \\
3 \text { translucent green/blue } \\
\text { bands, with A berg Group } \\
\text { II cruciform brooch, etc }\end{array}$ & $\begin{array}{l}\text { West } 1988,33-4 \text {, } \\
\text { fig } 76.52 . F 5 ; \\
\text { Bury St Edmunds } \\
\text { Museum }\end{array}$ \\
\hline West Stow & Suffolk & $\begin{array}{l}5 \text { th- } \\
7 \text { th c }\end{array}$ & $\begin{array}{l}\text { Settlement: globular } \\
\text { brownish eyes with } \\
\text { white centres }\end{array}$ & $\begin{array}{l}\text { West } 1985,73-4 ; \\
\text { Bury St Edmunds } \\
\text { Museum }\end{array}$ \\
\hline Wasperton & Warks & $\begin{array}{l}\text { mid- } \\
6 \text { th c }\end{array}$ & $\begin{array}{l}\text { F1081: } 2406,30 \text { and } 40 \text {, } \\
2 \text { globulars with small } \\
\text { red dots }\end{array}$ & $\begin{array}{l}\text { Publication in } \\
\text { preparation (M Carver); } \\
\text { Warwick Museum }\end{array}$ \\
\hline $\begin{array}{l}\text { Colling- } \\
\text { bourne } \\
\text { Ducis }\end{array}$ & Wilts & $\begin{array}{l}\text { late } \\
5 \text { th- } \\
6 \text { th c }\end{array}$ & $\begin{array}{l}\text { Grave } 20: 2 \text { globulars } \\
\text { with random thin red } \\
\text { lines with } 2 \text { saucer } \\
\text { brooches }\end{array}$ & $\begin{array}{l}\text { Gingell } 1975,85, \\
\text { fig } 18.7 ; \\
\text { Devizes Museum }\end{array}$ \\
\hline $\begin{array}{l}\text { Peters- } \\
\text { finger }\end{array}$ & Wilts & $\begin{array}{l}\text { late } \\
5 \text { th- } \\
6 \text { th c }\end{array}$ & $\begin{array}{l}\text { Grave } 25: 2 \text { cylinders } \\
\text { and } 1 \text { globular with } \\
\text { reddish girth bands or } \\
\text { waves with } 3 \text { button } \\
\text { brooches, } 1 \text { bow brooch, } \\
\text { etc }\end{array}$ & $\begin{array}{l}\text { Leeds and Shortt 1953, } \\
21-2, \text { pl III.94; } \\
\text { Salisbury Museum }\end{array}$ \\
\hline Sewerby & Yorks & $\begin{array}{l}\text { mid- } \\
6 \text { th c }\end{array}$ & $\begin{array}{l}\text { Grave } 12: 2 \text { annulars with } \\
\text { equidistant reddish } \\
\text { spots (class C4) with } \\
\text { Åberg Group IVb cruciform } \\
\text { brooch, etc }\end{array}$ & $\begin{array}{l}\text { Hirst } 1985,68 \text {, } \\
\text { figs } 23 \text { and } 36.1 \mathrm{e}\end{array}$ \\
\hline
\end{tabular}




\section{SCHEDUle 5}

\section{GREEN AND TURQUOISE GLASS BEADS}

MoNochrome

i Green annular, globular and short cylinder beads (opaque or translucent)

ii Green cylinder beads (opaque or translucent): (a) round section

(b) square and polygonal section

(c) polyhedral section: no schedule

iii Green short cylinder beads, singles or doubles

iv Green barrel-shaped and biconical beads

v Green melon, sub-melon and 'nicked' beads (see Schedule liii for light blue-green translucent melon and sub-melon beads)

vi Green segmented or wound beads (see Map 17)

\section{POLYCHROME}

vii Green beads with crossed waves, with or without dots (opaque or translucent), singles or doubles

viii Green opaque or translucent beads: various shapes with waves or zigzags (excluding crossed waves)

ix Miscellaneous green decorated beads (excluding crossed waves, waves and zigzags)

$\mathbf{x} \quad$ Turquoise beads (opaque or translucent)

\section{MONOCHROME}

\section{i Green anNular, globular and SHORT CYLINDER BEADS}

(OPAQUE OR TRANSLUCENT)

\begin{tabular}{|c|c|c|c|c|}
\hline Kempston & Beds & $\begin{array}{l}\text { 6th } c \\
\text { prob } \\
\text { 6th c }\end{array}$ & $\begin{array}{l}\text { Grave 16: opaque bottle } \\
\text { green, etc; } \\
\text { three bright irregular } \\
\text { translucent }\end{array}$ & $\begin{array}{l}\text { Fitch 1864; } \\
\text { BM 91 6-24 } 53 \text { and } \\
916-2455\end{array}$ \\
\hline $\begin{array}{l}\text { Long } \\
\text { Wittenham }\end{array}$ & Berks & $\begin{array}{l}\text { 5th- } \\
\text { 6th c } \\
\text { late } \\
5 \text { th- } \\
6 \text { th c }\end{array}$ & $\begin{array}{l}\text { Grave } 52: 3 \text { translucent } \\
\text { with disc brooches; } \\
\text { Grave } 96: 1 \text { dark } \\
\text { translucent with small-long } \\
\text { brooch }\end{array}$ & Akerman 1860 \\
\hline
\end{tabular}




\begin{tabular}{|c|c|c|c|c|}
\hline Wallingford & Berks & $\begin{array}{l}\text { late } \\
\text { 5th- } \\
\text { 6th c } \\
\text { 2nd } \\
\text { half } \\
\text { 5th c }\end{array}$ & $\begin{array}{l}\text { Grave 11: small annulars } \\
\text { in translucent glass with } 2 \\
\text { small-long brooches and pot; } \\
\text { Grave 15: } 2 \text { opaque } \\
\text { annulars with applied } \\
\text { saucer brooch (floriate/ } \\
\text { anchor cross) pair } \\
\text { and } 2 \text { quoit brooches }\end{array}$ & $\begin{array}{l}\text { Leeds 1938, 98-9, pl Vll; } \\
\text { Welch 1975; Evison 1978; } \\
\text { Böhme 1986; Ashmolean } \\
\text { Museum 1938.1232 }\end{array}$ \\
\hline Bishopstone & Bucks & $\begin{array}{l}\text { prob } \\
\text { early } \\
\text { 6th c }\end{array}$ & $\begin{array}{l}\text { Necklace: globular with } \\
\text { other beads and saucer } \\
\text { brooches }\end{array}$ & $\begin{array}{l}\text { Lowndes } 1870 \text { and } \\
1878 ; \\
\text { Aylesbury Museum }\end{array}$ \\
\hline $\begin{array}{l}\text { Newport } \\
\text { Pagnell }\end{array}$ & Bucks & $\begin{array}{l}\text { prob } \\
\text { 6th c }\end{array}$ & $\begin{array}{l}\text { Many translucent and opaque } \\
\text { annulars }\end{array}$ & $\begin{array}{l}\text { Meaney 1964, 58; } \\
\text { Aylesbury Museum }\end{array}$ \\
\hline Barrington & Cambs & $\begin{array}{l}5 \text { th- } \\
7 \text { th } c\end{array}$ & $\begin{array}{l}\text { One opaque annular; } \\
\text { one opaque globular (Plate 4) } \\
\text { with mixed beads }\end{array}$ & $\begin{array}{l}\text { Fox } 1923,250-5 ; \\
\text { Beck Collection, } \\
\text { Cambridge Museum; } \\
\text { Ashmolean Museum }\end{array}$ \\
\hline $\begin{array}{l}\text { Linton } \\
\text { Heath }\end{array}$ & Cambs & $\begin{array}{l}5 \text { th- } \\
6 \text { th } c\end{array}$ & $\begin{array}{l}\text { Three small globular beads } \\
\text { with others and } 2 \text { small-long } \\
\text { brooches }\end{array}$ & $\begin{array}{l}\text { Neville 1854; } \\
\text { Cambridge Museum }\end{array}$ \\
\hline $\begin{array}{l}\text { Little } \\
\text { Wilbraham }\end{array}$ & Cambs & $\begin{array}{l}5 \text { th- } \\
7 \text { th } c\end{array}$ & $\begin{array}{l}\text { Unassociated group: } \\
1 \text { opaque green }\end{array}$ & $\begin{array}{l}\text { Neville 1852; } \\
\text { Ashmolean Museum } \\
1909.313\end{array}$ \\
\hline $\begin{array}{l}\text { Waterbeach, } \\
\text { Car Dyke }\end{array}$ & Cambs & $\begin{array}{l}\text { prob } \\
5 \text { th- } \\
6 \text { th c }\end{array}$ & $\begin{array}{l}\text { Grubenhaus: thick annular } \\
\text { green }\end{array}$ & Lethbridge 1927 \\
\hline $\begin{array}{l}\text { Norton-on- } \\
\text { Tees }\end{array}$ & Durham & $\begin{array}{l}\text { 6th c } \\
\text { 6th c } \\
\text { mid- } \\
\text { 6th c } \\
\text { 6th c }\end{array}$ & $\begin{array}{l}\text { Grave } 7: 5 \text { annulars with } \\
\text { B clasps, etc; } \\
\text { Grave } 44: 1 \text { bead with } \\
\text { annular brooch, etc; } \\
\text { Grave } 63: 1 \text { annular and } \\
1 \text { barrel with } \AA \text { berg Group } \\
\text { Va cruciform brooch, etc; } \\
\text { Grave } 113: 1 \text { globular with } \\
\text { annular brooch, etc }\end{array}$ & $\begin{array}{l}\text { Sherlock and Welch } 1992 \text {, } \\
42-7,125,152,163-5 \\
\text { and } 192 ; \text { figs } 34.7 .1 \text {, } \\
47.44 .1,52.63 .1 \text { and } \\
64.113 .1\end{array}$ \\
\hline $\begin{array}{l}\text { Great } \\
\text { Chesterford }\end{array}$ & Essex & $\begin{array}{l}\text { late } \\
\text { 5th- } \\
\text { 6th c } \\
\text { late } \\
\text { 5th- } \\
\text { 6th c } \\
\text { 6th c }\end{array}$ & $\begin{array}{l}\text { Grave 27: } 2 \text { 'discs' (B25 } \\
\text { and B29) with small-long } \\
\text { brooch, etc; } \\
\text { Grave 79: } 2 \text { 'discs' (B18 and } \\
\text { B29) with iron penannular } \\
\text { brooch, girdle hanger, etc; } \\
\text { Grave 97: } 2 \text { 'discs' (B25 and } \\
\text { B29) with saucer brooch } \\
\text { pair, etc; } \\
\text { Grave 103: } 3 \text { 'discs' (B18, } \\
\text { B29 and B36) with Style I } \\
\text { applied saucer brooch pair, } \\
\text { etc; }\end{array}$ & $\begin{array}{l}\text { Evison 1994, 12, 14, } \\
45-6,95-6,103, \\
105,105-6 \text { and } 114-15 \text {, } \\
\text { figs } 26.27 .2 \mathrm{~b} \text { and } \mathrm{c} \text {, } \\
37.79 .2 \mathrm{c} \text { and } \mathrm{d} \text {, } \\
\text { 41.97.3e and } \mathrm{f} \\
\text { 42.103.3d, e and } \mathrm{f}, \\
\text { and 58.C5.2d; } \\
\text { BM }\end{array}$ \\
\hline
\end{tabular}




\begin{tabular}{|c|c|c|c|c|}
\hline & & $\begin{array}{l}\text { 5th- } \\
\text { 6th } c\end{array}$ & Cremation 5: 1 'disc' (B24) & \\
\hline Mucking II & Essex & $\begin{array}{l}\text { 5th- } \\
6 \text { th c } \\
\text { first } \\
\text { half } \\
5 \text { th c }\end{array}$ & $\begin{array}{l}\text { Many annulars, a few } \\
\text { globulars, wide range of } \\
\text { colour; eg, } \\
\text { Grave } 989: 1 \text { annular with } \\
\text { Glaston-Mucking type and } \\
\text { supporting-arm brooches, } \\
\text { faceted carinated pottery } \\
\text { bowl, etc }\end{array}$ & $\begin{array}{l}\text { Evison 1981,138-9, } \\
\text { figs } 4 \text { and } 5 \\
\text { BM }\end{array}$ \\
\hline $\begin{array}{l}\text { Springfield } \\
\text { Lyons }\end{array}$ & Essex & $\begin{array}{l}\text { prob } \\
\text { 6th c }\end{array}$ & Two examples & $\begin{array}{l}\text { Publication in } \\
\text { preparation (S Tyler); BM }\end{array}$ \\
\hline Lechlade & Glos & 6th $\mathrm{c}$ & $\begin{array}{l}\text { Grave 176: mid green } \\
\text { 'disc' with annular or } \\
\text { penannular brooch pair }\end{array}$ & $\begin{array}{l}\text { Boyle et al forthcoming; } \\
\text { Cirencester Museum }\end{array}$ \\
\hline Alton & Hants & $\begin{array}{l}\text { 5th- } \\
\text { 6th } c\end{array}$ & $\begin{array}{l}\text { Grave 9: } 3 \text { 'discs', } \\
2 \text { translucent (B09, B10 and } \\
\text { B11) with iron knife, etc }\end{array}$ & $\begin{array}{l}\text { Evison } 1988 \mathrm{a}, 16,44 \\
73 \text {, figs } 4 \text { and } 25.9 .1 \mathrm{c}-\mathrm{e} \\
\text { Hampshire Museums }\end{array}$ \\
\hline Droxford & Hants & $\begin{array}{l}\text { 5th- } \\
\text { 6th c }\end{array}$ & $\begin{array}{l}\text { Grave 20: } 2 \text { annulars (class } \\
\text { XII) with knife and buckle; } \\
\text { Grave } 32 \text { : annular (class } \\
\text { XII) with knife, etc }\end{array}$ & $\begin{array}{l}\text { Aldsworth } 1978,124, \\
128,132 \text { and } 173, \\
\text { figs } 24.20 .26 \text { and } 27 \\
\text { and } 28.32 .11 \text {; } \\
\text { Hampshire Museums }\end{array}$ \\
\hline $\begin{array}{l}\text { Bowcombe } \\
\text { Down }\end{array}$ & $\begin{array}{l}\text { Isle of } \\
\text { Wight }\end{array}$ & $\begin{array}{l}\text { prob } \\
\text { 6th c }\end{array}$ & $\begin{array}{l}\text { One translucent annular } \\
\text { (type 6.6?) }\end{array}$ & $\begin{array}{l}\text { Arnold 1982, colour } \\
\text { pl frontispiece; } \\
\text { ex Carisbrooke Castle }\end{array}$ \\
\hline $\begin{array}{l}\text { St Neots, } \\
\text { Lords Bridge }\end{array}$ & Hunts & $\begin{array}{l}\text { prob } \\
\text { 6th c }\end{array}$ & $\begin{array}{l}\text { Grave: large annular } \\
\text { translucent rich green }\end{array}$ & BM $195610-21$ and 2 \\
\hline $\begin{array}{l}\text { Beakes- } \\
\text { bourne }\end{array}$ & Kent & $\begin{array}{l}5 \text { th- } \\
\text { late } \\
\text { 6th c }\end{array}$ & $\begin{array}{l}\text { Grave 22: } 1 \text { annular } \\
\text { translucent }\end{array}$ & $\begin{array}{l}\text { Meaney 1964, 109; } \\
\text { Canterbury Museum } \\
\text { RM 7517-7522 }\end{array}$ \\
\hline $\begin{array}{l}\text { Breach } \\
\text { Down }\end{array}$ & Kent & $\begin{array}{l}\text { 7th- } \\
8 \text { th c }\end{array}$ & $\begin{array}{l}\text { Necklace with annulars and } \\
\text { globulars }\end{array}$ & $\begin{array}{l}\text { Conyngham and Akerman } \\
\text { 1844; BM 79 5-24 } 89\end{array}$ \\
\hline $\begin{array}{l}\text { Dover, } \\
\text { Buckland }\end{array}$ & Kent & $\begin{array}{l}475- \\
525 \\
525- \\
575 \\
625- \\
650 \\
650- \\
675\end{array}$ & $\begin{array}{l}\text { Grave 20: more than 1 bead } \\
\text { that is 'disc'-shaped (B27); } \\
\text { Grave 23: } 1 \text { 'disc' (B27); } \\
\text { Grave 55: } 1 \text { globular (B32); } \\
\text { Grave 6: } 1 \text { annular/'disc' } \\
\text { translucent (B26); } \\
\text { Grave 134: } 2 \text { annulars/ } \\
\text { globulars/'discs' (B27); } \\
\text { Grave 155: } 2 \text { annulars/ } \\
\text { globulars/'discs' (B27); } \\
\text { Grave 75: } 1 \text { globular (B28) }\end{array}$ & $\begin{array}{l}\text { Evison } 1987,73,80 \text {, } \\
220,222,225,231, \\
217,245,250 \text { and } 235 \text {; } \\
\text { figs } 12.5 \mathrm{~d}, 15.23 .4 \mathrm{c} \text {, } \\
\text { 19.4e, } 30.1 \mathrm{e}, 7.10 \mathrm{~d}, \\
55.134 .2 \mathrm{~d}, 60.155 .2 \mathrm{~b}, \\
38.75 .1 \mathrm{e} \text {; } \mathrm{MM}\end{array}$ \\
\hline
\end{tabular}




\begin{tabular}{|c|c|c|c|c|}
\hline Faversham & Kent & $\begin{array}{l}\text { 6th- } \\
7 \text { th c }\end{array}$ & $\begin{array}{l}\text { Numbers of muddy or } \\
\text { clear green }\end{array}$ & $\begin{array}{l}\text { Meaney 1964, 118-19; } \\
\text { Ashmolean Museum }\end{array}$ \\
\hline Folkestone & Kent & $\begin{array}{l}\text { prob } \\
\text { 6th c }\end{array}$ & $\begin{array}{l}\text { No. 14: } 1 \text { greenish grey } \\
\text { annular }\end{array}$ & $\begin{array}{l}\text { Meaney 1964, 120-1; } \\
\text { Folkestone Museum }\end{array}$ \\
\hline Holborough & Kent & $\begin{array}{l}\text { 7th- } \\
8 \text { th } \mathrm{c}\end{array}$ & Two barrel-shaped & $\begin{array}{l}\text { Evison } 1956,126 \text {, } \\
\text { fig } 20.3 \mathrm{~h} \text {; } \\
\text { BM } 19475-2358\end{array}$ \\
\hline Howletts & Kent & $\begin{array}{l}\text { prob } \\
\text { 6th c }\end{array}$ & $\begin{array}{l}\text { Globular, bright grass } \\
\text { green, ?jade }\end{array}$ & $\begin{array}{l}\text { Smith 1918; } \\
\text { BM 1936 5-11 }\end{array}$ \\
\hline $\begin{array}{l}\text { Kingston or } \\
\text { Barfreston }\end{array}$ & Kent & 7th $\mathrm{c}$ & $\begin{array}{l}\text { Approx } 40 \text { small annulars } \\
\text { (marked Kingston ' } F \text { ') }\end{array}$ & $\begin{array}{l}\text { Faussett 1856; } \\
\text { Liverpool Museum } 6327\end{array}$ \\
\hline $\begin{array}{l}\text { Lyminge } \\
\text { II }\end{array}$ & Kent & $\begin{array}{l}\text { first } \\
\text { half } \\
\text { 6th c } \\
\text { 6th c }\end{array}$ & $\begin{array}{l}\text { Grave } 16: 2 \text { small bottle } \\
\text { green annulars with } \\
1 \text { radiate-headed, } 1 \text { button } \\
\text { brooch and D bracteate, etc; } \\
\text { Grave 24: annular trans- } \\
\text { lucent grass-green and } c .12 \\
\text { little green translucent } \\
\text { beads with small equal-arm } \\
\text { brooch pair, etc }\end{array}$ & $\begin{array}{l}\text { Warhurst 1955, } \\
\text { 15,18, pl VIIb.1; } \\
\text { Maidstone Museum }\end{array}$ \\
\hline Orpington & Kent & $\begin{array}{l}5 \text { th- } \\
6 \text { th c }\end{array}$ & $\begin{array}{l}\text { Grave 32: very small } \\
\text { globular with } 2 \text { pierced } \\
\text { Roman coins }\end{array}$ & $\begin{array}{l}\text { Tester 1968, 139-41, } \\
\text { fig 6.32a.iv; } \\
\text { Orpington Museum }\end{array}$ \\
\hline Ozingell & Kent & $\begin{array}{l}\text { prob } \\
\text { 6th c }\end{array}$ & Two globulars & $\begin{array}{l}\text { Meaney 1964, 131; } \\
\text { Powell-Cotton Museum }\end{array}$ \\
\hline Polhill & Kent & 7th $\mathrm{c}$ & $\begin{array}{l}\text { Grave } 37: 3 \text { barrels with } \\
\text { jewelled disc brooch, knife, } \\
\text { etc; } \\
\text { Grave } 102: 4 \text { barrels with } \\
5 \text { silver wire rings, etc }\end{array}$ & $\begin{array}{l}\text { Hawkes and Philp } 1973 \text {, } \\
176,185,193,207 \\
\text { fig } 55.517 \text { and } 527\end{array}$ \\
\hline Sarre & Kent & $\begin{array}{l}\text { prob } \\
\text { late } \\
\text { 6th c } \\
7 \text { th c }\end{array}$ & $\begin{array}{l}\text { two annulars with } \\
\text { amethysts, gold coins, etc }\end{array}$ & Meaney $1964,135-6$ \\
\hline Stowting & Kent & & Dark blue/green globular & $\begin{array}{l}\text { Smith 1846a; Brent 1867; } \\
\text { Beck Collection, } \\
\text { Cambridge Museum } \\
147.1861\end{array}$ \\
\hline Westbere & Kent & $\begin{array}{l}\text { prob } \\
\text { 7th c }\end{array}$ & $\begin{array}{l}\text { Translucent olive green } \\
\text { globulars }\end{array}$ & $\begin{array}{l}\text { Jessup 1946; } \\
\text { Canterbury Museum } \\
\text { RM 6422, } 6425 \text { and } 6426\end{array}$ \\
\hline Saxby & Leics & & One globular and 1 annular & $\begin{array}{l}\text { Publication in } \\
\text { preparation (J Timby) }\end{array}$ \\
\hline $\begin{array}{l}\text { Castle } \\
\text { Bytham }\end{array}$ & Lincs & 7th $\mathrm{c}$ & $\begin{array}{l}\text { String with } 2 \text { annulars } \\
\text { with quoit brooch }\end{array}$ & $\begin{array}{l}\text { Akerman 1855a, 26-7, } \\
\text { pl XII.4 }\end{array}$ \\
\hline
\end{tabular}




\begin{tabular}{|c|c|c|c|c|}
\hline Cleatham & Lincs & & Grave 30: 2 very dark & $\begin{array}{l}\text { Publication in } \\
\text { preparation (K Leahy); } \\
\text { Scunthorpe Museum }\end{array}$ \\
\hline Fonaby & Lincs & $\begin{array}{l}\text { late } \\
5 \text { th- } \\
6 \text { th c }\end{array}$ & $\begin{array}{l}\text { Grave 3: yellowish green } \\
\text { barrel with } 2 \text { annular } \\
\text { brooches, etc; } \\
\text { Grave } 32: 2 \text { opaque } \\
\text { globulars with Åberg } \\
\text { Group II cruciform brooch } \\
\text { and small-long brooch, etc }\end{array}$ & $\begin{array}{l}\text { Cook } 1981,18,34, \\
\text { figs } 4.3 .1 .64 \text { and } \\
11.32 .1 .5, \text { pl VIIA; } \\
\text { Scunthorpe Museum }\end{array}$ \\
\hline Laceby & Lincs & $\begin{array}{l}\text { 6th- } \\
7 \text { th c }\end{array}$ & $\begin{array}{l}\text { Group: small annulars } \\
\text { with amber beads; } \\
\text { and another bead }\end{array}$ & $\begin{array}{l}\text { Myres } 1951,89 \text { and } 98 ; \\
\text { Thompson 1956, 184-9; } \\
\text { Lincoln Museum }\end{array}$ \\
\hline ‘Lincs' & Lincs & & $\begin{array}{l}\text { Necklace 11: } 1 \text { grass green } \\
\text { opaque globular }\end{array}$ & Scunthorpe Museum \\
\hline $\begin{array}{l}\text { Nettleton } \\
\text { Caistor }\end{array}$ & Lincs & $\begin{array}{l}\text { late } \\
\text { 6th- } \\
\text { 7th c }\end{array}$ & One dark green & $\begin{array}{l}\text { Meaney 1964, 160; } \\
\text { Lincoln Museum }\end{array}$ \\
\hline $\begin{array}{l}\text { South } \\
\text { Willingham }\end{array}$ & Lincs & & $\begin{array}{l}\text { One large greenish gold } \\
\text { annular }\end{array}$ & $\begin{array}{l}\text { Meaney } 1964,165 \\
\text { BM 76 2-12 12-15 }\end{array}$ \\
\hline $\begin{array}{l}\text { Welbeck } \\
\text { Hill }\end{array}$ & Lincs & $\begin{array}{l}\text { late } \\
5 \text { th- } \\
\text { 6th c }\end{array}$ & $\begin{array}{l}\text { Grave 64: dark bottle } \\
\text { green annular with } 2 \text { small- } \\
\text { long brooches }\end{array}$ & $\begin{array}{l}\text { Publication in } \\
\text { preparation ( } G \text { Taylor); } \\
\text { Scunthorpe Museum }\end{array}$ \\
\hline $\begin{array}{l}\text { Welbourne, } \\
\text { High Dyke }\end{array}$ & Lincs & & Four opaque annulars & $\begin{array}{l}\text { Bruce } 1880,76 \text {, no. } 305 \text {; } \\
\text { Alnwick Museum }\end{array}$ \\
\hline Brooke & Norfolk & $\begin{array}{l}\text { prob } \\
\text { 6th c }\end{array}$ & $\begin{array}{l}\text { One irregular wound } \\
\text { globular, etc, with amber } \\
\text { beads }\end{array}$ & $\begin{array}{l}\text { Meaney 1964, 170; } \\
\text { BM 1866-69 }\end{array}$ \\
\hline $\begin{array}{l}\text { Morning } \\
\text { Thorpe }\end{array}$ & Norfolk & 6th c & $\begin{array}{l}\text { Grave } 360: 1 \text { annular with } \\
\text { annular brooch pair, } \\
\text { C clasps, etc; } \\
\text { Grave } 378: 3 \text { annulars with } \\
\text { annular brooch pair, B7 } \\
\text { clasps, etc; } \\
\text { and many other examples }\end{array}$ & $\begin{array}{l}\text { Green et al } 1987 \text {, } \\
139-40 \text { and } 148 \text {, } \\
\text { figs } 421.360 . \text { Fvi and } \\
436.378 . \text { Ev; } \\
\text { Norwich Museum }\end{array}$ \\
\hline Spong Hill & Norfolk & $\begin{array}{l}\text { 6th c } \\
\text { mid- } \\
\text { 6th } \mathrm{c} \\
\text { later } \\
\text { 5th- } \\
\text { 6th } \mathrm{c}\end{array}$ & $\begin{array}{l}\text { Grave } 5: 2 \text { globulars, } \\
1 \text { bluish-green annular } \\
\text { and } 1 \text { annular with } 2 \text { small- } \\
\text { long brooches, A clasps, } \\
\text { etc; } \\
\text { Grave } 12: 2 \text { globulars } \\
\text { with } 3 \text { annular brooches; } \\
\text { Grave } 22: 2 \text { annulars with } \\
\text { Aberg Group II and IVa } \\
\text { cruciform brooches; } \\
\text { Grave } 26: 2 \text { globulars } \\
\text { and } 1 \text { annular with Åberg } \\
\text { Group I cruciform brooch, }\end{array}$ & $\begin{array}{l}\text { Hills et al } 1984 \text {, } \\
55,61,70,74,90 \text { and } \\
90-1, \text { figs } 73.3 b \text { and } 6 c \text {, } \\
75.12 .4,79.5 .8 \text { and } 33 \text {, } \\
83.1,92.10 \text { and } 94.5\end{array}$ \\
\hline
\end{tabular}




\begin{tabular}{|c|c|c|c|c|}
\hline & & $\begin{array}{l}\text { mid- } \\
6 \text { th } \mathrm{c} \\
6 \text { th } \mathrm{c}\end{array}$ & $\begin{array}{l}\text { stamped pot, etc; } \\
\text { Grave } 38: 2 \text { translucent } \\
\text { bluish-green barrels with } \\
1 \text { square-headed and } 2 \\
\text { annular brooches, clasps, } \\
\text { etc; } \\
\text { Grave 39: } 8 \text { annulars } \\
\text { with Group IVa cruciform } \\
\text { and annular brooches, etc; } \\
\text { Cremation } 2462: 1 \text { globular } \\
\text { in stamped and bossed urn }\end{array}$ & $\begin{array}{l}\text { Hills et al } 1987 \\
\text { 49, fig } 106.2462 / 1 \\
\text { Norfolk Arch Unit }\end{array}$ \\
\hline Brixworth & Northants & $\begin{array}{l}6 \text { th- } \\
7 \text { th } \mathrm{c}\end{array}$ & $\begin{array}{l}\text { One annular with other } \\
\text { beads }\end{array}$ & $\begin{array}{l}\text { Smith } 1902,245-6 ; \\
\text { Northampton Museum }\end{array}$ \\
\hline Holdenby & Northants & $\begin{array}{l}\text { 6th- } \\
7 \text { th } c\end{array}$ & $\begin{array}{l}\text { Five very small opaque } \\
\text { with other beads }\end{array}$ & $\begin{array}{l}\text { Leeds 1909; } \\
\text { Northampton Museum }\end{array}$ \\
\hline $\begin{array}{l}\text { Howick } \\
\text { Heugh }\end{array}$ & $\begin{array}{l}\text { North- } \\
\text { umber- } \\
\text { land }\end{array}$ & & $\begin{array}{l}\text { Grave } 6: 2 \text { green annulars } \\
\text { with knife }\end{array}$ & $\begin{array}{l}\text { Keeney } 1939,120-8 ; \\
\text { Cramp and Miket } 1982 \text {, } \\
5-6, \text { fig } 4.3 .2 ; \\
\text { Newcastle Museum } \\
1935.20\end{array}$ \\
\hline $\begin{array}{l}\text { Holme } \\
\text { Pierrepont }\end{array}$ & Notts & & $\begin{array}{l}\text { Quarry site: several dark } \\
\text { green translucent }\end{array}$ & $\begin{array}{l}\text { Myres 1977, Corpus } \\
\text { no. 2093, fig 303; } \\
\text { BM 1931 3-13 }\end{array}$ \\
\hline $\begin{array}{l}\text { Empingham } \\
\text { II }\end{array}$ & Rutland & $\begin{array}{l}5 \text { th- } \\
7 \text { th } c\end{array}$ & $\begin{array}{l}\text { Grave 98B: annular } \\
\text { translucent; } \\
\text { Grave 129: annular }\end{array}$ & $\begin{array}{l}\text { Timby 1996; } \\
\text { Oakham Museum }\end{array}$ \\
\hline $\begin{array}{l}\text { Bury St } \\
\text { Edmunds, } \\
\text { Westgarth } \\
\text { Gardens }\end{array}$ & Suffolk & $\begin{array}{l}5 \text { th- } \\
6 \text { th } c\end{array}$ & $\begin{array}{l}\text { Grave } 48: 1 \text { 'disc' with } \\
2 \text { small-long brooches, } \\
\text { clasps, etc }\end{array}$ & $\begin{array}{l}\text { West 1988, 32, } \\
\text { fig 73.E.15; } \\
\text { Bury St } \\
\text { Edmunds Museum }\end{array}$ \\
\hline $\begin{array}{l}\text { Holywell } \\
\text { Row }\end{array}$ & Suffolk & $\begin{array}{l}5 \text { th- } \\
7 \text { th c }\end{array}$ & One opaque globular & $\begin{array}{l}\text { Lethbridge 1931; } \\
\text { Cambridge Museum Z7128 }\end{array}$ \\
\hline Icklingham & Suffolk & & $\begin{array}{l}\text { Several opaque and } \\
\text { translucent annulars }\end{array}$ & $\begin{array}{l}\text { Meaney 1964, 131; } \\
\text { Warwick Museum A } 1439\end{array}$ \\
\hline Ipswich & Suffolk & $\begin{array}{l}\text { prob } \\
6 \text { th- } \\
7 \text { th c }\end{array}$ & One or 2 annulars & $\begin{array}{l}\text { Layard 1907, pl XXXII, and } \\
\text { 1909, pl VII; } \\
\text { Ipswich Museum }\end{array}$ \\
\hline Lakenheath & Suffolk & $\begin{array}{l}\text { prob } \\
6 \text { th- } \\
7 \text { th c }\end{array}$ & $\begin{array}{l}\text { Miscellaneous collection: } \\
\text { small annular opaque grass } \\
\text { green; } \\
1 \text { large annular golden } \\
\text { green translucent }\end{array}$ & $\begin{array}{l}\text { Meaney 1964, 230; } \\
\text { BM } 191012-2211 ; \\
\text { Beck Collection, } \\
\text { Cambridge Museum } \\
47.1834 \text { b }\end{array}$ \\
\hline $\begin{array}{l}\text { Little } \\
\text { Eriswell }\end{array}$ & Suffolk & 6 th c & $\begin{array}{l}\text { Grave 27: globular clear } \\
\text { green overlying opaque } \\
\text { yellow with } 2 \text { ring brooches }\end{array}$ & $\begin{array}{l}\text { Hutchinson 1966; } \\
\text { Bury St Edmunds } \\
\text { Museum }\end{array}$ \\
\hline
\end{tabular}




\begin{tabular}{|c|c|c|c|c|}
\hline West Stow & Suffolk & $\begin{array}{l}6 \text { th- } \\
7 \text { th c } \\
5 \text { th c } \\
5 \text { th c } \\
\text { late } \\
6 \text { th c }\end{array}$ & $\begin{array}{l}\text { Cemetery: } 1 \text { unpolished } \\
\text { emerald green annular; } \\
1 \text { small annular; } \\
1 \text { dark green 'disc'; } \\
1 \text { dark green barrel } \\
\text { Settlement: } \\
\text { SFB15: } 1 \text { dark olive green } \\
\text { translucent 'disc' (SF243); } \\
\text { SFB63: } 1 \text { green 'disc' } \\
\text { (SF2003); } \\
\text { SFB37: } 1 \text { dark blue-green } \\
\text { translucent globular } \\
\text { (SF972); } \\
\text { SFB44: } 1 \text { turquoise } \\
\text { translucent globular } \\
\text { (SF1169); } \\
\text { WF6, 12: } 1 \text { dark green } \\
\text { 'disc' (SF471) }\end{array}$ & $\begin{array}{l}\text { West 1985, 74; } \\
\text { Ashmolean Museum } \\
\text { 1909.424; } \\
\text { Bury St Edmunds Museum } \\
\text { Thetford Museum; } \\
\text { Thetford Museum; } \\
\text { West 1985, 23, 49, 34, } 37 \\
\text { and } 73, \text { figs } 72.8,207.5 \text {, } \\
\text { 129.10 and 150.6; } \\
\text { Bury St Edmunds } \\
\text { Museum }\end{array}$ \\
\hline Guildown & Surrey & $\begin{array}{l}\text { prob } \\
6 \text { th } c\end{array}$ & $\begin{array}{l}\text { Grave } 185 \text { necklace: } \\
1 \text { light green annular }\end{array}$ & $\begin{array}{l}\text { Lowther } 1931,10,43 \text {, } \\
\text { pl IX.3; } \\
\text { Guildford Museum }\end{array}$ \\
\hline Alfriston & Sussex & $\begin{array}{l}\text { late } \\
5 \text { th- } \\
6 \text { th c } \\
5 \text { th- } \\
\text { early } \\
7 \text { th c }\end{array}$ & $\begin{array}{l}\text { Grave } 43 \text { : globular beads } \\
\text { with others on silver wire } \\
\text { rings and } 2 \text { larger very dark } \\
\text { annulars; } \\
\text { Grave } 68: 10 \text { small bright } \\
\text { green irregular annulars } \\
\text { with quoit brooch, etc; } \\
\text { Grave } 77: 1 \text { translucent } \\
\text { grass green annular } \\
\text { with knife, etc; } \\
\text { Grave } 79: 2 \text { large grass } \\
\text { green translucent annulars }\end{array}$ & $\begin{array}{l}\text { Griffith } 1915,203 \text {, } \\
\text { pl XXII.4; } \\
\text { Welch } 1983,82 \text { and } 84 ; \\
\text { Lewes Museum }\end{array}$ \\
\hline $\begin{array}{l}\text { Apple } \\
\text { Down I }\end{array}$ & Sussex & $\begin{array}{l}\text { first } \\
\text { half } \\
6 \text { th c } \\
\text { early } \\
\text { 6th c } \\
\text { prob } \\
\text { 6th c } \\
\text { first } \\
\text { half } \\
6 \text { th c }\end{array}$ & $\begin{array}{l}\text { Grave 10: translucent } \\
\text { bottle green annular with } \\
\text { cast saucer brooch pair; } \\
\text { Grave } 13: 1 \text { opaque } \\
\text { annular with cast saucer } \\
\text { brooch, etc; } \\
\text { Grave } 90: 1 \text { opaque annular } \\
\text { and } 1 \text { opaque globular; } \\
\text { Grave } 128: 3 \text { annulars, } \\
\text { ?translucent, small and many } \\
\text { very small with button } \\
\text { brooch, etc }\end{array}$ & $\begin{array}{l}\text { Down and Welch } 1990 \text {, } \\
35,36,46 \text { and } 51,162-3 \text {, } \\
\text { figs } 2.17,2.19-20, \\
2.32 \text { and } 2.40-41, \\
\text { pl } 40.3 ; \\
\text { Chichester Museum }\end{array}$ \\
\hline Highdown & Sussex & $\begin{array}{l}\text { prob } \\
6 \text { th } c\end{array}$ & $\begin{array}{l}\text { Unknown grave: } 2 \\
\text { opaque globulars; } \\
2 \text { annulars different }\end{array}$ & $\begin{array}{l}\text { Read } 1895 \text { and } 1896 \text {; } \\
\text { Welch } 1983,84 ; \\
\text { Worthing Museum } \\
3484 \text { and } 3481\end{array}$ \\
\hline
\end{tabular}




\begin{tabular}{|c|c|c|c|c|}
\hline $\begin{array}{l}\text { Bidford- } \\
\text { on-Avon }\end{array}$ & Warks & $\begin{array}{l}5 \text { th- } \\
\text { 7th c }\end{array}$ & $\begin{array}{l}55 \text { minute beads like pin } \\
\text { heads }\end{array}$ & $\begin{array}{l}\text { Humphreys et al } 1923 \text {, } \\
\text { 105, pl XVIII; } \\
\text { Stratford Museum }\end{array}$ \\
\hline Wasperton & Warks & 6th $c$ & $\begin{array}{l}\text { F1579: } 3 \text { bright grass green } \\
\text { with applied saucer } \\
\text { brooches }\end{array}$ & $\begin{array}{l}\text { Publication in } \\
\text { preparation (M Carver); } \\
\text { Warwick Museum }\end{array}$ \\
\hline $\begin{array}{l}\text { Colling- } \\
\text { bourne Ducis }\end{array}$ & Wilts & $\begin{array}{l}\text { first } \\
\text { half } \\
\text { 6th c }\end{array}$ & $\begin{array}{l}\text { Grave } 31 \text { : very small with } \\
\text { button brooch pair, finger } \\
\text { rings, etc }\end{array}$ & $\begin{array}{l}\text { Gingell 1975, 90, fig 20; } \\
\text { Devizes Museum }\end{array}$ \\
\hline $\begin{array}{l}\text { Harnham } \\
\text { Hill }\end{array}$ & Wilts & 6th $c$ & $\begin{array}{l}15 \text { annular beads in } \\
\text { green or blue }\end{array}$ & $\begin{array}{l}\text { Akerman 1853; } \\
\text { Jackson 1854; } \\
\text { BM } 53 \text { 12-14 } 31\end{array}$ \\
\hline $\begin{array}{l}\text { Pewsey } \\
\text { Blackpatch }\end{array}$ & Wilts & $\begin{array}{l}5 \text { th- } \\
6 \text { th } c\end{array}$ & $\begin{array}{l}\text { Grave 18: annular dark } \\
\text { olive green; } \\
\text { Grave 102: ditto }\end{array}$ & $\begin{array}{l}\text { Publication in } \\
\text { preparation (K Annable); } \\
\text { Devizes Museum }\end{array}$ \\
\hline $\begin{array}{l}\text { near } \\
\text { Driffield }\end{array}$ & Yorks & & Large annular & Hull Museum \\
\hline Sewerby & Yorks & $\begin{array}{l}\text { mid- } \\
\text { 6th c }\end{array}$ & $\begin{array}{l}\text { Grave 8: dark green } \\
\text { annulars (A la) with Åberg } \\
\text { Group IVa cruciform } \\
\text { brooch, annular brooch pair; } \\
\text { Grave 28: annular, double } \\
\text { annular (Plate } 4 \text { ) and small } \\
\text { barrelswith Åberg Group IVa } \\
\text { cruciform brooch }\end{array}$ & $\begin{array}{l}\text { Hirst } 1985,62-4 \text {, } \\
\text { figs } 34.6 \mathrm{~b} \text {, } \\
42.28 .2 \mathrm{~b} \text {, e and } \mathrm{f}\end{array}$ \\
\hline $\begin{array}{l}\text { West } \\
\text { Heslerton }\end{array}$ & Yorks & $\begin{array}{l}\text { prob } \\
\text { 6th c }\end{array}$ & A number & $\begin{array}{l}\text { Publication in } \\
\text { preparation (D Powlesland) }\end{array}$ \\
\hline 'Yorks' & Yorks & 7th $\mathrm{c}$ & $\begin{array}{l}\text { One large globular; with } \\
\text { miscellaneous beads }\end{array}$ & Hull Museum \\
\hline \multirow[t]{2}{*}{$\begin{array}{l}\text { Dalmeny } \\
\text { Hound } \\
\text { Point }\end{array}$} & $\begin{array}{l}\text { Scotland: } \\
\text { West } \\
\text { Lothian }\end{array}$ & $\begin{array}{l}\text { prob } \\
\text { 7th } c\end{array}$ & $\begin{array}{l}\text { Cist: necklace with mixed } \\
\text { colours }\end{array}$ & $\begin{array}{l}\text { Brown 1915b; Brown } \\
\text { 1915a (IV), 812-13, fig 29; } \\
\text { Edinburgh Museum }\end{array}$ \\
\hline & ii GreEN & CYLII & $\begin{array}{l}\text { ER BEADS (OPAQUE OR T } \\
\text { (a) ROUND SECTION }\end{array}$ & RANSLUCENT): \\
\hline $\begin{array}{l}\text { Long } \\
\text { Wittenham }\end{array}$ & Berks & 6th $c$ & Grave 98: 1 translucent & $\begin{array}{l}\text { Akerman 1860; } \\
\text { BM }\end{array}$ \\
\hline $\begin{array}{l}\text { Linton } \\
\text { Heath B }\end{array}$ & Cambs & $\begin{array}{l}5 \text { th- } \\
\text { 6th c }\end{array}$ & $\begin{array}{l}\text { Large translucent bright } \\
\text { emerald green with } 2 \text { small- } \\
\text { long brooches; } \\
\text { another }\end{array}$ & $\begin{array}{l}\text { Neville } 1854 \text {; } \\
\text { Cambridge Museum } \\
48.1598 \text { and } \\
48.1551\end{array}$ \\
\hline $\begin{array}{l}\text { Norton- } \\
\text { on-Tees }\end{array}$ & Durham & $\begin{array}{l}\text { late } \\
5 \text { th- } \\
\text { 6th c } \\
6 \text { th c }\end{array}$ & $\begin{array}{l}\text { Grave 1: } 1 \text { bead (A5a) } \\
\text { with Áberg Group II } \\
\text { cruciform brooch, etc; } \\
\text { Grave 4: } 1 \text { bead (A5a) } \\
\text { with annular brooch pair, }\end{array}$ & $\begin{array}{l}\text { Vyner 1984; } \\
\text { Sherlock and Welch } 1992 \text {, } \\
121,123 \text { and } 133 \text {, } \\
\text { figs } 33.1 .1,33.3 .1 \\
\text { and } 38.1\end{array}$ \\
\hline
\end{tabular}




\begin{tabular}{|c|c|c|c|c|}
\hline & & $\begin{array}{l}\text { later } \\
\text { 6th c }\end{array}$ & $\begin{array}{l}\text { class B7 clasps, etc; } \\
\text { Grave 22: with Class C2 } \\
\text { cruciform brooch, etc }\end{array}$ & \\
\hline $\begin{array}{l}\text { Great } \\
\text { Chesterford }\end{array}$ & Essex & $\begin{array}{l}\text { late } \\
5 \text { th- } \\
6 \text { th c }\end{array}$ & $\begin{array}{l}\text { Grave } 37: 2 \text { opaque dark } \\
\text { emerald (CO1) with Hahn- } \\
\text { heim-type radiate-headed } \\
\text { and small-long brooch, etc; } \\
\text { Grave 1: } 1 \text { short cylinder } \\
\text { (B26) with small-long } \\
\text { brooch pair, etc }\end{array}$ & $\begin{array}{l}\text { Evison } 1994,14,16 \text {, } \\
46,97 \text { and } 90 ; \\
\text { figs } 28.4 \mathrm{Ah}, 15.1 .3 \mathrm{~b} ; \\
\mathrm{BM}\end{array}$ \\
\hline $\begin{array}{l}\text { Springfield } \\
\text { Lyons }\end{array}$ & Essex & & $\begin{array}{l}\text { Feature no. 3923: pale } \\
\text { green opaque }\end{array}$ & $\begin{array}{l}\text { Publication in } \\
\text { preparation ( } \mathrm{S} \text { Tyler); BM }\end{array}$ \\
\hline Lechlade & Glos & 7th $c$ & $\begin{array}{l}\text { Grave 145/2: bright } \\
\text { 'coiled cylinder' }\end{array}$ & $\begin{array}{l}\text { Boyle et al forthcoming; } \\
\text { Cirencester Museum }\end{array}$ \\
\hline $\begin{array}{l}\text { Canterbury, } \\
\text { Cakebread } \\
\text { Robey V }\end{array}$ & Kent & c. 400 & $\begin{array}{l}\text { From multiple burial: } \\
6 \text { varying sizes }\end{array}$ & $\begin{array}{l}\text { Information from } \\
\text { T Tatton-Brown and } \\
\text { P Garrard }\end{array}$ \\
\hline $\begin{array}{l}\text { Dover, } \\
\text { Buckland }\end{array}$ & Kent & $\begin{array}{l}475- \\
525 \\
575- \\
625\end{array}$ & $\begin{array}{l}\text { Grave 20: more than } 1 \\
\text { cylinder (B31) (Plate } 4) \text {; } \\
\text { Grave 30: } 1 \text { cylinder } \\
\text { (B31) }\end{array}$ & $\begin{array}{l}\text { Evison } 1987,73,80, \\
220 \text { and } 224, \text { figs } 12.5 \mathrm{~g} \\
\text { and 18.4i; } \\
\text { BM }\end{array}$ \\
\hline $\begin{array}{l}\text { Gilton or } \\
\text { Kingston }\end{array}$ & Kent & $\begin{array}{l}\text { prob } \\
\text { 6th- } \\
7 \text { th c }\end{array}$ & With pointed ends & $\begin{array}{l}\text { Faussett } 1856,1-34, \mathrm{pl} \text { VI; } \\
\text { Liverpool Museum }\end{array}$ \\
\hline Stowting & Kent & & $\begin{array}{l}\text { One opaque light emerald } \\
\text { and } 2 \text { long and narrow with } \\
\text { pointed ends and several } \\
\text { other examples }\end{array}$ & $\begin{array}{l}\text { Smith 1846a; } \\
\text { Brent 1867; } \\
\text { Beck Collection, } \\
\text { Cambridge Museum } \\
47.1800,1801,1804,1805\end{array}$ \\
\hline Fonaby & Lincs & $\begin{array}{l}\text { mid- } \\
\text { 6th } c \\
\text { 6th } c\end{array}$ & $\begin{array}{l}\text { Grave } 31: 1 \text { long trans- } \\
\text { lucent pale green with } \\
\text { Áberg Group IV cruciform } \\
\text { brooch, etc; } \\
\text { Grave } 41: 1 \text { translucent } \\
\text { green cylinder with } \\
\text { chatelaine and keys, etc, in } \\
\text { multiple grave }\end{array}$ & $\begin{array}{l}\text { Cook } 1981,32 \text { and } 38, \\
\text { figs } 10.31 .1 .12 \text { and } \\
14.41 .1 .1 ; \\
\text { Scunthorpe Museum }\end{array}$ \\
\hline $\begin{array}{l}\text { Welbeck } \\
\text { Hill }\end{array}$ & Lincs & $\begin{array}{l}\text { mid- } \\
\text { 6th } c\end{array}$ & $\begin{array}{l}\text { Grave 64: } 1 \text { opaque with } 2 \\
\text { Áberg Group IVb cruci- } \\
\text { form brooches, etc }\end{array}$ & $\begin{array}{l}\text { Publication in } \\
\text { preparation (G Taylor); } \\
\text { Cook 1981, 78; } \\
\text { Scunthorpe Museum }\end{array}$ \\
\hline Brooke & Norfolk & $\begin{array}{l}\text { prob } \\
6 \text { th } c\end{array}$ & Two opaque yellowish green & $\begin{array}{l}\text { Meaney } 1964,170 \text {; } \\
\text { BM 1866-69 }\end{array}$ \\
\hline $\begin{array}{l}\text { Morning } \\
\text { Thorpe }\end{array}$ & Norfolk & $\begin{array}{l}\text { mid- } \\
6 \text { th } c\end{array}$ & $\begin{array}{l}\text { Grave } 96: 3 \text { cylinders with } \\
\text { Aberg Group IVa cruciform } \\
\text { brooch, small-long brooch } \\
\text { pair, B19 clasps, etc; } \\
\text { and others }\end{array}$ & $\begin{array}{l}\text { Green } \text { et al 1987, 63, } \\
\text { fig } 327 . \text { Hiv; } \\
\text { Norwich Museum }\end{array}$ \\
\hline
\end{tabular}




\begin{tabular}{|c|c|c|c|c|}
\hline Spong Hill & Norfolk & 6th $c$ & $\begin{array}{l}\text { Grave } 42: 2 \text { tapering } \\
\text { perforation with small-long } \\
\text { brooch pair, stamped pot }\end{array}$ & $\begin{array}{l}\text { Hills et al } 1984, \\
\text { 95-7, fig 98.42.3; } \\
\text { Norfolk Arch Unit }\end{array}$ \\
\hline $\begin{array}{l}\text { South } \\
\text { Shields }\end{array}$ & $\begin{array}{l}\text { North- } \\
\text { umber- } \\
\text { land }\end{array}$ & $\begin{array}{l}\text { prob } \\
\text { 4th- } \\
5 \text { th c }\end{array}$ & $\begin{array}{l}\text { Several with miscellaneous } \\
\text { beads of late Roman type }\end{array}$ & $\begin{array}{l}\text { Information from } \\
\text { R Miket; } \\
\text { South Shields Museum }\end{array}$ \\
\hline $\begin{array}{l}\text { Little } \\
\text { Eriswell }\end{array}$ & Suffolk & 6th $c$ & $\begin{array}{l}\text { Grave } 27 \text { : emerald green } \\
\text { with } 2 \text { ring brooches }\end{array}$ & $\begin{array}{l}\text { Hutchinson 1966; } \\
\text { Bury St Edmunds Museum }\end{array}$ \\
\hline Highdown & Sussex & $\begin{array}{l}\text { 5th- } \\
\text { 6th } c\end{array}$ & $\begin{array}{l}\text { Necklace: } 4 \text { very small } \\
\text { cylinders }\end{array}$ & $\begin{array}{l}\text { Read } 1895 \text { and } 1896 ; \\
\text { Welch } 1983,84 ; \\
\text { Worthing Museum }\end{array}$ \\
\hline $\begin{array}{l}\text { Peters- } \\
\text { finger }\end{array}$ & Wilts & $\begin{array}{l}\text { prob } \\
\text { 6th } c\end{array}$ & $\begin{array}{l}\text { Grave } 62: 1 \text { thick opaque } \\
\text { emerald green cylinder } \\
(172 a)\end{array}$ & $\begin{array}{l}\text { Leeds and Shortt } 1953, \\
\text { 41, pl IV.172.LXII; } \\
\text { Salisbury Museum }\end{array}$ \\
\hline \multirow[t]{2}{*}{ Sewerby } & Yorks & $\begin{array}{l}\text { mid- } \\
\text { 6th } c\end{array}$ & $\begin{array}{l}\text { Grave } 28: 1 \text { with pointed } \\
\text { ends (A5a) with } \AA \text { berg } \\
\text { Group IVa cruciform } \\
\text { brooch }\end{array}$ & $\begin{array}{l}\text { Hirst } 1985,66 \text {, } \\
\text { fig } 42.28 .2 j\end{array}$ \\
\hline & ii GreEN & \multicolumn{3}{|c|}{$\begin{array}{l}\text { CYLINDER BEADS (OPAQUE OR TRANSLUCENT): } \\
\text { (b) SQUARE OR POLYGONAL SECTION }\end{array}$} \\
\hline $\begin{array}{l}\text { Linton } \\
\text { Heath B }\end{array}$ & Cambs & $\begin{array}{l}\text { prob } \\
\text { 6th } c\end{array}$ & $\begin{array}{l}\text { Long rectangular-sectioned } \\
\text { faded green }\end{array}$ & $\begin{array}{l}\text { Neville 1854; } \\
\text { Cambridge Museum } 48.1598\end{array}$ \\
\hline $\begin{array}{l}\text { Norton- } \\
\text { on-Tees }\end{array}$ & Durham & $\begin{array}{l}\text { late } \\
5 \text { th- } \\
6 \text { th c }\end{array}$ & $\begin{array}{l}\text { Grave 1: } 1 \text { emerald green } \\
\text { square section (A5b) with } \\
\text { Åberg Group II cruciform } \\
\text { brooch, clasp, etc }\end{array}$ & $\begin{array}{l}\text { Vyner 1984; } \\
\text { Sherlock and Welch 1992, } \\
121 \text {, fig 33.1.1 }\end{array}$ \\
\hline Barfreston & Kent & 7 th $c$ & Hexagonal emerald green & $\begin{array}{l}\text { Faussett } 1856,135-43 \\
\text { Liverpool Museum }\end{array}$ \\
\hline $\begin{array}{l}\text { Dover, } \\
\text { Buckland }\end{array}$ & Kent & $\begin{array}{l}575- \\
625 \\
650- \\
675\end{array}$ & $\begin{array}{l}\text { Grave 38: } 1 \text { flat hexagonal } \\
\text { blue-green (B37); } \\
\text { Grave 133: 1 pentagonal } \\
\text { light green (B25) }\end{array}$ & $\begin{array}{l}\text { Evison 1987, } 73,80 \text {, } \\
227 \text { and } 245, \text { figs } 22.4 d \\
\text { and } 5.133 .2 \mathrm{~d} \text {, colour pl III } \\
\text { B25 133/2d; BM }\end{array}$ \\
\hline Faversham & Kent & $\begin{array}{l}5 \text { th- } \\
7 \text { th } c\end{array}$ & $\begin{array}{l}\text { One pentagonal dull green } \\
\text { and } 1 \text { lighter; } \\
1 \text { hexagonal opaque }\end{array}$ & $\begin{array}{l}\text { Meaney 1964, 118-19; } \\
\text { Ashmolean Museum } \\
1909.155 \text { and } 1909.168\end{array}$ \\
\hline Finglesham & Kent & $\begin{array}{l}\text { first } \\
\text { half } \\
\text { 6th c }\end{array}$ & $\begin{array}{l}\text { Grave D3: broken-off } \\
\text { hexagonal light green with } \\
\text { great square-headed brooch, } \\
\text { radiate-headed brooch, etc }\end{array}$ & $\begin{array}{l}\text { Chadwick 1958, 12, } \\
\text { fig } 5 \text { d; } \\
\text { Deal Museum }\end{array}$ \\
\hline Howletts & Kent & $\begin{array}{l}\text { prob } \\
\text { 6th } c\end{array}$ & $\begin{array}{l}\text { Grave 142: } 1 \text { pentagonal } \\
\text { emerald green (Plate 4) }\end{array}$ & $\begin{array}{l}\text { Smith 1918; } \\
\text { BM } 1936 \text { 5-11 }\end{array}$ \\
\hline 'Kent' & Kent & & Necklace: 1 square section & $\begin{array}{l}\text { Canterbury Museum } \\
\text { RM } 2620\end{array}$ \\
\hline
\end{tabular}




\begin{tabular}{|c|c|c|c|c|}
\hline Quarrington & Lincs & $\begin{array}{l}\text { prob } \\
6 \text { th } c\end{array}$ & Three square-sectioned & $\begin{array}{l}\text { Bruce } 1880,76, \text { no. } 303 \text {; } \\
\text { Alnwick Museum }\end{array}$ \\
\hline Kenninghall & Norfolk & $\begin{array}{l}5 \text { th- } \\
7 \text { th } c\end{array}$ & $\begin{array}{l}\text { Two pentagonal opaque; } \\
\text { hexagonal }\end{array}$ & $\begin{array}{l}\text { Manning } 1872 a, 292 \text {, and } \\
1872 b ; \\
\text { BM } 83 \text { 7-2 31; } \\
\text { Norwich Museum }\end{array}$ \\
\hline Spong Hill & Norfolk & $\begin{array}{l}\text { 6th } c \\
6 \text { th } c\end{array}$ & $\begin{array}{l}\text { Grave } 18: 2 \text { square-sectioned } \\
\text { (Plate 4) with great } \\
\text { square-headed brooch, etc; } \\
\text { Grave } 44: 1 \text { square- } \\
\text { sectioned yellow-green } \\
\text { with annular brooch pair, } \\
\text { etc }\end{array}$ & $\begin{array}{l}\text { Hills et al } 1984 \text {, } \\
67 \text { and } 98, \text { figs } 77.18 .4 \\
\text { and } 99.44 .7 ; \\
\text { Norfolk Arch Unit }\end{array}$ \\
\hline $\begin{array}{l}\text { Mitchell's } \\
\text { Hill }\end{array}$ & Suffolk & $\begin{array}{l}\text { 6th- } \\
7 \text { th } c\end{array}$ & $\begin{array}{l}\text { Square or hexagonal } \\
\text { section, bluish-green }\end{array}$ & $\begin{array}{l}\text { Meaney 1964, 231; } \\
\text { Bury St Edmunds } \\
\text { Museum }\end{array}$ \\
\hline Sewerby & Yorks & $\begin{array}{l}\text { later } \\
\text { 6th } c\end{array}$ & $\begin{array}{l}\text { Grave } 49 \text { : Strings I - } \\
1 \text { diamond-sectioned pale } \\
\text { green - and II - } 2 \\
\text { square-sectioned dark } \\
\text { green with } 1 \text { pale green } \\
\text { (A5b) - with Class C2 florid } \\
\text { cruciform brooch, etc }\end{array}$ & $\begin{array}{l}\text { Hirst } 1985,66 \text {, fig } 52 \text {, } \\
\text { Strings I.b, II.b and II.c }\end{array}$ \\
\hline $\begin{array}{l}\text { West } \\
\text { Heslerton }\end{array}$ & Yorks & & $\begin{array}{l}\text { Grave 1A17: square- } \\
\text { sectioned }\end{array}$ & $\begin{array}{l}\text { Publication in } \\
\text { preparation (D Powlesland) }\end{array}$ \\
\hline
\end{tabular}

ii Green cylinder beads (Opaque or translucent):

(c) POLYHEDRAL SECTION: no schedule (Plate 4)

iii Green Short cylinder beAds, Singles or doubles

\begin{tabular}{|c|c|c|c|c|}
\hline $\begin{array}{l}\text { Chamber- } \\
\text { lain's } \\
\text { Barn II }\end{array}$ & Beds & $\begin{array}{l}\text { mid- } \\
\text { late } \\
7 \text { th c }\end{array}$ & $\begin{array}{l}\text { Grave 39: large 'ring- } \\
\text { shaped' opaque green with } \\
\text { silver union pin set, silver } \\
\text { wire rings, etc }\end{array}$ & $\begin{array}{l}\text { Hyslop 1963, 181, } \\
\text { fig 13d; } \\
\text { Luton Museum }\end{array}$ \\
\hline Kempston & Beds & $\begin{array}{l}\text { prob } \\
\text { 6th c }\end{array}$ & $\begin{array}{l}\text { Grave 16: several opaque } \\
\text { emerald green }\end{array}$ & $\begin{array}{l}\text { Fitch 1864; } \\
\text { BM } 916-24 \text { and } 53\end{array}$ \\
\hline $\begin{array}{l}\text { Dorchester, } \\
\text { Trumpet } \\
\text { Major }\end{array}$ & Dorset & $\begin{array}{l}\text { 6th- } \\
7 \text { th } c\end{array}$ & Pale opaque green & $\begin{array}{l}\text { Sparey-Green 1984, } \\
149 \text {, fig } 13.8\end{array}$ \\
\hline $\begin{array}{l}\text { Canterbury, } \\
\text { Mint Yard }\end{array}$ & Kent & $\begin{array}{l}\text { prob } \\
5 \text { th- } \\
7 \text { th c }\end{array}$ & Two blue green & $\begin{array}{l}\text { Information from } \mathrm{T} \\
\text { Tatton-Brown and } \mathrm{P} \text { Garrard }\end{array}$ \\
\hline $\begin{array}{l}\text { Crundale } \\
\text { Down }\end{array}$ & Kent & & $\begin{array}{l}\text { Some beads, possibly } \\
\text { mixed up with Sarre beads }\end{array}$ & $\begin{array}{l}\text { Faussett } 1856,177-98 ; \\
\text { BM } 936-1196\end{array}$ \\
\hline $\begin{array}{l}\text { Dover, } \\
\text { Buckland }\end{array}$ & Kent & $\begin{array}{l}475- \\
525\end{array}$ & $\begin{array}{l}\text { Grave 20: } 1 \text { cylinder and } \\
\text { fragments (B31); }\end{array}$ & $\begin{array}{l}\text { Evison } 1987,73,80 \text {, } \\
220,223-4,224,235 \text {, }\end{array}$ \\
\hline
\end{tabular}




\begin{tabular}{|c|c|c|c|c|}
\hline & & $\begin{array}{l}575- \\
625 \\
675- \\
700\end{array}$ & $\begin{array}{l}\text { Grave 29: } 1 \text { double (B30); } \\
\text { Grave 30: } 1 \text { cylinder (B31); } \\
\text { Grave 110: } 2 \text { cylinders } \\
\text { (B29) and } 1 \text { green-blue } \\
\text { (B42); } \\
\text { Grave 160: } 3 \text { cylinders } \\
\text { (B29); } \\
\text { Grave } 75: 2 \text { cylinders } \\
\text { (B29); } \\
\text { Grave } 127: 1 \text { cylinder } \\
\text { (B29) }\end{array}$ & $\begin{array}{l}241,243 \text { and } 251 \text {, } \\
\text { figs } 12.5 \mathrm{~g}, 17.2 \mathrm{i}, 18.4 \mathrm{i} \text {, } \\
38.75 .1 \mathrm{f}, 49.110 .12 \mathrm{~b} \text { and } \mathrm{c} \text {, } \\
52.127 .1 \mathrm{~d} \text { and } 62.160 .2 \mathrm{~b} \text {, } \\
\text { colour pl III (B29); } \\
\text { BM }\end{array}$ \\
\hline Folkestone & Kent & $\begin{array}{l}\text { prob } \\
\text { 6th c }\end{array}$ & (?) No. 32: 1 example & $\begin{array}{l}\text { Meaney } 1964,120-1 \\
\text { Folkestone Museum }\end{array}$ \\
\hline $\begin{array}{l}\text { Lyminge } \\
\text { II }\end{array}$ & Kent & $\begin{array}{l}\text { first } \\
\text { half } \\
\text { 6th } c\end{array}$ & $\begin{array}{l}\text { Grave } 16 \text { : opaque emerald } \\
\text { with radiate and button } \\
\text { brooches, etc }\end{array}$ & $\begin{array}{l}\text { Warhurst 1955, 15; } \\
\text { Maidstone Museum }\end{array}$ \\
\hline $\begin{array}{l}\text { Milton } \\
\text { Regis }\end{array}$ & Kent & 7th $\mathrm{c}$ & $\begin{array}{l}\text { Necklace: } 9 \text { opaque } \\
\text { unstratified beads }\end{array}$ & $\begin{array}{l}\text { Hawkes and Grove } 1963 \text {, } \\
\text { 32, fig 2, no. } 4 \text {; } \\
\text { Maidstone Museum }\end{array}$ \\
\hline Polhill & Kent & 7th $\mathrm{c}$ & $\begin{array}{l}\text { Grave 37: several (no. } 517 \text { ) } \\
\text { with keystone garnet disc } \\
\text { brooch, amethyst bead, etc }\end{array}$ & $\begin{array}{l}\text { Hawkes and Philp 1973, } \\
176 \text { and 207, fig 55.517 }\end{array}$ \\
\hline $\begin{array}{l}\text { Rochester II, } \\
\text { Watts } \\
\text { Avenue }\end{array}$ & Kent & & One emerald green & $\begin{array}{l}\text { Meaney 1964, 134; } \\
\text { Rochester Museum }\end{array}$ \\
\hline $\begin{array}{l}\text { Sibertswold } \\
\text { or Barfreston }\end{array}$ & Kent & $\begin{array}{l}\text { 6th- } \\
7 \text { th } c\end{array}$ & Grave 16: from earring & $\begin{array}{l}\text { Faussett } 1856,105 \\
\text { Liverpool Museum }\end{array}$ \\
\hline Stodmarsh & Kent & 6th $c$ & Opaque dirty green & $\begin{array}{l}\text { Akerman 1855b, 179-81, } \\
\text { pl XVI }\end{array}$ \\
\hline Fonaby & Lincs & 6th $c$ & $\begin{array}{l}\text { Grave } 41: 1 \text { translucent } \\
\text { with spearhead, etc }\end{array}$ & $\begin{array}{l}\text { Cook 1981, 38, } \\
\text { fig 14.41.1.1; } \\
\text { Scunthorpe Museum }\end{array}$ \\
\hline Kenninghall & Norfolk & $\begin{array}{l}\text { 6th- } \\
7 \text { th } c\end{array}$ & $\begin{array}{l}\text { Inhumation with necklace: } \\
1 \text { bluish-green translucent }\end{array}$ & $\begin{array}{l}\text { Manning } 1872 \mathrm{a}, 292 \text {, and } \\
1872 \mathrm{~b} \text {; } \\
\text { BM } 837-231\end{array}$ \\
\hline $\begin{array}{l}\text { Holme } \\
\text { Pierre- } \\
\text { pont }\end{array}$ & Notts & $\begin{array}{l}\text { prob } \\
\text { 6th c }\end{array}$ & $\begin{array}{l}\text { Large string: emerald green, } \\
\text { indirectly dated by brooches }\end{array}$ & $\begin{array}{l}\text { Smith 1906, 195-6; } \\
\text { BM }\end{array}$ \\
\hline $\begin{array}{l}\text { Standlake } \\
\text { Down }\end{array}$ & Oxon & $\begin{array}{l}\text { early } \\
7 \text { th } c\end{array}$ & $\begin{array}{l}\text { Grave } 24: 4 \text { very small } \\
\text { apple green cylinders }\end{array}$ & $\begin{array}{l}\text { Dickinson 1973, 244-5, } \\
\text { fig 5.1.n; } \\
\text { Ashmolean Museum }\end{array}$ \\
\hline $\begin{array}{l}\text { Empingham } \\
\text { II }\end{array}$ & Rutland & $\begin{array}{l}5 \text { th- } \\
7 \text { th c }\end{array}$ & Grave 114: 1 opaque & $\begin{array}{l}\text { Timby 1996; } \\
\text { Oakham Museum }\end{array}$ \\
\hline Camerton & Somerset & $\begin{array}{l}\text { prob } \\
\text { 7th } \mathrm{c}\end{array}$ & $\begin{array}{l}\text { Grave } 57 ; \\
\text { Grave } 97 ; \\
\text { Grave } 98\end{array}$ & $\begin{array}{l}\text { Horne } 1933,46,57, \\
60-1 \text { and } 61 ; \\
\text { Taunton Museum }\end{array}$ \\
\hline
\end{tabular}




\section{Locations of beads illustrated in Plates 1 to 8}

Where the site of the illustrated bead is marked as being uncertain, this is because these drawings were not identified by the author (Guido) but are assumed to represent one of the examples in the schedule. Drawings without a top view were drawn many years ago and the bead is no longer easily available. For the beads illustrated in the plates, the following list gives both their schedule reference and their provenance (where known).

PLATE 1
li Mucking II, Essex
lii Mucking II, Essex
liii Site uncertain; Fonaby,
Lincolnshire
liv Upton Snodsbury,
Worcestershire; Site
uncertain
1v Lechlade,
Gloucestershire
1via Mucking II, Essex
lvib Site uncertain;
Holywell Row, Suffolk;
Colchester, Essex
Ivii Bradwell Roman villa,
Buckinghamshire;
Alfriston, Sussex
1viii Mucking II, Essex
lix Harwell, Berkshire;
Wallingford, Berkshire
2i Mucking II, Essex
2ii Faversham, Kent;
Fonaby, Lincolnshire
2iii Harnham Hill,
Wiltshire; Site uncertain
2iv Charlton, Wiltshire

Plate 2

2va Andover, Hampshire;

Alfriston, Sussex

$2 \mathrm{vb}$ Wolstonbury, Sussex

2via Cheverell, Wiltshire

2vib No example illustrated

2vic Richborough, Kent

2viia Mucking II, Essex;

Barrington,

Cambridgeshire

2viib Richborough, Kent;

Silchester, Hampshire

2viii Barnsley Park,

Gloucestershire

2ix Little Wilbraham,

Cambridgeshire;

Stretton-on-Fosse,

Warwickshire

$2 x$ Lechlade,

Gloucestershire

2xi Uncertain site; Kenninghall, Norfolk

Plate 3

2xii Cirencester,

Gloucestershire;

Petersfinger, Wiltshire

2xiii Sewerby, Yorkshire 2xiv Probus, Cornwall

2xv Abingdon, Berkshire; Mucking II, Essex

2xvi No example illustrated

$3 i$ Mucking II, Essex;

Faversham, Kent

3ii Faversham, Kent; Linton Heath, Cambridgeshire

3iiia Morning Thorpe,

Norfolk; Morning Thorpe,

Norfolk

3iiib Faversham, Kent

3iiic Wheatley, Oxfordshire

3iiid Faversham, Kent;

Faversham, Kent

3iv Mucking II, Essex;

Mucking I, Essex

3v Puddlehill, Bedfordshire;

Chamberlain's Barn I,

Bedfordshire; Ipswich,

Suffolk

3vi Morning Thorpe,

Norfolk

Plate 4

4i Mucking II, Essex

4iia Site uncertain

4iib Kent, site uncertain;

Linton Heath $B$,

Cambridgeshire

4iic Standlake, Oxfordshire

4iii Polhill, Kent;

Chamberlain's Barn II,

Bedfordshire

4iv Norfolk, site uncertain;

Site uncertain

4v Stowting, Kent; Kent, unlocated; Kent, unlocated

4vi Site uncertain; Site uncertain; Site uncertain

4vii No example illustrated

$5 i$ Sewerby, Yorkshire;

Sewerby, Yorkshire;

Barrington,

Cambridgeshire

5iia Dover, Buckland, Kent

5iib Spong Hill, Norfolk;

Howletts, Kent

5iic Site uncertain

5iii Site uncertain

Siv Site uncertain; Site

uncertain

5v Site uncertain

5vi Dover, Buckland, Kent 5vii Lyminge II, Kent;

Fonaby, Lincolnshire

5viii Riby Park,

Lincolnshire; Morning

Thorpe, Norfolk

5 ix No example illustrated

5x Faversham, King's Field,

Kent; South Willingham,

Lincolnshire;

Chamberlain's Barn II,

Bedfordshire

Plate 5

6i Stretton-on-Fosse,

Warwickshire; Dover,

Buckland, Kent

6ii Dover, Buckland, Kent;

Lakenheath, Suffolk;

Horton Kirby I, Kent;

Site uncertain

6iii Site uncertain;

Kingston, Kent

6iv Pewsey Blackpatch,

Wiltshire

6v Alfriston, Sussex

6vi Site uncertain

6vii Polhill, Kent; Gilton,

Kent

6viii Chamberlain's Barn II,

Bedfordshire

6ix Site uncertain

6x Morning Thorpe,

Norfolk

6xi Kempston, Bedfordshire

6xii Lechlade,

Gloucestershire

6xiii Sibertswold, Kent

6xiv Kingston, Kent

6xv Andover, Hampshire

Plate 6

7 Mucking II, Essex;

Lechlade, Gloucestershire;

Great Chesterford, Essex

8i Mucking II, Essex; Site

uncertain; Faversham,

Kent

8ii Faversham, Kent; Dover, Buckland, Kent

8iii Spong Hill, Norfolk;

Mucking II, Essex

8iv Faversham, Kent;

?Faversham, Kent

8v Sewerby, Yorkshire;

Westbere, Kent 8vi Great Chesterford, Essex

8vii Andover, Hampshire;

Dover, Buckland, Kent

8viii Little Wilbraham,

Cambridgeshire

8ix Stretton-on-Fosse,

Warwickshire

8x Mucking II, Essex;

Mucking II, Essex

8xi Selmeston, Sussex

8xii Chamberlain's Barn I,

Bedfordshire; Faversham,

Kent

8xiii Site uncertain

Plate 7

8xiva Mucking II, Essex;

Mucking II, Essex

8xivb Site uncertain;

Faversham, Kent

8xivc Faversham, Kent

$8 x v$ Dover, Buckland, Kent

$8 x v i \quad$ Site uncertain

8xviia Dover, Buckland,

Kent; Dover, Buckland,

Kent

8 xviib York, Yorkshire

8 xviiia Howletts, Kent

8 xviiib Faversham, Kent

8xviiic Little Wilbraham,

Cambridgeshire; Mucking

II, Essex

8xixa Haslingfield,

Cambridgeshire

$8 \mathrm{xixb}$ Site uncertain

9 Polhill, Kent; Site uncertain

Plate 8

10a Ipswich, Suffolk; Site uncertain; Site uncertain; Cassington, Oxfordshire

10b Dover, Buckland, Kent; Dover, Buckland, Kent; Mucking II, Essex; Dover, Buckland, Kent; Sewerby, Yorkshire

1la Site uncertain

$11 \mathrm{~b}$ Sewerby, Yorkshire; Saltburn, Yorkshire

12 Hartlip, Kent; Salisbury, Wiltshire; Cannington, Somerset

13 Mucking II, Essex; Mucking II, Essex 


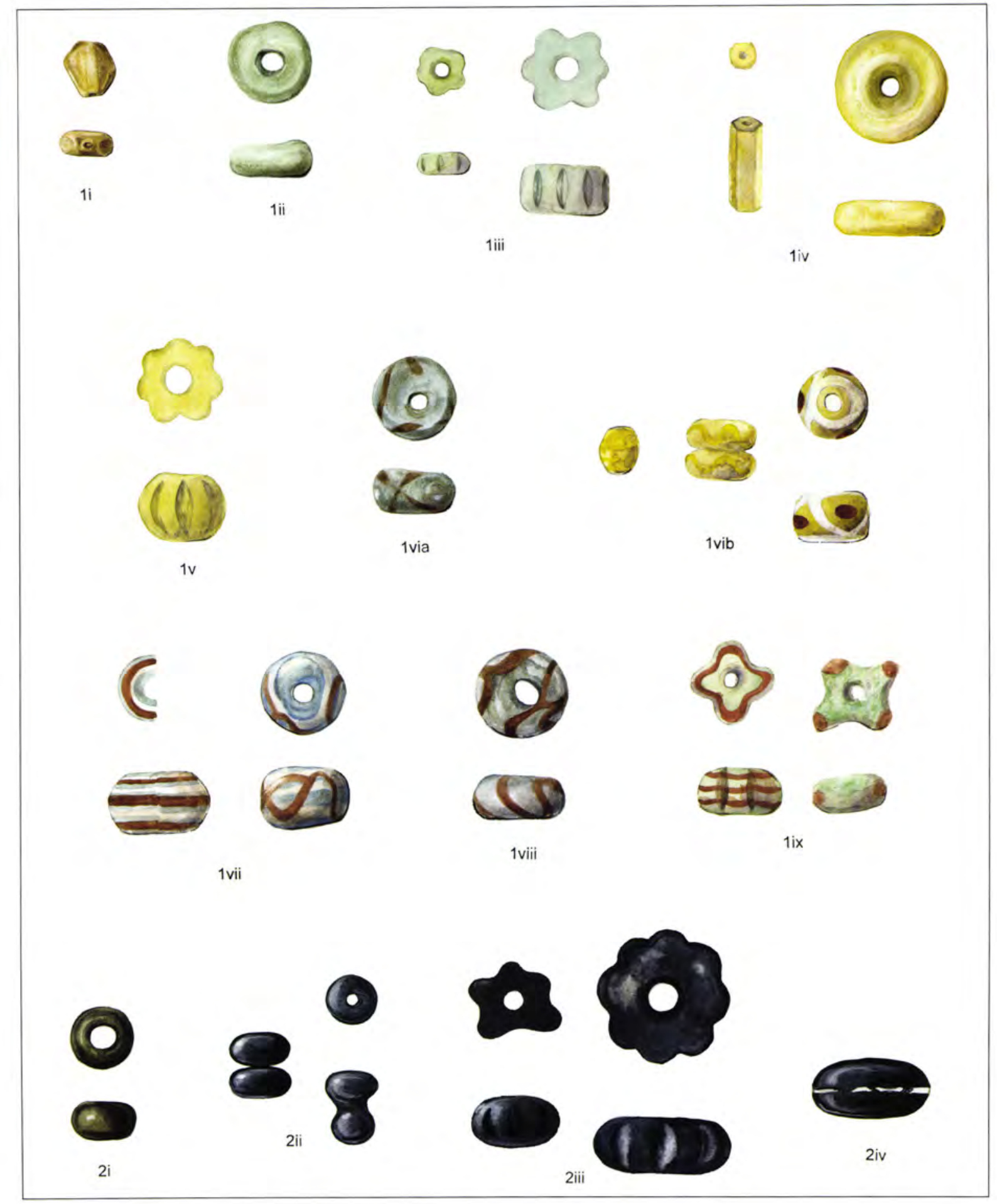

Plate 1 Colourless and pale translucent glass beads (Schedule 1i-ix); 'black' glass beads (Schedule 2i-iv) 


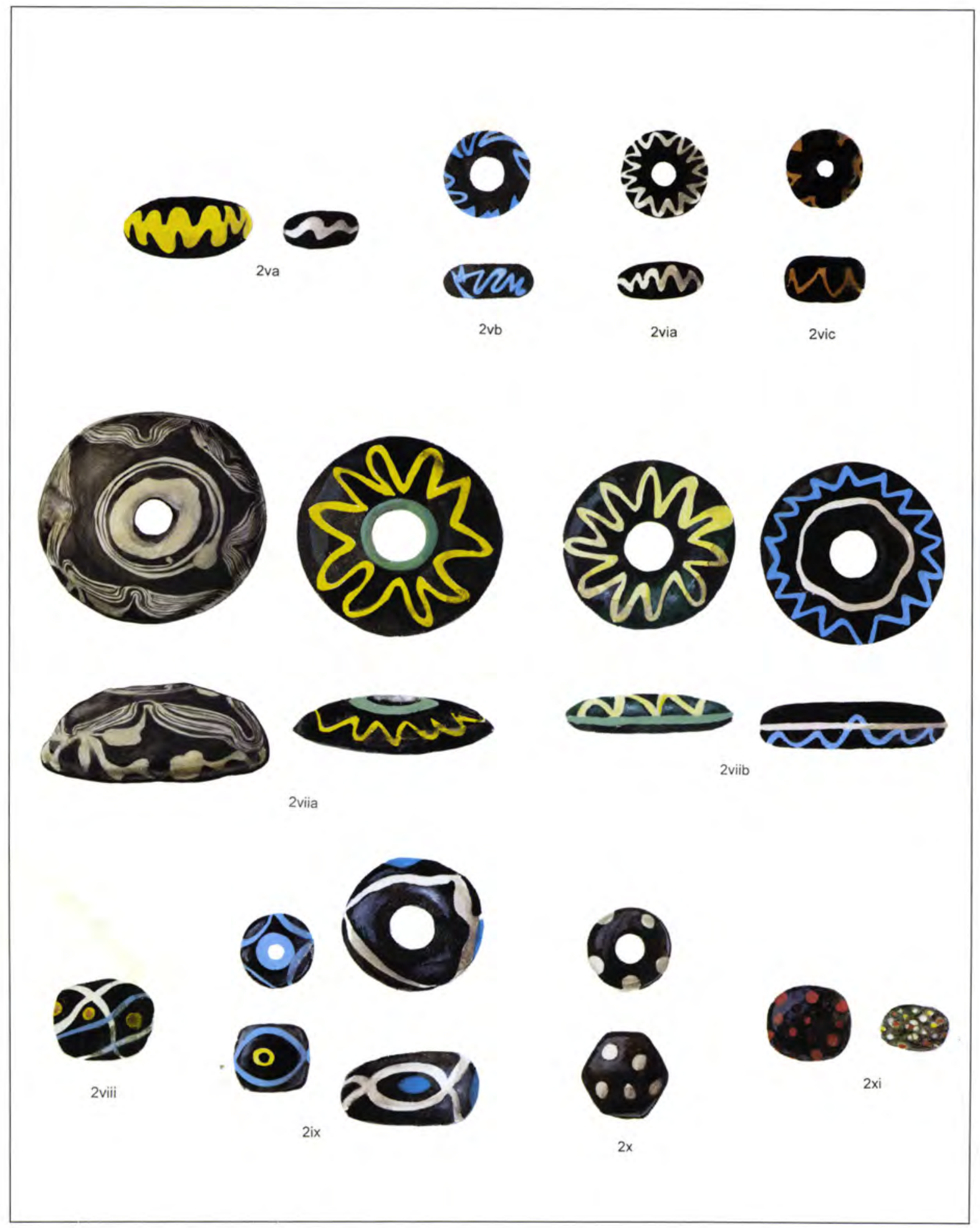

Plate 2 'Black'glass bead: ; jochedule 2v-xi) 


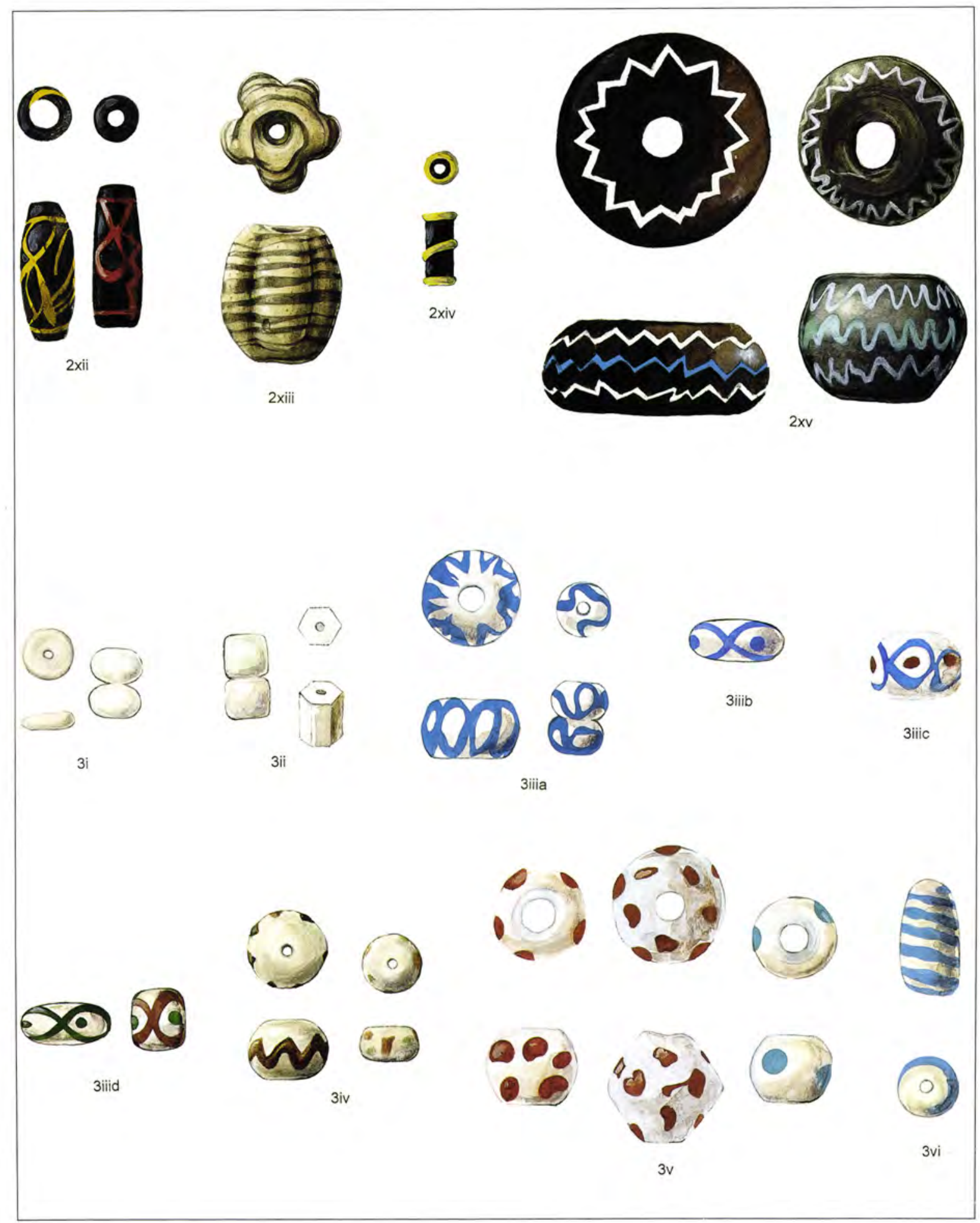

Plate 3 'Black' glass beads (Schedule 2xii-xv); white (or biue-white) glass beads (Schedule 3i-vi) 


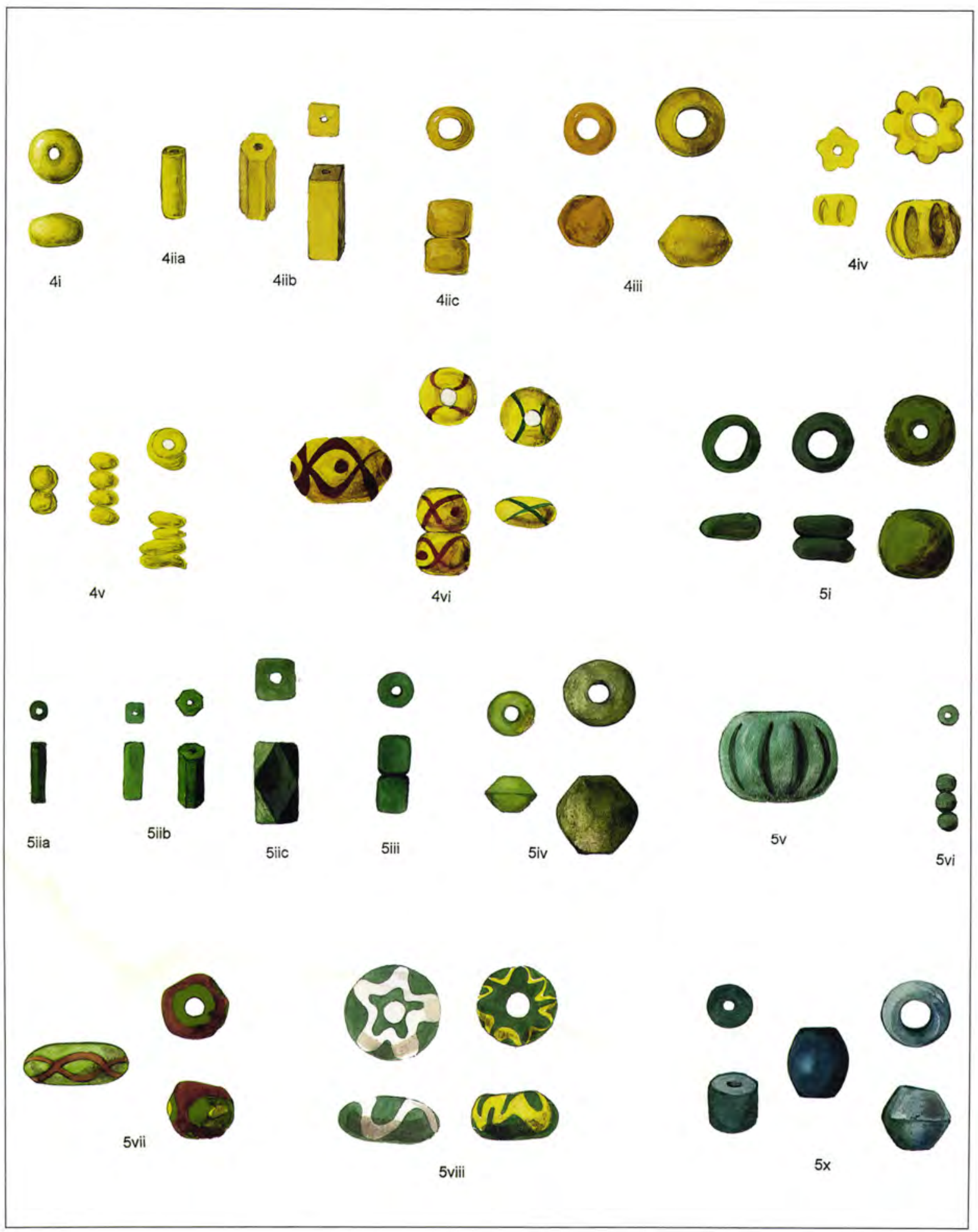

Plate 4 Opaque yellow glass beads (Schedule 4i-vi); green and turquoise glass beads (Schedule 5i-viii and $x$ ) 


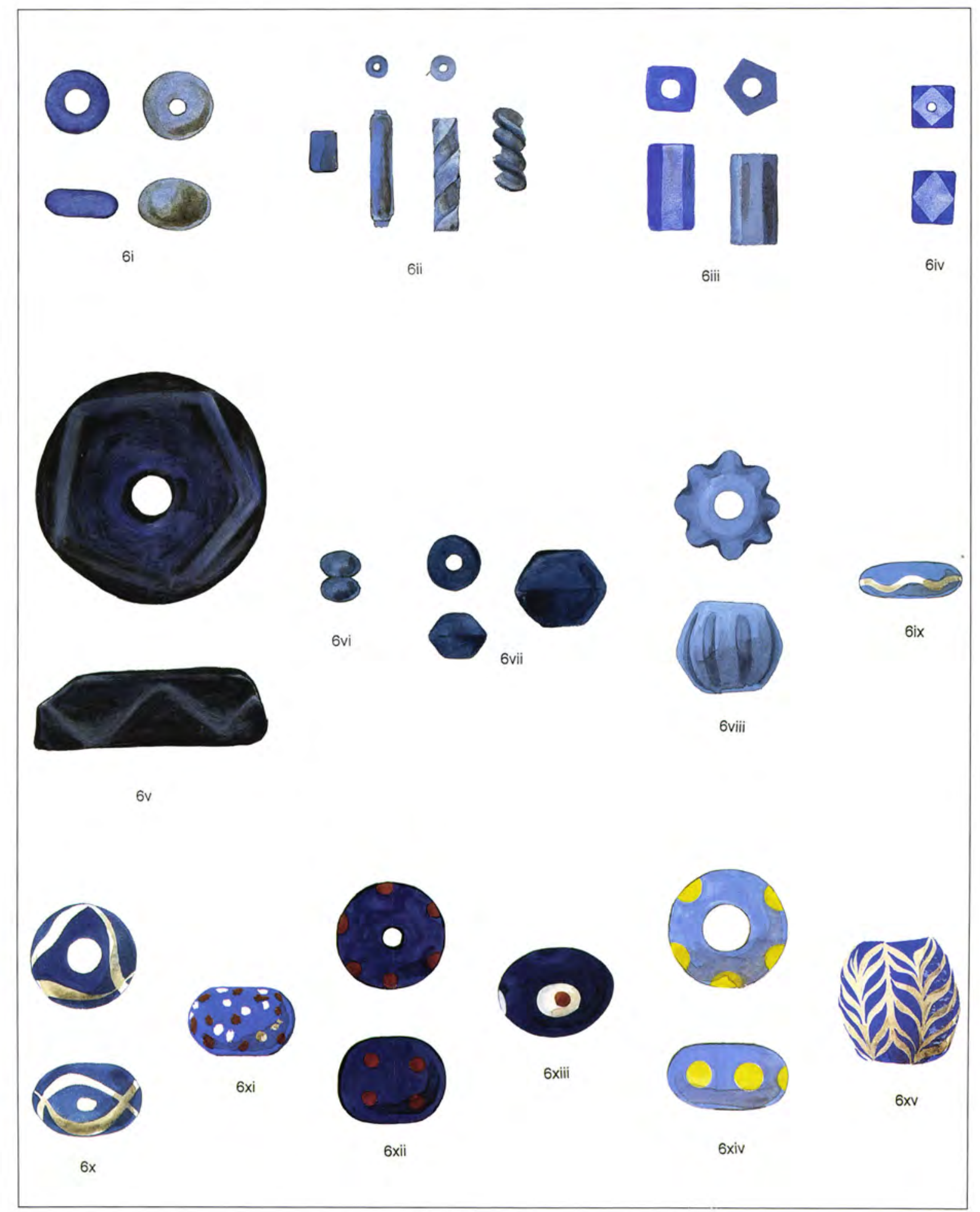

Plate 5 Blue glass beads (Schedule 6i-xv) 


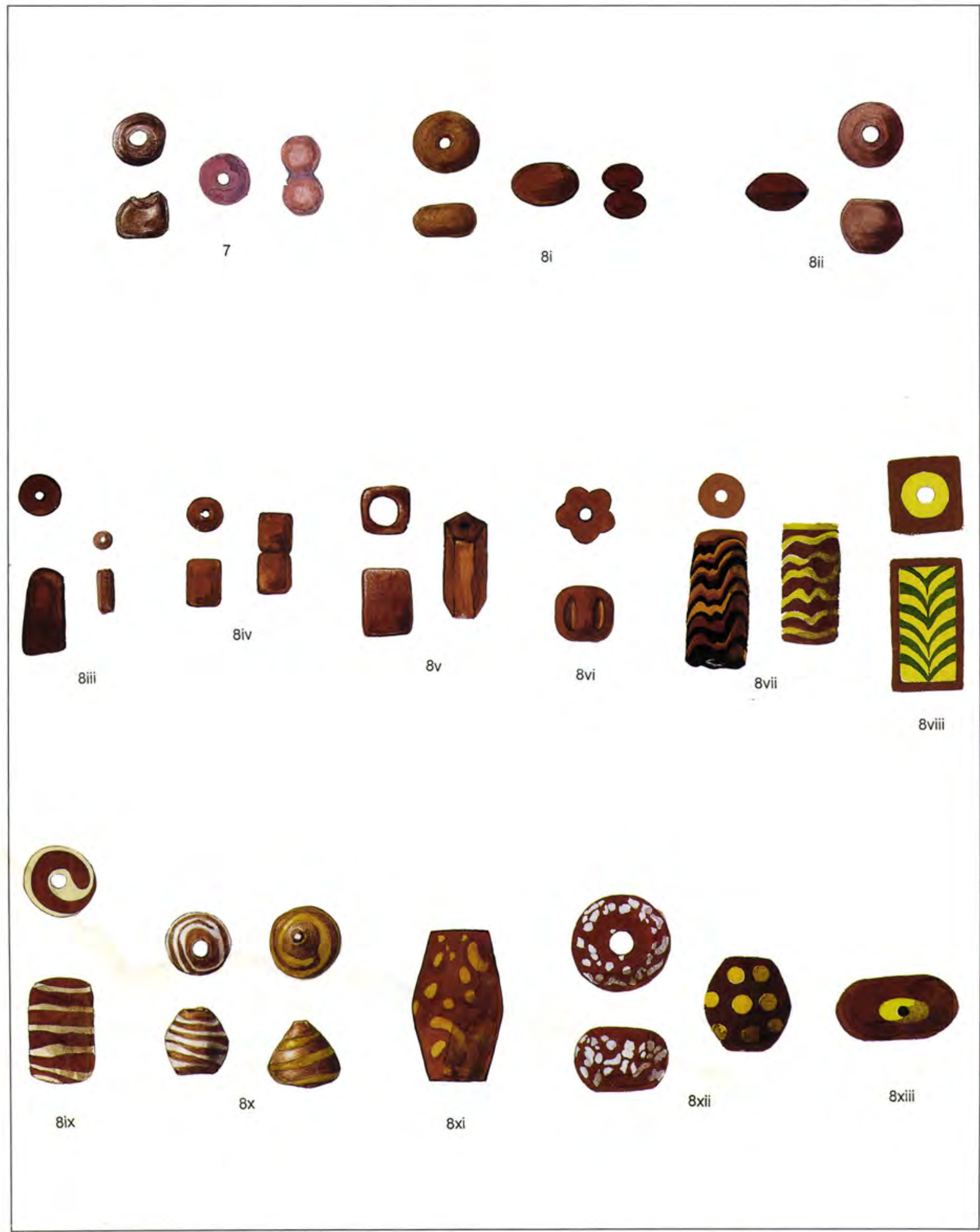

Plate 6 Crimson or pink glass beads (Schedule 7); terracotta or brick-red glass beads (Schedule 8i-xiii) 


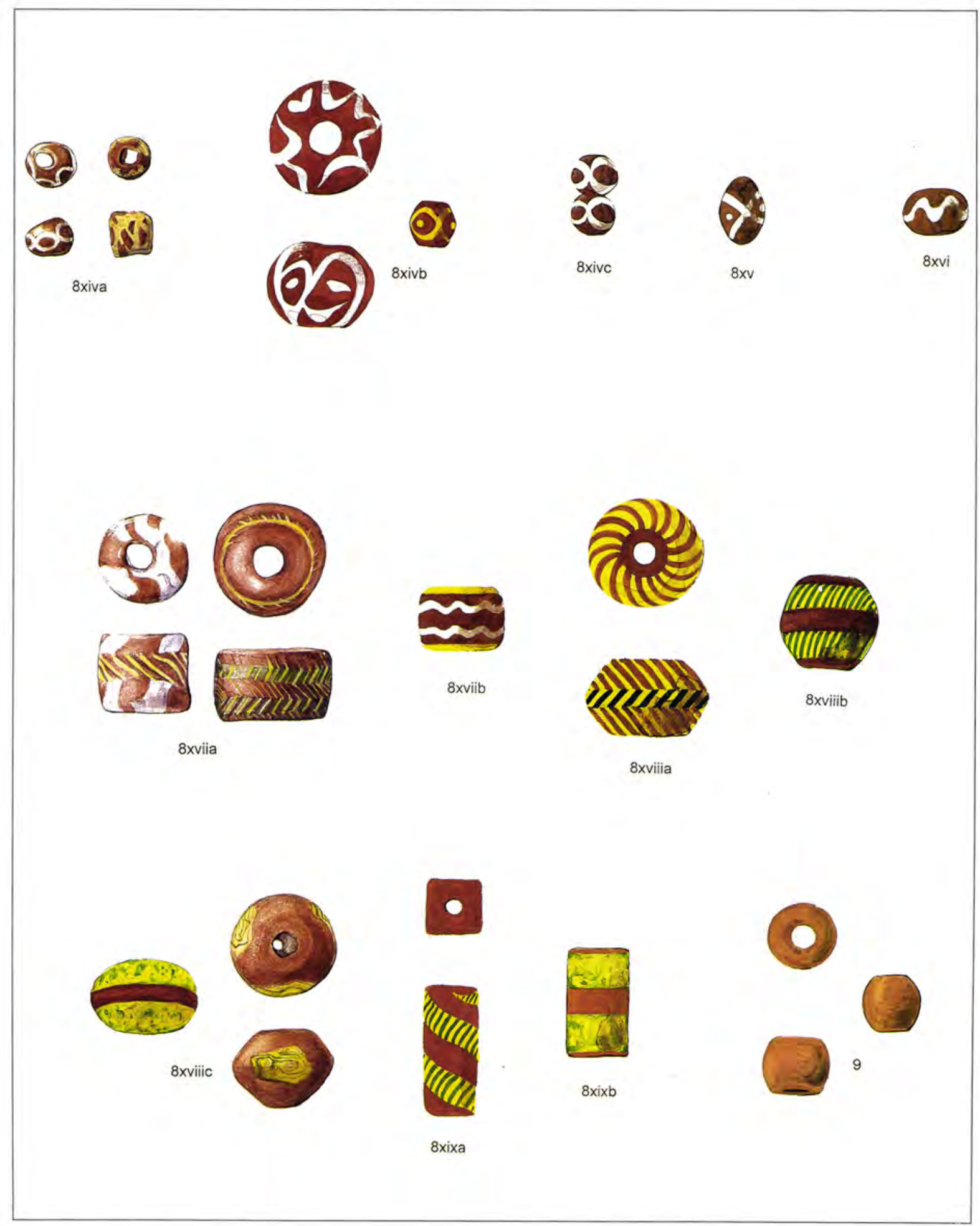

Plate 7 Terracotta or brick-red glass beads (Schedule 8xiv-xix); opaque orange glass beads (Schedule 9) 


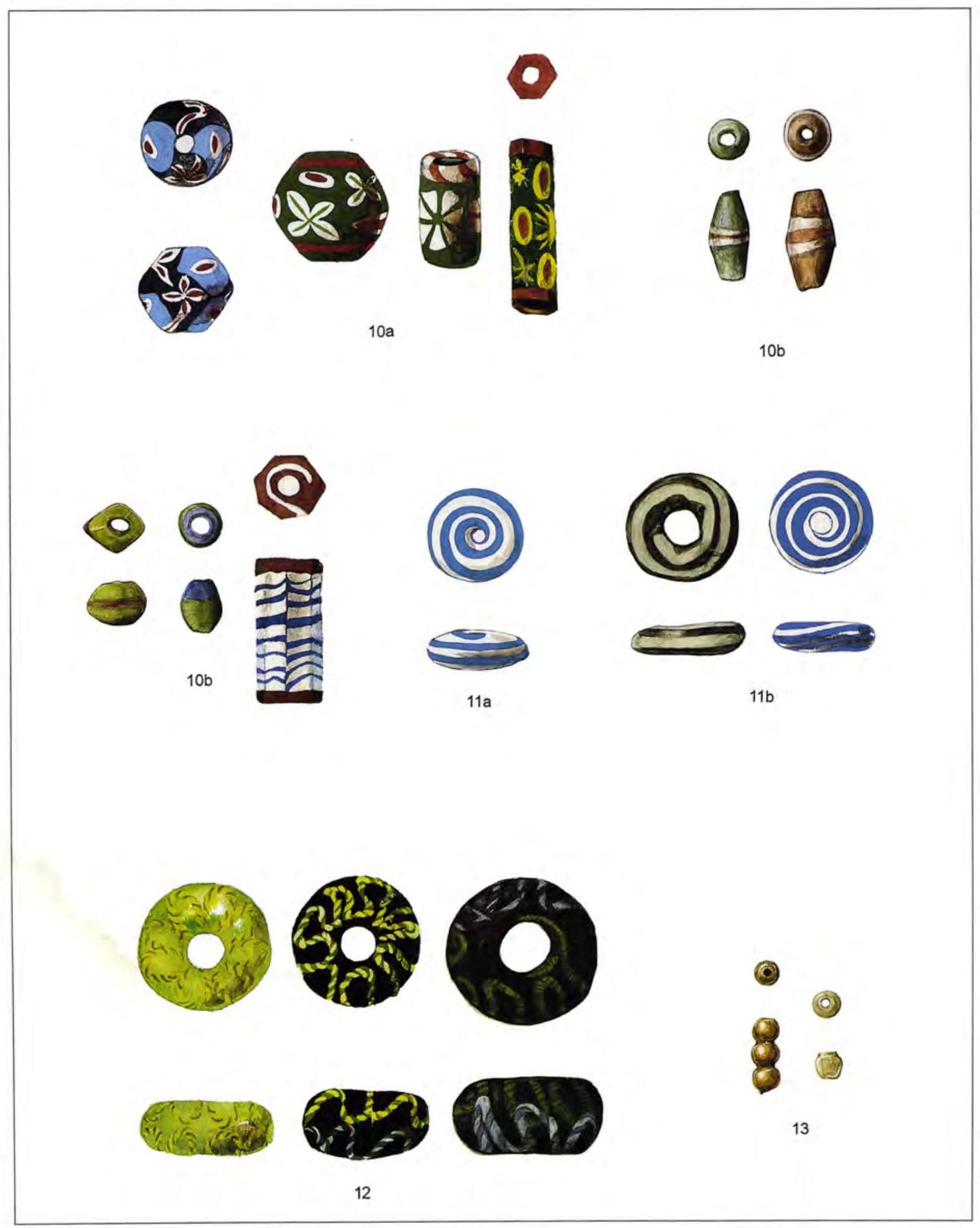

Plate 8 Millefiori and mosaic glass beads (Schedule 10); annular glass beads with coloured surface spirals (Schedule 11); annular glass beads with fine twist decoration (Schedule 12); gold and silver-in-glass beads (Schedule 13) 



\begin{tabular}{|c|c|c|c|c|}
\hline Ipswich & Suffolk & $\begin{array}{l}\text { prob } \\
6 \text { th- } \\
\text { 7th c }\end{array}$ & & $\begin{array}{l}\text { Layard 1907, pl XXXI, and } \\
\text { 1909; } \\
\text { Ipswich Museum }\end{array}$ \\
\hline West Stow & Suffolk & $\begin{array}{l}\text { 5th- } \\
7 \text { th } c\end{array}$ & $\begin{array}{l}\text { Cemetery: } 1 \text { small opaque; } \\
\text { and } 1 \text { other }\end{array}$ & $\begin{array}{l}\text { West 1985, 74; } \\
\text { Ashmolean Museum } \\
\text { 1909.419a; } \\
\text { Bury St Edmunds Museum }\end{array}$ \\
\hline Mitcham & Surrey & $\begin{array}{l}\text { prob } \\
\text { 6th } c\end{array}$ & $\begin{array}{l}\text { Grave 129: single; } \\
\text { Grave 62: double }\end{array}$ & $\begin{array}{l}\text { Bidder and Morris } 1959, \\
69,64,110-12, \\
\text { pl XVI.129 and } 62 ; \\
\text { Cambridge Museum }\end{array}$ \\
\hline $\begin{array}{l}\text { Apple } \\
\text { Down I }\end{array}$ & Sussex & $\begin{array}{l}\text { first } \\
\text { half } \\
\text { 6th c }\end{array}$ & $\begin{array}{l}\text { Grave 128: short cylinders } \\
\text { and barrel with button } \\
\text { brooch }\end{array}$ & $\begin{array}{l}\text { Down and Welch } 1990 \text {, } \\
51,163 \text {, fig 2.41; } \\
\text { Chichester Museum }\end{array}$ \\
\hline $\begin{array}{l}\text { Everthorpe } \\
\text { Hall }\end{array}$ & Yorks & & Necklace: 1 grass green & $\begin{array}{l}\text { Meaney 1964, 288; } \\
\text { Hull Museum }\end{array}$ \\
\hline $\begin{array}{l}\text { Painsthorpe } \\
\text { Wold I }\end{array}$ & Yorks & 7th $\mathrm{c}$ & $\begin{array}{l}\text { Group: } 5 \text { examples with } \\
\text { brooch, etc }\end{array}$ & $\begin{array}{l}\text { Mortimer } 1905 \text {, } \\
\text { pl XXXIV, fig } 278 \text {; } \\
\text { Hull Museum }\end{array}$ \\
\hline 'Yorks' & $\begin{array}{l}\text { Yorks } \\
\text { iv }\end{array}$ & EEN & RREL-SHAPED AND BICOI & Hull Museum \\
\hline $\begin{array}{l}\text { Chamber- } \\
\text { lain's } \\
\text { Barn II }\end{array}$ & Beds & $\begin{array}{l}\text { mid- } \\
\text { late } \\
7 \text { th c }\end{array}$ & $\begin{array}{l}\text { Grave 9: several opaque } \\
\text { barrels; } \\
\text { Grave } 57 \text { : opaque green } \\
\text { biconical }\end{array}$ & $\begin{array}{l}\text { Hyslop } 1963,173 \text { and } \\
187, \text { figs } 9 \mathrm{e}-\mathrm{g} \\
\text { and } 17 \mathrm{~b} ; \\
\text { Luton Museum }\end{array}$ \\
\hline $\begin{array}{l}\text { Little } \\
\text { Chester }\end{array}$ & Derbys & $\begin{array}{l}\text { 6th- } \\
7 \text { th } c\end{array}$ & $\begin{array}{l}\text { Three yellowish green } \\
\text { slightly biconical }\end{array}$ & $\begin{array}{l}\text { Wheeler } 1985,304 \text {; } \\
\text { publication in } \\
\text { preparation } \\
\text { (C Sparey-Green) }\end{array}$ \\
\hline Lechlade & Glos & $\begin{array}{l}\text { 7th } \mathrm{c} \\
7 \text { th } \mathrm{c}\end{array}$ & $\begin{array}{l}\text { Grave } 89 / 2: 1 \text { semi-opaque } \\
\text { bright green bicone; } \\
\text { Grave } 84: 2 \text { large bright } \\
\text { green-blue barrels with } \\
\text { gold pendant }\end{array}$ & $\begin{array}{l}\text { Boyle et al forthcoming; } \\
\text { Cirencester Museum }\end{array}$ \\
\hline Horndean & Hants & 7th $\mathrm{c}$ & $\begin{array}{l}\text { Grave S27: child with } \\
\text { bronze wire ring }\end{array}$ & $\begin{array}{l}\text { Knocker } 1957,132-3 \text {, } \\
136 \text {, fig 15.S27.2; } \\
\text { Portsmouth Museums }\end{array}$ \\
\hline $\begin{array}{l}\text { Breach } \\
\text { Down }\end{array}$ & Kent & $\begin{array}{l}\text { 7th- } \\
\text { 8th } c\end{array}$ & Several barrels or biconicals & $\begin{array}{l}\text { Conyngham and Akerman } \\
\text { 1844; BM 79 5-24 }\end{array}$ \\
\hline $\begin{array}{l}\text { Canterbury, } \\
\text { Cakebread } \\
\text { Robey V }\end{array}$ & Kent & $c .400$ & $\begin{array}{l}\text { From multiple burial: } \\
6 \text { biconicals of varying } \\
\text { sizes }\end{array}$ & $\begin{array}{l}\text { Information from } \mathrm{T} \\
\text { Tatton-Brown and P Garrard }\end{array}$ \\
\hline $\begin{array}{l}\text { Dover, } \\
\text { Buckland }\end{array}$ & Kent & $\begin{array}{l}575- \\
625\end{array}$ & Grave 30: 1 bicone (B33); & $\begin{array}{l}\text { Evison } 1987,73-4,80, \\
224,220,245 \text { and } 244,\end{array}$ \\
\hline
\end{tabular}




\begin{tabular}{|c|c|c|c|c|}
\hline & & $\begin{array}{l}625- \\
650 \\
650- \\
675\end{array}$ & $\begin{array}{l}\text { Grave 18: } 2 \text { green-blue } \\
\text { barrels (B41); } \\
\text { Grave 132: } 2 \text { green-blue } \\
\text { barrels (B41); } \\
\text { Grave 129: } 1 \text { bicone (B33) } \\
\text { and } 1 \text { dark green-blue (B44) }\end{array}$ & $\begin{array}{l}\text { figs } 18.4 \mathrm{~h}, 11.18 .2 \mathrm{~d} \text {, } \\
54.132 .2 \mathrm{c} \text { and } 53.5 \mathrm{e} \text { and } \mathrm{f} \\
\mathrm{BM}\end{array}$ \\
\hline Faversham & Kent & $\begin{array}{l}7 \text { th } c \\
5 \text { th- } \\
7 \text { th c }\end{array}$ & $\begin{array}{l}\text { Pale green barrel with } \\
\text { amethyst bead; } \\
\text { dark green biconical }\end{array}$ & $\begin{array}{l}\text { Smith } 1860 \text {; } \\
\text { Ashmolean Museum } \\
1909.163\end{array}$ \\
\hline Monkton & Kent & $\begin{array}{l}\text { mid- } \\
\text { 7th c }\end{array}$ & $\begin{array}{l}\text { Grave 3: short cylinder and } \\
\text { bicone with composite } \\
\text { disc brooch, amethyst, etc }\end{array}$ & $\begin{array}{l}\text { Hawkes and Hogarth 1974, } \\
59 \text {, fig } 5 \mathrm{~h} \text { and i; } \\
\text { Ashmolean Museum }\end{array}$ \\
\hline Polhill & Kent & 7th $c$ & $\begin{array}{l}\text { Grave } 37: 2 \text { bicones (Group } \\
517 \text { ) with keystone garnet } \\
\text { disc brooch, amethyst } \\
\text { bead, etc }\end{array}$ & $\begin{array}{l}\text { Hawkes and Philp 1973, } \\
176 \text { and 207, fig 55.517 }\end{array}$ \\
\hline Riby Park & Lincs & 7th $\mathrm{c}$ & $\begin{array}{l}\text { Two grass green biconicals } \\
\text { with } 7 \text { th }-c \text { pot, etc }\end{array}$ & $\begin{array}{l}\text { Meaney } 1964,161 \\
\text { Lincoln Museum }\end{array}$ \\
\hline West Stow & Suffolk & $\begin{array}{l}5 \text { th- } \\
\text { 7th } c\end{array}$ & $\begin{array}{l}\text { Cemetery: little biconicals } \\
\text { in light opaque green }\end{array}$ & $\begin{array}{l}\text { West } 1985,74 ; \\
\text { Bury St Edmunds Museum }\end{array}$ \\
\hline 'Yorks' & Yorks & & $\begin{array}{l}\text { One possible barrel, dark } \\
\text { green }\end{array}$ & Hull Museum \\
\hline $\begin{array}{l}\text { Dalmeny } \\
\text { Hound } \\
\text { Point }\end{array}$ & $\begin{array}{l}\text { Scotland: } \\
\text { West } \\
\text { Lothian }\end{array}$ & $\begin{array}{l}\text { prob } \\
\text { 7th c }\end{array}$ & $\begin{array}{l}\text { Cist: necklace with dark } \\
\text { green opaque biconical }\end{array}$ & $\begin{array}{l}\text { Brown 1915b; Brown } \\
\text { 1915a (IV), 812-13, fig 29; } \\
\text { Edinburgh Museum }\end{array}$ \\
\hline \multirow[t]{2}{*}{$\begin{array}{l}\text { Mochrum } \\
\text { Castle } \\
\text { Island }\end{array}$} & $\begin{array}{l}\text { Scotland: } \\
\text { Wigton }\end{array}$ & & Two blue-green biconicals & $\begin{array}{l}\text { Radford 1950,62, } \\
\text { nos } 3 \text { and 4; } \\
\text { c/o Lord David Stuart, } \\
\text { Port William, Wigton }\end{array}$ \\
\hline & $\begin{array}{l}\text { v Gre } \\
\text { (see Sched }\end{array}$ & $\begin{array}{l}\text { EN ME } \\
\text { dule } 1 \mathrm{i}\end{array}$ & $\begin{array}{l}\text { ON, SUB-MELON AND 'NI } \\
\text { for light blue-green trat } \\
\text { and sub-melon beads) }\end{array}$ & $\begin{array}{l}\text { KED' BEADS } \\
\text { slucent melon }\end{array}$ \\
\hline $\begin{array}{l}\text { Long } \\
\text { Wittenham }\end{array}$ & Berks & $\begin{array}{l}5 \text { th- } \\
\text { 6th } c\end{array}$ & $\begin{array}{l}\text { Grave } 52: 2 \text { opaque melons } \\
\text { with disc brooches; } \\
\text { Grave } 53\end{array}$ & $\begin{array}{l}\text { Akerman 1860; } \\
\text { BM }\end{array}$ \\
\hline Wallingford & Berks & $\begin{array}{l}5 \text { th- } \\
6 \text { th } c\end{array}$ & $\begin{array}{l}\text { Grave 11: } 2 \text { dark green } \\
\text { long sub-melons with } 2 \\
\text { small-long brooches }\end{array}$ & $\begin{array}{l}\text { Leeds } 1938,97, \mathrm{pl} \mathrm{III;} \\
\text { Ashmolean Museum }\end{array}$ \\
\hline $\begin{array}{l}\text { Norton- } \\
\text { on-Tees }\end{array}$ & Durham & 6th $c$ & $\begin{array}{l}\text { Grave } 7: 1 \text { sub-melon } \\
\text { opaque yellow/green bead } \\
\text { with wrist clasps, etc; } \\
\text { Grave } 87: 1 \text { sub-melon } \\
\text { blue-green bead }\end{array}$ & $\begin{array}{l}\text { Sherlock and Welch } 1992 \text {, } \\
125 \text { and } 181 \text {, figs } 34.7 .1 \text { and } \\
59.87 .1\end{array}$ \\
\hline
\end{tabular}




\begin{tabular}{|c|c|c|c|c|}
\hline $\begin{array}{l}\text { Great } \\
\text { Chesterford }\end{array}$ & Essex & 6th $c$ & $\begin{array}{l}\text { Grave 62: 4-lobed sub- } \\
\text { melon yellow and green } \\
\text { mixed glass (B17) with } \\
\text { small square-headed } \\
\text { brooch pair, etc }\end{array}$ & $\begin{array}{l}\text { Evison 1994, 14, 101, } \\
\text { fig 33.62.41; BM }\end{array}$ \\
\hline Mucking II & Essex & $\begin{array}{l}\text { 5th- } \\
6 \text { th c } \\
\text { 5th- } \\
\text { 6th c }\end{array}$ & $\begin{array}{l}\text { Grave } 648: 3 \text { flattish } \\
\text { notched with small-long } \\
\text { brooch pair, kidney-shaped } \\
\text { buckle loop, etc; } \\
\text { Grave } 845: 1 \text { green-yellow } \\
\text { translucent with disc } \\
\text { brooch pair; } \\
\text { Grave 860: blue-green with } \\
\text { small-long brooch pair, etc }\end{array}$ & $\begin{array}{l}\text { Hirst and Clark forthcoming; } \\
\mathrm{BM}\end{array}$ \\
\hline $\begin{array}{l}\text { Beakes- } \\
\text { bourne }\end{array}$ & Kent & $\begin{array}{l}\text { 6th- } \\
7 \text { th c }\end{array}$ & $\begin{array}{l}\text { Two opaque green } \\
\text { sub-melons }\end{array}$ & $\begin{array}{l}\text { Meaney 1964, 109; } \\
\text { Canterbury Museum }\end{array}$ \\
\hline $\begin{array}{l}\text { Dover, } \\
\text { Buckland }\end{array}$ & Kent & $\begin{array}{l}650- \\
675 \\
675- \\
750\end{array}$ & $\begin{array}{l}\text { Grave 129: } 1 \text { dark green- } \\
\text { blue (B43); } \\
\text { Grave 141: } 1 \text { dark green- } \\
\text { blue (B43) }\end{array}$ & $\begin{array}{l}\text { Evison } 1987,74,80 \text {, } \\
244,248, \text { figs } 53.129 .5 \mathrm{~d}, \\
58.141 .6 \mathrm{~b} \text {; } \\
\text { BM }\end{array}$ \\
\hline Quarrington & Lincs & & $\begin{array}{l}\text { Rather long and badly made } \\
\text { sub-melon }\end{array}$ & $\begin{array}{l}\text { Bruce } 1880,76, \text { no. } 303 \text {; } \\
\text { Alnwick Museum }\end{array}$ \\
\hline Ruskington & Lincs & $\begin{array}{l}\text { prob } \\
\text { 6th } c\end{array}$ & One grass green sub-melon & $\begin{array}{l}\text { Meaney 1964, 161-2; } \\
\text { Lincoln Museum }\end{array}$ \\
\hline Illington & Norfolk & 6th $c$ & $\begin{array}{l}\text { Urn 221: } 1 \text { large } \\
\text { translucent green in } \\
\text { linear-decorated pot }\end{array}$ & $\begin{array}{l}\text { Davison et al } 1993 \text {, } \\
\text { 37, fig } 47.221 \mathrm{a} ; \\
\text { Norwich Museum }\end{array}$ \\
\hline $\begin{array}{l}\text { Morning } \\
\text { Thorpe }\end{array}$ & Norfolk & $\begin{array}{l}\text { mid- } \\
6 \text { th c } \\
\text { mid- } \\
6 \text { th } c \\
\text { late } \\
5 \text { th- } \\
6 \text { th c } \\
\text { prob } \\
6 \text { th c }\end{array}$ & $\begin{array}{l}\text { Grave 133: sub-melon with } \\
\text { Åberg Group IV cruciform } \\
\text { brooch, etc; } \\
\text { Grave 358: sub-melon with } \\
\text { Aberg Group IV cruciform } \\
\text { brooch, etc; } \\
\text { Grave 362: } 2 \text { sub-melons } \\
\text { with Åberg Group II or III, } \\
\text { etc; } \\
\text { Grave 309: } 1 \text { sub-melon; } \\
\text { and possibly others }\end{array}$ & $\begin{array}{l}\text { Green et al } 1987, \\
73,138,140 \text { and } 120 \text {, } \\
\text { figs } 341 . \text { Hiv, } 417 . \mathrm{Dv} \text {, } \\
424 . \mathrm{Lv} \text { and } 395 . \mathrm{Avi} \text {; } \\
\text { Norwich Museum }\end{array}$ \\
\hline 'Norfolk' & Norfolk & & $\begin{array}{l}\text { Green opaque with } \\
7 \text { segments }\end{array}$ & Norwich Museum \\
\hline Spong Hill & Norfolk & $\begin{array}{l}\text { mid- } \\
\text { 6th } c\end{array}$ & $\begin{array}{l}\text { Grave } 38: 2 \text { small sub- } \\
\text { melons with square- } \\
\text { headed brooch, etc; } \\
\text { Grave } 39: 4 \text { sub-melons } \\
\text { with Åberg Group IVa } \\
\text { cruciform brooch, etc }\end{array}$ & $\begin{array}{l}\text { Hills et al } 1984, \\
90 \text { and } 90-1 \text {, } \\
\text { figs } 92.10 \text { a and } 94.5 ; \\
\text { Norfolk Arch Unit }\end{array}$ \\
\hline
\end{tabular}




\begin{tabular}{|c|c|c|c|c|}
\hline Cassington & Oxon & & $\begin{array}{l}\text { Disturbed grave: } \\
1 \text { greenish sub-melon }\end{array}$ & $\begin{array}{l}\text { Leeds and Riley } 1942,64, \\
\text { pl VIB; } \\
\text { Ashmolean Museum }\end{array}$ \\
\hline $\begin{array}{l}\text { Empingham } \\
\text { II }\end{array}$ & Rutland & $\begin{array}{l}\text { 5th- } \\
7 \text { th c }\end{array}$ & $\begin{array}{l}\text { Grave 73: } 1 \text { sub-melon, } \\
\text { bottle-green }\end{array}$ & $\begin{array}{l}\text { Timby 1996; } \\
\text { Oakham Museum }\end{array}$ \\
\hline Camerton & Somerset & 7th $\mathrm{c}$ & $\begin{array}{l}\text { Grave 97: well-made light } \\
\text { green melon }\end{array}$ & $\begin{array}{l}\text { Horne } 1933,46,60-1 \text {; } \\
\text { Taunton Museum }\end{array}$ \\
\hline $\begin{array}{l}\text { Bury St } \\
\text { Edmunds, } \\
\text { Westgarth } \\
\text { Gardens }\end{array}$ & Suffolk & $\begin{array}{l}\text { 5th- } \\
\text { 6th } c\end{array}$ & $\begin{array}{l}\text { Grave } 48: 1 \text { small sub- } \\
\text { melon with } 2 \text { small-long } \\
\text { brooches }\end{array}$ & $\begin{array}{l}\text { West 1988, 32, fig 73; } \\
\text { Bury St Edmunds } \\
\text { Museum }\end{array}$ \\
\hline Wasperton & Warks & $\begin{array}{l}\text { mid- } \\
\text { late } \\
\text { 6th c }\end{array}$ & One long sub-melon & $\begin{array}{l}\text { Publication in } \\
\text { preparation (M Carver); } \\
\text { Warwick Museum }\end{array}$ \\
\hline Saltburn & Yorks & $\begin{array}{l}6 \text { th- } \\
7 \text { th c }\end{array}$ & $\begin{array}{l}\text { One green translucent } \\
\text { 5-lobed sub-melon }\end{array}$ & $\begin{array}{l}\text { Gallagher } 1987,16,25 \text {, } \\
\text { fig } 5.38 \text {; } \\
\text { Middlesbrough Museum }\end{array}$ \\
\hline 'Yorks' & Yorks & & $\begin{array}{l}\text { Two dark translucent sub- } \\
\text { melons }\end{array}$ & York Museum \\
\hline
\end{tabular}

\section{vi Green Segmented or wound beads (see Map 17)}

\begin{tabular}{|c|c|c|c|c|}
\hline Mucking II & Essex & $\begin{array}{l}5 \text { th- } \\
6 \text { th c }\end{array}$ & $\begin{array}{l}\text { Grave 860: } 2 \text { segments pale } \\
\text { blue-green with small- } \\
\text { long brooch pair, etc }\end{array}$ & $\begin{array}{l}\text { Hirst and Clark forthcomin } \\
\text { BM }\end{array}$ \\
\hline Lechlade & Glos & 6th c & $\begin{array}{l}\text { Grave 190: bright blue- } \\
\text { green miniature segmented } \\
\text { and annular }\end{array}$ & $\begin{array}{l}\text { Boyle et al forthcoming; } \\
\text { Cirencester Museum }\end{array}$ \\
\hline $\begin{array}{l}\text { Dover, } \\
\text { Buckland }\end{array}$ & Kent & $\begin{array}{l}475- \\
525 \\
575 \\
625\end{array}$ & $\begin{array}{l}\text { Grave 46: } 9 \text { doubles, } \\
\text { blue-green (B35) and } 14 \\
\text { globulars (C04); } \\
\text { Grave 1: } 12 \text { doubles } \\
\text { (B35), } 3 \text { triples (Plate 4), } \\
\text { blue-green (B36) and } \\
\text { 13 globulars (C04); } \\
\text { Grave 29: } 2 \text { globulars } \\
\text { (C04); } \\
\text { Grave 32: } 10 \text { doubles } \\
\text { (B35), } 1 \text { triple, blue-green } \\
\text { (B36) and } 24 \text { globulars } \\
\text { (C04); } \\
\text { Grave 35: } 22 \text { globulars } \\
\text { (C04); } \\
\text { Grave 6: globular (C04) }\end{array}$ & $\begin{array}{l}\text { Evison } 1987,73,75,80-1 \text {, } \\
216,217,223-4,225,226 \\
\text { and } 229, \text { figs } 5.41, \mathrm{~m} \text { and } \mathrm{s} \text {, } \\
7.10 \mathrm{~g}, 19.4 \mathrm{f}, \mathrm{g} \text { and } \mathrm{i} \text {, } \\
21.5 \mathrm{i}, 26.46 .3 \mathrm{e} \text { and } \mathrm{f} \\
\mathrm{BM}\end{array}$ \\
\hline
\end{tabular}

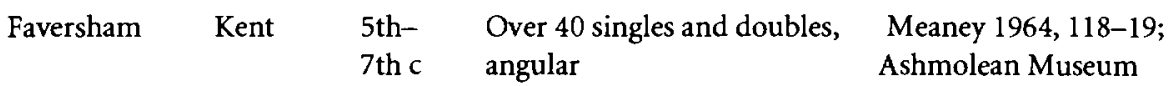




\begin{tabular}{|c|c|c|c|c|}
\hline $\begin{array}{l}\text { Faversham, } \\
\text { King's Field }\end{array}$ & Kent & $\begin{array}{l}5 \text { th- } \\
7 \text { th c }\end{array}$ & 16 examples, angular & $\begin{array}{l}\text { Meaney } 1964,118-19 \\
\text { Rochester Museum }\end{array}$ \\
\hline Folkestone & Kent & $\begin{array}{l}\text { prob } \\
\text { 6th } c\end{array}$ & $\begin{array}{ll}\text { No. } 4 & \text { \} all } \\
\text { No. } 11 & \text { \} angular, } \\
\text { No. } 19 & \text { \} over } \\
\text { No. } 27 & 40 \\
\text { No. } 32 & \text { J examples; } \\
\text { No. } 32: \text { some double; } \\
\text { others }\end{array}$ & $\begin{array}{l}\text { Meaney } 1964,120-1 ; \\
\text { Folkestone Museum; } \\
\text { Maidstone Museum }\end{array}$ \\
\hline 'Kent' & Kent & $\begin{array}{l}\text { prob } \\
6 \text { th- } \\
7 \text { th c }\end{array}$ & $\begin{array}{l}\text { A number of small } \\
\text { single segments and } \\
1 \text { double-segmented }\end{array}$ & Canterbury Museum \\
\hline Stowting & Kent & $\begin{array}{l}\text { prob } \\
6 \text { th c }\end{array}$ & $\begin{array}{l}\text { Necklace: } 2 \text { or } 3 \text { opaque } \\
\text { bottle green }\end{array}$ & $\begin{array}{l}\text { Meaney } 1964,137-8 \\
\text { Maidstone Museum }\end{array}$ \\
\hline Westbere & Kent & $\begin{array}{l}\text { 6th- } \\
7 \text { th c }\end{array}$ & $\begin{array}{l}\text { Several opaque grass } \\
\text { green singles, perhaps } \\
\text { once joined, angular }\end{array}$ & $\begin{array}{l}\text { Jessup 1946; } \\
\text { Canterbury Museum }\end{array}$ \\
\hline Fonaby & Lincs & $\begin{array}{l}\text { 6th- } \\
7 \text { th c }\end{array}$ & $\begin{array}{l}\text { Much larger than usual, } \\
\text { 2-segmented }\end{array}$ & $\begin{array}{l}\text { Cook 1981; } \\
\text { Scunthorpe Museum }\end{array}$ \\
\hline Brixworth & Northants & $\begin{array}{l}\text { prob } \\
6 \text { th c }\end{array}$ & $\begin{array}{l}\text { One translucent } \\
\text { 2-segmented }\end{array}$ & $\begin{array}{l}\text { Smith } 1902,245 ; \\
\text { Northampton Museum }\end{array}$ \\
\hline Yeavering & $\begin{array}{l}\text { North- } \\
\text { umber- } \\
\text { land }\end{array}$ & $\begin{array}{l}\text { prob } \\
\text { mid- } \\
6 \text { th- } \\
7 \text { th c }\end{array}$ & $\begin{array}{l}\text { Settlement: } 2 \text {-segmented } \\
\text { (unbroken) translucent } \\
\text { blue-green and 4-segmented } \\
\text { (broken) semi-translucent }\end{array}$ & Hope-Taylor 1977 \\
\hline $\begin{array}{l}\text { Standlake } \\
\text { Down }\end{array}$ & Oxon & $\begin{array}{l}\text { early } \\
7 \text { th c }\end{array}$ & $\begin{array}{l}\text { Grave 24: double-segment } \\
\text { greenish-yellow, angular }\end{array}$ & $\begin{array}{l}\text { Dickinson 1973, 244-5, } \\
\text { fig 5.1.l; } \\
\text { Ashmolean Museum }\end{array}$ \\
\hline Brandon & Suffolk & $\begin{array}{l}\text { mid- } \\
7 \text { th- } \\
9 \text { th c }\end{array}$ & $\begin{array}{l}\text { Cemetery and settlement: } \\
\text { miscellaneous find of } \\
\text { small triple bead }\end{array}$ & $\begin{array}{l}\text { Carr et al 1988; } \\
\text { publication in } \\
\text { preparation ( } \mathrm{R} \text { Carr) }\end{array}$ \\
\hline West Stow & Suffolk & $\begin{array}{l}5 \text { th- } \\
7 \text { th c }\end{array}$ & $\begin{array}{l}\text { Cemetery: } 1 \text { double- } \\
\text { segmented (as Faversham } \\
\text { necklace above) }\end{array}$ & $\begin{array}{l}\text { West } 1985,74, \\
\text { fig } 275.30 ; \\
\text { Bury St Edmunds Museum }\end{array}$ \\
\hline Alfriston & Sussex & $\begin{array}{l}5 \text { th- } \\
6 \text { th c }\end{array}$ & $\begin{array}{l}\text { Grave } 68 \\
\text { Grave } 79\end{array}$ & $\begin{array}{l}\text { Griffith and Salzmann } 1914 ; \\
\text { Welch } 1983,84 ; \\
\text { Lewes Museum }\end{array}$ \\
\hline $\begin{array}{l}\text { Apple } \\
\text { Down I }\end{array}$ & Sussex & $\begin{array}{l}\text { first } \\
\text { half } \\
\text { 6th c } \\
\text { first } \\
\text { half } \\
\text { 6th c }\end{array}$ & $\begin{array}{l}\text { Grave 10: } 1 \text { segment only } \\
\text { opaque green with cast } \\
\text { saucer brooch pair, etc; } \\
\text { Grave } 128: \text { a number of } \\
\text { single and double segments } \\
\text { with button brooch }\end{array}$ & $\begin{array}{l}\text { Down and Welch } 1990 \text {, } \\
35,51 \text { and } 162-3 \text {, } \\
\text { fig } 2.17 \text { and } 2.40-41 \text {; } \\
\text { Chichester Museum }\end{array}$ \\
\hline Highdown & Sussex & $\begin{array}{l}\text { prob } \\
6 \text { th c }\end{array}$ & $\begin{array}{l}\text { Approx } 30 \text { small emerald } \\
\text { green, some quadruple }\end{array}$ & $\begin{array}{l}\text { Read } 1895 \text { and } 1896 ; \\
\text { Welch } 1983,84 ; \\
\text { Worthing Museum }\end{array}$ \\
\hline
\end{tabular}




$\begin{array}{lllll}\begin{array}{l}\text { Colling- } \\ \text { bourne Ducis }\end{array} & \text { Wilts } & \begin{array}{l}\text { first } \\ \text { half } \\ \text { 6th c }\end{array} & \begin{array}{l}\text { Grave 31: several very } \\ \text { small emerald green with } \\ \text { button brooch pair, etc }\end{array} & \begin{array}{l}\text { Gingell 1975, 90, fig 20; } \\ \text { Devizes Museum }\end{array} \\ \begin{array}{l}\text { Harnham } \\ \text { Hill }\end{array} & \text { Wilts } & \begin{array}{l}\text { prob } \\ \text { 6th c }\end{array} & \begin{array}{l}\text { One small translucent blue- } \\ \text { green 2-segmented }\end{array} & \begin{array}{l}\text { Akerman 1853; } \\ \text { Jackson 1854 }\end{array} \\ \begin{array}{l}\text { Lydiard } \\ \text { Tregoze }\end{array} & \text { Wilts } & \begin{array}{l}\text { prob } \\ \text { 6th c }\end{array} & \begin{array}{l}\text { Odd segments, very small } \\ \text { with saucer brooches }\end{array} & \begin{array}{l}\text { Goddard 1895; } \\ \text { Devizes Museum; } \\ \text { BM 53 12-14 30 }\end{array} \\ \begin{array}{l}\text { Woodyates, } \\ \text { Oakley Down }\end{array} & \text { Wilts } & \text { prob } & \begin{array}{l}\text { Barrow 1: 1 triple and 10 } \\ \text { possible segments, all } \\ \text { opaque }\end{array} & \text { Devizes Museum } \\ \begin{array}{l}\text { West } \\ \text { Heslerton }\end{array} & \text { Yorks } & \text { 6th c } & \begin{array}{l}\text { Grave 1 HE 20: 17 } \\ \text { 2-segmented and 7 }\end{array} & \begin{array}{l}\text { Publication in } \\ \text { preparation (D Powlesland) }\end{array} \\ & & & \begin{array}{l}\text { 3-segmented, all } \\ \text { translucent }\end{array} & \end{array}$

\section{POLYCHROME}

vii Green beads With Crossed Waves, With or Without dots (OPAQUE OR TRANSLUCENT), SINGLES OR DOUBLES

\begin{tabular}{|c|c|c|c|c|}
\hline $\begin{array}{l}\text { Hasling- } \\
\text { field }\end{array}$ & Cambs & $\begin{array}{l}\text { prob } \\
\text { 6th c }\end{array}$ & $\begin{array}{l}\text { Small translucent, waves } \\
\text { in yellow and dots }\end{array}$ & $\begin{array}{l}\text { Fox 1923, 255-9; } \\
\text { Ashmolean Museum } \\
1909.233\end{array}$ \\
\hline $\begin{array}{l}\text { Springfield } \\
\text { Lyons }\end{array}$ & Essex & & $\begin{array}{l}\text { Grave 4752: rather barrel- } \\
\text { shaped with terracotta } \\
\text { waves and yellow dots; } \\
\text { another pale green with } \\
\text { white waves and red dots; } \\
\text { Grave } 4758 \text { : annular } \\
\text { opaque white crossed } \\
\text { waves and } 3 \text { red dots }\end{array}$ & $\begin{array}{l}\text { Publication in } \\
\text { preparation (S Tyler); } \\
\text { BM }\end{array}$ \\
\hline $\begin{array}{l}\text { Dover, } \\
\text { Buckland }\end{array}$ & Kent & $\begin{array}{l}575- \\
625\end{array}$ & $\begin{array}{l}\text { Grave } 35 \text { : barrel-shaped and } \\
\text { yellow waves (D50) }\end{array}$ & $\begin{array}{l}\text { Evison } 1987,77,82, \\
226, \text { fig } 21.5 \mathrm{k}, \\
\text { colour pl IV (D50); BM }\end{array}$ \\
\hline 'Kent' & Kent & & $\begin{array}{l}\text { Two beads with terracotta } \\
\text { waves and yellow dots }\end{array}$ & Canterbury Museum \\
\hline $\begin{array}{l}\text { Lyminge } \\
\text { II }\end{array}$ & Kent & 6th $c$ & $\begin{array}{l}\text { Grave 24: terracotta waves } \\
\text { (Plate 4) with equal-arm } \\
\text { brooch pair, etc }\end{array}$ & $\begin{array}{l}\text { Warhurst } 1955,18, \\
\text { pl VIIb, no. } 1\end{array}$ \\
\hline Fonaby & Lincs & 6th $\mathrm{c}$ & $\begin{array}{l}\text { Grave 25: terracotta wave } \\
\text { and yellow dots (Plate 4) } \\
\text { with girdle-hangers }\end{array}$ & $\begin{array}{l}\text { Cook } 1981,28 \text {, } \\
\text { fig } 9.1 .5 ; \\
\text { Scunthorpe Museum }\end{array}$ \\
\hline Ruskington & Lincs & $\begin{array}{l}\text { prob } \\
\text { 6th- } \\
\text { 7th c }\end{array}$ & $\begin{array}{l}\text { Cylindrical, yellow waves } \\
\text { and dots }\end{array}$ & $\begin{array}{l}\text { Meaney 1964, 161-2; } \\
\text { Lincoln Museum }\end{array}$ \\
\hline
\end{tabular}




\begin{tabular}{|c|c|c|c|c|}
\hline Brooke & Norfolk & $\begin{array}{l}\text { prob } \\
\text { 6th c }\end{array}$ & $\begin{array}{l}\text { Light translucent with } \\
\text { terracotta waves }\end{array}$ & $\begin{array}{l}\text { Meaney 1964, 170; } \\
\text { BM 1866-69 }\end{array}$ \\
\hline $\begin{array}{l}\text { Morning } \\
\text { Thorpe }\end{array}$ & Norfolk & $\begin{array}{l}\text { 6th- } \\
7 \text { th } c\end{array}$ & $\begin{array}{l}\text { Several with white or } \\
\text { terracotta waves and spots; } \\
\text { eg, Grave } 30: 3 \text { beads }\end{array}$ & $\begin{array}{l}\text { Green et al 1987, eg, } \\
\text { 42-3, fig 303.30.Dix; } \\
\text { Norwich Museum }\end{array}$ \\
\hline Spong Hill & Norfolk & $\begin{array}{l}\text { mid- } \\
6 \text { th c } \\
\text { late } \\
\text { 5th- } \\
\text { early } \\
6 \text { th c } \\
5 \text { th- } \\
7 \text { th c }\end{array}$ & $\begin{array}{l}\text { Grave 39: terracotta waves } \\
\text { with Aberg Group IVa } \\
\text { cruciform brooch, etc; } \\
\text { Grave } 46 \text { : yellow waves and } \\
\text { terracotta dots with Åberg } \\
\text { Group II cruciform and } \\
\text { equal-arm brooches, etc; } \\
\text { Cremation 1653: white } \\
\text { waves and dots }\end{array}$ & $\begin{array}{l}\text { Hills et al } 1984 \text {, } \\
90-1 \text { and } 100 \text {, figs } 94 \\
\text { and 102.46; }\end{array}$ \\
\hline Duston & Northants & $\begin{array}{l}\text { prob } \\
\text { 6th- } \\
\text { 7th c }\end{array}$ & White waves and yellow & $\begin{array}{l}\text { George 1903; } \\
\text { Northampton Museum }\end{array}$ \\
\hline West Stow & Suffolk & $\begin{array}{l}5 \text { th- } \\
7 \text { th c }\end{array}$ & $\begin{array}{l}\text { Cemetery: white waves } \\
1909.419 a\end{array}$ & $\begin{array}{l}\text { West } 1985,74-5 ; \\
\text { Ashmolean Museum }\end{array}$ \\
\hline $\begin{array}{l}\text { Apple } \\
\text { Down I }\end{array}$ & Sussex & $\begin{array}{l}\text { prob } \\
\text { 6th c }\end{array}$ & $\begin{array}{l}\text { Grave 117: double bead, } \\
\text { blue waves on pale green; } \\
\text { Grave } 134: 2 \text { light green } \\
\text { with blue }\end{array}$ & $\begin{array}{l}\text { Down and Welch } 1990 \text {, } \\
49 \text {, fig 2.36-37, and } \\
\text { 51, fig 2.42-43; } \\
\text { Chichester Museum }\end{array}$ \\
\hline
\end{tabular}

viii GREEN OPAQUE OR TRANSLUCENT BEADS: VARIOUS SHAPES WITH WAVES OR ZIGZAGS (EXCLUDING CROSSED WAVES)

\begin{tabular}{|c|c|c|c|c|}
\hline $\begin{array}{l}\text { Chamber- } \\
\text { lain's } \\
\text { Barn II }\end{array}$ & Beds & $\begin{array}{l}\text { mid- } \\
\text { late } \\
7 \text { th c }\end{array}$ & $\begin{array}{l}\text { Grave 8: slightly biconical } \\
\text { translucent light green with } \\
\text { yellow zigzags }\end{array}$ & $\begin{array}{l}\text { Hyslop 1963, 173, } \\
\text { fig 8c; } \\
\text { Luton Museum }\end{array}$ \\
\hline Harwell & Berks & $\begin{array}{l}\text { 5th- } \\
6 \text { th } c\end{array}$ & $\begin{array}{l}\text { Grave 4: necklace with } \\
4 \text { very dark green or black } \\
\text { globular with white wave } \\
\text { with disc brooch pair }\end{array}$ & $\begin{array}{l}\text { Kirk and Marshall 1956, } \\
27,33-4, \\
\text { fig 10h.3-6, pl IB; } \\
\text { Ashmolean Museum }\end{array}$ \\
\hline $\begin{array}{l}\text { Hasling- } \\
\text { field }\end{array}$ & Cambs & $\begin{array}{l}\text { prob } \\
\text { 6th- } \\
7 \text { th c }\end{array}$ & $\begin{array}{l}\text { Globular pale opaque green } \\
\text { with grass green waves; } \\
\text { thick annular with white } \\
\text { wave and red streak }\end{array}$ & $\begin{array}{l}\text { Fox } 1923,255-9 ; \\
\text { BM: no number; } \\
\text { Hull Museum }\end{array}$ \\
\hline $\begin{array}{l}\text { Linton } \\
\text { Heath B }\end{array}$ & Cambs & $\begin{array}{l}\text { 5th- } \\
\text { 6th } c\end{array}$ & $\begin{array}{l}\text { Two translucent globulars, } \\
\text { grass green with opaque } \\
\text { yellow waves with } 2 \text { small- } \\
\text { long brooches }\end{array}$ & $\begin{array}{l}\text { Neville 1854; } \\
\text { Cambridge Museum }\end{array}$ \\
\hline Mucking II & Essex & $\begin{array}{l}\text { 5th- } \\
\text { 6th } c\end{array}$ & $\begin{array}{l}\text { Grave } 845: 2 \text { globulars in } \\
\text { dark green with cream } \\
\text { waves with disc brooch pair }\end{array}$ & $\begin{array}{l}\text { Hirst and Clark forthcoming; } \\
\text { BM }\end{array}$ \\
\hline Alton & Hants & $\begin{array}{l}\text { first } \\
\text { half } \\
7 \text { th c }\end{array}$ & $\begin{array}{l}\text { Grave 39: annular olive } \\
\text { green translucent with } \\
\text { yellow zigzag }\end{array}$ & $\begin{array}{l}\text { Evison 1988a, 80-1, } \\
\text { fig 33.39.1g; } \\
\text { Hampshire Museums }\end{array}$ \\
\hline
\end{tabular}




\begin{tabular}{|c|c|c|c|c|}
\hline Droxford & Hants & $\begin{array}{l}\text { late } \\
5 \text { th- } \\
\text { 6th c }\end{array}$ & $\begin{array}{l}\text { Grave 20: cube, translucent } \\
\text { yellow trails (class VI) } \\
\text { (not seen) }\end{array}$ & $\begin{array}{l}\text { Aldsworth } 1978,124 \text {, } \\
172, \text { fig } 24.13 ; \\
\text { Winchester Museum }\end{array}$ \\
\hline Faversham & Kent & $\begin{array}{l}5 \text { th- } \\
7 \text { th c }\end{array}$ & $\begin{array}{l}\text { Translucent greenish annular } \\
\text { with white waves overlaid } \\
\text { by green band; } \\
\text { short cylindrical translucent } \\
\text { greenish with heavy yellow } \\
\text { zigzags }\end{array}$ & $\begin{array}{l}\text { Meaney 1964, 118-19; } \\
\text { Ashmolean Museum } \\
1909.169\end{array}$ \\
\hline $\begin{array}{l}\text { Horton } \\
\text { Kirby I }\end{array}$ & Kent & & $\begin{array}{l}\text { Square-sectioned cylinder } \\
\text { with yellow wave on } 2 \\
\text { facets and red on others }\end{array}$ & $\begin{array}{l}\text { Meaney } 1964,124 ; \\
\text { Maidstone Museum }\end{array}$ \\
\hline Sarre & Kent & 7 th $c$ & $\begin{array}{l}\text { ?Dark green opaque with } \\
\text { yellow wave and line }\end{array}$ & Brent $1868, \mathrm{pl}$ VIII \\
\hline Riby Park & Lincs & 7th $c$ & $\begin{array}{l}\text { Translucent blue-green } \\
\text { annular with white wave } \\
\text { (Plate 5) }\end{array}$ & $\begin{array}{l}\text { Phillips } 1934,145 \text { and } 148 ; \\
\text { Myres } 1951,88 \text { and } 98 ; \\
\text { Lincoln Museum }\end{array}$ \\
\hline $\begin{array}{l}\text { Morning } \\
\text { Thorpe }\end{array}$ & Norfolk & $\begin{array}{l}\text { prob } \\
\text { 6th- } \\
7 \text { th c }\end{array}$ & $\begin{array}{l}\text { Grave 309: } 2 \text { annulars with } \\
\text { yellow zigzags } \\
\text { (Plate 5) }\end{array}$ & $\begin{array}{l}\text { Green et al 1987, 120, } \\
\text { fig 396.Aviii; } \\
\text { Norwich Museum }\end{array}$ \\
\hline $\begin{array}{l}\text { Cadbury- } \\
\text { Congresbury }\end{array}$ & Somerset & $\begin{array}{l}5 \text { th- } \\
6 \text { th } c\end{array}$ & $\begin{array}{l}\text { Atypical squat, straight- } \\
\text { sided pale blue-green with } \\
\text { yellow wave and girth band: } \\
\text { Anglo-Saxon period, but } \\
\text { not Anglo-Saxon in } \\
\text { cultural terms }\end{array}$ & $\begin{array}{l}\text { Rahtz et al 1992, } \\
143-4 \text {, fig } 99\end{array}$ \\
\hline Alfriston & Sussex & $\begin{array}{l}5 \text { th- } \\
6 \text { th } c\end{array}$ & $\begin{array}{l}\text { Grave 51: annular very } \\
\text { dark with white waves }\end{array}$ & $\begin{array}{l}\text { Griffith and Salzmann } 1914 \\
\text { 42; Welch 1983, 84; } \\
\text { Lewes Museum }\end{array}$ \\
\hline $\begin{array}{l}\text { Apple } \\
\text { Down I }\end{array}$ & Sussex & $\begin{array}{l}\text { prob } \\
\text { 6th c }\end{array}$ & $\begin{array}{l}\text { Grave 107: rather drum- } \\
\text { shaped, dark green with } \\
\text { yellow band at top and } \\
\text { bottom and zigzag between } \\
\text { with another bead, more } \\
\text { translucent in blue with } \\
\text { same pattern }\end{array}$ & $\begin{array}{l}\text { Down and Welch } 1990 \text {, } \\
48,165, \text { fig } 2.35 \\
\text { colour pl 40.9; } \\
\text { Chichester Museum }\end{array}$ \\
\hline \multirow[t]{2}{*}{ Sewerby } & Yorks & $\begin{array}{l}\text { mid- } \\
\text { 6th } c\end{array}$ & $\begin{array}{l}\text { Grave 8: large annular with } \\
\text { white (C2a) with Åberg } \\
\text { Group IVa cruciform } \\
\text { brooch, etc }\end{array}$ & $\begin{array}{l}\text { Hirst } 1985,68 \text {, } \\
\text { fig } 34.6-65 \mathrm{~h}\end{array}$ \\
\hline & $\begin{array}{c}\mathrm{ix} 1 \\
(\mathrm{EXCLU}\end{array}$ & \multicolumn{3}{|c|}{$\begin{array}{l}\text { MISCELLANEOUS GREEN DECORATED BEADS } \\
\text { UDING CROSSED WAVES, WAVES AND ZIGZAGS) }\end{array}$} \\
\hline Wallingford & Berks & $\begin{array}{l}5 \text { th- } \\
6 \text { th } c\end{array}$ & $\begin{array}{l}\text { Grave 11: interrupted line } \\
\text { in white like black egs }\end{array}$ & $\begin{array}{l}\text { Leeds } 1938,97, \mathrm{pl} \mathrm{III;} \\
\text { Ashmolean Museum }\end{array}$ \\
\hline $\begin{array}{l}\text { Linton } \\
\text { Heath B }\end{array}$ & Cambs & $\begin{array}{l}\text { prob } \\
\text { 6th c }\end{array}$ & $\begin{array}{l}\text { dark ?green ground and } \\
\text { crossed bands of yellow } \\
\text { and green twists }\end{array}$ & $\begin{array}{l}\text { Neville 1854; } \\
\text { Cambridge Museum }\end{array}$ \\
\hline
\end{tabular}




\begin{tabular}{|c|c|c|c|c|}
\hline $\begin{array}{l}\text { Little } \\
\text { Wilbraham }\end{array}$ & Cambs & $\begin{array}{l}\text { prob } \\
\text { 6th } c\end{array}$ & $\begin{array}{l}\text { Two beads similar to } \\
\text { Linton Heath above }\end{array}$ & $\begin{array}{l}\text { Lethbridge and Carter } \\
\text { 1926; see also Neville 1852; } \\
\text { Cambridge Museum }\end{array}$ \\
\hline $\begin{array}{l}\text { Little } \\
\text { Chester }\end{array}$ & Derbys & & $\begin{array}{l}\text { Grave: with group of } 5 \\
\text { long cylinders with cable } \\
\text { of green and yellow } \\
\text { decoration on light green, } \\
\text { ?imitation of millefiori }\end{array}$ & $\begin{array}{l}\text { Wheeler 1985, 304; } \\
\text { publication in } \\
\text { preparation } \\
\text { (C Sparey-Green) }\end{array}$ \\
\hline $\begin{array}{l}\text { Norton- } \\
\text { on-Tees }\end{array}$ & Durham & $\begin{array}{l}\text { 6th- } \\
7 \text { th } c\end{array}$ & $\begin{array}{l}\text { Green ground with white } \\
\text { girth band and ?blue and } \\
\text { white diagonal bands }\end{array}$ & $\begin{array}{l}\text { Sherlock and Welch 1992, } \\
\text { 196, fig } 67.1\end{array}$ \\
\hline Mucking II & Essex & $\begin{array}{l}\text { 2nd } \\
\text { half } \\
5 \text { th c } \\
\text { 5th- } \\
\text { 6th c }\end{array}$ & $\begin{array}{l}\text { Grave 334: } 2 \text { cylinders } \\
\text { with red stripe and yellow } \\
\text { zigzag, with disc brooch } \\
\text { pair and inlaid iron buckle; } \\
\text { Grave } 397: 1 \text { large } \\
\text { biconical/globular in blue- } \\
\text { green with yellow zigzag } \\
\text { and } 5 \text { horizontal red stripes } \\
\text { with small-long brooch; } \\
\text { Grave } 637 \text { : annular with } \\
\text { white spots/band around } \\
\text { girth with quoit brooch, } \\
\text { equal-arm brooch and tubular } \\
\text { fitting; } \\
\text { Grave } 845: 1 \text { lobed and } \\
1 \text { annular ditto, with disc } \\
\text { brooch pair }\end{array}$ & $\begin{array}{l}\text { Hirst and Clark forthcoming; } \\
\text { BM }\end{array}$ \\
\hline $\begin{array}{l}\text { Springfield } \\
\text { Lyons }\end{array}$ & Essex & $\begin{array}{l}\text { prob } \\
\text { 6th c }\end{array}$ & $\begin{array}{l}\text { Grave 4758: barrel with } \\
\text { yellow and red dots and trails } \\
\text { (late pit context } 7350 \text { ); } \\
\text { Grave } 4882: 8 \text { translucent } \\
\text { with yellow decoration; } \\
\text { Grave } 6033: 2 \text { translucent } \\
\text { pale green with white and } \\
\text { brown stripes and bands; } \\
\text { Grave } 6044 \text { : globular pale } \\
\text { opaque with alternate red } \\
\text { and yellow dots }\end{array}$ & $\begin{array}{l}\text { Publication in } \\
\text { preparation (S Tyler); } \\
\text { BM }\end{array}$ \\
\hline $\begin{array}{l}\text { Dover, } \\
\text { Buckland }\end{array}$ & Kent & $\begin{array}{l}650- \\
675\end{array}$ & $\begin{array}{l}\text { Grave 30: biconical with } \\
\text { white band with central } \\
\text { red (D58), cf blue type; } \\
\text { Grave 59: barrel green } \\
\text { with blue translucent end } \\
\text { (D59); } \\
\text { Grave 129: annular light } \\
\text { olive green with dark blue } \\
\text { blob at one end (D61) }\end{array}$ & $\begin{array}{l}\text { Evison } 1987,77-8,82 \text {, } \\
224-5,232-3 \text { and } 244 \text {, } \\
\text { figs } 18.4 t, 33.3 \mathrm{p} \text {, and } \\
53.129 .5 \mathrm{i}, \text { colour } \\
\text { pl IV (D58, } 59 \text { and } 61) \text {; } \\
\text { BM }\end{array}$ \\
\hline
\end{tabular}




\begin{tabular}{|c|c|c|c|c|}
\hline Faversham & Kent & $\begin{array}{l}5 \text { th- } \\
7 \text { th } c\end{array}$ & $\begin{array}{l}\text { Thick annular dark } \\
\text { translucent with bands } \\
\text { underlying red bands; } \\
\text { globular opaque } 3 \text { eyes } \\
\text { with red ring round black } \\
\text { spot; } \\
\text { pentagonal cylinder } \\
\text { emerald green with oval } \\
\text { yellow spots; } \\
\text { large annular with red } \\
\text { central band and white } \\
\text { mosaic triangles }\end{array}$ & $\begin{array}{l}\text { Meaney 1964, 118-19; } \\
\text { Ashmolean Museum } \\
\text { 1909.143; } \\
\text { Ashmolean Museum } \\
\text { 1909.171; } \\
\text { Beck Collection, } \\
\text { Cambridge Museum }\end{array}$ \\
\hline Gilton & Kent & $\begin{array}{l}\text { prob } \\
\text { 6th- } \\
7 \text { th c }\end{array}$ & $\begin{array}{l}\text { Two almost identical with } \\
\text { Westbere (see below); } \\
\text { slightly biconical globular } \\
\text { yellowish green with white } \\
\text { eyes surrounded by } \\
\text { red rings }\end{array}$ & $\begin{array}{l}\text { Faussett 1856, pl V; } \\
\text { Liverpool Museum }\end{array}$ \\
\hline Harrietsham & Kent & $\begin{array}{l}\text { 7th- } \\
\text { 8th c }\end{array}$ & $\begin{array}{l}\text { Opaque green cylinder } \\
\text { central groove with vague } \\
\text { red lines }\end{array}$ & $\begin{array}{l}\text { Meaney } 1964,123 \\
\text { Maidstone Museum }\end{array}$ \\
\hline $\begin{array}{l}\text { Lyminge } \\
\text { II }\end{array}$ & Kent & $\begin{array}{l}\text { first } \\
\text { half } \\
\text { 6th c }\end{array}$ & $\begin{array}{l}\text { Grave 39: roughly globular } \\
\text { with irregular red and yellow } \\
\text { spots, with saucer and small } \\
\text { square-headed brooch } \\
\text { pairs, etc }\end{array}$ & $\begin{array}{l}\text { Warhurst } 1955,27 \text {, } \\
\text { pl VIIb, no. } 2\end{array}$ \\
\hline Orpington & Kent & 5 th $\mathrm{c}$ & $\begin{array}{l}\text { Grave } 68 \text { : large melon, } \\
\text { rather flat with red and } \\
\text { yellow markings with } \\
\text { applied saucer brooch pair }\end{array}$ & $\begin{array}{l}\text { Tester } 1969,49 \text {, } \\
\text { fig 5.68c; } \\
\text { Orpington Museum }\end{array}$ \\
\hline Ozingell & Kent & $\begin{array}{l}5 \text { th- } \\
7 \text { th } c\end{array}$ & $\begin{array}{l}\text { One annular and } 1 \text { globular } \\
\text { with mosaic insets in yellow, } \\
\text { terracotta and white }\end{array}$ & Powell-Cotton Museum \\
\hline Polhill & Kent & 7 th $c$ & $\begin{array}{l}\text { Grave 102: opaque squarish } \\
\text { with scatter of red and } \\
\text { yellow dots (group 527) } \\
\text { with silver wire rings, etc }\end{array}$ & $\begin{array}{l}\text { Hawkes and Philp 1973, } \\
185,207 \text {, fig } 55.527\end{array}$ \\
\hline Rochester & Kent & $\begin{array}{l}\text { prob } \\
\text { 6th c }\end{array}$ & $\begin{array}{l}\text { Child's necklace: all small, } \\
1 \text { slightly hexagonal, } \\
\text { yellow chevrons }\end{array}$ & $\begin{array}{l}\text { Meaney 1964, 134; } \\
\text { Skipton Museum }\end{array}$ \\
\hline $\begin{array}{l}\text { Sibertswold } \\
\text { or } \\
\text { Barfreston }\end{array}$ & Kent & $\begin{array}{l}\text { 6th- } \\
7 \text { th c }\end{array}$ & $\begin{array}{l}\text { Annular translucent olive } \\
\text { green with blue and white } \\
\text { striped scrolls }\end{array}$ & $\begin{array}{l}\text { Faussett 1856; } \\
\text { Liverpool Museum }\end{array}$ \\
\hline Westbere & Kent & 7 th $\mathrm{c}$ & $\begin{array}{l}\text { Three short strings: drop } \\
\text { bead in opaque grass green } \\
\text { with yellow end } \\
\text { (see Gilton above) }\end{array}$ & $\begin{array}{l}\text { Jessup 1946; } \\
\text { Canterbury Museum }\end{array}$ \\
\hline
\end{tabular}




\begin{tabular}{|c|c|c|c|c|}
\hline Riby Park & Lincs & 7th $c$ & $\begin{array}{l}\text { Three opaque blue-green } \\
\text { globular with terracotta } \\
\text { rings round yellow dots }\end{array}$ & $\begin{array}{l}\text { Phillips } 1934,148 \text { and 154; } \\
\text { Myres 1951, 88 and 98; } \\
\text { Lincoln Museum }\end{array}$ \\
\hline Ruskington & Lincs & & $\begin{array}{l}\text { Green cylinder with band } \\
\text { of yellow chevrons }\end{array}$ & $\begin{array}{l}\text { Meaney 1964, 161-2; } \\
\text { Lincoln Museum }\end{array}$ \\
\hline $\begin{array}{l}\text { Morning } \\
\text { Thorpe }\end{array}$ & Norfolk & $\begin{array}{l}\text { first } \\
\text { half } \\
\text { 6th c } \\
\text { 6th c }\end{array}$ & $\begin{array}{l}\text { Grave } 30: 2 \text { with red and } \\
\text { white bands with Aberg } \\
\text { Group II and III cruciform } \\
\text { brooches, B clasps, etc; } \\
\text { Grave } 337: 1 \text { with white } \\
\text { decoration with small-long } \\
\text { brooch pair, stamped pots, } \\
\text { etc }\end{array}$ & $\begin{array}{l}\text { Green et al } 1987,42-3 \text {, } \\
\text { and 131, figs } 304 \text {.Dxii and } \\
\text { 407.Lx; } \\
\text { Norwich Museum }\end{array}$ \\
\hline Spong Hill & Norfolk & $\begin{array}{l}\text { prob } \\
5 \text { th c }\end{array}$ & $\begin{array}{l}\text { Cremation } 1245: \text { bead } \\
\text { broken and melted by heat } \\
\text { terracotta eyes in white } \\
\text { rings in bossed urn }\end{array}$ & $\begin{array}{l}\text { Hills } 1977,48, \\
\text { fig 127.1245; } \\
\text { Norfolk Arch Unit }\end{array}$ \\
\hline Holdenby & Northants & $\begin{array}{l}\text { prob } \\
6 \text { th- } \\
7 \text { th c }\end{array}$ & $\begin{array}{l}\text { Cube-shaped with each } \\
\text { face having yellow eyes } \\
\text { in white rings }\end{array}$ & $\begin{array}{l}\text { Leeds } 1909 ; \\
\text { Northampton Museum }\end{array}$ \\
\hline Rollright & Oxon & $\begin{array}{l}6 \text { th- } \\
7 \text { th } c\end{array}$ & $\begin{array}{l}\text { Odd finds near burial } \\
\text { close to Rollright Stones: } \\
\text { opaque annular with } \\
\text { yellow bands at top and } \\
\text { bottom and spaced yellow } \\
\text { spots }\end{array}$ & $\begin{array}{l}\text { Information from } \\
\text { Oxford Archaeological } \\
\text { Unit }\end{array}$ \\
\hline $\begin{array}{l}\text { Little } \\
\text { Eriswell }\end{array}$ & Suffolk & $\begin{array}{l}\text { 6th- } \\
7 \text { th } c\end{array}$ & $\begin{array}{l}\text { Yellowish green with white } \\
\text { bands }\end{array}$ & $\begin{array}{l}\text { Hutchinson 1966; } \\
\text { Bury St Edmunds Museum }\end{array}$ \\
\hline Selmeston & Sussex & $\begin{array}{l}\text { 5th- } \\
7 \text { th c }\end{array}$ & $\begin{array}{l}\text { Rather biconical blue-green } \\
\text { with parallel white bands } \\
\text { all over }\end{array}$ & $\begin{array}{l}\text { Welch } 1983,84 \text {, } \\
\text { fig } 49 a \text {, no. } 4 \text {; } \\
\text { Lewes Museum }\end{array}$ \\
\hline $\begin{array}{l}\text { Peters- } \\
\text { finger }\end{array}$ & Wilts & 6th $c$ & $\begin{array}{l}\text { Grave } 62 \text { : small cylinder } \\
\text { with terracotta band } \\
\text { wound round it }\end{array}$ & $\begin{array}{l}\text { Leeds and Shortt } 1953, \\
41, \text { pl IV: } 172 \text { LXII; } \\
\text { Salisbury Museum }\end{array}$ \\
\hline Sewerby & Yorks & $\begin{array}{l}\text { prob } \\
\text { 6th c }\end{array}$ & $\begin{array}{l}\text { Grave } 29 \text { : annular with } \\
3 \text { terracotta spots equally } \\
\text { spaced (C4) with } 2 \text { annular } \\
\text { brooches }\end{array}$ & $\begin{array}{l}\text { Hirst 1985, 68, } \\
\text { fig 43.29.1-60g }\end{array}$ \\
\hline \multicolumn{5}{|c|}{ TURQUOISE BEADS (OPAQUE OR TRANSLUCENT) } \\
\hline $\begin{array}{l}\text { Chamber- } \\
\text { lain's } \\
\text { Barn II }\end{array}$ & Beds & $\begin{array}{l}\text { mid- } \\
\text { late } \\
7 \text { th c }\end{array}$ & $\begin{array}{l}\text { Grave 9: translucent } \\
\text { biconical (Plate 4); } \\
\text { Grave 39: translucent } \\
\text { biconical }\end{array}$ & $\begin{array}{l}\text { Hyslop } 1963,173,181 \text {, } \\
\text { figs } 9 \mathrm{~d}, 13 \mathrm{c} \text { and } \mathrm{g} ; \\
\text { Luton Museum }\end{array}$ \\
\hline
\end{tabular}




\begin{tabular}{|c|c|c|c|c|}
\hline Kempston & Beds & $\begin{array}{l}5 \text { th- } \\
7 \text { th c }\end{array}$ & $\begin{array}{l}\text { Small bright annular; } \\
\text { Grave 16: large dark } \\
\text { annular; } \\
\text { another unmarked grave: } \\
1 \text { globular }\end{array}$ & $\begin{array}{l}\text { Fitch 1864; } \\
\text { BM } 916-2453 \text {; } \\
\text { BM } 916-24 \text { 53; } \\
\text { BM } 916-2451\end{array}$ \\
\hline Frilford & Berks & & $\begin{array}{l}\text { Three biconicals, very } \\
\text { slightly translucent }\end{array}$ & $\begin{array}{l}\text { Bradford and Goodchild } \\
\text { 1939; BM OA } 4880\end{array}$ \\
\hline $\begin{array}{l}\text { Little } \\
\text { Wilbraham }\end{array}$ & Cambs & $\begin{array}{l}5 \text { th- } \\
7 \text { th c }\end{array}$ & Two globulars & $\begin{array}{l}\text { Lethbridge and Carter 1926; } \\
\text { Neville 1852; } \\
\text { Cambridge Museum }\end{array}$ \\
\hline Lechlade & Glos & $\begin{array}{l}\text { mid- } \\
\text { late } \\
7 \text { th } c\end{array}$ & $\begin{array}{l}\text { Grave } 84: 2 \text { bright green- } \\
\text { blue barrels; } \\
\text { Grave } 98 \text { : bright green- } \\
\text { blue biconical bead; } \\
\text { Grave } 138 \text { : small lobed } \\
\text { bright green-blue with union } \\
\text { pins, etc }\end{array}$ & $\begin{array}{l}\text { Boyle et al forthcoming; } \\
\text { Cirencester Museum }\end{array}$ \\
\hline Andover & Hants & $\begin{array}{l}5 \text { th- } \\
6 \text { th } c\end{array}$ & $\begin{array}{l}\text { Grave 50: } 3 \text { annulars } \\
\text { pale turquoise blue with } \\
\text { small-long brooch, etc }\end{array}$ & $\begin{array}{l}\text { Cook and Dacre } 1985 \text {, } \\
38,80-2, \text { fig } 63.10, \\
14 \text { and } 27, \text { colour pl } \\
\text { frontispiece; } \\
\text { Hampshire Museums }\end{array}$ \\
\hline $\begin{array}{l}\text { Breach } \\
\text { Down }\end{array}$ & Kent & & $\begin{array}{l}\text { Two small irregular } \\
\text { translucent }\end{array}$ & $\begin{array}{l}\text { Conyngham and Akerman } \\
1844 ; \mathrm{BM} 795-24\end{array}$ \\
\hline $\begin{array}{l}\text { Dover, } \\
\text { Buckland }\end{array}$ & Kent & $\begin{array}{l}475- \\
525 \\
575- \\
625\end{array}$ & $\begin{array}{l}\text { Grave } 46: 88 \text { beads (B34 } \\
\text { and B35); } \\
\text { Grave 1: } 27 \text { beads (B34); } \\
\text { Grave 29: } 3 \text { beads (B34); } \\
\text { Grave 32: } 46 \text { beads (B34, } \\
\text { B35 and B36); } \\
\text { Grave } 35: 1 \text { bead (B34) }\end{array}$ & $\begin{array}{l}\text { Evison } 1987,73,80 \text {, } \\
216,223-4,225 \text { and } 226 \text {, } \\
\text { figs } 5.4 \mathrm{k}, 17.2 \mathrm{j} \text {, } \\
19.4 \mathrm{e}, \mathrm{f} \text { and } \mathrm{g}, 21.5 \mathrm{f} \text {, and } \\
26.46 .3 \mathrm{~d} \text { and } \mathrm{e} \text {; } \\
\text { BM }\end{array}$ \\
\hline $\begin{array}{l}\text { Faversham, } \\
\text { King's Field }\end{array}$ & Kent & $\begin{array}{l}5 \text { th- } \\
7 \text { th } c\end{array}$ & $\begin{array}{l}\text { Necklace: } c .40 \text { segments; } \\
1 \text { short (Plate } 4 \text { ) and } \\
1 \text { double cylinder; } \\
\text { barrel with yellow crossed } \\
\text { waves }\end{array}$ & $\begin{array}{l}\text { Meaney 1964, 118-19; } \\
\text { Rochester Museum; } \\
\text { Ashmolean Museum; } \\
\text { Beck Collection, } \\
\text { Cambridge Museum }\end{array}$ \\
\hline Howletts & Kent & $\begin{array}{l}\text { prob } \\
\text { 6th c }\end{array}$ & Rich translucent annular & $\begin{array}{l}\text { Smith 1918; } \\
\text { BM 1936 5-11 } 144\end{array}$ \\
\hline Kingston & Kent & $\begin{array}{l}\text { prob } \\
7 \text { th } c\end{array}$ & $\begin{array}{l}\text { One biconical, } 1 \text { long } \\
\text { biconical, } 1 \text { small lobed and } \\
\text { short cylinder }\end{array}$ & $\begin{array}{l}\text { Faussett 1856, 35-94; } \\
\text { Liverpool Museum } 6327\end{array}$ \\
\hline Monkton & Kent & 7 th $\mathrm{c}$ & Grave 24: 3 biconicals & $\begin{array}{l}\text { Hawkes and Hogarth } 1974 \text {, } \\
59, \text { fig } 5 j-1 \text {; } \\
\text { Ashmolean Museum }\end{array}$ \\
\hline $\begin{array}{l}\text { Sitting- } \\
\text { bourne, } \\
\text { Rondeau Estate }\end{array}$ & Kent & $\begin{array}{l}\text { prob } \\
\text { 6th } c\end{array}$ & One globular and 1 melon & $\begin{array}{l}\text { Payne 1880; } \\
\text { Meaney 1964, 129; } \\
\text { BM } 83 \text { 12-13 } 644\end{array}$ \\
\hline
\end{tabular}


The Glass Beads of Anglo-Saxon England

\begin{tabular}{|c|c|c|c|c|}
\hline Stodmarsh & Kent & $\begin{array}{l}\text { prob } \\
\text { 6th c }\end{array}$ & One melon & $\begin{array}{l}\text { Akerman 1855b, 179-81, } \\
\text { pl XVI; } \\
\text { BM 54 12-2 } 17\end{array}$ \\
\hline Wingham & Kent & 7th $c$ & $\begin{array}{l}\text { One globular and dark } \\
\text { opaque barrel }\end{array}$ & $\begin{array}{l}\text { Meaney 1964, 140-1; } \\
\text { BM 54 12-2 } 18 \text { and } 20\end{array}$ \\
\hline $\begin{array}{l}\text { Nettleton } \\
\text { Caistor }\end{array}$ & Lincs & $\begin{array}{l}\text { prob } \\
\text { 6th- } \\
\text { 7th c }\end{array}$ & One annular small opaque & $\begin{array}{l}\text { Meaney 1964, } 160 \\
\text { Lincoln Museum }\end{array}$ \\
\hline Riby Park & Lincs & 7 th $\mathrm{c}$ & $\begin{array}{l}\text { One large annular with white } \\
\text { wave, } 1 \text { with eyes }\end{array}$ & $\begin{array}{l}\text { Phillips } 1934 ; \\
\text { Myres 1951, } 88 \text { and 98; } \\
\text { Lincoln Museum }\end{array}$ \\
\hline $\begin{array}{l}\text { South } \\
\text { Willingham }\end{array}$ & Lincs & & $\begin{array}{l}\text { Two barrel beads (Plate } 4 \text { ), } \\
1 \text { very small annular with } \\
\text { cruciform brooch }\end{array}$ & $\begin{array}{l}\text { Meaney } 1964,165 \\
\text { BM OA } 5057 \text { and } \\
\text { BM } 762-1212-15\end{array}$ \\
\hline Syston Park & Lincs & $\begin{array}{l}\text { prob } \\
\text { 6th- } \\
\text { 7th c }\end{array}$ & Large light melon & $\begin{array}{l}\text { Akerman 1855a, 41-2, } \\
\text { pl XXI; } \\
\text { BM (18) } 1111-92\end{array}$ \\
\hline $\begin{array}{l}\text { Bury St } \\
\text { Edmunds, } \\
\text { Westgarth } \\
\text { Gardens }\end{array}$ & Suffolk & 6th $c$ & $\begin{array}{l}\text { Grave } 31: 1 \text { polyhedral } \\
\text { with stamped pot }\end{array}$ & $\begin{array}{l}\text { West } 1988,28, \\
\text { fig } 68 ; \\
\text { Bury St Edmunds } \\
\text { Museum }\end{array}$ \\
\hline Lakenheath & Suffolk & $\begin{array}{l}\text { prob } \\
\text { 6th- } \\
\text { 7th c }\end{array}$ & $\begin{array}{l}\text { One short cylinder and some } \\
\text { very small segmented }\end{array}$ & $\begin{array}{l}\text { Meaney 1964, 230; } \\
\text { Beck Collection, } \\
\text { Cambridge Museum }\end{array}$ \\
\hline $\begin{array}{l}\text { Mitchell's } \\
\text { Hill }\end{array}$ & Suffolk & $\begin{array}{l}\text { prob } \\
\text { 6th } c\end{array}$ & $\begin{array}{l}\text { One cylinder with polygonal } \\
\text { section, } 1 \text { melon and semi- } \\
\text { translucent globular }\end{array}$ & $\begin{array}{l}\text { Meaney } 1964,231 ; \\
\text { Bury St Edmunds } \\
\text { Museum }\end{array}$ \\
\hline $\begin{array}{l}\text { Kingston- } \\
\text { by-Lewes }\end{array}$ & Sussex & $\begin{array}{l}\text { 6th- } \\
7 \text { th } c\end{array}$ & Rather biconical & $\begin{array}{l}\text { Craddock } 1979,99, \text { pl 12; } \\
\text { Welch } 1983,83 \text {, fig } 73 \mathrm{e} ; \\
\text { Lewes Museum }\end{array}$ \\
\hline Driffield & Yorks & & $\begin{array}{l}\text { With other beads: } \\
1 \text { globular }\end{array}$ & $\begin{array}{l}\text { Mortimer 1905; } \\
\text { Hull Museum }\end{array}$ \\
\hline
\end{tabular}




\title{
SCHEDULE 6
}

\section{BLUE GLASS BEADS}

\author{
MoNochrome \\ i Blue annular, globular and short barrel-shaped beads: no schedule \\ ii Blue cylinder beads, round, long or short \\ iii Blue cylinder beads, square or polygonal section, long or short \\ iv Blue diamond-faceted, cube-shaped or oblong beads \\ v Blue or 'black' large, flat polygonal beads: no schedule \\ vi Blue segmented beads \\ vii Blue biconical beads (opaque or translucent) \\ viii Blue melon, sub-melon and lobed beads (opaque or translucent) (see Map 18) \\ Polychrome \\ ix Blue beads with white or cream waves, or zigzags \\ $\mathrm{x} \quad$ Blue beads with crossed waves, with or without dots \\ xi Blue beads with white and coloured specks (see Map 19) \\ xii Blue beads with red spots (see Map 20) \\ xiii Blue beads with red centred white eyes \\ xiv Blue beads with white or yellow spots (see Map 20) \\ xv Miscellaneous blue decorated beads
}

\section{MONOCHROME}

i BLUE ANNULAR, GLOBULAR AND SHORT BARREL-SHAPED BEADS: no schedule (Plate 5)

ii Blue CYlinder BEADS, ROUND, LONG OR SHORT

$\begin{array}{llrll}\begin{array}{l}\text { Frilford } \\ \text { Linton }\end{array} & \text { Berks } & \text { 5th c } & \begin{array}{l}\text { Grave 196: translucent, } \\ \text { cut ends }\end{array} & \begin{array}{l}\text { Brown 1975, 293; } \\ \text { Ashmolean Museum }\end{array} \\ \begin{array}{l}\text { Heath B } \\ \text { Norton- } \\ \text { on-Tees }\end{array} & \text { Cambs } & \text { 6th c } & \begin{array}{l}\text { Very weathered, } \\ \text { pointed ends }\end{array} & \begin{array}{l}\text { Neville 1854; } \\ \text { Cambridge Museum }\end{array} \\ & & \text { Durham } & \text { mid- } \\ \text { 6th c } & \begin{array}{l}\text { Grave 63: with Aberg } \\ \text { Group IVa cruciform } \\ \text { brooch, B clasps, etc; } \\ \text { Grave 94: with annular } \\ \text { brooch pair, B clasps, etc }\end{array} & \begin{array}{l}\text { Sherlock and Welch 1992, } \\ \text { 6th c }\end{array} \\ & & & 60.94 .1\end{array}$




\begin{tabular}{|c|c|c|c|c|}
\hline $\begin{array}{l}\text { Great } \\
\text { Chesterford }\end{array}$ & Essex & 6th c & $\begin{array}{l}\text { Grave } 18: \text { one (C03), with } \\
\text { disc brooch pair, B clasps, } \\
\text { etc; } \\
\text { Grave } 29: 3 \text { with pinched } \\
\text { ends ( } 02-03 \text { ), with B } \\
\text { clasps, etc; } \\
\text { Grave } 37 \text { : cut ends (C02), } \\
\text { with radiate and small-long } \\
\text { brooches, etc; } \\
\text { Grave } 62: 2 \text { (C03), with } \\
\text { small square-headed } \\
\text { brooch pair, etc }\end{array}$ & $\begin{array}{l}\text { Evison } 1994,16,94,96, \\
97,101 \text {, figs } 26.29 .5 \mathrm{~d} \text { and } \mathrm{e}, \\
28.37 .4 \mathrm{i}, 33.62 .4 \mathrm{~m} \text {; } \\
\mathrm{BM}\end{array}$ \\
\hline $\begin{array}{l}\text { Springfield } \\
\text { Lyons }\end{array}$ & Essex & & Grave 2806: pointed ends & $\begin{array}{l}\text { Publication in } \\
\text { preparation (S Tyler); } \\
\text { BM }\end{array}$ \\
\hline Lechlade & Glos & $\begin{array}{l}6 \text { th c } \\
5 \text { th- } \\
6 \text { th c }\end{array}$ & $\begin{array}{l}\text { Grave } 18: \text { many with great } \\
\text { square-headed brooch and } \\
\text { and saucer brooch pair, etc; } \\
\text { Grave } 163 \text { : greenish-blue } \\
\text { with disc brooch pair }\end{array}$ & $\begin{array}{l}\text { Boyle et al forthcoming; } \\
\text { Cirencester Museum }\end{array}$ \\
\hline Alton & Hants & 6th c & $\begin{array}{l}\text { Grave } 12 \text { : translucent } \\
\text { with saucer brooch pair, } \\
\text { etc }\end{array}$ & $\begin{array}{l}\text { Evison 1988a, } 74 \text {, } \\
\text { fig 26.4id; } \\
\text { Hampshire Museums }\end{array}$ \\
\hline Llanigon & Herefords & 6th $\mathrm{c}$ & $\begin{array}{l}\text { Secondary in barrow: } \\
1 \text { example, cut ends, } \\
\text { with other beads }\end{array}$ & $\begin{array}{l}\text { Morgan and Marshall 1921; } \\
\text { Hereford Museum }\end{array}$ \\
\hline $\begin{array}{l}\text { Barfreston } \\
\text { or Kingston }\end{array}$ & Kent & & One dark blue long, cut ends & $\begin{array}{l}\text { Faussett 1856; } \\
\text { Liverpool Museum }\end{array}$ \\
\hline $\begin{array}{l}\text { Beakes- } \\
\text { bourne }\end{array}$ & Kent & $\begin{array}{l}\text { prob } \\
\text { 6th c }\end{array}$ & Grave 24: short translucent & $\begin{array}{l}\text { Meaney 1964, 109; } \\
\text { Canterbury Museum }\end{array}$ \\
\hline Canterbury & Kent & & $\begin{array}{l}\text { Miscellaneous: } 1 \text { long cut } \\
\text { end }\end{array}$ & $\begin{array}{l}\text { Brent Collection } 2183 \text {, } \\
\text { Canterbury Museum }\end{array}$ \\
\hline Coombe & Kent & 6 th $c$ & $\begin{array}{l}\text { Cremation necklace: } \\
\text { translucent, possibly } \\
\text { with square-headed } \\
\text { brooch, etc }\end{array}$ & $\begin{array}{l}\text { Davidson and Webster } \\
\text { 1967, 20-1, fig 5, pl VII.D; } \\
\text { Saffron Walden Museum }\end{array}$ \\
\hline $\begin{array}{l}\text { Dover, } \\
\text { Buckland }\end{array}$ & Kent & $\begin{array}{l}525- \\
575 \\
575 \\
625\end{array}$ & $\begin{array}{l}\text { Grave } 13: 1 \text { drawn, } \\
1 \text { pointed and } 1 \text { twisted } \\
\text { (C01); } \\
\text { Grave 20: } 4 \text { drawn (C01); } \\
\text { Grave } 48: 4 \text { drawn (C01); } \\
\text { Grave 15: } 6 \text { beads (C01); } \\
\text { Grave 92: } 2 \text { beads (C01); } \\
\text { Grave 1: } 3 \text { short } \\
\text { cylinders (B46) (Plate 5); } \\
\text { Grave } 30: \text { translucent with } \\
\text { pointed ends (B51); }\end{array}$ & $\begin{array}{l}\text { Evison } 1987,75,81,219, \\
220,230,219,237-8,216, \\
224,227 \text { and } 233, \\
\text { figs } 9.3 \mathrm{e}, 12.5 \mathrm{n}, \\
27.4 \mathrm{i}, 11.15 .7 \mathrm{~b}, 42.3 \mathrm{c} \text { and } \mathrm{k} \text {, } \\
5.4 \mathrm{n}, 18.4 \mathrm{j}, 22.4 \mathrm{e} \text { and } \\
34.3 \mathrm{n} \text {; BM }\end{array}$ \\
\hline
\end{tabular}


Grave 38: translucent with pointed ends ( $\mathrm{CO1})$;

Grave 60: 2 beads (C01)

\begin{tabular}{|c|c|c|c|c|}
\hline $\begin{array}{l}\text { Dover, } \\
\text { Priory Hill }\end{array}$ & Kent & $\begin{array}{l}\text { late } \\
\text { 6th } c\end{array}$ & Two long thin, cut ends & $\begin{array}{l}\text { Meaney } 1964,117 \\
\text { Dover Museum }\end{array}$ \\
\hline $\begin{array}{l}\text { Eastry, } \\
\text { Buttsole }\end{array}$ & Kent & $\begin{array}{l}\text { prob } \\
\text { 6th c }\end{array}$ & Two translucent & $\begin{array}{l}\text { Hawkes 1979; } \\
\text { Canterbury Museum }\end{array}$ \\
\hline Faversham & Kent & $\begin{array}{l}5 \text { th- } \\
7 \text { th } c\end{array}$ & Five examples & $\begin{array}{l}\text { Meaney 1964, } 118-19 \\
\text { Ashmolean Museum }\end{array}$ \\
\hline Finglesham & Kent & $\begin{array}{l}\text { prob } \\
\text { 6th c } \\
\text { first } \\
\text { half } \\
\text { 6th c }\end{array}$ & $\begin{array}{l}\text { Grave A3: one, with amber } \\
\text { bead, etc; } \\
\text { Grave D3: translucent } \\
\text { with rounded ends, with } \\
\text { great square-headed } \\
\text { brooch, etc }\end{array}$ & $\begin{array}{l}\text { Chadwick } 1958,10,12 \text {, } \\
\text { fig } 5 \text { p and e, pl III; } \\
\text { Deal Museum }\end{array}$ \\
\hline Folkestone & Kent & $\begin{array}{l}\text { prob } \\
\text { 6th c }\end{array}$ & $\begin{array}{l}\text { One short opaque mid- } \\
\text { blue; and another }\end{array}$ & $\begin{array}{l}\text { Folkestone Museum; } \\
\text { Maidstone Museum }\end{array}$ \\
\hline $\begin{array}{l}\text { Gilton or } \\
\text { Kingston }\end{array}$ & Kent & $\begin{array}{l}\text { 6th- } \\
\text { 7th c }\end{array}$ & $\begin{array}{l}\text { 'Strings', unclear if } \\
\text { necklaces; } \\
\text { another with pointed end } \\
\text { and } 1 \text { marked } \\
\text { 'Kingston } 6333 \text { ' }\end{array}$ & $\begin{array}{l}\text { Faussett 1856, pl VI; } \\
\text { Maidstone Museum; } \\
\text { Liverpool Museum } \\
6123\end{array}$ \\
\hline Harrietsham & Kent & & Two examples & $\begin{array}{l}\text { Meaney 1964, 123; } \\
\text { Maidstone Museum }\end{array}$ \\
\hline $\begin{array}{l}\text { Horton } \\
\text { Kirby I }\end{array}$ & Kent & $\begin{array}{l}\text { prob } \\
\text { 6th c }\end{array}$ & $\begin{array}{l}\text { Long slightly twisted cut } \\
\text { ends (Plate } 5 \text { ), with saucer } \\
\text { brooches and other beads }\end{array}$ & $\begin{array}{l}\text { Meaney } 1964,124 ; \\
\text { Maidstone Museum }\end{array}$ \\
\hline $\begin{array}{l}\text { Lyminge } \\
\text { II }\end{array}$ & Kent & $\begin{array}{l}\text { first } \\
\text { half } \\
\text { 6th c }\end{array}$ & $\begin{array}{l}\text { Grave } 16: 3 \text { long different } \\
\text { sizes, cut ends with } \\
\text { Hahnheim-type radiate } \\
\text { brooch, button brooch and } \\
\text { D bracteate, etc }\end{array}$ & $\begin{array}{l}\text { Warhurst 1955, 15; } \\
\text { Maidstone Museum }\end{array}$ \\
\hline Polhill & Kent & $\begin{array}{l}6 \text { th } c \\
\text { or } \\
7 \text { th } c\end{array}$ & $\begin{array}{l}\text { Grave } 41 \text { : possible } \\
\text { earlier bead survival on a } \\
\text { 7th-century string with } 8 \\
\text { other beads }\end{array}$ & $\begin{array}{l}\text { Hawkes and Philp } 1973 \\
177,207 \text {, fig } 55.521\end{array}$ \\
\hline Sarre & Kent & $\begin{array}{l}5 \text { th- } \\
7 \text { th } c \\
6 \text { th } c\end{array}$ & $\begin{array}{l}\text { One 'string', not necessarily } \\
\text { associated; } \\
\text { Grave CCLX: } 3 \text { with } \\
\text { pointed ends, with saucer } \\
\text { brooch }\end{array}$ & $\begin{array}{l}\text { Meaney 1964, 135-6; } \\
\text { BM 93 6-1 218; } \\
\text { Brent 1868, 316-17, } \\
\text { pl VIII.CCLX }\end{array}$ \\
\hline Stowting & Kent & $\begin{array}{l}\text { prob } \\
\text { 6th c }\end{array}$ & $\begin{array}{l}\text { Three short opaque with } \\
\text { cut ends; } \\
3 \text { long various blues with } \\
\text { cut ends }\end{array}$ & $\begin{array}{l}\text { Meaney } 1964,137-8 ; \\
\text { Beck Collection, } \\
\text { Cambridge Museum; } \\
\text { Maidstone Museum }\end{array}$ \\
\hline
\end{tabular}




\begin{tabular}{|c|c|c|c|c|}
\hline $\begin{array}{l}\text { Castle } \\
\text { Bytham }\end{array}$ & Lincs & $\begin{array}{l}\text { prob } \\
\text { 7th c }\end{array}$ & One bead with quoit brooch & $\begin{array}{l}\text { Akerman 1855a, 26-7, } \\
\text { pl XII.4 }\end{array}$ \\
\hline Fonaby & Lincs & $\begin{array}{l}5 \text { th- } \\
6 \text { th } c \\
5 \text { th- } \\
6 \text { th } c \\
\text { mid- } \\
6 \text { th } c\end{array}$ & $\begin{array}{l}\text { Grave 32: with } \AA \text { berg } \\
\text { Group II cruciform brooch, } \\
\text { etc; } \\
\text { Grave 28: translucent with } \\
\text { small-long brooch, etc; } \\
\text { Grave } 31: 2 \text { with pointed } \\
\text { end, with Aberg Group IV } \\
\text { cruciform and annular } \\
\text { brooches, etc; } \\
\text { Grave } 43: 4 \text { translucent } \\
\text { with rounded ends, with } \\
\text { Åberg Group IVa and other } \\
\text { cruciform brooches, etc }\end{array}$ & $\begin{array}{l}\text { Cook } 1981,34,30,32 \\
\text { and } 40, \text { figs } 11.2 .2 \text {, } \\
10.28 .3 .1, \\
10.31 .1 \text { and } 15, \\
\text { and } 16.1 .19,43-5 ; \\
\text { Scunthorpe Museum }\end{array}$ \\
\hline Laceby & Lincs & $\begin{array}{l}\text { prob } \\
\text { 6th c }\end{array}$ & $\begin{array}{l}\text { Group: } 1 \text { dark cylinder } \\
\text { with many amber beads }\end{array}$ & $\begin{array}{l}\text { Myres } 1951,89 \text { and } 98 ; \\
\text { Thompson 1956, 184-9; } \\
\text { Lincoln Museum }\end{array}$ \\
\hline Quarrington & Lincs & $\begin{array}{l}\text { prob } \\
\text { 6th c }\end{array}$ & & $\begin{array}{l}\text { Bruce } 1880,76, \text { nos } 303-4 \text {; } \\
\text { Alnwick Museum }\end{array}$ \\
\hline Brooke & Norfolk & $\begin{array}{l}\text { prob } \\
\text { 6th c }\end{array}$ & & $\begin{array}{l}\text { Meaney 1964, 170; } \\
\text { BM 1866-69 }\end{array}$ \\
\hline $\begin{array}{l}\text { Morning } \\
\text { Thorpe }\end{array}$ & Norfolk & $\begin{array}{l}\text { 6th c } \\
\text { 6th c } \\
\text { mid- } \\
\text { 6th c } \\
\text { 6th c } \\
\text { 6th c }\end{array}$ & $\begin{array}{l}\text { Grave } 92: 2 \text { beads with } \\
\text { annular brooch and bucket } \\
\text { pendants, etc; } \\
\text { Grave 106: bead with } \\
\text { annular brooches, etc; } \\
\text { Grave 337: bead with } \\
\text { small-long brooch pair, etc; } \\
\text { Grave 358: } 3 \text { beads with } \\
\text { Aberg Group IV cruciform } \\
\text { brooch, B clasps, etc; } \\
\text { Grave 369: bead with } \\
\text { B clasps, brooches, etc; } \\
\text { Grave } 378 \text { : one with B } \\
\text { clasps, annular brooch } \\
\text { pair, etc }\end{array}$ & $\begin{array}{l}\text { Green } \text { et al } 1987,62,65 \text {, } \\
\text { 131, 138, } 143 \text { and } 148 \text {, } \\
\text { figs } 326.92 . \mathrm{Cx} \text {, } \\
\text { 331.106.Jv, } 407 . \mathrm{Lii} \text {, } \\
\text { 417.Biii and Dvii, } \\
\text { 427.Lii and 436.378.Eii; } \\
\text { Norwich Museum }\end{array}$ \\
\hline Spong Hill & Norfolk & 6th c & $\begin{array}{l}\text { Grave } 24: 8 \text { beads with } \\
\text { great square-headed } \\
\text { brooch, etc; } \\
\text { Grave } 37: 2 \text { beads with } \\
\text { B clasps, annular brooch } \\
\text { pair, etc; } \\
\text { Grave } 38: 2 \text { beads with } \\
\text { plain square-headed } \\
\text { brooch, etc; } \\
\text { Grave } 44: 1 \text { with annular } \\
\text { brooch, etc }\end{array}$ & $\begin{array}{l}\text { Hills et al } 1984,73,86, \\
89-90 \text { and } 99.44 .7, \\
\text { figs } 80.24 .2,90.37 .6, \\
92.7 b \text { and } 10 a \text {, and } 99.44 .7 \text {; } \\
\text { Norfolk Arch Unit }\end{array}$ \\
\hline
\end{tabular}




\begin{tabular}{|c|c|c|c|c|}
\hline $\begin{array}{l}\text { South } \\
\text { Shields }\end{array}$ & $\begin{array}{l}\text { North- } \\
\text { umber- } \\
\text { land }\end{array}$ & & $\begin{array}{l}\text { Marked 'various': some } \\
\text { look late Roman or } \\
\text { 5th-century? }\end{array}$ & $\begin{array}{l}\text { Information from } \\
\text { R Miket; } \\
\text { South Shields Museum }\end{array}$ \\
\hline $\begin{array}{l}\text { Standlake } \\
\text { Down }\end{array}$ & Oxon & $\begin{array}{l}\text { early } \\
7 \text { th } c\end{array}$ & $\begin{array}{l}\text { Grave } 24 \text { : one with other } \\
\text { beads, etc }\end{array}$ & $\begin{array}{l}\text { Dickinson 1973, 244-5, } \\
\text { fig 5.1; Ashmolean Museum }\end{array}$ \\
\hline $\begin{array}{l}\text { Empingham } \\
\text { II }\end{array}$ & Rutland & $\begin{array}{l}5 \text { th- } \\
7 \text { th c }\end{array}$ & $\begin{array}{l}\text { Graves } 6,22,49,79,85 \mathrm{~A}, \\
85 \mathrm{~B}, 105,129: \text { cylinders } \\
\text { and } 8 \text { short cylinders }\end{array}$ & $\begin{array}{l}\text { Timby 1996; } \\
\text { Oakham Museum }\end{array}$ \\
\hline Camerton & Somerset & $\begin{array}{l}\text { prob } \\
7 \text { th } c\end{array}$ & $\begin{array}{l}\text { Grave 31: with infant } \\
\text { burial }\end{array}$ & $\begin{array}{l}\text { Horne } 1933,46 \text { and } 48 ; \\
\text { Taunton Museum }\end{array}$ \\
\hline $\begin{array}{l}\text { Holywell } \\
\text { Row }\end{array}$ & Suffolk & $\begin{array}{l}\text { mid- } \\
6 \text { th c } \\
\text { prob } \\
6 \text { th c }\end{array}$ & $\begin{array}{l}\text { Grave } 37: 4 \text { beads with } \\
\text { Aberg Group IV cruciform } \\
\text { brooch; } \\
\text { Grave 35: } 1 \text { with } 2 \text { annular } \\
\text { brooches, etc }\end{array}$ & $\begin{array}{l}\text { Lethbridge 1931, } 19 \text {, } \\
\text { figs } 9.6 \text { and 11.C.3; } \\
\text { Cambridge Museum } \\
\text { Z } 7128\end{array}$ \\
\hline Lakenheath & Suffolk & & $\begin{array}{l}\text { One bead, pointed ends } \\
\text { (Plate 5) }\end{array}$ & $\begin{array}{l}\text { Meaney 1964,230; } \\
\text { Beck Collection, } \\
\text { Cambridge Museum }\end{array}$ \\
\hline $\begin{array}{l}\text { Little } \\
\text { Eriswell }\end{array}$ & Suffolk & $\begin{array}{l}\text { 6th- } \\
7 \text { th } c\end{array}$ & $\begin{array}{l}\text { Graves } 2 \text { and 27: with } \\
\text { pinched ends and with } \\
\text { ring brooches }\end{array}$ & $\begin{array}{l}\text { Hutchinson 1966; } \\
\text { Bury St Edmunds } \\
\text { Museum }\end{array}$ \\
\hline Snape & Suffolk & $\begin{array}{l}\text { prob } \\
\text { 6th c }\end{array}$ & $\begin{array}{l}\text { Grave 0841: } 1 \text { with pinched } \\
\text { ends }\end{array}$ & $\begin{array}{l}\text { Filmer-Sankey and } \\
\text { Pestell forthcoming }\end{array}$ \\
\hline West Stow & Suffolk & $\begin{array}{l}\text { 5th- } \\
7 \text { th c }\end{array}$ & $\begin{array}{l}\text { Cemetery: } 1 \text { long trans- } \\
\text { lucent, cut ends (and others) }\end{array}$ & $\begin{array}{l}\text { West 1985, 74; } \\
\text { Ashmolean Museum } \\
\text { 1909.419a }\end{array}$ \\
\hline Guildown & Surrey & $\begin{array}{l}\text { 5th- } \\
\text { 6th c }\end{array}$ & Grave 78 & $\begin{array}{l}\text { Lowther 1931, 12, 36, } \\
\text { pl IX.7; } \\
\text { Guildford Museum }\end{array}$ \\
\hline Mitcham & Surrey & $\begin{array}{l}\text { 5th- } \\
\text { 7th c }\end{array}$ & One example & $\begin{array}{l}\text { Bidder and Morris 1959; } \\
\text { 110-12; } \\
\text { Cambridge Museum }\end{array}$ \\
\hline Alfriston & Sussex & $\begin{array}{l}\text { 5th- } \\
\text { 6th } c\end{array}$ & $\begin{array}{l}\text { Grave } 77 \text { : long hexagonal; } \\
\text { Context (?): } 1 \text { hexagonal } \\
\text { and } 1 \text { twisted (AJ7) }\end{array}$ & $\begin{array}{l}\text { Griffith } 1915,203 \text {; } \\
\text { Welch } 1983,83 \text { and } 372 \text {; } \\
\text { Lewes Museum }\end{array}$ \\
\hline $\begin{array}{l}\text { Apple } \\
\text { Down I }\end{array}$ & Sussex & $\begin{array}{l}\text { 5th- } \\
\text { 6th } c\end{array}$ & $\begin{array}{l}\text { Grave 18: translucent, } \\
\text { broken at each end, } \\
\text { with tubular mount, etc }\end{array}$ & $\begin{array}{l}\text { Down and Welch } 1990,37, \\
162, \text { fig } 2.22 .18 .8 \\
\text { colour pl 41.45; } \\
\text { Chichester Museum }\end{array}$ \\
\hline Highdown & Sussex & $\begin{array}{l}\text { prob } \\
\text { 6th c }\end{array}$ & $\begin{array}{l}\text { Necklace: a few very } \\
\text { small beads with cut } \\
\text { ends }\end{array}$ & $\begin{array}{l}\text { Welch 1983; } \\
\text { Worthing Museum } 3486\end{array}$ \\
\hline Wasperton & Warks & $\begin{array}{l}\text { late } \\
\text { 5th- } \\
\text { mid- } \\
\text { 6th c }\end{array}$ & $\begin{array}{l}\text { A number, some with } \\
\text { pointed ends }\end{array}$ & $\begin{array}{l}\text { Publication in } \\
\text { preparation } \\
\text { (M Carver); } \\
\text { Warwick Museum }\end{array}$ \\
\hline
\end{tabular}




\begin{tabular}{|c|c|c|c|c|}
\hline $\begin{array}{l}\text { Lydiard } \\
\text { Tregoze }\end{array}$ & Wilts & 6th $c$ & $\begin{array}{l}\text { With } 2 \text { inhumations: } \\
\text { beads, etc }\end{array}$ & $\begin{array}{l}\text { Goddard 1895; } \\
\text { Devizes Museum }\end{array}$ \\
\hline $\begin{array}{l}\text { Winter- } \\
\text { bourne } \\
\text { Gunner }\end{array}$ & Wilts & 6 th $\mathrm{c}$ & $\begin{array}{l}\text { Grave } 7 \text { : larger translucent } \\
\text { with cut ends }\end{array}$ & $\begin{array}{l}\text { Musty and Stratton 1964; } \\
\text { Salisbury Museum }\end{array}$ \\
\hline Driffield & Yorks & $\begin{array}{l}\text { prob } \\
\text { 6th } c\end{array}$ & $\begin{array}{l}\text { Necklace of } 3 \text { rows: } 10 \\
\text { beads }\end{array}$ & $\begin{array}{l}\text { Mortimer 1905; } \\
\text { Hull Museum }\end{array}$ \\
\hline 'E Yorks' & Yorks & 6th $c$ & Necklace: 10 beads & $\begin{array}{l}\text { Mortimer 1905, pl CXIII; } \\
\text { Hull Museum }\end{array}$ \\
\hline Hessle II & Yorks & & $\begin{array}{l}\text { With other beads and } \\
\text { pottery }\end{array}$ & $\begin{array}{l}\text { Meaney 1964, 291; } \\
\text { Hull Museum records }\end{array}$ \\
\hline $\begin{array}{l}\text { Painsthorpe } \\
\text { Wold I }\end{array}$ & Yorks & 7th $\mathrm{c}$ & $\begin{array}{l}\text { Group with wound } \\
\text { slatey opaque }\end{array}$ & $\begin{array}{l}\text { Mortimer 1905, pl XXXIV; } \\
\text { Hull Museum }\end{array}$ \\
\hline Sewerby & Yorks & $\begin{array}{l}\text { mid- } \\
6 \text { th } c \\
\text { 6th } c\end{array}$ & $\begin{array}{l}\text { Grave } 15: 2 \text { with pointed } \\
\text { ends (A5a) with \&̊berg } \\
\text { Group IVa cruciform } \\
\text { brooch, etc; } \\
\text { Grave } 54: 2 \text { more (A5a) } \\
\text { with annular brooches, etc }\end{array}$ & $\begin{array}{l}\text { Hirst } 1985,66 \\
\text { figs } 37 b \text { and } 55 a\end{array}$ \\
\hline $\begin{array}{l}\text { West } \\
\text { Heslerton }\end{array}$ & Yorks & 6th $c$ & $\begin{array}{l}\text { Graves } 1 \text { A } 17: 3 \text {; } \\
1 \text { B } 8: 1 ; \\
2 \text { BA 78: } 1 \text {; } \\
1 \text { HE 12: } 1 \text {; and } \\
3 \text { other graves }\end{array}$ & $\begin{array}{l}\text { Publication in } \\
\text { preparation } \\
\text { (D Powlesland) }\end{array}$ \\
\hline 'Yorks' & Yorks & 6th $c$ & $\begin{array}{l}\text { Group: } 2 \text { examples } \\
\text { with other beads }\end{array}$ & Hull Museum \\
\hline $\begin{array}{l}\text { Clatchard } \\
\text { Craig }\end{array}$ & $\begin{array}{l}\text { Scotland: } \\
\text { Fife }\end{array}$ & $\begin{array}{l}\text { 6th- } \\
\text { 8th } c\end{array}$ & $\begin{array}{l}\text { Perhaps slight collar } \\
\text { at } 1 \text { end }\end{array}$ & $\begin{array}{l}\text { Close-Brooks 1986; } \\
\text { Edinburgh Museum }\end{array}$ \\
\hline iii & Blue $\mathrm{C}$ & YLINDER & $\begin{array}{l}\text { BEADS, SQUARE OR POI } \\
\text { LONG OR SHORT }\end{array}$ & GONAL SECTION, \\
\hline Frilford I & Berks & $\begin{array}{l}\text { prob } \\
5 \text { th- } \\
6 \text { th c }\end{array}$ & $\begin{array}{l}\text { Cemetery adjacent to a } \\
\text { Roman cemetery: } \\
\text { opaque hexagonal bead }\end{array}$ & $\begin{array}{l}\text { Akerman } 1865 ; \\
\text { Rolleston } 1869 ; \\
\text { BM OA } 4880\end{array}$ \\
\hline $\begin{array}{l}\text { Breach } \\
\text { Downs }\end{array}$ & Kent & $\begin{array}{l}\text { 7th- } \\
\text { early } \\
\text { 8th } c\end{array}$ & $\begin{array}{l}\text { Rather large semi- } \\
\text { translucent bright } \\
\text { pentagonal bead }\end{array}$ & $\begin{array}{l}\text { Conyngham and Akerman } \\
1844 ; \\
\text { BM 79 5-24 } 89\end{array}$ \\
\hline $\begin{array}{l}\text { Dover, } \\
\text { Buckland }\end{array}$ & Kent & $\begin{array}{l}575- \\
625 \\
625- \\
650\end{array}$ & $\begin{array}{l}\text { Grave 1: pentagonal (B47); } \\
\text { Grave 62: } 4 \text { pentagonals } \\
\text { (B47) }\end{array}$ & $\begin{array}{l}\text { Evison } 1987,74,216 \text { and } \\
234, \text { figs } 5.4 q \text { and } 35.62 .4 j \\
\text { BM }\end{array}$ \\
\hline Faversham & Kent & & $\begin{array}{l}\text { One square section, } \\
\text { dark glass }\end{array}$ & $\begin{array}{l}\text { Meaney } 1964,118-19 ; \\
\text { Ashmolean Museum } \\
1909.143\end{array}$ \\
\hline
\end{tabular}




\begin{tabular}{|c|c|c|c|c|}
\hline Finglesham & Kent & $\begin{array}{l}\text { first } \\
\text { half } \\
6 \text { th c }\end{array}$ & $\begin{array}{l}\text { Grave D3: long translucent } \\
\text { square section with great } \\
\text { square-headed brooch, etc }\end{array}$ & $\begin{array}{l}\text { Chadwick } 1958,12 \text {, fig } 5 \text { b, } \\
\text { pl III; } \\
\text { Deal Museum }\end{array}$ \\
\hline ‘Kent' & Kent & & $\begin{array}{l}\text { Several with long square } \\
\text { section }\end{array}$ & Canterbury Museum \\
\hline Kingston & Kent & $\begin{array}{l}\text { prob } \\
7 \text { th c }\end{array}$ & $\begin{array}{l}\text { Mid-blue pentagonal } \\
\text { (Plate 5) }\end{array}$ & $\begin{array}{l}\text { Faussett 1856, 35-94; } \\
\text { Liverpool Museum } 6327\end{array}$ \\
\hline Monkton & Kent & & One square-section & $\begin{array}{l}\text { Hawkes and Hogarth 1974; } \\
\text { Perkins and Hawkes 1984; } \\
\text { Ashmolean Museum }\end{array}$ \\
\hline $\begin{array}{l}\text { Morning } \\
\text { Thorpe }\end{array}$ & Norfolk & $\begin{array}{l}\text { 6th- } \\
7 \text { th c }\end{array}$ & $\begin{array}{l}\text { Grave } 375: 3 \text { pentagonal } \\
\text { roughly tapering with } \\
\text { scutiform pendant, etc }\end{array}$ & $\begin{array}{l}\text { Green } \text { et al } 1987,146 \text {, } \\
\text { fig } 434 . \text { Cii; } \\
\text { Norwich Museum }\end{array}$ \\
\hline $\begin{array}{l}\text { Mitchell's } \\
\text { Hill }\end{array}$ & Suffolk & $\begin{array}{l}\text { prob } \\
6 \text { th- } \\
7 \text { th c }\end{array}$ & $\begin{array}{l}\text { Long square section with } \\
\text { signs of red core at one end }\end{array}$ & $\begin{array}{l}\text { Meaney } 1964,231 \\
\text { Bury St Edmunds } \\
\text { Museum }\end{array}$ \\
\hline Snape & Suffolk & $\begin{array}{l}6 \text { th- } \\
7 \text { th } \mathrm{c}\end{array}$ & One pentagonal & $\begin{array}{l}\text { Filmer-Sankey and } \\
\text { Pestell forthcoming }\end{array}$ \\
\hline $\begin{array}{l}\text { Trimley St } \\
\text { Mary (near } \\
\text { Felixstowe) }\end{array}$ & Suffolk & & $\begin{array}{l}\text { No context: atypical } \\
\text { pentagonal bead }\end{array}$ & $\begin{array}{l}\text { Information from Tim } \\
\text { Sapwell, Norwich }\end{array}$ \\
\hline Mitcham & Surrey & $\begin{array}{l}\text { prob } \\
\text { 6th c }\end{array}$ & $\begin{array}{l}\text { Grave } 62: 1 \text { long square } \\
\text { section opaque; and another } \\
\text { from cemetery }\end{array}$ & $\begin{array}{l}\text { Bidder and Duckworth 1906; } \\
\text { Bidder and Morris 1959, } \\
64,110 \text {, pl XVI.62; } \\
\text { Cambridge Museum }\end{array}$ \\
\hline Selmeston & Sussex & $\begin{array}{l}\text { prob } \\
\text { 6th- } \\
7 \text { th c }\end{array}$ & $\begin{array}{l}\text { Translucent cobalt blue } \\
\text { pentagonal }\end{array}$ & $\begin{array}{l}\text { Welch } 1983,83 \text {, fig } 49 \text {; } \\
\text { Lewes Museum }\end{array}$ \\
\hline $\begin{array}{l}\text { Harnham } \\
\text { Hill }\end{array}$ & Wilts & 6th c & $\begin{array}{l}\text { Long opaque mid-blue } \\
\text { square section }\end{array}$ & $\begin{array}{l}\text { Akerman 1853; } \\
\text { Jackson 1854; } \\
\text { BM } 53 \text { 12-14 }\end{array}$ \\
\hline Sewerby & Yorks & $\begin{array}{l}\text { late } \\
\text { 6th c }\end{array}$ & $\begin{array}{l}\text { Grave 49: } 6 \text { long pale blue } \\
\text { square section (A5b) with } \\
\text { Class } C 2 \text { square-headed } \\
\text { cruciform brooch, etc }\end{array}$ & $\begin{array}{l}\text { Hirst } 1985,66 \text {, fig } 52 \text {, } \\
\text { String I.a and String Il.e }\end{array}$ \\
\hline iv & Blue $D$ & MOND & FACETED, CUBE-SHAPED & OR OBLONG BEADS \\
\hline $\begin{array}{l}\text { Springfield } \\
\text { Lyons }\end{array}$ & Essex & 6th $\mathrm{c}$ & $\begin{array}{l}\text { Grave 4882: translucent } \\
\text { cube with cruciform and } \\
\text { disc brooches }\end{array}$ & $\begin{array}{l}\text { Publication in } \\
\text { preparation (S Tyler); } \\
\text { BM }\end{array}$ \\
\hline Faversham & Kent & $\begin{array}{l}\text { 6th- } \\
7 \text { th } c\end{array}$ & $\begin{array}{l}\text { One oblong (also in pink } \\
\text { and green) }\end{array}$ & $\begin{array}{l}\text { Meaney 1964, 118-19; } \\
\text { Ashmolean Museum }\end{array}$ \\
\hline Finglesham & Kent & $\begin{array}{l}\text { first } \\
\text { half } \\
6 \text { th c }\end{array}$ & $\begin{array}{l}\text { Grave D3: light blue } \\
\text { translucent oblong with } \\
\text { great square-headed } \\
\text { brooch, etc }\end{array}$ & $\begin{array}{l}\text { Chadwick 1958, 12, fig 5c, } \\
\text { pl III; } \\
\text { Deal Museum }\end{array}$ \\
\hline
\end{tabular}


The Glass Beads of Anglo-Saxon England

\begin{tabular}{|c|c|c|c|c|}
\hline Howletts & Kent & 6th $c$ & $\begin{array}{l}\text { Translucent small } \\
\text { diamond-shaped }\end{array}$ & $\begin{array}{l}\text { Smith 1918; } \\
\text { BM 1936 5-11 } 144\end{array}$ \\
\hline Sarre & Kent & $\begin{array}{l}\text { prob } \\
\text { 6th } c\end{array}$ & String: dark blue oblong & $\begin{array}{l}\text { Meaney } 1964,135-6 ; \\
\text { BM 93 6-1 } 218\end{array}$ \\
\hline Stowting & Kent & $\begin{array}{l}\text { prob } \\
\text { 6th c }\end{array}$ & $\begin{array}{l}\text { Two mid-blue translucent } \\
\text { oblongs }\end{array}$ & $\begin{array}{l}\text { Meaney } 1964,137-8 ; \\
\text { Beck Collection, } \\
\text { Cambridge Museum; } \\
\text { Maidstone Museum }\end{array}$ \\
\hline Laceby & Lincs & $\begin{array}{l}6 \text { th- } \\
7 \text { th } c\end{array}$ & $\begin{array}{l}\text { Group: dark blue or } \\
\text { black cube }\end{array}$ & $\begin{array}{l}\text { Meaney } 1964,157 \\
\text { Lincoln Museum }\end{array}$ \\
\hline West Stow & Suffolk & $\begin{array}{l}5 \text { th- } \\
7 \text { th } c\end{array}$ & $\begin{array}{l}\text { Cemetery: at least one } \\
\text { cobalt blue translucent } \\
\text { and many very small jet } \\
\text { beads }\end{array}$ & $\begin{array}{l}\text { West } 1985,74 ; \\
\text { Bury St Edmunds } \\
\text { Museum }\end{array}$ \\
\hline Alfriston & Sussex & $\begin{array}{l}\text { late } \\
5 \text { th- } \\
6 \text { th c }\end{array}$ & $\begin{array}{l}\text { Grave 68: atypical dark } \\
\text { squarish with facets with } \\
\text { plain quoit brooch, etc }\end{array}$ & $\begin{array}{l}\text { Griffith and Salzmann } 1914 \\
\text { 47, pl I.1; Welch 1983, } 83 \text {, } \\
\text { fig 32c; Lewes Museum }\end{array}$ \\
\hline $\begin{array}{l}\text { Pewsey } \\
\text { Blackpatch }\end{array}$ & Wilts & $\begin{array}{l}\text { prob } \\
\text { 6th c }\end{array}$ & $\begin{array}{l}\text { Grave 53: bright blue } \\
\text { translucent cube } \\
\text { (Plate 5) }\end{array}$ & $\begin{array}{l}\text { Publication in } \\
\text { preparation (K Annable); } \\
\text { Devizes Museum }\end{array}$ \\
\hline $\begin{array}{l}\text { Londes- } \\
\text { borough }\end{array}$ & Yorks & $\begin{array}{l}\text { mid- } \\
6 \text { th } c\end{array}$ & $\begin{array}{l}\text { Grave 7: various poorly } \\
\text { described faceted beads } \\
\text { with Åberg Group IVa } \\
\text { cruciform brooch, etc }\end{array}$ & $\begin{array}{l}\text { Swanton } 1964,275 \text {, } \\
\text { fig } 8.6 \text {; } \\
\text { Cramp and Miket 1982, } \\
\text { 7, no. 4.2, fig 5; } \\
\text { Newcastle Museum }\end{array}$ \\
\hline Sewerby & Yorks & $\begin{array}{l}\text { late } \\
\text { 6th c }\end{array}$ & $\begin{array}{l}\text { Grave 49: pale blue slightly } \\
\text { faceted oblong (A5b) with } \\
\text { Class C2 square-headed } \\
\text { cruciform brooch, etc }\end{array}$ & $\begin{array}{l}\text { Hirst } 1985,66 \text {, fig 52, } \\
\text { String II.e }\end{array}$ \\
\hline \multirow[t]{3}{*}{$\begin{array}{l}\text { York, } \\
\text { Sycamore } \\
\text { Terrace }\end{array}$} & Yorks & $\begin{array}{l}3 \text { rd- } \\
\text { 4th c }\end{array}$ & $\begin{array}{l}\text { Many very small blue } \\
\text { opaque faceted with early } \\
\text { Christian bone inscription, } \\
\text { glass flagon, etc }\end{array}$ & $\begin{array}{l}\text { RCHM 1962, } \\
73 \text {, fig } 58\end{array}$ \\
\hline & \multirow[t]{2}{*}{ v Blue } & \multirow{2}{*}{\multicolumn{3}{|c|}{$\begin{array}{l}\text { OR 'BLACK' LARGE, FLAT POLYGONAL BEADS: } \\
\text { no schedule (Plate 5) }\end{array}$}} \\
\hline & & & & \\
\hline Kempston & Beds & $\begin{array}{l}\text { prob } \\
\text { 6th c }\end{array}$ & $\begin{array}{l}\text { Grave 16: } 2 \text { double } \\
\text { translucent rather } \\
\text { squared segments; } \\
\text { Grave 16: triple }\end{array}$ & $\begin{array}{l}\text { Fitch 1864; } \\
\text { BM } 916-2453 \text { and } 55\end{array}$ \\
\hline $\begin{array}{l}\text { Linton } \\
\text { Heath B }\end{array}$ & Cambs & $\begin{array}{l}\text { prob } \\
\text { 6th c }\end{array}$ & $\begin{array}{l}\text { Double string: } \\
1 \text { double bead }\end{array}$ & $\begin{array}{l}\text { Neville 1854; } \\
\text { Cambridge Museum }\end{array}$ \\
\hline $\begin{array}{l}\text { Norton- } \\
\text { on-Tees }\end{array}$ & Durham & 6th c & $\begin{array}{l}\text { Grave } 70: 1 \text { double with } \\
\text { annular brooch, B clasps, } \\
\text { etc }\end{array}$ & $\begin{array}{l}\text { Sherlock and Welch 1992, } \\
171 \text {, fig } 55.70 .2\end{array}$ \\
\hline
\end{tabular}




\begin{tabular}{|c|c|c|c|c|}
\hline $\begin{array}{l}\text { Great } \\
\text { Chesterford }\end{array}$ & Essex & $\begin{array}{l}\text { prob } \\
6 \text { th c }\end{array}$ & $\begin{array}{l}\text { Grave 31: one triple } \\
\text { bright translucent (B37) }\end{array}$ & $\begin{array}{l}\text { Evison } 1994,15,96 \text {, } \\
\text { fig } 27.31 .4 c ; \mathrm{BM}\end{array}$ \\
\hline Alton & Hants & $\begin{array}{l}5 \text { th- } \\
6 \text { th } c\end{array}$ & $\begin{array}{l}\text { Grave 9: one double } \\
\text { dark blue translucent }\end{array}$ & $\begin{array}{l}\text { Evison 1988a, 73, fig } 25.1 \text {; } \\
\text { Hampshire Museums }\end{array}$ \\
\hline Llanigon & $\begin{array}{l}\text { Here- } \\
\text { fords }\end{array}$ & $\begin{array}{l}\text { prob } \\
6 \text { th c }\end{array}$ & $\begin{array}{l}\text { Double opaque with other } \\
\text { beads }\end{array}$ & $\begin{array}{l}\text { Morgan and Marshall 1921; } \\
\text { Hereford Museum }\end{array}$ \\
\hline $\begin{array}{l}\text { Beakes- } \\
\text { bourne II }\end{array}$ & Kent & $\begin{array}{l}5 \text { th- } \\
6 \text { th c }\end{array}$ & Grave 22: 3 examples & $\begin{array}{l}\text { Meaney } 1964,109 \\
\text { Canterbury Museum }\end{array}$ \\
\hline $\begin{array}{l}\text { Dover, } \\
\text { Buckland }\end{array}$ & Kent & $\begin{array}{l}475- \\
525\end{array}$ & $\begin{array}{l}\text { Grave } 13: 1 \text { beaded and } \\
1 \text { twisted }(\mathrm{CO}-03) \text {; } \\
\text { Grave } 48: 1 \text { broken with } \\
5 \text { segments }(\mathrm{C} 02)\end{array}$ & $\begin{array}{l}\text { Evison } 1987,75,219,230 \text {, } \\
\text { figs } 9.3 \mathrm{f} \text { and } \mathrm{g}, 27.4 \mathrm{j} ; \\
\mathrm{BM}\end{array}$ \\
\hline Faversham & Kent & $\begin{array}{l}5 \text { th- } \\
7 \text { th c }\end{array}$ & $\begin{array}{l}\text { Several doubles and } \\
\text { one triple }\end{array}$ & $\begin{array}{l}\text { Meaney 1964, 118-19; } \\
\text { Ashmolean Museum }\end{array}$ \\
\hline Gilton & Kent & 7th $\mathrm{c}$ & About 7 fragments & $\begin{array}{l}\text { Faussett } 1856, \text { pls V and } \\
\text { VI; Liverpool Museum }\end{array}$ \\
\hline Orpington & Kent & $\begin{array}{l}5 \text { th- } \\
6 \text { th c }\end{array}$ & $\begin{array}{l}\text { Grave 58: one double with } \\
\text { disc brooch pair, etc }\end{array}$ & $\begin{array}{l}\text { Tester 1969, 44-6, } \\
\text { fig 4.58b; } \\
\text { Orpington Museum }\end{array}$ \\
\hline Polhill & Kent & 7th $\mathrm{c}$ & $\begin{array}{l}\text { Grave } 41 \text { : about } 7 \text { segments, } \\
\text { dark blue }\end{array}$ & $\begin{array}{l}\text { Hawkes and Philp 1973, } 177 \\
\text { 207, fig } 55.520\end{array}$ \\
\hline Sarre & Kent & $\begin{array}{l}\text { prob } \\
7 \text { th c }\end{array}$ & Group XCVII: 1 triple & $\begin{array}{l}\text { Brent } 1868 \\
\text { pl VIII.XCVII }\end{array}$ \\
\hline $\begin{array}{l}\text { Castle } \\
\text { Bytham }\end{array}$ & Lincs & 7th c & $\begin{array}{l}\text { One blue } 2 \text {-segmented with } \\
\text { quoit brooch }\end{array}$ & $\begin{array}{l}\text { Akerman 1855a, 26-7, } \\
\text { pl XII.4 }\end{array}$ \\
\hline Dragonby & Lincs & & $\begin{array}{l}\text { Small bright cobalt blue } \\
\text { triple }\end{array}$ & Lincoln Museum \\
\hline Sleaford & Lincs & $\begin{array}{l}\text { late } \\
5 \text { th- } \\
6 \text { th c }\end{array}$ & $\begin{array}{l}\text { Pale blue 2-segmented } \\
\text { with clasp }\end{array}$ & $\begin{array}{l}\text { Thomas 1887; } \\
\text { BM }\end{array}$ \\
\hline $\begin{array}{l}\text { Morning } \\
\text { Thorpe }\end{array}$ & Norfolk & $\begin{array}{l}\text { mid- } \\
6 \text { th } c\end{array}$ & $\begin{array}{l}\text { Grave 133: badly made } \\
\text { with Aberg Group IV } \\
\text { cruciform brooch, etc }\end{array}$ & $\begin{array}{l}\text { Green et al } 1987,73 \text {, } \\
\text { fig } 341.133 . \text { Hvi; } \\
\text { Norwich Museum }\end{array}$ \\
\hline $\begin{array}{l}\text { Spong } \\
\text { Hill }\end{array}$ & Norfolk & $\begin{array}{l}6 \text { th } c \\
6 \text { th } c\end{array}$ & $\begin{array}{l}\text { Grave 12: } 2 \text { doubles with } \\
\text { annular brooches; } \\
\text { Grave } 37: \text { badly made } \\
\text { triple with annular brooches } \\
\text { and B clasps }\end{array}$ & $\begin{array}{l}\text { Hills et al } 1984,61,86 \text {, } \\
\text { figs } 75.12 .4 \text { and } 90.37 .6 \text {; } \\
\text { Norfolk Arch Unit }\end{array}$ \\
\hline $\begin{array}{l}\text { Empingham } \\
\text { II }\end{array}$ & Rutland & $\begin{array}{l}5 \text { th- } \\
7 \text { th } c\end{array}$ & $\begin{array}{l}\text { Grave 85A: } 1 \text { double- } \\
\text { segmented bead }\end{array}$ & $\begin{array}{l}\text { Timby 1996; } \\
\text { Oakham Museum }\end{array}$ \\
\hline $\begin{array}{l}\text { Bury St } \\
\text { Edmunds, } \\
\text { Westgarth } \\
\text { Gardens }\end{array}$ & Suffolk & 6th $\mathrm{c}$ & $\begin{array}{l}\text { Grave 48: I double } \\
\text { translucent with small- } \\
\text { long brooches, clasps, etc }\end{array}$ & $\begin{array}{l}\text { West } 1988,32 \text {, } \\
\text { fig } 73 . \text { E 14; } \\
\text { Bury St Edmunds } \\
\text { Museum }\end{array}$ \\
\hline
\end{tabular}




\begin{tabular}{|c|c|c|c|c|}
\hline $\begin{array}{l}\text { Apple } \\
\text { Down I }\end{array}$ & Sussex & $\begin{array}{l}\text { first } \\
\text { half } \\
\text { 6th c }\end{array}$ & $\begin{array}{l}\text { Grave } 128: 1 \text { double and } \\
\text { many segments with } \\
\text { button brooch }\end{array}$ & $\begin{array}{l}\text { Down and Welch } 1990 \text {, } \\
51,164-5 \text {, fig } 2.41 ; \\
\text { Chichester Museum }\end{array}$ \\
\hline Highdown & Sussex & $\begin{array}{l}\text { prob } \\
\text { 6th c }\end{array}$ & $\begin{array}{l}\text { Atypical, about } 32 \text { small } \\
\text { doubles and triples }\end{array}$ & $\begin{array}{l}\text { Welch 1983, 83; } \\
\text { Worthing Museum } 3486\end{array}$ \\
\hline $\begin{array}{l}\text { Painsthorpe } \\
\text { Wold I }\end{array}$ & Yorks & 7 th $c$ & $\begin{array}{l}\text { Slatey-blue opaque, } \\
5 \text { to } 6 \text { segments twisted }\end{array}$ & $\begin{array}{l}\text { Mortimer 1905; } \\
\text { Hull Museum }\end{array}$ \\
\hline Saltburn & Yorks & $\begin{array}{l}\text { prob } \\
6 \text { th- } \\
7 \text { th c }\end{array}$ & Double, ?triple & $\begin{array}{l}\text { Gallagher } 1987,16,25 \text {, } \\
\text { fig } 5.41 \text {; } \\
\text { Middlesbrough Museum }\end{array}$ \\
\hline Sewerby & Yorks & $\begin{array}{l}\text { mid- } \\
6 \text { th } c\end{array}$ & $\begin{array}{l}\text { Grave 28: } 1 \text { small double } \\
\text { (A2b) with Åberg Group } \\
\text { IV cruciform brooch }\end{array}$ & Hirst 1985,66 , fig $42.28 . \mathrm{h}$ \\
\hline $\begin{array}{l}\text { West } \\
\text { Heslerton }\end{array}$ & Yorks & $\begin{array}{l}\text { prob } \\
\text { 6th c }\end{array}$ & $\begin{array}{l}\text { Grave } 1 \text { B 10: triple } \\
\text { with annular brooches; } \\
\text { Grave } 1 \text { B 17: double; } \\
\text { Grave } 1 \text { B 104: double } \\
\text { with annular brooch, etc }\end{array}$ & $\begin{array}{l}\text { Publication in } \\
\text { preparation } \\
\text { (D Powlesland) }\end{array}$ \\
\hline
\end{tabular}

vii Blue biconical Beads (OPAQUe or tRANSLUCENT)

\begin{tabular}{|c|c|c|c|c|}
\hline $\begin{array}{l}\text { Long } \\
\text { Wittenham }\end{array}$ & Berks & $\begin{array}{l}\text { 5th- } \\
6 \text { th } c\end{array}$ & $\begin{array}{l}\text { Grave } 142: 2 \text { translucent } \\
\text { with small-long brooches }\end{array}$ & Akerman 1860 \\
\hline Barrington & Cambs & $\begin{array}{l}\text { prob } \\
6 \text { th } c\end{array}$ & $\begin{array}{l}\text { Grave 10: very small } \\
\text { opaque }\end{array}$ & $\begin{array}{l}\text { Foster } 1880,16,32 \text {, } \\
\text { pl VI.3; } \\
\text { Beck Collection, } \\
\text { Cambridge Museum } \\
\text { 1 B } 47.183\end{array}$ \\
\hline $\begin{array}{l}\text { Hasling- } \\
\text { field }\end{array}$ & Cambs & $\begin{array}{l}5 \text { th- } \\
7 \text { th c }\end{array}$ & $\begin{array}{l}\text { Miscellaneous string: } \\
\text { more than } 1 \text { bead }\end{array}$ & $\begin{array}{l}\text { Fox 1923, 255-9; } \\
\text { Ashmolean Museum } \\
\text { 1909.233 }\end{array}$ \\
\hline $\begin{array}{l}\text { Springfield } \\
\text { Lyons }\end{array}$ & Essex & $\begin{array}{l}\text { 5th- } \\
6 \text { th } \mathrm{c}\end{array}$ & $\begin{array}{l}\text { Grave } 4882: 2 \text { translucent } \\
\text { and opaque with disc } \\
\text { brooches, etc }\end{array}$ & $\begin{array}{l}\text { Publication in } \\
\text { preparation (S Tyler); } \\
\text { BM }\end{array}$ \\
\hline $\begin{array}{l}\text { Winchester, } \\
\text { Lower } \\
\text { Brook } \\
\text { Street }\end{array}$ & Hants & $\begin{array}{l}\text { late } \\
7 \text { th- } \\
\text { 8th c }\end{array}$ & $\begin{array}{l}\text { Grave 23: small and thin } \\
\text { translucent with gold } \\
\text { pendants and silver wire } \\
\text { rings }\end{array}$ & $\begin{array}{l}\text { Hawkes } 1990 \mathrm{~b}, 632 \text {, } \\
\text { fig } 168.1996 \text {, } \\
\text { colour pl XLVII }\end{array}$ \\
\hline $\begin{array}{l}\text { Canterbury, } \\
\text { Cakebread } \\
\text { Robey V }\end{array}$ & Kent & c. 400 & $\begin{array}{l}\text { From multiple burial: } \\
3 \text { beads of varying sizes }\end{array}$ & $\begin{array}{l}\text { Information } \mathrm{T} \text { Tatton- } \\
\text { Brown and } \mathrm{P} \text { Garrard }\end{array}$ \\
\hline Faversham & Kent & $\begin{array}{l}\text { 5th- } \\
7 \text { th } c\end{array}$ & Four rather greenish-blue & $\begin{array}{l}\text { Meaney 1964, 118-19; } \\
\text { Ashmolean Museum } \\
1909.163\end{array}$ \\
\hline Gilton & Kent & $\begin{array}{l}\text { prob } \\
7 \text { th } c\end{array}$ & $\begin{array}{l}\text { With other beads: large } \\
\text { (Plate 5) }\end{array}$ & $\begin{array}{l}\text { Faussett 1856; } \\
\text { Liverpool Museum M } 6123\end{array}$ \\
\hline
\end{tabular}




\begin{tabular}{|c|c|c|c|c|}
\hline Howletts & Kent & 6th $c$ & Cobalt blue opaque & $\begin{array}{l}\text { Smith 1918; } \\
\text { BM } 1936 \text { 5-11 } 142\end{array}$ \\
\hline Kingston & Kent & $\begin{array}{l}\text { prob } \\
\text { 7the }\end{array}$ & $\begin{array}{l}\text { Mixed beads: } 1 \text { large with } \\
\text { flat top and bottom and } \\
\text { others }\end{array}$ & $\begin{array}{l}\text { Faussett 1856; } \\
\text { Liverpool Museum } \\
\text { M 6327: 'Kingston F' }\end{array}$ \\
\hline Polhill & Kent & 7th $\mathrm{c}$ & $\begin{array}{l}\text { Grave 41: } 2 \text { beads; } \\
\text { Grave 95: } 1 \text { bead (Plate 5) }\end{array}$ & $\begin{array}{l}\text { Hawkes and Philp 1973, 177, } \\
184,207 \text {, fig } 55.520 \text { and } 526\end{array}$ \\
\hline Stowting & Kent & & Very small & $\begin{array}{l}\text { Smith } 1846 a \text {; Brent } 1867 \text {; } \\
\text { Beck Collection, } \\
\text { Cambridge Museum } \\
47.1809\end{array}$ \\
\hline Thurnham & Kent & 7 th $\mathrm{c}$ & Two examples & Beck 1940 \\
\hline Laceby & Lincs & $\begin{array}{l}\text { 6th- } \\
\text { 7th } \mathrm{c}\end{array}$ & One or 2 small dark blue & $\begin{array}{l}\text { Thompson 1956, 184-9; } \\
\text { Lincoln Museum }\end{array}$ \\
\hline 'Lincs' & Lincs & & Mixed beads: 1 wide & Grantham Museum \\
\hline $\begin{array}{l}\text { Morning } \\
\text { Thorpe }\end{array}$ & Norfolk & 6th $\mathrm{c}$ & $\begin{array}{l}\text { Grave } 360: 1 \text { small wide } \\
\text { bead with Class } C \text { clasps, } \\
\text { annular brooch pair, etc }\end{array}$ & $\begin{array}{l}\text { Green et al 1987, } 140 \text {, } \\
\text { fig } 421 . F v ; \\
\text { Norwich Museum }\end{array}$ \\
\hline Guildown & Surrey & 6th $c$ & Grave 185 & $\begin{array}{l}\text { Lowther } 1931,11-12,43 \text {, } \\
\text { pl IX.3; } \\
\text { Guildford Museum }\end{array}$ \\
\hline \multirow[t]{2}{*}{ Wasperton } & Warks & $\begin{array}{l}\text { late } \\
\text { 5th } \mathrm{c}\end{array}$ & $\begin{array}{l}\text { F1579.3305 with applied } \\
\text { saucer brooches }\end{array}$ & $\begin{array}{l}\text { Publication in } \\
\text { preparation (M Carver); } \\
\text { Warwick Museum }\end{array}$ \\
\hline & BLUE N & \multicolumn{3}{|c|}{$\begin{array}{l}\text { MELON, SUB-MELON AND LOBED BEA } \\
\text { TRANSLUCENT) (see Map 18) }\end{array}$} \\
\hline $\begin{array}{l}\text { Chamber- } \\
\text { lain's } \\
\text { Barn II }\end{array}$ & Beds & $\begin{array}{l}\text { mid- } \\
\text { late } \\
7 \text { th } c\end{array}$ & $\begin{array}{l}\text { Grave } 40 \text { : large dark } \\
\text { translucent } 8 \text { lobes } \\
\text { (Plate 5) }\end{array}$ & $\begin{array}{l}\text { Hyslop 1963, 181, } \\
\text { fig 14e; } \\
\text { Luton Museum }\end{array}$ \\
\hline Kempston & Beds & & Small dark flattish: 4 lobes & $\begin{array}{l}\text { Fitch 1864; } \\
\text { BM 91 6-24 } 54\end{array}$ \\
\hline Wallingford & Berks & $\begin{array}{l}\text { 2nd } \\
\text { half } \\
5 \text { th c }\end{array}$ & $\begin{array}{l}\text { Grave } 15 \text { with applied } \\
\text { saucer brooch (floriate/ } \\
\text { anchor cross) pair and } 2 \\
\text { quoit brooches, etc }\end{array}$ & $\begin{array}{l}\text { Leeds 1938, 98-9, pl VII; } \\
\text { Welch 1975, 92; Evison } \\
\text { 1978; Böhme 1986, 545-7, } \\
\text { 571; Ashmolean Museum }\end{array}$ \\
\hline $\begin{array}{l}\text { Linton } \\
\text { Heath B }\end{array}$ & Cambs & $\begin{array}{l}\text { 6th- } \\
7 \text { th } c\end{array}$ & $\begin{array}{l}\text { Necklace: pale powder } \\
\text { blue, very damaged }\end{array}$ & $\begin{array}{l}\text { Neville 1854; } \\
\text { Cambridge Museum }\end{array}$ \\
\hline $\begin{array}{l}\text { Norton- } \\
\text { on-Tees }\end{array}$ & Durham & $\begin{array}{l}\text { 6th c } \\
\text { mid- } \\
\text { 6th c }\end{array}$ & $\begin{array}{l}\text { Grave 7: } 2 \text { translucent } \\
\text { with B clasps, etc; } \\
\text { Grave 102: } 1 \text { translucent } \\
\text { with A } 8 \text { berg Group IVa } \\
\text { cruciform brooch, etc }\end{array}$ & $\begin{array}{l}\text { Sherlock and Welch } 1992 \text {, } \\
125 \text { and } 186 \text {, figs } 34.7 .1 \\
\text { and } 62.102 .1\end{array}$ \\
\hline
\end{tabular}




\begin{tabular}{|c|c|c|c|}
\hline $\begin{array}{l}\text { Great } \\
\text { Chesterford }\end{array}$ & Essex & 6th $c$ & $\begin{array}{l}\text { Grave 103: flat bluish } \\
\text { opaque (B49) with applie } \\
\text { saucer brooch pair }\end{array}$ \\
\hline Lechlade & Glos & 6th $c$ & $\begin{array}{l}\text { Grave } 47 \text { : sub-melon witl } \\
\text { saucer brooch }\end{array}$ \\
\hline Alton & Hants & $\begin{array}{l}5 \text { th } c \\
5 \text { th- } \\
6 \text { th } c\end{array}$ & $\begin{array}{l}\text { Grave } 27: 1 \text { translucent } \\
\text { melon; } \\
\text { Grave } 33 \text { : with disc } \\
\text { pendant, etc }\end{array}$ \\
\hline
\end{tabular}

\begin{tabular}{|c|c|c|c|}
\hline Droxford & Hants & $\begin{array}{l}\text { late } \\
\text { 5th- } \\
\text { 6th c }\end{array}$ & $\begin{array}{l}\text { Grave 20: } 5 \text { translucent } \\
\text { beads (Class IIIB); } \\
\text { Grave 21: } 4 \text { beads (IIIB); } \\
\text { Grave 32: } 1 \text { bead (IIIB) }\end{array}$ \\
\hline $\begin{array}{l}\text { Winchester, } \\
\text { Lower } \\
\text { Brook } \\
\text { Street }\end{array}$ & Hants & $\begin{array}{l}\text { late } \\
\text { 7th- } \\
\text { 8th c }\end{array}$ & $\begin{array}{l}\text { Grave 23: large hole and } \\
\text { smaller lobes than usual } \\
\text { with gold pendants and } \\
\text { silver wire rings }\end{array}$ \\
\hline $\begin{array}{l}\text { Chessell } \\
\text { Down }\end{array}$ & $\begin{array}{l}\text { Isle of } \\
\text { Wight }\end{array}$ & 6th $c$ & $\begin{array}{l}\text { Small globular blue melon } \\
\text { (types } 11.3 \text { and } 4 \text { ) }\end{array}$ \\
\hline $\begin{array}{l}\text { Barfreston } \\
\text { or } \\
\text { Sibertswold }\end{array}$ & Kent & $\begin{array}{l}\text { 6th- } \\
7 \text { th c }\end{array}$ & $\begin{array}{l}\text { Two dark (?blue) flattish } \\
\text { 7-lobed }\end{array}$ \\
\hline $\begin{array}{l}\text { Beakes- } \\
\text { bourne }\end{array}$ & Kent & 7th $\mathrm{c}$ & Large flattish translucent \\
\hline Faversham & Kent & $\begin{array}{l}5 \text { th- } \\
7 \text { th c }\end{array}$ & $\begin{array}{l}\text { Several; } 2 \text { are dark } \\
\text { translucent irregular } \\
\text { 7-lobed }\end{array}$ \\
\hline Sarre & Kent & & $\begin{array}{l}\text { Small dark bead with } \\
\text { yellow above }\end{array}$ \\
\hline Stowting & Kent & 7th $c$ & Bright blue translucent bead \\
\hline Thurnham & Kent & $\begin{array}{l}\text { prob } \\
\text { 7th c }\end{array}$ & One melon \\
\hline $\begin{array}{l}\text { Castle } \\
\text { Bytham }\end{array}$ & Lincs & $\begin{array}{l}\text { prob } \\
\text { 7th c }\end{array}$ & $\begin{array}{l}\text { One double melon with } \\
\text { quoit brooch }\end{array}$ \\
\hline Fonaby & Lincs & 6th $\mathrm{c}$ & $\begin{array}{l}\text { Grave } 25: 1 \text { opaque with } \\
\text { girdle hangers, etc }\end{array}$ \\
\hline
\end{tabular}

Syston Lincs 6th- String: 2 with other beads

Park

Lincs $\quad 6$ th-

7 th $\mathrm{c}$
Evison 1994, 15, 46, 106, fig 42.103.3m;

BM 1964 7-2 328

Boyle et al forthcoming; Cirencester Museum

Evison 1988a, 78, 79, figs $30.1 \mathrm{c}$ and $33.8 \mathrm{~b}$; Hampshire Museums

Cook and Dacre 1985, 35, fig 59.20 and 42 , and colour frontispiece; Hampshire Museums

Aldsworth 1978, 124, 132,172 , figs 24.10 , 25.13 and 28.32.9; Hampshire Museums

Hawkes 1990b, 632, fig 168.2002, colour pl XLVII

Arnold 1982, 49-50, 121, colour frontispiece; $\mathrm{BM}$

Faussett 1856, pl V; Liverpool Museum M 6625.

Faussett 1856, pl V.4 and Diary V, 13, fig 3; Liverpool Museum

Meaney 1964, 118-19;

Ashmolean Museum

Meaney 1964, 135-6;

BM OA 4904

Meaney 1964, 137-8

Beck 1940

Akerman 1855a, 26-7, pl XII.4

Cook 1981, 28-30, fig 9.1.4; Scunthorpe Museum

Akerman 1855a, 41-2, pl XXI;

BM (18) 11 11-92 


\begin{tabular}{|c|c|c|c|c|}
\hline $\begin{array}{l}\text { Morning } \\
\text { Thorpe }\end{array}$ & Norfolk & $\begin{array}{l}\text { 6th- } \\
7 \text { th c }\end{array}$ & $\begin{array}{l}\text { Grave 309: } 2 \text { with drum } \\
\text { bead, etc; }\end{array}$ & $\begin{array}{l}\text { Green et al 1987, 120, } \\
\text { fig 396.309.Avii; } \\
\text { Norwich Museum }\end{array}$ \\
\hline $\begin{array}{l}\text { Spong } \\
\text { Hill }\end{array}$ & Norfolk & 6th $c$ & $\begin{array}{l}\text { Grave 37: } 1 \text { small with B } \\
\text { clasps, annular brooch } \\
\text { pair, etc }\end{array}$ & $\begin{array}{l}\text { Hills et al } 1984,86 \text {, } \\
\text { fig } 90.38 .6 \text {; } \\
\text { Norfolk Arch Unit }\end{array}$ \\
\hline Thornham & Norfolk & 7th $c$ & Opaque blue & $\begin{array}{l}\text { Norwich Museum } \\
\text { records }\end{array}$ \\
\hline Brixworth & Northants & $\begin{array}{l}\text { 6th- } \\
7 \text { th c }\end{array}$ & Necklace: 1 opaque blue & $\begin{array}{l}\text { Smith 1902, 245-6; } \\
\text { Northampton Museum }\end{array}$ \\
\hline Duston & Northants & $\begin{array}{l}5 \text { th- } \\
7 \text { th c }\end{array}$ & & $\begin{array}{l}\text { George 1903; } \\
\text { Northampton Museum }\end{array}$ \\
\hline $\begin{array}{l}\text { Cassington, } \\
\text { Purwell } \\
\text { Farm }\end{array}$ & Oxon & 6th $c$ & $\begin{array}{l}\text { Translucent dark blue } \\
\text { with other beads including } \\
\text { millefiori beads }\end{array}$ & $\begin{array}{l}\text { Leeds and Riley } 1942,64 \text {, } \\
\text { pl VIB; } \\
\text { Ashmolean Museum }\end{array}$ \\
\hline $\begin{array}{l}\text { Empingham } \\
\text { II }\end{array}$ & Rutland & $\begin{array}{l}\text { 5th- } \\
7 \text { th c }\end{array}$ & & $\begin{array}{l}\text { Timby 1996; } \\
\text { Oakham Museum }\end{array}$ \\
\hline Ipswich & Suffolk & $\begin{array}{l}\text { 6th- } \\
7 \text { th c }\end{array}$ & A few & $\begin{array}{l}\text { Layard } 1907 \text { and 1909; } \\
\text { Ipswich Museum }\end{array}$ \\
\hline $\begin{array}{l}\text { Apple } \\
\text { Down I }\end{array}$ & Sussex & $\begin{array}{l}\text { prob } \\
\text { 6th c }\end{array}$ & $\begin{array}{l}\text { Grave } 38: 2 \text { translucent } \\
\text { sub-melons }\end{array}$ & $\begin{array}{l}\text { Down and Welch } 1990,40 \text {, } \\
\text { 162, fig 2.24.38.1, } \\
\text { colour pl 40.6; } \\
\text { Chichester Museum }\end{array}$ \\
\hline Selmeston & Sussex & $\begin{array}{l}\text { 5th- } \\
7 \text { th } c\end{array}$ & & $\begin{array}{l}\text { Welch } 1983,83 \text {, fig } 49 \text { a.5; } \\
\text { Lewes Museum }\end{array}$ \\
\hline Sewerby & Yorks & 6th c & $\begin{array}{l}\text { Grave 41: translucent with } \\
6 \text { lobes (A4a) with annular } \\
\text { brooch pair, etc; } \\
\text { Grave 19: translucent with } \\
9 \text { lobes (A4c) with great } \\
\text { square-headed brooch, etc }\end{array}$ & $\begin{array}{l}\text { Hirst } 1985,66 \text {, figs } 48 b \\
\text { and } 39.10\end{array}$ \\
\hline $\begin{array}{l}\text { West } \\
\text { Heslerton }\end{array}$ & Yorks & $\begin{array}{l}\text { prob } \\
\text { 6th } c\end{array}$ & Grave 188 & $\begin{array}{l}\text { Publication in } \\
\text { preparation (D Powlesland) }\end{array}$ \\
\hline 'Yorks' & Yorks & $\begin{array}{l}\text { 6th- } \\
7 \text { th } c\end{array}$ & A few & $\begin{array}{l}\text { Yorkshire Museum } \\
\text { 1977.7.1734; } \\
\text { BM } 5315 \text { and } 16\end{array}$ \\
\hline
\end{tabular}

\section{POLYCHROME}

ix Blue beAds With WHITE OR CREAM WAVES, OR Zigzags

$\begin{array}{lllll}\text { Chamber- } & \text { Beds } & \begin{array}{l}\text { mid- } \\ \text { late }\end{array} & \begin{array}{l}\text { Grave 9: irregular } \\ \text { wave on translucent }\end{array} & \begin{array}{l}\text { Hyslop 1963, 173, } \\ \text { fig 9b; }\end{array} \\ \text { Barn II } & & \text { 7th c } & \text { light blue } & \text { Luton Museum } \\ \text { Cow Low } & \text { Derbys } & \text { 7th c } & & \text { Ozanne 1963, 28-9 }\end{array}$




\begin{tabular}{|c|c|c|c|c|}
\hline $\begin{array}{l}\text { Norton- } \\
\text { on-Tees }\end{array}$ & Durham & $\begin{array}{l}\text { late } \\
5 \text { th- } \\
\text { early } \\
6 \text { th } c\end{array}$ & $\begin{array}{l}\text { Grave 1: very irregular } \\
\text { opaque blue with } \AA \text { berg } \\
\text { Group II cruciform brooch, } \\
\text { etc }\end{array}$ & $\begin{array}{l}\text { Vyner 1984; } \\
\text { Sherlock and Welch 1992, } \\
\text { 121, fig 33.1.1 }\end{array}$ \\
\hline Cirencester & Glos & & $\begin{array}{l}\text { Miscellaneous: small } \\
\text { dark trail with } \\
\text { yellow zigzag }\end{array}$ & $\begin{array}{l}\text { Brown 1976, 25, } \\
\text { fig 3.2.10; } \\
\text { Cirencester Museum } \\
\text { C } 917\end{array}$ \\
\hline $\begin{array}{l}\text { Dover, } \\
\text { Buckland }\end{array}$ & Kent & $\begin{array}{l}650- \\
675\end{array}$ & $\begin{array}{l}\text { Grave 133: one (D08) } \\
\text { (Plate 5) with amethyst } \\
\text { beads, etc }\end{array}$ & $\begin{array}{l}\text { Evison } 1987,76,82,245 \text {, } \\
\text { fig 55.133.1c, colour } \\
\text { pl III (D08); BM }\end{array}$ \\
\hline Faversham & Kent & $\begin{array}{l}\text { 5th- } \\
7 \text { th c }\end{array}$ & $\begin{array}{l}\text { Annular with white } \\
\text { zigzag }\end{array}$ & $\begin{array}{l}\text { Meaney 1964, 118-19; } \\
\text { Ashmolean Museum } \\
1909.155\end{array}$ \\
\hline Kingston & Kent & $\begin{array}{l}\text { prob } \\
\text { 7th c }\end{array}$ & & $\begin{array}{l}\text { Faussett MS Diary III 33; } \\
\text { Liverpool Museum M } 6166\end{array}$ \\
\hline Sibertswold & Kent & 7 th $c$ & $\begin{array}{l}\text { Grave 172: very dark } \\
\text { blue with yellow } \\
\text { spacious wave }\end{array}$ & $\begin{array}{l}\text { Faussett 1856, 130-2; } \\
\text { Faussett MS Diary IV, 48; } \\
\text { Liverpool Museum M } 6519\end{array}$ \\
\hline Saxby & Leics & & $\begin{array}{l}\text { One globular with white } \\
\text { waves }\end{array}$ & $\begin{array}{l}\text { Publication in } \\
\text { preparation (J Timby) }\end{array}$ \\
\hline $\begin{array}{l}\text { Castle } \\
\text { Bytham }\end{array}$ & Lincs & $\begin{array}{l}\text { prob } \\
\text { 7th c }\end{array}$ & $\begin{array}{l}\text { One with white zigzags } \\
\text { with quoit brooch }\end{array}$ & $\begin{array}{l}\text { Akerman 1855a, 26-7, } \\
\text { pl XII.4 }\end{array}$ \\
\hline Sleaford & Lincs & $\begin{array}{l}\text { prob } \\
\text { 6th c }\end{array}$ & $\begin{array}{l}\text { Grave 191: ?on } \\
\text { pendant with greenish wave }\end{array}$ & $\begin{array}{l}\text { Thomas } 1887,401 \text {, } \\
\text { pl XXIV.3; BM }\end{array}$ \\
\hline Kenninghall & Norfolk & $\begin{array}{l}\text { 5th- } \\
\text { 6th c }\end{array}$ & String: 1 small bead & $\begin{array}{l}\text { Manning } 1872 a, 292 \text {, and } \\
\text { 1872b; } \\
\text { BM } 837-231\end{array}$ \\
\hline $\begin{array}{l}\text { Spong } \\
\text { Hill }\end{array}$ & Norfolk & $\begin{array}{l}\text { 5th- } \\
\text { 6th c }\end{array}$ & $\begin{array}{l}\text { Cremation stray finds: } \\
2 \text { beads and glass vessel } \\
\text { sherds }\end{array}$ & $\begin{array}{l}\text { Hills and Penn 1981, 66, } \\
\text { fig 166.241; } \\
\text { Norfolk Arch Unit }\end{array}$ \\
\hline $\begin{array}{l}\text { Empingham } \\
\text { II }\end{array}$ & Rutland & $\begin{array}{l}5 \text { th- } \\
7 \text { th c }\end{array}$ & One translucent melon & $\begin{array}{l}\text { Timby 1996; } \\
\text { Oakham Museum }\end{array}$ \\
\hline Brandon & Suffolk & $\begin{array}{l}\text { mid- } \\
\text { 7th- } \\
9 \text { th c }\end{array}$ & $\begin{array}{l}\text { Settlement: globular } \\
\text { with irregular wave }\end{array}$ & $\begin{array}{l}\text { Carr et al 1988; } \\
\text { publication in } \\
\text { preparation (R Carr) }\end{array}$ \\
\hline $\begin{array}{l}\text { Mitchell's } \\
\text { Hill }\end{array}$ & Suffolk & $\begin{array}{l}\text { 6th- } \\
7 \text { th } c\end{array}$ & $\begin{array}{l}\text { Two translucent } \\
\text { annulars with white wave }\end{array}$ & $\begin{array}{l}\text { Meaney 1964, 231; } \\
\text { Bury St Edmunds } \\
\text { Museum }\end{array}$ \\
\hline West Stow & Suffolk & $\begin{array}{l}5 \text { th- } \\
7 \text { th } c\end{array}$ & $\begin{array}{l}\text { Cemetery: broken wave } \\
\text { on translucent cobalt } \\
\text { blue bead }\end{array}$ & $\begin{array}{l}\text { West } 1985,74 ; \\
\text { Ashmolean Museum } \\
1909.424\end{array}$ \\
\hline Guildown & Surrey & 6th $c$ & $\begin{array}{l}\text { Grave 206: with square- } \\
\text { headed brooch pair, etc }\end{array}$ & $\begin{array}{l}\text { Lowther } 1931,11,44 \text {, } \\
\text { pl IX.1; } \\
\text { Guildford Museum } \\
\text { AS } 7391\end{array}$ \\
\hline
\end{tabular}




$\begin{array}{lllll}\begin{array}{l}\text { Kingston- } \\ \text { by-Lewes }\end{array} & \text { Sussex } & \text { 6th c } & \text { Annular } & \begin{array}{l}\text { Craddock 1979, pl 12; } \\ \text { Welch 1983, fig 73f; } \\ \text { Lewes Museum }\end{array} \\ \text { Selmeston } & \text { Sussex } & \begin{array}{l}\text { prob } \\ \text { 6th c }\end{array} & \begin{array}{l}\text { Necklace: small } \\ \text { annular with amber } \\ \text { and glass beads }\end{array} & \begin{array}{l}\text { Welch 1983, fig 49; } \\ \text { Lewes Museum }\end{array} \\ \begin{array}{l}\text { Broadway } \\ \text { Hill }\end{array} & \text { Worcs } & \begin{array}{l}\text { late } \\ \text { 5th- } \\ \text { 6th c }\end{array} & \begin{array}{l}\text { Disturbed finds from } \\ \text { inhumation graves }\end{array} & \text { Cook 1958,68 } \\ & & & \end{array}$

X Blue beads with CRossed waves, With or without dots

$\begin{array}{lllll}\begin{array}{l}\text { Dover, } \\ \text { Buckland }\end{array} & \text { Kent } & \begin{array}{l}575- \\ 625\end{array} & \begin{array}{l}\text { Grave 29: globular } \\ \text { dark translucent with } \\ \text { darker blue swags, } \\ \text { crossed waves and red } \\ \text { dots (D44); }\end{array} & \begin{array}{l}\text { Evison 1987, 77, 82, } \\ \text { 223-4 and 245, } \\ \text { figs 17.2q and 54.132.2d; }\end{array} \\ \text { BM }\end{array}$

\begin{tabular}{|c|c|c|c|c|}
\hline Sewerby & Yorks & 6th $c$ & $\begin{array}{l}\text { Grave 19: globular with } \\
\text { white waves and red spots } \\
\text { (C3) with great square- } \\
\text { headed brooch, etc }\end{array}$ & Hirst 1985,68 , fig 39.9 \\
\hline $\begin{array}{l}\text { West } \\
\text { Heslerton }\end{array}$ & Yorks & & $\begin{array}{l}\text { One with yellow wave and } \\
\text { dot }\end{array}$ & $\begin{array}{l}\text { Publication in } \\
\text { preparation (D Powlesland) }\end{array}$ \\
\hline$x i$ & BLUE BEADS & WITH & WHITE AND COLOURED & SPECKS (see Map 19) \\
\hline Kempston & Beds & $\begin{array}{l}\text { prob } \\
6 \text { th } c\end{array}$ & $\begin{array}{l}\text { Grave } 16: 7 \text { varied } \\
\text { shapes, translucent, with } \\
\text { terracotta and white specks }\end{array}$ & $\begin{array}{l}\text { Fitch } 1864 ; \\
\text { BM } 916-2451 \text { and } 53\end{array}$ \\
\hline
\end{tabular}




\begin{tabular}{|c|c|c|c|}
\hline $\begin{array}{l}\text { Long } \\
\text { Wittenham }\end{array}$ & Berks & $\begin{array}{l}5 \text { th- } \\
6 \text { th } c\end{array}$ & $\begin{array}{l}\text { Grave 53: } 1 \text { translucent } \\
\text { with red and white specks, } \\
\text { another with white } \\
\text { specks, both with } \\
\text { saucer brooches; } \\
\text { Grave 142: } 6 \text { translucent } \\
\text { with varied coloured specks } \\
\text { and pair small-long brooches }\end{array}$ \\
\hline
\end{tabular}

Hasling- Cambs 6th $\mathrm{c}$

field

Colchester Essex

Mucking II Essex

Springfield Essex

Lyons

Chessell Isle of

Down Wight

Faversham Kent 5th-

7 th $\mathrm{c}$

Lyminge Kent 6th c

II

Saxby Leics

Morning Norfolk 5th-

Thorpe

5 th-

6th c

5 th-

6th $c$

6th c

5 th-

6th c

2nd

half

5th c

6th c
With miscellaneous

beads: 2 flattened

above and below with

white and terracotta specks

prob Yellow specks

Grave 584: 2 beads with

2 small-long brooches, etc;

Grave 843: 2 globulars with

red and white crumbs with

small square-headed

brooch pair, glass claw

beaker, etc;

Grave 845: 5 globulars with

white and red crumbs with

disc brooch pair;

Grave 924B: green-blue

with red, white and yellow

specks with 2 applied

brooches, Kempston type

glass cone beaker

Grave 6033: light blue

with terracotta specks

Annular with white, green and red (type 6.17)

Annular with red and white

Grave 24: with red spots

with equal-arm brooches

Globular with red, white and black specks

Grave 90: white and

terracotta with Åberg

Group II cruciform

brooches, B clasp, etc;
Fox 1923, 255-9;

Ashmolean Museum

Information from

P Crummy

Hirst and Clark forthcoming;

BM

Publication in

preparation (S Tyler);

BM

Arnold 1982, 50, 120, colour frontispiece; $\mathrm{BM}$

Meaney 1964, 118-19;

Ashmolean Museum

Warhurst 1955, 18, pl VIIb.1;

Maidstone Museum

Publication in preparation (J Timby)

Green et al 1987, 60, 42-3, 65-6 and 73,

figs 324.Axxii, 303.Dviii, 334.N/Oviii and 341.133.Hv; 


\begin{tabular}{|c|c|c|c|c|}
\hline & & $\begin{array}{l}\text { first } \\
\text { half } \\
\text { 6th c }\end{array}$ & $\begin{array}{l}\text { Grave 30: } 1 \text { with } \\
\text { white specks and } 1 \text { white and } \\
\text { terracotta specks with } \\
\text { Aberg Group II and III } \\
\text { brooches, etc; } \\
\text { Grave 108: } 2 \text { with terracotta } \\
\text { green and white specks with } \\
\text { annular brooch pair, } \\
\text { B clasps, etc; } \\
\text { Grave } 133 \text { : white terracotta } \\
\text { and green with Aberg Group } \\
\text { IV brooch, etc }\end{array}$ & Norwich Museum \\
\hline $\begin{array}{l}\text { Spong } \\
\text { Hill }\end{array}$ & Norfolk & $\begin{array}{l}5 \text { th- } \\
6 \text { th c } \\
\text { late } \\
5 \text { th- } \\
6 \text { th c } \\
\text { mid- } \\
6 \text { th c }\end{array}$ & $\begin{array}{l}\text { Cremation 1952: very burnt } \\
\text { with terracotta specks; } \\
\text { Grave 5: green and terracotta } \\
\text { with small-long brooch } \\
\text { pair, A clasps, etc; } \\
\text { Grave 39: green and white } \\
\text { with Aberg Group IV } \\
\text { cruciform brooch, etc }\end{array}$ & $\begin{array}{l}\text { Hills and Penn 1981, 44, } \\
\text { fig 165.1952; } \\
\text { Hills et al } 1984,55 \text { and } \\
90-1, \text { figs } 73.6 \mathrm{c} \text { and } \\
94.5 \text {; } \\
\text { Norfolk Arch Unit }\end{array}$ \\
\hline Duston & Northants & $\begin{array}{l}\text { 6th- } \\
7 \text { th } c\end{array}$ & $\begin{array}{l}\text { One with red and white } \\
\text { specks }\end{array}$ & $\begin{array}{l}\text { George 1903; } \\
\text { Northampton Museum }\end{array}$ \\
\hline $\begin{array}{l}\text { Mitchell's } \\
\text { Hill }\end{array}$ & Suffolk & & $\begin{array}{l}\text { One with terracotta, } \\
\text { blue and white specks }\end{array}$ & $\begin{array}{l}\text { Meaney 1964,231; } \\
\text { Bury St Edmunds } \\
\text { Museum }\end{array}$ \\
\hline Wasperton & Warks & 6th $c$ & $\begin{array}{l}\text { Dark translucent with } \\
\text { red and white specks }\end{array}$ & $\begin{array}{l}\text { Publication in } \\
\text { preparation (M Carver); } \\
\text { Warwick Museum }\end{array}$ \\
\hline $\begin{array}{l}\text { Peters- } \\
\text { finger }\end{array}$ & Wilts & 6th $c$ & $\begin{array}{l}\text { Grave 29: light translucent } \\
\text { appearing 'black' with red, } \\
\text { yellow and blue specks } \\
\text { with applied saucer } \\
\text { brooch pair, etc }\end{array}$ & $\begin{array}{l}\text { Leeds and Shortt } 1953 \text {, } \\
\text { 24-5, pl III.104; } \\
\text { Salisbury Museum }\end{array}$ \\
\hline $\begin{array}{l}\text { Broadway } \\
\text { Hill }\end{array}$ & Worcs & $\begin{array}{l}\text { late } \\
\text { 5th- } \\
\text { 6th } c\end{array}$ & $\begin{array}{l}\text { Disturbed finds from } \\
\text { inhumation graves: } \\
2 \text { with light blue or white } \\
\text { specks and } 2 \text { with red spots } \\
\text { as well }\end{array}$ & $\begin{array}{l}\text { Cook 1958, 68, } \\
\text { fig } 9.8\end{array}$ \\
\hline Driffield & Yorks & & $\begin{array}{l}\text { One with light blue, } \\
\text { red and white specks }\end{array}$ & $\begin{array}{l}\text { Mortimer 1905; } \\
\text { Hull Museum }\end{array}$ \\
\hline Sewerby & Yorks & $\begin{array}{l}\text { first } \\
\text { half } \\
\text { 6th c }\end{array}$ & $\begin{array}{l}\text { Grave 35: green and red } \\
\text { specks (C5b) with Aberg } \\
\text { Group III cruciform } \\
\text { brooch, etc }\end{array}$ & Hirst 1985,68 , fig $44 a$ \\
\hline $\begin{array}{l}\text { Cilgwyn } \\
\text { Pontfaen }\end{array}$ & $\begin{array}{l}\text { Wales: } \\
\text { Brecon }\end{array}$ & & $\begin{array}{l}\text { Stray find: red and white } \\
\text { specks on royal blue }\end{array}$ & $\begin{array}{l}\text { Information from } \\
\text { G C Boon }\end{array}$ \\
\hline
\end{tabular}


xii Blue beads With Red spots (see Map 20)

$\begin{array}{llll}\text { Barrington } & \text { Cambs } & \begin{array}{l}5 \text { th- } \\ 7 \text { th c }\end{array} & \begin{array}{l}\text { Mixed collection: } \\ \text { slightly biconical }\end{array} \\ \begin{array}{llll}\text { Little } \\ \text { Wilbraham }\end{array} & \text { Cambs } & \begin{array}{l}5 \text { th- } \\ 7 \text { th c }\end{array} & \begin{array}{l}\text { Unassociated group: } \\ \text { slightly biconical; } \\ \text { possibly 7th century }\end{array} \\ \text { Lechlade } & \text { Glos } & \text { 6th c } & \begin{array}{l}\text { Grave 17: blue with spots } \\ \text { with keystone garnet disc } \\ \text { brooch, etc (Plate 5); } \\ \text { Grave 101: globular (as } \\ \text { grave 17 above) with } \\ \text { applied saucer brooch }\end{array} \\ & & 6 \text { th c } & \\ \text { Dover, } & \text { Kent } & 650- & \begin{array}{l}\text { Grave 157: annular with } \\ \text { few spots }\end{array}\end{array}$

$\begin{array}{llll}\text { Harrietsham } & \text { Kent } & \begin{array}{l}\text { prob } \\ \text { 7th c }\end{array} & \begin{array}{l}\text { Necklace: small annular } \\ \text { wide hole }\end{array} \\ \text { Lyminge } & \text { Kent } & \text { first } & \text { Grave 39: 2 annulars } \\ \text { half } & \text { and 2 globulars with } \\ \text { 6th c } & \begin{array}{l}\text { small square-headed } \\ \text { and saucer brooch pairs; }\end{array} \\ & & \text { first } & \text { Grave 24: with other beads } \\ \text { half } & \text { and with equal-arm brooches } \\ & & \text { 6th c }\end{array}$

\begin{tabular}{|c|c|c|c|c|}
\hline Polhill & Kent & 7th $c$ & $\begin{array}{l}\text { Grave 102: dark blue or } \\
\text { black (?6th-century), } \\
\text { survival }\end{array}$ & $\begin{array}{l}\text { Hawkes and Philp 1973, } \\
185,207 \text {, fig 55.527 }\end{array}$ \\
\hline Sarre & Kent & $\begin{array}{l}5 \text { th- } \\
7 \text { th c }\end{array}$ & $\begin{array}{l}\text { Two beads slightly } \\
\text { biconical with white } \\
\text { girth line, atypical }\end{array}$ & $\begin{array}{l}\text { Meaney 1964, 135-6; } \\
\text { BM 93 6-1 218 }\end{array}$ \\
\hline Spong Hill & Norfolk & $\begin{array}{l}\text { 5th- } \\
\text { 6th c }\end{array}$ & $\begin{array}{l}\text { Stray find: globular } \\
\text { slightly dished top, } \\
\text { messy spots }\end{array}$ & $\begin{array}{l}\text { Hills } 1977,67, \\
\text { fig } 127.179 ; \\
\text { Norfolk Arch Unit }\end{array}$ \\
\hline $\begin{array}{l}\text { Bury St } \\
\text { Edmunds, } \\
\text { Westgarth } \\
\text { Gardens }\end{array}$ & Suffolk & $\begin{array}{l}\text { 5th- } \\
\text { 6th c }\end{array}$ & $\begin{array}{l}\text { Grave 52: annular with red } \\
\text { spot ? with Åberg Group } \\
\text { II cruciform brooch, etc }\end{array}$ & $\begin{array}{l}\text { West 1988, 33-4, } \\
\text { figs 76-77; Bury St } \\
\text { Edmunds Museum }\end{array}$ \\
\hline Ashtead & Surrey & 7 th $c$ & $\begin{array}{l}\text { S14: slightly barrel-shaped } \\
\text { with pairs of red spots } \\
\text { with amethyst beads }\end{array}$ & $\begin{array}{l}\text { Poulton } 1989,70,95-6 \text {, } \\
\text { fig } 3.15 \mathrm{a}\end{array}$ \\
\hline Guildown & Surrey & $\begin{array}{l}5 \text { th- } \\
\text { 6th c }\end{array}$ & Grave 185 & $\begin{array}{l}\text { Lowther } 1931,11-12,43 \text {, } \\
\text { pl IX.3; } \\
\text { Guildford Museum }\end{array}$ \\
\hline Alfriston & Sussex & $\begin{array}{l}\text { 5th- } \\
\text { 6th c }\end{array}$ & $\begin{array}{l}\text { Grave } 87 \text { : several } \\
\text { with saucer brooch pair; }\end{array}$ & $\begin{array}{l}\text { Griffith } 1915,147 \text {; } \\
\text { Griffith and Salzmann 1914, }\end{array}$ \\
\hline
\end{tabular}

Evison 1987, 77, 82, 250, fig 61.157.If, colour pl IV (D56); BM

Maidstone Museum AS 356

Warhurst 1955, 27, 18, pl VIIb.2;

Maidstone Museum

Fox 1923, 250-2;

Neville 1852;

Fox 1923, 260-2

Boyle et al forthcoming; Cirencester Museum 
$\begin{array}{ll}\text { first } & \text { Grave 28: } 2 \text { with great } \\ \text { half } & \text { square-headed brooch, } \\ \text { 6th c } & \text { etc; } \\ \text { first } & \text { Grave 47: with small } \\ \text { half } & \text { square-headed brooch } \\ \text { 6th c } & \text { pair, etc; } \\ \text { 6th c } & \text { Grave 64: with amber beads }\end{array}$

Xiii Blue beads With Red-CENTRED White Eyes

\begin{tabular}{|c|c|c|c|c|}
\hline & xiii & LUE B & DS WITH RED-CENTRED & WHITE EYES \\
\hline Barrington & Cambs & $\begin{array}{l}\text { 5th- } \\
7 \text { th } c\end{array}$ & $\begin{array}{l}\text { Thick annular with } 3 \\
\text { equidistant eyes }\end{array}$ & $\begin{array}{l}\text { Fox } 1923,250-2 ; \\
\text { Ashmolean Museum }\end{array}$ \\
\hline $\begin{array}{l}\text { Hasling- } \\
\text { field }\end{array}$ & Cambs & $\begin{array}{l}\text { prob } \\
7 \text { th c }\end{array}$ & $\begin{array}{l}\text { Small annular with } \\
\text { blue-grey ground }\end{array}$ & $\begin{array}{l}\text { Fox } 1923,255-9 ; \\
\text { Mortimer Collection, } \\
\text { Hull Museum }\end{array}$ \\
\hline Alton & Hants & 7 th $c$ & $\begin{array}{l}\text { Grave } 39: \text { white and red } \\
\text { ring spot }\end{array}$ & $\begin{array}{l}\text { Evison } 1988 \mathrm{a}, 80-1 \text {, } \\
\text { fig } 33.1 \mathrm{~m} \text {; } \\
\text { Hampshire Museums }\end{array}$ \\
\hline Faversham & Kent & $\begin{array}{l}\text { 5th- } \\
7 \text { th c }\end{array}$ & $\begin{array}{l}\text { Dark blue globular } \\
\text { with } 3 \text { well-made eyes }\end{array}$ & $\begin{array}{l}\text { Meaney } 1964,118-19 \\
\text { Ashmolean Museum } \\
1909.170\end{array}$ \\
\hline Sarre & Kent & $\begin{array}{l}\text { prob } \\
\text { 6th c }\end{array}$ & $\begin{array}{l}\text { Miscellaneous } \\
\text { collection }\end{array}$ & $\begin{array}{l}\text { Meaney } 1964,135-6 \text {; } \\
\text { BM OA } 4904\end{array}$ \\
\hline $\begin{array}{l}\text { Sibertswold } \\
\text { or } \\
\text { Barfreston }\end{array}$ & Kent & $\begin{array}{l}\text { 6th- } \\
7 \text { th } c\end{array}$ & $\begin{array}{l}\text { Three eyes (Plate } 5 \text { ), } \\
\text { as Faversham above }\end{array}$ & $\begin{array}{l}\text { Faussett 1856, pl V; } \\
\text { Liverpool Museum }\end{array}$ \\
\hline $\begin{array}{l}\text { Morning } \\
\text { Thorpe }\end{array}$ & Norfolk & $\begin{array}{l}\text { 6th } c \\
6 \text { th } c\end{array}$ & $\begin{array}{l}\text { Grave 216: with annular } \\
\text { brooch pair, etc; } \\
\text { Grave 288: with great } \\
\text { square-headed brooch, etc }\end{array}$ & $\begin{array}{l}\text { Green et al } 1987 \text {, } \\
93 \text { and } 115, \\
\text { figs } 366 . J v i i i \\
\text { and } 389.288 . F v i i i ; \\
\text { Norwich Museum }\end{array}$ \\
\hline Brandon & Suffolk & $\begin{array}{l}\text { mid- } \\
7 \text { th- } \\
9 \text { th c }\end{array}$ & $\begin{array}{l}\text { Settlement: well-made } \\
\text { eyes }\end{array}$ & $\begin{array}{l}\text { Carr et al } 1988 ; \\
\text { publication in } \\
\text { preparation (R D Carr) }\end{array}$ \\
\hline Ipswich & Suffolk & $\begin{array}{l}\text { 6th- } \\
7 \text { th } c\end{array}$ & Small example & $\begin{array}{l}\text { Layard } 1907 \text { and } 1909 \text {, } \\
\text { pl XXXI; } \\
\text { Ipswich Museum }\end{array}$ \\
\hline
\end{tabular}

xiv Blue beads With White or yellow spots (see Map 20)

$\begin{array}{lllll}\text { Alton } & \text { Hants } & \text { 7th c } & \begin{array}{l}\text { Grave 39: slightly } \\ \text { biconical }\end{array} & \begin{array}{l}\text { Evison 1988a, 80-1, } \\ \text { fig 33.10; } \\ \text { Hampshire Museums }\end{array} \\ \text { Longthorpe } & \text { Hunts } & & \begin{array}{l}\text { Stray find: early Roman } \\ \text { fort, but also early Anglo- } \\ \text { Saxon mixed rite cemetery }\end{array} & \begin{array}{l}\text { Frere and St Joseph 1974, } \\ 122\end{array} \\ \begin{array}{llll}\text { Chessell } \\ \text { Down }\end{array} & \begin{array}{l}\text { Isle of } \\ \text { Wight }\end{array} & \begin{array}{l}\text { prob } \\ \text { 6th c }\end{array} & \text { Grave 228: annular (6.18) } & \begin{array}{l}\text { Arnold 1982, 50, 120, } \\ \text { colour frontispiece; BM }\end{array}\end{array}$

36, 41 and 45;

Welch 1983, 83, figs 36c, $23 \mathrm{~b}$ and $30 \mathrm{a}$;

Lewes Museum 


\begin{tabular}{|c|c|c|c|c|}
\hline Faversham & Kent & $\begin{array}{l}5 \text { th- } \\
7 \text { th } c\end{array}$ & $\begin{array}{l}\text { Biconical with white } \\
\text { spots }\end{array}$ & $\begin{array}{l}\text { Meaney 1964, 118-19; } \\
\text { Pitt-Rivers Collection, } \\
\text { Salisbury Museum 3D 2A } 9\end{array}$ \\
\hline Kingston & Kent & $\begin{array}{l}\text { prob } \\
\text { 7th } c\end{array}$ & $\begin{array}{l}\text { Large light blue } \\
\text { annular with spaced } \\
\text { yellow spots (Plate 5) }\end{array}$ & $\begin{array}{l}\text { Faussett Diary } \\
\text { III, 58, fig 1; } \\
\text { Liverpool Museum }\end{array}$ \\
\hline $\begin{array}{l}\text { Castle } \\
\text { Bytham }\end{array}$ & Lincs & $\begin{array}{l}\text { prob } \\
7 \text { th } c\end{array}$ & $\begin{array}{l}\text { With white spots, } \\
\text { with quoit brooch }\end{array}$ & $\begin{array}{l}\text { Akerman 1855a, 26-7, } \\
\text { pl XII.4 }\end{array}$ \\
\hline $\begin{array}{l}\text { Morning } \\
\text { Thorpe }\end{array}$ & Norfolk & $\begin{array}{l}\text { 6th- } \\
7 \text { th } c\end{array}$ & $\begin{array}{l}\text { Grave } 284 \text { : biconical } \\
\text { with well-defined spots }\end{array}$ & $\begin{array}{l}\text { Green et al 1987, 113, } \\
\text { fig } 387.284 . C i v ; \\
\text { Norwich Museum }\end{array}$ \\
\hline Ipswich & Suffolk & $\begin{array}{l}\text { 6th- } \\
7 \text { th } c\end{array}$ & $\begin{array}{l}\text { Big biconical with } \\
\text { well-made white spots }\end{array}$ & $\begin{array}{l}\text { Layard } 1907 \text { and 1909; } \\
\text { pl XXXI: Ipswich Museum }\end{array}$ \\
\hline Alfriston & Sussex & $\begin{array}{l}\text { 5th- } \\
6 \text { th } c\end{array}$ & $\begin{array}{l}\text { Grave } 87 \text { : globular } \\
\text { with cast saucer } \\
\text { brooch pair }\end{array}$ & $\begin{array}{l}\text { Griffith } 1915,204 ; \\
\text { Welch } 1983,83 \text {, fig 36c; } \\
\text { Lewes Museum }\end{array}$ \\
\hline $\begin{array}{l}\text { Apple } \\
\text { Down I }\end{array}$ & Sussex & $\begin{array}{l}\text { prob } \\
\text { 6th c }\end{array}$ & $\begin{array}{l}\text { Grave 107: translucent } \\
\text { biconical with white spots }\end{array}$ & $\begin{array}{l}\text { Down and Welch } 1990, \\
48, \text { fig } 2.35 \text {, colour pl } 40.8 \\
\text { and 17; Chichester Museum }\end{array}$ \\
\hline Wasperton & Warks & $\begin{array}{l}\text { 5th- } \\
\text { 6th c } \\
\text { mid- } \\
\text { late } \\
6 \text { th } c\end{array}$ & $\begin{array}{l}\text { Dark blue with saucer } \\
\text { brooches; } \\
\text { some biconical peacock } \\
\text { blue with saucer brooch }\end{array}$ & $\begin{array}{l}\text { Publication in } \\
\text { preparation (M Carver); } \\
\text { Warwick Museum }\end{array}$ \\
\hline $\begin{array}{l}\text { Market } \\
\text { Lavington }\end{array}$ & Wilts & & $\begin{array}{l}\text { Grave 11: biconical } \\
\text { with yellow spots }\end{array}$ & $\begin{array}{l}\text { Wessex Trust for } \\
\text { Archaeology }\end{array}$ \\
\hline $\begin{array}{l}\text { Wykeham } \\
\text { Grange, } \\
\text { near York }\end{array}$ & Yorks & & $\begin{array}{l}\text { Semi-translucent annular } \\
\text { with } 4 \text { well-spaced spots }\end{array}$ & BM $924-2156$ \\
\hline
\end{tabular}

xv Miscellaneous blue decorated beads

$\begin{array}{lllll}\begin{array}{l}\text { Little } \\ \text { Wilbraham }\end{array} & \text { Cambs } & \begin{array}{l}\text { 5th- } \\ 7 \text { th } \mathrm{c}\end{array} & \begin{array}{l}\text { Cube-shaped with irregular } \\ \text { yellow eyes }\end{array} & \text { Fox 1923, 260-2 } \\ \text { Escomb } & \text { Durham } & \begin{array}{l}\text { prob } \\ \text { 7th- } \\ 8 \text { th c }\end{array} & \begin{array}{l}\text { Church nave foundations: } \\ \text { with yellow band top and } \\ \text { bottom (cf Harrietsham } \\ \text { below) }\end{array} & \begin{array}{l}\text { Pocock and Wheeler 1971, } \\ \text { 23, fig 10 }\end{array} \\ \begin{array}{l}\text { Great } \\ \text { Chesterford }\end{array} & \text { Essex } & \begin{array}{l}\text { mid- } \\ \text { 6th c }\end{array} & \begin{array}{l}\text { Grave 37: irregular } \\ \text { bead (D49) with whitish } \\ \text { roundel with Hahnheim- } \\ \text { type radiate brooch, etc }\end{array} & \begin{array}{l}\text { Evison 1994, 18, 46, } \\ \text { BM 1964 7-2 178 }\end{array} \\ \begin{array}{l}\text { Springfield } \\ \text { Lyons }\end{array} & \text { Essex } & & \begin{array}{l}\text { Grave 6573: translucent } \\ \text { annular with pale blue } \\ \text { spots }\end{array} & \begin{array}{l}\text { Publication in } \\ \text { preparation (S Tyler); } \\ \text { BM }\end{array}\end{array}$




\begin{tabular}{|c|c|c|c|c|}
\hline Alton & Hants & 7 th $\mathrm{c}$ & $\begin{array}{l}\text { Grave 39: } 1 \text { annular with } \\
\text { terracotta rings in white } \\
\text { dots (D14) }\end{array}$ & $\begin{array}{l}\text { Evison } 1988 \mathrm{a}, 17,80-1 \text {, } \\
\text { fig } 33.39 .1 \mathrm{~m} \text {; } \\
\text { Hampshire Museums }\end{array}$ \\
\hline Andover & Hants & 6th c & $\begin{array}{l}\text { Grave } 44 \text { : two clear bright } \\
\text { blue with white feathering } \\
\text { (Plate 5) }\end{array}$ & $\begin{array}{l}\text { Cook and Dacre } 1985,35 \text {, } \\
83 \text {, fig } 59.25 \text { and } 27, \text { colour } \\
\text { frontispiece; } \\
\text { Hampshire Museums }\end{array}$ \\
\hline $\begin{array}{l}\text { Chessell } \\
\text { Down }\end{array}$ & $\begin{array}{l}\text { Isle of } \\
\text { Wight }\end{array}$ & $\begin{array}{l}\text { prob } \\
6 \text { th c }\end{array}$ & $\begin{array}{l}\text { Annular with white } \\
\text { parallel lines }(6.9)\end{array}$ & $\begin{array}{l}\text { Arnold } 1982,50,120, \\
\text { colour frontispiece; } \mathrm{BM}\end{array}$ \\
\hline $\begin{array}{l}\text { Beakes- } \\
\text { bourne }\end{array}$ & Kent & $\begin{array}{l}\text { prob } \\
6 \text { th- } \\
7 \text { th c }\end{array}$ & $\begin{array}{l}\text { Grave 33: two long } \\
\text { square-sectioned with } \\
\text { bands of blue and yellow }\end{array}$ & Canterbury Museum \\
\hline Harrietsham & Kent & & $\begin{array}{l}\text { Biconical with white } \\
\text { line top and bottom and } \\
\text { zigzag between (cf } \\
\text { Escomb above) }\end{array}$ & Maidstone Museum \\
\hline Howletts & Kent & 6th $\mathrm{c}$ & $\begin{array}{l}\text { Small globular with } \\
\text { white girth band }\end{array}$ & $\begin{array}{l}\text { Smith 1918; } \\
\text { BM 1936 5-11 } 143\end{array}$ \\
\hline $\begin{array}{l}\text { Castle } \\
\text { Bytham }\end{array}$ & Lincs & $\begin{array}{l}\text { prob } \\
7 \text { th } \mathrm{c}\end{array}$ & $\begin{array}{l}\text { Large squarish bead with } \\
2 \text { rows of white zigzags } \\
\text { with quoit brooch }\end{array}$ & $\begin{array}{l}\text { Akerman 1855a, 26-7, } \\
\text { pl XII.4 }\end{array}$ \\
\hline Fonaby & Lincs & 6 th c & $\begin{array}{l}\text { Grave } 3 \text { : blue and white } \\
\text { feathered cylinder }\end{array}$ & $\begin{array}{l}\text { Cook } 1981,18 \\
\text { fig } 4.1 .17, \text { pl VIIA; } \\
\text { Scunthorpe Museum }\end{array}$ \\
\hline Spong Hill & Norfolk & $\begin{array}{l}5 \text { th- } \\
6 \text { th c }\end{array}$ & $\begin{array}{l}\text { Cremation 1784: distorted } \\
\text { by burning, large } \\
\text { with white and blue rings }\end{array}$ & $\begin{array}{l}\text { Hills and Penn } 1981,34 \text {, } \\
\text { fig } 165.1784 \text {; } \\
\text { Norfolk Arch Unit }\end{array}$ \\
\hline Welton & Northants & & $\begin{array}{l}\text { Slate blue gadrooned } \\
\text { with horizontal white } \\
\text { lines around }\end{array}$ & $\begin{array}{l}\text { Dryden } 1885,337 ; \\
\text { Meaney 1964, 196-7; } \\
\text { Northampton Museum }\end{array}$ \\
\hline $\begin{array}{l}\text { Bury St } \\
\text { Edmunds, } \\
\text { Westgarth } \\
\text { Gardens }\end{array}$ & Suffolk & 6th $\mathrm{c}$ & $\begin{array}{l}\text { Grave } 16: 2 \text { beads } \\
\text { with green/yellow } \\
\text { twist on ?dark blue } \\
\text { with small-long brooches } \\
\text { and clasps }\end{array}$ & $\begin{array}{l}\text { West } 1988,24 \text {, } \\
\text { fig } 64.9 \text { and } 10 ; \\
\text { Bury St Edmunds } \\
\text { Museum }\end{array}$ \\
\hline Ipswich & Suffolk & $\begin{array}{l}6 \text { th- } \\
7 \text { th } c\end{array}$ & $\begin{array}{l}\text { Large biconical with } \\
\text { white parallel lines } \\
\text { under red spots }\end{array}$ & $\begin{array}{l}\text { Layard 1907, pl XXXI, and } \\
1909 ; \\
\text { Ipswich Museum }\end{array}$ \\
\hline $\begin{array}{l}\text { Apple } \\
\text { Down I }\end{array}$ & Sussex & $\begin{array}{l}\text { prob } \\
6 \text { th } c\end{array}$ & $\begin{array}{l}\text { Grave 107: yellow top and } \\
\text { bottom and zigzags with } \\
\text { similar bead in green glass } \\
\text { and yellow ornament }\end{array}$ & $\begin{array}{l}\text { Down and Welch } 1990 \text {, } \\
48,162, \text { fig } 2.35, \text { colour } \\
\text { pl } 40.7 \text {; } \\
\text { Chichester Museum }\end{array}$ \\
\hline Wasperton & Warks & $\begin{array}{l}\text { mid- } \\
6 \text { th } c\end{array}$ & $\begin{array}{l}\text { Two slatey blue globular } \\
\text { with } 2 \text { terracotta bands } \\
\text { and with saucer brooches }\end{array}$ & $\begin{array}{l}\text { Publication in } \\
\text { preparation (M Carver); } \\
\text { Warwick Museum }\end{array}$ \\
\hline
\end{tabular}


The Glass Beads of Anglo-Saxon England

Saltburn Yorks 6th- Large annular with

Gallagher 1987, 16, 25,

7th c irregular white girth

fig 5.33;

line and rough waves

Middlesbrough Museum 


\title{
SCHEDULE 7
}

\section{CRIMSON OR PINK GLASS BEADS}

\author{
All SHAPES, MOSTly ANNULAR, GLOBUlaR AND SHORT BARREL-SHAPED \\ (SOME BIPARTITE) (see Map 21)
}

\begin{tabular}{|c|c|c|c|c|}
\hline Kempston & Beds & $\begin{array}{l}5 \text { th- } \\
7 \text { th c }\end{array}$ & $\begin{array}{l}\text { Large almost opaque; } \\
21 \text { pink annulars; } \\
1 \text { annular; } \\
\text { Grave } 16: 3 \text { small and } \\
2 \text { large annulars }\end{array}$ & $\begin{array}{l}\text { BM } 916-2452 ; \\
\text { BM } 916-2454 ; \\
\text { BM } 916-2451 ; \\
\text { Fitch 1864; } \\
\text { BM } 916-2453\end{array}$ \\
\hline Luton I & Beds & $\begin{array}{l}\text { early } \\
\text { 6th c }\end{array}$ & $\begin{array}{l}\text { Grave } 27 \text { : with saucer } \\
\text { brooches, etc, and other } \\
\text { necklaces: many annular } \\
\text { and some segmented }\end{array}$ & $\begin{array}{l}\text { Austin } 1928,28 \text {, } \\
\text { pl XXX; } \\
\text { Luton Museum }\end{array}$ \\
\hline $\begin{array}{l}\text { Ely, } \\
\text { Cratendune }\end{array}$ & Cambs & $\begin{array}{l}\text { later } \\
5 \text { th- } \\
7 \text { th c }\end{array}$ & $\begin{array}{l}\text { Atypical large } \\
\text { amulet or spindle } \\
\text { whorl with white rosette }\end{array}$ & $\begin{array}{l}\text { Fowler 1948; } \\
\text { Cambridge Museum }\end{array}$ \\
\hline $\begin{array}{l}\text { Hasling- } \\
\text { field }\end{array}$ & Cambs & $\begin{array}{l}\text { 5th- } \\
\text { 7th c }\end{array}$ & $\begin{array}{l}30 \text { annular and a few } \\
\text { globulars }\end{array}$ & $\begin{array}{l}\text { Meaney 1964, 66-7; } \\
\text { Birmingham Museum } \\
\text { A386/73 }\end{array}$ \\
\hline $\begin{array}{l}\text { Linton } \\
\text { Heath }\end{array}$ & Cambs & $\begin{array}{l}\text { prob } \\
\text { 6th } c\end{array}$ & $\begin{array}{l}\text { Miscellaneous collection: } \\
5 \text { to } 6 \text { translucent pink } \\
\text { annulars }\end{array}$ & $\begin{array}{l}\text { Neville 1854; } \\
\text { Cambridge Museum }\end{array}$ \\
\hline $\begin{array}{l}\text { Little } \\
\text { Wilbraham }\end{array}$ & Cambs & $\begin{array}{l}\text { 5th- } \\
\text { 7th c }\end{array}$ & $\begin{array}{l}\text { Globular appearing black } \\
\text { with specks }\end{array}$ & $\begin{array}{l}\text { Cambridge Museum } \\
19481462\end{array}$ \\
\hline $\begin{array}{l}\text { Feering, } \\
\text { Kelvedon }\end{array}$ & Essex & $\begin{array}{l}\text { prob } \\
\text { early } \\
7 \text { th c }\end{array}$ & $\begin{array}{l}\text { Not seen: ref given by } \\
\text { Mrs S Tyler }\end{array}$ & $\begin{array}{l}\text { Anon } 1888,124 ; \\
\text { Colchester Museum }\end{array}$ \\
\hline $\begin{array}{l}\text { Great } \\
\text { Chesterford }\end{array}$ & Essex & $\begin{array}{l}\text { 5th- } \\
\text { 6th c }\end{array}$ & $\begin{array}{l}\text { Grave } 37 \text { : very irregular, } \\
\text { dark, probably globular } \\
\text { with Hahnheim-type } \\
\text { radiate brooch, etc; } \\
\text { Grave } 45: 2 \text { double } \\
\text { globular (Plate } 6 \text { ) and } \\
\text { possibly others } \\
\text { burnt (B07/B08) with } \\
\text { square-headed small-long } \\
\text { brooch pair, etc; }\end{array}$ & $\begin{array}{l}\text { Evison } 1994,14-18,46 \text {, } \\
97,98,109 \text { and } 114 \text {, } \\
\text { figs } 28.37 .4 \mathrm{Aj} \text {, } \\
29.45 .3 \mathrm{f} \text { and } \mathrm{g}, \\
49.127 .5 \\
\text { 57.159.1b and c; } \\
\text { BM } 19647-2178 \text {; } \\
\text { BM } 19647-2199 \text {; } \\
\text { BM } 19647-2402 \text {; }\end{array}$ \\
\hline
\end{tabular}




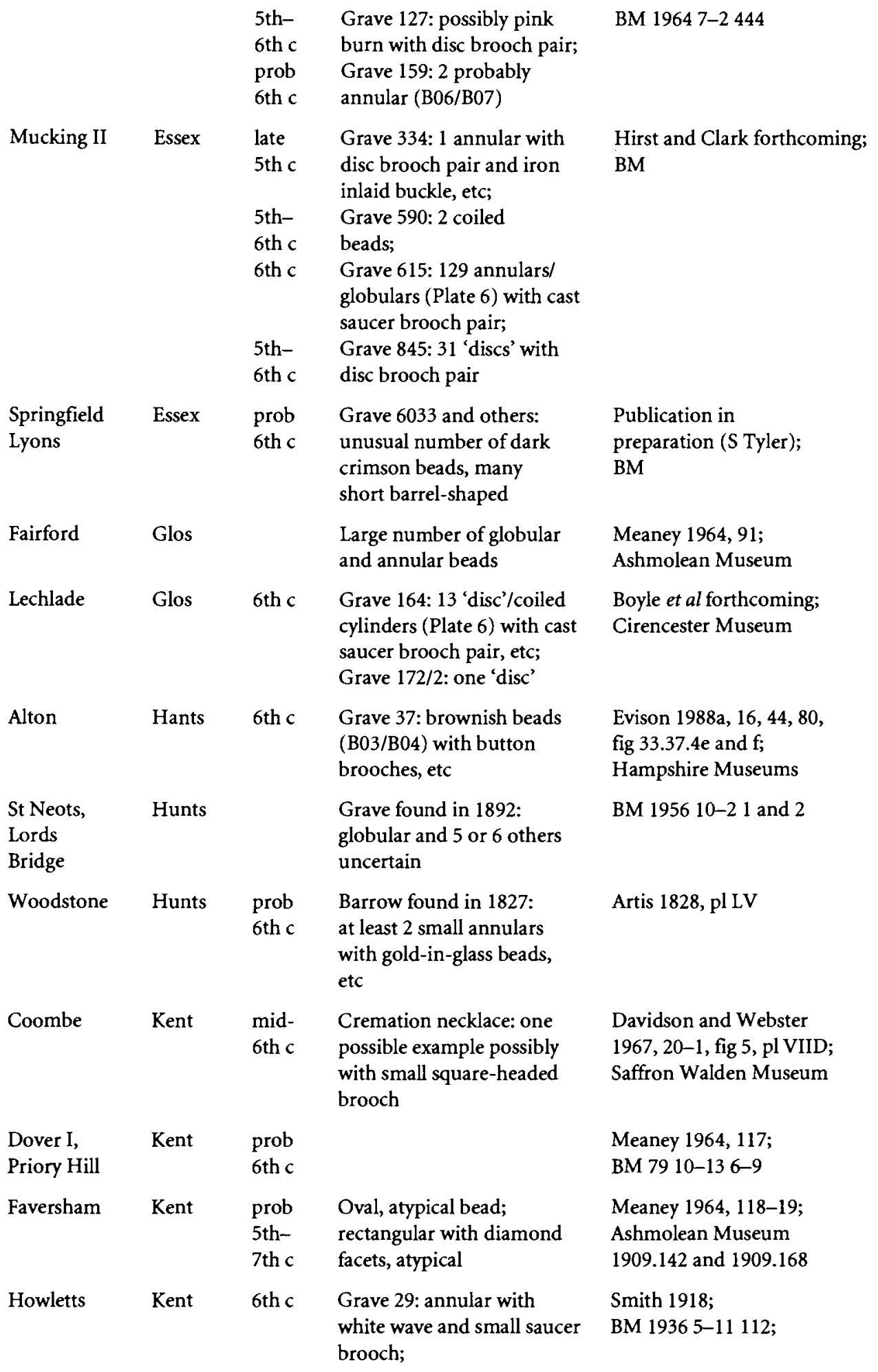




\begin{tabular}{|c|c|c|c|c|}
\hline & & $\begin{array}{l}\text { prob } \\
\text { 6th c }\end{array}$ & $\begin{array}{l}\text { Grave } 142: 1 \text { very dark } \\
\text { with gold-in-glass beads; } \\
1 \text { very dark annular, } \\
\text { ?pink, wound }\end{array}$ & $\begin{array}{l}\text { BM } 19365-11143 ; \\
\text { BM 1936 5-11 } 145\end{array}$ \\
\hline $\begin{array}{l}\text { Lyminge } \\
\text { II }\end{array}$ & Kent & 6th $c$ & $\begin{array}{l}\text { Grave } 16: 1 \text { small shapeless } \\
\text { bead with Hahnheim-type } \\
\text { radiate and button brooches, } \\
\text { and bracteate, etc }\end{array}$ & $\begin{array}{l}\text { Warhurst 1955, 15; } \\
\text { Maidstone Museum }\end{array}$ \\
\hline $\begin{array}{l}\text { Milton } \\
\text { Regis (near) }\end{array}$ & Kent & & $\begin{array}{l}\text { Grave 3: marvered green } \\
\text { and crimson }\end{array}$ & $\begin{array}{l}\text { Rigold and Webster } 1970, \\
4\end{array}$ \\
\hline Sarre & Kent & $\begin{array}{l}\text { 6th- } \\
7 \text { th c }\end{array}$ & $\begin{array}{l}\text { Necklace: } 2 \text { small annulars } \\
\text { with amethysts, gold coins } \\
\text { with AD } 641 \text { tpq, millefiori } \\
\text { pendant, etc; } \\
\text { miscellaneous find: } \\
1 \text { medium globular with } \\
\text { collar at one end, which } \\
\text { suggests once double bead }\end{array}$ & Brent 1863 and 1866 \\
\hline $\begin{array}{l}\text { Sibertswold } \\
\text { or } \\
\text { Barfreston }\end{array}$ & Kent & & $\begin{array}{l}\text { Two annulars translucent } \\
\text { pink/amber colour }\end{array}$ & $\begin{array}{l}\text { Faussett } 1856, \mathrm{pl} \mathrm{V} \text {; } \\
\text { Liverpool Museum } \\
\text { M } 6625\end{array}$ \\
\hline Cleatham & Lincs & $\begin{array}{l}\text { 5th- } \\
\text { 6th c }\end{array}$ & Grave $34: 2$ annular beads & $\begin{array}{l}\text { Information K Leahy; } \\
\text { Scunthorpe Museum }\end{array}$ \\
\hline Fonaby & Lincs & 6th $c$ & $\begin{array}{l}\text { Grave 32: pink glass used } \\
\text { for crossed waves with } \\
\text { Aberg Group III cruciform } \\
\text { brooch, etc }\end{array}$ & $\begin{array}{l}\text { Cook 1981, 34, } \\
\text { fig 11.32.3; } \\
\text { Scunthorpe Museum }\end{array}$ \\
\hline Laceby & Lincs & $\begin{array}{l}\text { 6th- } \\
7 \text { th c }\end{array}$ & $\begin{array}{l}\text { One annular and other beads } \\
\text { on wire }\end{array}$ & $\begin{array}{l}\text { Myres } 1951,89 \text { and } 98 ; \\
\text { Thompson 1956, 184-9; } \\
\text { Lincoln Museum }\end{array}$ \\
\hline $\begin{array}{l}\text { Lincoln, } \\
\text { Burton Rd }\end{array}$ & Lincs & & $\begin{array}{l}\text { Two pink globular in group } \\
\text { of } 4 \text { beads }\end{array}$ & $\begin{array}{l}\text { Lincoln Museum } \\
\text { (presented by A Ross) }\end{array}$ \\
\hline Quarrington & Lincs & $\begin{array}{l}\text { prob } \\
6 \text { th } c\end{array}$ & $\begin{array}{l}\text { Dark pinkish brown } \\
\text { globular }\end{array}$ & $\begin{array}{l}\text { Bruce } 1880,76, \text { no. } 303 \text {; } \\
\text { Alnwick Castle Museum }\end{array}$ \\
\hline Searby & Lincs & $\begin{array}{l}\text { prob } \\
5 \text { th- } \\
6 \text { th c }\end{array}$ & $\begin{array}{l}\text { Necklace mixed beads: } \\
1 \text { annular }\end{array}$ & $\begin{array}{l}\text { Smith 1852a, 234; } \\
\text { Smith 1861, 137-40; } \\
\text { Meaney 1964, 162; } \\
\text { BM 93 6-18 24 }\end{array}$ \\
\hline $\begin{array}{l}\text { Welbeck } \\
\text { Hill }\end{array}$ & Lincs & $\begin{array}{l}5 \text { th- } \\
6 \text { th } \mathrm{c}\end{array}$ & $\begin{array}{l}\text { Grave } 64: 1 \text { pink and a half } \\
\text { bead }\end{array}$ & $\begin{array}{l}\text { Publication in } \\
\text { preparation (G Taylor); } \\
\text { Scunthorpe Museum }\end{array}$ \\
\hline Kenninghall & Norfolk & $\begin{array}{l}5 \text { th- } \\
7 \text { th c }\end{array}$ & $\begin{array}{l}\text { Large collection: } 1 \text { pink } \\
\text { melon, abnormal }\end{array}$ & $\begin{array}{l}\text { Manning } 1872 \mathrm{a}, 292 \text {, and } \\
1872 \mathrm{~b} ; \\
\text { BM } 837-230\end{array}$ \\
\hline $\begin{array}{l}\text { Morning } \\
\text { Thorpe }\end{array}$ & Norfolk & $\begin{array}{l}\text { mid- } \\
\text { 6th } c\end{array}$ & $\begin{array}{l}\text { Grave 208: } 2 \text { with Åberg } \\
\text { Group IV cruciform } \\
\text { brooch, etc }\end{array}$ & $\begin{array}{l}\text { Green } \text { et al } 1987,89-90 \text {, } \\
\text { fig } 361.208 . \text { Evi; } \\
\text { Norwich Museum }\end{array}$ \\
\hline
\end{tabular}




\begin{tabular}{|c|c|c|c|c|}
\hline $\begin{array}{l}\text { Northwold } \\
\text { Brookville }\end{array}$ & Norfolk & & Three purple annulars & Information M Reeve \\
\hline Spong Hill & Norfolk & $\begin{array}{l}\text { 5th- } \\
6 \text { th } c \\
6 \text { th } c \\
6 \text { th } c \\
\text { mid- } \\
\text { 6th } c\end{array}$ & $\begin{array}{l}\text { Grave 5: many annular } \\
\text { and other beads with small- } \\
\text { long brooch pair, A clasps; } \\
\text { Grave 12: single and double } \\
\text { with annular brooches, etc; } \\
\text { Grave 18: with great } \\
\text { square-headed brooch, etc; } \\
\text { Grave 22: many annular } \\
\text { and some globular collared, } \\
\text { ?once bipartite with Aberg } \\
\text { Group IV cruciform } \\
\text { brooch, etc; } \\
\text { Grave } 26: \text { pink annular with } \\
\text { Aberg Group I cruciform } \\
\text { brooch, etc; } \\
\text { Grave } 37: 1 \text { pink and green } \\
\text { glass, etc, with annular } \\
\text { brooch pair, B clasps, etc; } \\
\text { Grave } 38: 2 \text { beads - } \\
1 \text { annular and } 1 \text { annular } \\
\text { wound with square- } \\
\text { headed brooch, etc; } \\
\text { Grave } 39: 1 \text { annular and } \\
\text { short square cylinder with } \\
\text { multi-facetted crystal, etc }\end{array}$ & $\begin{array}{l}\text { Hills et al } 1984,54-5 \text {, } \\
61,67,70,75,86,90, \\
90-1, \text { figs } 73.3 \mathrm{a}, 75.4 \text {, } \\
77.18 .4,79.5 \\
83.5 \mathrm{~b}, 90.37 .6 \\
92.10 \mathrm{~h}, 94.5 \\
\text { Norfolk Arch Unit }\end{array}$ \\
\hline $\begin{array}{l}\text { Standlake } \\
\text { Down }\end{array}$ & Oxon & $\begin{array}{l}\text { early } \\
7 \text { th c }\end{array}$ & $\begin{array}{l}\text { Grave 24: oval with many } \\
\text { other beads }\end{array}$ & $\begin{array}{l}\text { Dickinson } 1973,244-5 \text {, } \\
252-4 \text {, fig } 5.1 \text {; } \\
\text { Ashmolean Museum }\end{array}$ \\
\hline $\begin{array}{l}\text { Empingham } \\
\text { II }\end{array}$ & Rutland & $\begin{array}{l}\text { 5th- } \\
\text { 7th } c\end{array}$ & $\begin{array}{l}\text { Grave } 37: \text { short cylinder } \\
\text { with square section; } \\
\text { Grave } 69: 1 \text { cylinder; } \\
\text { Grave 98B: } 2 \text { annulars }\end{array}$ & $\begin{array}{l}\text { Timby 1996; } \\
\text { Oakham Museum }\end{array}$ \\
\hline $\begin{array}{l}\text { Bury St } \\
\text { Edmunds, } \\
\text { Westgarth } \\
\text { Gardens }\end{array}$ & Suffolk & 6th $c$ & $\begin{array}{l}\text { Grave 16: two cubes with } \\
\text { green and yellow bands with } \\
2 \text { small-long brooches, } \\
\text { B clasps, etc }\end{array}$ & $\begin{array}{l}\text { West } 1988,24 \text {, } \\
\text { fig } 64.9 \text { and } 10 \text {; } \\
\text { Bury St Edmunds } \\
\text { Museum }\end{array}$ \\
\hline $\begin{array}{l}\text { Fornham } \\
\text { All } \\
\text { Saints }\end{array}$ & Suffolk & & $\begin{array}{l}\text { Two globulars, perhaps } \\
\text { broken double }\end{array}$ & BM $19584-61$ \\
\hline $\begin{array}{l}\text { Holywell } \\
\text { Row }\end{array}$ & Suffolk & 6th $c$ & $\begin{array}{l}\text { Two very worn pink } \\
\text { annulars }\end{array}$ & $\begin{array}{l}\text { Lethbridge 1931, 1-46; } \\
\text { Cambridge Museum }\end{array}$ \\
\hline Lakenheath & Suffolk & $\begin{array}{l}\text { 6th- } \\
\text { 7th } c\end{array}$ & $\begin{array}{l}\text { Nine globular or annular; } \\
1 \text { or } 2 \text { originally double? }\end{array}$ & $\begin{array}{l}\text { Meaney 1964, 230; } \\
\text { BM 1910 12-22 } 11 \text { and } 9\end{array}$ \\
\hline $\begin{array}{l}\text { Mitchell's } \\
\text { Hill }\end{array}$ & Suffolk & $\begin{array}{l}\text { prob } \\
\text { 6th } c\end{array}$ & Three once joined? & $\begin{array}{l}\text { Meaney } 1964,231 \\
\text { Bury St Edmunds } \\
\text { Museum }\end{array}$ \\
\hline
\end{tabular}




\begin{tabular}{|c|c|c|c|c|}
\hline West Stow & Suffolk & $\begin{array}{l}5 \text { th- } \\
7 \text { th } \mathrm{c}\end{array}$ & $\begin{array}{l}\text { Cemetery: } 24 \text { short } \\
\text { cylindrical translucent } \\
\text { pink on string but not } \\
\text { necessarily associated; } \\
\text { Cemetery: large slightly } \\
\text { biconical dark with claret } \\
\text { colour }\end{array}$ & $\begin{array}{l}\text { West } 1985,74-5 ; \\
\text { Bury St Edmunds } \\
\text { Museum; } \\
\text { Ashmolean Museum } \\
\text { 1909.424 }\end{array}$ \\
\hline Guildown & Surrey & $\begin{array}{l}5 \text { th- } \\
6 \text { th c } \\
\text { prob } \\
6 \text { th c }\end{array}$ & $\begin{array}{l}\text { Grave } 77: 1 \text { small bead on } \\
\text { earring with disc brooch } \\
\text { pair, etc; } \\
\text { Grave 185: } 1 \text { annular } \\
\text { and } 1 \text { globular }\end{array}$ & $\begin{array}{l}\text { Lowther } 1931,11-12,35 \\
\text { and } 43, \text { pl IX.2 and 3; } \\
\text { Guildford Museum }\end{array}$ \\
\hline Mitcham & Surrey & $\begin{array}{l}\text { prob } \\
6 \text { th } c\end{array}$ & $\begin{array}{l}\text { Grave 197: some annular } \\
\text { pink or black uncertain }\end{array}$ & $\begin{array}{l}\text { Bidder and Morris } 1959 \text {, } \\
73,110, \text { pl XVI.197; } \\
\text { Cambridge Museum }\end{array}$ \\
\hline Alfriston & Sussex & $\begin{array}{l}5 \text { th- } \\
\text { mid- } \\
6 \text { th c }\end{array}$ & $\begin{array}{l}\text { Grave } 77 \text { (and possibly } \\
\text { Grave } 43 \text { ): } 1 \text { annular; } \\
\text { Grave } 68: 24 \text { annulars } \\
\text { of various sizes and } \\
1 \text { ?originally double } \\
\text { with quoit brooch }\end{array}$ & $\begin{array}{l}\text { Griffith } 1915,203 ; \\
\text { Griffith and Salzmann 1914, } \\
40-1 \text { and } 47 \text {, pl I.1; } \\
\text { Welch } 1983 ; \\
\text { Lewes Museum }\end{array}$ \\
\hline $\begin{array}{l}\text { Apple } \\
\text { Down I }\end{array}$ & Sussex & 6 th c & $\begin{array}{l}\text { Grave 13: several; } \\
\text { Grave 90: dark; } \\
\text { Grave 134: brown/crimson, } \\
\text { etc }\end{array}$ & $\begin{array}{l}\text { Down and Welch } 1990 \text {, } \\
\text { 162-5; } \\
\text { Chichester Museum }\end{array}$ \\
\hline Highdown & Sussex & $\begin{array}{l}\text { prob } \\
6 \text { th } c\end{array}$ & $\begin{array}{l}\text { One very weathered } \\
\text { ?annular on bracelet } \\
\text { with other beads }\end{array}$ & $\begin{array}{l}\text { Read } 1895 \text { and } 1896 \text {; } \\
\text { Welch } 1983 \text {; } \\
\text { Worthing Museum } 3482\end{array}$ \\
\hline Wasperton & Warks & $\begin{array}{l}\text { 6th c } \\
\text { mid- } \\
6 \text { th } c \\
\text { 6th } c\end{array}$ & $\begin{array}{l}\text { F1579 } 3305: 16 \text { annulars } \\
\text { and } 5 \text { globulars; } \\
\text { F3100 } 3559 / 31 \text { : } 1 \text { globular } \\
\text { and } 2 \text { annular with saucer } \\
\text { brooch; } \\
\text { F3107 } 3616: 4 \text { annulars } \\
\text { with cruciform brooch }\end{array}$ & $\begin{array}{l}\text { Publication in } \\
\text { preparation (M Carver); } \\
\text { Warwick Museum }\end{array}$ \\
\hline Charlton & Wilts & 6 th $c$ & Grave 24: 1 annular & $\begin{array}{l}\text { Davies } 1984,120-1 \text {, } \\
139-40, \text { fig } 9 \mathrm{~N} ; \\
\text { Salisbury Museum }\end{array}$ \\
\hline $\begin{array}{l}\text { Harnham } \\
\text { Hill }\end{array}$ & Wilts & $\begin{array}{l}5 \text { th- } \\
6 \text { th c }\end{array}$ & $\begin{array}{l}\text { One, possibly } 2 \text {, annulars; } \\
1 \text { pink annular with amber } \\
\text { beads; } \\
15 \text { blue and green and } 1 \text { pink } \\
\text { annular and possibly another }\end{array}$ & $\begin{array}{l}\text { Akerman } 1853 \text {; } \\
\text { Jackson } 1854 ; \\
\text { BM } 58 \text { 2-14 } 31 \\
\text { BM } 546-159 ; \\
\text { BM } 5312-1431\end{array}$ \\
\hline $\begin{array}{l}\text { Peters- } \\
\text { finger }\end{array}$ & Wilts & $\begin{array}{l}\text { prob } \\
6 \text { th } c\end{array}$ & $\begin{array}{l}\text { Grave } 62: 1 \text { annular } \\
\text { appearing 'black' }\end{array}$ & $\begin{array}{l}\text { Leeds and Shortt } 1953, \\
41, \text { pl IV.172; } \\
\text { Salisbury Museum }\end{array}$ \\
\hline
\end{tabular}


The Glass Beads of Anglo-Saxon England

\begin{tabular}{|c|c|c|c|c|}
\hline $\begin{array}{l}\text { Pewsey } \\
\text { Blackpatch }\end{array}$ & Wilts & $\begin{array}{l}\text { late } \\
5 \text { th- } \\
\text { 6th c } \\
\text { late } \\
\text { 6th c } \\
\text { late } \\
5 \text { th- } \\
\text { early } \\
\text { 6th c } \\
\text { 6th c } \\
\text { 6th c }\end{array}$ & $\begin{array}{l}\text { Grave } 2: 7 \text { translucent } \\
\text { annular, ivory purse frame, } \\
\text { etc; } \\
\text { Grave } 18: 1 \text { annular opaque } \\
\text { with pot and bone comb; } \\
\text { Grave } 35: 3 \text { small annular } \\
\text { with toilet set; }\end{array}$ & $\begin{array}{l}\text { Publication in } \\
\text { preparation (K Annable) } \\
\text { Devizes Museum }\end{array}$ \\
\hline $\begin{array}{l}\text { Broadway } \\
\text { Hill }\end{array}$ & Worcs & $\begin{array}{l}\text { late } \\
\text { 5th- } \\
\text { 6th c }\end{array}$ & $\begin{array}{l}\text { Disturbed finds from } \\
\text { inhumation graves: } \\
14 \text { brownish beads }\end{array}$ & Cook 1958, 68 \\
\hline Sewerby & Yorks & $\begin{array}{l}\text { mid- } \\
\text { 6th } c\end{array}$ & $\begin{array}{l}\text { Grave 28: annular (Ala) } \\
\text { with Åberg Group IVa }\end{array}$ & $\begin{array}{l}\text { Hirst } 1985,62-4, \\
\text { figs } 22 \text { and } 42.28 .2-50 \mathrm{~d}\end{array}$ \\
\hline Whitby & Yorks & & Straight-sided & $\begin{array}{l}\text { Beck 1943; Peers and } \\
\text { Radford } 1943\end{array}$ \\
\hline 'Yorks' & Yorks & & $\begin{array}{l}\text { Miscellaneous: } 1 \text { slightly } \\
\text { biconical }\end{array}$ & Yorkshire Museum \\
\hline
\end{tabular}




\title{
SCHEDULE 8
}

\section{TERRACOTTA OR BRICK-RED \\ GLASS BEADS}

\author{
MONOCHROME \\ i Terracotta annular, globular and short cylinder beads, singles and doubles \\ ii Terracotta small biconical and true barrel-shaped beads \\ iii Terracotta cylinder beads \\ iv Terracotta short cylinder beads, singles and doubles \\ v Terracotta cylinder beads with square or panelled polygonal sections \\ vi Terracotta melon and sub-melon beads (see Map 22)

\section{Polychrome} \\ vii Terracotta cylinder beads with coloured combing (including green and yellow) \\ (see Map 23) \\ viii Terracotta cylinder beads with square or polygonal sections and with coloured \\ combing (including green and yellow) (see Map 24) \\ ix Terracotta cylinder beads with wound bands \\ $\mathbf{x}$ Terracotta globular beads with white or yellow wound lines \\ xi Terracotta tall biconical beads with random yellow lines \\ xii Terracotta beads with specks or spots \\ xiii Miscellaneous terracotta beads with eyes \\ xiv Terracotta beads: \\ (a) with white or yellow crossed waves, without dots (see above and below) \\ (b) with white or yellow crossed waves and dots \\ (c) doubles with white or yellow crossed waves (some with dots) \\ xv Terracotta oval beads, some barrel-shaped, with white or yellow crossed waves, \\ with or without dots \\ xvi Terracotta beads with single wave or zigzag \\ xvii Terracotta drum-shaped beads: \\ (a) with herringbone and fine twist decoration, including green and yellow \\ (b) with related decoration, some with plain zones and some with imitation \\ twists \\ xviii Terracotta, mainly bun-shaped, beads:
}

(a) with twisted decoration (reticella) and related (excluding green and yellow) (see Map 25) 
(b) with green and yellow twisted bands (see Map 26)

(c) with green and yellow marbling (see Map 27)

xix Terracotta cylinder beads, round, square or polygonal sections:

(a) with green and yellow bands, panels, etc, with various motifs (see Map 28)

(b) with green and yellow marbling (see Map 28)

\section{MONOCHROME}

\section{i TerRacotTa ANNULAR, globUlar AND SHORT CYLINDER BEADS, SINGLES AND DOUBLES}

\begin{tabular}{|c|c|c|c|c|}
\hline Kempston & Beds & $\begin{array}{l}\text { prob } \\
\text { 6th c }\end{array}$ & $\begin{array}{l}\text { Grave 16: about } 15 \\
\text { annulars }\end{array}$ & $\begin{array}{l}\text { Fitch 1864; } \\
\text { BM 91 6-24 52 }\end{array}$ \\
\hline Harwell & Berks & $\begin{array}{l}\text { 5th- } \\
\text { 6th c }\end{array}$ & $\begin{array}{l}\text { Grave } 4: 2 \text { annulars with } \\
\text { disc brooch pair }\end{array}$ & $\begin{array}{l}\text { Kirk and Marshall 1956, } \\
27,33-4, \\
\text { fig 10h.11-12, pl IB; } \\
\text { Ashmolean Museum }\end{array}$ \\
\hline Wallingford & Berks & $\begin{array}{l}5 \text { th- } \\
\text { late } \\
6 \text { th c }\end{array}$ & $\begin{array}{l}\text { Grave 13: several; } \\
\text { Grave } 21\end{array}$ & $\begin{array}{l}\text { Leeds 1938, } 98 ; \\
\text { Harden 1940; } \\
\text { Ashmolean Museum }\end{array}$ \\
\hline Bishopstone & Bucks & $\begin{array}{l}\text { early } \\
\text { 6th } c\end{array}$ & $\begin{array}{l}\text { Six annulars with } \\
\text { saucer brooches }\end{array}$ & $\begin{array}{l}\text { Lowndes } 1878, \mathrm{pl} \mathrm{II} \\
\text { Aylesbury Museum }\end{array}$ \\
\hline Barrington & Cambs & $\begin{array}{l}5 \text { th- } \\
7 \text { th c }\end{array}$ & Five annulars & $\begin{array}{l}\text { Fox 1923, 250-2; } \\
\text { Ashmolean Museum }\end{array}$ \\
\hline $\begin{array}{l}\text { Linton } \\
\text { Heath B }\end{array}$ & Cambs & $\begin{array}{l}\text { 6th- } \\
7 \text { th } c\end{array}$ & $\begin{array}{l}\text { One annular; } \\
\text { about } 8 \text { globulars }\end{array}$ & $\begin{array}{l}\text { Neville 1854; } \\
\text { Cambridge Museum }\end{array}$ \\
\hline $\begin{array}{l}\text { Little } \\
\text { Wilbraham }\end{array}$ & Cambs & $\begin{array}{l}\text { prob } \\
\text { 6th- } \\
7 \text { th c }\end{array}$ & $\begin{array}{l}\text { Unassociated group: } \\
1 \text { globular }\end{array}$ & $\begin{array}{l}\text { Neville 1852; } \\
\text { Fox 1923, 260-2; } \\
\text { Cambridge Museum }\end{array}$ \\
\hline $\begin{array}{l}\text { Waterbeach, } \\
\text { Car Dyke }\end{array}$ & Cambs & $\begin{array}{l}\text { prob } \\
\text { 6th } c\end{array}$ & $\begin{array}{l}\text { Grubenhaus. } 1 \text { or } 2 \\
\text { annulars }\end{array}$ & $\begin{array}{l}\text { Lethbridge 1927, 145, } \\
\text { fig } 4.1\end{array}$ \\
\hline $\begin{array}{l}\text { Norton- } \\
\text { on-Tees }\end{array}$ & Durham & $\begin{array}{l}\text { mid- } \\
\text { 6th c }\end{array}$ & $\begin{array}{l}\text { Grave } 35 \text { : } 1 \text { barrel-shaped } \\
\text { with annular brooch pair, } \\
\text { B18c clasps, etc; } \\
\text { Grave } 40: 1 \text { barrel-shaped } \\
\text { with annular brooch pair, } \\
\text { penannular brooch, B18c } \\
\text { clasps, bracelet pair, etc; } \\
\text { Grave 102: Group } 2 \text { annular } \\
\text { with Åberg Group IVa } \\
\text { cruciform brooch, etc }\end{array}$ & $\begin{array}{l}\text { Sherlock and Welch } 1992 \\
145,149 \text { and } 186 \text {, } \\
\text { figs } 43.35 .1,45.40 .1 \\
\text { and } 62.102 .1\end{array}$ \\
\hline $\begin{array}{l}\text { Great } \\
\text { Chesterford }\end{array}$ & Essex & $\begin{array}{l}\text { mid- } \\
\text { 6th c } \\
\text { late } \\
\text { 5th- } \\
\text { 6th c }\end{array}$ & $\begin{array}{l}\text { Grave 20: } 3 \text { discs (B02) } \\
\text { with Áberg Group IVa } \\
\text { cruciform brooch, etc; } \\
\text { Grave 29: } 1 \text { disc (B02) with } \\
\text { penannular brooch(es), } \\
\text { B7 clasps, etc; }\end{array}$ & $\begin{array}{l}\text { Evison 1994, 14, 46, } 94, \\
96,100,103,105-6,112, \\
114-15, \text { figs } 24.20 .4 \mathrm{c}, \\
26.29 .5 \mathrm{c}, 27.31 .4 \mathrm{a}, \\
33.56 .2 \mathrm{a}, 37.79 .2 \mathrm{a}, \\
42.103 .3 \mathrm{a}, 55.148 .1 \mathrm{a},\end{array}$ \\
\hline
\end{tabular}




\begin{tabular}{|c|c|c|c|c|}
\hline & & $\begin{array}{l}\text { 5th- } \\
6 \text { th c } \\
\text { prob } \\
6 \text { th } c\end{array}$ & $\begin{array}{l}\text { Grave 31: } 4 \text { small discs } \\
\text { (B01) with bracelet, etc; } \\
\text { Grave 56: } 1 \text { small disc } \\
\text { (B01) with nails and beads; } \\
\text { Grave 79: } 2 \text { small discs } \\
\text { (B01) with penannular } \\
\text { brooch, girdle hanger shaft, } \\
\text { etc; } \\
\text { Grave 103: } 7 \text { small } \\
\text { globulars/discs (B01) with } \\
\text { Style I applied saucer } \\
\text { brooch pair, etc; } \\
\text { Grave 148: } 1 \text { disc (B02) } \\
\text { with small-long brooch, } \\
\text { stamped pottery bowl, etc; } \\
\text { Cremation 5: several, } \\
\text { 1 very small globular/disc } \\
\text { (B01) and } 12 \text { discs (B02) in } \\
\text { plain pottery urn }\end{array}$ & $\begin{array}{l}\text { 58.C5.2a and b; } \\
\text { BM } 19647-2\end{array}$ \\
\hline Mucking II & Essex & $\begin{array}{l}\text { 7th c } \\
\text { 6th } \mathrm{c} \\
\text { 6th } \mathrm{c} \\
\text { first } \\
\text { half } \\
\text { 5th } \mathrm{c}\end{array}$ & $\begin{array}{l}\text { Various, including: } \\
\text { Grave 608: } 2 \text { globulars; } \\
\text { Grave 690B: } 4 \text { globulars } \\
\text { with button brooch; } \\
\text { Grave 960B: } 1 \text { globular; } \\
\text { Grave 989: } 1 \text { globular with } \\
\text { Glaston-Mucking and } \\
\text { supporting-arm brooches and } \\
\text { faceted carinated pottery } \\
\text { bowl }\end{array}$ & $\begin{array}{l}\text { Evison 1981, 138-9, } \\
\text { figs } 4 \text { and } 5 \\
\text { BM }\end{array}$ \\
\hline $\begin{array}{l}\text { Springfield } \\
\text { Lyons }\end{array}$ & Essex & 6th $c$ & $\begin{array}{l}\text { Grave 4752: } 2 \text { globulars and } \\
\text { 1 annular; } \\
\text { Grave 4899: } 1 \text { globular; } \\
\text { Grave 4909: } 1 \text { globular; } \\
\text { Grave 6033: } 2 \text { annulars }\end{array}$ & $\begin{array}{l}\text { Publication in } \\
\text { preparation (S Tyler); } \\
\text { BM }\end{array}$ \\
\hline Fairford & Glos & $\begin{array}{l}\text { prob } \\
\text { 6th c }\end{array}$ & Annular & $\begin{array}{l}\text { Smith 1852b; } \\
\text { Meaney 1964, 91; } \\
\text { BM } 19267-71 \text { and } 2\end{array}$ \\
\hline Lechlade & Glos & 6th $c$ & $\begin{array}{l}\text { Grave 176: 'disc' with } \\
\text { annular/penannular } \\
\text { brooches }\end{array}$ & $\begin{array}{l}\text { Boyle et al forthcoming; } \\
\text { Cirencester Museum }\end{array}$ \\
\hline Andover & Hants & $\begin{array}{l}\text { prob } \\
\text { 6th c }\end{array}$ & $\begin{array}{l}\text { Grave 9: } 2 \text { short cylinders } \\
\text { with iron annular brooch } \\
\text { pair, etc }\end{array}$ & $\begin{array}{l}\text { Cook and Dacre } 1985,26 \text {, } \\
\text { 82, fig 44.64; } \\
\text { Hampshire Museums }\end{array}$ \\
\hline Droxford & Hants & $\begin{array}{l}\text { 5th- } \\
6 \text { th } c\end{array}$ & $\begin{array}{l}\text { Grave 20: } 2 \text { small annulars } \\
\text { (Class } \mathrm{Xa} \text { and } \mathrm{b} \text { ) }\end{array}$ & $\begin{array}{l}\text { Aldsworth } 1978,124 \text {, } \\
173, \text { fig } 24.22 \text { and } 23 \text {; } \\
\text { Winchester Museum }\end{array}$ \\
\hline $\begin{array}{l}\text { Winchester, } \\
\text { Lower Brook } \\
\text { Street }\end{array}$ & Hants & $\begin{array}{l}\text { later } \\
\text { 7th- } \\
\text { 8th } c\end{array}$ & Grave 23: 2 thick annulars & $\begin{array}{l}\text { Hawkes } 1990 \mathrm{~b}, 622 \text {, } \\
625-6 \text { and } 632, \\
\text { fig } 168.1999 \text { and } 2003 \text {, } \\
\text { colour pl XLVII }\end{array}$ \\
\hline
\end{tabular}




\begin{tabular}{|c|c|c|c|c|}
\hline $\begin{array}{l}\text { St Neots, } \\
\text { Lords } \\
\text { Bridge }\end{array}$ & Hunts & & $\begin{array}{l}\text { Grave found in } 1892: \\
4 \text { globulars }\end{array}$ & BM $195610-21$ and 2 \\
\hline $\begin{array}{l}\text { Beakes- } \\
\text { bourne }\end{array}$ & Kent & $\begin{array}{l}\text { prob } \\
\text { 7th } c\end{array}$ & One annular & $\begin{array}{l}\text { Faussett 1856; } \\
\text { Liverpool Museum }\end{array}$ \\
\hline $\begin{array}{l}\text { Breach } \\
\text { Downs }\end{array}$ & Kent & $\begin{array}{l}\text { 6th- } \\
\text { 7th } \mathrm{c}\end{array}$ & One globular & $\begin{array}{l}\text { Conyngham and Akerman } \\
1844, \mathrm{pl} \mathrm{I} ; \mathrm{BM} 795-2189\end{array}$ \\
\hline Coombe & Kent & $\begin{array}{l}\text { mid- } \\
\text { 6th c }\end{array}$ & $\begin{array}{l}\text { Cremation necklace: } \\
\text { globular and annular } \\
\text { possibly with small } \\
\text { square-headed brooch }\end{array}$ & $\begin{array}{l}\text { Davidson and Webster } 1967, \\
20-1 \text {, fig } 5 \mathrm{~g}-\mathrm{i} \text {, pl VIID; } \\
\text { Saffron Walden Museum }\end{array}$ \\
\hline $\begin{array}{l}\text { Dover, } \\
\text { Buckland }\end{array}$ & Kent & $\begin{array}{l}625- \\
650 \\
650- \\
675 \\
\\
675- \\
700 \\
700- \\
750\end{array}$ & $\begin{array}{l}\text { Grave 29: } 1 \text { annular/disc } \\
\text { (B01); } \\
\text { Grave } 30: 12 \text { annulars/discs } \\
\text { (B01); } \\
\text { Grave 38: } 1 \text { annular/disc } \\
\text { (B01); } \\
\text { Grave 59: } 6 \text { globulars/discs } \\
\text { (B01); } \\
\text { Grave 18: } 5 \text { barrels (B02); } \\
\text { Grave 67: } 3 \text { barrels (B02); } \\
\text { Grave 155: } 2 \text { annulars/discs } \\
\text { (B01); } \\
\text { Grave 141: } 1 \text { disc (B01); } \\
\text { Grave } 75: 2 \text { discs (B01) }\end{array}$ & $\begin{array}{l}\text { Evison } 1987,72,79,223 \text {, } \\
224,227,232,220,235 \text {, } \\
250,248 \text { and } 235 \text {, } \\
\text { figs } 17.2 \mathrm{c}, 18.4 \mathrm{~b}, 22.4 \mathrm{~b} \text {, } \\
33.3 \mathrm{~b}, 11.18 .2 \mathrm{~b}, 37.67 .1 \mathrm{e} \text {, } \\
60.155 .2 \mathrm{a}, 58.141 .6 \mathrm{a} \text {, } \\
38.75 .1 \mathrm{~d} ; \\
\mathrm{BM}\end{array}$ \\
\hline Faversham & Kent & $\begin{array}{l}5 \text { th- } \\
7 \text { th c }\end{array}$ & $\begin{array}{l}\text { Lots of small annulars and } \\
\text { globulars among } \\
\text { miscellaneous beads; } \\
4 \text { double beads (Plate 6) }\end{array}$ & $\begin{array}{l}\text { Meaney 1964, 118-19; } \\
\text { Beck Collection, } \\
\text { Cambridge Museum } 47.1824\end{array}$ \\
\hline $\begin{array}{l}\text { Folkestone, } \\
\text { Dover Hill }\end{array}$ & Kent & $\begin{array}{l}\text { prob } \\
\text { 6th } c\end{array}$ & $\begin{array}{l}\text { No. } 14 \text { : various; } \\
\text { No. ?32: } 2 \text { examples }\end{array}$ & $\begin{array}{l}\text { Meaney } 1964,120-1 \\
\text { Folkestone Museum }\end{array}$ \\
\hline $\begin{array}{l}\text { Gilton or } \\
\text { Kingston }\end{array}$ & Kent & $\begin{array}{l}\text { 6th- } \\
7 \text { th } c\end{array}$ & $\begin{array}{l}\text { A number of annulars and } \\
\text { a few globulars and other } \\
\text { forms }\end{array}$ & $\begin{array}{l}\text { Faussett } 1856 \text {, pls V and VI; } \\
\text { Liverpool Museum }\end{array}$ \\
\hline Holborough & Kent & $\begin{array}{l}\text { late } \\
\text { 7th- } \\
\text { 8th c }\end{array}$ & $\begin{array}{l}\text { One globular at least among } \\
47 \text { beads }\end{array}$ & $\begin{array}{l}\text { Evison } 1956,126 \text {, } \\
\text { fig 20.3j; } \\
\text { BM } 19475-2358\end{array}$ \\
\hline $\begin{array}{l}\text { Horton } \\
\text { Kirby I }\end{array}$ & Kent & & Six annulars & $\begin{array}{l}\text { Meaney 1964, 124; } \\
\text { Maidstone Museum } \\
\text { AS } 200\end{array}$ \\
\hline Howletts & Kent & & $\begin{array}{l}\text { Grave 142: } 1 \text { small barrel- } \\
\text { shaped }\end{array}$ & $\begin{array}{l}\text { Smith 1918; } \\
\text { BM 1936 5-11 }\end{array}$ \\
\hline $\begin{array}{l}\text { Lyminge } \\
\text { II }\end{array}$ & Kent & 6th $c$ & $\begin{array}{l}\text { Grave } 24: 2 \text { small with } \\
\text { equal-arm brooch pair, etc; }\end{array}$ & $\begin{array}{l}\text { Warhurst } 1955,18,27 \\
\text { pl VIIb.1 and 2; }\end{array}$ \\
\hline
\end{tabular}


6th $c$

Monkto

Orpington Ken

Ozingell

Polhill

Rochester, Kent

Watts

Avenue

Sarre

Stowting

Westbere

Cleatham

Lincs

prob

6th $\mathrm{c}$

Fonaby

Lincs

6th c

6th $\mathrm{c}$

Nettleton

Lincs

Caistor

mid-

prob

6th c

5th-

6th c

prob

7 th $\mathrm{C}$

7 th $\mathrm{c}$

7 th $\mathrm{c}$

6th-

7 th $\mathrm{c}$

7 th $\mathrm{c}$

prob

6th c

prob

6th-

7th c

prob

7th c

prob 6th $\mathrm{c}$
Grave 39: 2 with squareheaded and saucer brooch pairs

Grave 3: globular or possibly barrel-shaped with composite disc brooch

Grave 41: globular with 2 finger rings

Grave 32: 1 or 2 in group

of 14 beads;

Grave 58: 2 annulars with

disc brooch pair

Several

Four beads, rather barrel-

shaped

late Group CXV;

Unsure background:

probably with other beads

and amethyst;

1 double

Grave 30: several;

Grave 9: several

Grave 3: 2 annulars with annular brooch, etc; Grave 32: 21 annulars with Ảberg Group III cruciform brooch, etc
Maidstone Museum

Hawkes and Hogarth 1974, 59 , fig 5.2e and $f$

Tester 1968,141 ,

fig 6.32a;

Tester 1969, 44-6,

fig $4.58 \mathrm{~b}$;

Orpington Museum

Meaney 1964, 131;

Powell-Cotton Museum

Hawkes and Philp 1973, 177,207 , fig 55.520

Meaney 1964, 134;

Rochester Museum

Brent 1868,

pl VII.CXV;

Smith 1860, pl II

Meaney 1964, 137-8;

Beck Collection,

Cambridge Museum 47.1808

Jessup 1946;

Canterbury Museum

RM 6422, 6425 and 6426

BM 54 12-2 18 and 30;

BM 79 5-24 51

Publication in preparation (K Leahy); Scunthorpe Museum

Cook 1981, 18, 34, figs 4.3.1.31 and 33, 11.32.1.3;

Scunthorpe Museum

Meaney 1964, 160;

Lincoln Museum 


\begin{tabular}{|c|c|c|c|c|}
\hline Ruskington & Lincs & $\begin{array}{l}\text { prob } \\
\text { 6th- } \\
7 \text { th c }\end{array}$ & $\begin{array}{l}\text { About } 10 \text { beads, including } \\
\text { some terracotta }\end{array}$ & $\begin{array}{l}\text { Meaney 1964, 161-2; } \\
\text { Lincoln Museum }\end{array}$ \\
\hline $\begin{array}{l}\text { South } \\
\text { Willingham }\end{array}$ & Lincs & $\begin{array}{l}\text { 6th- } \\
7 \text { th } c\end{array}$ & $\begin{array}{l}\text { Small group: globular or } \\
\text { barrel-shaped }\end{array}$ & BM OA 5057 \\
\hline $\begin{array}{l}\text { Syston } \\
\text { Park }\end{array}$ & Lincs & $\begin{array}{l}\text { prob } \\
\text { 6th- } \\
\text { 7th c }\end{array}$ & Five doubles & $\begin{array}{l}\text { Akerman 1855a, 41-2, } \\
\text { pl XXI; } \\
\text { BM (18) } 1111-92\end{array}$ \\
\hline Waddington & Lincs & & $\begin{array}{l}\text { Single female burial: } \\
2 \text { annulars on necklace }\end{array}$ & $\begin{array}{l}\text { Petch 1957, 19; } \\
\text { Lincoln Museum }\end{array}$ \\
\hline Brooke & Norfolk & 6th $c$ & Very small globular & $\begin{array}{l}\text { Meaney 1964, 170; } \\
\text { BM }\end{array}$ \\
\hline $\begin{array}{l}\text { Castle } \\
\text { Acre }\end{array}$ & Norfolk & $\begin{array}{l}\text { 5th- } \\
\text { 6th } c\end{array}$ & One annular & $\begin{array}{l}\text { Meaney 1964, 172-3; } \\
\text { Norwich Museum }\end{array}$ \\
\hline $\begin{array}{l}\text { Morning } \\
\text { Thorpe }\end{array}$ & Norfolk & $\begin{array}{l}\text { mid- } \\
\text { 6th } \mathrm{c} \\
6 \text { th } \mathrm{C}\end{array}$ & $\begin{array}{l}\text { Grave 30: } 5 \text { beads with } \\
\text { Aberg Group II cruciform } \\
\text { brooch pair, B clasps, etc; } \\
\text { Grave } 91: 1 \text { barrel-shaped } \\
\text { with } \AA \text { berg Group IVa } \\
\text { cruciform brooch, etc; } \\
\text { and a number from other } \\
\text { graves }\end{array}$ & $\begin{array}{l}\text { Green et al } 1987,42-3 \text {, } \\
\text { 61, etc, figs } 303.30 . \mathrm{Ci} \text {, } \\
\text { 325.Ev, etc; } \\
\text { Norwich Museum }\end{array}$ \\
\hline Spong Hill & Norfolk & $\begin{array}{l}5 \text { th- } \\
6 \text { th } c\end{array}$ & $\begin{array}{l}\text { Grave 22: several annulars } \\
\text { and globulars with } \AA \text { berg } \\
\text { Group IVa cruciform } \\
\text { brooch, etc; } \\
\text { Grave } 26: 2 \text { annulars with } \\
\text { Aberg Group I cruciform } \\
\text { brooch, stamped pot, etc }\end{array}$ & $\begin{array}{l}\text { Hills et al } 1984,70 \\
\text { and } 74, \text { figs } 79.5 .9,30 \text {, } \\
31 \text { and 55-8, and } 83.1 \text {; } \\
\text { Norfolk Arch Unit }\end{array}$ \\
\hline
\end{tabular}

Corbridge North- late Necklace: several annulars

Knowles and Forster 1909, umber- 5th- with 2 Aberg Group II 407 ; land 6th $\mathrm{c}$ cruciform brooches

Bury St

Edmunds,

Westgarth

Suffolk prob

Grave 14: 1 globular

6th c

Gardens

Ipswich

Suffolk prob

6th-

Several annulars and globulars

7 th $\mathrm{c}$

Lakenheath Suffolk

Several small annulars

$\begin{array}{llll}\begin{array}{l}\text { Mitchell's } \\ \text { Hill }\end{array} & \text { Suffolk } & \begin{array}{l}\text { ?late } \\ \text { 6th- } \\ \text { 7th c }\end{array} & \text { About 6 globulars } \\ \text { Guildown } & \text { Surrey } & \begin{array}{l}\text { prob } \\ \text { 6th c }\end{array} & \begin{array}{l}\text { Grave 77: several annulars; } \\ \text { Grave 78: several small } \\ \text { annulars }\end{array}\end{array}$

Corbridge Museum

West 1988, 24,

fig 62.14.3;

Bury St Edmunds

Museum

Layard 1907, pl XXXIII, and 1909, pl VI; Ipswich Museum

Meaney 1964, 230; BM 1919 12-22 11

Meaney 1964, 231; Bury St Edmunds Museum

Lowther 1931, 11, 12, 35 and 36, pl IX.2 and 7; Guildford Museum 


\begin{tabular}{|c|c|c|c|c|}
\hline Mitcham & Surrey & $\begin{array}{l}5 \text { th- } \\
6 \text { th } c\end{array}$ & $\begin{array}{l}\text { Grave 62: } 1 \text { annular; } \\
\text { Grave 117: } 1 \text { globular }\end{array}$ & $\begin{array}{l}\text { Bidder and Duckworth } \\
1906 ; \\
\text { Bidder and Morris 1959, } \\
64,68,110 \text { and } 112 \text {, } \\
\text { pl XVI.62 and } 117 \text {; } \\
\text { Cambridge Museum } \\
54.391\end{array}$ \\
\hline Alfriston & Sussex & $\begin{array}{l}\text { 6th c } \\
5 \text { th- } \\
6 \text { th c }\end{array}$ & $\begin{array}{l}\text { Grave } 43 \text { : globular on } \\
\text { bracelet; } \\
\text { Grave 68: } 5 \text { annulars with } \\
\text { quoit brooch; } \\
\text { Grave } 77: 1 \text { annular }\end{array}$ & $\begin{array}{l}\text { Griffith and Salzmann } \\
1914,39-41 \text { and } 47 \text {, } \\
\text { pls XI.3 and I.1; } \\
\text { Griffith } 1915,203 \text {; } \\
\text { Welch } 1983,85-6 \text {, } \\
\text { figs 20a and 32c; } \\
\text { Lewes Museum }\end{array}$ \\
\hline $\begin{array}{l}\text { Apple } \\
\text { Down I }\end{array}$ & Sussex & $\begin{array}{l}\text { prob } \\
\text { 6th c }\end{array}$ & $\begin{array}{l}\text { Grave 90: } 1 \text { annular; } \\
\text { Grave 107: small annular }\end{array}$ & $\begin{array}{l}\text { Down and Welch } 1990 \text {, } \\
46,48,162, \text { figs } 2.32 \text { and } \\
2.35 \text {, colour pl } 40.15 ; \\
\text { Chichester Museum }\end{array}$ \\
\hline Highdown & Sussex & $\begin{array}{l}\text { prob } \\
\text { 6th } c \\
5 \text { th- } \\
6 \text { th } c\end{array}$ & $\begin{array}{l}\text { Grave } 10: 2 \text { or } 3 \text { globulars; } \\
\text { small collection on } \\
\text { necklace: several globulars } \\
\text { and } 1 \text { double }\end{array}$ & $\begin{array}{l}\text { Read 1895; } \\
\text { Welch 1983, 85-6, fig 92i; } \\
\text { Worthing Museum } \\
3488 \text { and } 3486\end{array}$ \\
\hline Wasperton & Warks & $\begin{array}{l}\text { prob } \\
\text { 6th c }\end{array}$ & Several small globulars & $\begin{array}{l}\text { Publication in } \\
\text { preparation (M Carver); } \\
\text { Warwick Museum }\end{array}$ \\
\hline $\begin{array}{l}\text { Colling- } \\
\text { bourne Ducis }\end{array}$ & Wilts & 6th $c$ & $\begin{array}{l}\text { Grave } 20 \text { : annular with cast } \\
\text { saucer brooch pair, etc }\end{array}$ & $\begin{array}{l}\text { Gingell } 1975,66-7, \\
85, \text { fig } 18.9 ; \\
\text { Devizes Museum }\end{array}$ \\
\hline $\begin{array}{l}\text { Harnham } \\
\text { Hill }\end{array}$ & Wilts & 6th $c$ & Annular & $\begin{array}{l}\text { Akerman } 1853 \\
\text { Jackson } 1854 \\
\text { BM } 5312-1430\end{array}$ \\
\hline $\begin{array}{l}\text { Peters- } \\
\text { finger }\end{array}$ & Wilts & 6th $c$ & $\begin{array}{l}\text { Grave } 25 \text { : about } 10 \\
\text { globulars and annulars with } \\
\text { miniature bow brooch and } \\
3 \text { button brooches, etc; } \\
\text { Grave } 62: 1 \text { annular }\end{array}$ & $\begin{array}{l}\text { Leeds and Shortt 1953, } \\
22 \text { and } 41, \text { pls III.94.XXV } \\
\text { and IV.172.LXII; } \\
\text { Salisbury Museum }\end{array}$ \\
\hline Driffield & Yorks & $\begin{array}{l}\text { prob } \\
\text { 6th c }\end{array}$ & C44: many globulars & $\begin{array}{l}\text { Mortimer 1905, 292; } \\
\text { Hull Museum }\end{array}$ \\
\hline Saltburn & Yorks & $\begin{array}{l}\text { 6th- } \\
7 \text { th } c\end{array}$ & $\begin{array}{l}\text { Necklace with globulars } \\
\text { and annulars }\end{array}$ & $\begin{array}{l}\text { Gallagher } 1987,25 \text {, fig } 6 \text {; } \\
\text { Hornsby 1912; } \\
\text { Middlesbrough Museum }\end{array}$ \\
\hline Sewerby & Yorks & 6 th $c$ & $\begin{array}{l}\text { Grave 29: } 2 \text { annulars (A1a) } \\
\text { with } 2 \text { annular brooches; } \\
\text { Grave } 15: 1 \text { medium } \\
\text { globular or barrel (A2c) } \\
\text { with } \AA \text { berg Group IVa } \\
\text { cruciform brooch, etc }\end{array}$ & $\begin{array}{l}\text { Hirst } 1985,62-6 \text {, figs } 37 a \\
\text { and } 43.29 . d\end{array}$ \\
\hline
\end{tabular}


The Glass Beads of Anglo-Saxon England

\begin{tabular}{|c|c|c|c|c|}
\hline $\begin{array}{l}\text { West } \\
\text { Heslerton }\end{array}$ & Yorks & 6th $\mathrm{c}$ & Some globulars and annulars & $\begin{array}{l}\text { Publication in } \\
\text { preparation (D Powlesland) }\end{array}$ \\
\hline near York & Yorks & & One annular & BM $5311-1516$ \\
\hline ii & Terracotta & SMALL & BICONICAL AND TRUE BA & ARREL-SHAPED BEADS \\
\hline Lechlade & Glos & $\begin{array}{l}\text { later } \\
\text { 6th } c \\
\text { mid- } \\
\text { later } \\
7 \text { th } c\end{array}$ & $\begin{array}{l}\text { Grave } 36 / 2 \text { : biconical with } \\
\text { knife; } \\
\text { Grave 187: barrel with } \\
\text { silver cross, etc }\end{array}$ & $\begin{array}{l}\text { Boyle et al forthcoming; } \\
\text { Cirencester Museum }\end{array}$ \\
\hline $\begin{array}{l}\text { Breach } \\
\text { Down }\end{array}$ & Kent & $\begin{array}{l}\text { 7th- } \\
8 \text { th } c\end{array}$ & $\begin{array}{l}\text { Several barrels with } \\
\text { brooches, etc }\end{array}$ & $\begin{array}{l}\text { Conyngham and Akerman } \\
\text { 1844, pl I; BM 79 5-24 }\end{array}$ \\
\hline $\begin{array}{l}\text { Crundale } \\
\text { Down }\end{array}$ & Kent & $\begin{array}{l}\text { prob } \\
\text { 6th- } \\
7 \text { th c }\end{array}$ & $\begin{array}{l}\text { One biconical, possibly } \\
\text { confused with some from } \\
\text { Sarre }\end{array}$ & $\begin{array}{l}\text { Faussett 1856, 177-98; } \\
\text { BM 93 6-1 } 196\end{array}$ \\
\hline $\begin{array}{l}\text { Dover I, } \\
\text { Priory Hill }\end{array}$ & Kent & $\begin{array}{l}\text { 5th- } \\
7 \text { th c }\end{array}$ & Several barrels & $\begin{array}{l}\text { Meaney } 1964,117 \\
\text { BM } 7910-137 \text { and } 8\end{array}$ \\
\hline $\begin{array}{l}\text { Dover, } \\
\text { Buckland }\end{array}$ & Kent & $\begin{array}{l}575- \\
625\end{array}$ & $\begin{array}{l}\text { Grave 1: } 1 \text { biconical (B03) } \\
\text { (Plate 6) }\end{array}$ & $\begin{array}{l}\text { Evison } 1987,72,79,216 \text {, } \\
\text { fig } 5.4 \mathrm{~g} ; \mathrm{BM}\end{array}$ \\
\hline Faversham & Kent & $\begin{array}{l}\text { 5th- } \\
7 \text { th c }\end{array}$ & $\begin{array}{l}\text { One biconical (Plate 6); } \\
2 \text { barrels }\end{array}$ & $\begin{array}{l}\text { Meaney 1964, 118-19; } \\
\text { Ashmolean Museum } \\
\text { 1909.163; } \\
\text { Ashmolean Museum } \\
1909.159\end{array}$ \\
\hline Gilton & Kent & & $\begin{array}{l}\text { Biconical, globular } \\
\text { and annular }\end{array}$ & $\begin{array}{l}\text { Faussett } 1856,1-34 \\
\text { Liverpool Museum }\end{array}$ \\
\hline Howletts & Kent & $\begin{array}{l}\text { 2nd } \\
\text { half } \\
\text { 6th } c\end{array}$ & Grave A: biconical & $\begin{array}{l}\text { Smith 1918; } \\
\text { BM }\end{array}$ \\
\hline Polhill & Kent & 7th c & $\begin{array}{l}\text { Grave } 41 \text { : biconical with } \\
2 \text { finger rings }\end{array}$ & $\begin{array}{l}\text { Hawkes and Philp 1973, } \\
177,207 \text {, fig } 5.520\end{array}$ \\
\hline Stowting & Kent & $\begin{array}{l}\text { prob } \\
\text { 6th- } \\
7 \text { th c }\end{array}$ & $\begin{array}{l}\text { One biconical; } \\
2 \text { barrels }\end{array}$ & $\begin{array}{l}\text { Beck Collection, } \\
\text { Cambridge Museum } 471811 \text {; } \\
\text { Maidstone Museum } \\
\text { AS } 300\end{array}$ \\
\hline Wingham & Kent & & Several barrels & $\begin{array}{l}\text { Meaney } 1964,140-1 ; \\
\text { BM 79 5-24 } 51\end{array}$ \\
\hline $\begin{array}{l}\text { South } \\
\text { Willingham }\end{array}$ & Lincs & $\begin{array}{l}6 \text { th- } \\
7 \text { th } c\end{array}$ & Two barrels & $\begin{array}{l}\text { Meaney } 1964,165 \\
\text { BM OA } 5057\end{array}$ \\
\hline $\begin{array}{l}\text { Morning } \\
\text { Thorpe }\end{array}$ & Norfolk & $\begin{array}{l}\text { later } \\
\text { 6th c }\end{array}$ & $\begin{array}{l}\text { Grave 342: taller and rather } \\
\text { larger biconical with } \\
\text { annular brooch pair, florid } \\
\text { brooch knob, etc }\end{array}$ & $\begin{array}{l}\text { Green et al 1987, } 133 \text {, } \\
\text { fig } 410.342 . L i i i ; \\
\text { Norwich Museum }\end{array}$ \\
\hline Icklingham & Suffolk & $\begin{array}{l}\text { late } \\
\text { 6th c }\end{array}$ & One barrel & $\begin{array}{l}\text { Meaney 1964, 231; } \\
\text { Bury St Edmunds Museum }\end{array}$ \\
\hline
\end{tabular}




\begin{tabular}{|c|c|c|c|c|}
\hline Ipswich & Suffolk & $\begin{array}{l}\text { late } \\
6 \text { th } c \\
\text { 6th- } \\
\text { 7th c }\end{array}$ & $\begin{array}{l}\text { One barrel; } \\
\text { one or } 2 \text { barrels }\end{array}$ & $\begin{array}{l}\text { Layard 1907, pls XXXII } \\
\text { and XXXIII, and 1909, } \\
\text { pls VII and VI; } \\
\text { Ipswich Museum }\end{array}$ \\
\hline & & iii & \multicolumn{2}{|c|}{ TERRACOTTA CYLINDER BEADS } \\
\hline Barrington & Cambs & $\begin{array}{l}\text { 5th- } \\
7 \text { th } c\end{array}$ & $\begin{array}{l}\text { Four various sizes among } \\
\text { mixed beads }\end{array}$ & $\begin{array}{l}\text { Fox 1923, 251-2; } \\
\text { Ashmolean Museum }\end{array}$ \\
\hline Mucking II & Essex & $\begin{array}{l}\text { first } \\
\text { half } \\
5 \text { th } c\end{array}$ & $\begin{array}{l}\text { Various, including: Grave } 989 \text { : } \\
\text { with Glaston-Mucking and } \\
\text { supporting-arm brooches, etc }\end{array}$ & $\begin{array}{l}\text { Hirst and Clark forthcoming; } \\
\text { Evison } 1981,138-9 \text {, } \\
\text { figs } 4 \text { and } 5 ; B M\end{array}$ \\
\hline Coombe & Kent & $\begin{array}{l}\text { mid- } \\
\text { 6th } c\end{array}$ & $\begin{array}{l}\text { Cremation: necklace, } \\
\text { possibly with small square- } \\
\text { headed brooch }\end{array}$ & $\begin{array}{l}\text { Davidson and Webster } \\
\text { 1967, 20-1, fig 5f, } \\
\text { pl VII.D; } \\
\text { BM } 186512-143\end{array}$ \\
\hline $\begin{array}{l}\text { Dover, } \\
\text { Buckland }\end{array}$ & Kent & $\begin{array}{l}650- \\
675\end{array}$ & Grave 6: 7 cylinders (B07) & $\begin{array}{l}\text { Evison } 1987,72,79,217 \text {, } \\
\text { fig 7.10f; BM }\end{array}$ \\
\hline $\begin{array}{l}\text { Gilton or } \\
\text { Kingston }\end{array}$ & Kent & $\begin{array}{l}\text { 6th- } \\
7 \text { th } c\end{array}$ & Several not very large & $\begin{array}{l}\text { Faussett 1856, 1-34; } \\
\text { Liverpool Museum }\end{array}$ \\
\hline Howletts & Kent & $\begin{array}{l}\text { 6th- } \\
\text { 7th } c\end{array}$ & Group 142: several & $\begin{array}{l}\text { Smith 1918; } \\
\text { BM 1936 5-11 }\end{array}$ \\
\hline $\begin{array}{l}\text { Castle } \\
\text { Bytham }\end{array}$ & Lincs & $\begin{array}{l}\text { prob } \\
\text { 7th c }\end{array}$ & One with quoit brooch & $\begin{array}{l}\text { Akerman 1855a, 26-7, } \\
\text { pl XII.4 }\end{array}$ \\
\hline 'E Anglia' & Norfolk & & $\begin{array}{l}\text { Group of unassociated } \\
\text { beads: } 6 \text { of various sizes }\end{array}$ & $\begin{array}{l}\text { Fitch Collection, Norwich } \\
\text { Museum } 427641-264\end{array}$ \\
\hline $\begin{array}{l}\text { Melton } \\
\text { Constable }\end{array}$ & Norfolk & $\begin{array}{l}\text { 6th- } \\
\text { 7th } c\end{array}$ & One rather large & $\begin{array}{l}\text { Woodward Collection } \\
1838, \text { Norwich Museum }\end{array}$ \\
\hline $\begin{array}{l}\text { Morning } \\
\text { Thorpe }\end{array}$ & Norfolk & $\begin{array}{l}5 \text { th- } \\
6 \text { th c }\end{array}$ & $\begin{array}{l}\text { Grave 90: with } 3 \text { Åberg } \\
\text { Group II cruciform } \\
\text { brooches, B clasps, etc }\end{array}$ & $\begin{array}{l}\text { Green et al 1987, } 60 \text {, } \\
\text { fig 324.Axiv; } \\
\text { Norwich Museum }\end{array}$ \\
\hline Spong Hill & Norfolk & 6th $c$ & $\begin{array}{l}\text { Grave } 42: 2 \text { cylinders with } \\
\text { square-headed small-long } \\
\text { brooch pair, B clasps, etc; } \\
\text { Grave } 56: 1 \text { cylinder with } \\
\text { annular brooch pair, etc } \\
\text { (Plate 6) }\end{array}$ & $\begin{array}{l}\text { Hills et al } 1984,95-6 \text {, } \\
109 \text {, figs } 98.42 .3 ; \\
\text { and 106.56.3; } \\
\text { Norfolk Arch Unit }\end{array}$ \\
\hline Mitcham & Surrey & $\begin{array}{l}\text { prob } \\
6 \text { th c }\end{array}$ & Grave 129: 1 broken & $\begin{array}{l}\text { Bidder and Morris } 1959 \text {, } \\
69,112 \text {, pl XVI.129; } \\
\text { Cambridge Museum } 54.392\end{array}$ \\
\hline Hessle II & Yorks & $\begin{array}{l}\text { prob } \\
\text { 6th c }\end{array}$ & $\begin{array}{l}\text { Badly made and rather large } \\
\text { with pots, etc, and many } \\
\text { amber beads }\end{array}$ & Hull Museum records \\
\hline
\end{tabular}




\section{iv TeRracotTA SHORT CYLINDER BEADS, SINGLES AND DOUBLES}

\begin{tabular}{|c|c|c|c|c|}
\hline $\begin{array}{l}\text { Chamber- } \\
\text { lain's } \\
\text { Barn II }\end{array}$ & Beds & $\begin{array}{l}\text { mid- } \\
\text { late } \\
\text { 7th c }\end{array}$ & Grave 8 & $\begin{array}{l}\text { Hyslop 1963, 173, fig 8b; } \\
\text { Luton Museum }\end{array}$ \\
\hline $\begin{array}{l}\text { Hasling- } \\
\text { field }\end{array}$ & Cambs & & $\begin{array}{l}\text { Miscellaneous groups } \\
\text { on strings }\end{array}$ & $\begin{array}{l}\text { Meaney 1964, 66-7; } \\
\text { Ashmolean Museum } \\
1909.333\end{array}$ \\
\hline Mucking II & Essex & $\begin{array}{l}\text { prob } \\
\text { 7th c }\end{array}$ & $\begin{array}{l}\text { Various, including } \\
\text { Grave } 608\end{array}$ & $\begin{array}{l}\text { Hirst and Clark forthcoming } \\
\text { BM }\end{array}$ \\
\hline $\begin{array}{l}\text { Springfield } \\
\text { Lyons }\end{array}$ & Essex & & Grave $6033(1)$ & $\begin{array}{l}\text { Publication in } \\
\text { preparation (S Tyler); } \\
\text { BM }\end{array}$ \\
\hline Lechlade & Glos & 6th $c$ & Grave 101: 2 & $\begin{array}{l}\text { Boyle et al forthcoming; } \\
\text { Cirencester Museum }\end{array}$ \\
\hline Andover & Hants & $\begin{array}{l}5 \text { th- } \\
\text { 6th c }\end{array}$ & $\begin{array}{l}\text { Grave 50: } 1 \text { large with } \\
\text { square-headed small-long } \\
\text { brooch, openwork Quoit } \\
\text { Brooch Style buckle, etc }\end{array}$ & $\begin{array}{l}\text { Cook and Dacre } 1985,82 \text {, } \\
\text { fig } 63.35 \text {; } \\
\text { Hampshire Museums }\end{array}$ \\
\hline $\begin{array}{l}\text { Beakes- } \\
\text { bourne }\end{array}$ & Kent & $\begin{array}{l}\text { 6th- } \\
7 \text { th } c\end{array}$ & & Faussett $1856, \mathrm{pl} \mathrm{V.4}$ \\
\hline $\begin{array}{l}\text { Breach } \\
\text { Down }\end{array}$ & Kent & $\begin{array}{l}\text { 6th- } \\
\text { 7th c }\end{array}$ & Only 1 bead & $\begin{array}{l}\text { Conyngham and Akerman } \\
1844 \text {, pl 1; } \\
\text { BM 79 5-24 } 29\end{array}$ \\
\hline $\begin{array}{l}\text { Chartham } \\
\text { Down }\end{array}$ & Kent & $\begin{array}{l}\text { prob } \\
\text { 6th c }\end{array}$ & Grave 14: 1 & $\begin{array}{l}\text { Faussett } 1856,160-76 \\
\text { Liverpool Museum } \\
\text { M } 6722\end{array}$ \\
\hline Coombe & Kent & $\begin{array}{l}\text { mid- } \\
\text { 6th c }\end{array}$ & $\begin{array}{l}\text { Cremation: necklace, } \\
\text { several, possibly with } \\
\text { small square-headed } \\
\text { brooch, etc }\end{array}$ & $\begin{array}{l}\text { Davidson and Webster } \\
\text { 1967, 20-1, fig } 5 \mathrm{~g} \text {, } \\
\text { pl VII.D; } \\
\text { Saffron Walden Museum }\end{array}$ \\
\hline $\begin{array}{l}\text { Dover, } \\
\text { Buckland }\end{array}$ & Kent & $\begin{array}{l}625- \\
650 \\
675- \\
700\end{array}$ & $\begin{array}{l}\text { Grave 1: } 4 \text { short cylinders } \\
\text { (B04); } \\
\text { Grave 29: } 1 \text { double (B09); } \\
\text { Grave 60: } 8 \text { short cylinders } \\
\text { (B04); } \\
\text { Grave 62: } 2 \text { short cylinders } \\
\text { (B04); } \\
\text { Grave 107: } 1 \text { short cylinder } \\
\text { (B04); } \\
\text { Grave 110: } 6 \text { short } \\
\text { cylinders (B05); } \\
\text { Grave 160: } 1 \text { short cylinder } \\
\text { (B05); } \\
\text { Grave 127: } 3 \text { short } \\
\text { cylinders (B05) }\end{array}$ & $\begin{array}{l}\text { Evison } 1987,72,79, \\
216,223-4,233,234, \\
241,251,243 \text {, figs } 5.4 \mathrm{i}, \\
17.2 \mathrm{~g}, 34.3 \mathrm{f}, 35.62 .4 \mathrm{f}, \\
48.107 .6 \mathrm{~b}, 49.110 .12 \mathrm{a}, \\
62.160 .2 \mathrm{a}, \\
52.127 .1 \mathrm{~b} \text { and } \mathrm{c} ; \mathrm{BM}\end{array}$ \\
\hline $\begin{array}{l}\text { Eastry, } \\
\text { Eastry House }\end{array}$ & Kent & $\begin{array}{l}\text { late } \\
\text { 6th } c\end{array}$ & $\begin{array}{l}\text { Grave: a number, with a } \\
\text { Gotlandic bow brooch }\end{array}$ & $\begin{array}{l}\text { Hawkes 1979, 86, 93, } \\
\text { fig 4.4; Deal Museum }\end{array}$ \\
\hline
\end{tabular}




\begin{tabular}{|c|c|c|c|c|}
\hline Faversham & Kent & $\begin{array}{l}5 \text { th- } \\
\text { 7th c }\end{array}$ & $\begin{array}{l}\text { A number, both singles } \\
\text { and doubles (Plate 6) }\end{array}$ & $\begin{array}{l}\text { Meaney 1964, 118-19; } \\
\text { Beck Collection, } \\
\text { Cambridge Museum; } \\
\text { Ashmolean Museum }\end{array}$ \\
\hline $\begin{array}{l}\text { Folkestone, } \\
\text { Dover Hill }\end{array}$ & Kent & $\begin{array}{l}\text { prob } \\
\text { 6th c }\end{array}$ & Nos 15 and 28: about 10 & $\begin{array}{l}\text { Meaney } 1964,120-1 ; \\
\text { Maidstone Museum }\end{array}$ \\
\hline $\begin{array}{l}\text { Gilton or } \\
\text { Kingston }\end{array}$ & Kent & $\begin{array}{l}\text { 6th- } \\
\text { 7th c }\end{array}$ & Several & $\begin{array}{l}\text { Faussett } 1856,1-34 ; \\
\text { Liverpool Museum }\end{array}$ \\
\hline Holborough & Kent & $\begin{array}{l}\text { late } \\
7 \text { th- } \\
8 \text { th c }\end{array}$ & Necklace: 1 & $\begin{array}{l}\text { Evison 1956, 126, } \\
\text { fig 20.3m; } \\
\text { BM } 1947 \text { 5-2 } 358\end{array}$ \\
\hline Howletts & Kent & $\begin{array}{l}\text { 2nd } \\
\text { half } \\
\text { 6the }\end{array}$ & No. 142: several & $\begin{array}{l}\text { Smith 1918; } \\
\text { BM } 19365-11\end{array}$ \\
\hline 'Kent' & Kent & & $\begin{array}{l}\text { Numbers of doubles } \\
\text { unprovenanced }\end{array}$ & Canterbury Museum \\
\hline $\begin{array}{l}\text { Milton } \\
\text { Regis }\end{array}$ & Kent & 7 th $c$ & Necklace: a number & $\begin{array}{l}\text { Hawkes and Grove } 1963 \text {, } \\
32 \text {, fig } 2.4\end{array}$ \\
\hline Polhill & Kent & 7th $c$ & $\begin{array}{l}\text { Grave 29: } 1 \text { bead }(516) ; \\
\text { Grave 37: } 1 \text { bead (517); } \\
\text { Grave 41: } 2 \text { beads }(520) ; \\
\text { Grave } 86: 1 \text { bead }(525) ; \\
\text { Grave } 95: 1 \text { bead ( } 526) ; \\
\text { Grave 102: } 4 \text { beads ( } 527) ; \\
\text { Unstratified: } 1 \text { bead (529) }\end{array}$ & $\begin{array}{l}\text { Hawkes and Philp } 1973 \text {, } \\
175,176,177,183,184 \\
\text { and } 185,207, \text { fig } 55.516, \\
517,520,525 \text {, } \\
526,527 \text { and } 529\end{array}$ \\
\hline Sarre & Kent & $\begin{array}{l}\text { late } \\
\text { 6th- } \\
\text { 7th c }\end{array}$ & $\begin{array}{l}\text { Group XXIII: } 1 \text { double; } \\
\text { Group LXXV: } 1 \text { double; } \\
\text { Group IV: single (?) }\end{array}$ & $\begin{array}{l}\text { Brent } 1868 \text {, pls VII.XXIII } \\
\text { and VIII.LXXV and IV }\end{array}$ \\
\hline Stowting & Kent & $\begin{array}{l}\text { prob } \\
6 \text { th- } \\
7 \text { th c }\end{array}$ & $\begin{array}{l}\text { One example; } \\
4 \text { examples }\end{array}$ & $\begin{array}{l}\text { Meaney 1964, 137-8; } \\
\text { Maidstone Museum }\end{array}$ \\
\hline Westbere & Kent & $\begin{array}{l}\text { 6th- } \\
7 \text { th } c\end{array}$ & $\begin{array}{l}\text { On } 1 \text { of } 3 \text { strings: } \\
1 \text { bead }\end{array}$ & $\begin{array}{l}\text { Jessup 1946; } \\
\text { Canterbury Museum } \\
\text { RM 6422, 6425, } 6426\end{array}$ \\
\hline Wingham & Kent & 7th $c$ & One double and 1 single & $\begin{array}{l}\text { Meaney } 1964,140-1 ; \\
\text { BM } 5412-218 \text { and } 20\end{array}$ \\
\hline $\begin{array}{l}\text { Castle } \\
\text { Bytham }\end{array}$ & Lincs & $\begin{array}{l}\text { prob } \\
\text { 6th- } \\
\text { 7th c }\end{array}$ & Several & $\begin{array}{l}\text { Akerman } 1855 a, 26-7 \text {, } \\
\text { pl XII.4 }\end{array}$ \\
\hline $\begin{array}{l}\text { Nettleton } \\
\text { Caistor }\end{array}$ & Lincs & $\begin{array}{l}\text { 6th- } \\
7 \text { th } c\end{array}$ & One example in group & $\begin{array}{l}\text { Meaney } 1964,160 \\
\text { Lincoln Museum }\end{array}$ \\
\hline Waddington & Lincs & & Single burial: 4 to 5 & $\begin{array}{l}\text { Petch 1957, 19; } \\
\text { Lincoln Museum }\end{array}$ \\
\hline $\begin{array}{l}\text { Welbeck } \\
\text { Hill }\end{array}$ & Lincs & $\begin{array}{l}5 \text { th- } \\
6 \text { th } c\end{array}$ & $\begin{array}{l}\text { One with } 2 \text { small-long } \\
\text { brooches }\end{array}$ & $\begin{array}{l}\text { Publication in } \\
\text { preparation (G Taylor); } \\
\text { Scunthorpe Museum }\end{array}$ \\
\hline
\end{tabular}




\begin{tabular}{|c|c|c|c|c|}
\hline $\begin{array}{l}\text { Morning } \\
\text { Thorpe }\end{array}$ & Norfolk & $\begin{array}{l}\text { 6th } c \\
\text { 6th } c\end{array}$ & $\begin{array}{l}\text { Grave 30: } 5 \text { beads with } \\
\text { Aberg Group II cruciform } \\
\text { brooch pair, B13b clasps, } \\
\text { etc; } \\
\text { Grave 288: } 7 \text { beads with } \\
\text { great square-headed brooch; } \\
\text { Grave 293: } 4 \text { beads with } \\
\text { annular brooch pair, B7 } \\
\text { clasps, etc; } \\
\text { Grave } 371: 2 \text { beads with } \\
\text { Aberg Group III cruciform } \\
\text { brooch, great square-headed } \\
\text { brooch, etc }\end{array}$ & $\begin{array}{l}\text { Green } \text { et al } 1987,42-3 \text {, } \\
115,116 \text { and } 145 ; \\
\text { figs } 303.30 . \mathrm{Ci} \text {, } \\
\text { 389.288.Fiii, } \\
\text { 390.Miii and } 431 . \text { Hiii; } \\
\text { Norwich Museum }\end{array}$ \\
\hline Corbridge & $\begin{array}{l}\text { North- } \\
\text { umber- } \\
\text { land }\end{array}$ & $\begin{array}{l}\text { 5th- } \\
\text { 6th c }\end{array}$ & $\begin{array}{l}\text { Two or } 3 \text { with Åberg } \\
\text { Group II cruciform brooches }\end{array}$ & $\begin{array}{l}\text { Knowles and Forster 1909, } \\
407 ; \\
\text { Corbridge Museum }\end{array}$ \\
\hline $\begin{array}{l}\text { Standlake } \\
\text { Down }\end{array}$ & Oxon & $\begin{array}{l}\text { early } \\
\text { 7th c }\end{array}$ & Grave 24: 3 doubles & $\begin{array}{l}\text { Dickinson } 1973,244-5 \text {, } \\
252-4 \text {, fig 5.1a; } \\
\text { Ashmolean Museum }\end{array}$ \\
\hline $\begin{array}{l}\text { Empingham } \\
\text { II }\end{array}$ & Rutland & $\begin{array}{l}\text { 5th- } \\
7 \text { th c }\end{array}$ & Grave 85A: short cylinder & $\begin{array}{l}\text { Timby 1996; } \\
\text { Oakham Museum }\end{array}$ \\
\hline Camerton & Somerset & 7 th $c$ & $\begin{array}{l}\text { Grave } 97 \text { on child's } \\
\text { necklace: } 1 \text { bead }\end{array}$ & $\begin{array}{l}\text { Horne } 1933,46,60-1 \\
\text { Taunton Museum }\end{array}$ \\
\hline $\begin{array}{l}\text { Bury St } \\
\text { Edmunds, } \\
\text { Westgarth } \\
\text { Gardens }\end{array}$ & Suffolk & 6th $c$ & Grave 31: 1 bead & $\begin{array}{l}\text { West 1988, } 28 \text {, } \\
\text { fig } 68.31 . A 13 \text {; } \\
\text { Bury St Edmunds } \\
\text { Museum }\end{array}$ \\
\hline Ipswich & Suffolk & $\begin{array}{l}\text { 6th- } \\
7 \text { th c }\end{array}$ & $\begin{array}{l}\text { Various strings: several } \\
\text { singles and } 1 \text { double }\end{array}$ & $\begin{array}{l}\text { Layard 1907, pl XXXII, and } \\
\text { 1909, pl VII; } \\
\text { Ipswich Museum }\end{array}$ \\
\hline West Stow & Suffolk & $\begin{array}{l}\text { prob } \\
\text { 6th c }\end{array}$ & Settlement: 1 bead & West 1985,73 \\
\hline Mitcham & Surrey & $\begin{array}{l}\text { prob } \\
\text { late } \\
\text { 6th c }\end{array}$ & $\begin{array}{l}\text { Grave } 117: 1 \text { bead; } \\
\text { Grave } 62: 1 \text { double with } \\
\text { signs of turquoise and several } \\
\text { doubles and singles }\end{array}$ & $\begin{array}{l}\text { Bidder and Duckworth } \\
\text { 1906; Bidder and Morris } \\
\text { 1959,68, 112, pl XVI; } \\
\text { Cambridge Museum }\end{array}$ \\
\hline Alfriston & Sussex & $\begin{array}{l}\text { 2nd } \\
\text { half } \\
\text { 6th } c\end{array}$ & Grave 64: 1 only & $\begin{array}{l}\text { Griffith and Salzmann 1914, } \\
\text { 45; Welch 1983, 85-6, } \\
\text { fig 30a; Lewes Museum }\end{array}$ \\
\hline $\begin{array}{l}\text { Apple } \\
\text { Down I }\end{array}$ & Sussex & $\begin{array}{l}\text { prob } \\
\text { 6th c }\end{array}$ & $\begin{array}{l}\text { Grave } 117: 1 \text { broken with } \\
\text { amber beads; } \\
\text { Grave 134: } 2 \text { beads with } \\
\text { amber beads }\end{array}$ & $\begin{array}{l}\text { Down and Welch } 1990,49, \\
51,162-4, \text { figs } 2.37 \\
\text { and } 2.43, \text { colour pl } 41.46 ; \\
\text { Chichester Museum }\end{array}$ \\
\hline $\begin{array}{l}\text { West } \\
\text { Heslerton }\end{array}$ & Yorks & $\begin{array}{l}\text { prob } \\
\text { 6th c }\end{array}$ & $\begin{array}{l}917: 2 \text { (associations not yet } \\
\text { known) }\end{array}$ & $\begin{array}{l}\text { Publication in } \\
\text { preparation (D Powlesland) }\end{array}$ \\
\hline
\end{tabular}


v TeRracotTa CYLINDER BEADS WITH SQUaRE OR PANELLED POLYGONAL SECTIONS

\begin{tabular}{|c|c|c|c|c|}
\hline Alton & Hants & 7 th $\mathrm{c}$ & $\begin{array}{l}\text { Grave 39: } 1 \text { square section } \\
\text { (B02) }\end{array}$ & $\begin{array}{l}\text { Evison } 1988 \mathrm{a}, 16,44, \\
80-1, \text { fig } 33.39 .1 \mathrm{f} \\
\text { Hampshire Museums }\end{array}$ \\
\hline $\begin{array}{l}\text { Dover, } \\
\text { Buckland }\end{array}$ & Kent & $\begin{array}{l}650- \\
675\end{array}$ & $\begin{array}{l}\text { Grave 155: } 1 \text { square section } \\
\text { (B08) }\end{array}$ & $\begin{array}{l}\text { Evison } 1987,72,79,250, \\
\text { fig } 60.155 .2 \mathrm{c} ; \mathrm{BM}\end{array}$ \\
\hline Faversham & Kent & $\begin{array}{l}6 \text { th- } \\
7 \text { th } c\end{array}$ & $\begin{array}{l}\text { One square section and } \\
1 \text { pentagonal }\end{array}$ & $\begin{array}{l}\text { Meaney } 1964,118-19 ; \\
\text { Ashmolean Museum }\end{array}$ \\
\hline Finglesham & Kent & $\begin{array}{l}\text { first } \\
\text { half } \\
\text { 6th } c\end{array}$ & $\begin{array}{l}\text { Grave D3: } 1 \text { square } \\
\text { section with great square- } \\
\text { headed brooch, etc }\end{array}$ & $\begin{array}{l}\text { Chadwick } 1958,12 \text {, } \\
\text { fig 5o; } \\
\text { Deal Museum }\end{array}$ \\
\hline Gilton & Kent & $\begin{array}{l}6 \text { th- } \\
7 \text { th c }\end{array}$ & & $\begin{array}{l}\text { Faussett 1856, pl V; } \\
\text { Liverpool Museum }\end{array}$ \\
\hline Howletts & Kent & $\begin{array}{l}\text { prob } \\
6 \text { th } c\end{array}$ & Group 142: 2 beads & $\begin{array}{l}\text { Smith 1918; } \\
\text { BM } 19365-11\end{array}$ \\
\hline Westbere & Kent & 7 th $\mathrm{c}$ & $\begin{array}{l}\text { On } 1 \text { of } 3 \text { strings: } \\
2 \text { pentagonal (Plate } 6)\end{array}$ & $\begin{array}{l}\text { Jessup 1946; } \\
\text { Canterbury Museum }\end{array}$ \\
\hline Wingham & Kent & 7 th $\mathrm{c}$ & $\begin{array}{l}\text { One thin square section } \\
\text { perhaps with amethysts }\end{array}$ & $\begin{array}{l}\text { Meaney } 1964,140-1 \\
\text { BM } 795-2451\end{array}$ \\
\hline Brooke & Norfolk & $\begin{array}{l}\text { 6th- } \\
\text { early } \\
7 \text { th c }\end{array}$ & & $\begin{array}{l}\text { Meaney 1964, 170; } \\
\text { BM 1866-69 }\end{array}$ \\
\hline $\begin{array}{l}\text { Morning } \\
\text { Thorpe }\end{array}$ & Norfolk & 6th $\mathrm{c}$ & $\begin{array}{l}\text { Grave } 359: 1 \text { bead with } \\
\text { great square-headed } \\
\text { brooch, etc }\end{array}$ & $\begin{array}{l}\text { Green } \text { et al } 1987,139 \text {, } \\
\text { fig } 421.259 . \text { Piii; } \\
\text { Norwich Museum }\end{array}$ \\
\hline Ipswich & Suffolk & $\begin{array}{l}\text { 6th- } \\
\text { early } \\
7 \text { th c }\end{array}$ & Necklace: 2 beads & $\begin{array}{l}\text { Layard } 1907 \text { and } \\
\text { 1909; } \\
\text { Ipswich Museum }\end{array}$ \\
\hline Snape & Suffolk & $\begin{array}{l}\text { prob } \\
7 \text { th } c\end{array}$ & $\begin{array}{l}\text { Grave } 0421: 2 \text { square- } \\
\text { sectioned }\end{array}$ & $\begin{array}{l}\text { Filmer-Sankey and } \\
\text { Pestell forthcoming }\end{array}$ \\
\hline Saltburn & Yorks & $\begin{array}{l}\text { 6th- } \\
7 \text { th } c\end{array}$ & $\begin{array}{l}\text { Long - as bead from } \\
\text { Finglesham above }\end{array}$ & $\begin{array}{l}\text { Gallagher 1987, } 25 \text {, } \\
\text { fig 5.39; } \\
\text { Middlesbrough Museum }\end{array}$ \\
\hline Sewerby & Yorks & 6th c & $\begin{array}{l}\text { Grave 16: Type A5c } \\
\text { (Plate 6) with clasps }\end{array}$ & $\begin{array}{l}\text { Hirst } 1985,66 \text {, } \\
\text { fig } 38.16 .3\end{array}$ \\
\hline $\begin{array}{l}\text { West } \\
\text { Heslerton }\end{array}$ & Yorks & $\begin{array}{l}\text { prob } \\
6 \text { th } c\end{array}$ & Miscellaneous graves & $\begin{array}{l}\text { Publication in } \\
\text { preparation (D Powlesland) }\end{array}$ \\
\hline vi & Terrac & TTA & LON AND SUB-MELON & Ds (see Map 22) \\
\hline $\begin{array}{l}\text { Great } \\
\text { Chesterford }\end{array}$ & Essex & $\begin{array}{l}5 \text { th- } \\
6 \text { th } c\end{array}$ & $\begin{array}{l}\text { Grave 79: sub-melon (B03) } \\
\text { (Plate 6) with iron } \\
\text { penannular brooch, girdle } \\
\text { hanger shaft fragment, etc }\end{array}$ & $\begin{array}{l}\text { Evison } 1994,14,46,103 \text {, } \\
\text { fig } 37.79 .2 \mathrm{~h} \text {; } \\
\text { BM } 19647-2268\end{array}$ \\
\hline
\end{tabular}




\begin{tabular}{|c|c|c|c|c|}
\hline Mucking II & Essex & $\begin{array}{l}\text { prob } \\
6 \text { th c }\end{array}$ & Grave 340: with buckle & $\begin{array}{l}\text { Hirst and Clark forthcoming } \\
\text { BM }\end{array}$ \\
\hline Fonaby & Lincs & $\begin{array}{l}\text { prob } \\
\text { 6th c }\end{array}$ & $\begin{array}{l}\text { Grave 3: } 1 \text { bead with } 2 \\
\text { annular brooches, etc }\end{array}$ & $\begin{array}{l}\text { Cook 1981, 18, fig 4.3.36, } \\
\text { pl VIIA; } \\
\text { Scunthorpe Museum }\end{array}$ \\
\hline Waddington & Lincs & $\begin{array}{l}\text { prob } \\
6 \text { th c }\end{array}$ & $\begin{array}{l}11 \text { inhumations in } 1947 \text { : } \\
1 \text { small bead }\end{array}$ & $\begin{array}{l}\text { Petch 1957, 19; } \\
\text { Lincoln Museum }\end{array}$ \\
\hline $\begin{array}{l}\text { Morning } \\
\text { Thorpe }\end{array}$ & Norfolk & $\begin{array}{l}\text { 5th- } \\
\text { 6th c } \\
\text { prob } \\
\text { 6th c }\end{array}$ & $\begin{array}{l}\text { Grave 328: } 1 \text { bead with } \\
\text { small-long brooch pair, } \\
\text { etc; } \\
\text { Grave } 383: 1 \text { smaller }\end{array}$ & $\begin{array}{l}\text { Green et al } 1987,128 \text { and } \\
149, \text { figs } 402.328 . \mathrm{Ci} \text { and } \\
\text { 438.383.Bii; } \\
\text { Norwich Museum }\end{array}$ \\
\hline Spong Hill & Norfolk & 6th $c$ & $\begin{array}{l}\text { Grave 18: } 2 \text { beads with } \\
\text { great square-headed and } \\
\text { small-long brooch, etc; } \\
\text { Grave 26: } 1 \text { bead with } \\
\text { Áberg Group I cruciform } \\
\text { brooch, etc }\end{array}$ & $\begin{array}{l}\text { Hills et al } 1984,67 \text { and } 74 \text {, } \\
\text { figs } 77.18 .4 \text { and } 83.1 \text {; } \\
\text { Norfolk Arch Unit }\end{array}$ \\
\hline $\begin{array}{l}\text { Bury St } \\
\text { Edmunds, } \\
\text { Westgarth } \\
\text { Gardens }\end{array}$ & Suffolk & 6th $c$ & $\begin{array}{l}\text { Grave } 48: 2 \text { small with } 2 \\
\text { small-long brooches, } \\
\text { B clasps, etc }\end{array}$ & $\begin{array}{l}\text { West } 1988,32 \text {, } \\
\text { fig } 73 . E 8-9 ; \\
\text { Bury St Edmunds } \\
\text { Museum }\end{array}$ \\
\hline Ipswich & Suffolk & $\begin{array}{l}\text { 6th- } \\
7 \text { th } c\end{array}$ & $\begin{array}{l}\text { Uncertain whether it is of } \\
\text { terracotta glass or amber }\end{array}$ & $\begin{array}{l}\text { Layard 1907, pl XXXII, and } \\
\text { 1909, pl VII; } \\
\text { Ipswich Museum }\end{array}$ \\
\hline Wasperton & Warks & $\begin{array}{l}\text { mid- } \\
6 \text { th } c\end{array}$ & $\begin{array}{l}\text { F1081: } 1 \text { sub-melon of } \\
5 \text { lobes with saucer } \\
\text { brooches, etc }\end{array}$ & $\begin{array}{l}\text { Publication in } \\
\text { preparation (M Carver); } \\
\text { Warwick Museum }\end{array}$ \\
\hline
\end{tabular}

\section{POLYCHROME}

vii Terracotta cylinder beads with square or Polygonal SECTIONS AND WITH COLOURED COMBING (INCLUDING GREEN AND YELLOW) (see Map 23)

\begin{tabular}{|c|c|c|c|c|}
\hline Reading & Berks & $\begin{array}{l}\text { 6th- } \\
7 \text { th } c\end{array}$ & $\begin{array}{l}\text { Two with yellow on central } \\
\text { black panel }\end{array}$ & $\begin{array}{l}\text { Stevens } 1894,156-7 \\
\text { Reading Museum }\end{array}$ \\
\hline $\begin{array}{l}\text { Newport } \\
\text { Pagnell }\end{array}$ & Bucks & $\begin{array}{l}\text { prob } \\
\text { 6th- } \\
7 \text { th c }\end{array}$ & Atypical, black or white & $\begin{array}{l}\text { Meaney } 1964,58 \\
\text { Aylesbury Museum }\end{array}$ \\
\hline Alton & Hants & 7th $\mathrm{c}$ & $\begin{array}{l}\text { Grave 39: yellow combing } \\
\text { (D05) with iron buckle and } \\
\text { knife }\end{array}$ & $\begin{array}{l}\text { Evison } 1988 \mathrm{a}, 17,44, \\
80-1, \text { fig } 33.39 .1 \mathrm{~h} ; \\
\text { Hampshire Museums }\end{array}$ \\
\hline Andover & Hants & $\begin{array}{l}\text { prob } \\
\text { 6th c }\end{array}$ & $\begin{array}{l}\text { Grave } 44: 4 \text { with yellow } \\
\text { or yellow and black (Plate } 6 \text { ) } \\
\text { with amber beads, } \\
\text { iron purse-mount, etc }\end{array}$ & $\begin{array}{l}\text { Cook and Dacre } 1985,35, \\
82 \text {, fig } 59.3 \text { and } 41,2 \text { and } 13 \text {, } \\
\text { colour frontispiece; } \\
\text { Hampshire Museums }\end{array}$ \\
\hline
\end{tabular}




\begin{tabular}{|c|c|c|c|c|}
\hline $\begin{array}{l}\text { Chessell } \\
\text { Down }\end{array}$ & $\begin{array}{l}\text { Isle of } \\
\text { Wight }\end{array}$ & 6th $\mathrm{c}$ & $\begin{array}{l}\text { One possible example, } \\
\text { badly illustrated (type } 4.4 \text { ) }\end{array}$ & $\begin{array}{l}\text { Arnold } 1982,49,120 \text {, } \\
\text { colour frontispiece; BM }\end{array}$ \\
\hline $\begin{array}{l}\text { Dover, } \\
\text { Buckland }\end{array}$ & Kent & $\begin{array}{l}625- \\
650\end{array}$ & $\begin{array}{l}\text { Grave } 1: 2 \text { with white } \\
\text { combing (D16); } \\
\text { Grave 59: } 4 \text { with yellow } \\
\text { (D14 and D15); } \\
\text { Grave 42: } 2 \text { with yellow } \\
\text { (D14) (Plate 6) }\end{array}$ & $\begin{array}{l}\text { Evison } 1987,76,82, \\
216,232-3 \text { and } 228-9 \text {, } \\
\text { figs } 5.4 \mathrm{u}, 33.3 \mathrm{j} \text { and } \mathrm{k} \text {, } \\
\text { and } 24.41 .1 \mathrm{li} \text {, colour } \\
\text { pl III.D14, } 15 \text { and } 16 \text {; } \\
\text { BM }\end{array}$ \\
\hline Faversham & Kent & $\begin{array}{l}5 \text { th- } \\
7 \text { th } c\end{array}$ & $\begin{array}{l}\text { Central yellow panels } \\
\text { with green combing }\end{array}$ & $\begin{array}{l}\text { Meaney 1964, 118-19; } \\
\text { Ashmolean Museum } \\
1909.146\end{array}$ \\
\hline Howletts & Kent & & $\begin{array}{l}\text { Two with dark central areas } \\
\text { with yellow }\end{array}$ & $\begin{array}{l}\text { Smith 1918; } \\
\text { BM } 1936 \text { 5-11 and 32-38 }\end{array}$ \\
\hline Sarre & Kent & $\begin{array}{l}\text { prob } \\
\text { 7th c }\end{array}$ & $\begin{array}{l}\text { With yellow, black and dark } \\
\text { green combing }\end{array}$ & $\begin{array}{l}\text { Meaney 1964, 135-6; } \\
\text { BM 93 6-1 218 }\end{array}$ \\
\hline Stowting & Kent & $\begin{array}{l}\text { prob } \\
\text { 6th- } \\
7 \text { th c }\end{array}$ & $\begin{array}{l}\text { Miscellaneous beads } \\
\text { with white combing }\end{array}$ & $\begin{array}{l}\text { Brent 1867; } \\
\text { Beck Collection, } \\
\text { Cambridge Museum }\end{array}$ \\
\hline $\begin{array}{l}\text { Barton-on- } \\
\text { Humber }\end{array}$ & Lincs & $\begin{array}{l}\text { mid- } \\
\text { 6th } c\end{array}$ & $\begin{array}{l}\text { Grave 115: } 2 \text { beads with } \\
\text { green and yellow twist with } \\
\text { annular brooch pair and } \\
\text { Áberg Group IVb cruciform } \\
\text { brooch, etc }\end{array}$ & $\begin{array}{l}\text { Drinkall and Foreman } \\
\text { forthcoming; } \\
\text { Scunthorpe Museum }\end{array}$ \\
\hline $\begin{array}{l}\text { Morning } \\
\text { Thorpe }\end{array}$ & Norfolk & $\begin{array}{l}\text { prob } \\
\text { 6th c }\end{array}$ & $\begin{array}{l}\text { Grave 309: } 2 \text { with yellow } \\
\text { with amber beads, etc }\end{array}$ & $\begin{array}{l}\text { Green et al } 1987,120 \text {, } \\
\text { fig 395.309.Aix; } \\
\text { Norwich Museum }\end{array}$ \\
\hline $\begin{array}{l}\text { Spong } \\
\text { Hill }\end{array}$ & Norfolk & $\begin{array}{l}\text { prob } \\
\text { 6th c }\end{array}$ & $\begin{array}{l}\text { Cremation stray find: } \\
\text { yellow and white combing }\end{array}$ & $\begin{array}{l}\text { Hills } 1977,28,67, \\
\text { fig } 127.27 ; \\
\text { Norfolk Arch Unit }\end{array}$ \\
\hline Duston & Northants & $\begin{array}{l}\text { prob } \\
6 \text { th- } \\
7 \text { th c }\end{array}$ & One with yellow combing & $\begin{array}{l}\text { George 1903; } \\
\text { Northampton Museum }\end{array}$ \\
\hline Lakenheath & Suffolk & $\begin{array}{l}\text { prob } \\
6 \text { th- } \\
7 \text { th c }\end{array}$ & $\begin{array}{l}\text { Green and yellow combing } \\
\text { with terracotta at each end }\end{array}$ & $\begin{array}{l}\text { Meaney 1964,230; } \\
\text { BM 1910 12-2311 }\end{array}$ \\
\hline $\begin{array}{l}\text { Apple } \\
\text { Down I }\end{array}$ & Sussex & $\begin{array}{l}\text { prob } \\
6 \text { th c }\end{array}$ & $\begin{array}{l}\text { Grave 117: } 1 \text { broken with } \\
\text { yellow }\end{array}$ & $\begin{array}{l}\text { Down and Welch } 1990 \text {, } \\
49,162-3, \text { fig } 2.37 ; \\
\text { Chichester Museum }\end{array}$ \\
\hline $\begin{array}{l}\text { West } \\
\text { Heslerton }\end{array}$ & Yorks & $\begin{array}{l}\text { prob } \\
\text { 6th c }\end{array}$ & $\begin{array}{l}\text { Grave number not } \\
\text { allocated: yellow combing }\end{array}$ & $\begin{array}{l}\text { Publication in } \\
\text { preparation (D Powlesland) }\end{array}$ \\
\hline viii & \multicolumn{4}{|c|}{$\begin{array}{l}\text { TERRACOTTA POLYGONAL CYLINDER BEADS WITH COLOUF } \\
\text { OMBING (INCLUDING GREEN AND YELLOW) (see Map 24) }\end{array}$} \\
\hline Barrington & Cambs & $\begin{array}{l}\text { prob } \\
6 \text { th- } \\
7 \text { th c }\end{array}$ & $\begin{array}{l}\text { Mixed beads: } 1 \text { rectangular } \\
\text { with side panels of green and } \\
\text { and yellow }\end{array}$ & $\begin{array}{l}\text { Fox 1923, 251-2; } \\
\text { Ashmolean Museum }\end{array}$ \\
\hline
\end{tabular}




\begin{tabular}{|c|c|c|c|c|}
\hline $\begin{array}{l}\text { Little } \\
\text { Wilbraham }\end{array}$ & Cambs & $\begin{array}{l}\text { prob } \\
\text { 6th c }\end{array}$ & $\begin{array}{l}\text { Two rectangular with } \\
\text { feathered green and yellow } \\
\text { combing on sides (Plate 6) }\end{array}$ & $\begin{array}{l}\text { Lethbridge and Carter } \\
\text { 1926; } \\
\text { Cambridge Museum }\end{array}$ \\
\hline 'Kent' & Kent & & $\begin{array}{l}\text { Two square-sectioned with } \\
\text { yellow }\end{array}$ & Canterbury Museum \\
\hline Sarre & Kent & $\begin{array}{l}\text { 6th- } \\
7 \text { th c }\end{array}$ & $\begin{array}{l}\text { Group XC: square- } \\
\text { sectioned with many } \\
\text { combed beads }\end{array}$ & Brent 1868, pl VIII \\
\hline Fonaby & Lincs & 6th c & $\begin{array}{l}\text { Grave 3: rectangular } \\
\text { section with slatey blue and } \\
\text { white combing on bands } \\
\text { alternating with terracotta }\end{array}$ & $\begin{array}{l}\text { Cook 1981, } 18, \\
\text { fig 4.3.1.17, } \\
\text { pl VIIA; } \\
\text { Scunthorpe Museum }\end{array}$ \\
\hline $\begin{array}{l}\text { South } \\
\text { Willingham }\end{array}$ & Lincs & $\begin{array}{l}\text { prob } \\
\text { 6th } c\end{array}$ & $\begin{array}{l}\text { Square section ?combed } \\
\text { in green, yellow, etc }\end{array}$ & $\begin{array}{l}\text { Meaney } 1964,165 \\
\text { BM } 762-1212-15\end{array}$ \\
\hline $\begin{array}{l}\text { Morning } \\
\text { Thorpe }\end{array}$ & Norfolk & $\begin{array}{l}\text { prob } \\
\text { 6th c }\end{array}$ & $\begin{array}{l}\text { Grave } 304: 2 \text { square- } \\
\text { sectioned green and yellow } \\
\text { with annular brooch pair, } \\
\text { penannular brooch, etc }\end{array}$ & $\begin{array}{l}\text { Green et al 1987, 119, } \\
\text { fig } 395 . \text { Gii; } \\
\text { Norwich Museum }\end{array}$ \\
\hline Duston & Northants & $\begin{array}{l}\text { prob } \\
\text { 6th- } \\
\text { 7th c }\end{array}$ & $\begin{array}{l}\text { One ?hexagonal with } \\
\text { ?combing }\end{array}$ & $\begin{array}{l}\text { George 1903; } \\
\text { Northampton Museum }\end{array}$ \\
\hline West Stow & Suffolk & $\begin{array}{l}5 \text { th- } \\
7 \text { th c }\end{array}$ & $\begin{array}{l}\text { Cemetery: rectangular with } \\
\text { panel on each long side } \\
\text { with green and yellow } \\
\text { combing }\end{array}$ & $\begin{array}{l}\text { West 1985, 74, fig } 276.3 \\
\text { (O28); } \\
\text { Ashmolean Museum }\end{array}$ \\
\hline Highdown & Sussex & $\begin{array}{l}\text { 5th- } \\
6 \text { th c }\end{array}$ & $\begin{array}{l}\text { Unknown grave: } \\
\text { atypical bead }\end{array}$ & $\begin{array}{l}\text { Read } 1895 \text { and } 1896 \text {; } \\
\text { Welch 1983, 85-6; } \\
\text { Worthing Museum }\end{array}$ \\
\hline Sewerby & Yorks & 6th $\mathrm{c}$ & $\begin{array}{l}\text { Grave 35: } 5 \text { beads (type } \\
\text { C6) with Åberg Group III } \\
\text { cruciform brooch, etc }\end{array}$ & Hirst 1985,66 , fig $44 b$ \\
\hline
\end{tabular}

ix TERRACOTTA CYLINDER BEADS WITH WOUND BANDS

\begin{tabular}{|c|c|c|c|c|}
\hline Wallingford & Berks & $\begin{array}{l}\text { 2nd } \\
\text { half } \\
\text { 5th } c\end{array}$ & $\begin{array}{l}\text { Grave 15: } 2 \text { yellow and } \\
\text { brown, atypical with } \\
\text { applied saucer brooch } \\
\text { (floriate/anchor cross) pair } \\
\text { and } 2 \text { quoit brooches, etc }\end{array}$ & $\begin{array}{l}\text { Leeds 1938, 89-99, } \\
\text { pl VII; Welch 1975; } \\
\text { Evison 1978; } \\
\text { Böhme 1986; } \\
\text { Ashmolean Museum }\end{array}$ \\
\hline $\begin{array}{l}\text { Fingringhoe, } \\
\text { Frog Hall } \\
\text { Farm }\end{array}$ & Essex & & $\begin{array}{l}\text { Unstratified finds: small, } \\
\text { yellow bands }\end{array}$ & $\begin{array}{l}\text { Information from } \\
\text { P Crummy }\end{array}$ \\
\hline $\begin{array}{l}\text { St Neots, } \\
\text { Lords } \\
\text { Bridge }\end{array}$ & Hunts & & One with yellow bands & BM $195610-21$ and 2 \\
\hline $\begin{array}{l}\text { Breach } \\
\text { Down }\end{array}$ & Kent & $\begin{array}{l}\text { prob } \\
\text { 7th c }\end{array}$ & $\begin{array}{l}\text { Two with white lines with } \\
\text { amethyst beads }\end{array}$ & $\begin{array}{l}\text { Mantell Collection, } \\
\text { BM 53 4-12 95-105 }\end{array}$ \\
\hline
\end{tabular}




\begin{tabular}{|c|c|c|c|c|}
\hline $\begin{array}{l}\text { Canterbury, } \\
\text { Rootes } \\
\text { Garage }\end{array}$ & Kent & & $\begin{array}{l}\text { Thin with yellow bands } \\
\text { bands }\end{array}$ & $\begin{array}{l}\text { Canterbury Museum } \\
8679\end{array}$ \\
\hline $\begin{array}{l}\text { Dover, } \\
\text { Buckland }\end{array}$ & Kent & $\begin{array}{l}575- \\
625 \\
625- \\
650\end{array}$ & $\begin{array}{l}\text { Grave 59: } 1 \text { with white } \\
\text { bands (D05); } \\
\text { Grave } 42: 3 \text { with white } \\
\text { bands (D05) }\end{array}$ & $\begin{array}{l}\text { Evison } 1987,76,82 \text {, } \\
232-3,228-9, \\
\text { figs } 33.3 \mathrm{i}, 24.42 .1 \mathrm{~g}, \\
\text { colour pl III.D05; } \\
\text { BM }\end{array}$ \\
\hline Faversham & Kent & $\begin{array}{l}\text { 6th- } \\
7 \text { th } c\end{array}$ & $\begin{array}{l}\text { Several with cream- } \\
\text { coloured bands, } \\
\text { 1 squarish section }\end{array}$ & $\begin{array}{l}\text { Meaney 1964, 118-19; } \\
\text { Ashmolean Museum }\end{array}$ \\
\hline $\begin{array}{l}\text { Folkestone, } \\
\text { Dover Hill }\end{array}$ & Kent & $\begin{array}{l}\text { prob } \\
\text { 6th } c\end{array}$ & $\begin{array}{l}\text { Nos } 4,11,28: 1 \text { in each } \\
\text { group with yellow bands }\end{array}$ & $\begin{array}{l}\text { Meaney 1964, 120-1; } \\
\text { Maidstone Museum; } \\
\text { Folkestone Museum }\end{array}$ \\
\hline Gilton & Kent & $\begin{array}{l}\text { 6th- } \\
7 \text { th } c\end{array}$ & $\begin{array}{l}\text { One pentagonal and possibly } \\
\text { more }\end{array}$ & $\begin{array}{l}\text { Faussett 1856, pl VI; } \\
\text { Liverpool Museum } \\
\text { M } 6123\end{array}$ \\
\hline 'Kent' & Kent & & Several with yellow bands & Canterbury Museum \\
\hline Stowting & Kent & & Several wound with yellow & $\begin{array}{l}\text { Meaney 1964, 137-8; } \\
\text { Maidstone Museum; } \\
\text { Beck Collection, } \\
\text { Cambridge Museum } 47.1800\end{array}$ \\
\hline $\begin{array}{l}\text { Morning } \\
\text { Thorpe }\end{array}$ & Norfolk & $\begin{array}{l}\text { prob } \\
\text { 6th c }\end{array}$ & $\begin{array}{l}\text { Grave 309: with white } \\
\text { bands with amber beads }\end{array}$ & $\begin{array}{l}\text { Green et al 1987, 120, } \\
\text { fig } 396.309 . \mathrm{Ax} \\
\text { Norwich Museum }\end{array}$ \\
\hline $\begin{array}{l}\text { Holme } \\
\text { Pierre- } \\
\text { pont }\end{array}$ & Notts & $\begin{array}{l}\text { prob } \\
\text { 6th c }\end{array}$ & $\begin{array}{l}\text { Short cylinder with yellow } \\
\text { bands }\end{array}$ & $\begin{array}{l}\text { Myres 1977, Corpus } \\
\text { no. 2093, fig 303; } \\
\text { BM 1931 3-13 }\end{array}$ \\
\hline Ipswich & Suffolk & $\begin{array}{l}6 \text { th- } \\
7 \text { th } c\end{array}$ & $\begin{array}{l}\text { Several with white or } \\
\text { yellow bands }\end{array}$ & $\begin{array}{l}\text { Layard 1907, pl XXXII, and } \\
\text { 1909, pl VII; } \\
\text { Ipswich Museum }\end{array}$ \\
\hline Highdown & Sussex & $\begin{array}{l}\text { prob } \\
\text { 6th c }\end{array}$ & $\begin{array}{l}\text { Unlocated grave: } 2 \text { with } \\
\text { yellow bands }\end{array}$ & $\begin{array}{l}\text { Read } 1895 \text { and } 1896 ; \\
\text { Welch 1983, 85-6; } \\
\text { Worthing Museum } 3484\end{array}$ \\
\hline $\begin{array}{l}\text { Stretton- } \\
\text { on- } \\
\text { Fosse }\end{array}$ & Warks & $\begin{array}{l}5 \text { th- } \\
6 \text { th } c\end{array}$ & $\begin{array}{l}\text { Two with yellow bands } \\
\text { with small-long brooch } \\
\text { (Plate 6) }\end{array}$ & $\begin{array}{l}\text { Information from W J } \\
\text { Ford; Gelling 1992, 31, } \\
40-1 \text {, fig } 21 \text {; } \\
\text { Warwick Museum }\end{array}$ \\
\hline Wasperton & Warks & $\begin{array}{l}\text { mid- } \\
\text { later } \\
6 \text { th } c\end{array}$ & F270: yellow band & $\begin{array}{l}\text { Publication in } \\
\text { preparation (M Carver); } \\
\text { Warwick Museum }\end{array}$ \\
\hline $\begin{array}{l}\text { West } \\
\text { Heslerton }\end{array}$ & Yorks & & Grave 2BA85: white bands & $\begin{array}{l}\text { Publication in } \\
\text { preparation (D Powlesland) }\end{array}$ \\
\hline
\end{tabular}


$\mathrm{x}$ Terracotta globular beads With White or yellow WOUND LINES

\begin{tabular}{|c|c|c|c|c|}
\hline Mucking II & Essex & $\begin{array}{l}\text { first } \\
\text { half } \\
5 \text { th c }\end{array}$ & $\begin{array}{l}\text { Various, including } \\
\text { Grave 924B: } 1 \text { bead with } \\
\text { applied saucer brooch pair, } \\
\text { Kempston cone beaker, etc; } \\
\text { Grave 989: } 2 \text { beads with } \\
\text { Glaston-Mucking and } \\
\text { supporting-arm brooches, } \\
\text { etc (Plate } 6 \text { ) }\end{array}$ & $\begin{array}{l}\text { Evison 1981, 138-9, } \\
\text { figs } 4 \text { and } 5 \\
\text { BM }\end{array}$ \\
\hline Lechlade & Glos & 6th $c$ & $\begin{array}{l}\text { Grave } 25 \text { : yellow spiral } \\
\text { with saucer brooch, } \\
2 \text { buckles, etc }\end{array}$ & $\begin{array}{l}\text { Boyle et al forthcoming; } \\
\text { Cirencester Museum }\end{array}$ \\
\hline $\begin{array}{l}\text { Canterbury, } \\
\text { Mint Yard }\end{array}$ & Kent & $\begin{array}{l}\text { 6th- } \\
\text { 8th c }\end{array}$ & $\begin{array}{l}\text { Two small globulars with } \\
\text { yellow bands, atypical }\end{array}$ & $\begin{array}{l}\text { Information from } \\
\mathrm{T} \text { Tatton-Brown } \\
\text { and P Garrard }\end{array}$ \\
\hline $\begin{array}{l}\text { Dover, } \\
\text { Buckland }\end{array}$ & Kent & $\begin{array}{l}575- \\
625 \\
625- \\
650\end{array}$ & $\begin{array}{l}\text { Grave 59: barrel with } \\
\text { yellow bands (D02); } \\
\text { Grave 42: barrel with } \\
\text { yellow bands (D02) }\end{array}$ & $\begin{array}{l}\text { Evison } 1987,76,82, \\
233-4,228-9, \\
\text { figs } 33.3 \mathrm{~h}, 24.42 .1 \mathrm{e} \\
\text { colour pl III.D02; BM }\end{array}$ \\
\hline $\begin{array}{l}\text { Gilton or } \\
\text { Kingston }\end{array}$ & Kent & $\begin{array}{l}\text { prob } \\
\text { 6th- } \\
\text { 7th c }\end{array}$ & $\begin{array}{l}\text { At least } 1 \text { example: } \\
\text { very small }\end{array}$ & $\begin{array}{l}\text { Faussett 1856, 1-34, } \\
\text { pl VI; } \\
\text { Liverpool Museum }\end{array}$ \\
\hline $\begin{array}{l}\text { mainly } \\
\text { Gilton }\end{array}$ & Kent & $\begin{array}{l}\text { prob } \\
\text { 6th- } \\
7 \text { th c }\end{array}$ & Several small examples & $\begin{array}{l}\text { Faussett 1856, pl V; } \\
\text { Liverpool Museum }\end{array}$ \\
\hline 'Kent' & Kent & $\begin{array}{l}\text { prob } \\
\text { 6th- } \\
7 \text { th c }\end{array}$ & $\begin{array}{l}\text { With miscellaneous beads: } \\
\text { one example }\end{array}$ & $\begin{array}{l}\text { Liverpool Museum } \\
\text { M } 7839\end{array}$ \\
\hline Stowting & Kent & $\begin{array}{l}\text { prob } \\
7 \text { th c }\end{array}$ & $\begin{array}{l}\text { Small atypical annular } \\
\text { bead }\end{array}$ & $\begin{array}{l}\text { Meaney 1964, 137-8; } \\
\text { Beck Collection, } \\
\text { Cambridge Museum }\end{array}$ \\
\hline Westbere & Kent & $\begin{array}{l}\text { prob } \\
\text { 7th c }\end{array}$ & $\begin{array}{l}\text { With } 3 \text { strings: } \\
1 \text { with white bands }\end{array}$ & $\begin{array}{l}\text { Jessup 1946, 11; } \\
\text { Canterbury Museum }\end{array}$ \\
\hline $\begin{array}{l}\text { London, } \\
\text { Dowgate } \\
\text { Hill }\end{array}$ & Middx & & Three examples & $\begin{array}{l}\text { Museum of London } \\
\text { (formerly Guildhall } \\
\text { Museum) } 3964\end{array}$ \\
\hline $\begin{array}{l}\text { Mitchell's } \\
\text { Hill }\end{array}$ & Suffolk & & One rather barrel-shaped & $\begin{array}{l}\text { Meaney } 1964,231 ; \\
\text { Bury St Edmunds } \\
\text { Museum }\end{array}$ \\
\hline Guildown & Surrey & $\begin{array}{l}5 \text { th- } \\
6 \text { th c }\end{array}$ & $\begin{array}{l}\text { Grave 78: with small-long } \\
\text { brooch pair, etc }\end{array}$ & $\begin{array}{l}\text { Lowther 1931, 12-13, } \\
\text { 36, pl IX.7; } \\
\text { Guildford Museum }\end{array}$ \\
\hline
\end{tabular}




\begin{tabular}{|c|c|c|c|c|}
\hline Mitcham & Surrey & $\begin{array}{l}\text { prob } \\
\text { 6th c }\end{array}$ & $\begin{array}{l}\text { Grave } 62 \text { with other beads; } \\
\text { Grave 117: white bands }\end{array}$ & $\begin{array}{l}\text { Bidder and Morris } 1959 \\
64,68,110-12, \\
\text { pl XVI.62 and } 117 ; \\
\text { Cambridge Museum }\end{array}$ \\
\hline Selmeston & Sussex & $\begin{array}{l}\text { prob } \\
5 \text { th- } \\
7 \text { th } \mathrm{c}\end{array}$ & Unassociated bead & $\begin{array}{l}\text { Welch 1983, 85-6, } \\
\text { fig } 49 \mathrm{a} \text {; } \\
\text { Lewes Museum }\end{array}$ \\
\hline
\end{tabular}

\section{xi Terracotta tall biconical beads With RANDOM} YELLOW LINES

\begin{tabular}{|c|c|c|c|c|}
\hline $\begin{array}{l}\text { Chamber- } \\
\text { lain's } \\
\text { Barn I }\end{array}$ & Beds & $\begin{array}{l}\text { late } \\
6 \text { th- } \\
\text { early } \\
7 \text { th c }\end{array}$ & $\begin{array}{l}\text { Grave 3: } 1 \text { example with } \\
\text { knife, etc }\end{array}$ & $\begin{array}{l}\text { Hyslop 1963, 167, } \\
\text { fig 4.31; } \\
\text { Luton Museum }\end{array}$ \\
\hline Willington & Derbys & $\begin{array}{l}\text { post- } \\
5 \text { th c }\end{array}$ & Grubenhaus 3: one bead & $\begin{array}{l}\text { Wheeler } 1979,131-3 \text {, } \\
215 \text {, fig } 90.24\end{array}$ \\
\hline Howletts & Kent & $\begin{array}{l}\text { mid- } \\
6 \text { th- } \\
7 \text { th c }\end{array}$ & $\begin{array}{l}\text { Grave A: } 1 \text { possible bead } \\
\text { with millefiori and other } \\
\text { beads }\end{array}$ & $\begin{array}{l}\text { Smith 1918; } \\
\text { BM } 1936 \text { 5-11 }\end{array}$ \\
\hline Stowting & Kent & 6th $c$ & $\begin{array}{l}\text { Small, rather doubtful } \\
\text { example }\end{array}$ & $\begin{array}{l}\text { Brent 1867; } \\
\text { Beck Collection, } \\
\text { Cambridge Museum }\end{array}$ \\
\hline
\end{tabular}

Kenninghall Norfolk 5th- On string: 1 more rounded, Manning 1872a, 292, and 7 th c but still biconical, with 1872b; millefioribead, etc $\quad$ BM 83 7-231

\begin{tabular}{|c|c|c|c|c|}
\hline Selmeston & Sussex & $\begin{array}{l}5 \text { th- }- \\
7 \text { th c }\end{array}$ & One bead (Plate 6) & $\begin{array}{l}\text { Welch 1983, 85-6, } \\
\text { fig } 49 \text { a; } \\
\text { Lewes Museum }\end{array}$ \\
\hline
\end{tabular}

\section{xii Terracotta beads with specks or spots}

\begin{tabular}{|c|c|c|c|c|}
\hline $\begin{array}{l}\text { Chamber- } \\
\text { lain's } \\
\text { Barn I }\end{array}$ & Beds & $\begin{array}{l}\text { late } \\
6 \text { th- } \\
\text { early } \\
\text { 7th c }\end{array}$ & $\begin{array}{l}\text { Grave 3: grey opaque } \\
\text { specks all over }\end{array}$ & $\begin{array}{l}\text { Hyslop } 1963,167 \text {, } \\
\text { fig } 4.3 \mathrm{~m} \text {; } \\
\text { Luton Museum }\end{array}$ \\
\hline Watchfield & Berks & $\begin{array}{l}\text { 5th- } \\
\text { 6th } c\end{array}$ & $\begin{array}{l}\text { Grave } 14 \text { : thick annular } \\
\text { with large equally placed } \\
\text { yellow spots ( } 83.59 \text { ) with } \\
2 \text { disc brooches, etc }\end{array}$ & $\begin{array}{l}\text { Scull } 1992,167,234-5 \text {, } \\
\text { fig } 31.83 .59 ; \\
\text { Woodstock Museum }\end{array}$ \\
\hline $\begin{array}{l}\text { Linton } \\
\text { Heath B }\end{array}$ & Cambs & $\begin{array}{l}\text { prob } \\
\text { 6th } c\end{array}$ & $\begin{array}{l}\text { Two small annular with } \\
\text { one or more yellow spots }\end{array}$ & $\begin{array}{l}\text { Neville 1854; } \\
\text { Cambridge Museum } \\
48.1598\end{array}$ \\
\hline Mucking II & Essex & 5 th $c$ & $\begin{array}{l}\text { Grave } 924 \mathrm{~B}: 1 \text { bead with } \\
\text { applied saucer brooch } \\
\text { pair, Kempston cone } \\
\text { beaker, etc }\end{array}$ & $\begin{array}{l}\text { Hirst and Clark forthcoming; } \\
\text { BM }\end{array}$ \\
\hline
\end{tabular}




\begin{tabular}{|c|c|c|c|c|}
\hline Lechlade & Glos & 6th $\mathrm{c}$ & $\begin{array}{l}\text { Grave } 25 \text { : white spots } \\
\text { with saucer brooch, etc }\end{array}$ & $\begin{array}{l}\text { Boyle et al forthcoming; } \\
\text { Cirencester Museum }\end{array}$ \\
\hline Droxford & Hants & $\begin{array}{l}\text { prob } \\
\text { 6th } c\end{array}$ & $\begin{array}{l}\text { Grave 30: annular with } \\
\text { yellow blobs (Class Xc) }\end{array}$ & $\begin{array}{l}\text { Aldsworth } 1978,128, \\
\text { 173, fig 27.30.13; } \\
\text { Hampshire Museums }\end{array}$ \\
\hline Faversham & Kent & $\begin{array}{l}\text { 5th- } \\
7 \text { th } c\end{array}$ & $\begin{array}{l}\text { Mixed collection: } 1 \text { with } \\
\text { pairs of yellow spots and } \\
\text { blue/white eyes; } \\
2 \text { examples big rather } \\
\text { biconical with regular } \\
\text { all-over yellow spots; } \\
\text { cube-shaped with yellow } \\
\text { edges and spot on each face }\end{array}$ & $\begin{array}{l}\text { Meaney } 1964,118-19 ; \\
\text { Beck Collection, } \\
\text { Cambridge Museum } \\
47.1822 \text { and } 47.1817 ; \\
\text { Ashmolean Museum } \\
1909.169 \text { and } 1909.143\end{array}$ \\
\hline Monkton & Kent & $\begin{array}{l}\text { 6th- } \\
\text { 7th c }\end{array}$ & $\begin{array}{l}\text { One small annular with } \\
\text { yellow spots }\end{array}$ & $\begin{array}{l}\text { Hawkes and Hogarth 1974; } \\
\text { Perkins and Hawkes 1984; } \\
\text { Ashmolean Museum } \\
1972.1427\end{array}$ \\
\hline Sarre & Kent & $\begin{array}{l}\text { 5th- } \\
7 \text { th c }\end{array}$ & $\begin{array}{l}\text { Group XCVIII: } 1 \text { small } \\
\text { with spots of grey/blue }\end{array}$ & $\begin{array}{l}\text { Brent } 1868 \text {, } \\
\text { pl VIII.XCVIII }\end{array}$ \\
\hline Laceby & Lincs & $\begin{array}{l}\text { 6th- } \\
\text { 7the }\end{array}$ & $\begin{array}{l}\text { Group of beads: cube- } \\
\text { shaped with yellow spot } \\
\text { on each face }\end{array}$ & $\begin{array}{l}\text { Meaney 1964, 157; } \\
\text { Lincoln Museum }\end{array}$ \\
\hline 'E Anglia' & Norfolk & & $\begin{array}{l}\text { Miscellaneous beads: } \\
1 \text { globular with white spots }\end{array}$ & $\begin{array}{l}\text { Norwich Museum: } \\
\text { marked 427 641-2 } 64\end{array}$ \\
\hline Spong Hill & Norfolk & 6th $\mathrm{c}$ & $\begin{array}{l}\text { Grave 22: few yellow spots } \\
\text { with Åberg Group IVa } \\
\text { cruciform brooch, etc }\end{array}$ & $\begin{array}{l}\text { Hills et al } 1984,70 \text {, } \\
\text { fig } 79.27,57 \text { and } 66 \text {; } \\
\text { Norfolk Arch Unit }\end{array}$ \\
\hline Brixworth & Northants & $\begin{array}{l}\text { prob } \\
6 \text { th } c\end{array}$ & $\begin{array}{l}\text { Necklace: } 1 \text { cube with } \\
\text { yellow spot on each face, } \\
\text { with many brooches }\end{array}$ & $\begin{array}{l}\text { Smith 1902, 245 6; } \\
\text { Northampton Museum }\end{array}$ \\
\hline Mitcham & Surrey & $\begin{array}{l}\text { prob } \\
\text { 6th } c\end{array}$ & $\begin{array}{l}\text { Grave 197: wedge-shaped } \\
\text { with yellow and green spots } \\
\text { (irregular); } \\
\text { Grave 117: annular with } \\
\text { pairs of yellow spots }\end{array}$ & $\begin{array}{l}\text { Bidder and Morris } 1959, \\
73,68,110-12, \\
\text { pl XVI.197 and 117; } \\
\text { Cambridge Museum }\end{array}$ \\
\hline Waspertion & Warks & $\begin{array}{l}\text { mid- } \\
\text { 6th } c\end{array}$ & $\begin{array}{l}\text { F1081: } 2 \text { annulars with row } \\
\text { of yellow spots around } \\
\text { centre with saucer brooch }\end{array}$ & $\begin{array}{l}\text { Publication in } \\
\text { preparation (M Carver); } \\
\text { Warwick Museum }\end{array}$ \\
\hline $\begin{array}{l}\text { Peters- } \\
\text { finger }\end{array}$ & Wilts & $\begin{array}{l}\text { 5th- } \\
\text { 6th } c\end{array}$ & $\begin{array}{l}\text { Grave 29: yellow edges and } \\
\text { green spot in each side } \\
\text { with applied saucer brooch } \\
\text { baseplates }\end{array}$ & $\begin{array}{l}\text { Leeds and Shortt } 1953 \text {, } \\
24-5 \text {, pl III.104.XXIX; } \\
\text { Salisbury Museum }\end{array}$ \\
\hline $\begin{array}{l}\text { West } \\
\text { Heslerton }\end{array}$ & Yorks & $\begin{array}{l}\text { prob } \\
\text { 6th } c\end{array}$ & $\begin{array}{l}\text { Group 917: annular with } \\
\text { four yellow spots }\end{array}$ & $\begin{array}{l}\text { Publication in } \\
\text { preparation (D Powlesland) }\end{array}$ \\
\hline
\end{tabular}




\begin{tabular}{|c|c|c|c|c|}
\hline $\begin{array}{l}\text { Chamber- } \\
\text { lain's } \\
\text { Barn I }\end{array}$ & Beds & $\begin{array}{l}\text { late } \\
6 \text { th- } \\
\text { early } \\
\text { 7th c }\end{array}$ & $\begin{array}{l}\text { Grave 3: } 1 \text { with white ring } \\
\text { round blue eye }\end{array}$ & $\begin{array}{l}\text { Hyslop 1963, } 167 \text {, } \\
\text { fig } 4.3 \mathrm{i} \text {; } \\
\text { Luton Museum }\end{array}$ \\
\hline Puddlehill & Beds & $c .600$ & $\begin{array}{l}\text { Grave 10: large with white } \\
\text { rings round eyes with cast } \\
\text { saucer brooch pair, etc }\end{array}$ & $\begin{array}{l}\text { Matthews and Hawkes } \\
1985,91 \text { and } 95 \text {, } \\
\text { figs } 38 \text { and } 40.15\end{array}$ \\
\hline $\begin{array}{l}\text { East } \\
\text { Shefford }\end{array}$ & Berks & $\begin{array}{l}\text { 5th- } \\
\text { 6th c }\end{array}$ & $\begin{array}{l}\text { Globular, blue and white } \\
\text { rings round terracotta }\end{array}$ & $\begin{array}{l}\text { Peake and Hooton 1915; } \\
\text { Newbury Museum }\end{array}$ \\
\hline Bishopstone & Bucks & $\begin{array}{l}\text { prob } \\
\text { 6th c }\end{array}$ & $\begin{array}{l}\text { Dark green eye surrounded } \\
\text { by yellow and then green } \\
\text { rings, with small-long } \\
\text { brooch, etc }\end{array}$ & $\begin{array}{l}\text { Lowndes } 1870 \text { and } \\
1878 ; \\
\text { Aylesbury Museum }\end{array}$ \\
\hline Barrington & Cambs & $\begin{array}{l}\text { prob } \\
\text { 6th c }\end{array}$ & $\begin{array}{l}\text { Annular with terracotta or } \\
\text { dark eye enclosed with } \\
\text { yellow }\end{array}$ & $\begin{array}{l}\text { Meaney 1964,60-1; } \\
\text { Beck Collection, } \\
\text { Cambridge Museum }\end{array}$ \\
\hline $\begin{array}{l}\text { Little } \\
\text { Wilbraham }\end{array}$ & Cambs & & $\begin{array}{l}\text { Annular with green eyes } \\
\text { surrounded with yellow }\end{array}$ & $\begin{array}{l}\text { Lethbridge and Carter } \\
\text { 1926; Cambridge Museum }\end{array}$ \\
\hline Mucking II & Essex & $\begin{array}{l}\text { mid- } \\
\text { 6th } c\end{array}$ & $\begin{array}{l}\text { Grave } 936: 3 \text { beads with } \\
\text { green and white spots with } \\
\text { small square-headed brooch } \\
\text { pair }\end{array}$ & $\begin{array}{l}\text { Hirst and Clark forthcoming; } \\
\text { BM }\end{array}$ \\
\hline 'Kent' & Kent & & $\begin{array}{l}\text { Four small barrel beads with } \\
\text { turquoise spots on white }\end{array}$ & $\begin{array}{l}\text { Faussett-Rolfe Collection, } \\
\text { Liverpool Museum }\end{array}$ \\
\hline Westbere & Kent & $\begin{array}{l}\text { prob } \\
\text { 7th } \mathrm{c}\end{array}$ & $\begin{array}{l}\text { On } 1 \text { of } 3 \text { strings: white } \\
\text { rings round blue eyes }\end{array}$ & $\begin{array}{l}\text { Jessup 1946; } \\
\text { Canterbury Museum } \\
\text { RM } 6422\end{array}$ \\
\hline Cleatham & Lincs & & $\begin{array}{l}\text { Grave 30: small barrel with } \\
\text { white round terracotta eye }\end{array}$ & $\begin{array}{l}\text { Publication in } \\
\text { preparation (K Leahy); } \\
\text { Scunthorpe Museum }\end{array}$ \\
\hline $\begin{array}{l}\text { Nettleton } \\
\text { Caistor }\end{array}$ & Lincs & $\begin{array}{l}\text { prob } \\
\text { 6th- } \\
7 \text { th c }\end{array}$ & $\begin{array}{l}\text { Large biconical with central } \\
\text { line and blue eyes in yellow } \\
\text { rings }\end{array}$ & Lincoln Museum \\
\hline Syston Park & Lincs & $\begin{array}{l}\text { 6th- } \\
7 \text { th } c\end{array}$ & $\begin{array}{l}\text { Two rather drum-shaped with } \\
\text { yellow rings round green or } \\
\text { terracotta eyes }\end{array}$ & $\begin{array}{l}\text { Akerman 1855a, 41-2, } \\
\text { pl XXI.4; } \\
\text { BM (18) } 1111-92\end{array}$ \\
\hline Spong Hill & Norfolk & $\begin{array}{l}\text { prob } \\
\text { 5th c }\end{array}$ & $\begin{array}{l}\text { Cremation 2132: burnt with } \\
\text { white round blue eyes in } \\
\text { bossed urn }\end{array}$ & $\begin{array}{l}\text { Hills and Penn 1981, } \\
\text { 56, fig 165.2132; } \\
\text { Norfolk Arch Unit }\end{array}$ \\
\hline Corbridge & $\begin{array}{l}\text { North- } \\
\text { umber- } \\
\text { land }\end{array}$ & $\begin{array}{l}\text { 5th- } \\
6 \text { th c }\end{array}$ & $\begin{array}{l}\text { Necklace: annular with } \\
\text { yellow round blue with } \\
\text { two Åberg Group } 11 \\
\text { cruciform brooches }\end{array}$ & $\begin{array}{l}\text { Knowles and Forster 1909, } \\
\text { 407; Corbridge Museum }\end{array}$ \\
\hline
\end{tabular}




$\begin{array}{lllll}\text { Ipswich } & \text { Suffolk } & \begin{array}{l}\text { 6th- } \\ 7 \text { th c }\end{array} & \begin{array}{l}\text { Annular with white round } \\ \text { blotched yellow and white } \\ \text { round blue eyes }\end{array} & \begin{array}{l}\text { Layard } 1907 \text { and } \\ 1909 ; \\ \text { Ipswich Museum }\end{array} \\ \begin{array}{l}\text { Pewsey } \\ \text { Blackpatch }\end{array} & \text { Wilts } & \begin{array}{l}\text { prob } \\ \text { 6th c }\end{array} & \begin{array}{l}\text { Grave 85: annular with } \\ \text { yellow round greenish eyes }\end{array} & \begin{array}{l}\text { Publication in } \\ \text { preparation (K Annable); } \\ \text { Devizes Museum }\end{array}\end{array}$

xiv Terracotta BeAds: (a) With WHITE OR Yellow CROSSED WAVES, Without Dots (see also double beads, type xivc, below)

\begin{tabular}{|c|c|c|c|c|}
\hline $\begin{array}{l}\text { Chamber- } \\
\text { lain's } \\
\text { Barn I }\end{array}$ & Beds & $\begin{array}{l}\text { late } \\
6 \text { th- } \\
\text { early } \\
\text { 7th c }\end{array}$ & $\begin{array}{l}\text { Grave 3: one with white } \\
\text { waves }\end{array}$ & $\begin{array}{l}\text { Hyslop } 1963,167 \text {, } \\
\text { fig } 4.3 \mathrm{k} ; \\
\text { Luton Museum }\end{array}$ \\
\hline Puddlehill & Beds & $c .600$ & $\begin{array}{l}\text { Grave } 10 \text { : several with } \\
\text { white waves with saucer } \\
\text { brooch pair, etc }\end{array}$ & $\begin{array}{l}\text { Matthews and Hawkes } \\
1985,91 \text { and } 95 \text {, } \\
\text { figs } 38 \text { and } 40.7-13\end{array}$ \\
\hline $\begin{array}{l}\text { Linton } \\
\text { Heath }\end{array}$ & Cambs & $\begin{array}{l}\text { prob } \\
\text { 6th } c\end{array}$ & $\begin{array}{l}\text { One yellow wave on double } \\
\text { necklace }\end{array}$ & $\begin{array}{l}\text { Neville 1854; } \\
\text { Cambridge Museum }\end{array}$ \\
\hline $\begin{array}{l}\text { Little } \\
\text { Chester }\end{array}$ & Derbys & $\begin{array}{l}\text { prob } \\
7 \text { th } c\end{array}$ & $\begin{array}{l}\text { Barrel-shaped beads with } \\
\text { yellow waves }\end{array}$ & $\begin{array}{l}\text { Wheeler } 1985,304 ; \\
\text { publication in } \\
\text { preparation } \\
\text { (C Sparey-Green) }\end{array}$ \\
\hline Mucking II & Essex & $\begin{array}{l}\text { 7th } c \\
7 \text { th } c \\
6 \text { th- } \\
7 \text { th } c\end{array}$ & $\begin{array}{l}\text { Grave } 608: 2 \text { annulars with } \\
\text { white waves (Plate 7); } \\
\text { Grave } 621: 2 \text { annulars with } \\
\text { white waves and } 1 \text { barrel } \\
\text { with yellow waves (Plate 7); } \\
\text { Grave } 962: 3 \text { barrels with } \\
\text { yellow waves, } 3 \text { barrels } \\
\text { with white waves and } 1 \\
\text { globular with blue-white } \\
\text { waves }\end{array}$ & $\begin{array}{l}\text { Hirst and Clark forthcoming; } \\
\text { BM }\end{array}$ \\
\hline Prittlewell & Essex & $\begin{array}{l}\text { late } \\
\text { 6th c }\end{array}$ & $\begin{array}{l}\text { Grave-group 32: white } \\
\text { waves }\end{array}$ & $\begin{array}{l}\text { Tyler 1988, 113, fig 15.3; } \\
\text { Southend Museum }\end{array}$ \\
\hline Lechlade & Glos & $\begin{array}{l}\text { 6th } c \\
7 \text { th } c\end{array}$ & $\begin{array}{l}\text { Grave 101: yellow waves; } \\
\text { Grave 197: white waves }\end{array}$ & $\begin{array}{l}\text { Boyle et al forthcoming; } \\
\text { Cirencester Museum }\end{array}$ \\
\hline Andover & Hants & $\begin{array}{l}\text { prob } \\
\text { 6th c }\end{array}$ & $\begin{array}{l}\text { Grave } 23: 3 \text { with white } \\
\text { waves with amber beads; } \\
\text { Grave } 44: 1 \text { cube with } \\
\text { yellow waves }\end{array}$ & $\begin{array}{l}\text { Cook and Dacre } 1985 \text {, } \\
29,35,82 \text {, } \\
\text { figs } 48.23 .2 \text { and } 3 \text {, and } 59.36 \text {, } \\
\text { colour frontispiece; } \\
\text { Hampshire Museums }\end{array}$ \\
\hline $\begin{array}{l}\text { Southampton } \\
\text { (Hamwic) }\end{array}$ & Hants & $\begin{array}{l}\text { late } \\
7 \text { th- } \\
9 \text { th c }\end{array}$ & $\begin{array}{l}\text { Rather drum-shaped with } \\
\text { yellow waves }\end{array}$ & $\begin{array}{l}\text { Publication in } \\
\text { preparation }\end{array}$ \\
\hline $\begin{array}{l}\text { Beakes- } \\
\text { bourne }\end{array}$ & Kent & $\begin{array}{l}\text { prob } \\
\text { 6th- } \\
\text { 7th c }\end{array}$ & Two with white waves & Faussett $1856, \mathrm{pl}$ V.4 \\
\hline
\end{tabular}




\begin{tabular}{|c|c|c|c|c|}
\hline $\begin{array}{l}\text { Canterbury, } \\
\text { Mint Yard }\end{array}$ & Kent & $\begin{array}{l}\text { 6th- } \\
8 \text { th } \mathrm{c}\end{array}$ & Several & $\begin{array}{l}\text { Information from } \\
\mathrm{T} \text { Tatton-Brown } \\
\text { and } \mathrm{P} \text { Garrard }\end{array}$ \\
\hline Coombe & Kent & $\begin{array}{l}\text { mid- } \\
\text { 6th c }\end{array}$ & $\begin{array}{l}\text { Cremation: necklace, white } \\
\text { waves, possibly with small } \\
\text { square-headed brooch, etc }\end{array}$ & $\begin{array}{l}\text { Davidson and Webster } \\
\text { 1967, 20-1, pl VII.D; } \\
\text { BM } 6512-143\end{array}$ \\
\hline $\begin{array}{l}\text { Dover, } \\
\text { Buckland }\end{array}$ & Kent & $\begin{array}{l}625- \\
650\end{array}$ & $\begin{array}{l}\text { Grave } 1: 2 \text { beads with } \\
\text { white waves and } 2 \text { beads } \\
\text { with white waves and } \\
\text { central band (D18 and D37); } \\
\text { Grave 30: barrels - } \\
1 \text { yellow (D30) and } 1 \text { yellow } \\
\text { with central line (D38); } \\
\text { Grave 32: } 4 \text { with yellow - } \\
1 \text { disc (D17), } 3 \text { short } \\
\text { cylinders (D32); } \\
\text { Grave 59: } 1 \text { barrel yellow } \\
\text { with central line (D38); } \\
\text { Grave 18: } 6 \text { short } \\
\text { cylinders }-4 \text { with yellow } \\
\text { (D32) and } 2 \text { with white } \\
\text { (D34); } \\
\text { Grave } 42: 1 \text { disc with white } \\
\text { (D19) }\end{array}$ & 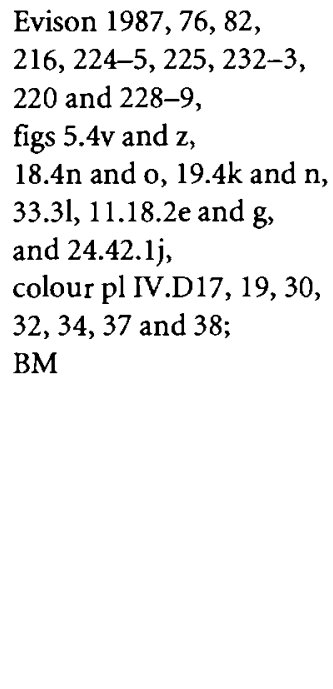 \\
\hline Faversham & Kent & $\begin{array}{l}5 \text { th- } \\
7 \text { th c }\end{array}$ & $\begin{array}{l}\text { Annular with white; } \\
\text { and others }\end{array}$ & $\begin{array}{l}\text { Meaney 1964, 118-19; } \\
\text { Beck Collection, } \\
\text { Cambridge Museum; } \\
\text { Ashmolean Museum }\end{array}$ \\
\hline $\begin{array}{l}\text { Folkestone, } \\
\text { Dover Hill }\end{array}$ & Kent & $\begin{array}{l}\text { prob } \\
5 \text { th- } \\
6 \text { th c }\end{array}$ & $\begin{array}{l}\text { No. } 15: c .10 \text { with white } \\
\text { waves }\end{array}$ & $\begin{array}{l}\text { Meaney } 1964,120-1 \\
\text { Maidstone Museum }\end{array}$ \\
\hline Harrietsham & Kent & $\begin{array}{l}\text { 7th- } \\
\text { 8th c }\end{array}$ & $\begin{array}{l}\text { Necklace with bead like an } \\
\text { 8th-c one from Escomb, } \\
\text { Co. Durham }\end{array}$ & Maidstone Museum \\
\hline Holborough & Kent & $\begin{array}{l}\text { late } \\
\text { 7th c }\end{array}$ & Necklace: 1 with yellow & $\begin{array}{l}\text { Evison } 1956,126 \text {, } \\
\text { fig 20.3g; } \\
\text { BM } 19475-2358\end{array}$ \\
\hline 'Kent' & Kent & & $\begin{array}{l}\text { Miscellaneous barrel- } \\
\text { shaped with yellow and } \\
\text { others, various }\end{array}$ & $\begin{array}{l}\text { Faussett-Rolfe Collection, } \\
\text { Liverpool Museum; } \\
\text { Canterbury Museum }\end{array}$ \\
\hline $\begin{array}{l}\text { Kingston or } \\
\text { Gilton }\end{array}$ & Kent & $\begin{array}{l}\text { 6th- } \\
\text { 7th c }\end{array}$ & $\begin{array}{l}\text { Many barrel-shaped, } \\
\text { mostly small white }\end{array}$ & $\begin{array}{l}\text { Faussett 1856; } \\
\text { Liverpool Museum }\end{array}$ \\
\hline Sarre & Kent & $\begin{array}{l}\text { prob } \\
\text { 7th c }\end{array}$ & $\begin{array}{l}\text { About } 12 \text { with white, mostly } \\
\text { barrel-shaped }\end{array}$ & $\begin{array}{l}\text { Brent } 1868 ; \\
\text { BM } 946-1218 ; \\
\text { and some in Liverpool } \\
\text { Museum }\end{array}$ \\
\hline
\end{tabular}




\begin{tabular}{|c|c|c|c|c|}
\hline Stowting & Kent & $\begin{array}{l}\text { prob } \\
\text { 6th- } \\
7 \text { th c }\end{array}$ & $\begin{array}{l}\text { Number of annular and } \\
\text { barrel-shaped, yellow } \\
\text { or white or green }\end{array}$ & $\begin{array}{l}\text { Smith 1846a; Brent 1867; } \\
\text { Beck Collection, } \\
\text { Cambridge Museum; } \\
\text { Maidstone Museum }\end{array}$ \\
\hline Westbere & Kent & & One annular with white & $\begin{array}{l}\text { Jessup 1946; } \\
\text { Canterbury Museum }\end{array}$ \\
\hline $\begin{array}{l}\text { London, } \\
\text { Bargate Hill }\end{array}$ & $\begin{array}{l}\text { City of } \\
\text { London }\end{array}$ & & Two with white & $\begin{array}{l}\text { Museum of London } \\
\text { (formerly Guildhall } \\
\text { Museum) }\end{array}$ \\
\hline $\begin{array}{l}\text { Morning } \\
\text { Thorpe }\end{array}$ & Norfolk & $\begin{array}{l}\text { prob } \\
\text { 6th c }\end{array}$ & $\begin{array}{l}\text { Grave } 322 \text { : with white, with } \\
\text { scutiform pendant, etc; } \\
\text { Grave } 375: 7 \text { with white, } \\
\text { with scutiform pendant; } \\
\text { Grave } 384 \text { : with white, with } \\
\text { annular brooch, etc }\end{array}$ & $\begin{array}{l}\text { Green et al 1987, } \\
\text { 126, 146-7, } \\
\text { figs 401.322.Evii, } \\
\text { 434.375.Cviii, } \\
\text { 438.384.Avi; } \\
\text { Norwich Museum }\end{array}$ \\
\hline Hepple & $\begin{array}{l}\text { North- } \\
\text { umber- } \\
\text { land }\end{array}$ & 7th $\mathrm{c}$ & $\begin{array}{l}\text { Rather biconical with } \\
\text { yellow }\end{array}$ & $\begin{array}{l}\text { Miket 1974, 276, fig 3; } \\
\text { Cramp and Miket 1982, } \\
\text { 4-5, fig 2.4; } \\
\text { Newcastle upon Tyne } \\
\text { Museum }\end{array}$ \\
\hline Ipswich & Suffolk & $\begin{array}{l}\text { 6th- } \\
7 \text { th } c\end{array}$ & $\begin{array}{l}\text { A number with white or } \\
\text { yellow }\end{array}$ & $\begin{array}{l}\text { Layard 1907, pl XXXI, and } \\
\text { 1909; } \\
\text { Ipswich Museum }\end{array}$ \\
\hline $\begin{array}{l}\text { Mitchell's } \\
\text { Hill }\end{array}$ & Suffolk & $\begin{array}{l}\text { prob } \\
6 \text { th- } \\
7 \text { th c }\end{array}$ & White & $\begin{array}{l}\text { Meaney 1964, 231; } \\
\text { Bury St Edmunds } \\
\text { Museum }\end{array}$ \\
\hline Snape & Suffolk & $\begin{array}{l}\text { prob } \\
\text { 6th c }\end{array}$ & $\begin{array}{l}\text { Grave } 0421: 2 \text { large and } \\
1 \text { small bun-shaped white }\end{array}$ & $\begin{array}{l}\text { Filmer-Sankey and } \\
\text { Pestell forthcoming }\end{array}$ \\
\hline Mitcham & Surrey & $\begin{array}{l}\text { prob } \\
\text { 6th c }\end{array}$ & $\begin{array}{l}\text { Grave 129: several barrels } \\
\text { yellow }\end{array}$ & $\begin{array}{l}\text { Bidder and Morris } 1959 \text {, } \\
69,110-12 \text {, pl XVI.129; } \\
\text { Cambridge Museum }\end{array}$ \\
\hline $\begin{array}{l}\text { Apple } \\
\text { Down I }\end{array}$ & Sussex & $\begin{array}{l}\text { prob } \\
\text { 6th c }\end{array}$ & $\begin{array}{l}\text { Grave } 107: \text { white with } \\
\text { amber beads; } \\
\text { Grave } 117: 3 \text { with white } \\
\text { with amber beads; } \\
\text { Grave } 141 \text { : white with } \\
\text { amber beads; } \\
\text { Area } 2: 9 \text { white }\end{array}$ & $\begin{array}{l}\text { Down and Welch } 1990 \text {, } \\
48,49,52 \text { and } 162-4 \text {, } \\
\text { figs } 2.35,2.37 \text {, } \\
2.45 .141 .2 \\
\text { colour pl } 40.14, \\
19,21 \text { and } 23 \text {; } \\
\text { Chichester Museum }\end{array}$ \\
\hline Saltburn & Yorks & $\begin{array}{l}\text { prob } \\
\text { 7th c }\end{array}$ & $\begin{array}{l}\text { One white and } 1 \text { yellow } \\
\text { barrel-shaped }\end{array}$ & $\begin{array}{l}\text { Gallagher 1987, 16, fig 5; } \\
\text { Middlesbrough Museum }\end{array}$ \\
\hline $\begin{array}{l}\text { West } \\
\text { Heslerton }\end{array}$ & Yorks & $\begin{array}{l}\text { prob } \\
\text { 6th c }\end{array}$ & 503: several & $\begin{array}{l}\text { Publication in } \\
\text { preparation (D Powlesland) }\end{array}$ \\
\hline
\end{tabular}


xiv TERRACOTTA BEADS: (b) WITH WHITE OR YELLOW CROSSED WAVES AND DOTS

\begin{tabular}{|c|c|c|c|c|}
\hline $\begin{array}{l}\text { Chamber- } \\
\text { lain's } \\
\text { Barn I }\end{array}$ & Beds & $\begin{array}{l}\text { late } \\
6 \text { th- } \\
\text { early } \\
7 \text { th c }\end{array}$ & $\begin{array}{l}\text { Grave 3: red, blue or white } \\
\text { dots }\end{array}$ & $\begin{array}{l}\text { Hyslop } 1963,167 \text {, } \\
\text { fig } 4.3 \mathrm{~h}-\mathrm{j} ; \\
\text { Luton Museum }\end{array}$ \\
\hline Wallingford & Berks & $\begin{array}{l}5 \text { th- } \\
\text { late } \\
6 \text { th } c\end{array}$ & Green dots & $\begin{array}{l}\text { Leeds 1938, 93-101; } \\
\text { Harden 1940, 164; } \\
\text { Reading Museum }\end{array}$ \\
\hline $\begin{array}{l}\text { Sunderland, } \\
\text { Pennywell } \\
\text { Estate }\end{array}$ & Durham & & $\begin{array}{l}\text { Stray find: white waves and } \\
\text { dots }\end{array}$ & $\begin{array}{l}\text { Newcastle upon Tyne: } \\
\text { unpublished }\end{array}$ \\
\hline Mucking II & Essex & $\begin{array}{l}\text { 2nd } \\
\text { half } \\
5 \text { th } c \\
\text { first } \\
\text { half } \\
\text { 5th } c\end{array}$ & $\begin{array}{l}\text { Grave 334: } 1 \text { bead with } \\
\text { disc brooch pair, amber } \\
\text { beads, inlaid buckle, etc; } \\
\text { Grave } 989: 5 \text { beads with } \\
\text { Glaston-Mucking and } \\
\text { supporting-arm brooches, } \\
\text { etc }\end{array}$ & $\begin{array}{l}\text { Evison } 1981,138-9 \text {, } \\
\text { figs } 4 \text { and } 5 \\
\text { BM }\end{array}$ \\
\hline Prittlewell & Essex & $\begin{array}{l}\text { late } \\
\text { 6th } c\end{array}$ & $\begin{array}{l}\text { Grave-group 32: crossed } \\
\text { waves in yellow and dots }\end{array}$ & $\begin{array}{l}\text { Tyler } 1988,113 \text {, fig } 15.25 \text {; } \\
\text { Southend Museum }\end{array}$ \\
\hline Lechlade & Glos & 6th $c$ & $\begin{array}{l}\text { Grave } 25: 3 \text { with white } \\
\text { waves and dots and } 4 \text { with } \\
\text { yellow waves and dots with } \\
\text { saucer brooch, etc }\end{array}$ & $\begin{array}{l}\text { Boyle et al forthcoming; } \\
\text { Cirencester Museum }\end{array}$ \\
\hline Droxford & Hants & $\begin{array}{l}\text { prob } \\
\text { Gth } c\end{array}$ & $\begin{array}{l}\text { Grave } 20 \text { : yellow crossed } \\
\text { waves and dots (Class IIc) }\end{array}$ & $\begin{array}{l}\text { Aldsworth } 1978,124 \text {, } \\
172, \text { fig } 24.8 ; \\
\text { Winchester Museum }\end{array}$ \\
\hline $\begin{array}{l}\text { Canterbury, } \\
\text { Mint Yard }\end{array}$ & Kent & $\begin{array}{l}\text { 6th- } \\
\text { 8th c }\end{array}$ & One single & $\begin{array}{l}\text { Information from } \\
\mathrm{T} \text { Tatton-Brown } \\
\text { and } \mathrm{P} \text { Garrard }\end{array}$ \\
\hline $\begin{array}{l}\text { Dover, } \\
\text { Buckland }\end{array}$ & Kent & $\begin{array}{l}575- \\
625\end{array}$ & $\begin{array}{l}\text { Grave 1: } 4 \text { rather barrel- } \\
\text { shaped with white (D48) and } \\
3 \text { with yellow (D46); } \\
\text { Grave 29: barrel with white } \\
\text { (D49); } \\
\text { Grave 30: } 3 \text { discs with } \\
\text { yellow (D40), } 2 \text { barrels } \\
\text { with white (D49); } \\
\text { Grave 59: disc with white } \\
\text { (D42), } 2 \text { barrels with } \\
\text { yellow (D47), barrel with } \\
\text { white (D49); } \\
\text { Grave 60: barrel with } \\
\text { white (D49); }\end{array}$ & $\begin{array}{l}\text { Evison } 1987,77,82 \text {, } \\
216,223-4,224-5 \text {, } \\
232-3,233,228-9 \text { and } 231 \text {, } \\
\text { figs 5.4z.( } 2 \text { and } 1), 17.2 \mathrm{r} \text {, } \\
18.4 \mathrm{p} \text { and } \mathrm{r}, 33.3 \mathrm{~m}, \mathrm{n} \\
\text { and } \mathrm{o}, 34.3 \mathrm{~s} \text {, } \\
24.42 .1 \mathrm{n}, \mathrm{p} \text { and } \mathrm{s}, 30.1 \mathrm{i}, \\
\text { colour pl IV.D40, } \\
42,46,48 \text { and } 54 \text {; } \\
\text { BM }\end{array}$ \\
\hline
\end{tabular}




\begin{tabular}{|c|c|c|c|c|}
\hline & & $\begin{array}{l}625- \\
650\end{array}$ & $\begin{array}{l}\text { Grave } 42 \text { : disc with yellow } \\
\text { (D40), } 3 \text { barrels with white } \\
\text { (D48) and short cylinder } \\
\text { with yellow (D54); } \\
\text { Grave 55: short cylinder } \\
\text { with yellow (D54) }\end{array}$ & \\
\hline $\begin{array}{l}\text { Eastry, } \\
\text { Eastry House }\end{array}$ & Kent & $\begin{array}{l}\text { late } \\
\text { 6th c }\end{array}$ & $\begin{array}{l}\text { Yellow swags and dots } \\
\text { with Gotlandic bow brooch }\end{array}$ & $\begin{array}{l}\text { Hawkes } 1979,86 \text { and } 93 \text {, } \\
\text { fig } 4.4 .18 \text { and } 29 ; \\
\text { Deal Museum }\end{array}$ \\
\hline Faversham & Kent & $\begin{array}{l}5 \text { th- } \\
7 \text { th c }\end{array}$ & $\begin{array}{l}\text { Various with barrel forms } \\
\text { and different colour wave } \\
\text { and dots (Plate } 7 \text { ) }\end{array}$ & $\begin{array}{l}\text { Meaney } 1964,118-19 \\
\text { Ashmolean Museum }\end{array}$ \\
\hline $\begin{array}{l}\text { Folkestone, } \\
\text { Dover Hill }\end{array}$ & Kent & $\begin{array}{l}\text { prob } \\
\text { 6thc }\end{array}$ & $\begin{array}{l}\text { Grave 15: a few singles; } \\
\text { Grave 28: } 2-3 \text { beads }\end{array}$ & $\begin{array}{l}\text { Meaney } 1964,120-1 \\
\text { Maidstone Museum }\end{array}$ \\
\hline $\begin{array}{l}\text { Gilton or } \\
\text { Kingston }\end{array}$ & Kent & $\begin{array}{l}\text { prob } \\
6 \text { th- } \\
7 \text { th c }\end{array}$ & $\begin{array}{l}\text { Mainly barrel-shaped and } \\
\text { white waves }\end{array}$ & $\begin{array}{l}\text { Faussett 1856; } \\
\text { Liverpool Museum }\end{array}$ \\
\hline Holborough & Kent & $\begin{array}{l}\text { late } \\
7 \text { th- } \\
8 \text { th c }\end{array}$ & & $\begin{array}{l}\text { Evison } 1956,126, \\
\text { fig } 20.3 \mathrm{c}-\mathrm{e} \text {; } \\
\mathrm{BM} 19475-2358\end{array}$ \\
\hline Monkton & Kent & $\begin{array}{l}6 \text { th- } \\
7 \text { th c }\end{array}$ & Grave 13 & $\begin{array}{l}\text { Hawkes and Hogarth } 1974 \text {, } \\
65,80 \text {, fig } 7.13 .1 \text {; } \\
\text { Ashmolean Museum }\end{array}$ \\
\hline Sarre & Kent & $\begin{array}{l}\text { prob } \\
7 \text { th c }\end{array}$ & $\begin{array}{l}\text { Barrel-shaped mostly, } \\
\text { several with white or } \\
\text { yellow }\end{array}$ & $\begin{array}{l}\text { Brent 1868; } \\
\text { Liverpool Museum; } \\
\text { BM 93 6-1 } 218\end{array}$ \\
\hline Stowting & Kent & $\begin{array}{l}6 \text { th- } \\
7 \text { th c }\end{array}$ & $\begin{array}{l}\text { Two barrel-shaped, } \\
\text { white wave and dots }\end{array}$ & $\begin{array}{l}\text { Meaney } 1964,137-8 \\
\text { Maidstone Museum }\end{array}$ \\
\hline Saxby & Leics & & $\begin{array}{l}\text { One globular with white } \\
\text { crossed wave and yellow dot }\end{array}$ & $\begin{array}{l}\text { Publication in } \\
\text { preparation (J Timby) }\end{array}$ \\
\hline $\begin{array}{l}\text { London, } \\
\text { Wandsworth } \\
\text { (Thames) }\end{array}$ & Surrey & & $\begin{array}{l}\text { Large biconical white } \\
\text { wave and yellow dots }\end{array}$ & $\begin{array}{l}\text { Museum of London } \\
\text { (formerly Guildhall } \\
\text { Museum) } 3955\end{array}$ \\
\hline Brooke & Norfolk & $\begin{array}{l}\text { prob } \\
\text { 6th c }\end{array}$ & $\begin{array}{l}\text { Large with white waves and } \\
\text { dots }\end{array}$ & $\begin{array}{l}\text { Meaney 1964, 170; } \\
\text { BM 1866-69 }\end{array}$ \\
\hline $\begin{array}{l}\text { Morning } \\
\text { Thorpe }\end{array}$ & Norfolk & 6 th c & $\begin{array}{l}\text { Grave } 108: 2 \text { with white and } \\
\text { yellow with annular brooch } \\
\text { pair and B clasps; } \\
\text { Grave } 216: 2 \text { all white with } \\
\text { annular brooch pair; } \\
\text { Grave 299: } 3 \text { all white with } \\
\text { annular brooch pair and B7 } \\
\text { clasps }\end{array}$ & $\begin{array}{l}\text { Green et al 1987,65-6, } \\
93 \text { and 118, figs 332.Gix, } \\
\text { 366.Jii and 393.299.Rii; } \\
\text { Norwich Museum }\end{array}$ \\
\hline Spong Hill & Norfolk & 6 th $\mathrm{c}$ & $\begin{array}{l}\text { Grave } 42: 1 \text { with white } \\
\text { waves and yellow dots with } \\
\text { small-long brooch pair, } \\
\text { B clasps, stamped pot, etc }\end{array}$ & $\begin{array}{l}\text { Hills et al 1984, 95-6, } \\
\text { fig } 98.42 .3 \text {; } \\
\text { Norfolk Arch Unit }\end{array}$ \\
\hline
\end{tabular}




\begin{tabular}{|c|c|c|c|c|}
\hline $\begin{array}{l}\text { Cassington, } \\
\text { Purwell Farm }\end{array}$ & Oxon & 6th $c$ & Three white waves and dots & $\begin{array}{l}\text { Leeds and Riley } 1942, \\
64, \mathrm{pl} \text { VIB; } \\
\text { Ashmolean Museum } \\
1942.146\end{array}$ \\
\hline Ipswich & Suffolk & $\begin{array}{l}\text { 6th- } \\
7 \text { th } c\end{array}$ & $\begin{array}{l}\text { A number of small barrels } \\
\text { with white waves and dots }\end{array}$ & $\begin{array}{l}\text { Layard } 1907 \text { and } \\
\text { 1909; Ipswich Museum }\end{array}$ \\
\hline Snape & Suffolk & $\begin{array}{l}\text { prob } \\
\text { 6th } c\end{array}$ & $\begin{array}{l}\text { Grave } 0421: 2 \text { with yellow; } \\
3 \text { with white }\end{array}$ & $\begin{array}{l}\text { Filmer-Sankey and } \\
\text { Pestell forthcoming }\end{array}$ \\
\hline Guildown & Surrey & $\begin{array}{l}\text { prob } \\
\text { 6th c }\end{array}$ & $\begin{array}{l}\text { Grave } 185: 1 \text { white wave and } \\
\text { dots }\end{array}$ & $\begin{array}{l}\text { Lowther 1931, 11-12, 43, } \\
\text { pl IX.3; } \\
\text { Guildford Museum }\end{array}$ \\
\hline Mitcham & Surrey & $\begin{array}{l}\text { prob } \\
\text { 6th c }\end{array}$ & $\begin{array}{l}\text { Grave 62: } 1 \text { all white and } 2 \\
\text { yellow; } \\
\text { Grave 197: yellow waves and } \\
\text { green dots }\end{array}$ & $\begin{array}{l}\text { Bidder and Morris } 1959 \text {, } \\
64,73,110-12, \\
\text { pl XVI.62 and } 197 \text {; } \\
\text { Cambridge Museum }\end{array}$ \\
\hline Alfriston & Sussex & $\begin{array}{l}\text { 5th- } \\
6 \text { th } c\end{array}$ & $\begin{array}{l}\text { Grave } 87: 1 \text { with yellow and } \\
1 \text { with white waves and dots } \\
\text { with } 5 \text { spiral cast saucer } \\
\text { brooch pair, etc }\end{array}$ & $\begin{array}{l}\text { Griffith and Salzmann 1914, } \\
\text { 204, pl XXII.2; Welch } \\
\text { 1983, 85-6, fig 36c; } \\
\text { Lewes Museum }\end{array}$ \\
\hline $\begin{array}{l}\text { Apple } \\
\text { Down I }\end{array}$ & Sussex & $\begin{array}{l}\text { prob } \\
\text { 6th } c\end{array}$ & $\begin{array}{l}\text { Grave } 134: 1 \text { white with } \\
\text { white waves and dots with } \\
\text { amber beads, etc, and } \\
\text { small barrels with yellow } \\
\text { waves and dots; } \\
\text { Area } 2 \text { : several with } \\
\text { yellow waves and dots; } \\
\text { Grave } 117 \text { : yellow waves } \\
\text { and dots with amber beads }\end{array}$ & $\begin{array}{l}\text { Down and Welch } 1990,51 \text {, } \\
49 \text { and } 162-5 \text {, figs } 2.43 \text { and } \\
2.37 \text {, colour pls } 40.22 \text { and } \\
41.35 \text { and } 40 \text {; } \\
\text { Chichester Museum }\end{array}$ \\
\hline Highdown & Sussex & $\begin{array}{l}\text { prob } \\
\text { 6th c }\end{array}$ & Two yellow and 3 white & $\begin{array}{l}\text { Welch 1983, 85-6; } \\
\text { Worthing Museum }\end{array}$ \\
\hline $\begin{array}{l}\text { Stretton- } \\
\text { on- } \\
\text { Fosse II }\end{array}$ & Warks & $\begin{array}{l}\text { 5th- } \\
\text { 6th } \mathrm{c}\end{array}$ & $\begin{array}{l}\text { Grave 47: white waves } \\
\text { and green dots with small- } \\
\text { long brooch }\end{array}$ & $\begin{array}{l}\text { Gelling } 1992,31 \text { and } 40-1 \text {, } \\
\text { fig 21; } \\
\text { Warwick Museum }\end{array}$ \\
\hline Mildenhall & Wilts & 6th $c$ & $\begin{array}{l}\text { White waves and dots } \\
\text { with saucer brooches, etc }\end{array}$ & $\begin{array}{l}\text { Meaney 1964, 271-2; } \\
\text { Devizes Museum }\end{array}$ \\
\hline $\begin{array}{l}\text { Pewsey } \\
\text { Blackpatch }\end{array}$ & Wilts & $\begin{array}{l}\text { prob } \\
\text { 6th } c\end{array}$ & $\begin{array}{l}\text { Grave 42: colours missing, } \\
\text { but yellow dots }\end{array}$ & $\begin{array}{l}\text { Publication in } \\
\text { preparation (K Annable); } \\
\text { Devizes Museum }\end{array}$ \\
\hline $\begin{array}{l}\text { Broadway } \\
\text { Hill }\end{array}$ & Worcs & 6th $c$ & $\begin{array}{l}\text { Grave 1: white wave and } \\
\text { yellow dots with cast } \\
\text { saucer brooch pair }\end{array}$ & $\begin{array}{l}\text { Cook 1958, 64, } \\
\text { fig } 4.12\end{array}$ \\
\hline
\end{tabular}

Cassington, Oxon 6th $\mathrm{c}$ Three white waves and dot Purwell Farm

Mucking II Essex 7th c Grave 608: double annular with blue-white waves;
Hirst and Clark forthcoming; BM 


\begin{tabular}{|c|c|c|c|c|}
\hline & & 7th $\mathrm{c}$ & $\begin{array}{l}\text { Grave } 621 \text { : } 1 \text { double } \\
\text { globular with yellow } \\
\text { waves and dots with wire } \\
\text { rings, wooden box, etc }\end{array}$ & \\
\hline Lechlade & Glos & 6th c & $\begin{array}{l}\text { Grave 25: one with yellow } \\
\text { and one with white waves } \\
\text { and dots with saucer brooch, } \\
\text { etc; } \\
\text { Grave 101: with white } \\
\text { waves }\end{array}$ & $\begin{array}{l}\text { Boyle et al forthcoming; } \\
\text { Cirencester Museum }\end{array}$ \\
\hline Droxford & Hants & $\begin{array}{l}\text { prob } \\
\text { 6th c }\end{array}$ & $\begin{array}{l}\text { Grave 20: grey waves } \\
\text { (Class IIa) }\end{array}$ & $\begin{array}{l}\text { Aldsworth 1978, } 124 \\
\text { and 172, fig 24.6; } \\
\text { Winchester Museum }\end{array}$ \\
\hline $\begin{array}{l}\text { Canterbury, } \\
\text { Mint Yard }\end{array}$ & Kent & $\begin{array}{l}\text { 6th- } \\
\text { 8th c }\end{array}$ & One example & $\begin{array}{l}\text { Information from } \\
\mathrm{T} \text { Tatton-Brown } \\
\text { and P Garrard }\end{array}$ \\
\hline $\begin{array}{l}\text { Dover, } \\
\text { Buckland }\end{array}$ & Kent & $\begin{array}{l}625- \\
650\end{array}$ & $\begin{array}{l}\text { Grave } 1 \text { : disc with white } \\
\text { waves (D20); } \\
\text { Grave } 30: 2 \text { discs with } \\
\text { white waves (D20) and } \\
4 \text { with yellow waves and } \\
\text { dots (D41); } \\
\text { Grave 18: short cylinder } \\
\text { with yellow wave (D33) }\end{array}$ & $\begin{array}{l}\text { Evison } 1987,76,82 \text {, } \\
216,224-5 \text { and } 220 \text {, } \\
\text { figs } 5.4 \mathrm{w}, 18.41 \text { and } q \text {, } \\
11.18 .2 \mathrm{f} ; \mathrm{BM}\end{array}$ \\
\hline $\begin{array}{l}\text { Eastry, } \\
\text { Eastry House }\end{array}$ & Kent & $\begin{array}{l}\text { late } \\
\text { 6th c }\end{array}$ & $\begin{array}{l}\text { White waves and spots } \\
\text { with Gotlandic bow brooch }\end{array}$ & $\begin{array}{l}\text { Hawkes } 1979,86 \text { and } 93 \text {, } \\
\text { fig 4.4.33; } \\
\text { Deal Museum }\end{array}$ \\
\hline Faversham & Kent & $\begin{array}{l}5 \text { th- } \\
7 \text { th c }\end{array}$ & A number (Plate 7) & $\begin{array}{l}\text { Meaney } 1964,118-19 \\
\text { Ashmolean Museum }\end{array}$ \\
\hline $\begin{array}{l}\text { Folkestone, } \\
\text { Dover Hill }\end{array}$ & Kent & $\begin{array}{l}\text { prob } \\
6 \text { th } c\end{array}$ & No. 15: more than one & $\begin{array}{l}\text { Meaney } 1964,120-1 \\
\text { Maidstone Museum }\end{array}$ \\
\hline 'Kent' & Kent & & $\begin{array}{l}\text { Unprovenanced: some } \\
\text { examples }\end{array}$ & $\begin{array}{l}\text { Canterbury Museum; } \\
\text { Faussett-Rolfe Collection, } \\
\text { Liverpool Museum } \\
\text { M } 7836 \text { and } 7839\end{array}$ \\
\hline Stowting & Kent & & White waves and no dots & $\begin{array}{l}\text { Meaney } 1964,137-8 \\
\text { Maidstone Museum }\end{array}$ \\
\hline Syston Park & Lincs & $\begin{array}{l}\text { prob } \\
6 \text { th- } \\
7 \text { th c }\end{array}$ & Irregular with white waves & $\begin{array}{l}\text { Akerman 1855a, 41-2, } \\
\text { pl XXI; } \\
\text { BM (18) } 1111-92\end{array}$ \\
\hline $\begin{array}{l}\text { Morning } \\
\text { Thorpe }\end{array}$ & Norfolk & 6th $c$ & $\begin{array}{l}\text { Grave 371: with white } \\
\text { waves with Åberg Group } \\
\text { III cruciform brooch and } \\
\text { great square-headed } \\
\text { brooch, etc }\end{array}$ & $\begin{array}{l}\text { Green et al 1987, 145, } \\
\text { fig } 431 . \text { Hvi; } \\
\text { Norwich Museum }\end{array}$ \\
\hline
\end{tabular}




$\begin{array}{lllll}\begin{array}{l}\text { Apple } \\ \text { Down I }\end{array} & \text { Sussex } & \begin{array}{l}\text { prob } \\ \text { 6th c }\end{array} & \begin{array}{l}\text { Grave 134: 1 with white } \\ \text { and 1 with yellow waves } \\ \text { with amber beads }\end{array} & \begin{array}{l}\text { Down and Welch 1990, 51, } \\ \text { 164, fig 2.43, } \\ \text { colour pl 40.33; } \\ \text { Chichester Museum }\end{array} \\ \text { Wasperton } & \text { Warks } & \begin{array}{l}\text { mid- } \\ \text { 6th c }\end{array} & \begin{array}{l}\text { F1081: yellow dots with } \\ \text { saucer brooches }\end{array} & \begin{array}{l}\text { Publication in } \\ \text { preparation (M Carver); } \\ \text { Warwick Museum }\end{array} \\ \begin{array}{l}\text { West } \\ \text { Heslerton }\end{array} & \text { Yorks } & & \text { At least one, probably more } & \begin{array}{l}\text { Publication in } \\ \text { preparation (D Powlesland) }\end{array}\end{array}$

XV TERRACOTTA OVAL BEADS, SOME BARREL-SHAPED, WITH WHITE OR YELLOW CROSSED WAVES, WITH OR WITHOUT DOTS

\begin{tabular}{|c|c|c|c|c|}
\hline Mucking II & Essex & $\begin{array}{l}\text { prob } \\
\text { 7th c }\end{array}$ & $\begin{array}{l}\text { Grave 608: } 1 \text { barrel with } \\
\text { yellow wave and spot }\end{array}$ & $\begin{array}{l}\text { Hirst and Clark forthcoming; } \\
\text { BM }\end{array}$ \\
\hline Prittlewell & Essex & $c .600$ & $\begin{array}{l}\text { Two with yellow waves and } \\
\text { dots, with saucer brooches }\end{array}$ & $\begin{array}{l}\text { Tyler 1988; } \\
\text { Southend Museum }\end{array}$ \\
\hline Alton & Hants & $\begin{array}{l}\text { prob } \\
7 \text { th c }\end{array}$ & $\begin{array}{l}\text { Grave } 39: 1 \text { with yellow and } \\
2 \text { with colour missing }\end{array}$ & $\begin{array}{l}\text { Evison 1988a, 17, 44, } \\
\text { 80-1, fig 33.39.1k and l; } \\
\text { Hampshire Museums }\end{array}$ \\
\hline $\begin{array}{l}\text { Beakes- } \\
\text { bourne }\end{array}$ & Kent & $\begin{array}{l}\text { prob } \\
7 \text { th } c\end{array}$ & $\begin{array}{l}\text { On small string: white } \\
\text { waves without dots }\end{array}$ & $\begin{array}{l}\text { Faussett 1856, pl V.4; } \\
\text { Liverpool Museum }\end{array}$ \\
\hline $\begin{array}{l}\text { Dover, } \\
\text { Buckland }\end{array}$ & Kent & $\begin{array}{l}575- \\
625\end{array}$ & $\begin{array}{l}\text { Grave 1: } 3 \text { with yellow } \\
\text { (D46) and } 4 \text { with white } \\
\text { (D48); } \\
\text { Grave 29: } 1 \text { with white } \\
\text { (D49); } \\
\text { Grave } 30: 3 \text { discs with } \\
\text { yellow (D40), } 2 \text { barrels } \\
\text { with white (D49); } \\
\text { Grave 59: } 2 \text { discs with } \\
\text { white (D42), } 2 \text { barrels with } \\
\text { yellow (D47) and } 1 \text { with } \\
\text { white (D49); } \\
\text { Grave 60: } 1 \text { barrel with } \\
\text { white (D49); } \\
\text { Grave } 42: 1 \text { disc with } \\
\text { yellow (D40), } 2 \text { barrels } \\
\text { with white (D49) and } 1 \text { short } \\
\text { cylinder with yellow } \\
\text { (D54); } \\
\text { Grave 55: } 1 \text { short cylinder } \\
\text { with yellow (D54) }\end{array}$ & $\begin{array}{l}\text { Evison } 1987,77,82 \text {, } \\
216,223-4,224-5,232-3 \text {, } \\
233,228-9 \text { and } 231 \text {, } \\
\text { figs } 5.42 .1 \text { and } 2,17.2 \mathrm{r} \text {, } \\
18.4 \mathrm{p} \text { and } \mathrm{r} \text {, } \\
33.3 \mathrm{~m}, \mathrm{n} \text { and } \mathrm{o}, 34.3 \mathrm{~s} \text {, } \\
24.42 .1 \mathrm{n}, \mathrm{r} \text { and } \mathrm{s}, 30.1 \mathrm{i} \text {, } \\
\text { colour pl IV.D40, } \\
42,46,48 \text { and } 54 ; \\
\text { BM }\end{array}$ \\
\hline Faversham & Kent & $\begin{array}{l}\text { prob } \\
6 \text { th- } \\
7 \text { th c }\end{array}$ & $\begin{array}{l}\text { Several with white or } \\
\text { yellow and dots }\end{array}$ & $\begin{array}{l}\text { Meaney } 1964,118-19 \\
\text { Ashmolean Museum } \\
1909.149,161,168,170,171 \\
\text { and } 191\end{array}$ \\
\hline $\begin{array}{l}\text { Folkestone, } \\
\text { Dover Hill }\end{array}$ & Kent & $\begin{array}{l}\text { prob } \\
\text { 6th c }\end{array}$ & No. 12: white waves and dots & $\begin{array}{l}\text { Meaney 1964, 120-1; } \\
\text { Maidstone Museum }\end{array}$ \\
\hline
\end{tabular}




\begin{tabular}{|c|c|c|c|c|}
\hline $\begin{array}{l}\text { Gilton or } \\
\text { Kingston }\end{array}$ & Kent & $\begin{array}{l}6 \text { th- } \\
7 \text { th c }\end{array}$ & Several & $\begin{array}{l}\text { Faussett } 1856,1-34, \\
\text { pls V and VI; } \\
\text { Liverpool Museum }\end{array}$ \\
\hline Holborough & Kent & $\begin{array}{l}\text { late } \\
7 \text { th- } \\
\text { 8th c }\end{array}$ & $\begin{array}{l}\text { Several with yellow waves } \\
\text { and } 1 \text { white with dots }\end{array}$ & $\begin{array}{l}\text { Evison } 1956,126 \text {, } \\
\text { fig 20c, d and e; } \\
\text { BM } 19475-2358\end{array}$ \\
\hline 'Kent' & Kent & $\begin{array}{l}\text { prob } \\
6 \text { th- } \\
7 \text { th c }\end{array}$ & $\begin{array}{l}\text { Numbers with or without } \\
\text { dots }\end{array}$ & $\begin{array}{l}\text { Probably ex-Faussett } \\
\text { Collection, Liverpool } \\
\text { Museum } 7487\end{array}$ \\
\hline Sarre & Kent & $\begin{array}{l}\text { late } \\
6 \text { th- } \\
7 \text { th c }\end{array}$ & $\begin{array}{l}\text { On ?necklace from old } \\
\text { find }\end{array}$ & $\begin{array}{l}\text { Meaney 1964, 135-6; } \\
\text { BM 93 6-1 } 218\end{array}$ \\
\hline Stowting & Kent & $\begin{array}{l}\text { prob } \\
7 \text { th } c\end{array}$ & $\begin{array}{l}\text { Two on ?associated group } \\
\text { or necklace with amethyst }\end{array}$ & $\begin{array}{l}\text { Meaney 1964, 137-8; } \\
\text { Maidstone Museum }\end{array}$ \\
\hline Ipswich & Suffolk & $\begin{array}{l}\text { 6th- } \\
7 \text { th c }\end{array}$ & $\begin{array}{l}\text { Several with yellow waves } \\
\text { and dots }\end{array}$ & $\begin{array}{l}\text { Layard 1907, pl XXXIII, and } \\
\text { 1909, pl VI; } \\
\text { Ipswich Museum }\end{array}$ \\
\hline Mitcham & Surrey & $\begin{array}{l}\text { mid- } \\
5 \text { th- } \\
6 \text { th c }\end{array}$ & $\begin{array}{l}\text { Grave } 62: 2 \text { examples with } \\
\text { yellow waves and dots }\end{array}$ & $\begin{array}{l}\text { Bidder and Duckworth } \\
\text { 1906; Bidder and Morris } \\
\text { 1959, 64, 110, pl XVI.62; } \\
\text { Cambridge Museum }\end{array}$ \\
\hline
\end{tabular}

\section{xvi Terracotta beads With SINGLE WAVE OR ZIGZaG}

\begin{tabular}{|c|c|c|c|c|}
\hline Frilford I & Berks & $\begin{array}{l}\text { prob } \\
\text { 6th } c\end{array}$ & $\begin{array}{l}\text { Globular with white wave } \\
\text { and central blue line }\end{array}$ & $\begin{array}{l}\text { Bradford and Goodchild } \\
\text { 1939; } \\
\text { Ashmolean Museum }\end{array}$ \\
\hline Andover & Hants & $\begin{array}{l}\text { prob } \\
\text { 6th } c\end{array}$ & $\begin{array}{l}\text { Grave } 44: 2 \text { with yellow } \\
\text { waves }\end{array}$ & $\begin{array}{l}\text { Cook and Dacre } 1985,35 \text {, } \\
82-3 \text {, fig } 59.24 \text { and } 40, \\
\text { colour frontispiece; } \\
\text { Hampshire Museums }\end{array}$ \\
\hline $\begin{array}{l}\text { Winchester, } \\
\text { Lower Brook } \\
\text { Street }\end{array}$ & Hants & $\begin{array}{l}\text { late } \\
\text { 7th } c\end{array}$ & $\begin{array}{l}\text { Grave 23: slightly biconical } \\
\text { with angular yellow wave }\end{array}$ & $\begin{array}{l}\text { Hawkes } 1990 \mathrm{~b}, 625-6,632 \text {, } \\
\text { fig } 168, \text { no. } 1993, \\
\text { colour pl XIVII }\end{array}$ \\
\hline $\begin{array}{l}\text { Chessell } \\
\text { Down }\end{array}$ & $\begin{array}{l}\text { Isle of } \\
\text { Wight }\end{array}$ & $\begin{array}{l}\text { prob } \\
\text { 6th c }\end{array}$ & $\begin{array}{l}\text { Annular with angular yellow } \\
\text { wave (type } 6.16 \text { ) }\end{array}$ & $\begin{array}{l}\text { Arnold } 1982,120 \text {, } \\
\text { colour frontispiece; BM }\end{array}$ \\
\hline $\begin{array}{l}\text { Dover, } \\
\text { Buckland }\end{array}$ & Kent & $\begin{array}{l}575- \\
625\end{array}$ & $\begin{array}{l}\text { Grave } 30: 1 \text { with angular } \\
\text { yellow wave (D06) }\end{array}$ & $\begin{array}{l}\text { Evison } 1987,76,82 \text {, } \\
224-5 \text {, fig } 18.4 \mathrm{k} \text {; colour } \\
\text { pl III.D06; BM }\end{array}$ \\
\hline $\begin{array}{l}\text { Folkestone, } \\
\text { Dover Hill }\end{array}$ & Kent & $\begin{array}{l}\text { prob } \\
\text { 6th } c\end{array}$ & $\begin{array}{l}\text { One globular with } 2 \text { yellow } \\
\text { and } 1 \text { white waves }\end{array}$ & $\begin{array}{l}\text { Meaney } 1964,120-1 \\
\text { Maidstone Museum }\end{array}$ \\
\hline $\begin{array}{l}\text { Horton } \\
\text { Kirby I }\end{array}$ & Kent & & $\begin{array}{l}\text { With white annular/ } \\
\text { globular }\end{array}$ & $\begin{array}{l}\text { Meaney 1964, 124; } \\
\text { Maidstone Museum }\end{array}$ \\
\hline 'Kent' & Kent & & One with yellow & Canterbury Museum \\
\hline
\end{tabular}




\begin{tabular}{|c|c|c|c|c|}
\hline Monkton & Kent & $\begin{array}{l}\text { 6th- } \\
7 \text { th } c\end{array}$ & $\begin{array}{l}\text { One thick annular/globular } \\
\text { with yellow wave }\end{array}$ & $\begin{array}{l}\text { Hawkes and Hogarth } 1 \\
\text { Perkins and Hawkes } 19 \\
\text { Ashmolean Museum }\end{array}$ \\
\hline Sarre & Kent & $\begin{array}{l}\text { prob } \\
\text { 7th c }\end{array}$ & $\begin{array}{l}\text { Group XCVIII: } 1 \text { angular } \\
\text { with white wave }\end{array}$ & Brent 1868 \\
\hline Syston Park & Lincs & $\begin{array}{l}\text { prob } \\
\text { 6th- } \\
7 \text { th c }\end{array}$ & $\begin{array}{l}\text { Supposedly on string: } \\
1 \text { annular with angular } \\
\text { wave }\end{array}$ & $\begin{array}{l}\text { Akerman 1855a, } 41-2 \text {, } \\
\text { pl XXI; } \\
\text { BM (18) 11-1192 }\end{array}$ \\
\hline Hornton & Oxon & $\begin{array}{l}\text { prob } \\
\text { late } \\
6 \text { th } c\end{array}$ & $\begin{array}{l}\text { Inhumation burial: whitish } \\
\text { wave on short rather } \\
\text { cylindrical bead; } \\
\text { ditto, but more globular }\end{array}$ & $\begin{array}{l}\text { Meaney } 1964,209 \\
\text { Banbury Museum } \\
\text { on loan from BM; } \\
\text { BM } 18863-23\end{array}$ \\
\hline $\begin{array}{l}\text { Empingham } \\
\text { II }\end{array}$ & Rutland & $\begin{array}{l}5 \text { th- } \\
7 \text { th c }\end{array}$ & $\begin{array}{l}\text { Three annulars with white } \\
\text { waves; } \\
2 \text { square cylinders with } \\
\text { yellow waves ( } 2 \text { different } \\
\text { boxes marked } 67 \text { and } 20 \text { ) }\end{array}$ & $\begin{array}{l}\text { Timby 1996; } \\
\text { Oakham Museum }\end{array}$ \\
\hline Alfriston & Sussex & $\begin{array}{l}5 \text { th- } \\
\text { early } \\
6 \text { th } c\end{array}$ & $\begin{array}{l}\text { Grave } 68 \text { : annular with } \\
\text { regular wave, with quoit } \\
\text { brooch, etc }\end{array}$ & $\begin{array}{l}\text { Griffiths and Salzmann } \\
\text { 1914, 46-7, pl I.1; } \\
\text { Welch 1983, 85-6; } \\
\text { Lewes Museum }\end{array}$ \\
\hline $\begin{array}{l}\text { xvii } \\
\text { AND }\end{array}$ & $\begin{array}{l}\text { Terrac } \\
\text { FINE T }\end{array}$ & IST D & $\begin{array}{l}\text { UM-SHAPED BEADS: (a) } \\
\text { ORATION, INCLUDING GI }\end{array}$ & $\begin{array}{l}\text { VITH HERRINGBONE } \\
\text { EEN AND YELLOW }\end{array}$ \\
\hline Kempston & Beds & 6 th $c$ & $\begin{array}{l}\text { Grave 16: small yellow and } \\
\text { green, badly made bands }\end{array}$ & $\begin{array}{l}\text { Fitch 1864; } \\
\text { BM 91 6-24 } 53\end{array}$ \\
\hline Puddlehill & Beds & $c .600$ & $\begin{array}{l}\text { Grave } 10: 3 \text { registers of } \\
\text { terracotta, yellow and black } \\
\text { on terracotta ground with } \\
\text { cast saucer brooch pair }\end{array}$ & $\begin{array}{l}\text { Matthews and Hawkes } \\
1985,91 \text { and } 95 \text {, } \\
\text { figs } 38 \text { and } 40.37\end{array}$ \\
\hline $\begin{array}{l}\text { Maidenhead, } \\
\text { Somerlease } \\
\text { Road }\end{array}$ & Berks & & $\begin{array}{l}\text { Five registers, twisted green } \\
\text { and yellow on terracotta }\end{array}$ & Reading Museum \\
\hline Wallingford & Berks & $\begin{array}{l}5 \text { th- } \\
6 \text { th c }\end{array}$ & $\begin{array}{l}\text { Near grave 13: } 5 \text { registers, } \\
\text { green and yellow on } \\
\text { terracotta }\end{array}$ & $\begin{array}{l}\text { Leeds 1938, 98; } \\
\text { Ashmolean Museum }\end{array}$ \\
\hline $\begin{array}{l}\text { Hasling- } \\
\text { field }\end{array}$ & Cambs & $\begin{array}{l}\text { prob } \\
\text { 6th c }\end{array}$ & $\begin{array}{l}\text { Two banded in green and } \\
\text { yellow }\end{array}$ & $\begin{array}{l}\text { Fox 1923, 256-7; } \\
\text { Ashmolean Museum } \\
1909.233\end{array}$ \\
\hline $\begin{array}{l}\text { Little } \\
\text { Wilbraham }\end{array}$ & Cambs & $\begin{array}{l}\text { prob } \\
6 \text { th- } \\
7 \text { th c }\end{array}$ & $\begin{array}{l}\text { Two dark green with } \\
\text { irregular bands of green and } \\
\text { yellow stripes }\end{array}$ & $\begin{array}{l}\text { Lethbridge and Carter } \\
\text { 1926; Neville 1852; } \\
\text { Cambridge Museum }\end{array}$ \\
\hline Cirencester & Glos & $\begin{array}{l}\text { 6th- } \\
7 \text { th c }\end{array}$ & $\begin{array}{l}\text { Two beads with white } \\
\text { zigzags and yellow ends on } \\
\text { terracotta; } \\
\text { another larger }\end{array}$ & $\begin{array}{l}\text { Brown 1976, } 25 \text {, } \\
\text { fig 3.2.13 and 14; } \\
\text { Cirencester Museum } \\
\text { (ex R Day Collection) }\end{array}$ \\
\hline
\end{tabular}




\begin{tabular}{|c|c|c|c|c|}
\hline Lechlade & Glos & 6th $c$ & $\begin{array}{l}\text { Grave } 101 \text { : with yellow, } \\
\text { white and slatey blue, } \\
\text { chevrons all over, with } \\
\text { applied saucer brooch }\end{array}$ & $\begin{array}{l}\text { Boyle et al forthcoming; } \\
\text { Cirencester Museum }\end{array}$ \\
\hline Andover & Hants & $\begin{array}{l}\text { prob } \\
\text { 6th c }\end{array}$ & $\begin{array}{l}\text { Grave } 44 \text { : } 1 \text { with } 3 \text { zones } \\
\text { of yellow on terracotta; } \\
1 \text { irregular lines green and } \\
\text { yellow or white on } \\
\text { terracotta, etc }\end{array}$ & $\begin{array}{l}\text { Cook and Dacre } 1985,35 \text {, } \\
83, \text { fig } 59.30,34 \text { and } 39, \\
\text { colour frontispiece; } \\
\text { Hampshire Museums }\end{array}$ \\
\hline Droxford & Hants & $\begin{array}{l}\text { prob } \\
\text { 6th c }\end{array}$ & $\begin{array}{l}\text { Grave 20: rather biconical } \\
\text { with black central core, } \\
\text { terracotta, red and yellow } \\
\text { herringbone all over } \\
\text { (Class V) }\end{array}$ & $\begin{array}{l}\text { Aldsworth } 1978,124 \\
\text { and 172, fig 24.12; } \\
\text { Winchester Museum }\end{array}$ \\
\hline $\begin{array}{l}\text { Chessell } \\
\text { Down }\end{array}$ & $\begin{array}{l}\text { Isle of } \\
\text { Wight }\end{array}$ & 6th $c$ & $\begin{array}{l}\text { Four beads } 3 \text { registers white, } \\
\text { yellow and terracotta, } \\
\text { another with green and } 2 \\
\text { others with zigzags and } 1 \\
\text { terracotta with white marks } \\
\text { (types } 8.1,8.3 \text { and } 8.4 \text { ) }\end{array}$ & $\begin{array}{l}\text { Arnold } 1982,49-50,121 \text {, } \\
\text { colour frontispiece; } \\
\text { BM } 6010-114 \text { and } 6, \\
\text { and } 677-29143 \text { and } 144\end{array}$ \\
\hline Barfreston & Kent & 7th $c$ & $\begin{array}{l}\text { Tumulus } 6: 2 \text { with tall or } \\
\text { shallow waves in terracotta, } \\
\text { yellow and white } \\
\text { with amethysts }\end{array}$ & $\begin{array}{l}\text { Faussett Collection, } \\
\text { Liverpool Museum }\end{array}$ \\
\hline $\begin{array}{l}\text { Beakes- } \\
\text { bourne II }\end{array}$ & Kent & & & $\begin{array}{l}\text { Meaney 1964, 109; } \\
\text { Canterbury Museum }\end{array}$ \\
\hline Broadstairs & Kent & & & Hurd 1913, 16 \\
\hline $\begin{array}{l}\text { Dover, } \\
\text { Buckland }\end{array}$ & Kent & $\begin{array}{l}525- \\
575 \\
575- \\
625\end{array}$ & $\begin{array}{l}\text { Grave 92: } 3 \text { zones terra- } \\
\text { cotta and yellow or green } \\
\text { and yellow (D67) (Plate 7); } \\
\text { Grave 93: probably sword- } \\
\text { bead; } 3 \text { zones terracotta, } \\
\text { opaque yellow and } \\
\text { light translucent green, } \\
\text { above and below terracotta anc } \\
\text { light translucent green } \\
\text { (D68); } \\
\text { Grave } 42: 3 \text { zones white } \\
\text { on terracotta diagonal band } \\
\text { and central twisted stripe in } \\
\text { terracotta and yellow (D66) } \\
\text { (Plate 7) }\end{array}$ & $\begin{array}{l}\text { Evison } 1987,78,82, \\
237-8,238 \text { and } 228-9, \\
\text { figs } 42.3 \mathrm{~d}, 43.1, \text { and } \\
24.42 .1 \mathrm{t} \text {, colour } \mathrm{pl} \\
\text { IV.D66, } 67 \text { and } 68 \text {; } \\
\text { BM } \\
\text { d }\end{array}$ \\
\hline $\begin{array}{l}\text { Gilton or } \\
\text { Kingston }\end{array}$ & Kent & $\begin{array}{l}\text { 6th- } \\
7 \text { th } c\end{array}$ & $\begin{array}{l}\text { Two diagonal bands of green } \\
\text { and yellow twist }\end{array}$ & $\begin{array}{l}\text { Faussett 1856, pl VI; } \\
\text { Liverpool Museum } \\
6123\end{array}$ \\
\hline 'Kent' & Kent & & $\begin{array}{l}\text { Whole surface with diagonal } \\
\text { black, yellow and terracotta } \\
\text { overlaid with finer diagonals } \\
\text { in opposite directions }\end{array}$ & Canterbury Museum \\
\hline
\end{tabular}




\begin{tabular}{|c|c|c|c|c|}
\hline Sarre & Kent & $\begin{array}{l}\text { prob } \\
\text { 6th c }\end{array}$ & $\begin{array}{l}\text { Five zones, yellow and } \\
\text { terracotta twists; } \\
\text { another with grey core, } \\
\text { terracotta with irregular } \\
\text { yellow and terracotta central } \\
\text { band }\end{array}$ & $\begin{array}{l}\text { Brent 1868, pl VIII; } \\
\text { BM } 932-18\end{array}$ \\
\hline Stodmarsh & Kent & $\begin{array}{l}\text { prob } \\
6 \text { th- } \\
7 \text { th c }\end{array}$ & $\begin{array}{l}\text { Five zones, mainly yellow } \\
\text { and terracotta herringbone, } \\
\text { with } 2 \text { yellow and black }\end{array}$ & $\begin{array}{l}\text { Akerman 1855b, 179-81, } \\
\text { pl XVI; } \\
\text { BM } 5412-217\end{array}$ \\
\hline Syston Park & Lincs & $\begin{array}{l}\text { prob } \\
\text { 6th- } \\
\text { 7th c }\end{array}$ & $\begin{array}{l}\text { One with white chevrons, } \\
\text { yellow band top and bottom }\end{array}$ & $\begin{array}{l}\text { Akerman 1855a, pl XXI; } \\
\text { BM } 1181-92\end{array}$ \\
\hline $\begin{array}{l}\text { Melton } \\
\text { Constable }\end{array}$ & Norfolk & & $\begin{array}{l}\text { Three zones, yellow and } \\
\text { terracotta }\end{array}$ & $\begin{array}{l}\text { S Woodward Collection, } \\
\text { Norwich Museum }\end{array}$ \\
\hline $\begin{array}{l}\text { Morning } \\
\text { Thorpe }\end{array}$ & Norfolk & $\begin{array}{l}\text { prob } \\
\text { 6th c }\end{array}$ & $\begin{array}{l}\text { Grave } 309: 3 \text { zones with } \\
\text { yellow and terracotta central } \\
\text { zone and second bead }\end{array}$ & $\begin{array}{l}\text { Green et al 1987, } 120 \text {, } \\
\text { fig } 396.309 . \mathrm{Axi} ; \\
\text { Norwich Museum }\end{array}$ \\
\hline Upton & Northants & & $\begin{array}{l}\text { Unstratified: black, } \\
\text { terracotta, yellow and green }\end{array}$ & $\begin{array}{l}\text { Information from } \\
\text { M Heyworth, } \\
\text { English Heritage }\end{array}$ \\
\hline $\begin{array}{l}\text { Cassington, } \\
\text { Purwell } \\
\text { Farm }\end{array}$ & Oxon & 6th $c$ & $\begin{array}{l}\text { One with } 3 \text { alternating zones } \\
\text { of yellow and terracotta; } \\
\text { possibly another }\end{array}$ & $\begin{array}{l}\text { Leeds and Riley } 1942 \text {, } \\
64, \text { pl VIB; } \\
\text { Ashmolean Museum }\end{array}$ \\
\hline $\begin{array}{l}\text { Bury St } \\
\text { Edmunds }\end{array}$ & Suffolk & & $\begin{array}{l}\text { Five zones, alternating } \\
\text { terracotta/yellow } \\
\text { and green/yellow }\end{array}$ & $\begin{array}{l}\text { Bury St Edmunds } \\
\text { Museum }\end{array}$ \\
\hline $\begin{array}{l}\text { Grundis- } \\
\text { burgh }\end{array}$ & Suffolk & & $\begin{array}{l}\text { Four zones, yellow, black, } \\
\text { terracotta and central plain } \\
\text { band in terracotta }\end{array}$ & $\begin{array}{l}\text { Fitch Collection, } \\
\text { Norwich Museum } \\
646.76 .94\end{array}$ \\
\hline Alfriston & Sussex & $\begin{array}{l}\text { prob } \\
\text { 6th } c\end{array}$ & $\begin{array}{l}\text { Grave } 64: 3 \text { zones, terra- } \\
\text { cotta central band with } \\
\text { yellow and black striped } \\
\text { band top and bottom } \\
\text { with amber beads }\end{array}$ & $\begin{array}{l}\text { Griffiths and Salzmann } \\
1914,45, \mathrm{pl} 1.2 ; \\
\text { Welch } 1983,85-6, \\
\text { fig } 30 \mathrm{a} ; \\
\text { Lewes Museum }\end{array}$ \\
\hline Selmeston & Sussex & $\begin{array}{l}\text { 6th- } \\
\text { 7th c }\end{array}$ & $\begin{array}{l}\text { Three zones, terracotta and } \\
\text { yellow above and below and } \\
\text { green and yellow centre band }\end{array}$ & $\begin{array}{l}\text { Welch } 1983,85-6 \text {, } \\
\text { fig 49a.3; } \\
\text { Lewes Museum }\end{array}$ \\
\hline Mildenhall & Wilts & 6th $c$ & $\begin{array}{l}\text { Two zones, yellow and terra- } \\
\text { cotta and terracotta band } \\
\text { top and bottom with } \\
\text { saucer brooches }\end{array}$ & $\begin{array}{l}\text { Meaney 1964, 270; } \\
\text { Devizes Museum }\end{array}$ \\
\hline $\begin{array}{l}\text { Upton } \\
\text { Snodsbury }\end{array}$ & Worcs & $\begin{array}{l}\text { late } \\
\text { 6th } c\end{array}$ & $\begin{array}{l}\text { Possibly a sword-bead: } \\
3 \text { zones, } 2 \text { yellow on } \\
\text { terracotta, central zone } \\
\text { black on terracotta }\end{array}$ & $\begin{array}{l}\text { Meaney 1964, 281; } \\
\text { Worcester Museum }\end{array}$ \\
\hline
\end{tabular}




\begin{tabular}{|c|c|c|c|c|}
\hline $\begin{array}{l}\text { Londes- } \\
\text { borough }\end{array}$ & Yorks & $\begin{array}{l}\text { mid- } \\
6 \text { th c }\end{array}$ & $\begin{array}{l}\text { Grave } 7: 3 \text { zones, black, } \\
\text { yellow and white alternated } \\
\text { with Åberg Group IVa } \\
\text { cruciform brooch, etc; } \\
\text { Grave } 4: 3 \text { zones, yellow } \\
\text { on terracotta herringbone } \\
\text { with great square-headed } \\
\text { brooch, etc }\end{array}$ & $\begin{array}{l}\text { Swanton } 1964,270-1 \text {, } \\
275 \text {, fig } 8.4 \text { and 6; } \\
\text { Newcastle Museum; }\end{array}$ \\
\hline Sancton & Yorks & $\begin{array}{l}\text { 5th- } \\
7 \text { th c }\end{array}$ & & $\begin{array}{l}\text { Myres and Southern 1973, } \\
\text { fig 1.2012; } \\
\text { Hull Museum }\end{array}$ \\
\hline $\begin{array}{l}\text { West } \\
\text { Heslerton }\end{array}$ & Yorks & & $\begin{array}{l}\text { Three zones, terracotta with } \\
\text { yellow herringbone }\end{array}$ & $\begin{array}{l}\text { Publication in } \\
\text { preparation (D Powlesland) }\end{array}$ \\
\hline Dunadd & $\begin{array}{l}\text { Scotland: } \\
\text { Argylls }\end{array}$ & $\begin{array}{l}\text { prob } \\
\text { 6th- } \\
\text { 7th c }\end{array}$ & $\begin{array}{l}\text { Fort: dark grey with white } \\
\text { chevrons with yellow top } \\
\text { and bottom lines: atypical } \\
\text { but probably an import } \\
\text { from Frankish continent }\end{array}$ & $\begin{array}{l}\text { Craw 1930, 119, } \\
\text { fig 3.5; } \\
\text { Edinburgh Museum }\end{array}$ \\
\hline
\end{tabular}

\section{xvii TerRacotTa DRUM-SHAPED beAds: (b) WITH RELATED DECORATION, SOME WITH PLAIN ZONES AND SOME WITH IMITATION TWISTS}

\begin{tabular}{|c|c|c|c|c|}
\hline $\begin{array}{l}\text { Chartham } \\
\text { Downs }\end{array}$ & Kent & & $\begin{array}{l}\text { No. 14: millefiori on terra- } \\
\text { cotta background (see } \\
\text { under millefiori) }\end{array}$ & $\begin{array}{l}\text { Faussett } 1856,162-76 \\
\text { Liverpool Museum }\end{array}$ \\
\hline $\begin{array}{l}\text { Dover, } \\
\text { Buckland }\end{array}$ & Kent & $\begin{array}{l}700- \\
750\end{array}$ & $\begin{array}{l}\text { Grave } 76 \text { : dark grey, } \\
\text { yellow and white, } 2 \text { short } \\
\text { cylinders (D13) }\end{array}$ & $\begin{array}{l}\text { Evison } 1987,76,82,236 \text {, } \\
\text { fig } 39.76 .1 \mathrm{~b} \text {, colour pl } \\
\text { III.D13; BM }\end{array}$ \\
\hline Sibertswold & Kent & $\begin{array}{l}\text { prob } \\
\text { 7th c }\end{array}$ & $\begin{array}{l}\text { Grave } 1772 / 1773 \text { : small } \\
\text { terracotta and white swags } \\
\text { round centre and yellow top } \\
\text { and bottom; another larger, } \\
\text { very damaged }\end{array}$ & $\begin{array}{l}\text { Mayer, ex Faussett } \\
\text { Collection, Liverpool } \\
\text { Museum } 6625\end{array}$ \\
\hline Wrotham II & Kent & & $\begin{array}{l}\text { With miscellaneous finds: } \\
\text { small, ?clear glass core, } \\
\text { terracotta and yellow waves } \\
\text { and yellow ends }\end{array}$ & $\begin{array}{l}\text { Anon 1920; } \\
\text { Meaney 1964, 141-2; } \\
\text { BM } 19275-122 \text { and } 3\end{array}$ \\
\hline 'Sussex’ & Sussex & & $\begin{array}{l}\text { On necklace: smokey } \\
\text { greenish yellow, } \\
\text { translucent, yellow wave } \\
\text { and yellow ends }\end{array}$ & $\begin{array}{l}\text { Welch } 1983,85-6 \text {, } \\
\text { fig } 47 c ; \\
\text { Lewes Museum }\end{array}$ \\
\hline $\begin{array}{l}\text { Salisbury, } \\
3 \text { Nether- } \\
\text { avon Road }\end{array}$ & Wilts & & $\begin{array}{l}\text { Grey ground with white } \\
\text { waves, yellow top and } \\
\text { bottom }\end{array}$ & Salisbury Museum \\
\hline Kilham & Yorks & 6th $c$ & $\begin{array}{l}\text { Green and yellow diagonal } \\
\text { stripes with thin terracotta } \\
\text { lines along centre and top }\end{array}$ & $\begin{array}{l}\text { Brown 1915a (IV), 806-8; } \\
\text { BM 76 2-12 } 10\end{array}$ \\
\hline
\end{tabular}




\begin{tabular}{|c|c|c|c|c|}
\hline York & Yorks & & $\begin{array}{l}\text { Terracotta with } 2 \text { lines of } \\
\text { white zigzags and yellow } \\
\text { top and bottom (Plate } 7)\end{array}$ & BM 946-1418 \\
\hline $\begin{array}{l}\text { near } \\
\text { Earlston }\end{array}$ & $\begin{array}{l}\text { Scotland: } \\
\text { Berwicks }\end{array}$ & & $\begin{array}{l}\text { Terracotta with } 3 \text { waves of } \\
\text { yellow }\end{array}$ & $\begin{array}{l}\text { Edinburgh Museum } \\
\text { FJ } 102\end{array}$ \\
\hline xviii & \multicolumn{4}{|c|}{$\begin{array}{l}\text { TERRACOTTA, MAINLY BUN-SHAPED, BEADS: (a) WITH TWISTED } \\
\text { DECORATION (reticella) AND RELATED } \\
\text { (EXCludiNG GREEN AND yelLOW) (see Map 25) }\end{array}$} \\
\hline Frilford I & Berks & $\begin{array}{l}\text { prob } \\
\text { 6th c }\end{array}$ & $\begin{array}{l}\text { Black background with } \\
\text { terracotta top and bottom } \\
\text { and central band with fine } \\
\text { yellow lines diagonally }\end{array}$ & $\begin{array}{l}\text { Rolleston 1869; } \\
\text { Bradford and Goodchild } \\
1939 ; \\
\text { BM } 672-419\end{array}$ \\
\hline Reading & Berks & & $\begin{array}{l}\text { Terracotta bands top, bottom } \\
\text { and centre, } 2 \text { yellow zones } \\
\text { with blue diagonal lines; } \\
\text { another similar but green } \\
\text { lines and overlaid by terra- } \\
\text { cotta crossed waves }\end{array}$ & $\begin{array}{l}\text { Stevens 1894; } \\
\text { Reading Museum }\end{array}$ \\
\hline Cirencester & Glos & & $\begin{array}{l}\text { Atypical with yellow } \\
\text { ?combing all over }\end{array}$ & $\begin{array}{l}\text { Brown } 1976,25 \text {, } \\
\text { fig 3.2.15; } \\
\text { Cirencester Museum } \\
\text { (ex R Day Collection) }\end{array}$ \\
\hline $\begin{array}{l}\text { Beakes- } \\
\text { bourne II }\end{array}$ & Kent & $\begin{array}{l}\text { early- } \\
\text { late } \\
\text { 6th c }\end{array}$ & $\begin{array}{l}\text { Grave } 24 \text { : terracotta with } \\
\text { yellow lines and buff bands } \\
\text { with black lines }\end{array}$ & $\begin{array}{l}\text { Meaney } 1964,109 ; \\
\text { Canterbury Museum } \\
\text { RM } 7538\end{array}$ \\
\hline Gilton & Kent & $\begin{array}{l}\text { prob } \\
\text { 6th- } \\
\text { 7th c }\end{array}$ & $\begin{array}{l}\text { Slightly biconical, very fine } \\
\text { yellow and black lines }\end{array}$ & $\begin{array}{l}\text { Faussett 1856; } \\
\text { Liverpool Museum } \\
4270\end{array}$ \\
\hline Howletts & Kent & 6th $c$ & $\begin{array}{l}\text { Yellow stripes on terracotta } \\
\text { and yellow central band with } \\
\text { black lines (Plate } 7 \text { ) }\end{array}$ & $\begin{array}{l}\text { Smith 1918; } \\
\text { BM } 19365-11143\end{array}$ \\
\hline Sarre & Kent & $\begin{array}{l}\text { prob } \\
\text { 6th- } \\
\text { 7th c }\end{array}$ & $\begin{array}{l}\text { Terracotta with } \\
\text { (?) chevrons; badly illustrated }\end{array}$ & Brent $1868, \mathrm{pl}$ VIII \\
\hline Stowting & Kent & $\begin{array}{l}\text { prob } \\
\text { 6th c }\end{array}$ & $\begin{array}{l}\text { Grave } 15: 3 \text { zones of } \\
\text { diagonal lines in terracotta } \\
\text { and hardly visible yellow }\end{array}$ & $\begin{array}{l}\text { Brown 1915a (IV), 712-15; } \\
\text { BM OA 368-9 }\end{array}$ \\
\hline $\begin{array}{l}\text { Barton-on- } \\
\text { Humber }\end{array}$ & Lincs & $\begin{array}{l}\text { mid- } \\
\text { 6th c }\end{array}$ & $\begin{array}{l}\text { Grave 115: several with } \\
\text { zigzag covering whole } \\
\text { bead, but with central } \\
\text { terracotta band with annular } \\
\text { brooch pair and Aberg } \\
\text { Group IVb cruciform } \\
\text { brooch; }\end{array}$ & $\begin{array}{l}\text { Drinkall and Foreman } \\
\text { forthcoming; } \\
\text { Scunthorpe Museum }\end{array}$ \\
\hline
\end{tabular}




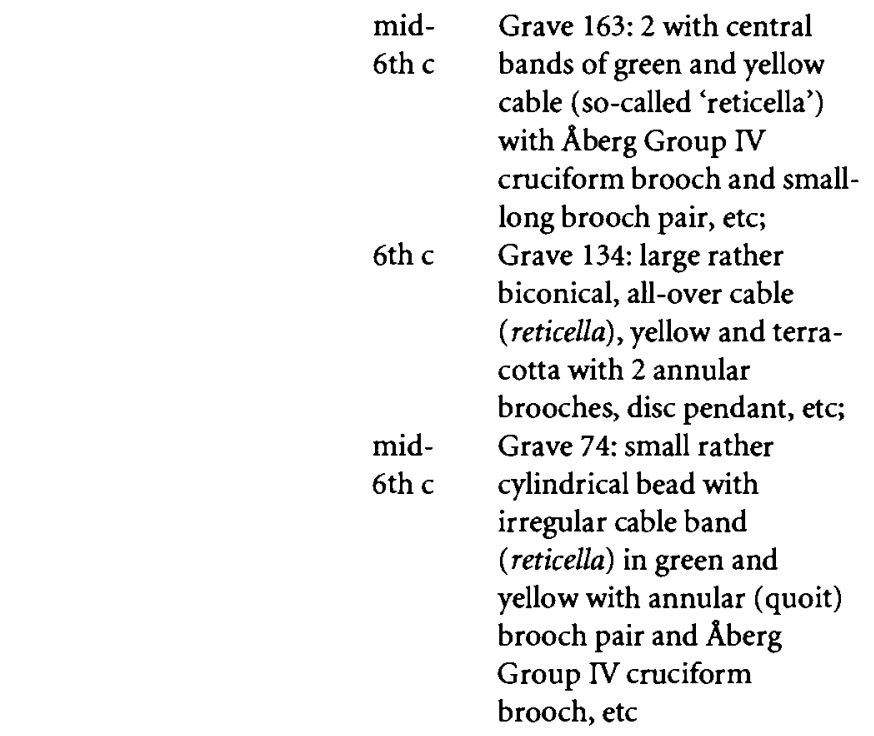

Syston Park Lincs

$\begin{array}{llll}\begin{array}{l}\text { Morning } \\ \text { Thorpe }\end{array} & \text { Norfolk } & \begin{array}{l}\text { prob } \\ \text { 6th c }\end{array} & \begin{array}{l}\text { Grave 299: with annular } \\ \text { brooches, B clasps, etc; } \\ \text { and other graves }\end{array} \\ \text { Spong Hill } \quad \text { Norfolk } & \begin{array}{l}\text { mid- } \\ \text { 6th c }\end{array} & \begin{array}{l}\text { Grave 39: } 1 \text { blue, green and } \\ \text { white (see also green and } \\ \text { yellow), with A berg Group } \\ \text { IVa cruciform brooch, etc }\end{array}\end{array}$

$\begin{array}{lll}\begin{array}{l}\text { Cassington, Oxon } \\ \text { Purwell }\end{array} & \begin{array}{l}\text { prob } \\ \text { 6th c }\end{array} & \begin{array}{l}\text { Atypical, yellow borders, } \\ \text { terracotta core and white and } \\ \text { Farm }\end{array} \\ & & \text { terracotta alternate swags }\end{array}$

Hoxne, Suffolk

Old Newton

Ipswich Suffolk 6th

7 th $\mathrm{c}$

Sutton Hoo Suffolk 7th

West Stow
On a string: biconical with rough yellow and terracotta zones (ta alternate swags

White ground with red and blue fine diagonal lines in 2 zones

Three zones, central band of yellow on terracotta and fine diagonal lines top and bottom; possibly others

Mound 7 surface find: yellow and brown cable (so-called 'reticella')

$$
\text { Cemetery: } 1 \text { biconical }
$$
with 3 zones
Suffolk late 6 th c

\author{
Akerman 1855a, 41-2, \\ pl XXI; \\ BM (18) 11 11-92
}

Green et al 1987, 117-18, fig 393.299.Riv;

Norwich Museum

Hills et al 1984, 90-1, fig 94.5;

Norfolk Arch Unit

Leeds and Riley 1942, 64, pl VIB;

Ashmolean Museum 1942.157

No records;

BM 1912 5-28 36

Layard 1907, pl XXXII, and 1909, pl VII;

Ipswich Museum

Selkirk 1990, 357;

publication in preparation (M Carver); BM

West 1985, 74, fig 276.5; Thetford Museum 
xviii TERRACOTTA, MAINLY BUN-SHAPED, BEADS: (b) WITH GREEN

AND YELLOW TWISTED BANDS (see Map 26)

\begin{tabular}{|c|c|c|c|c|}
\hline Kempston & Beds & $\begin{array}{l}\text { 6th- } \\
7 \text { th c }\end{array}$ & $\begin{array}{l}\text { Grave } 16: 1 \text { with central } \\
\text { line in terracotta and green } \\
\text { and yellow diagonal lines }\end{array}$ & $\begin{array}{l}\text { Fitch 1864; } \\
\text { BM 91 6-24 } 53\end{array}$ \\
\hline Barrington & Cambs & $\begin{array}{l}5 \text { th- } \\
7 \text { th c }\end{array}$ & $\begin{array}{l}\text { Mixed beads: } 2 \text { bands of } \\
\text { green and yellow diagonal } \\
\text { lines and central terracotta } \\
\text { band }\end{array}$ & $\begin{array}{l}\text { Fox 1923, 251-2; } \\
\text { Ashmolean Museum }\end{array}$ \\
\hline $\begin{array}{l}\text { Hasling- } \\
\text { field }\end{array}$ & Cambs & $\begin{array}{l}\text { 6th- } \\
7 \text { th c }\end{array}$ & $\begin{array}{l}\text { Miscellaneous collection } \\
\text { on string: } 3 \text { beads similar } \\
\text { to Barrington above }\end{array}$ & $\begin{array}{l}\text { Fox } 1923,255-9 ; \\
\text { Ashmolean Museum } \\
1909.238\end{array}$ \\
\hline $\begin{array}{l}\text { Linton } \\
\text { Heath B }\end{array}$ & Cambs & $\begin{array}{l}\text { late } \\
\text { 5th- } \\
6 \text { th c }\end{array}$ & $\begin{array}{l}\text { Two with green and yellow } \\
\text { diagonal bands and central } \\
\text { terracotta band, with } 2 \\
\text { small-long brooches and } 1 \\
\text { uncertain brooch }\end{array}$ & $\begin{array}{l}\text { Neville 1854; } \\
\text { Cambridge Museum }\end{array}$ \\
\hline $\begin{array}{l}\text { Little } \\
\text { Wilbraham }\end{array}$ & Cambs & $\begin{array}{l}\text { prob } \\
\text { 6th- } \\
\text { 7th c }\end{array}$ & $\begin{array}{l}\text { Miscellaneous beads: } \\
1 \text { with central herringbone } \\
\text { in green and yellow; } 2 \\
\text { larger with central terracotta } \\
\text { band; other related beads }\end{array}$ & $\begin{array}{l}\text { Neville 1852; } \\
\text { Ashmolean Museum } \\
1909.313\end{array}$ \\
\hline $\begin{array}{l}\text { Little } \\
\text { Chester }\end{array}$ & Derbys & $\begin{array}{l}\text { 6th- } \\
7 \text { th c }\end{array}$ & $\begin{array}{l}\text { Single band yellow and green } \\
\text { central band }\end{array}$ & $\begin{array}{l}\text { Wheeler } 1985,304 \text {; } \\
\text { publication in } \\
\text { preparation } \\
\text { (C Sparey-Green) }\end{array}$ \\
\hline Lechlade & Glos & 6th $c$ & $\begin{array}{l}\text { Grave 180: atypical, with } \\
5 \text { zones, terracotta with } \\
\text { yellow, and yellow with blue } \\
\text { chevrons with amber beads }\end{array}$ & $\begin{array}{l}\text { Boyle et al forthcoming; } \\
\text { Cirencester Museum }\end{array}$ \\
\hline Droxford & Hants & $\begin{array}{l}\text { prob } \\
\text { 6th c }\end{array}$ & $\begin{array}{l}\text { No. } 59: 2 \text { parallel lines in } \\
\text { green and yellow (class V) }\end{array}$ & $\begin{array}{l}\text { Aldsworth } 1978,138 \text { and } \\
172 \text {, fig } 36.59 ; \mathrm{BM}\end{array}$ \\
\hline $\begin{array}{l}\text { Beakes- } \\
\text { bourne }\end{array}$ & Kent & $\begin{array}{l}5 \text { th- } \\
\text { 6th c }\end{array}$ & $\begin{array}{l}\text { Grave 33: crossed green } \\
\text { and yellow bands }\end{array}$ & $\begin{array}{l}\text { Meaney 1964, 109; } \\
\text { Canterbury Museum }\end{array}$ \\
\hline Faversham & Kent & $\begin{array}{l}5 \text { th- } \\
7 \text { th } c\end{array}$ & $\begin{array}{l}\text { Three with } 2 \text { green and } \\
\text { yellow bands (Plate } 7 \text { ); } \\
1 \text { annular with } 1 \text { central } \\
\text { band; } \\
\text { unassociated: yellow and } \\
\text { green bands of diagonal } \\
\text { stripes and terracotta central } \\
\text { band }\end{array}$ & $\begin{array}{l}\text { Meaney 1964, 118-19; } \\
\text { Ashmolean Museum } \\
1909.143 \text { and 144; } \\
\text { Beck Collection, } \\
\text { Cambridge Museum }\end{array}$ \\
\hline 'Kent' & Kent & & $\begin{array}{l}\text { Two beads with central terra- } \\
\text { cotta band and green and } \\
\text { yellow diagonals each side }\end{array}$ & $\begin{array}{l}\text { Canterbury Museum } \\
\text { RM } 2620\end{array}$ \\
\hline
\end{tabular}




\begin{tabular}{|c|c|c|c|c|}
\hline Stodmarsh & Kent & $\begin{array}{l}\text { prob } \\
\text { 6th c }\end{array}$ & $\begin{array}{l}\text { Two bands of green and } \\
\text { yellow }\end{array}$ & $\begin{array}{l}\text { Akerman 1855b, 179-81, } \\
\text { pl I; BM 54 12-2 } 17\end{array}$ \\
\hline $\begin{array}{l}\text { Barton-on- } \\
\text { Humber }\end{array}$ & Lincs & $\begin{array}{l}\text { mid- } \\
\text { 6th } c\end{array}$ & $\begin{array}{l}\text { Grave 163: } 2 \text { small with } \\
\text { cabled (so-called 'reticella') } \\
\text { bands with small-long } \\
\text { brooch pair and Åberg } \\
\text { Group IV cruciform brooch }\end{array}$ & $\begin{array}{l}\text { Drinkall and Foreman } \\
\text { forthcoming; } \\
\text { Scunthorpe Museum }\end{array}$ \\
\hline Fonaby & Lincs & $\begin{array}{l}\text { mid- } \\
\text { 6th } c\end{array}$ & $\begin{array}{l}\text { Grave } 31: 2 \text { bands of green } \\
\text { and yellow, with } \AA \text { berg } \\
\text { Group IV cruciform } \\
\text { brooch; } \\
\text { Grave } 39 \text { : similar; } \\
\text { several others without } \\
\text { grave numbers }\end{array}$ & $\begin{array}{l}\text { Cook } 1981,32 \text { and } 38 \text {, } \\
\text { figs } 10.31 .7,14.39 .7, \\
18.10 \text { and } 19.14 ; \\
\text { Scunthorpe Museum }\end{array}$ \\
\hline Quarrington & Lincs & & $\begin{array}{l}\text { Terracotta with central band } \\
\text { between green and yellow }\end{array}$ & $\begin{array}{l}\text { Bruce } 1880,76 \text {, no. } 303 \text {; } \\
\text { Alnwick Museum }\end{array}$ \\
\hline $\begin{array}{l}\text { Welbeck } \\
\text { Hill }\end{array}$ & Lincs & $\begin{array}{l}5 \text { th- } \\
6 \text { th c }\end{array}$ & $\begin{array}{l}\text { Grave } 64: 2 \text { with central } \\
\text { terracotta band between } \\
\text { yellow and green diagonal } \\
\text { lines }\end{array}$ & $\begin{array}{l}\text { Publication in } \\
\text { preparation (G Taylor); } \\
\text { Scunthorpe Museum }\end{array}$ \\
\hline $\begin{array}{l}\text { Morning } \\
\text { Thorpe }\end{array}$ & Norfolk & $\begin{array}{l}\text { first } \\
\text { half } \\
\text { 6th c } \\
\text { 5th- } \\
\text { 6th c } \\
\text { prob } \\
\text { 6th c } \\
\text { first } \\
\text { half } \\
\text { 6th c } \\
\text { first } \\
\text { half } \\
\text { 6th c }\end{array}$ & $\begin{array}{l}\text { Grave 16: } 4 \text { beads with a } \\
\text { florid cruciform brooch, } \\
\text { small-long brooch pair, } \\
\text { B7 clasps, etc; } \\
\text { Grave } 30: 4 \text { beads with } 1 \\
\text { Aberg Group III and } 2 \\
\text { Group II cruciform } \\
\text { brooches, B13b clasps, } \\
\text { etc; } \\
\text { Grave 90: } 4 \text { beads with } 3 \\
\text { Aberg Group II cruciform } \\
\text { brooches, B18 clasps, etc; } \\
\text { Grave } 231: 2 \text { beads with } \\
\text { small-long brooch pair; } \\
\text { Grave } 304: 3 \text { beads with } \\
\text { penannular brooch and } \\
\text { annular brooch pair; } \\
\text { Grave } 346: 4 \text { beads with } \\
\text { Åberg Group II cruciform } \\
\text { brooch and } 2 \text { small-long } \\
\text { brooches; } \\
\text { Grave } 370: 4 \text { beads with } \\
\text { Aberg Group III cruciform } \\
\text { brooch, small-long brooch } \\
\text { pair, etc }\end{array}$ & $\begin{array}{l}\text { Green } \text { et al } 1987 \text {, } \\
37-8,42-3,60,98 \text {, } \\
119,134 \text { and } 144 \text {, } \\
\text { figs } 298 \text {.Eiv and vii, } \\
\text { 304.Dxxvi and xxvii, } \\
\text { 324.Axxiv, 371.Cviii, } \\
\text { 395.304.Giv and v, } \\
\text { 411.Evii and 430.Eiii; } \\
\text { Norwich Museum }\end{array}$ \\
\hline Spong Hill & Norfolk & $\begin{array}{l}\text { first } \\
\text { half } \\
\text { 6th c }\end{array}$ & $\begin{array}{l}\text { Grave } 46: 1 \text { with } \AA \text { berg } \\
\text { Group II cruciform brooch, } \\
\text { Anglian equal-arm brooch } \\
\text { pair, B clasps, etc; }\end{array}$ & $\begin{array}{l}\text { Hills et al } 1984,100 \\
\text { and } 109, \text { figs } 102.46 .23 \\
\text { and } 106.56 .3 ; \\
\text { Norfolk Arch Unit }\end{array}$ \\
\hline
\end{tabular}


6th c

Grave 56: 3 ( 1 badly made)

with annular brooch pair,

etc

\begin{tabular}{|c|c|c|c|c|}
\hline $\begin{array}{l}\text { Holme } \\
\text { Pierre- } \\
\text { pont }\end{array}$ & Notts & & $\begin{array}{l}\text { Four small beads with central } \\
\text { terracotta band }\end{array}$ & $\begin{array}{l}\text { Smith 1906, 195; } \\
\text { Myres 1977, Corpus } \\
\text { no. 2093, fig 303; } \\
\text { BM } 19313-13\end{array}$ \\
\hline $\begin{array}{l}\text { Bury St } \\
\text { Edmunds, } \\
\text { Westgarth } \\
\text { Gardens }\end{array}$ & Suffolk & 6th $c$ & $\begin{array}{l}\text { Grave } 16: 5 \text { beads with } 2 \\
\text { small-long brooches, } \\
\text { B clasps, etc; } \\
\text { Grave } 44: 1 \text { bead; } \\
\text { Grave } 48: 1 \text { with } 2 \text { small- } \\
\text { long brooches, B clasps, } \\
\text { etc }\end{array}$ & $\begin{array}{l}\text { West } 1988,24,31 \text { and } 32 \text {, } \\
\text { figs } 64 . \text { D4-8, } 72.44 . A 6 \\
\text { and } 73 . E 19 ; \\
\text { Bury St Edmunds } \\
\text { Museum }\end{array}$ \\
\hline Ipswich & Suffolk & $\begin{array}{l}\text { 6th- } \\
7 \text { th c }\end{array}$ & Several examples & $\begin{array}{l}\text { Layard } 1907 \text { and 1909; } \\
\text { Ipswich Museum }\end{array}$ \\
\hline Lakenheath & Suffolk & & $\begin{array}{l}\text { Two beads with single cables } \\
\text { green and yellow }\end{array}$ & $\begin{array}{l}\text { Meaney 1964,230; } \\
\text { BM } 191012-2211\end{array}$ \\
\hline $\begin{array}{l}\text { Mitchell's } \\
\text { Hill }\end{array}$ & Suffolk & & Several & $\begin{array}{l}\text { Meaney 1964, 231; } \\
\text { Bury St Edmunds } \\
\text { Museum }\end{array}$ \\
\hline West Stow & Suffolk & $\begin{array}{l}5 \text { th- } \\
7 \text { th c }\end{array}$ & $\begin{array}{l}\text { Cemetery: crossed bands } \\
\text { of green and yellow }\end{array}$ & $\begin{array}{l}\text { Smith 1852a, 165-7, } \\
\text { pl XXXIX; } \\
\text { West 1985, 74, fig 276.1; } \\
\text { Cambridge Museum }\end{array}$ \\
\hline Alfriston & Sussex & $\begin{array}{l}\text { first } \\
\text { half } \\
\text { 6th c }\end{array}$ & $\begin{array}{l}\text { Grave } 90: 1 \text { wound bead } \\
\text { and } 1 \text { crossed band with a } \\
\text { 6-spiral cast saucer brooch }\end{array}$ & $\begin{array}{l}\text { Griffiths 1915, 205; } \\
\text { Welch 1983, 85-6; } \\
\text { Lewes Museum AJ/90 }\end{array}$ \\
\hline Wasperton & Warks & 6th $c$ & $\begin{array}{l}\text { Biconical with } 2 \text { wide bands } \\
\text { with central terracotta band, } \\
\text { with saucer brooch }\end{array}$ & $\begin{array}{l}\text { Publication in } \\
\text { preparation (M Carver); } \\
\text { Warwick Museum }\end{array}$ \\
\hline $\begin{array}{l}\text { Peters- } \\
\text { finger }\end{array}$ & Wilts & 6th $c$ & $\begin{array}{l}\text { Grave } 29: 2 \text { green and } \\
\text { yellow bands, with } 2 \\
\text { applied saucer brooch } \\
\text { bases, etc }\end{array}$ & $\begin{array}{l}\text { Leeds and Shortt 1953, } \\
\text { 24-5, pl III.104.XXIX; } \\
\text { Salisbury Museum }\end{array}$ \\
\hline $\begin{array}{l}\text { Upton } \\
\text { Snodsbury }\end{array}$ & Worcs & $\begin{array}{l}\text { late } \\
\text { 6th c }\end{array}$ & Small with central band & $\begin{array}{l}\text { Ponting 1867; } \\
\text { Worcester Museum }\end{array}$ \\
\hline
\end{tabular}

xviii Terracotta, MAINLY BUN-SHAPED, BeAds: (c) WITH GREEN AND YELLOW MARBLING (see Map 27)

\begin{tabular}{|c|c|c|}
\hline Barrington & Cambs & $\begin{array}{l}5 \text { th- } \\
7 \text { th c }\end{array}$ \\
\hline
\end{tabular}




\begin{tabular}{|c|c|c|c|c|}
\hline $\begin{array}{l}\text { Little } \\
\text { Wilbraham }\end{array}$ & Cambs & $\begin{array}{l}\text { prob } \\
\text { late } \\
6 \text { th- } \\
7 \text { th c }\end{array}$ & $\begin{array}{l}\text { One marbled above and } \\
\text { below with terracotta } \\
\text { central band (Plate } 7) \text {; smaller } \\
\text { with same yellow and green } \\
\text { marble; } 1 \text { typical with roundels, } \\
\text { with square-headed } \\
\text { brooches, etc }\end{array}$ & $\begin{array}{l}\text { Lethbridge and Carter } 1926 \text {; } \\
\text { Neville 1852; } \\
\text { Cambridge Museum }\end{array}$ \\
\hline $\begin{array}{l}\text { Little } \\
\text { Chester }\end{array}$ & Derbys & $\begin{array}{l}\text { 6th- } \\
7 \text { th c }\end{array}$ & Grave group & $\begin{array}{l}\text { Wheeler } 1985,304 \text {; } \\
\text { publication in } \\
\text { preparation } \\
\text { (C Sparey-Green) }\end{array}$ \\
\hline $\begin{array}{l}\text { Colchester, } \\
\text { Butt Lane }\end{array}$ & Essex & & $\begin{array}{l}\text { Elongated, ?barrel-shaped } \\
\text { central band }\end{array}$ & $\begin{array}{l}\text { Akerman 1852, pl V.5; } \\
\text { Colchester Museum }\end{array}$ \\
\hline Colchester & Essex & & $\begin{array}{l}\text { Large, terracotta central } \\
\text { band }\end{array}$ & $\begin{array}{l}\text { Bought from } \\
\text { Revd J H Pollaxfen; } \\
\text { BM 70 4-2 } 344\end{array}$ \\
\hline $\begin{array}{l}\text { Great } \\
\text { Chesterford }\end{array}$ & Essex & $\begin{array}{l}\text { first } \\
\text { half } \\
6 \text { th c }\end{array}$ & $\begin{array}{l}\text { Grave } 120: 2 \text { very weather- } \\
\text { ed terracotta wave and } \\
\text { weathered roundel on } \\
\text { marbling (D05), with cast } \\
\text { saucer brooch pair; } \\
\text { Grave 127: central band } \\
\text { terracotta and terracotta } \\
\text { wave on all-over marbling } \\
\text { (D43), with disc brooch } \\
\text { pair }\end{array}$ & $\begin{array}{l}\text { Evison } 1994,17,46,108 \text { and } \\
\text { 109, figs } 46.120 .3 \mathrm{c} \text { and } \\
49.127 .5 \mathrm{e} ; \\
\text { BM } 19647-2369 ; \\
\text { BM } 19647-2402\end{array}$ \\
\hline
\end{tabular}

\begin{tabular}{|c|c|c|c|c|}
\hline Mucking II & Essex & 6th $c$ & $\begin{array}{l}\text { Grave 936: globular with } 3 \\
\text { large marbled spots (Plate } 7 \text { ), } \\
\text { with small square-headed } \\
\text { brooches }\end{array}$ & $\begin{array}{l}\text { Hirst and Clark forthcomi } \\
\text { BM }\end{array}$ \\
\hline $\begin{array}{l}\text { Bowcombe } \\
\text { Down }\end{array}$ & $\begin{array}{l}\text { Isle of } \\
\text { Wight }\end{array}$ & $\begin{array}{l}\text { prob } \\
\text { 6th c }\end{array}$ & $\begin{array}{l}\text { Thin annular with badly } \\
\text { made marbling }\end{array}$ & $\begin{array}{l}\text { Arnold } 1982,89-96 ; \\
\text { (formerly in Carisbrooke } \\
\text { Castle Museum) }\end{array}$ \\
\hline Howletts & Kent & & $\begin{array}{l}\text { Small globular with terra- } \\
\text { cotta crossed waves on } \\
\text { a marbled background }\end{array}$ & $\begin{array}{l}\text { Smith 1918; } \\
\text { BM } 1936 \text { 5-11 } 143\end{array}$ \\
\hline Quarrington & Lincs & & $\begin{array}{l}\text { Wide marbled band; } \\
\text { another with roundels } \\
\text { of marbling }\end{array}$ & $\begin{array}{l}\text { Bruce } 1880,76, \text { no. } 303 \text {; } \\
\text { Alnwick Museum }\end{array}$ \\
\hline $\begin{array}{l}\text { South } \\
\text { Willingham }\end{array}$ & Lincs & $\begin{array}{l}\text { 6th- } \\
7 \text { th } c\end{array}$ & $\begin{array}{l}\text { Two with central terracotta } \\
\text { band between marbling }\end{array}$ & $\begin{array}{l}\text { Meaney } 1964,165 \\
\text { BM OA } 5057\end{array}$ \\
\hline Holdenby & Northants & $\begin{array}{l}\text { 6th- } \\
7 \text { th } c\end{array}$ & $\begin{array}{l}\text { Necklace: } 2 \text { beads, terra- } \\
\text { cotta bands between } \\
\text { marbling }\end{array}$ & $\begin{array}{l}\text { Anon 1901; } \\
\text { Leeds } 1909\end{array}$ \\
\hline
\end{tabular}




\begin{tabular}{|c|c|c|c|c|}
\hline $\begin{array}{l}\text { Holme } \\
\text { Pierrepont }\end{array}$ & Notts & $\begin{array}{l}\text { prob } \\
\text { 6th- } \\
7 \text { th c }\end{array}$ & $\begin{array}{l}\text { One cube-shaped with } \\
\text { marbled roundel; } \\
1 \text { small rather drum-shaped } \\
\text { with central terracotta band, } \\
\text { with brooches }\end{array}$ & $\begin{array}{l}\text { Smith 1906, 195; } \\
\text { Myres 1977, Corpus } \\
\text { no. 2093, fig 303; } \\
\text { BM } 1931 \text { 3-13 }\end{array}$ \\
\hline $\begin{array}{l}\text { Bury St } \\
\text { Edmunds, } \\
\text { Westgarth } \\
\text { Gardens }\end{array}$ & Suffolk & $\begin{array}{l}\text { prob } \\
\text { 6th } c\end{array}$ & $\begin{array}{l}\text { Grave 14: small marbled; } \\
\text { Grave 44: with roundel }\end{array}$ & $\begin{array}{l}\text { West 1988, } 24 \text { and } 31 \text {, } \\
\text { figs } 61.14 . A 4 \text { and } 72.44 . A 5 ; \\
\text { Bury St Edmunds } \\
\text { Museum }\end{array}$ \\
\hline Hoxne & Suffolk & $\begin{array}{l}\text { prob } \\
\text { 6th } c\end{array}$ & $\begin{array}{l}\text { One with marbled roundels } \\
\text { and } 1 \text { globular marbled }\end{array}$ & $\begin{array}{l}\text { Meaney 1964, 228; } \\
\text { BM } 19125-2836\end{array}$ \\
\hline $\begin{array}{l}\text { Mitchell's } \\
\text { Hill }\end{array}$ & Suffolk & $\begin{array}{l}\text { prob } \\
\text { 6th } c\end{array}$ & $\begin{array}{l}\text { One with marbled roundels } \\
\text { and } 1 \text { ditto, but smaller }\end{array}$ & $\begin{array}{l}\text { Meaney 1964, 231; } \\
\text { Bury St Edmunds } \\
\text { Museum }\end{array}$ \\
\hline Alfriston & Sussex & $\begin{array}{l}\text { first } \\
\text { half } \\
\text { 6th } c\end{array}$ & $\begin{array}{l}\text { Grave } 43 \text { : with other beads } \\
\text { and with great square- } \\
\text { headed brooch, etc }\end{array}$ & $\begin{array}{l}\text { Griffiths and Salzmann } \\
\text { 1914, 39-41, pl I.9; } \\
\text { Welch 1983, 85-6, } \\
\text { fig 21a; Lewes Museum }\end{array}$ \\
\hline $\begin{array}{l}\text { Harnham } \\
\text { Hill }\end{array}$ & Wilts & $\begin{array}{l}\text { prob } \\
\text { 6th- } \\
\text { 7th c }\end{array}$ & $\begin{array}{l}\text { Two beads with central terra- } \\
\text { cotta band and } 1 \text { with } \\
\text { roundels of marbling }\end{array}$ & $\begin{array}{l}\text { Akerman } 1853 \\
\text { Jackson } 1854 \\
\text { BM }\end{array}$ \\
\hline $\begin{array}{l}\text { Pewsey } \\
\text { Blackpatch }\end{array}$ & Wilts & 6th c & $\begin{array}{l}\text { Grave 53: terracotta with } \\
\text { all-over marbling in yellow } \\
\text { and green }\end{array}$ & $\begin{array}{l}\text { Publication in } \\
\text { preparation (K Annable); } \\
\text { Devizes Museum }\end{array}$ \\
\hline
\end{tabular}

xix TerracotTa CYLINDER BEADS, ROUND, SQUARE OR POLYGonal SECTIONS: (a) WITH GREEN AND YELLOW BANDS, PANELS, ETC, WITH VARIOUS MOTIFS (see Map 28)

\begin{tabular}{|c|c|c|c|c|}
\hline Barrington & Cambs & $\begin{array}{l}5 \text { th- } \\
7 \text { th c }\end{array}$ & $\begin{array}{l}\text { Mixed beads: } 1 \text { round with } \\
\text { wound stripes, } 1 \text { square- } \\
\text { sectioned with herringbone } \\
\text { panels }\end{array}$ & $\begin{array}{l}\text { Fox 1923, 251-2; } \\
\text { Ashmolean Museum }\end{array}$ \\
\hline $\begin{array}{l}\text { Hasling- } \\
\text { field }\end{array}$ & Cambs & & $\begin{array}{l}\text { Miscellaneous beads on } \\
\text { string: } 1 \text { with wound bands } \\
\text { of cabling (Plate } 7 \text { ), } 1 \text { central } \\
\text { panel, } 1 \text { very small with } \\
\text { central terracotta band }\end{array}$ & $\begin{array}{l}\text { Fox } 1923,255-9 ; \\
\text { Ashmolean Museum }\end{array}$ \\
\hline $\begin{array}{l}\text { Little } \\
\text { Wilbraham }\end{array}$ & Cambs & $\begin{array}{l}5 \text { th- } \\
7 \text { th c }\end{array}$ & $\begin{array}{l}\text { Unassociated group: } \\
2 \text { square-sectioned with } \\
\text { central terracotta panel with } \\
\text { herringbone in green and } \\
\text { yellow, } 1 \text { square-sectioned } \\
\text { with green and yellow } \\
\text { border, } 3 \text { round-sectioned } \\
\text { with irregular green and } \\
\text { yellow banded decoration }\end{array}$ & $\begin{array}{l}\text { Neville } 1852 \text { (with } \\
\text { coloured illustrations); } \\
\text { Cambridge Museum }\end{array}$ \\
\hline
\end{tabular}




$\begin{array}{lllll}\begin{array}{l}\text { Great } \\ \text { Chester- }\end{array} & \text { Essex } & \begin{array}{l}\text { 2nd } \\ \text { ford }\end{array} & \begin{array}{l}\text { Grave 113:3 with wound } \\ \text { bands in green and yellow }\end{array} & \begin{array}{l}\text { Evison 1994, 17, 46, } \\ \text { 106, fig 43.113.3b; } \\ \text { 5th c }\end{array} \\ & & \begin{array}{l}\text { (D09) with applied saucer } \\ \text { brooch pair (floriate/anchor } \\ \text { cross design) }\end{array} & \text { BM 1964 7-2 342 }\end{array}$

\begin{tabular}{|c|c|c|c|c|}
\hline Mucking II & Essex & 6th $c$ & $\begin{array}{l}\text { Grave 552: square- } \\
\text { sectioned with lozenge- } \\
\text { shaped Frankish brooches }\end{array}$ & $\begin{array}{l}\text { Hirst and Clark forthce } \\
\text { BM }\end{array}$ \\
\hline $\begin{array}{l}\text { Springfield } \\
\text { Lyons }\end{array}$ & Essex & $\begin{array}{l}\text { prob } \\
7 \text { th } c\end{array}$ & $\begin{array}{l}\text { Late pit cutting with a } \\
\text { grave (context } 7350 \text { ): } \\
\text { find } 3924\end{array}$ & $\begin{array}{l}\text { Publication in } \\
\text { preparation (S Tyler); } \\
\text { BM }\end{array}$ \\
\hline $\begin{array}{l}\text { Chessell } \\
\text { Down }\end{array}$ & $\begin{array}{l}\text { Isle of } \\
\text { Wight }\end{array}$ & $\begin{array}{l}\text { prob } \\
6 \text { th } c\end{array}$ & $\begin{array}{l}\text { Several round with bands } \\
\text { of yellow and green (types } \\
8.1,8.3 \text { and } 8.4 \text { ) }\end{array}$ & $\begin{array}{l}\text { Arnold } 1982,50,121 \text {, } \\
\text { colour frontispiece }\end{array}$ \\
\hline $\begin{array}{l}\text { Beakes- } \\
\text { bourne II }\end{array}$ & Kent & $\begin{array}{l}\text { prob } \\
\text { 6th c }\end{array}$ & $\begin{array}{l}\text { Grave 33: round with } \\
\text { yellow and green stripes }\end{array}$ & $\begin{array}{l}\text { Meaney } 1964,109 ; \\
\text { Canterbury Museum }\end{array}$ \\
\hline Faversham & Kent & $\begin{array}{l}\text { 5th- } \\
\text { 7th c }\end{array}$ & $\begin{array}{l}\text { Three round with green and } \\
\text { yellow striped bands, } \\
2 \text { square }\end{array}$ & $\begin{array}{l}\text { Meaney 1964, 118-19; } \\
\text { Ashmolean Museum } \\
1909.143\end{array}$ \\
\hline Howletts & Kent & 6th $c$ & $\begin{array}{l}\text { One square-sectioned with } \\
\text { irregular green and yellow } \\
\text { design, } 1 \text { very small with } \\
\text { terracotta showing at one } \\
\text { end, green and yellow stripes }\end{array}$ & $\begin{array}{l}\text { Smith 1918; } \\
\text { BM } 19365-11143 \\
\text { and } 144\end{array}$ \\
\hline $\begin{array}{l}\text { Barton-on- } \\
\text { Humber }\end{array}$ & Lincs & $\begin{array}{l}\text { mid- } \\
\text { 6th c }\end{array}$ & $\begin{array}{l}\text { Grave } 115: 2 \text { zones of } \\
\text { green and yellow twist with } \\
\text { annular brooch pair and } \\
\text { Aberg Group IVb cruciform } \\
\text { brooch, etc; } \\
\text { Grave 74: with annular } \\
\text { (quoit) brooch pair and } \\
\text { Aberg Group IV cruciform } \\
\text { brooch, etc }\end{array}$ & $\begin{array}{l}\text { Drinkall and Foreman } \\
\text { forthcoming; } \\
\text { Scunthorpe Museum }\end{array}$ \\
\hline
\end{tabular}

Fonaby Lincs $\quad 6$ th c

Grave 25: rather pear-

Cook 1981, 28, 30 and 48, shaped with central striped band with girdle hangers;

5th- Grave 28: 2 ditto with

figs 9.1.7, 10.28.3.3,

19.15 and 16, pl VIIB;

Scunthorpe Museum

6 th c small-long brooch, etc;

unstratified: 2 with

central striped band

$\begin{array}{llll}\begin{array}{l}\text { Welbeck } \\ \text { Hill }\end{array} & \text { Lincs } & \begin{array}{l}\text { 5th- } \\ \text { 6th c }\end{array} & \begin{array}{l}\text { Grave 64: } 2 \text { diagonal bands } \\ \text { of green and yellow stripes, } \\ \text { with 2 cruciform brooches }\end{array} \\ \text { Castle Acre } & \text { Norfolk } & & \begin{array}{l}\text { Several examples not seen } \\ \text { by author }\end{array} \\ \text { Illington } & \text { Norfolk } & \begin{array}{l}\text { prob } \\ \text { 6th c }\end{array} & \begin{array}{l}\text { Cremation 5: panelled or } \\ \text { striped, square section } \\ \text { with stamped urn }\end{array}\end{array}$

Publication in preparation ( $G$ Taylor); Scunthorpe Museum

Meaney 1964, 172-3;

Norwich Museum

Davison et al 1993, 22, fig 47.5a;

Norwich Museum 


\begin{tabular}{|c|c|c|c|c|}
\hline $\begin{array}{l}\text { Morning } \\
\text { Thorpe }\end{array}$ & Norfolk & $\begin{array}{l}\text { prob } \\
\text { 6th c } \\
\text { mid- } \\
\text { 6th c }\end{array}$ & $\begin{array}{l}\text { Very numerous and varied, } \\
\text { for example: } \\
\text { Grave } 362: 2 \text { beads with } \\
\text { Aberg Group II or III } \\
\text { cruciform brooch, etc; } \\
\text { Grave } 96: 1 \text { with Åberg } \\
\text { Group IV cruciform brooch, } \\
\text { etc; some others with blue } \\
\text { glass as well }\end{array}$ & $\begin{array}{l}\text { Green et al } 1987,140-2, \\
63 \text {, figs } 424 . \mathrm{Lix} \text { and } 327 . \mathrm{Hv} \\
\text { Norwich Museum }\end{array}$ \\
\hline Spong Hill & Norfolk & $\begin{array}{l}\text { prob } \\
\text { 6th c } \\
\text { 6th c }\end{array}$ & $\begin{array}{l}\text { Grave } 56: 3 \text { with central } \\
\text { band with annular brooch } \\
\text { pair, etc; } \\
\text { Grave } 18: 2 \text { striped green } \\
\text { and yellow, } 1 \text { with panels } \\
\text { with great square-headed } \\
\text { brooch, etc }\end{array}$ & $\begin{array}{l}\text { Hills et al } 1984,109,67 \text {, } \\
\text { figs } 106.3 \text { and } 77.18 .4 \\
\text { Norfolk Arch Unit }\end{array}$ \\
\hline $\begin{array}{l}\text { Holme } \\
\text { Pierre- } \\
\text { pont }\end{array}$ & Notts & $\begin{array}{l}\text { prob } \\
\text { 6th- } \\
7 \text { th c }\end{array}$ & $\begin{array}{l}\text { Cube-shaped with round } \\
\text { panels: dated by brooches, } \\
\text { etc }\end{array}$ & $\begin{array}{l}\text { Meaney 1964, 200; } \\
\text { BM 1931 3-13 }\end{array}$ \\
\hline $\begin{array}{l}\text { Bury St } \\
\text { Edmunds, } \\
\text { Westgarth } \\
\text { Gardens }\end{array}$ & Suffolk & $\begin{array}{l}\text { first } \\
\text { half } \\
6 \text { th c } \\
6 \text { th c } \\
\text { prob } \\
\text { 6th c }\end{array}$ & $\begin{array}{l}\text { Grave } 61: 2 \text { round smallish } \\
\text { with stripes with Aberg } \\
\text { Group III cruciform } \\
\text { brooch, etc; } \\
\text { Grave } 16: 3 \text { larger with } \\
\text { wound stripes with small- } \\
\text { long brooch pair, B clasps; } \\
\text { Grave } 44: 1 \text { with roughly } \\
\text { wound stripes }\end{array}$ & $\begin{array}{l}\text { West 1988, 36, 24, 31, } \\
\text { figs 81.61.I.1-2, } \\
\text { 64.D4-6, 72.44.5; } \\
\text { Bury St Edmunds } \\
\text { Museum }\end{array}$ \\
\hline $\begin{array}{l}\text { Holywell } \\
\text { Row }\end{array}$ & Suffolk & $\begin{array}{l}\text { 6th- } \\
7 \text { th c } \\
\text { prob } \\
\text { 6th } c\end{array}$ & $\begin{array}{l}\text { Eight square-sectioned with } \\
\text { bands of green and yellow } \\
\text { cabling; } \\
2 \text { examples banded in } \\
\text { terracotta, yellow and green }\end{array}$ & $\begin{array}{l}\text { Lethbridge 1931; } \\
\text { Cambridge Museum }\end{array}$ \\
\hline Lakenheath & Suffolk & $\begin{array}{l}5 \text { th- } \\
7 \text { th c }\end{array}$ & $\begin{array}{l}\text { Square-section with terra- } \\
\text { cotta panel, (?) striped or } \\
\text { marbled }\end{array}$ & $\begin{array}{l}\text { Meaney 1964,230; } \\
\text { BM } 191012-229 \text { and } 11\end{array}$ \\
\hline $\begin{array}{l}\text { Mitchell's } \\
\text { Hill }\end{array}$ & Suffolk & $\begin{array}{l}5 \text { th- } \\
7 \text { th c }\end{array}$ & $\begin{array}{l}\text { One large round with cabling } \\
\text { around and another with } \\
\text { cable overlying }\end{array}$ & $\begin{array}{l}\text { Meaney 1964, 231; } \\
\text { Bury St Edmunds } \\
\text { Museum }\end{array}$ \\
\hline Snape & Suffolk & 6th $c$ & $\begin{array}{l}\text { Grave 357: several with } \\
\text { cruciform brooch; } \\
\text { Grave } 0421: 6 \text { or } 7 \text { round } \\
\text { or square section with } \\
\text { cabled stripes }\end{array}$ & $\begin{array}{l}\text { Filmer-Sankey 1990, 350; } \\
\text { Filmer-Sankey and } \\
\text { Pestell forthcoming }\end{array}$ \\
\hline $\begin{array}{l}\text { West } \\
\text { Stow }\end{array}$ & Suffolk & $\begin{array}{l}5 \text { th- } \\
7 \text { th } c\end{array}$ & $\begin{array}{l}\text { Cemetery: } 1 \text { large round } \\
\text { with stripes all over it; } \\
1 \text { square-sectioned ditto } \\
\text { bead; } \\
\text { Settlement: } 1 \text { round with } \\
\text { yellow and green band }\end{array}$ & $\begin{array}{l}\text { West } 1985,73-4 \text {, } \\
\text { fig } 276.2,3 \text { and } 4 \text {; } \\
\text { Ashmolean Museum; } \\
\text { Bury St Edmunds } \\
\text { Museum }\end{array}$ \\
\hline
\end{tabular}




$\begin{array}{lllll}\begin{array}{l}\text { Churchover, } \\ \text { Bensford } \\ \text { Bridge }\end{array} & \text { Warks } & \text { 6th c } & \begin{array}{l}\text { One with yellow and green } \\ \text { striped bands }\end{array} & \begin{array}{l}\text { Bloxam 1884; } \\ \text { Warwick Museum }\end{array} \\ \begin{array}{l}\text { Stretton- } \\ \text { on- } \\ \text { Fosse }\end{array} & \text { Warks } & \text { 6th c } & \begin{array}{l}\text { Two square-sectioned with } \\ \text { striped bands }\end{array} & \begin{array}{l}\text { Gelling 1992, 31, 40-1, } \\ \text { fig 21; } \\ \text { Wasperton }\end{array} \\ \text { Warwick Museum }\end{array}$

\section{xix Terracotta Cylinder beads, Round, SQUare or POlygonal SECTIONS: (b) WITH GREEN AND YELLOW MARBLING (see Map 28)}

\begin{tabular}{|c|c|c|c|c|}
\hline $\begin{array}{l}\text { Newport } \\
\text { Pagnell }\end{array}$ & Bucks & $\begin{array}{l}\text { prob } \\
\text { 6th c }\end{array}$ & Short cylinder, marbled & $\begin{array}{l}\text { Meaney 1964,58; } \\
\text { Aylesbury Museum, } \\
\text { site record CAS } 1373\end{array}$ \\
\hline $\begin{array}{l}\text { Linton } \\
\text { Heath B }\end{array}$ & Cambs & $\begin{array}{l}\text { 5th- } \\
\text { 6th } c\end{array}$ & $\begin{array}{l}\text { One small with marbled } \\
\text { bands, with } 2 \text { small-long } \\
\text { brooches }\end{array}$ & $\begin{array}{l}\text { Neville 1854; } \\
\text { Cambridge Museum }\end{array}$ \\
\hline $\begin{array}{l}\text { Colchester, } \\
\text { Bull Lane }\end{array}$ & Essex & & $\begin{array}{l}\text { No details, found } 1847 \text { : } \\
\text { band of terracotta round } \\
\text { centre }\end{array}$ & Akerman 1852 , pl V.5 \\
\hline $\begin{array}{l}\text { Great } \\
\text { Chesterford }\end{array}$ & Essex & $\begin{array}{l}5 \text { th- } \\
\text { 6th c } \\
6 \text { th c }\end{array}$ & $\begin{array}{l}\text { Grave 120: short cylinder, } \\
\text { central band terracotta } \\
\text { (D05) (Plate 7) with cast } \\
\text { saucer brooch pair; } \\
\text { Grave 34: ditto - very } \\
\text { small with marbling and } \\
\text { terracotta crossed waves } \\
\text { (D08); } \\
\text { Grave 127: marbled with } \\
\text { terracotta crossed wave and } \\
\text { blue dot (D43) with disc } \\
\text { brooch pair; } \\
\text { Grave 62: small with terra- } \\
\text { cotta crossed wave or } \\
\text { marbling (D24) with small } \\
\text { square-headed brooch pair }\end{array}$ & $\begin{array}{l}\text { Evison } 1994,17-18,46 \text {, } \\
108,109 \text { and } 101 \text {, } \\
\text { figs } 46.120 .3 \mathrm{c} \text {, } \\
27.34 .2 \mathrm{~b}, 49.127 .5 \mathrm{e} \\
\text { and } 33.62 .4 \mathrm{r} \text {; } \\
\text { BM } 19647-2369 \text {; } \\
\text { BM } 19647-2402 \text {; } \\
\text { BM } 19647-2235\end{array}$ \\
\hline
\end{tabular}




\begin{tabular}{|c|c|c|c|c|}
\hline $\begin{array}{l}\text { Bowcombe } \\
\text { Down }\end{array}$ & $\begin{array}{l}\text { Isle of } \\
\text { Wight }\end{array}$ & 6th $c$ & Flat cylinder with marbling & $\begin{array}{l}\text { Arnold } 1982,89-96 ; \\
\text { old drawing from } \\
\text { Carisbrooke Castle }\end{array}$ \\
\hline Howletts & Kent & 6th $c$ & $\begin{array}{l}\text { Roughly pentagonal with } \\
\text { terracotta band and marbling }\end{array}$ & $\begin{array}{l}\text { Smith 1918; } \\
\text { BM 1936 5-11 } 143\end{array}$ \\
\hline $\begin{array}{l}\text { Syston } \\
\text { Park }\end{array}$ & Lincs & $\begin{array}{l}\text { 6th- } \\
7 \text { th } c\end{array}$ & $\begin{array}{l}\text { String, unassociated: } \\
\text { marbled with terracotta } \\
\text { central band }\end{array}$ & $\begin{array}{l}\text { Akerman I855a, 41-2, } \\
\text { pl XXI.2; } \\
\text { BM (18) } 1111-92\end{array}$ \\
\hline Kenninghall & Norfolk & $\begin{array}{l}\text { 6th- } \\
\text { early } \\
7 \text { th c }\end{array}$ & $\begin{array}{l}\text { Very small, central band } \\
\text { marbled }\end{array}$ & $\begin{array}{l}\text { Manning } 1872 a \text { and } \\
1872 b ; \\
\text { BM } 837-231\end{array}$ \\
\hline Northwold & Norfolk & & $\begin{array}{l}\text { One round and } 1 \text { square- } \\
\text { sectioned }\end{array}$ & $\begin{array}{l}\text { Akerman } 1852,50 \\
\text { pl V.1-4; now lost }\end{array}$ \\
\hline Spong Hill & Norfolk & $\begin{array}{l}5 \text { th- } \\
\text { 6th } c\end{array}$ & $\begin{array}{l}\text { Several with uncertain } \\
\text { bands; } \\
\text { Grave } 5 \text { : cuboid with } \\
\text { marbled panels with small- } \\
\text { long brooch pair, A clasps }\end{array}$ & $\begin{array}{l}\text { Hills et al 1984, 54-5, } \\
\text { fig } 73.3 \mathrm{~b} \text {; } \\
\text { Norfolk Arch Unit }\end{array}$ \\
\hline Duston & Northants & $\begin{array}{l}\text { prob } \\
6 \text { th- } \\
7 \text { th c }\end{array}$ & $\begin{array}{l}\text { One example and possibly } \\
\text { another }\end{array}$ & $\begin{array}{l}\text { George 1903; } \\
\text { Northampton Museum }\end{array}$ \\
\hline $\begin{array}{l}\text { Empingham } \\
\text { II }\end{array}$ & Rutland & $\begin{array}{l}5 \text { th- } \\
7 \text { th c }\end{array}$ & $\begin{array}{l}\text { Grave 16: mixed green and } \\
\text { yellow zones with } \\
\text { terracotta body; } \\
\text { others not drawn }\end{array}$ & $\begin{array}{l}\text { Timby 1996; } \\
\text { Oakham Museum }\end{array}$ \\
\hline $\begin{array}{l}\text { Bury St } \\
\text { Edmunds, } \\
\text { Westgarth } \\
\text { Gardens }\end{array}$ & Suffolk & 6th $c$ & $\begin{array}{l}\text { Grave } 44: 1 \text { round with } \\
\text { marbled bands; } \\
\text { Grave } 61: 2 \text { with marbled } \\
\text { bands with } \AA \text { berg Group } \\
\text { III cruciform brooch, etc }\end{array}$ & $\begin{array}{l}\text { West } 1988,31 \text { and } 36 ; \\
\text { figs } 72.44 . \text { A5 } \\
\text { and } 81.62 .1 .1-2 ; \\
\text { Bury St Edmunds } \\
\text { Museum }\end{array}$ \\
\hline $\begin{array}{l}\text { Holywell } \\
\text { Row }\end{array}$ & Suffolk & $\begin{array}{l}\text { prob } \\
\text { 6th } \mathrm{c}\end{array}$ & Round with marbled band & $\begin{array}{l}\text { Lethbridge 1931; } \\
\text { Cambridge Museum }\end{array}$ \\
\hline Lakenheath & Suffolk & $\begin{array}{l}\text { prob } \\
\text { 6th- } \\
7 \text { th c }\end{array}$ & $\begin{array}{l}\text { Atypical squarish with } \\
\text { diamond-shaped panel }\end{array}$ & $\begin{array}{l}\text { Meaney 1964, 230; } \\
\text { BM } 191012-22 \text { 9-11 }\end{array}$ \\
\hline $\begin{array}{l}\text { Stretton- } \\
\text { on- } \\
\text { Fosse }\end{array}$ & Warks & $\begin{array}{l}\text { prob } \\
6 \text { th c }\end{array}$ & One square section & $\begin{array}{l}\text { Gelling 192, 31, 40-1, } \\
\text { fig 21; } \\
\text { Warwick Museum }\end{array}$ \\
\hline $\begin{array}{l}\text { Peters- } \\
\text { finger }\end{array}$ & Wilts & $\begin{array}{l}5 \text { th- } \\
\text { 6th c }\end{array}$ & $\begin{array}{l}\text { Grave 29: a messy pattern, } \\
\text { with } 2 \text { applied brooch bases }\end{array}$ & $\begin{array}{l}\text { Leeds and Shortt 1953, } \\
\text { 24-5, pl III.104.XXIX; } \\
\text { Salisbury Museum }\end{array}$ \\
\hline
\end{tabular}




\section{Schedule 9}

\section{Opaque orange glass BeAds}

Shapes mostly barRel AND CONICAL (see Map 29)

\begin{tabular}{|c|c|c|c|c|}
\hline $\begin{array}{l}\text { Hasling- } \\
\text { field }\end{array}$ & Cambs & $\begin{array}{l}\text { prob } \\
\text { 6th- } \\
\text { 7th c }\end{array}$ & Short cylinder & $\begin{array}{l}\text { Meaney } 1964,66-7 \\
\text { many museums }\end{array}$ \\
\hline $\begin{array}{l}\text { Norton- } \\
\text { on-Tees }\end{array}$ & Durham & $\begin{array}{l}\text { mid- } \\
\text { 6th } c\end{array}$ & $\begin{array}{l}\text { Grave } 63: 1 \text { with } \AA \text { berg } \\
\text { Group IVa cruciform } \\
\text { brooch, etc }\end{array}$ & $\begin{array}{l}\text { Sherlock and Welch } 1992 \text {, } \\
165 \text {, fig } 52.63 .1\end{array}$ \\
\hline Mucking II & Essex & $\begin{array}{l}\text { prob } \\
7 \text { th c }\end{array}$ & $\begin{array}{l}\text { Graves } 608 \text { and } 621: 6 \\
\text { barrel beads }\end{array}$ & $\begin{array}{l}\text { Hirst and Clark } \\
\text { forthcoming }\end{array}$ \\
\hline $\begin{array}{l}\text { Dover, } \\
\text { Buckland }\end{array}$ & Kent & $\begin{array}{l}575- \\
625 \\
625- \\
650 \\
650- \\
675\end{array}$ & $\begin{array}{l}\text { Grave 1: } 1 \text { squat } \\
\text { barrel-shaped; } \\
\text { Grave 18: } 2 \text { beads; } \\
\text { Grave 55: } 1 \text { bead; } \\
\text { Grave 53: } 4 \text { beads; } \\
\text { Grave 67: } 4 \text { beads; } \\
\text { Grave 132: } 2 \text { beads; } \\
\text { Grave 133: } 5 \text { beads; } \\
\text { Grave 134: } 2 \text { beads; } \\
\text { Grave 157: } 2 \text { beads }\end{array}$ & $\begin{array}{l}\text { Evison 1987, 61-2, 72, } \\
79-80 \text { (types B10-11), } \\
\text { figs 5.4f, 11.18.2c, } \\
30.1 \mathrm{e}, 28.53 .1 \mathrm{~b}, \\
37.67 .1 \mathrm{f}, 54.132 .2 \mathrm{~b}, \\
55.133 .1 \mathrm{~b} \text { and } 2 \mathrm{~b}, \\
55.134 .2 \mathrm{e}, 61.157 .1 \mathrm{e} \\
\text { BM }\end{array}$ \\
\hline Faversham & Kent & $\begin{array}{l}5 \text { th- } \\
7 \text { th } c\end{array}$ & $\begin{array}{l}\text { Mixed beads: } 4 \text { or } 5 \\
\text { examples }\end{array}$ & $\begin{array}{l}\text { Meaney 1964, 118-19; } \\
\text { Pitt-Rivers Collection, } \\
\text { Salisbury Museum } \\
\text { 3D2A1 }\end{array}$ \\
\hline Kingston & Kent & 7 th $c$ & $\begin{array}{l}\text { Grave } 241 \text { : biconical } \\
\text { and globular }\end{array}$ & $\begin{array}{l}\text { Faussett 1856, } 83 \\
\text { Liverpool Museum }\end{array}$ \\
\hline $\begin{array}{l}\text { Milton } \\
\text { Regis }\end{array}$ & Kent & 7th $\mathrm{c}$ & $\begin{array}{l}\text { Unstratified string: } \\
2 \text { examples with } \\
\text { short cylinder }\end{array}$ & $\begin{array}{l}\text { Hawkes and Grove } 1963 \text {, } \\
32 \text {, fig } 2 \text {, no. } 4\end{array}$ \\
\hline Monkton & Kent & $\begin{array}{l}\text { mid- } \\
7 \text { th } c\end{array}$ & $\begin{array}{l}\text { Grave } 3: 2 \text { 'barrels' } \\
\text { with composite disc } \\
\text { brooch, etc }\end{array}$ & $\begin{array}{l}\text { Hawkes and Hogarth 1974, } \\
59 \text {, fig 5.c and d }\end{array}$ \\
\hline Polhill & Kent & 7th $\mathrm{c}$ & $\begin{array}{l}\text { Grave } 38 \text { : large barrel- } \\
\text { shaped (Plate } 7 \text { ) with pin, } \\
2 \text { finger-rings and knife }\end{array}$ & $\begin{array}{l}\text { Hawkes and Philp 1973, } \\
176,192-3 \text { and } 207 \text {, } \\
\text { fig } 55.519\end{array}$ \\
\hline
\end{tabular}




\begin{tabular}{|c|c|c|c|c|}
\hline Sarre & Kent & 7th $\mathrm{c}$ & $\begin{array}{l}\text { Necklace with amethyst } \\
\text { beads and gold coin } \\
\text { pendants, etc: at least } 3\end{array}$ & $\begin{array}{l}\text { Smith 1860, pl II; } \\
\text { Brown 1915a (III), } \\
\text { opp 353, colour pl B.I }\end{array}$ \\
\hline $\begin{array}{l}\text { Sibertswold } \\
\text { or } \\
\text { Barfreston }\end{array}$ & Kent & $\begin{array}{l}6 \text { th- } \\
7 \text { th } c\end{array}$ & $\begin{array}{l}\text { About } 8 \text { examples: } \\
\text { barrel, globular, etc }\end{array}$ & $\begin{array}{l}\text { Faussett } 1856, \mathrm{pl} \mathrm{V;} \\
\text { Liverpool Museum }\end{array}$ \\
\hline Corbridge & $\begin{array}{l}\text { North- } \\
\text { umber- } \\
\text { land }\end{array}$ & $\begin{array}{l}5 \text { th- } \\
6 \text { th c }\end{array}$ & $\begin{array}{l}\text { Necklace: illustration } \\
\text { unclear, with } 2 \text { Åberg } \\
\text { Group II cruciform } \\
\text { brooches }\end{array}$ & $\begin{array}{l}\text { Knowles and Forster } \\
1909,407 ; \\
\text { Corbridge Museum }\end{array}$ \\
\hline West Stow & Suffolk & $\begin{array}{l}5 \text { th- } \\
7 \text { th } c\end{array}$ & $\begin{array}{l}\text { Cemetery: } 3 \text { barrels } \\
\text { (C8, O1, T2); } \\
\text { Settlement (SFB 43): } \\
1 \text { biconical (SF3006) }\end{array}$ & $\begin{array}{l}\text { West } 1985,37 \text { and } 73-4 \text {, } \\
\text { figs } 275.23 \text { and } 147.3 \text {; } \\
\text { Cambridge Museum; } \\
\text { Ashmolean Museum } \\
\text { 1909.419a; } \\
\text { Thetford Museum }\end{array}$ \\
\hline $\begin{array}{l}\text { Apple } \\
\text { Down I }\end{array}$ & Sussex & 7th $\mathrm{c}$ & $\begin{array}{l}\text { Grave } 151: 1 \text { barrel } \\
\text { with pin and knife }\end{array}$ & $\begin{array}{l}\text { Down and Welch } 1990,54 \\
\text { and } 164 \text {, fig } 2.46 .151 .1, \\
\text { pl 41.24; } \\
\text { Chichester Museum }\end{array}$ \\
\hline 'Sussex’ & Sussex & & Necklace of 6 beads & $\begin{array}{l}\text { Welch } 1983,85 \text {, fig } 47 c \text {; } \\
\text { Lewes Museum }\end{array}$ \\
\hline $\begin{array}{l}\text { York, } \\
\text { Coppergate }\end{array}$ & Yorks & & Several & Personal information \\
\hline
\end{tabular}




\section{SCHEDUle 10}

\section{MILLEFIORI AND MOSAIC \\ GLASS BEADS}

Globular and Cylindrical (see Plate 8 and Map 30)

(a) MILLEFIORI BEADS

\begin{tabular}{|c|c|c|c|c|}
\hline $\begin{array}{l}\text { Chamber- } \\
\text { lain's } \\
\text { Barn II }\end{array}$ & Beds & $\begin{array}{l}\text { mid- } \\
\text { late } \\
7 \text { th c }\end{array}$ & $\begin{array}{l}\text { Grave 32: globular } \\
\text { with red and white eyes, etc, } \\
\text { with quoit brooch, etc }\end{array}$ & $\begin{array}{l}\text { Hyslop } 1963,179 \text {, } \\
\text { fig } 12.32 . h ; \\
\text { Luton Museum }\end{array}$ \\
\hline Abingdon & Berks & 6th $c$ & $\begin{array}{l}\text { Grave B5: one millefiori } \\
\text { with cast saucer brooch } \\
\text { pair }\end{array}$ & $\begin{array}{l}\text { Leeds and Harden } 1936 \text {, } \\
32,58 \text {, pl VI.5; } \\
\text { shmolean Museum }\end{array}$ \\
\hline $\begin{array}{l}\text { Ower, } \\
\text { Clearal } \\
\text { Point }\end{array}$ & Dorset & $\begin{array}{l}\text { 6th- } \\
7 \text { th } c\end{array}$ & $\begin{array}{l}\text { Fragment of globular bead: } \\
\text { blue core with red and black }\end{array}$ & $\begin{array}{l}\text { Guido } 1987,100-1 \text {, } \\
\text { fig } 55.245\end{array}$ \\
\hline $\begin{array}{l}\text { Chessell } \\
\text { Down }\end{array}$ & $\begin{array}{l}\text { Isle of } \\
\text { Wight }\end{array}$ & 6th $c$ & $\begin{array}{l}\text { One unprovenanced large } \\
\text { barrel-shaped bead }\end{array}$ & $\begin{array}{l}\text { Arnold } 1982,50,71 \text { and } \\
121 \text { (no. } 233, \text { type } 16.2 \text { ), } \\
\text { colour frontispiece; } \\
\text { BM } 67-29.142\end{array}$ \\
\hline $\begin{array}{l}\text { Barfreston } \\
\text { or } \\
\text { Sibertswold }\end{array}$ & Kent & $\begin{array}{l}\text { 6th- } \\
7 \text { th } c\end{array}$ & Two badly made or burnt & $\begin{array}{l}\text { Faussett } 1856,228, \mathrm{pl} \mathrm{V;} \\
\text { Liverpool Museum }\end{array}$ \\
\hline $\begin{array}{l}\text { Chartham } \\
\text { Down }\end{array}$ & Kent & 7th $c$ & $\begin{array}{l}\text { Chartham Barrow 14: } \\
\text { drum-shaped with an } \\
\text { amethyst pendant and beads } \\
\text { on silver wire rings, etc }\end{array}$ & $\begin{array}{l}\text { Faussett 1856, 170; } \\
\text { Liverpool Museum }\end{array}$ \\
\hline $\begin{array}{l}\text { Dover, } \\
\text { Buckland }\end{array}$ & Kent & $\begin{array}{l}575- \\
625 \\
650- \\
675\end{array}$ & $\begin{array}{l}\text { Grave 38: globular; } \\
\text { Grave 53: globular; } \\
\text { Grave 132: } 1 \text { globular } \\
\text { and } 1 \text { cylinder }\end{array}$ & $\begin{array}{l}\text { Evison } 1987,63-5,78 \\
\text { and } 82(\mathrm{D} 62-\mathrm{D} 65) \text {, figs } \\
22.38 .4 \mathrm{~g}, 28.53 .1 \mathrm{c} \text { and } \\
54.132 .2 \mathrm{e} \text { and } \mathrm{f} \text {, colour } \\
\text { pl IV.D62, 63, 64, 65; } \\
\text { BM }\end{array}$ \\
\hline Faversham & Kent & $\begin{array}{l}\text { 6th- } \\
7 \text { th } c\end{array}$ & Two globulars & $\begin{array}{l}\text { Meaney 1964, 118-19; } \\
\text { Beck Collection, } \\
\text { Cambridge Museum }\end{array}$ \\
\hline
\end{tabular}




\begin{tabular}{|c|c|c|c|c|}
\hline Gilton & Kent & $\begin{array}{l}\text { 6th- } \\
7 \text { th } c\end{array}$ & One cylinder and 2 globulars & $\begin{array}{l}\text { Faussett } 1856,228, \\
\text { pls V and VI; } \\
\text { Liverpool Museum }\end{array}$ \\
\hline $\begin{array}{l}\text { Gilton or } \\
\text { Kingston }\end{array}$ & Kent & $\begin{array}{l}\text { 6th- } \\
7 \text { th } c\end{array}$ & Cylinder & $\begin{array}{l}\text { Faussett 1856, } 228, \\
\text { pl VI; } \\
\text { Liverpool Museum }\end{array}$ \\
\hline Howletts & Kent & $\begin{array}{l}\text { prob } \\
\text { 6th c } \\
\text { 2nd } \\
\text { half } \\
\text { 6th c }\end{array}$ & $\begin{array}{l}\text { Large globular; } \\
\text { Grave A: small cylinder }\end{array}$ & $\begin{array}{l}\text { Smith 1918; } \\
\text { BM 1936 5-11 143; } \\
\text { BM } 1936 \text { 5-11 }\end{array}$ \\
\hline 'Kent' & Kent & & $\begin{array}{l}\text { Barrel-shaped and } 1 \\
\text { cylinder-shaped }\end{array}$ & Canterbury Museum \\
\hline Sarre & Kent & $\begin{array}{l}\text { 6th- } \\
7 \text { th } c\end{array}$ & $\begin{array}{l}\text { Two cylinders; } \\
\text { Group XIX: large globular, } \\
\text { barrel-shaped and cylinder; } \\
\text { globular }\end{array}$ & $\begin{array}{l}\text { Brent } 1868,7-8, \text { pls VII } \\
\text { and VIII; } \\
\text { BM OA } 4904\end{array}$ \\
\hline $\begin{array}{l}\text { Caistor } \\
\text { by Norwich }\end{array}$ & Norfolk & 7th c & Globular & $\begin{array}{l}\text { Meaney 1964, 171-2; } \\
\text { Norwich Museum }\end{array}$ \\
\hline Kenninghall & Norfolk & 6th $c$ & Globular & $\begin{array}{l}\text { Manning } 1872,292 \text {, and } \\
1872 \text { b; } \\
\text { BM } 837-232\end{array}$ \\
\hline $\begin{array}{l}\text { Cassington, } \\
\text { Purwell } \\
\text { Farm }\end{array}$ & Oxon & $\begin{array}{l}\text { prob } \\
\text { 6th c }\end{array}$ & $\begin{array}{l}\text { Disturbed grave: } \\
\text { hexagonal cylinder } \\
\text { (Plate } 8 \text { ) }\end{array}$ & $\begin{array}{l}\text { Leeds and Riley } 1942,64 \text {, } \\
\text { pl VIB; } \\
\text { Ashmolean Museum }\end{array}$ \\
\hline Ipswich & Suffolk & $\begin{array}{l}\text { 6th- } \\
7 \text { th } c\end{array}$ & $\begin{array}{l}\text { Several globulars } \\
\text { (Plate 8) and } \\
\text { cylinders }\end{array}$ & $\begin{array}{l}\text { Layard 1907, pls XXXII and } \\
\text { XXXIII, and 1909, } \\
\text { pls VII and VI; } \\
\text { Ipswich Museum }\end{array}$ \\
\hline $\begin{array}{l}\text { Mitchell's } \\
\text { Hill }\end{array}$ & Suffolk & $\begin{array}{l}6 \text { th- } \\
7 \text { th } c\end{array}$ & Two globulars & $\begin{array}{l}\text { Meaney 1964, 231; } \\
\text { Bury St Edmunds } \\
\text { Museum }\end{array}$ \\
\hline
\end{tabular}




\title{
SCHEDULE 11
}

\section{ANNULAR GLASS BEADS WITH COLOURED SURFACE SPIRALS}

\author{
(see Map 31)
}

\section{(a) WHITE ANNULAR BEADS WITH LIGHT-BLUE SURFACE SPIRALS (Plate 8)}

\begin{tabular}{|c|c|c|c|c|}
\hline $\begin{array}{l}\text { Chamber- } \\
\text { lain's } \\
\text { Barn II }\end{array}$ & Beds & $\begin{array}{l}\text { mid- } \\
\text { late } \\
\text { 7th c }\end{array}$ & $\begin{array}{l}\text { Grave 32: 'ring-shaped' } \\
\text { bead with quoit brooch, } \\
\text { etc }\end{array}$ & $\begin{array}{l}\text { Hyslop 1963, 179, } \\
\text { fig 12.32.g; } \\
\text { Luton Museum }\end{array}$ \\
\hline Harrold & Beds & 7th $c$ & Not seen: cited by Evison & $\begin{array}{l}\text { Eagles and Evison } 1970 \text {, } \\
\text { fig } 13 j\end{array}$ \\
\hline Kempston & Beds & $\begin{array}{l}\text { prob } \\
\text { 6th c }\end{array}$ & $\begin{array}{l}\text { Grave } 16: 2 \text { beads of } \\
\text { this type stuck together } \\
\text { and probably wasters }\end{array}$ & $\begin{array}{l}\text { Fitch 1864; Smith 1868a, } \\
\text { 166-72; Smith 1868b; } \\
\text { Smith 1904, 176-84; } \\
\text { BM } 916-2453\end{array}$ \\
\hline $\begin{array}{l}\text { East } \\
\text { Shefford }\end{array}$ & Berks & $\begin{array}{l}\text { 5th- } \\
\text { 6th c }\end{array}$ & $\begin{array}{l}\text { Grave 13: with disc brooch } \\
\text { pair and amber beads }\end{array}$ & $\begin{array}{l}\text { Peake and Hooton 1915, } \\
\text { 114-15; Newbury Museum }\end{array}$ \\
\hline $\begin{array}{l}\text { Hasling- } \\
\text { field }\end{array}$ & Cambs & $\begin{array}{l}\text { 5th- } \\
7 \text { th c }\end{array}$ & One bead & $\begin{array}{l}\text { Fox 1923, 255-9; } \\
\text { Ashmolean Museum }\end{array}$ \\
\hline $\begin{array}{l}\text { Shudy } \\
\text { Camps }\end{array}$ & Cambs & 7th $\mathrm{c}$ & Two examples & $\begin{array}{l}\text { Lethbridge 1936; } \\
\text { Cambridge Museum }\end{array}$ \\
\hline $\begin{array}{l}\text { Colchester, } \\
\text { Guildford } \\
\text { Road } \\
\text { (Site K) }\end{array}$ & Essex & $\begin{array}{l}\text { first } \\
\text { half } \\
\text { 5th c }\end{array}$ & $\begin{array}{l}\text { Grave group 2: with a } \\
\text { cruciform brooch of } \\
\text { Witmarsum Type }\end{array}$ & $\begin{array}{l}\text { Crummy 1981, 11-12, } \\
\text { fig } 13.7 ; \\
\text { Colchester Museum }\end{array}$ \\
\hline Lechlade & Glos & 6th $c$ & $\begin{array}{l}\text { Grave 130: with cast saucer } \\
\text { brooches }\end{array}$ & $\begin{array}{l}\text { Boyle et al forthcoming; } \\
\text { Cirencester Museum }\end{array}$ \\
\hline $\begin{array}{l}\text { Breach } \\
\text { Down }\end{array}$ & Kent & $\begin{array}{l}\text { 7th- } \\
8 \text { th c }\end{array}$ & $\begin{array}{l}\text { Tumulus burial: badly } \\
\text { recorded }\end{array}$ & $\begin{array}{l}\text { Conyngham and Akerman } \\
\text { 1844, pl opp p } 49 \text {; } \\
\text { BM 79 5-24 } 89\end{array}$ \\
\hline $\begin{array}{l}\text { Dover, } \\
\text { Buckland }\end{array}$ & Kent & $\begin{array}{l}575- \\
625\end{array}$ & Grave 59 & $\begin{array}{l}\text { Evison } 1987,76,232 \text {, } \\
\text { fig 33.3g, colour pl } \\
\text { III.D01; BM }\end{array}$ \\
\hline
\end{tabular}




\begin{tabular}{|c|c|c|c|c|}
\hline Faversham & Kent & $\begin{array}{l}5 \text { th- } \\
7 \text { th c }\end{array}$ & & Smith 1860,46 , pl V.9 \\
\hline $\begin{array}{l}\text { Gilton or } \\
\text { Kingston }\end{array}$ & Kent & $\begin{array}{l}\text { prob } \\
6 \text { th- } \\
7 \text { th c }\end{array}$ & Mixed beads: 2 examples & $\begin{array}{l}\text { Faussett } 1856,228, \text { pl VI; } \\
\text { Liverpool Museum }\end{array}$ \\
\hline 'Kent' & Kent & & With unlocated collection & Canterbury Museum \\
\hline Sarre & Kent & & Miscellaneous collection & $\begin{array}{l}\text { Meaney 1964, 135-6; } \\
\text { BM } 8312-13644\end{array}$ \\
\hline $\begin{array}{l}\text { Sitting- } \\
\text { bourne, } \\
\text { Rondeau } \\
\text { Estate }\end{array}$ & Kent & $\begin{array}{l}\text { late } \\
6 \text { th- } \\
7 \text { th c }\end{array}$ & $\begin{array}{l}\text { Small example with } \\
\text { amethyst drops, etc }\end{array}$ & $\begin{array}{l}\text { Payne } 1880 \text { and } 1881 \text {; } \\
\text { BM }\end{array}$ \\
\hline Stodmarsh & Kent & $\begin{array}{l}\text { prob } \\
5 \text { th- } \\
6 \text { th c }\end{array}$ & With drum bead, etc & $\begin{array}{l}\text { Akerman 1855b; } \\
\text { BM } 54 \text { 12-2 } 17\end{array}$ \\
\hline $\begin{array}{l}\text { Castle } \\
\text { Bytham }\end{array}$ & Lincs & $\begin{array}{l}\text { prob } \\
7 \text { th c }\end{array}$ & $\begin{array}{l}\text { One bead: perhaps } \\
\text { the same bead } \\
\text { as above }\end{array}$ & $\begin{array}{l}\text { Akerman } 1855 a, 26-7 \text {, } \\
\text { pl XII.4; } \\
\text { Scunthorpe Museum } \\
\text { no. } 11\end{array}$ \\
\hline Cleatham & Lincs & $\begin{array}{l}\text { prob } \\
6 \text { th c }\end{array}$ & Grave 30 & $\begin{array}{l}\text { Publication in } \\
\text { preparation (K Leahy); } \\
\text { Scunthorpe Museum }\end{array}$ \\
\hline Fonaby & Lincs & 6th $\mathrm{c}$ & $\begin{array}{l}\text { Grave 3: 'oblate' with } \\
2 \text { annular brooches, etc }\end{array}$ & $\begin{array}{l}\text { Cook } 1981,18, \\
\text { fig 4.3.1.23; } \\
\text { Scunthorpe Museum }\end{array}$ \\
\hline $\begin{array}{l}\text { Welbourne, } \\
\text { High Dyke }\end{array}$ & Lincs & $\begin{array}{l}\text { prob } \\
6 \text { th c }\end{array}$ & $\begin{array}{l}\text { Cemetery near Roman } \\
\text { road: } 1 \text { bead }\end{array}$ & $\begin{array}{l}\text { Bruce } 1880,76, \text { no. } 305 ; \\
\text { Alnwick Museum }\end{array}$ \\
\hline $\begin{array}{l}\text { Bergh } \\
\text { Apton }\end{array}$ & Norfolk & $\begin{array}{l}\text { prob } \\
\text { 6th c }\end{array}$ & Grave 82 : with plain pot & $\begin{array}{l}\text { Green and Rogerson } 1978 \text {, } \\
48 \text {, fig } 99.82 . \text { Bii; } \\
\text { Norwich Museum }\end{array}$ \\
\hline Dunham & Norfolk & & $\begin{array}{l}\text { No adequate information: } \\
\text { small example }\end{array}$ & Norwich Museum \\
\hline Kenninghall & Norfolk & $\begin{array}{l}5 \text { th- } \\
7 \text { th c }\end{array}$ & $\begin{array}{l}\text { Inhumation burial in } \\
\text { cemetery }\end{array}$ & $\begin{array}{l}\text { Manning } 1872 a, 292 \text {, and } \\
1872 b\end{array}$ \\
\hline $\begin{array}{l}\text { Morning } \\
\text { Thorpe }\end{array}$ & Norfolk & 6 th $\mathrm{c}$ & $\begin{array}{l}\text { Grave } 30 \text { : with Åberg } \\
\text { Group II (pair) and Group III } \\
\text { cruciform brooches, etc }\end{array}$ & $\begin{array}{l}\text { Green et al 1987, } \\
\text { 43, fig 303.30.Div; } \\
\text { Norwich Museum }\end{array}$ \\
\hline Spong Hill & Norfolk & $\begin{array}{l}6 \text { th } c \\
5 \text { th- } \\
6 \text { th c }\end{array}$ & $\begin{array}{l}\text { Grave 45: with Åberg } \\
\text { Group IV cruciform } \\
\text { brooch, etc; } \\
\text { Cremation 33: from } \\
\text { stamped urn; } \\
\text { Cremation 62: from } \\
\text { plain urn with Åberg } \\
\text { Group II cruciform } \\
\text { brooch(es); }\end{array}$ & $\begin{array}{l}\text { Hills } 1977,37 \text { and } 38 \text {, } \\
\text { fig } 127.33 \text { and } 62 ;\end{array}$ \\
\hline
\end{tabular}




\begin{tabular}{|c|c|c|c|c|}
\hline & & 6th $c$ & $\begin{array}{l}\text { Cremation 2144: from } \\
\text { stamped urn }\end{array}$ & $\begin{array}{l}\text { 57, fig } 165.2144 ; \\
\text { Norfolk Arch Unit }\end{array}$ \\
\hline Corbridge & $\begin{array}{l}\text { North- } \\
\text { umber- } \\
\text { land }\end{array}$ & $\begin{array}{l}5 \text { th- } \\
6 \text { th c }\end{array}$ & $\begin{array}{l}\text { Necklace near } 2 \text { Åerg } \\
\text { Group II cruciform } \\
\text { brooches }\end{array}$ & $\begin{array}{l}\text { Knowles and Forster } \\
\text { 1909, 407; } \\
\text { Corbridge Museum }\end{array}$ \\
\hline $\begin{array}{l}\text { Holme } \\
\text { Pierre- } \\
\text { pont }\end{array}$ & Notts & $\begin{array}{l}\text { prob } \\
\text { 6th c }\end{array}$ & With brooches, etc & $\begin{array}{l}\text { Myres 1977, Corpus } \\
\text { no. 2093, fig } 303\end{array}$ \\
\hline $\begin{array}{l}\text { Empingham } \\
\text { II }\end{array}$ & Rutland & $\begin{array}{l}5 \text { th- } \\
6 \text { th } c \\
6 \text { th } c\end{array}$ & $\begin{array}{l}\text { Grave } 67 \text { : with } 2 \\
\text { small-long brooches; } \\
\text { Grave } 73 \text { : with swastika and } \\
\text { cruciform brooches }\end{array}$ & $\begin{array}{l}\text { Timby 1996; } \\
\text { Oakham Museum }\end{array}$ \\
\hline $\begin{array}{l}\text { Bury St } \\
\text { Edmunds, } \\
\text { Westgarth } \\
\text { Gardens }\end{array}$ & Suffolk & 6th $c$ & $\begin{array}{l}\text { Grave } 48 \text { : with } 2 \text { small- } \\
\text { long brooches and B clasps, } \\
\text { etc }\end{array}$ & $\begin{array}{l}\text { West } 1988,32 \text {, } \\
\text { fig } 73 . E 20 ; \\
\text { Bury St Edmunds } \\
\text { Museum }\end{array}$ \\
\hline $\begin{array}{l}\text { Holywell } \\
\text { Row }\end{array}$ & Suffolk & $\begin{array}{l}\text { 6th } c \\
5 \text { th- } \\
6 \text { th } c\end{array}$ & $\begin{array}{l}\text { Grave } 39 \text { : with } 2 \text { small- } \\
\text { long brooches, etc; } \\
\text { Grave } 47 \text { : with } 5 \text {-star } \\
\text { applied saucer brooch }\end{array}$ & $\begin{array}{l}\text { Lethbridge } 1931,21 \\
\text { and } 25 \text {, figs } 10 . A .51 \text { and } \\
11 . F .1 ; \\
\text { Cambridge Museum }\end{array}$ \\
\hline West Stow & Suffolk & $\begin{array}{l}5 \text { th- } \\
7 \text { th } \mathrm{c}\end{array}$ & $\begin{array}{l}\text { Cemetery: } 1 \text { 'disc' } \\
\text { (blue with white spiral?) }\end{array}$ & $\begin{array}{l}\text { West } 1985,72 \text { and } \\
74 \text { (type 027), fig } 276.6 \text {; } \\
\text { Ashmolean Museum } \\
1909.24\end{array}$ \\
\hline Guildown & Surrey & $\begin{array}{l}\text { 6th } \mathrm{c} \\
6 \text { th } \mathrm{c}\end{array}$ & $\begin{array}{l}\text { Grave } 185: \text { with stamp- } \\
\text { ornamented pot; } \\
\text { Grave } 206: \text { with square- } \\
\text { headed brooch pair }\end{array}$ & $\begin{array}{l}\text { Lowther } 1931,11,43 \text {, } \\
\text { 44, pl IX.1 and 3; } \\
\text { Guildford Museum }\end{array}$ \\
\hline $\begin{array}{l}\text { Pewsey } \\
\text { Blackpatch }\end{array}$ & Wilts & 6th $c$ & Grave 53 & $\begin{array}{l}\text { Publication in } \\
\text { preparation (K Annable); } \\
\text { Devizes Museum }\end{array}$ \\
\hline Winklebury & Wilts & & $\begin{array}{l}\text { One example with } 4 \text { other } \\
\text { Anglo-Saxon beads }\end{array}$ & $\begin{array}{l}\text { Pitt-Rivers 1888, 266, } \\
\text { pl CL.29; } \\
\text { Pitt-Rivers Collection, } \\
\text { Salisbury Museum }\end{array}$ \\
\hline $\begin{array}{l}\text { Winter- } \\
\text { bourne } \\
\text { Stoke II }\end{array}$ & Wilts & & $\begin{array}{l}\text { Secondary burial in } \\
\text { barrow with cremation }\end{array}$ & $\begin{array}{l}\text { Hoare } 1812,119 ; \\
\text { Devizes Museum }\end{array}$ \\
\hline Kilham & Yorks & $\begin{array}{l}\text { prob } \\
5 \text { th- } \\
6 \text { th c }\end{array}$ & $\begin{array}{l}\text { Grave 1: spiral } \\
\text { colour lost }\end{array}$ & $\begin{array}{l}\text { Brown 1915a (IV), } \\
\text { 806-8 }\end{array}$ \\
\hline
\end{tabular}




\section{(b) ANNULAR BEADS WITH SURFACE SPIRALS IN OTHER COLOURS}

\begin{tabular}{|c|c|c|c|c|}
\hline $\begin{array}{l}\text { Newport } \\
\text { Pagnell }\end{array}$ & Bucks & $\begin{array}{l}\text { prob } \\
\text { 6th c }\end{array}$ & $\begin{array}{l}\text { On an amulet: white } \\
\text { with black spiral }\end{array}$ & $\begin{array}{l}\text { Meaney } 1964,58 \\
\text { Aylesbury Museum }\end{array}$ \\
\hline Fairford & Glos & & $\begin{array}{l}\text { Terracotta with yellow } \\
\text { spiral }\end{array}$ & $\begin{array}{l}\text { Meaney } 1964,91 ; \\
\text { Ashmolean Museum }\end{array}$ \\
\hline Lechlade & Glos & $\begin{array}{l}\text { 7th } \mathrm{c} \\
6 \text { th } \mathrm{c} \\
6 \text { th } \mathrm{c}\end{array}$ & $\begin{array}{l}\text { Grave } 25 \text { : terracotta } \\
\text { with yellow spiral with } \\
\text { cast saucer brooch; } \\
\text { Grave } 36 / 2 \text { : opaque } \\
\text { green-blue with white } \\
\text { spiral with knife; } \\
\text { Grave } 41 \text { : yellow with } \\
\text { spiral with disc brooch } \\
\text { pair; } \\
\text { Grave } 160 \text { : black with } \\
\text { white spiral with pin, etc }\end{array}$ & $\begin{array}{l}\text { Boyle et al forthcoming; } \\
\text { Cirencester Museum }\end{array}$ \\
\hline $\begin{array}{l}\text { Breach } \\
\text { Down }\end{array}$ & Kent & $\begin{array}{l}\text { 7th- } \\
8 \text { th } c\end{array}$ & $\begin{array}{l}\text { Black ground, white } \\
\text { spirals }\end{array}$ & $\begin{array}{l}\text { Conyngham and Akermar } \\
\text { 1844; BM 79 5-24 } 89\end{array}$ \\
\hline Canterbury & Kent & $\begin{array}{l}\text { prob } \\
\text { 6th c }\end{array}$ & $\begin{array}{l}\text { ?Sword-bead: large, } \\
\text { bun-shaped, dark with } \\
\text { white spiral }\end{array}$ & $\begin{array}{l}\text { Evans Collection, } \\
\text { Ashmolean Museum }\end{array}$ \\
\hline Faversham & Kent & $\begin{array}{l}5 \text { th- } \\
7 \text { th c }\end{array}$ & $\begin{array}{l}\text { Almost translucent } \\
\text { green with white spiral }\end{array}$ & $\begin{array}{l}\text { Meaney 1964, 118-19; } \\
\text { Ashmolean Museum }\end{array}$ \\
\hline $\begin{array}{l}\text { Gilton } \\
\text { or } \\
\text { Kingston }\end{array}$ & Kent & $\begin{array}{l}\text { prob } \\
\text { 6th- } \\
7 \text { th c }\end{array}$ & $\begin{array}{l}\text { Blue with white } \\
\text { spiral }\end{array}$ & $\begin{array}{l}\text { Faussett } 1856,228, \mathrm{pl} \mathrm{VI} \text {; } \\
\text { Liverpool Museum } \\
6123\end{array}$ \\
\hline Polhill & Kent & $\begin{array}{l}\text { a few } \\
\text { 6th } \\
\text { and } \\
7 \text { th } c\end{array}$ & $\begin{array}{l}\text { Grave } 95 \text { : white spiral on } \\
\text { blue with buckle and knife, } \\
\text { etc; } \\
\text { Grave } 102 \text { : white spiral on } \\
\text { black and on blue with faint } \\
\text { red spiral inlay with silver } \\
\text { rings, etc; } \\
\text { unstratified bead group: } \\
\text { white spiral on green-blue }\end{array}$ & $\begin{array}{l}\text { Hawkes and Philp } 1973 \text {, } \\
184,185 \text { and } 207, \\
\text { fig } 55.526,528 \text { and } 529\end{array}$ \\
\hline Sarre & Kent & $\begin{array}{l}5 \text { th- } \\
7 \text { th c }\end{array}$ & $\begin{array}{l}\text { Slatey blue with white } \\
\text { spirals }\end{array}$ & $\begin{array}{l}\text { Meaney 1964, 135-6; } \\
\text { BM 93 6-1 } 218\end{array}$ \\
\hline $\begin{array}{l}\text { Sitting- } \\
\text { bourne, } \\
\text { Rondeau } \\
\text { Estate }\end{array}$ & Kent & & $\begin{array}{l}\text { Turquoise with white } \\
\text { spiral }\end{array}$ & $\begin{array}{l}\text { Payne } 1880 \text { and } \\
1881 ; \\
\text { BM } 8812-13644\end{array}$ \\
\hline
\end{tabular}




$\begin{array}{llll}\begin{array}{l}\text { Barton-on- } \\ \text { Humber }\end{array} & \text { Lincs } & \text { 7th c } & \begin{array}{l}\text { Grave 101: very weathered, } \\ \text { colours uncertain, but } \\ \text { probably white spiral } \\ \text { on dark ground, with } \\ \text { annular brooch and } \\ \text { chatelaine, etc }\end{array} \\ \text { Fonaby } & \text { Lincs } & 6 \text { th c } & \begin{array}{l}\text { Grave 3: blue with ?white } \\ \text { spiral }\end{array}\end{array}$

$\begin{array}{llll}\begin{array}{l}\text { Welbourne, } \\ \text { High Dyke }\end{array} & \text { Lincs } & \begin{array}{c}\text { prob } \\ \text { 6th c }\end{array} & \text { Blue with white spiral } \\ \text { Ronaldsway } & \begin{array}{l}\text { Isle of } \\ \text { Man }\end{array} & & \begin{array}{l}\text { Dark blue/green with } \\ \text { white spiral }\end{array} \\ \text { Fakenham } & \text { Norfolk } & & \begin{array}{l}\text { Translucent colourless } \\ \text { with white spiral, atypical }\end{array} \\ \begin{array}{l}\text { Morning } \\ \text { Thorpe }\end{array} & \text { Norfolk } & \begin{array}{l}\text { mid- } \\ \text { 6th c }\end{array} & \begin{array}{l}\text { Grave 358: blue with } \\ \text { white spiral, with } \\ \text { Aberg Group IVa } \\ \text { cruciform brooch, etc }\end{array}\end{array}$

Spong Hill Norfolk 5th c Cremation 2064: 1 blue with white spiral with linear-decorated urn

\begin{tabular}{|c|c|c|c|}
\hline Chesters & $\begin{array}{l}\text { North- } \\
\text { umber- } \\
\text { land }\end{array}$ & $\begin{array}{l}\text { prob } \\
\text { late } \\
\text { Rom- } \\
\text { an }\end{array}$ & $\begin{array}{l}\text { Roman fort: light green } \\
\text { translucent, colour missing } \\
\text { from spiral, irregular- } \\
\text { shaped, atypical }\end{array}$ \\
\hline $\begin{array}{l}\text { Empingham } \\
\text { II }\end{array}$ & Rutland & $\begin{array}{l}5 \text { th- } \\
7 \text { th c }\end{array}$ & $\begin{array}{l}\text { Grave } 5: 1 \text { green with } \\
\text { white spiral; } \\
\text { unstratified: } 1 \text { blue with } \\
\text { yellow spiral }\end{array}$ \\
\hline
\end{tabular}

Brandon Suffolk mid- Settlement: 1 black with 7th- white spiral, 9th c 1 reddish with white

West Stow Suffolk $\quad 5$ th-

7th $\mathrm{c}$

Cemetery: 1 'disc' dark green with white trails (see type (a) above)

$\begin{array}{llll}\text { Alfriston } & \text { Sussex } & \begin{array}{l}\text { 5th- } \\ \text { 6th } \mathrm{c}\end{array} & \begin{array}{l}\text { Translucent blue with } \\ \text { white now missing }\end{array}\end{array}$

'Sussex' Sussex

Charlton
Wilts
One with blue spiral on black ground

late Burial 86: greenish spiral 6th- translucent bottle glass
Drinkall and Foreman forthcoming;

Scunthorpe Museum

Cook 1981, 18,

fig 4.3.1.23;

Scunthorpe Museum

Bruce 1880, 76, no. 305;

Alnwick Museum

Anon 1937, fig 3

Norwich Museum

Green et al 1987, 139, fig 419.Uv; Norwich Museum

Hills et al 1981, 52, fig 165.2064; Norfolk Arch Unit

Chesters Museum

Timby 1996;

Oakham Museum

Carr et al 1988; information from R D Carr, Suffolk Arch Unit

West 1985,72 and 74 (type O26), fig 276.6; Ashmolean Museum 1909.24

Griffith and Salzmann 1914, pl I.3;

Welch 1983, 83;

Lewes Museum

Welch 1983, 84, fig 47c;

Lewes Museum

Davies 1984, 122,

fig 12D;

Salisbury Museum 


$\begin{array}{lllll}\text { Kilham } & \text { Yorks } & \text { 6th c } & \begin{array}{l}\text { Grave 1: translucent green/ } \\ \text { blue with groove missing }\end{array} & \begin{array}{l}\text { Brown 1915a (III), } \\ \text { 306-8 }\end{array} \\ \text { Saltburn } & \text { Yorks } & \begin{array}{l}\text { 6th- } \\ 7 \text { th c }\end{array} & \begin{array}{l}\text { Blue with white spiral } \\ \text { (Plate 8) (with } \\ \text { amethysts, etc) }\end{array} & \begin{array}{l}\text { Gallagher 1987, 16, } \\ 25, \text { fig 5; } \\ \text { Middlesbrough Museum }\end{array} \\ \text { Sewerby } & \text { Yorks } & \text { 6th c } & \begin{array}{l}\text { Grave 35A: 1 bead, dark } \\ \text { green and white (type Cla) } \\ \text { (Plate 8) }\end{array} & \begin{array}{l}\text { Hirst 1985, 66, } \\ \text { fig 45.G35A.1 }\end{array} \\ \text { Whitby } & \text { Yorks } & \begin{array}{l}\text { Two pale grey with dark } \\ \text { prob }\end{array} & \text { Peers and Radford 1943 } \\ & & \text { 9th c } & \text { green spirals } & \end{array}$




\title{
ANNular glass BeADS WiTH FINE TWIST DECORATION
}

\author{
(see Map 32)
}

\begin{tabular}{|c|c|c|c|c|}
\hline Burwell & Cambs & 7 th $\mathrm{c}$ & $\begin{array}{l}\text { Grave 26: translucent } \\
\text { green with } 2 \text { twists, } 1 \text { blue } \\
\text { and green and } 1 \text { green } \\
\text { and yellow with wire loop } \\
\text { for use as pendant }\end{array}$ & $\begin{array}{l}\text { Lethbridge 1926a, pl I, } \\
\text { fig } 2 \text {; } \\
\text { Cambridge Museum }\end{array}$ \\
\hline $\begin{array}{l}\text { Shudy } \\
\text { Camps }\end{array}$ & Cambs & 7th $\mathrm{c}$ & $\begin{array}{l}\text { Grave } 104 \text { (by child's } \\
\text { head): no colour details; } \\
\text { Grave 11: similar bead }\end{array}$ & $\begin{array}{l}\text { Lethbridge 1936, } 25 \text { and 5, } \\
\text { fig 4.I; } \\
\text { Cambridge Museum }\end{array}$ \\
\hline $\begin{array}{l}\text { Gilton } \\
\text { or } \\
\text { Kingston }\end{array}$ & Kent & 7th $c$ & $\begin{array}{l}\text { Blackish with } 1 \text { red and } \\
1 \text { blue twist }\end{array}$ & $\begin{array}{l}\text { Faussett } 1856,228, \\
\text { pls V and VI; } \\
\text { Liverpool Museum }\end{array}$ \\
\hline Hartlip & Kent & & $\begin{array}{l}\text { Roman villa stray find: } \\
\text { light translucent green } \\
\text { with opaque yellow } \\
\text { twist (Plate 8) }\end{array}$ & $\begin{array}{l}\text { Smith } 1846 \mathrm{~b} \text { and } \\
1852 \mathrm{c} ; \\
\text { Maidstone Museum }\end{array}$ \\
\hline $\begin{array}{l}\text { Milton- } \\
\text { next- } \\
\text { Sittingbourne }\end{array}$ & Kent & $\begin{array}{l}\text { late } \\
\text { 7th c }\end{array}$ & $\begin{array}{l}\text { Two examples, no colours } \\
\text { given }\end{array}$ & Vallance 1848 \\
\hline $\begin{array}{l}\text { Sibertswold } \\
\text { or } \\
\text { Barfreston }\end{array}$ & Kent & & $\begin{array}{l}\text { Graves } 17 \text { and 133: } \\
2 \text { very similar - brownish } \\
\text { yellow with yellow twist } \\
\text { and rich bottle glass with } \\
\text { yellow }\end{array}$ & $\begin{array}{l}\text { Faussett } 1856,105 \text { and } \\
\text { 123, pl V; Faussett MS } \\
\text { Diary IV, 8, fig 1; } \\
\text { Liverpool Museum }\end{array}$ \\
\hline $\begin{array}{l}\text { Brafield- } \\
\text { on-Green }\end{array}$ & Northants & & $\begin{array}{l}\text { Iron Age site stray find: } \\
\text { half dark blue/green, } \\
\text { opaque red and yellow } \\
\text { twists and circumferential } \\
\text { band }\end{array}$ & $\begin{array}{l}\text { Northampton Museum } \\
\text { D272, 1952-8.3 }\end{array}$ \\
\hline Cannington & Somerset & 7 th $\mathrm{c}$ & $\begin{array}{l}\text { Grave } 405 \text { : rich green/blue } \\
\text { translucent with } 3 \text { twists: } \\
1 \text { blue and white, } 1 \text { red and } \\
\text { white, } 1 \text { yellow and green } \\
\text { (Plate } 8 \text { ), with dated brooch }\end{array}$ & $\begin{array}{l}\text { Publication in } \\
\text { preparation (P Rahtz) }\end{array}$ \\
\hline
\end{tabular}




\begin{tabular}{|c|c|c|c|c|}
\hline $\begin{array}{l}\text { Holywell } \\
\text { Row }\end{array}$ & Suffolk & 7th $\mathrm{C}$ & $\begin{array}{l}\text { Grave } 26: \text { green with } \\
\text { blue and white twist } \\
\text { with silver wire ring }\end{array}$ & $\begin{array}{l}\text { Lethbridge } 1931,50-2 \text {, } \\
\text { fig } 24.2 \text {; } \\
\text { Cambridge Museum }\end{array}$ \\
\hline $\begin{array}{l}\text { Fetcham, } \\
\text { Hawks Hill }\end{array}$ & Surrey & $\begin{array}{l}6 \text { th- } \\
7 \text { th c }\end{array}$ & $\begin{array}{l}\text { Green/blue translucent } \\
\text { with same glass twisted } \\
\text { with opaque yellow }\end{array}$ & $\begin{array}{l}\text { Smith 1907, 124, } \\
\text { fig } 3\end{array}$ \\
\hline Salisbury & Wilts & & $\begin{array}{l}\text { From a collection: } 1 \text { green } \\
\text { and yellow twist and blue } \\
\text { and white on dark ground } \\
\text { (Plate } 8 \text { ) }\end{array}$ & $\begin{array}{l}\text { Unpublished: } \\
\text { Salisbury Museum }\end{array}$ \\
\hline $\begin{array}{l}\text { Swallow- } \\
\text { cliffe } \\
\text { Down }\end{array}$ & Wilts & $\begin{array}{l}\text { 2nd } \\
\text { half } \\
7 \text { th c }\end{array}$ & $\begin{array}{l}\text { Barrow: translucent blue/ } \\
\text { green with same glass } \\
\text { twisted with opaque white }\end{array}$ & $\begin{array}{l}\text { Speake } 1989,50-2 \text {, } \\
\text { figs } 43 \text { and } 46 ; \\
\text { Salisbury Museum }\end{array}$ \\
\hline $\begin{array}{l}\text { Hawnby } \\
\text { Moor, } \\
\text { Sunny Bank }\end{array}$ & $\begin{array}{l}\text { Yorks } \\
\text { (NR) }\end{array}$ & 7 th $\mathrm{c}$ & $\begin{array}{l}\text { Tumulus: green/blue } \\
\text { translucent with same glass } \\
\text { and yellow twist, mounted } \\
\text { on bronze ring with } \\
\text { hanging-bowl, etc }\end{array}$ & $\begin{array}{l}\text { Denny } 1866,497-9 ; \\
\text { Smith 1912, 96, figs } 16 \text { and } \\
\text { 17; Brown 1915a (IV), } 809 \\
\text { and 473-4; } \\
\text { Meaney 1964, 290-1; } \\
\text { BM } 823-2346\end{array}$ \\
\hline $\begin{array}{l}\text { Denholm } \\
\text { Hill, } \\
\text { Cavers }\end{array}$ & $\begin{array}{l}\text { Scotland: } \\
\text { Roxbs }\end{array}$ & & $\begin{array}{l}\text { Probably an Anglian site: } \\
\text { translucent yellow with } \\
\text { opaque red and dark twist }\end{array}$ & $\begin{array}{l}\text { Edinburgh Museum } \\
\text { FJ } 120\end{array}$ \\
\hline
\end{tabular}




\section{SChedule 13}

\section{GOLD AND SILVER-IN-GLASS BEADS (partial schedules only) (Plate 8)}

\begin{tabular}{|c|c|c|c|c|}
\hline Kempston & Beds & $\begin{array}{l}\text { prob } \\
\text { 6th } c\end{array}$ & Grave 16: several & $\begin{array}{l}\text { Fitch 1864; } \\
\text { BM } 916-2453 \text { and } 54\end{array}$ \\
\hline Barrington & Cambs & 6th $\mathrm{c}$ & Two or 3 beads & $\begin{array}{l}\text { Fox 1923,250-5; } \\
\text { Smith 1868a }\end{array}$ \\
\hline $\begin{array}{l}\text { Hasling- } \\
\text { field }\end{array}$ & Cambs & $\begin{array}{l}\text { 5th- } \\
7 \text { th } c\end{array}$ & Some & Fox $1923,255-9$ \\
\hline $\begin{array}{l}\text { Linton } \\
\text { Heath B }\end{array}$ & Cambs & & Two segments & $\begin{array}{l}\text { Neville 1854; } \\
\text { Cambridge Museum }\end{array}$ \\
\hline $\begin{array}{l}\text { Great } \\
\text { Chesterford }\end{array}$ & Essex & 6th $c$ & $\begin{array}{l}\text { Grave } 62: 3 \text { to } 4 \text { wide-spaced } \\
\text { with small square-headed } \\
\text { brooch pair, etc }\end{array}$ & $\begin{array}{l}\text { Evison } 1994,16,46 \\
101, \text { fig } 33.4 n-p \\
B M\end{array}$ \\
\hline Mucking II & Essex & $\begin{array}{l}\text { first } \\
\text { half } \\
\text { 5th c }\end{array}$ & $\begin{array}{l}\text { Grave 989: gold-in-glass } \\
\text { beads with Glaston- } \\
\text { Mucking and supporting-arm } \\
\text { brooches, etc }\end{array}$ & $\begin{array}{l}\text { Hirst and Clark forthcoming; } \\
\text { Evison 1981, 138-9, } \\
\text { figs } 4 \text { and 5; } \\
\text { BM }\end{array}$ \\
\hline Lechlade & Glos & $\begin{array}{l}\text { 6th } c \\
5 \text { th- } \\
\text { 6th } c\end{array}$ & $\begin{array}{l}\text { Grave 10: } 34 \text { beads with } \\
\text { cast saucer brooch pair, } \\
\text { etc; } \\
\text { Grave 18: } 129 \text { beads with } \\
\text { great square-headed brooch, } \\
\text { cast saucer brooch pair, } \\
\text { etc; } \\
\text { Grave } 41: 27 \text { beads with } \\
\text { disc brooch pair, etc; } \\
\text { Grave } 90: 2 \text { beads with } \\
\text { applied saucer brooch pair, } \\
\text { etc; } \\
\text { Grave 136: } 1 \text { bead with } \\
\text { Style I fittings, etc; } \\
\text { Grave 163: } 12 \text { beads with } \\
\text { disc brooch pair, etc }\end{array}$ & $\begin{array}{l}\text { Boyce et al forthcoming; } \\
\text { Cirencester Museum }\end{array}$ \\
\hline $\begin{array}{l}\text { Bowcombe } \\
\text { Down }\end{array}$ & $\begin{array}{l}\text { Isle of } \\
\text { Wight }\end{array}$ & $\begin{array}{l}5 \text { th- } \\
6 \text { th } c\end{array}$ & & $\begin{array}{l}\text { Arnold 1982, 89-96, } \\
121 \text { (type } 14 \text { ) }\end{array}$ \\
\hline
\end{tabular}




\begin{tabular}{|c|c|c|c|c|}
\hline Woodstone & Hunts & $\begin{array}{l}\text { prob } \\
6 \text { th c }\end{array}$ & $\begin{array}{l}\text { Barrow excavated in } 1827 \\
\text { near cemetery: several }\end{array}$ & $\begin{array}{l}\text { Artis } 1828, \text { pl LV; } \\
\text { BM 6-2 } 113\end{array}$ \\
\hline $\begin{array}{l}\text { Breach } \\
\text { Down }\end{array}$ & Kent & $\begin{array}{l}7 \text { th- } \\
8 \text { th c }\end{array}$ & $\begin{array}{l}\text { Barrow cemetery, } \\
\text { possible Christian burials }\end{array}$ & $\begin{array}{l}\text { Conyngham and Akerman } \\
1844 ; \text { BM 79 5-24 } 89\end{array}$ \\
\hline $\begin{array}{l}\text { Beakes- } \\
\text { bourne II }\end{array}$ & Kent & $\begin{array}{l}5 \text { th- } \\
\text { late } \\
6 \text { th c }\end{array}$ & Graves 11 and 22 & $\begin{array}{l}\text { Meaney } 1964,109 ; \\
\text { Canterbury Museum }\end{array}$ \\
\hline Coombe & Kent & $\begin{array}{l}\text { mid } \\
6 \text { th } \mathrm{c}\end{array}$ & $\begin{array}{l}\text { Cremation burial: perhaps } \\
\text { with small-square-headed } \\
\text { brooch }\end{array}$ & $\begin{array}{l}\text { Davidson and Webster } \\
\text { 1967, 19; } \\
\text { Saffron Walden Museum }\end{array}$ \\
\hline $\begin{array}{l}\text { Dover, } \\
\text { Buckland }\end{array}$ & Kent & $\begin{array}{l}475- \\
525 \\
575- \\
625 \\
650- \\
675\end{array}$ & $\begin{array}{l}\text { Graves } 13,20,46 \text { and } 48 ; \\
\text { Graves } 32 \text { and } 35 ; \\
\text { Grave } 6 ; \\
\text { total no. of beads on this } \\
\text { site: } 112\end{array}$ & $\begin{array}{l}\text { Evison } 1987,75 \text { (types } \\
\text { C10, C11, C12, C13), } \\
\text { colour pl III.C10; } \\
\text { BM }\end{array}$ \\
\hline $\begin{array}{l}\text { Eastry, } \\
\text { Buttsole }\end{array}$ & Kent & 6th $c$ & Tumuli: unusual objects & $\begin{array}{l}\text { Meaney 1964, 113; } \\
\text { Canterbury Museum }\end{array}$ \\
\hline Faversham & Kent & $\begin{array}{l}\text { 6th- } \\
7 \text { th } \mathrm{c}\end{array}$ & $\begin{array}{l}\text { From } 1 \text { or more old } \\
\text { excavations: several }\end{array}$ & $\begin{array}{l}\text { Meaney 1964, 118-19; } \\
\text { Ashmolean Museum } \\
\text { 1909. } 161\end{array}$ \\
\hline $\begin{array}{l}\text { Folkestone, } \\
\text { Dover Hill }\end{array}$ & Kent & $\begin{array}{l}\text { prob } \\
6 \text { th } c\end{array}$ & $\begin{array}{l}\text { No. } 11 ; \\
\text { No. } 19 ; \\
\text { No. } 27 \\
\text { No. } 32\end{array}$ & $\begin{array}{l}\text { Meaney } 1964,120-1 ; \\
\text { Maidstone Museum and } \\
\text { Folkestone Museum }\end{array}$ \\
\hline Gilton & Kent & & $\begin{array}{l}\text { Group of } 13 \text { rather greenish } \\
\text { beads }\end{array}$ & $\begin{array}{l}\text { Faussett } 1856,1-34 \\
\text { Liverpool Museum } 6191\end{array}$ \\
\hline Howletts & Kent & 6 th $c$ & Several & $\begin{array}{l}\text { Smith 1918; } \\
\text { BM 1936 5-11 145, etc }\end{array}$ \\
\hline $\begin{array}{l}\text { Lyminge } \\
\text { II }\end{array}$ & Kent & 6 th $\mathrm{c}$ & $\begin{array}{l}\text { Grave 16: about } 20 \text { with } \\
\text { Hahnheim-type radiate } \\
\text { brooch, button brooch and } \\
\text { D bracteate, etc; } \\
\text { Grave } 39: \text { a few with cast } \\
\text { saucer brooch and small } \\
\text { square-headed brooch } \\
\text { pairs, etc }\end{array}$ & $\begin{array}{l}\text { Warhurst 1955, 15, } 27, \\
\text { pl VIIb.2; } \\
\text { Maidstone Museum }\end{array}$ \\
\hline $\begin{array}{l}\text { Rochester } \\
\text { II }\end{array}$ & Kent & $\begin{array}{l}\text { prob } \\
6 \text { th } c\end{array}$ & Child's necklace & $\begin{array}{l}\text { Meaney } 1964,134 \\
\text { Skipton Museum }\end{array}$ \\
\hline Stodmarsh & Kent & $\begin{array}{l}\text { 6th- } \\
7 \text { th } \mathrm{c}\end{array}$ & $\begin{array}{l}\text { On necklace with ?silver } \\
\text { drum bead, surface spiral } \\
\text { bead, etc }\end{array}$ & $\begin{array}{l}\text { Akerman 1855b, 179-81, } \\
\text { pl XVI; } \\
\text { Canterbury Museum; } \\
\text { BM } 5412-217\end{array}$ \\
\hline
\end{tabular}




\begin{tabular}{|c|c|c|c|c|}
\hline Stowting & Kent & $\begin{array}{l}\text { prob } \\
\text { early } \\
6 \text { th } c\end{array}$ & $\begin{array}{l}\text { Graves } 12,18,19 \text { and } 20 \text { : } \\
\text { a number, some very fine } \\
\text { and double or triple }\end{array}$ & $\begin{array}{l}\text { Smith 1846a, 399; } \\
\text { Brent 1867, 412-14; } \\
\text { Beck Collection, } \\
\text { Cambridge Museum } \\
\text { 47.1805; } \\
\text { Maidstone Museum }\end{array}$ \\
\hline Westbere & Kent & $\begin{array}{l}\text { prob } \\
7 \text { th c }\end{array}$ & Necklace: 2 & $\begin{array}{l}\text { Jessup 1946, 16, pl II; } \\
\text { Canterbury Museum }\end{array}$ \\
\hline Fonaby & Lincs & 6 th $c$ & $\begin{array}{l}\text { Grave } 3: 6 \text { with annular } \\
\text { brooches; } \\
\text { Grave } 28: 14 \text { with small- } \\
\text { long brooch; } \\
\text { Grave } 43: 6 \text { with } 2 \text { Aberg } \\
\text { Group IVa and ?V cruciform } \\
\text { brooches, etc }\end{array}$ & $\begin{array}{l}\text { Cook } 1981,18,30 \text { and } \\
40, \text { figs } 4.3 .1(9,25 \text {, } \\
26,27,54,55) \text {, } \\
10.28 .2 \text { and } 16.1 .40-42 \text {, } \\
46-48 ; \\
\text { Scunthorpe Museum }\end{array}$ \\
\hline Ruskington & Lincs & $\begin{array}{l}\text { prob } \\
6 \text { th c }\end{array}$ & & $\begin{array}{l}\text { Meaney 1964, 161-2; } \\
\text { Lincoln Museum }\end{array}$ \\
\hline Brooke & Norfolk & $\begin{array}{l}\text { prob } \\
6 \text { th c }\end{array}$ & One bead & $\begin{array}{l}\text { Meaney 1964, 170; } \\
\text { BM }\end{array}$ \\
\hline $\begin{array}{l}\text { Hunstanton } \\
\text { Park }\end{array}$ & Norfolk & & At least 1 example & $\begin{array}{l}\text { Meaney 1964, 176; } \\
\text { Norwich Museum }\end{array}$ \\
\hline Kenninghall & Norfolk & $\begin{array}{l}5 \text { th- } \\
7 \text { th c }\end{array}$ & One bead & $\begin{array}{l}\text { Manning } 1872 \mathrm{a}, 272 \text {, and } \\
1872 \mathrm{~b} \text {; } \\
\text { BM } 837-230 \text { and } 31\end{array}$ \\
\hline $\begin{array}{l}\text { Morning } \\
\text { Thorpe }\end{array}$ & Norfolk & 6 th c & $\begin{array}{l}\text { Grave 6: } 2 \text { with annular } \\
\text { brooch pair and clasps; } \\
\text { Grave 92: } 4 \text { with annular } \\
\text { brooch, etc; } \\
\text { Grave 106: } 1 \text { with } 2 \\
\text { annular brooches; } \\
\text { Grave } 253: 2 \text { with } \AA \text { berg } \\
\text { Group IVa cruciform } \\
\text { brooch, etc }\end{array}$ & $\begin{array}{l}\text { Green et al } 1987, \\
36,62,65 \text { and } 106, \\
\text { figs } 296.6 . \text { Eiii, } \\
\text { 326.92.Cviii and ix, } \\
\text { 331.106.Jvi } \\
\text { and 381.253.Riii; } \\
\text { Norwich Museum }\end{array}$ \\
\hline
\end{tabular}

\begin{tabular}{|c|c|c|c|c|}
\hline Spong Hill & Norfolk & $\begin{array}{l}5 \text { th- } \\
6 \text { th c } \\
6 \text { th c } \\
\text { 6th c }\end{array}$ & $\begin{array}{l}\text { Grave } 24: 4 \text { with great } \\
\text { square-headed brooch; } \\
\text { Grave 26: } 41 \text { with Åberg } \\
\text { Group I cruciform brooch; } \\
\text { Grave } 38: 11 \text { with small- } \\
\text { long brooch and annular } \\
\text { brooch pair, etc; } \\
\text { Grave } 57: 1 \text { with Åberg } \\
\text { Group V cruciform brooch }\end{array}$ & $\begin{array}{l}\text { Hills et al } 1984 \text {, } \\
72-3,74,89-90,112, \\
\text { figs } 80.24 .2,83.1 \text {, } \\
92.8 b \text { and } 10 a \text {, and } 108.10 \\
\text { Norfolk Arch Unit }\end{array}$ \\
\hline Holdenby & Northants & $\begin{array}{l}\text { prob } \\
\text { early } \\
7 \text { th c }\end{array}$ & Many beads & $\begin{array}{l}\text { Leeds } 1909 ; \\
\text { Northampton Museum }\end{array}$ \\
\hline $\begin{array}{l}\text { Marston } \\
\text { St Lawrence }\end{array}$ & Northants & $\begin{array}{l}\text { later } \\
6 \text { th } c\end{array}$ & & $\begin{array}{l}\text { Dryden } 1849 \text { and } 1885 ; \\
\text { Peterborough Museum }\end{array}$ \\
\hline
\end{tabular}




\begin{tabular}{|c|c|c|c|c|}
\hline Wheatley & Oxon & $\begin{array}{l}\text { 6th- } \\
7 \text { th } c\end{array}$ & Grave 27 & $\begin{array}{l}\text { Leeds 1916; } \\
\text { Ashmolean Museum }\end{array}$ \\
\hline $\begin{array}{l}\text { Empingham } \\
\text { II }\end{array}$ & Rutland & $\begin{array}{l}\text { 5th- } \\
7 \text { th c }\end{array}$ & $\begin{array}{l}23 \text { graves contain such } \\
\text { beads }\end{array}$ & $\begin{array}{l}\text { Timby } 1996,48 \\
\text { Oakham Museum }\end{array}$ \\
\hline $\begin{array}{l}\text { Bury St } \\
\text { Edmunds, } \\
\text { Westgarth } \\
\text { Gardens }\end{array}$ & Suffolk & $\begin{array}{l}\text { prob } \\
\text { 6th c }\end{array}$ & $\begin{array}{l}\text { Grave } 57: 1 \text { double and } \\
2 \text { single }\end{array}$ & $\begin{array}{l}\text { West } 1988,35 \text {, fig } \\
79.57 .1,3 \text { and } 4 ; \\
\text { Bury St Edmunds } \\
\text { Museum }\end{array}$ \\
\hline $\begin{array}{l}\text { Little } \\
\text { Eriswell }\end{array}$ & Suffolk & 6th $\mathrm{c}$ & Graves 27 and 33 & $\begin{array}{l}\text { Hutchinson 1966; } \\
\text { Bury St Edmunds } \\
\text { Museum }\end{array}$ \\
\hline West Stow & Suffolk & $\begin{array}{l}5 \text { th- } \\
7 \text { th c }\end{array}$ & Cemetery: on 2 necklaces; & $\begin{array}{l}\text { West } 1985,74, \\
\text { fig } 275.18 \text { and } 19 ; \\
\text { Bury St Edmunds } \\
\text { Museum; } \\
\text { Ashmolean Museum; } \\
\text { West } 1985,73 \text {; } \\
\text { Bury St Edmunds } \\
\text { Museum }\end{array}$ \\
\hline Highdown & Sussex & 5th c & $\begin{array}{l}\text { Grave } 36: 2 \text { segments, } \\
\text { perhaps once joined with } \\
\text { small-long brooches }\end{array}$ & $\begin{array}{l}\text { Read 1895, 377; } \\
\text { Welch 1983, 82-5, } \\
\text { fig } 101 \mathrm{~b} ; \\
\text { Worthing Museum } 3481\end{array}$ \\
\hline $\begin{array}{l}\text { Bidford- } \\
\text { on-Avon }\end{array}$ & Warks & $\begin{array}{l}\text { 6th- } \\
\text { 7th } \mathrm{c}\end{array}$ & & $\begin{array}{l}\text { Humphreys et al 1923, } \\
\text { pl XVIII, and } \\
\text { 1924, pl LVIII; } \\
\text { Stratford-upon-Avon } \\
\text { Museum }\end{array}$ \\
\hline Wasperton & Warks & $\begin{array}{l}\text { late } \\
5 \text { th- } \\
6 \text { th c }\end{array}$ & Many 6th-century necklaces & $\begin{array}{l}\text { Publication in } \\
\text { preparation (M Carver); } \\
\text { Warwick Museum }\end{array}$ \\
\hline Charlton & Wilts & $\begin{array}{l}\text { 5th- } \\
\text { 6th c } \\
\text { 6th c }\end{array}$ & $\begin{array}{l}\text { Burial 24: } 2 \text { beads } \\
\text { (Class V); } \\
\text { Burial 94: } 4 \text { beads (Class } \\
\text { V) with amber beads }\end{array}$ & $\begin{array}{l}\text { Davies } 1984,120-2,122 \text {, } \\
139-40 \text {, figs } 9 \text { and } 11 E ; \\
\text { Salisbury Museum }\end{array}$ \\
\hline $\begin{array}{l}\text { Colling- } \\
\text { bourne } \\
\text { Ducis }\end{array}$ & Wilts & $\begin{array}{l}\text { 6th c } \\
\text { first } \\
\text { half } \\
\text { 6th c }\end{array}$ & $\begin{array}{l}\text { Grave 20: with cast saucer } \\
\text { brooch pair, etc; } \\
\text { Grave } 31 \text { : with button } \\
\text { brooch pair and } 3 \text { finger } \\
\text { rings, etc }\end{array}$ & $\begin{array}{l}\text { Gingell } 1975,85 \text { and } 90 \text {, } \\
\text { fig } 20.7 ; \\
\text { Devizes Museum }\end{array}$ \\
\hline $\begin{array}{l}\text { Lydiard } \\
\text { Tregoze }\end{array}$ & Wilts & $\begin{array}{l}\text { 6th } c \\
\text { etc }\end{array}$ & Several with saucer brooch, & $\begin{array}{l}\text { Goddard 1895; } \\
\text { Devizes Museum }\end{array}$ \\
\hline $\begin{array}{l}\text { Pewsey } \\
\text { Blackpatch }\end{array}$ & Wilts & $\begin{array}{l}\text { 5th- } \\
\text { 6th } c\end{array}$ & $\begin{array}{l}\text { Grave 53: a few and possibly } \\
\text { others }\end{array}$ & $\begin{array}{l}\text { Publication in } \\
\text { preparation (K Annable); } \\
\text { Devizes Museum }\end{array}$ \\
\hline
\end{tabular}


The Glass Beads of Anglo-Saxon England

\begin{tabular}{|c|c|c|c|c|}
\hline $\begin{array}{l}\text { Winter- } \\
\text { bourne } \\
\text { Gunner }\end{array}$ & Wilts & 6th $c$ & $\begin{array}{l}\text { Grave } 8: 20+\text { with Style I } \\
\text { applied saucer brooch } \\
\text { pair }\end{array}$ & $\begin{array}{l}\text { Musty and Stratton 1964, } \\
\text { 93, fig 7.b5 and 6; } \\
\text { Salisbury Museum }\end{array}$ \\
\hline $\begin{array}{l}\text { Woodyates, } \\
\text { Oakley } \\
\text { Down }\end{array}$ & Wilts & $\begin{array}{l}\text { prob } \\
\text { 6th c }\end{array}$ & Barrow I & $\begin{array}{l}\text { Devizes Museum 199, } \\
200 \text { and } 222 \mathrm{~b}\end{array}$ \\
\hline Driffield & Yorks & & & $\begin{array}{l}\text { Mortimer 1905, pls CXII and } \\
\text { CXIII; Hull Museum }\end{array}$ \\
\hline Saltburn & Yorks & $\begin{array}{l}\text { prob } \\
\text { 6th c }\end{array}$ & Now lost & Gallagher 1987,25, no. 97 \\
\hline Sewerby & Yorks & 6th $c$ & $\begin{array}{l}\text { Grave 15: } 2 \text { doubles with } \\
\text { Åberg Group IVa } \\
\text { cruciform brooch, etc; } \\
\text { Grave } 28: 5 \text { singles with } \\
\text { Åberg Group IVa cruciform } \\
\text { brooch, etc; } \\
\text { Grave } 49: 3 \text { singles and } 9 \\
\text { doubles with Class C2 } \\
\text { square-headed cruciform } \\
\text { brooch, etc }\end{array}$ & $\begin{array}{l}\text { Hirst 1985, } 66 \text { (Group B), } \\
\text { figs } 37 . c, 42.28 \text {, } \\
\text { 51i, 52, Strings I.d and e, and } \\
\text { II.f }\end{array}$ \\
\hline 'Yorks' & Yorks & & Some brownish gold & $\begin{array}{l}\text { Mortimer } 1905 \text {, pls CXII and } \\
\text { CXIII; Hull Museum }\end{array}$ \\
\hline $\begin{array}{l}\text { Dinas } \\
\text { Powys }\end{array}$ & $\begin{array}{l}\text { Wales: } \\
\text { Glam }\end{array}$ & $\begin{array}{l}5 \text { th- } \\
7 \text { th c }\end{array}$ & $\begin{array}{l}\text { Hillfort: post-Roman } \\
\text { occupation }\end{array}$ & $\begin{array}{l}\text { Alcock 1963, 186, } \\
\text { fig } 41.5\end{array}$ \\
\hline
\end{tabular}




\section{Bibliography}

Åberg, N 1926. The Anglo-Saxons in England during the early centuries after the invasion, Cambridge

Ahrens, C 1983. 'Der Befund des spätsächsischen Gräberfeldes Ketzendorf bei Buxtehude', Hammaburg, NF 5, 9-50

Akerman, J Y 1852. 'Remarks on a coloured drawing of some ancient beads, executed by Benjamin Nightingale, Esq., from specimens in his possession', Archaeologia, 34, 46-50, $\mathrm{pl} \mathrm{v}$

Akerman, J Y 1853. 'An account of excavations in an Anglo-Saxon burial ground at Harnham Hill, near Salisbury', Archaeologia, $35,259-78$

Akerman, J Y 1855a. Remains of Pagan Saxondom, London

Akerman, J Y 1855b. "Notes of antiquarian researches in the summer and autumn of 1854', Archaeologia, 36, 175-86

Akerman, J Y 1860. 'Report on researches in an Anglo-Saxon cemetery at Long Wittenham, Berkshire, in 1859', Archaeologia, 38, 327-52, pls XVII-Xx

Akerman, J Y 1865. 'Report of excavations in an ancient cemetery at Frilford, near Abingdon, Berks.', Proc Soc Antiq London, 2nd series, 3 (1864-7), 136-41

Alcock, L 1963. Dinas Powys: an Iron Age, Dark Age and Early Medieval Settlement in Glamorgan, Cardiff

Aldsworth, F R 1978. 'Droxford Anglo-Saxon cemetery', Proc Hampshire Fld Club Archaeol Soc, 35, 93-182

Aleksejva, E M J 1975. 'Antonyje busy severnogo Pricernomor'ja (Ancient beads of the northern Black Sea coast area)', USSR Archaeology/Arch. SSSR G1-12, 27-9, Moscow

Almgren, O 1923. Studien über nordeuropäische Fibelformen der ersten nachchristlichen Jahrhunderte: mit Berücksichtigung der provinzialrömischen und südrussischen Formen, Mannus Bibliothek, 32, Leipzig

Ament, H 1976. 'Chronologische Untersuchungen an fränkischen Gräberfeldern der jüngeren Merowingerzeit im Rheinland', Bericht der Römische-Germanischen Kommission, 57, 285-336
Ament, H 1980. 'The Germanic tribes in Europe', in The Northern World (ed D M Wilson), 49-70, London

Andrae, R 1973. 'Mosaikaugenperlen. Untersuchungen zur Verbreitung und Datierung karolingerzeitlicher Millefioriglasperlen in Europa', Acta Praehistorica et Archaeologica, 4, 101-98

Anon 1870-1. 'Cimetière belgo-romaine à Bioulx', Annales de la Société Archéologique de Namur, 11, 354-8

Anon 1888. 'The Essex Field Club visit to Colchester, Lexden, Layer Marney, etc., May 19th to 21st 1888', Essex Natur, 2, 115-36

Anon 1895. 'Nos fouilles 1895-1896', Annales de la Société Archéologique de Namur 21, 365-76 Anon 1900. 'Nos fouilles 1900-1902', Annales de la Société Archéologique de Namur 24, 464-72

Anon 1901. 'Saxon cemetery at Holdenby', J Northamptonshire Natur Hist Soc, 11 (1901-2), 5-7

Anon 1920. 'Researches and Discoveries in Kent, 1915-1919', Archaeol Cantiana, 34, 153-62

Anon 1937. 'A list of ancient beads found in Man', J Manx Mus, 3, no. 53 (December 1937), 237, pl 117A and B

Antoniewiez, J 1963. 'Wyniki bada przeprowadzonych w latach 1958-1960 na cmentarzysku w miejscowo ci Szwajcaria, PoW Suwa ki (Results of the 1958-1960 excavations of the Swajcaria cemetery, Suwa ki District)', Wiadomosci Archeologiczne, 29, 166-92 (English summary, 191-2)

Arbman, H 1940-3. Birka: Untersuchungen und Studien 1. Die Gräber, Stockholm

Arnold, C J 1980. 'Wealth and social structure: a matter of life and death in Anglo-Saxon cemeteries', in Anglo-Saxon Cemeteries 1979 (ed P Rahtz et al), 81-142, Oxford

Arnold, C J 1982. The Anglo-Saxon Cemeteries of the Isle of Wight, London

Artis, E T 1828. The Durobrivae of Antoninus identified and illustrated in a series of plates exhibiting the excavated remains of that Roman station in the vicinity of Castor, Northamptonshire, London

Arwidsson, G 1942. Vendelstile, Email und Glas im 7.-8. Jahrhundert, Valsgärdestudien I, Uppsala 
Aspinall, A and Warren, S E (eds) 1983. Proceedings of the 22nd International Symposium on Archaeometry and Archaeological Prospection, Bradford 1982, Bradford

Austin, W 1928. 'A Saxon cemetery at Luton, Beds.', Antiq J, 8, 177-92

Bassett, S (ed) 1989. The Origins of the Anglo-Saxon Kingdoms, Leicester

Bateson, J D and Hedges, R E M 1975. 'The scientific analysis of a group of Roman-age enamelled brooches', Archaeometry, 17, $177-90$

Bayley, J 1987. 'Qualitative analyses of some of the beads [Buckland, Dover cemetery]', in Dover: The Buckland Anglo-Saxon Cemetery (V I Evison), 182-9, London

Bayley, J and Wilthew, P 1986. 'Qualitative and Semiquantitative analyses of glass beads', Proceedings of the 24th International Archaeometry Symposium, Smithsonian Institution, 14.5.84-18.5.84, 55-62, Smithsonian Institution Press, Washington DC

Beck, H C 1928. 'Classification and nomenclature of beads and pendants', Archaeologia, 77, 1-76

Beck, H C 1933. 'Notes on three beads from Camerton cemetery', Antiq J, 13, 169-70

Beck, H C 1943. 'The Beads', in 'The Saxon monastery of Whitby' (C Peers and C A R Radford), 84

Beck, R T 1940. 'Unrecorded Saxon cemetery at Thurnham, Kent', Antiq J, 20, 380-2

Bencard, M, Ambrosiani, K, Jørgensen, L B, Madsen, H B, Nielsen, I and Näsman, U 1978. 'Wikingerzeitliches Handwerk in Ribe. Eine U'bersicht', Acta Archaeologica, 49, 113-38

Bencard, M and Jørgensen, L B 1990. 'The foundation of Ribe', Antiquity, 64, 576-83

Benea, D 1983. 'Die Glaswerkstätten von Tibiscum und der Perlenexport im 2-4 Jahrhundert', Apulum (Alba Intra), 21, 115-20

Benea, D 1997. 'Gie Glasperlenwerkstatt von Tibiscum und die Handelsbeziehungen mit dem Barbaricum', in Perlen. Archäologie, Techniken, Analysen. Akten des Internationalen Perlensymposiums in Mannheim vom 11. bis 14. November 1994 (eds U von Freeden and A Wieczorek), 279-92
Bennett, P 1980. 'Interim report on excavations in 1980 by the Canterbury Archaeological Trust: 4. 68-69a Stour Street', Archaeol Cantiana, 96, 406-10

Bequet, A 1886. 'Le cimetière romano-franc de Jamiolle', Annales de la Société Archéologique de Namur, 16, 392-4

Bequet, A 1895. 'Le cimetière franc de PryEssai historique', Annales de la Société Archéologique de Namur, 21, 311-36

Besborodov, M A 1957. 'A chemical and technical study of ancient Russian glasses and refractories', J Soc Glass Technol, 41, no. 199, $168 \mathrm{~T}-184 \mathrm{~T}$

Bidder, H F and Duckworth, W L H 1906. 'Excavations in an Anglo-Saxon burial ground at Mitcham, Surrey', Archaeologia, $60,49-68$

Bidder, H F and Morris, J, 1959. 'The Anglo-Saxon cemetery at Mitcham', Surrey Archaeol Collect, 56, 51-131

Biek, L 1983. 'The ethnic factor in archaeotechnology', in Proceedings of the 22nd International Symposium on Archaeometry and Archaeological Prospection, Bradford 1982 (eds A Aspinall and S E Warren), 303-15

Biek, L and Bayley, J 1979. 'Glass and other vitreous materials', World Archaeol, 11, no. 1, 1-25

Biek, L, Butcher, S A, Carruthers, T G, Rooksby, H P, Warren, S E, Crummett, J G, Hedges, R E M and Kaczmarczyk, A 1980. 'Enamels and glass pastes on Roman-period "bronzes" found at Nornour, Isles of Scilly', in Proceedings of the 16th International Symposium on Archaeometry and Archaeological Prospection, Edinburgh 1976 (eds E A Slater and J O Tate), 50-79, Edinburgh

Biek, L, Bayley, J and Gilmore, G 1985. 'Scientific examination of the glass beads', in An Anglo-Saxon Inhumation Cemetery at Sewerby, Yorkshire (S M Hirst), 77-85

Biek, $L$ and Kay, P J 1982. 'Evidence of glass melting', in Excavations at Catsgore 1970-1973: a Romano-British Village (ed R Leech), 132-3, Western Archaeological Trust, Bristol

Bimson, M 1983. 'Coloured glass and millefiori in the Sutton Hoo grave deposit', in The Sutton Hoo Ship-Burial (ed R Bruce-Mitford), 3, 924-44, London 
Bloemers, J H F, Louwe Kooijmans, L P and Sarfatij, H 1981. Verleden Land. Archeologische opgravingen in Nederland, Amsterdam

Bloxham, M H 1884. On certain ancient British, Roman, and Anglo-Saxon pagandom remains, mostly sepulchral, found in Warwickshire, chiefly in the region of Rugby

Böhme, H W 1974. Germanische Grabfunde des 4. bis 5. Jahrhunderts zwischen unterer Elbe und Loire, Münchener Beiträge zur Vor- und Frühgeschichte, 19, Munich

Böhme, H W 1976. 'Das Land zwischen Elb- und Wesermündung vom 4 bis $6 \mathrm{Jh}$. Die Sachsen und ihre Beziehungen zum römischen Westen', in Führer zu vor- und frühgeschichtlichen Denkmälern, 29: Das Elb-Weser Dreieck I, 205-26, Mainz

Böhme, H W 1986. 'Das Ende der Römerherrschaft in Britannien und die angelsächsische Besiedlung Englands im 5. Jahrhundert', Jahrbuch des Römisch-Germanischen Zentralmuseums Mainz, 33, 466-574

Böhme, H W 1987. 'Gallien in der Spätantike', Jahrbuch des Römisch-Germanischen Zentralmuseums Mainz, 34, 770-3

Böhner, K 1958. Die fränkischen Altertümer des Trierer Landes, RGK Germanische Denkmäler der Völkerwanderungszeit, Serie B, Band 1, Berlin

Boon, G C 1959. 'The latest objects from Silchester, Hants.', Medieval Archaeol, 3, 79-88

Boon, G C 1966. 'Gilt glass beads from Caerleon and elsewhere', Bull Board Celtic Stud, 22 (1966-8), pt l, 104-9, pl vi

Boon, G C 1977. 'Gold-in-glass beads from the ancient world', Britannia, 8, 193-207

Boulanger, C 1902-5. Le mobilier funéraire Gallo-Romain et Franc en Picardie et en Artois, Paris

Boyle, A, Miles, D and Palmer, S forthcoming. The Anglo-Saxon Cemetery at Butler's Field, Lechlade, Gloucestershire

Bradford, J S P and Goodchild, R G 1939. 'Excavations at Frilford, Berks., 1937-8', Oxoniensia, 4, 1-70

Brenan, J 1991. Hanging Bowls and their Contexts, BAR Brit Ser, 220, Oxford

Brent, J 1863. 'Account of the Society's researches in the Saxon cemetery at Sarr', Archaeol Cantiana, 5, 305-22
Brent, J 1866. 'Account of the Society's researches in the Anglo-Saxon cemetery at Sarr', Archaeol Cantiana, 6, 157-85

Brent, J 1867. 'Researches in an Anglo-Saxon cemetery at Stowting, in Kent during the autumn of 1866', Archaeologia, 41, 409-20

Brent, J 1868. 'Account of the Society's researches in the Anglo-Saxon cemetery at Sarr', Archaeol Cantiana, 7, 307-21, pls viI-XIv

Briggs, C S 1985. 'A neglected Viking burial with beads from Kilmainham, Dublin, discovered in 1847', Medieval Archaeol, 29, 94-109

Brill, R H 1970. 'The chemical interpretation of the texts', in Glass and Glassmaking in Ancient Mesopotamia (A L Oppenheim, R H Brill, D Barag and A van Saldern), 105-28, Corning, New York

Brinch Madsen, H 1975. 'En nordjysk kvindegrav fra omkring 400 e. Kr', Hikuin, 2, 123-30

Brown, D 1975. 'A fifth-century burial at Kingsholm', in 'Excavations at Gloucester, third interim report, Kingsholm 1966-75' (H Hurst), 290-4

Brown, D 1976. 'Archaeological evidence for the Anglo-Saxon period', in Studies in the Archaeology and History of Cirencester (ed A McWhirr), BAR Brit Ser, 30, 19-45, Oxford

Brown, D 1981. 'Swastika patterns', in Angles, Saxons and Jutes (ed V I Evison), 227-40, Oxford

Brown, G B 1915a. The Arts in Early England III-IV: Saxon Art and Industry in the Pagan Period, London

Brown, G B 1915b. 'Notes on a necklace of glass beads found in a cist in Dalmeny Park, South Queensferry', Proc Soc Antiq Scoth 49 (1914-15), 332-8

Bruce, J C 1880. A Descriptive Catalogue of Antiquities, chiefly British, at Alnwick Castle, Newcastle upon Tyne

Bruce-Mitford, R 1974. Aspects of Anglo-Saxon Archaeology, London

Buckley, D G 1980. Archaeology of Essex to $A D$ 1500, CBA Res Rep, 34, London

Buckley, D G and Hedges, I D 1987. The Bronze Age and Saxon Settlements at Springfield Lyons, Essex: an interim report, Essex County Council Occas Pap, 5, Chelmsford

Bushe-Fox, J P 1926. First Report on the Excavation of the Roman Fort at Richborough, 
Kent, Rep Res Comm Soc Antiq London, 5, London

Bushe-Fox, J P 1928. Second Report on the Excavation of the Roman Fort at Richborough, Kent, Rep Res Comm Soc Antiq London, 7, London

Bushe-Fox, J P 1932. Third Report on the Excavation of the Roman Fort at Richborough, Kent, Rep Res Comm Soc Antiq London, 10, Oxford

Bushe-Fox, J P 1949. Fourth Report on the Excavation of the Roman Fort at Richborough, Kent, Rep Res Comm Soc Antiq London, 16, Oxford

Callander, J G 1921. 'Report on the excavation of Dun Beag, a broch near Struan, Skye', Proc Soc Antiq Scotl, 55 (1920-1), 110-31

Callmer, J 1977. Trade Beads and Bead Trade in Scandinavia ca.800-1000 AD, Acta Archaeologica Lundensia, S. in Quarto, 11, Lund

Callmer, J, Henderson, J and Näsman, U forthcoming. The Viking Glass from Ahus and Ribe

Campbell, E and Lane, A 1993. 'Celtic and Germanic interaction in Dalriada: the 7th-century metalworking site at Dunadd', in The Age of Migrating Ideas: Early Medieval Art in Northern Britain and Ireland: Proceedings of the Second International Conference on Insular Art held in the National Museums of Scotland in Edinburgh, 3-6 January 1991

(eds S R M Spearman and J Higgitt), 52-63, Edinburgh

Campbell, E and Lane, A forthcoming. Excavations at Dunadd, Mid-Argyll, 1980 and 1981

Carr, R D, Tester, A and Murphy, P 1988. 'The Middle-Saxon settlement at Staunch Meadow, Brandon', Antiquity, 62, 371-7

Carver, M O H 1992. 'The Anglo-Saxon cemetery at Sutton Hoo: an interim report', in The Age of Sutton Hoo: the Seventh Century in North-western Europe (ed M O H Carver), 343-71, Woodbridge

Chadwick, S E 1958. 'The Anglo-Saxon cemetery at Finglesham, Kent: a reconsideration', Medieval Archaeo L, 2, 1-71

Charleston, R J 1963. " "Glass cakes" ' as raw materials and articles of trade', J Glass Stud, 5, 54-67

Chenet, G 1920. 'Anciennes verreries d'Argonne', Bulletin archéologique du Comité des Travaux Historiques et Scientifiques, 253-86, Paris

Christlein, R 1966. Das alamannische Reihengräberfeld von Marktoberdorf im Allgäu, Materialhefte zur bayerischen Vorgeschichte, Heft 21, Kallmünz

Clarke, G 1979. The Roman Cemetery at Lankhills, Pre-Roman and Roman Winchester, Winchester Studies 2, Oxford

Close-Brooks, J 1986. 'Excavations at Clatchard Craig, Fife', Proc Soc Antiq Scoth 116, 117-84

Clough, T H McK, Dornier, A and Rutland, R A 1975. Anglo-Saxon and Viking Leicestershire including Rutland, Leicester

Conyngham, A and Akerman, J Y 1844. 'An account of the opening and examination of a considerable number of tumuli on Breach Downs, in the county of Kent', Archaeologia, $30,47-56$

Cook, A M 1981. The Anglo-Saxon Cemetery at Fonaby, Lincolnshire, Occas Pap Lincolnshire Hist Archaeol, 6, Sleaford

Cook, A M and Dacre, M W 1985. Excavations at Portway, Andover 1973-1975, Oxford Univ Comm Archaeol Monogr, 4, Oxford

Cook, J M 1958. 'An Anglo-Saxon cemetery at Broadway Hill, Broadway, Worcestershire', Antiq J, 38, 58-84

Cosack, E 1982. Das sächsische Gräberfeld bei Liebenau, Kr. Nienburg (Weser), RGK Germanische Denkmäler der Völkerwanderungszeit, Serie A, Band 15, Berlin

Cotton, A R 1933. 'Saxon discoveries at Fetcham', Antiq J, 13, 48-51

Craddock, J 1979. 'The Anglo-Saxon cemetery at Saxonbury, Lewes, East Sussex', Sussex Archaeol Collect, 117, 85-102

Cramp, R J 1970a. 'Glass finds from the Anglo-Saxon monastery at Monkwearmouth and Jarrow', Studies in Glass History and Design, 8th International Congress on Glass, London 1968, 16-19, Sheffield

Cramp, R J 1970b. 'Decorated window-glass and millefiori from Monkwearmouth', Antiq J, 50, 327-35

Cramp, R J 1975. 'Window-glass from the monastic site of Jarrow', J Glass Stud, 17, 88-96

Cramp, R J and Miket, R 1982. Catalogue of the Anglo-Saxon and Viking Antiquities in the Museum of Antiquities, Newcastle upon Tyne, Newcastle upon Tyne 
Craw, J H 1930. 'Excavations at Dunadd and at other sites on the Poltalloch Estates, Argyll', Proc Soc Antiq Scotl, 64, 111-46

Crawford, G 1981. 'Wasperton, Warwickshire', W Midlands Archaeol, 24, 121-9

Crawford, G 1982. 'Excavations at Wasperton: Second Interim Report', W Midlands Archaeol, 25, 30-44

Crawford, G 1983. 'Excavations at Wasperton: Third Interim Report', W Midlands Archaeol, 26, 15-28

Crummy, N 1983. The Roman Small Finds from Excavations in Colchester 1971-9, Colchester Archaeol Rep, 2, Colchester

Crummy, P 1981. Aspects of Anglo-Saxon and Norman Colchester, Colchester Archaeological Report, 1, CBA Res Rep, 39, London

Cunliffe, B 1968. Fifth Report on the Excavations of the Roman Fort at Richborough, Kent, Rep Res Comm Soc Antiq London, 23, London

Cunnington, M E and Goddard, E H 1934. Catalogue of Antiquities in the Museum of the Wiltshire Archaeological and Natural History Society at Devizes Part II, Devizes

Curle, J 1911. A Roman Frontier Post and its People: the Fort of Newstead in the Parish of Melrose, Glasgow

Dannheimer, H 1962. Die germanischen Funde der späten Kaiserzeit und des frühen

Mittelalters in Mittelfranken, RGK

Germanische Denkmäler der Völkerwanderungszeit, Serie A, Band 7, Berlin

Davidson, H R E and Webster, L E 1967. 'The Anglo-Saxon burial at Coombe (Woodnesborough), Kent', Medieval Archaeol, 2, 1-41

Davies, S M 1984. 'The excavation of an Anglo-Saxon cemetery and some prehistoric pits at Charlton Plantation near Downton', Wiltshire Archaeol Natur Hist Mag, 79, 109-54

Davison, A, Green, B and Milligan, W 1993. Illington: a Study of a Breckland Parish and its Anglo-Saxon Cemetery, E Anglian Archaeol Rep, 63, Norwich

Delamain, P 1892. Le cimetière d'Herpes, Société archéologique et historique de la Charente, Angoulême

Denny, H 1866. 'Tumuli on the Hambledon Hills, near Thirsk', Proc Geol Soc and
Polytechnic Soc W Riding Yorkshire, 4 (1859-68), 488-502

Diaconu, G 1969. 'Das Gräberfeld von Mogoşani (Kreis Dîmboviţa)', Dacia, 13, 367-402

Dickinson, T M 1973. 'Excavations at Standlake Down in 1954', Oxoniensia, 38, 239-57

Dickinson, T M 1976. 'The Anglo-Saxon burial sites of the upper Thames region'. Unpublished DPhil thesis, Oxford

Dickinson, T M 1979. 'On the origin and chronology of the early Anglo-Saxon disc brooch', in Anglo-Saxon Studies in Archaeology and History, 1 (eds S C Hawkes, D Brown and J Campbell), BAR Brit Ser, 72, 39-80, Oxford

Dickinson, T M 1980. Review in Archaeol J, 187, 485-8

Dierkens, A 1981. Les deux cimetières mérovingiens de Franchimont (Prov. de Namur). Fouilles de 1877-78, Namur

Drinkall, $\mathrm{G}$ and Foreman, $\mathrm{M}$ forthcoming. The Anglo-Saxon Cemetery at Castledyke South, Barton-on-Humber, Sheffield

Down, A and Welch, M G 1990. Chichester Excavations 7: Apple Down and the Mardens, Chichester

Dryden, $\mathrm{H}$ 1849. 'An account of a discovery of early Saxon remains at Barrow Furlong, on the Hill Farm, in the parish of Marston St Lawrence, in the county of Northampton', Archaeologia, 33, 326-34, pls XII-XIII

Dryden, H 1885. 'Excavation of an ancient burial ground at Marston St Lawrence, co. Northampton', Archaeologia, 48, 327-39, pls XXIII-XXV

Eagles, B N and Evison, V I 1970. 'Excavations at Harrold, Bedfordshire, 1951-3', Bedfordshire Archaeol 5, 17-55

Elgee, $\mathrm{F}$ and Elgee, $\mathrm{H} \mathrm{W}$ 1933. The Archaeology of Yorkshire, London

van Es, W A and Ypey, J 1977. 'Das Grab der "Prinzessin" von Zweeloo und seine Bedeutung im Rahmen des Gräberfeldes', Studien zur Sachsenforschung, 1, 97-126

Esmonde Cleary, A S 1989. The Ending of Roman Britain, London

Evison, V I 1955. 'Anglo-Saxon finds near Rainham, Essex, with a study of drinking horns', Archaeologia, 96, 159-95, pls LIx-LXIx

Evison, V I 1956. 'An Anglo-Saxon cemetery at Holborough, Kent', Archaeol Cantiana, 70, 84-141 
Evison, V I 1965. The Fifth-Century Invasions South of the Thames, London

Evison, V I 1967. 'The Dover Ring Sword and other sword rings and beads', Archaeologia, 101, 63-108

Evison, V I 1972. 'Glass cone beakers of the "Kempston" type', J Glass Stud, 14, 48-66

Evison, V I 1975. 'Sword rings and beads', Archaeologia, 105, 303-15

Evison, V I 1978. 'Early Anglo-Saxon applied disc brooches in England Part II', Antiq J, 58, 260-78

Evison, V I 1981. 'Distribution maps and England in the first two phases', in Angles, Saxons and Jutes (ed V I Evison), 126-67, Oxford

Evison, V I 1982a. 'Anglo-Saxon glass claw-beakers', Archaeologia, 107, 43-76

Evison, V I 1982b. 'Bichrome glass vessels of the seventh and eighth centuries', Studien zur Sachsenforschung, 3, 7-21

Evison, V I 1983. 'Some distinctive glass vessels of the post-Roman period', J Glass Stud, 25, 87-93

Evison, V I 1987. Dover: The Buckland Anglo-Saxon Cemetery, Hist Build Monuments Comm Archaeol Rep, 3, London

Evison, V I 1988a. An Anglo-Saxon Cemetery at Alton, Hampshire, Hampshire Fld Club Archaeol Soc Monogr, 4, Winchester

Evison, V I 1988b. 'Some Vendel, Viking and Saxon glass', in Trade and Exchange in Prehistory, Acta Archaeologica Lundensia, series in $8^{\circ}, 16$ (eds B Hårdh, L Larsson, D Olavson and R Petre), 237-45, Lund

Evison, V I 1994. An Anglo-Saxon Cemetery at Great Chesterford, Essex, CBA Res Rep, 91, York

Faussett, B 1856. Inventorium Sepulchrale: an account of some antiquities dug up at Gilton, Kingston, Sibertswold, Barfriston, Beakesbourne, Chartham, and Crundale, in the County of Kent, from $A D 1757$ to $A D 1773$ (edited by C R Smith), London

Fennell, K R 1964. 'The Anglo-Saxon cemetery at Loveden Hill, Lincs., and its signification in relation to the Dark Age settlement of the East Midlands'. Unpublished PhD thesis, Nottingham

Fennell, K R forthcoming. The Anglo-Saxon Cemetery at Loveden Hill, Hough-on-the-Hill, Lincolnshire

Filmer-Sankey, W 1988. From the Dust of Earth
Returning: Excavations of the Anglo-Saxon Cemetery at Snape, Suffolk

Filmer-Sankey, W 1990. 'Snape', Curr Archaeoh, $118,348-52$

Filmer-Sankey, $\mathrm{W}$ and Pestell, T forthcoming. Snape Anglo-Saxon Cemetery Excavations and Surveys 1824-1991, E Anglian Archaeol Rep

Fitch, S E 1864. 'Discovery of Saxon remains at Kempston', Rep Associated Architect Societies, 7, 269-99

Forbes, R J 1966. Studies in Ancient Technology, $v, 112-236$, Leiden

Foster, W K 1880. 'Account of the excavation of an Anglo-Saxon cemetery at Barrington, Cambridgeshire', Cambridge Antiq Comm, 5 (1880-4), 5-32, pls I-XII

Fowler, G 1948. 'Cratendune: a problem of the Dark Ages', Proc Cambridge Antiq Soc, 41, 70-3, pls xviII-xx

Fox, C 1923. The Archaeology of the Cambridge Region, Cambridge

Franken, M 1944. Die Alamannen zwischen Iller und Lech, RGK Germanische Denkmäler der Völkerwanderungszeit, Serie A, Band 5, Berlin

von Freeden, U and Wieczorek, A (eds) 1997. Perlen. Archäologie, Techniken, Analysen. Akten des Internationalen Perlensymposiums in Mannheim vom 11. bis 14. November 1994, Bonn

Fremersdorf, F 1955. Das fränkische Reihengräberfeld Köln-Mungersdorf, RGK Germanische Denkmäler der Völkerwanderungszeit, Serie A, Band 6, Frankfurt

Frere, S S and St Joseph, J K 1974. 'The Roman fortress at Longthorpe', Britannia, 5, 1-129

Gallagher, D B 1987. 'The Anglo-Saxon cemetery of Hob Hill, Saltburn', Yorkshire Archaeol J, $59,9-27$

Gam, T 1990. 'Perlemager af fag', Skalk, no. 1, 12-15

Gardner, W and Savory, H N 1964. Dinorben: $a$ hill fort occupied in early Iron Age and Roman times, Cardiff

Gasparetto, A 1965. 'Les fouilles de Torcello et leur apport à l'histoire de la verrerie de la Venetie dans la Haut Moyen Age', Proceedings of the 7th International Congress on Glass. paper no. 239, Brandeis, New York

Gasparetto, A 1967. 'A proposito dell'officina vetraria Torcellana - Forni e sistemi difusioni antichi', J Glass Stud, 9, 50-75 
Gelling, M 1992. The West Midlands in the Early Middle Ages, Leicester

George, T 1903. 'Exhibited a series of bronze ornaments from Anglo-Saxon burials at Duston', Proc Soc Antiq London, 2nd series, 19 (1901-3), 310-14

Gillam, J 1979. 'Romano-Saxon pottery: an alternative interpretation', in The End of Roman Britain (ed P J Casey), BAR Brit Ser, 71, 103-18, Oxford

Gillies, K J S 1982. 'The application of x-ray photoelectron spectroscopy in archaeology'. Unpublished $\mathrm{PhD}$ thesis, London

Gingell, C 1975. "The excavation of an early Anglo-Saxon cemetery at Collingbourne Ducis', Wiltshire Archaeol Natur Hist Mag, 70-1 (1975-6), 61-98

Goddard, E H 1895. 'Notes on objects from a Saxon interment at Basset Down', Wiltshire Archaeol Natur Hist Mag, 28, 1894-6, 104-8

Goethert-Polaschek, K 1980. Römische Gläser im Rheinischen Landesmuseum Trier, Trier

Goldstein, S M 1979. Pre-Roman and Early Roman Glass in the Corning Museum of Glass, Corning, New York

Gollub, S 1970. 'Ein neuer fränkischer Friedhof bei Newel, Krs. Trier', Trierer Zeitschrift, 33, 57-124

Green, B and Rogerson, A 1978. The Anglo-Saxon Cemetery at Bergh Apton, Norfolk, E Anglian Archaeol Rep, 7, Gressenhall

Green, B, Rogerson, A and White, S 1987. The Anglo-Saxon Cemetery at Morning Thorpe, Norfolk, E Anglian Archaeol Rep, 36, Gressenhall

Green, L H and Hart, A F 1987. 'Colour and chemical composition in ancient glass: an examination of some Roman and Wealden glass by means of ultraviolet-visible-infra-red spectroscopy and electron microprobe analysis', J Archaeol Sci, 14, 271-82

Green, M J 1975. The Bradwell Roman Villa. First Interim Report, Milton Keynes Devel Corp Occas Pap Archaeol, 1, Milton Keynes

Griffith, A F 1915. 'An Anglo-Saxon cemetery at Alfriston, Sussex: supplemental paper', Sussex Archaeol Collect, 57, 197-210

Griffith, A F and Salzmann, L F 1914. 'An Anglo-Saxon cemetery at Alfriston, Sussex', Sussex Archaeol Collect, 56, 16-53

Guido, M 1978. The Glass Beads of the Prehistoric and Roman Periods in Britain and Ireland,
Rep Res Comm Soc Antiq London, 35, London

Guido, M 1979. 'Glass beads [Lankhills]', in The Roman Cemetery at Lankhills, Pre-Roman and Roman Winchester (G Clarke), 292-4 and 297-300

Guido, M 1985. 'The Glass beads

[Kilmainham]', in 'A neglected Viking burial with beads from Kilmainham, Dublin, discovered in 1847' (C S Briggs), 101-3

Guido, M 1986. 'Glass bead [Clatchard Craig]', in 'Excavations at Clatchard Craig, Fife' (J Close-Brooks), 167

Guido, M 1987. 'The coloured glass [Ower]', in 'The excavation of a late Iron Age trading settlement and Romano-British pottery production site at Ower, Dorset' (P J Woodward), 100-1

Guido, M 1989. 'Comment [Swallowcliffe Down]', in A Saxon Bed Burial on Swallowcliffe Down (G Speake), 51-2

Guido, M 1992. 'Beads [Cadbury Congresbury]', in Cadbury Congresbury 1968-73. A

late/post-Roman hilltop settlement in Somerset (P Rahtz et al), 143-4

Guido, $M$ forthcoming (a). 'The glass beads from Cakebread Robey V, Canterbury', The Archaeology of Canterbury monogr rep ser

Guido, $M$ forthcoming (b). 'The glass beads from Mint Yard, Canterbury', The Archaeology of Canterbury monogr rep ser

Guido, $M$ and Welch, M G forthcoming. 'Indirect evidence for glass-bead manufacture in early Anglo-Saxon England', in Glass in Britain and Ireland, AD 350-1100 (ed J Price), Brit Mus Occas Pap, London

Haevernick, T E 1960. Die Glasarmringe und Ringperlen der Mittel- und Spätlatènezeit auf dem europäischen Festland, Bonn

Haith, C 1988. 'Un nouveau regard sur le cimetière d'Herpes (Charente)', Revue archéologique de Picardie, 3-4, 71-80

Hamlin, A and Lynn, C 1988. Pieces of the Past, Belfast

Harden, D B 1940. 'Notes and News: Wallingford, Berks.', Oxoniensia, 5, 164

Harden, D B 1956. 'Glass vessels in Britain and Ireland AD 400-1000', in Dark Age Britain: Studies presented to E. T. Leeds (ed D B Harden), 132-67, London

Harden, D B 1968a. 'The Canosa group of Hellenistic glasses in the British Museum', J Glass Stud, 10, 21-47 
Harden, D B 1968b. 'Ancient glass I: pre-Roman', Archaeol J, 125, 46-72

Harden, D B 1969. 'Ancient glass II: Roman', Archaeol J, 126, 44-77

Harden, D B 1971. 'Ancient glass III: post-Roman', Archaeol J, 128, 78-117

Härke, H 1992. Angelsächsische Waffengräber des 5. bis 7.Jahrhunderts, Cologne

Hässler, H-J 1983a. 'Das sächsische Gräberfeld bei Liebenau, Kreis Nienburg (Weser) Teil 2', Studien zur Sachsenforschung, 5.1

Hässler, H-J 1983b. 'Das sächsische Gräberfeld bei Liebenau, Kreis Nienburg (Weser) Teil 3', Studien zur Sachsenforschung, 5.2

Hässler, H-J 1985. 'Das sächsische Gräberfeld bei Liebenau, Kreis Nienburg (Weser) Teil 4', Studien zur Sachsenforschung, 5.3

Hawkes, S C 1969. 'Early Anglo-Saxon Kent', Archaeol J, 126, 186-9

Hawkes, S C 1973. 'The dating and social significance of the burials in the Polhill cemetery', in 'The Anglo-Saxon cemetery at Polhill, Dunton Green, Kent' (S C Hawkes and B J Philp), 186-201

Hawkes, S C 1974. 'The Monkton brooch', Antiq J, 54, 245-56

Hawkes, S C 1979. 'Eastry in Anglo-Saxon Kent: its importance and a newly found grave', in Anglo-Saxon Studies in Archaeology and History, 1 (eds S C Hawkes, D Brown and J Campbell), BAR Brit Ser, 72, 81-113, Oxford

Hawkes, S C 1981. 'The bronze objects (Guildford Road Estate, Colchester)', in Aspects of Anglo-Saxon and Norman Colchester (P Crummy), 10-11

Hawkes, S C 1990a. 'Bryan Faussett and the Faussett Collection: an assessment', in Anglo-Saxon Cemeteries: A Reappraisal (ed E Southworth), 1-24, Liverpool

Hawkes, S C 1990b. 'The Anglo-Saxon necklace from Lower Brook Street', in Object and Economy in Medieval Winchester. Artefacts from Medieval Winchester (ed M Biddle), 622-32, pls XLVI-XLVII

Hawkes, S C and Grove, L R A 1963. 'Finds from a seventh-century Anglo-Saxon cemetery at Milton Regis', Archaeol Cantiana, 78, 22-38

Hawkes, S C and Hogarth, A C 1974. 'The Anglo-Saxon cemetery at Monkton, Thanet. Report on the rescue excavations of May/June 1971', Archaeol Cantiana, 101, 49-89
Hawkes, S C and Philp, B J 1973. 'The Anglo-Saxon cemetery at Polhill, Dunton Green, Kent', in Excavations in West Kent 1960-1970 (ed B J Philp), 164-214, Dover

Hawkes, S C and Pollard, M 1981. 'The gold bracteates from sixth-century Anglo-Saxon graves in Kent, in the light of a new find from Finglesham', Frühmittelalterliches Studien, 15, 316-70

Hawthorne, J G and Smith, C S 1963. On Divers Arts. The Treatise of Theophilus, Chicago

Heather, P and Matthews, J 1991. The Goths in the Fourth Century, Liverpool

Hencken, H O'N 1950. 'Lagore Crannog: an Irish royal residence of the 7 th to 10 th centuries AD', Proc Roy Ir Acad, C, 53, 1-247

Henderson, J 1985. 'The raw materials of early glass production', Oxford J Archaeoh 4 (3), 267-91

Henderson, J 1986. Section on 'Beads and rings' in Finds from Parliament Street and other sites in the City Centre (D Tweddle), 209-27

Henderson, J 1987a. 'The nature of the early Christian glass industry in Ireland: some evidence from Dunmisk Fort, Co. Tyrone', Ulster J Archaeol, 51, 115-26

Henderson, J 1987b. "The Iron Age of "Loughey" and Meare: some inferences from glass analysis', Antiq J, 67, 29-42

Henderson, J 1988. 'Glass production and Bronze Age Europe', Antiquity, 62, no. 236, 435-51

Henderson, J 1989. 'The scientific analysis of ancient glass and its archaeological interpretation', in Scientific Analysis in Archaeology and its Interpretation (ed J Henderson), 30-58, Oxford

Henderson, J 1990. 'The scientific investigation of the glass beads from the Apple Down Anglo-Saxon cemetery', in Chichester Excavations 7: Apple Down and the Mardens (A Down and M G Welch), 156-65

Henderson, J 1991. 'Industrial specialisation in late Iron Age Britain and Europe', Archaeol J, $148,104-48$

Henderson, J 1993. 'Aspects of early medieval glass production in Britain', Annales du $12 e$ Congrès de l'Association Internationale pour l'Histoire du Verre, Vienna 26th-31st August 1991, 247-59, Amsterdam

Henderson, J and Holand, I 1992. 'The glass from Borg, an early medieval chieftain's farm 
in northern Norway', Medieval Archaeol, 36, 29-58

Henderson, J and Ivens, R 1992. 'Dunmisk and glass-making in early Christian Ireland', Antiquity, 66, 52-64

Henderson, J and Warren, S E 1981. 'X-ray fluoresence analysis of Iron Age glass: beads from Meare and Glastonbury lake villages', Archaeometry, 23, 83-94

Henderson, J and Warren, S E 1983. 'Analysis of prehistoric lead glass', in Proceedings of the 22nd International Symposium on Archaeometry and Archaeological Prospection, Bradford 1982 (eds A Aspinall and S E Warren), 168-80

Henry, F 1938. 'Deux objets de bronze irlandais au Musée des Antiquités Nationales', Préhistoire, 6, 65-91

Herrmann, J 1980. 'The northern Slavs', in The Northern World (ed D M Wilson), 183-206, London

Hills, C 1977. The Anglo-Saxon Cemetery at Spong Hill, North Elmham: Part I Catalogue of Cremations, E Anglian Archaeol Rep, 6, Gressenhall

Hills, C and Hurst, H 1989. 'A Goth at Gloucester?', Antiq J, 69, 154-8

Hills, C and Penn, K 1981. The Anglo-Saxon Cemetery at Spong Hill, North Elmham: Part II Catalogue of Cremations, E Anglian Archaeol Rep, 11, Gressenhall

Hills, C, Penn, K and Rickett, R 1984. The Anglo-Saxon Cemetery at Spong Hill, North Elmham: Part III Catalogue of Inhumations, E Anglian Archaeol Rep, 21, Gressenhall Hills, C, Penn, K and Rickett, R 1987. The Anglo-Saxon Cemetery at Spong Hill, North Elmham: Part IV Catalogue of Cremations, E Anglian Archaeol Rep, 34, Gressenhall

Hills, C, Penn, K and Rickett, R 1994. The Anglo-Saxon Cemetery at Spong Hill, North Elmham: Part V Catalogue of Cremations, E Anglian Archaeol Rep, 67, Gressenhall

Hilton, S 1980. The Anglo-Saxon Cemeteries at Horton Kirby, Kent. Unpublished BA dissertation, Institute of Archaeology, London

Hines, J 1984. The Scandinavian Character of Anglian England in the pre-Viking Period, BAR Brit Ser, 124, Oxford

Hinton, D A 1993. 'A smith's hoard from Tattershall Thorpe, Lincolnshire', Anglo-Saxon Engh 22, 147-67
Hirst, S M 1985. An Anglo-Saxon Inhumation Cemetery at Sewerby, Yorkshire, York Univ Archaeol Publ, 4, York

Hirst, S M and Biek, L 1981. 'Investigation of a glass-bead assemblage from an Anglo-Saxon cemetery near York', Revue d'Archéométrie, Supplément, 139-46

Hirst, S M and Clark, D forthcoming. Excavations at Mucking 3: The Anglo-Saxon Cemeteries, Engl Heritage Archaeol Rep

Hoare, R C 1812. The Ancient History of Wiltshire, Vol.1 South Wiltshire, London

Holleyman, G A 1935. 'Romano-British site on Wolstonbury Hill', Sussex Archaeol Collect, $76,35-45$

Hooke, D 1986. The Anglo-Saxon Landscape: the Kingdom of the Hwicce, Manchester

Hope-Taylor, B 1977. Yeavering: An Anglo-British Centre of Early Northumbria, Department Environment Archaeol Rep, 7, London

Horne, E 1928. 'Saxon cemetery at Camerton, Somerset', Proc Somersetshire Archaeol Natur Hist Soc, 74, 61-70

Horne, E 1933. 'An Anglo-Saxon cemetery at Camerton, Somerset, Part II', Proc Somersetshire Archaeol Natur Hist Soc, 79, $39-63$

Hornsby, W 1912. 'An Anglian cemetery at Hob Hill, near Saltburn', Yorkshire Archaeol J, 22, 131-7

Hruby, V 1955. Staré Mesto: velkomoravské pohrebište 'na valách', Monumenta Archaeologia 3, Prague

Huband, H R 1925. 'Farnham discoveries during 1924', Surrey Archaeol Collect, 36, 123-4

Huggett, J W 1988. 'Imported grave goods and the early Anglo-Saxon economy', Medieval Archaeol 32, 63-96

Hughes, M J 1972. 'A technical study of opaque red glass of the Iron Age in Britain', Proc Prehist Soc, 38, 98-107

Hughes, T McK 1901. 'Report [Hunstanton]', Proc Soc Antiq London, 2nd series, 18 (1899-1901), 310-21

Humphreys, J, Ryland, J W, Barnard, E A B, Wellstood, F C and Barnett, T G 1923. 'An Anglo-Saxon cemetery at Bidford-on-Avon, Warwickshire', Archaeologia, 73, 89-116 Humphreys, J, Ryland, J W, Wellstood, F C, Barnard, E A B and Barnett, T G 1924. 'An Anglo-Saxon cemetery at Bidford-on-Avon, 
Warwickshire. Second report on the excavations', Archaeologia, 74, 271-88

Hurd, H 1913. Some Notes on Recent Archaeological Discoveries at Broadstairs, Broadstairs

Hurk, L J A M van den 1980. 'The tumuli from the Roman period of Esch, Province of North Brabant, IV', Berichten van de Rijksdienst voor het Oudheidkundig Bodemonderzoek, 30, 367-92

Hurst, H 1975. 'Excavations at Gloucester, third interim report, Kingsholm 1966-75', Antiq J, 55, 267-94

Hutchinson, P 1966. 'The Anglo-Saxon cemetery at Little Eriswell, Suffolk', Proc Cambridge Antiq Soc, 54, 1-32

Hyslop, M 1963. 'Two Anglo-Saxon cemeteries at Chamberlain's Barn, Leighton Buzzard, Bedfordshire', Archaeol J, 120, 161-200

Iverson, $M$ and Näsman, U 1977. 'Smykkefund fra Eketorp-II', Kuml, 85-104 (English summary, 99-101)

Jackson, D A, Harding, D W and Myres, J N L 1969. 'The Iron Age and Anglo-Saxon site at Upton, Northants.', Antiq J, 49, 202-21

Jackson, J E 1854. 'Anglo-Saxon cemetery at Harnham Hill, near Salisbury', Wiltshire Archaeol Natur Hist Mag, 1, 196-208

James, E 1988. The Franks, Oxford

Jensen, S 1991. The Vikings of Ribe, Ribe

Jessup, R F 1946. 'An Anglo-Saxon cemetery at Westbere, Kent', Antiq J, 26, 11-21

Jewitt, L 1884. Half Hours among (with) some English Antiquities, 2nd edn, London Johnson, F 1926. 'The Chapel of St Clement at Brundall, Norfolk', Norfolk Archaeoh, 22, 194-203

Jones, M U 1980. 'Mucking and the early Saxon rural settlement in Essex', in Archaeology of Essex to AD 1500 (D G Buckley), 82-96

Keeney, G S 1939. 'A pagan Anglian cemetery at Howick, Northumberland', Archaeol Aeliana, 4th series, 16, 120-8, pl XvI

Kennett, D 1983. 'The earliest male grave at Kempston', Medieval Archaeol, 16, 88-91

Kirk, J R and Marshall, K 1956. 'A Saxon cemetery near the village of Harwell, Berks.', Oxoniensia, 21, 22-34

Kiss, A 1975. Avar Finds in the Hungarian National Museum, Budapest

Klaveren, $\mathrm{H}$ W van 1988. 'De vroegmiddeleeuwse nederzettung van
Rijnsburg'. Unpublished doctoral dissertation, IPP, University of Amsterdam

Klaveren, H W van 1993. Personal communication to $M$ Hegener, dated 1 June and forwarded to $M$ Guido

Knocker, G M 1957. 'Early burials and an Anglo-Saxon cemetery at Snell's Corner, near Horndean, Hampshire', Proc Hampshire Fld Club Archaeol Soc, 19, 117-70

Knowles, W H and Forster, R H 1909.

'Corstopitum: report on the excavations in 1908', Archaeol Aeliana, 3rd series, 5, 305-424

Koch, U 1974. 'Mediterrane und fränkische Glasperlen des 6 und 7 Jahrhunderts aus Finnland', in Studien zur vor- und frühgeschichtlichen Archäologie (eds G Kossack and G Ulbert), Münchner Beiträge zur Vor- und Frühgeschichte I-II, 495-520, Munich

Koch, U 1977. Das Reihengräberfeld bei Schretzheim, RGK Germanische Denkmäler der Völkerwanderungszeit, Serie A, Band 12-13, Berlin

Koch, U 1997. 'Polychrome Perlen in Württemberg/Nordbaden', in Perlen. Archäologie, Techniken, Analysen. Akten des Internationalen Perlensymposiums in Mannheim vom 11. bis 14. November 1994 (eds U von Freeden and A Wieczorek), 143-8, Taf. 5-12

La Baume, P 1967. Das fränkische Gräberfeld von Junkersdorf bei Köln, RGK Germanische Denkmäler der Völkerwanderungszeit, Serie $B$, Band 3, Berlin

Lawlor, H C 1925. The Monastery of St. Machaoi of Nendrum, Belfast Natur Hist Phil Soc, Belfast

Layard, N F 1907. 'An Anglo-Saxon cemetery in Ipswich', Archaeologia, 60, part 2, 325-52, colour pls XxxI, XxxII and XxxIII

Layard, N F 1909. 'Anglo-Saxon cemetery, Hadleigh Road, Ipswich', Proc Suffolk Inst Archaeol Hist, 13, 1-19, colour pls vi and vir Leahy, K 1993. 'The Anglo-Saxon settlement of Lindsey', in Pre-Viking Lindsey (ed A Vince), 29-44, Lincoln

Leciejewicz, L, Tabaczy'nska, E and Tabaczy'nski, S 1977. Torcello. Scavi 1961-62, Istituto Nazionale d'Archeologia e Storia deli'Arte, Monografie 3, Rome 
Leeds, E T 1909. 'Anglo-Saxon cemetery at Holdenby', J Northamptonshire Natur Hist Soc Fld Club, 15 (1909-11), 90-9

Leeds, E T 1916. 'An Anglo-Saxon cemetery at Wheatley, Oxfordshire', Proc Soc Antiq, 2nd series, 29 (1916-17), 48-65

Leeds, E T 1938. 'An Anglo-Saxon cemetery at Wallingford, Berkshire', Berkshire Archaeol J, 42, 93-101

Leeds, E T and Atkinson, R J C 1944. 'An Anglo-Saxon cemetery at Nassington, Northants.', Antiq J, 24, 100-28

Leeds, E T and Bradford, J S P 1942. 'Archaeological notes (Abingdon, Berks., Saxton Road)', Oxoniensia, 7, 102-4

Leeds, E T and Harden, D B 1936. The Anglo-Saxon Cemetery at Abingdon, Berkshire, Oxford

Leeds, E T and Riley, M 1942. 'Two early Saxon cemeteries at Cassington, Oxon.', Oxoniensia, 7, 61-70

Leeds, E T and Shortt, H de S 1953. An Anglo-Saxon Cemetery at Petersfinger, near Salisbury, Wilts., Salisbury

Lethbridge, T C 1925. 'The Anglo-Saxon cemetery, Burwell, Cambs.', Proc Cambridge Antiq Soc, 27 (1924-5), 72-9

Lethbridge, T C 1926a. 'Anglo-Saxon cemetery at Burwell, Cambs., Part II', Proc Cambridge Antiq Soc, 28 (1925-6), 116-23

Lethbridge, T C 1926b. 'Excavations in the Anglo-Saxon cemetery at Little Wilbraham', Proc Cambridge Antiq Soc, 29 (1926-7), 95-104

Lethbridge, T C 1927. 'An Anglo-Saxon hut on the Car Dyke, at Waterbeach', Antiq J, 7, $141-6$

Lethbridge, T C 1931. Recent Excavations in Anglo-Saxon Cemeteries in Cambridgeshire and Suffolk, Cambridge Antiq Soc, Quarto Publications, New Series 3, Cambridge

Lethbridge, T C 1936. A Cemetery at Shudy Camps, Cambridgeshire, Cambridge Antiq Soc, Quarto Publications, New Series 5, Cambridge

Lethbridge, T C and Carter, H G 1926.

'Excavations in the Anglo-Saxon cemetery at Little Wilbraham', Proc Cambridge Antiq Soc, 29 (1926-7), 95-104

Lindenschmit, L 1880-9. Handbuch der deutschen Altertumskunde, 1 Die Alterthümer der merovingischen Zeit, Brunswick
Lindqvist, S 1926. 'Vendelkulturen Ålder och Ursprung', Kungl. Vitterhets Historie och Antikvitets Akademiens Handlingar, 36, part $1,1-202$

Loeschcke, S 1915. 'Zur angeblich römischen Glashü̈tte auf der Hochmark bei Cordel', Römisch-Germanischen Korrespondenzblatt, 8, 49-57

Loeschcke, S 1925. 'Frühchristliche Werkstätte für Glasschmuck in Trier', Trierer Heimatbuch, Trier

Longworth, I H and Kinnes, I A 1980. Sutton Hoo Excavations, 1966, 1968-70, Brit Mus Occas Pap, 23, London

Lowndes, C 1870. 'Discovery of Anglo-Saxon remains in Stone', Rec Buckinghamshire, 3, 164

Lowndes, C 1878. 'On the discovery of Anglo-Saxon relics in Stone', Rec Buckinghamshire, 5, 23-5

Lowther, A W G 1931. 'The Saxon cemetery at Guildown, Surrey', Surrey Archaeol Collect, 29, 1-50

Lowther, A W G 1933. 'The Saxon cemetery at Guildown, Surrey', Surrey Archaeol Collect, 31, 119-22

Lundström, A 1976. 'Bead making in Scandinavia in the early Middle Ages', Early Medieval Studies, 9, Antikvariskt Arkiv, 61, 3-19

Lundström, A 1981. 'Survey of the glass from Helgö', in Excavations at Helgö, 7 (eds A Lundström and H Clarke), 1-38, Stockholm

Lynn, C J 1988. 'Excavations at 46-48 Scotch Street, Armagh, 1979-80', Ulster J Archaeol, $51,69-84$

Manning, C R 1872a. 'Kenninghall', Norfolk Archaeol, 7, 289-99

Manning, C R 1872b. 'Exhibited a collection of Saxon antiquities ... found at Kenninghall', Norfolk Archaeol, 7, 358

Matthews, C L and Hawkes, S C 1985. 'Early Saxon settlements and burials on Puddlehill, near Dunstable, Bedfordshire', Anglo-Saxon Studies in Archaeology and History, 4 (eds S C Hawkes, J Campbell and D Brown), 59-115, Oxford

Matthys, A 1975. 'Middeleewse Verzamelingen van het Gruuthuse Museum (Brugge): Merovingische Voorwerpen - Middeleeuws Aardewerk', Centre national de recherches archéologiques en Belgique: Répertoires 
archéologiques, Série B: les collections, 10, Brussels

McKinley, J 1994. The Anglo-Saxon Cemetery at Spong Hill, North Elmham: Part VIII The Cremations, E Anglian Archaeol Rep, 69, Gressenhall

Meaney, A 1964. A Gazetteer of Early Anglo-Saxon Burial Sites, London

Meaney, A 1981. Anglo-Saxon Amulets and Curing Stones, BAR Brit Ser, 96, Oxford

Menghin, W 1983. Das Schwert im frühen Mittelalter, Stuttgart

Mertens, J and van Impe, L 1971. 'Het laat-romeins Gravfeld van Oudenburg', Archaeologia Belgica, 135

Miket, R 1974. ' "Undoubted Anglian burials" from Hepple, Northumberland', Archaeol Aeliana, 5th series, 2, 275-80

Miket, R 1980. 'A re-statement of evidence for Bernician Anglo-Saxon burials', in Anglo-Saxon Cemeteries 1979 (P Rahtz et al), 289-305

Miles, D and Palmer, S 1986. Invested in Mother Earth, Oxford

Millard, L, Jarman, S and Hawkes, S C 1969. 'Anglo-Saxon burials near the Lord of the Manor, Ramsgate. New light on the site of Ozengell?', Archaeol Cantiana, 84, 9-30

Mommsen, $\mathrm{T}$ 1895. C. Iulii Solini collectanea rerum memorabilia, Berlin

Money, W 1890. 'Exhibited ... relics found at East Shefford', Proc Soc Antig London, 2nd series, 13 (1889-91), 107-8

Moorey, P R S 1985. Materials and manufacture in Ancient Mesopotamia: the evidence of Archaeology and Art: metals and metal-work, glazed materials and glass, BAR Int Ser, 237, Oxford

Morgan, W E T and Marshall, G 1921. 'Report on the excavation of a long barrow at Llanigon, co. Brecon,' Trans Woolhope Natur Fld Club (1921-3), 30-40

Morris, J 1959. 'Anglo-Saxon Surrey', Surrey Archaeol Coll 56, 132-58

Mortimer, J R 1905. Forty Years' Researches in British and Saxon Burial Mounds of East Yorkshire, including Romano-British Discoveries, London

Müller-Wille, M, Meier, D and Kroll, H 1988. 'The transformation of rural society, economy and landscape during the first millennium AD: archaeological and palaeobotanical contributions from northern
Germany and southern Scandinavia', Geografiska Annaler, 70, 53-68

Musty, J and Stratton, J E D 1964. 'A Saxon cemetery at Winterbourne Gunner, near Salisbury', Wiltshire Archaeol Natur Hist Mag, 59, 86-109

Myres, J N L 1951. 'The Anglo-Saxon pottery of Lincolnshire', Archaeol J, 108, 65-99

Myres, J N L 1977. A Corpus of Anglo-Saxon Pottery of the Pagan Period, Cambridge

Myres, J N L 1986. The English Settlements, Oxford History of England 1b, Oxford Myres, J N L and Green, B 1973. The Anglo-Saxon Cemeteries of Caistor-by-Norwich and Markshall, Norfolk, Rep Res Comm Soc Antiq London, 30, London

Myres, J N L and Southern, W H 1973. The Anglo-Saxon Cremation Cemetery at Sancton, East Yorkshire, Hull Mus Pub, 218, Kingston upon Hull

Näsman, U 1984. 'Vendel period glass from Eketorp II, Oland, Sweden. On glass and trade from the late 6 th to the late 8 th centuries AD', Acta Archaeologica, 55, 55-116

Neville, R C 1852. Saxon Obsequies: illustrated by ornaments and weapons discovered by the Rt. Hon. R. C. Neville in a cemetery near Little Wilbraham, London

Neville, R C 1854. 'Anglo-Saxon cemetery on Linton Heath, Cambridgeshire, excavated January, 1853', Archaeol J, 11, 95-115

Newton, R G 1978. 'Colouring agents used by medieval glassmakers', Glass Technol, 19, no. 3, 59-60

Newton, R G 1980. 'Recent views on ancient glasses', Glass Technol, 21, no. 4, 173-83

Nielsen, J N and Rasmussen, M 1986. Sejlflod en jernalderlandsby ved Limfjorden, Aalborg

O'Riordain, S P 1941-2. 'The excavation of a large earthern ring-fort at Garranes, Co. Cork', Proc Roy Ir Acad, C, 47, 77-150

Owen-Crocker, G R 1986. Dress in Anglo-Saxon England, Manchester

Ozanne, A 1963. 'The Peak dwellers', Medieval Archaeol, 6-7 (1962-3), 15-52

Panhuysen, T, Boyens, P, Dijkman, W, and Hulst, R 1990. 'Opgravingen door het Gemeentelijk Oudheidkundig Bodemonderzoek, Maastricht (GOBM)', in Archeologische Kroniek van Limburg over 1990 (ed H Stoepker), Publ Soc Hist Limbourg, $126,214-30$ 
Payne, G 1880. 'Account of recent discoveries of Roman and Saxon remains near Sittingbourne', Proc Soc Antiq London, 2nd series, 8 (1879-81), 275-6

Payne, G 1881. 'Note on the contents of nine Saxon graves examined by the side of the road through Sittingbourne', Proc Soc Antiq London, 2nd series, 8 (1879-81), 506-8

Peake, $\mathrm{H}$ and Hooton, E A 1915. 'Saxon graveyard at East Shefford, Berks.', J Roy Anthropol Inst, 45, 92-130, pls $1 \mathrm{II}-\mathrm{IX}$

Peers, C and Radford, C A R 1943. 'The Saxon monastery of Whitby', Archaeologia, 89, 27-88

Perkins, D R J and Hawkes, S C 1984. 'The Thanet Gas Pipeline Phase I and II (Monkton parish), 1982', Archaeol Cantiana, 101, 83-114

Petch, D F 1957. 'Archaeological notes for 1956'. Rep Pap Architect Archaeol Soc County of Lincoln, New Series, 7, 1-26

Phillips, C J 1941. Glass: the miracle maker. Its history, technology and applications

Phillips, C W 1934. 'The present state of archaeology in Lincolnshire. Part II', Archaeol Journal, 91, 97-187

Philp, B 1973. Excavations in West Kent 1960-1970, Dover

Pilloy, J 1895. Etudes sur les anciens lieux de sépulture dans l'Aisne, II, Saint-Quentin

Pirling, R 1966. Das römisch-fränkische Gräberfeld von Krefeld-Gellep, RGK Germanische Denkmäler der Völkerwanderungszeit, Serie B, Band 2, Berlin

Pirling, R 1974. Das römisch-fränkische Gräberfeld von Krefeld-Gellep 1960-63, RGK Germanische Denkmäler der Völkerwanderungszeit, Serie B, Band 8, Berlin

Pirling, R 1978. Das römisch-fränkische Gräberfeld von Krefeld-Gellep 1964-65, RGK Germanische Denkmäler der Völkerwanderungszeit, Serie B, Band 10, Berlin

Pirling, R 1989. Das römisch-fränkische Gräberfeld von Krefeld-Gellep 1966-1974, RGK Germanische Denkmäler der Völkerwanderungszeit, Serie B, Band 13, Berlin

Pitt-Rivers, A R 1888. 'Excavations in British barrows and Anglo-Saxon cemetery, Winkelbury Hill', in Excavations in
Cranbourne Chase near Rushmore on the Borders of Dorset and Wilts. 1880-1888, vol 11 Pitt-Rivers, A R 1898. Excavations in Cranbourne Chase near Rushmore on the Borders of Dorset and Wilts., vol Iv

Pocock, $M$ and Wheeler, H 1971. 'Excavations at Escomb Church, County Durham, 1968', J Brit Archaeol Ass, 3rd series, 34, 11-29

Ponting, W 1867. 'A discovery of Saxon interments at Upton Snodsbury', Archaeol J, 24, 351-3

Poulton, R 1989. 'Rescue excavations on an early Saxon cemetery at the former Goblin Works, Ashtead near Leatherhead', Surrey Archaeol Collect, 79, 67-97

Price, J 1975. 'The Glass', in The Bradwell Roman Villa. First Interim Report (M J Green), 12-14, fig 33

Price, J 1988. 'Romano-British glass bangles from east Yorkshire', in Recent Research in Roman Yorkshire: Studies in honour of Mary Kitson Clark (Mrs. Derwas Chitty) (eds J Price, P R Wilson, C S Briggs and S J Hardman), BAR Brit Ser, 193, 339-66, Oxford

Raddatz, K 1958. 'Zu den "magischen" Schwertanhängern des Thorsberger Moorfundes', Offa, 16, 81-4

Radford, C A R 1950. 'Castle Loch, Mochrum', Trans Dumfriesshire Galloway Natur Hist Antiq Soc, 28 (1949-50), 41-63

Rahtz, P et al forthcoming. Cannington Cemetery: Excavations 1962-3 of Prehistoric, Roman, post-Roman and later features at Cannington Park Quarry, near Bridgwater, Somerset, Brittania Monogr Ser

Rahtz, P, Dickinson, T and Watts, L 1980. Anglo-Saxon Cemeteries 1979, BAR Brit Ser, 82, Oxford

Rahtz, P, Woodward, A, Burrow, I, Everton, A, Watts, L, Leach, P, Hirst, S, Fowler, P and Gardner, K 1992. Cadbury Congresbury 1968-73. A late/post-Roman hilltop settlement in Somerset, BAR Brit Ser, 223, Oxford

RCHM 1962. An Inventory of the Historical Monuments in the City of York I: Eburacum Roman York, Roy Comm Hist Monuments Engl Inventories, London

Read, C H 1895. 'On excavations in a cemetery of South Saxons on High Down, Sussex', Archaeologia, 54, 369-82 
Read, C H 1896. 'Further excavations in an Anglo-Saxon cemetery at High Down, Sussex', Archaeologia, 55, 203-14

Reynolds, P K Baillie 1938. Excavations on the Site of the Roman Fort of Kanovium at Caerhun, Caernarvonshire. Collected reports on the excavations for the years 1926-1929 and on the pottery and other objects found, Cardiff

Richards, J D 1987. The Significance of Form and Decoration of Anglo-Saxon Cremation Urns, BAR Brit Ser, 166, Oxford

Richborough vols i to v: see Bushe-Fox, J P, 1926, 1928, 1932 and 1949, and Cunliffe, B, 1968 , above

Rigold, S E and Webster, L E 1970. 'Three Anglo-Saxon disc brooches', Archaeol Cantiana, 85, 1-18

Roberts, W I 1982. Romano-Saxon Pottery, BAR Brit Ser, 106, Oxford

Rogers, N S H 1993. Anglian and Other Finds from 46-54 Fishergate, The Archaeology of York, The Small Finds, 17/9, York

Rolleston, G 1869. 'Researches and excavations carried on in an ancient cemetery at Frilford, near Abingdon, Berks., in the years 1867-1868', Archaeologia, 42, 417-85

Rolleston, G 1880. 'Further researches in an Anglo-Saxon cemetery at Frilford, with remarks on the northern limit of Anglo-Saxon cremation in England', Archaeologia, 45, 405-10

Rooksby, H P 1962. 'Opacifiers in opal glasses', G. E. C. J Sci Technoh 29 (1), 20-6

Rooksby, H P 1964. 'A yellow cubic lead tin oxide opacifier in ancient glasses', Physics and Chemistry of Glasses, 5 (1), 20-5

Rouse, E C 1931. 'An Anglo-Saxon glass bead: found at Chalfont St Peter', Rec Buckinghamshire, 12 (1927-33), 267-8

Sablerolles, Y, Henderson, J and Dijkman, W 1997.'Early medieval glass bead making in Maastricht (Jordenstraat 30), The Netherlands. An archaeological and scientific investigation', in Perlen. Archäologie, Techniken, Analysen. Akten des Internationalen Perlensymposiums in Mannheim vom 11. bis 14. November 1994 (eds U von Freeden and A Wieczorek), 293-313

Sage, W 1984. Das Reihengräberfeld von Altenerding in Oberbayern, 1, Germanische Denkmäler der Völkerwanderungszeit, Serie A, Band 14, Berlin
Sarfatij, H 1977. 'Die Frühgeschichte von Rijnsburg, ein historisch-archäologischer Bericht', Ex Horreo (Cingula 1v), 290-302

Sayre, E V 1963. 'The intentional use of antimony and manganese in ancient glasses', in VIth International Congress on Glass: Advances in Glass Technology (eds F R Matson and G E Rindone), part 2, 263-82, New York

Sayre, E V and Smith, R W 1967. 'Some materials of glass manufacturing in antiquity', in Archaeological Chemistry: a symposium (ed M Levey), Third Symposium on Archaeological Chemistry, Atlantic City (NJ), 279-312, Philadelphia

Sayre, E V and Smith, R W 1974. 'Analytical studies of ancient Egyptian glass', in Recent Advances in Science and Technology of Materials (ed A Bishay), Cairo Solid State Conference, Recent Advances in Science and Technology of Materials, 3, 47-70, New York Scull, C 1985. 'Further evidence from East Anglia for enamelling on early Anglo-Saxon metalwork', Anglo-Saxon Stud Archaeol Hist, 4, 117-24

Scull, C 1992. 'Excavations and survey at Watchfield, Oxfordshire, 1983-92', Archaeol J. 149, 124-281

Selkirk, A 1990. 'Sutton Hoo', Curr Archaeol, $118,353-8$

Sellner, C, Oel, H and Camera, B 1979. 'Untersuchung alter Gläser (Waldglas) auf Zusammenhang von Zusammensetzung, Farbe und Schmelzatmosphare mit der Elektronenspektroskopie und der Elektronenspinresonanz (ESR)', Glastechniche Berichte, 52, 255-64

Sherlock, D R 1977. 'Annular blue glass beads in Anglo-Saxon graves'. Unpublished BA dissertation, Institute of Archaeology, London

Sherlock, S and Welch, M G 1992. An Anglo-Saxon Cemetery at Norton, Cleveland, CBA Res Rep, 82, London

Siegmann, M 1997. 'Die Perlen des frühmittelalterlichen Gräberfeldes von Liebenau, Kr. Nienburg/Weser (Niedersachsen)', in Perlen. Archäologie, Techniken, Analysen. Akten des Internationalen Perlensymposiums in Mannheim vom 11. bis 14. November 1994 (eds U von Freeden and A Wieczorek), $133-42$ 
Siegmund, F 1982. 'Zum Belegungsablauf auf dem fränkischen Gräberfeld von Krefeld-Gellep', Jahrbuch des Römisch-Germanischen Zentralmuseums Mainz, 29, 249-70

Smith, C R 1846a. 'On some Anglo-Saxon remains, discovered at Stowting in the county of Kent', Archaeologia, 31, 398-403

Smith, C R 1846b. 'Visit to Hartlip, Kent', J Brit Archaeol Ass, 1, 314-16

Smith, C R 1852a. 'Anglo-Saxon and Frankish remains', in Collectanea Antiqua, Etchings and Notices of Ancient Remains, illustrative of the Habits, Customs and History of Past Ages, 2 (ed C R Smith), 203-48, pls XLIX-LVIII, London

Smith, C R 1852b. 'Notes on Saxon sepulchral remains found at Fairford, Gloucestershire', Archaeologia, 34, 77-82, pl x

Smith, C R 1852c. 'Roman villa at Hartlip, Kent', in Collectanea Antiqua, Etchings and Notices of Ancient Remains, illustrative of the Habits, Customs and History of Past Ages, 2 (ed C R Smith), 1-24, pls 1-Ix, London

Smith, C R 1854. 'Anglo-Saxon remains discovered at Ozingell, Kent', in Collectanea Antiqua, Etchings and Notices of Ancient Remains, illustrative of the Habits, Customs and History of Past Ages, 3 (ed C R Smith), $1-18$, pls I-vI, London

Smith, C R 1860. 'On Anglo-Saxon remains discovered recently in various places in Kent', Archaeol Cantiana, 3, 35-46, pls 1-v

Smith, C R 1861. 'Anglo-Saxon remains found in Kent and Lincolnshire', in Collectanea Antiqua, Etchings and Notices of Ancient Remains, illustrative of the Habits, Customs and History of Past Ages, 5 (ed C R Smith), 129-40, pls XI-XIII, London

Smith, C R 1868a. 'Anglo-Saxon remains recently discovered in Kent, in Cambridgeshire, and in some other counties', in Collectanea Antiqua, Etchings and Notices of Ancient Remains, illustrative of the Habits, Customs and History of Past Ages, 6 (ed C R Smith), 136-72, pls xxII-xxxIv, London

Smith, C R 1868b. 'Anglo-Saxon remains discovered in Bedfordshire', in Collectanea Antiqua, Etchings and Notices of Ancient Remains, illustrative of the Habits, Customs and History of Past Ages, 6 (ed C R Smith), 201-21, pls xxx1X-xLIV, London

Smith, R A 1901a. 'Anglo-Saxon remains', in The
Victoria History of the County of Norfolk, , 325-51, Westminster

Smith, R A 1901b. "Note (Report) by R A Smith, Near Leatherhead (Fetcham parish)', Proc Soc Antiq London, 2nd series, 18 (1899-1901), 253-8

Smith, R A 1902. 'Anglo-Saxon remains', in The Victoria History of the County of Northampton, I, 223-56, Westminster

Smith, R A 1904. 'Anglo-Saxon remains', in The Victoria History of the County of Bedford, I, 175-90, Westminster

Smith, R A 1906. 'Anglo-Saxon remains', in The Victoria History of the County of Nottingham, I, 193-205, London

Smith, R A 1907. 'Recent and former discoveries at Hawkshill', Surrey Archaeol Collect, 20, 119-28

Smith, R A 1911. 'Anglo-Saxon remains', in The Victoria History of the County of Suffolk, I, 325-55, London

Smith, R A 1912. 'Anglo-Saxon remains', in The Victoria History of the County of York, II, 73-109, London

Smith, R A 1918. 'Prehistoric and Anglo-Saxon remains discovered by Captain L. Moysey at Howletts, near Bridge, Kent', Proc Soc Antiq London, 2nd series, 30 (1917-18), 102-13

Smith R A 1923. A Guide to the Anglo-Saxon and Foreign Teutonic Antiquities in the Department of British and Medieval Antiquities, British Museum, London

Sparey-Green, C 1984. 'Early Anglo-Saxon burials at the "Trumpet Major" Public House, Alington Avenue, Dorchester', Proc Dorset Natur Hist Archaeol Soc, 106, 149-52

Speake, G 1989. A Saxon Bed Burial on Swallowcliffe Down, Engl Heritage Archaeol Rep, 10, London

Stawiarska, T 1974. 'Paciorki szklane $\mathrm{z}$ okresu wplywow rzymskich wyslepujace w kulturze zachodniobaltyjskiej (Glass beads from Roman influence period in the west Baltic culture)', Archeologia Polski, 19, 177-233 (English summary, 229-33)

Stawiarska, T 1984. 'Szk a $z$ okresu wp ywów rzymskich $z$ Pó nocnej polski: studium technologiczne (Glasses from the Roman period excavated in northern Poland: a chemical assays)', Polska Akademia Nauk: Bibliotheca Antiqua, xıx (English summary, 149-56), Wroclaw 
Stevens, J 1894. 'The discovery of a Saxon burial place near Reading', J Brit Archaeol Ass, 50, 150-7

Stone, J F S and Tildesley, M L 1932. 'Saxon interments on Roche Court Down, Winterslow', Wiltshire Archaeol Natur Hist Mag, 45 (1930-2), 568-99

Stuart, J 1865. 'Notices of a group of artificial islands in the Loch of Dowalton, Wigtonshire, and of other artificial islands or "Crannogs" throughout Scotland', Proc Soc Antiq Scotl, 6 (1864-6), 114-78

Swanton, M J 1964. 'An Anglian cemetery at Londesborough in East Yorkshire', Yorkshire Archaeol J, 41 (1963-6), 262-86

Tabacynska, C 1968. 'Remarks on the origin of the Venetian glassmaking centre', Proceedings of the 8th International Congress on Glass: Studies in Glass History and Design, 20-3

Tangl, M 1955. S. Bonifatii et Lullii Epistolae, Monumenta Germaniae Historica: Epistolae Selectae, I, Berlin

Tempelmann-Mạczyńska, M 1985. Die Perlen der römischen Kaiserzeit und der frühen Phase der Völkerwanderungszeit im mitteleuropäischen Barbaricum, RG Forschungen Band 43, Frankfurt-am-Main

Tester, P J 1968. 'An Anglo-Saxon cemetery at Orpington. First Interim Report', Archaeol Cantiana, 83, 125-50

Tester, P J 1969. 'Excavations at Fordcroft, Orpington', Archaeol Cantiana, 84, 39-77

Thomas, G W 1887. 'On excavations in an Anglo-Saxon cemetery at Sleaford in Lincolnshire', Archaeologia, 50, 383-406, pls XxIII-Xxv

Thompson, F H 1956. 'Anglo-Saxon sites in Lincolnshire: unpublished material and recent discoveries', Antiq J, 36, 181-99

Timby, J R 1996. The Anglo-Saxon Cemetery at Empingham II, Rutland, Oxford

Turner, W E S 1956. 'Studies of ancient glass and glass-making processes. Part III. The chronology of glass-making constituents', J Soc Glass Technol, 40, 277T-300T

Turner, W E S and Rooksby, H P 1959. 'A study of the opalising agents in ancient glasses throughout three thousand four hundred years', Glastechnische Berichte, 8, 17-28

Turner, W E S and Rooksby, H P 1961. 'Further historical studies based on $\mathrm{X}$-ray diffraction methods of the reagents employed in making opal and opaque glasses', Jahrbuch des
Römisch-Germanischen Zentralmuseums

Mainz, 8, 1-16

Tweddle, D 1986. Finds from Parliament Street and other sites in the City Centre, The Archaeology of York, The Small Finds 17/4, London

Tyler, S 1988. 'The Anglo-Saxon cemetery at Prittlewell, Essex: an analysis of the grave goods', Essex Archaeol Hist, 19, 91-116

Vallance, W 1848. 'Anglo-Saxon antiquities discovered at Sittingbourne, Kent, from 1825 to 1828', in Collectanea Antiqua, Etchings and Notices of Ancient Remains, illustrative of the Habits, Customs and History of Past Ages, 1 (ed C R Smith), 97-106, pls xxxvi-xxxviII, London

Veeck, W 1931. Die Alamannen in Württemberg, RGK Germanische Denkmäler der Völkerwanderungszeit, Serie A, Band 1, Berlin

Venclová, N 1971. 'Prave ké sklo v Ceskoslovensku', Annonces de la Société Tchécoslovaque d'Archéologie, 13 (4), 55-74

Venclová, N 1981. 'La Tène violet glass in Bohemia', Praehistorica, 8, 217-20

Vyner, B E 1984. 'A Saxon burial from Norton-on-Tees, Cleveland', Medieval Archaeol, 28, 173-5

Walker, T J 1899. 'Notes on two Anglo-Saxon burial-places at Peterborough', J Brit Archaeol Ass, New Series, 5, 343-9

Warhurst, A 1955. 'The Jutish cemetery at Lyminge', Archaeol Cantiana, 69, 1-40

Webster, G 1951. 'An Anglo-Saxon urnfield at South Elkington, Louth, Lincolnshire', Archaeol J, 108, 25-59

Webster, G 1981. 'The excavation of a Romano-British rural establishment at Barnsley Park: Part I c.AD 140-360', Trans Bristol Gloucestershire Archaeol Soc, 99, 21-77

Webster, G and Smith, L 1982. 'Excavation of a Romano-British rural establishment at Barnsley Park, Gloucestershire, 1961-1979: Part II c.AD 360-400+', Trans Bristol Gloucestershire Archaeol Soc, 100, 65-189

Webster, L and Backhouse, J 1991. The Making of England: Anglo-Saxon Art and Culture AD 600-900, London

Wedlake, W J 1982. The Excavation of the Shrine of Apollo at Nettleton, Wiltshire, 1956-71, Rep Res Comm Soc Antiq London, 40, London 
Weinberg, G D 1969. 'Glass manufacture in Hellenistic Rhodes', Archaiologikon Deltion, 24, 143-51

Welander, R D E, Batey, C and Cowie, T G 1987. 'A Viking burial from Kneep, Uig, Isle of Lewis', Proc Soc Antiq Scoth, 117, 149-74

Welch, M G 1975. 'Mitcham Grave 205 and the chronology of applied brooches with floriate cross decoration', Antiq J, 55, 86-95

Welch, M G 1983. Early Anglo-Saxon Sussex, BAR Brit Ser, 112, Oxford

Welch, M G 1991. 'Contacts across the Channel between the fifth and seventh centuries: $a$ review of the archaeological evidence', Studien zur Sachsenforschung, 7, 261-9

Welch, M G 1992. The English Heritage Book of Anglo-Saxon England, London

Werner, A E and Bimson, M 1967. 'Technical report on the glass gaming pieces', in 'A La Tène III burial at Welwyn Garden City' (I Stead), Archaeologia, 101, 16-17

Werner, J 1953. Das alamannische Gräberfeld von Bülach, Monographien zur Ur- und Frühgeschichte der Schweiz, Band 9, Basel

Werner, J 1956. Beiträge zur Archäologie des Attila-Reiches, Abhandlungen der Bayerischen Akademie der Wissenschaften, Philosophisch-Historische Klasse, Neue Folge 38, Munich

West, S E 1985. West Stow: the Anglo-Saxon Village, E Anglian Archaeol Rep, 24, Bury St Edmunds

West, S E 1988. The Anglo-Saxon Cemetery at Westgarth Gardens, Bury St Edmunds, Suffolk: Catalogue, E Anglian Archaeol Rep, 38, Bury St Edmunds

Westell, W P 1931. 'A Romano-British cemetery at Baldock, Herts.', Archaeol J, 88, 247-301

Weyl, W A 1962. Coloured Glasses, Sheffield

Wheeler, H 1979. 'Excavation at Willington, Derbyshire, 1970-1972', Derbyshire Archaeol J, 99, 58-220

Wheeler, H 1985. 'Conclusion: the development of Roman Derby', Derbyshire Archaeol J, 105, 300-4

Wheeler, R E M 1924. Segontium and the Roman occupation of Wales, London

Wheeler, R E M 1943. Maiden Castle, Dorset, Rep Res Comm Soc Antiq London, 12, London

Wheeler, R E M and Wheeler, T V 1936. Verulamium: a Belgic and Two Roman Cities, Rep Res Comm Soc Antiq London, 11, London

Whitelock, D 1979. English Historical Documents I: c.500-1042, 2nd edn, London

Wilson, D M 1956. 'The initial excavation of an Anglo-Saxon cemetery at Melbourn, Cambridgeshire', Proc Cambridge Antiq Soc, 49, 29-41

Wilthew, P 1988. 'Examination and analysis of the glass beads', in An Anglo-Saxon Cemetery at Alton, Hampshire (V I Evison), 52-8

Winkelmann, W 1959. 'Ein münzdatiertes Grab des 7. Jahrhunderts $\mathrm{n}$. Chr. aus Hemer, $\mathrm{Kr}$. Iserlohn', Germania, 37, 303-6

Woodward, P J 1987. 'The excavation of a late Iron Age trading settlement and Romano-British pottery production site at Ower, Dorset', in Romano-British Industries in Purbeck, Dorset Natur Hist Archaeol Soc Monogr Ser, 6

Wylie, W M 1852. The Fairford Graves: a record of researches in an Anglo-Saxon burial-place in Gloucestershire, Oxford

Youngs, S 1989. The Work of Angels: Masterpieces of Celtic Metalwork, 6th-9th centuries $A D$, London

Ypey, J 1962. 'Die Funde aus dem frühmittelalterlichen Gräberfeld Huinerveld bei Putten im Museum Nairac in Barneveld', Berichten van de Rijksdienst voor het Oudheidkundig Bodemonderzoek, 12, 99-152

Zdvarko, M 1971. Wissenschaftliche Mitteilungen der Bósnisch-Herzogowinischen Landesmuseum, Sarajevo

\section{RECENT PUBLICATIONS}

Boyle, A, Jennings, D, Miles, D and Palmer, S 1998. The Anglo-Saxon Cemetery at Butler's Field, Lechlade, Gloucestershire. Vol 1: Prehistoric and Roman Activity and Anglo-Saxon Grave Catalogue, Oxford [see references to Boyle et al forthcoming]

Drinkall, G and Foreman, M 1998. The Anglo-Saxon Cemetery at Castledyke South, Barton-on-Humber, Sheffield [see references to Drinkall and Foreman forthcoming] 